\title{
2010
}

Matthias Schumann Lutz M. Kolbe Michael H. Breitner Arne Frerichs (Hg.)

Multikonferenz Wirtschaftsinformatik 2010
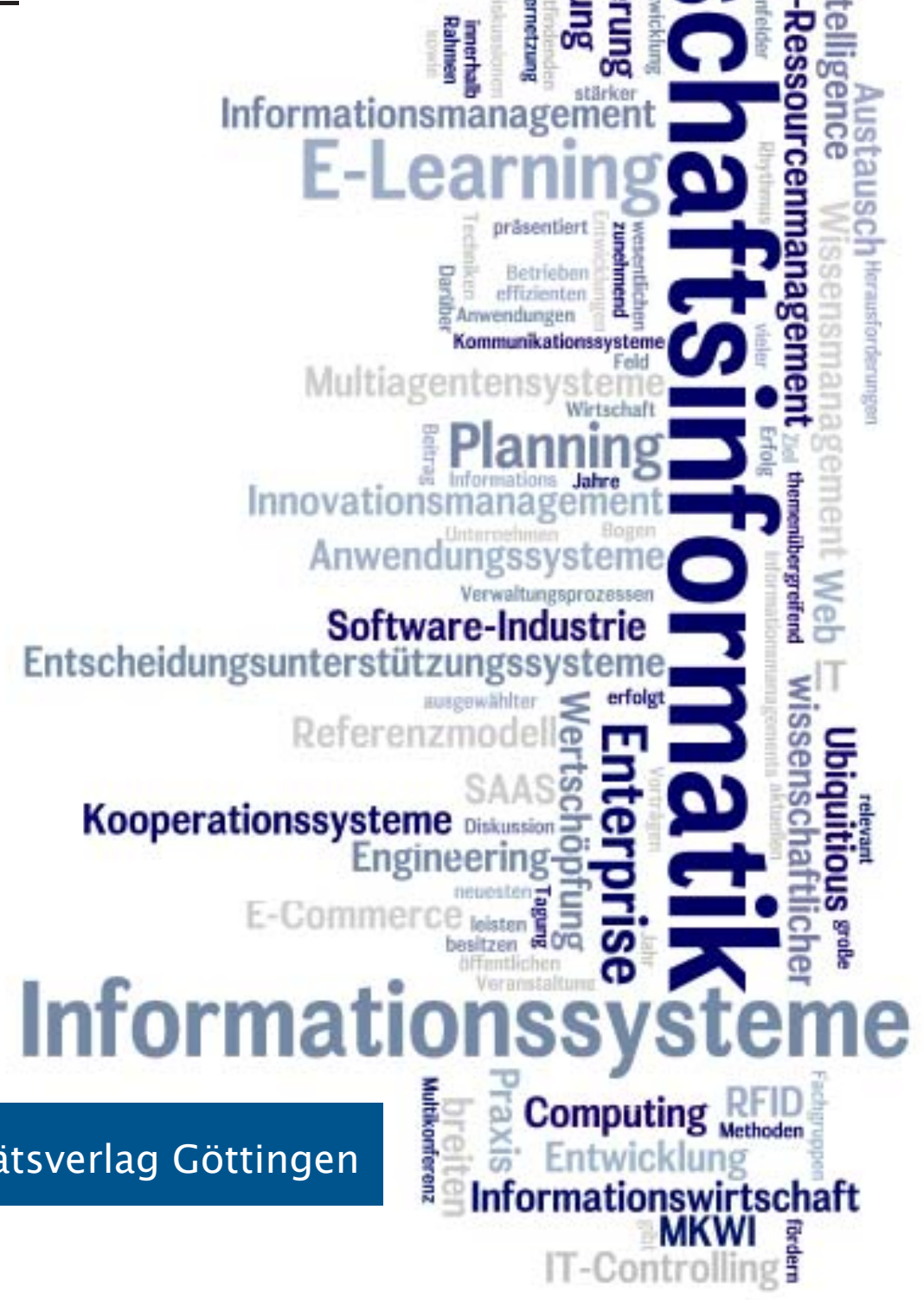

Informationsmanagementent

$[-100$ prasentient

Planning watio Resource
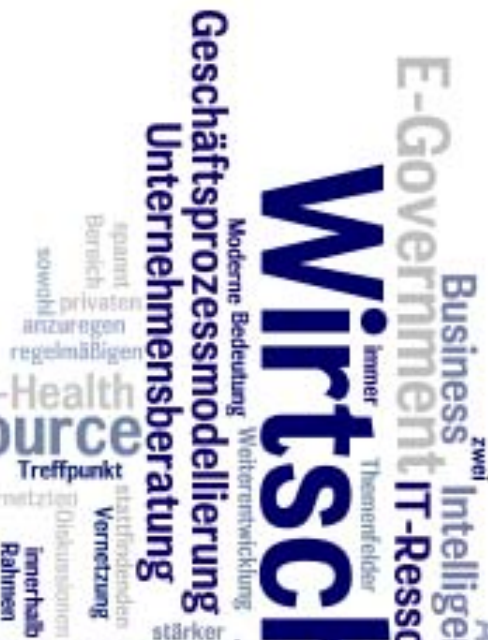

Innovationsmanagement

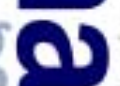

क्ञ

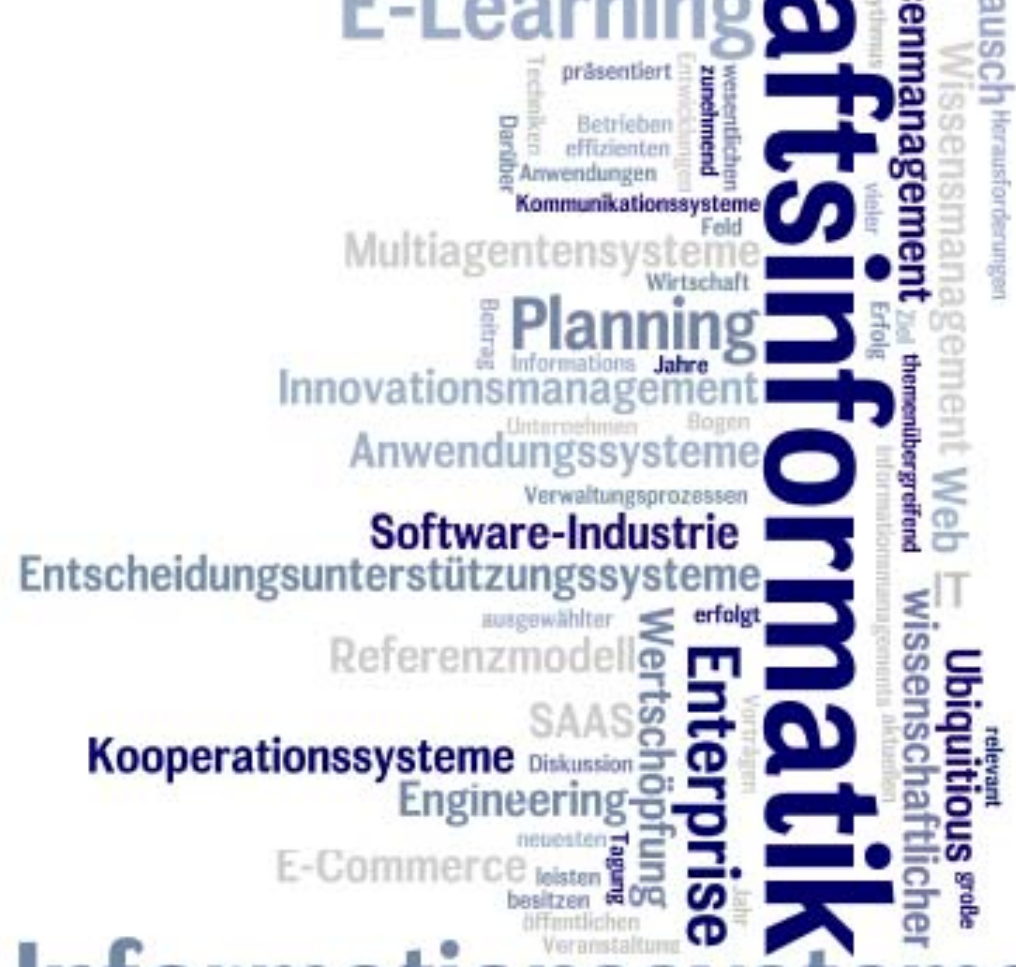
Informationssy steme 

Matthias Schumann, Lutz M. Kolbe, Michael H. Breitner, Arne Frerichs (Hg.) Multikonferenz Wirtschaftsinformatik 2010

This work is licensed under the Creative Commons License 2.0 "by-nd", allowing you to download, distribute and print the document in a few copies for private or educational use, given that the document stays unchanged and the creator is mentioned. You are not allowed to sell copies of the free version.

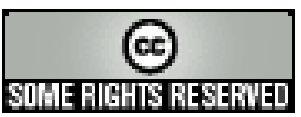


erschienen im Universitätsverlag Göttingen 2010 
Matthias Schumann, Lutz M. Kolbe, Michael H. Breitner, Arne Frerichs (Hg.)

\section{Multikonferenz \\ Wirtschaftsinformatik \\ 2010}

Göttingen, 23.-25. Februar 2010

Kurzfassungen der Beiträge

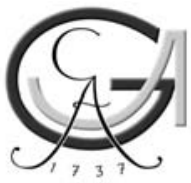

Universitätsverlag Göttingen 2010 


\section{Bibliographische Information der Deutschen Nationalbibliothek}

Die Deutsche Nationalbibliothek verzeichnet diese Publikation in der Deutschen Nationalbibliographie; detaillierte bibliographische Daten sind im Internet über $<$ http://dnb.ddb.de $>$ abrufbar.

\section{Kontakt}

e-mail: organisation@mkwi2010.de

www: http://www.mkwi2010.de

Dieses Buch ist auch als freie Onlineversion über die Homepage des Verlags sowie über den OPAC der Niedersächsischen Staats- und Universitätsbibliothek (http://www.sub.uni-goettingen.de) erreichbar und darf gelesen, heruntergeladen sowie als Privatkopie ausgedruckt werden. Es gelten die Lizenzbestimmungen der Onlineversion. Es ist nicht gestattet, Kopien oder gedruckte Fassungen der freien Onlineversion zu veräußern.

Satz und Layout: Arne Frerichs

Umschlaggestaltung: Margo Bargheer unter Verwendung einer www.wordle.net Grafik

(C) 2010 Universitätsverlag Göttingen

http://univerlag.uni-goettingen.de

ISBN: 978-3-941875-31-9 
Dieser Band enthält Kurzfassungen der Beiträge zur MKWI 2010.

Die Vollbeiträge finden Sie auf der beigelegten CD-ROM.

Darüber hinaus sind die Vollversionen der Beiträge auf dem wissenschaftlichen Publikationenserver (GoeScholar) der Georg-August-Universität Göttingen und über die Webseite des Universitätsverlags unter

http/ / wdbdocsubgndodd/ univelag 2010/ nkwi/

online verfügbar und in die Literaturnachweissysteme eingebunden. 



\section{Vorwort zur Multikonferenz Wirtschaftsinformatik 2010}

Informations- und Kommunikationstechnologien (IKT) sind aus dem betrieblichen Alltag nicht mehr wegzudenken. Sie sind in vielen Unternehmen ein Erfolgsfaktor und sind für Unternehmenskooperationen sowie -vernetzung notwendig. Moderne Kommunikationslösungen bilden das Rückgrat für global agierende Unternehmen. Hinzu kommt, dass auch private Haushalte immer stärker IKT einsetzen und z. B. die IKT-gestützte Vernetzung zwischen Dienstleistungsunternehmen und Konsumenten wächst. Die damit verbundenen wissenschaftlichen Themengebiete werden von der Wirtschaftsinformatik bearbeitet.

Die Multikonferenz Wirtschaftaftsinformatik (MKWI) ist der etablierte zweijährige Treffpunkt der Wirtschaftsinformatik-Community, der vom 23.02. bis 25.02.2010 an der Georg-August-Universität Göttingen stattfand. In 27 verschiedenen Teilkonferenzen sowie Plenumsveranstaltungen hatten die Teilnehmer die Möglichkeit, ihre Fragestellungen in den Fachgruppen zu diskutieren sowie neuste Ergebnisse zwischen Wissenschaft und Praxis auszutauschen.

Neben etablierten und sich stabil weiterentwickelnden Themenfeldern, wie z. B. Enterprise Architecture Management, Multiagentensystemen, Business Intelligence, E-Learning oder Informationssystemen in verschiedenen Wirtschaftszweigen und Branchen, greifen Teilkonferenzen auch neue und aktuelle Anforderungen und Fragestellungen der Praxis auf. Beispielhaft können als Themenfelder das IT-Ressourcenmanagement, Web 2.0-Technologien, die Integration von Produkt und Dienstleistung oder Integriertes Ertrags- und Risikomanagement in automatisierten Geschäftsprozessen genannt werden.

Der vorliegende Tagungsband mit den Kurzbeiträgen sowie die Langfassungen der Beiträge auf der beigefügten $\mathrm{CD}$ oder im Internet unter der Adresse http://webdoc.sub.gwdg.de/univerlag/2010/mkwi/ geben damit einerseits einen guten Überblick über die in der Wirtschaftsinformatik zurzeit behandelten Themenfelder. Andererseits bieten sie tiefen Einblick in den aktuellen Stand der wissenschaftlichen Diskussion der einzelnen Themen.

Insgesamt sind für die wissenschaftlichen Vorträge der Tagung 333 Beiträge eingereicht worden. Von diesen wurden 183 zur Publikation im Tagungsband und zur Präsentation auf der MKWI akzeptiert. Die Teilkonferenzleitungen haben mit Hilfe ihrer Programmkomitees aufwändige, mindestens zweifach anonyme Begutachtungsprozesse durchgeführt. Die nachfolgende Tabelle zeigt die Zahl der Einreichungen für die jeweilige Teilkonferenz sowie die Zahl der für die Tagung akzeptierten Beiträge und daraus abgeleitet die Annahmequote. 
Tabelle 1: Übersicht der Tracks und Teilkonferenzen (ohne MMS 2010)

\begin{tabular}{|c|c|c|c|}
\hline & Eingereicht & Akzeptiert & $\begin{array}{l}\text { Annahme- } \\
\text { quote }\end{array}$ \\
\hline \multicolumn{4}{|l|}{ Management und Methoden } \\
\hline $\begin{array}{l}\text { Conceptual Analysis and Ontological Modelling in Information Sys- } \\
\text { tems }\end{array}$ & 6 & 3 & $50 \%$ \\
\hline Enterprise Architecture Management & 17 & 9 & $52,94 \%$ \\
\hline IT- und Geschäftsstrategien in der Globalisierung & 9 & 2 & $22.22 \%$ \\
\hline IT Performance Management / IT-Controlling & 15 & 9 & $60 \%$ \\
\hline IT-Ressourcenmanagement & 7 & 3 & $42,86 \%$ \\
\hline Lern-Service-Engineering & 7 & $4(+3)^{1}$ & $57,15 \%$ \\
\hline Software-Industrie & 17 & 9 & $52,94 \%$ \\
\hline Unternehmensberatung im IT-Umfeld & 10 & 6 & $60 \%$ \\
\hline \multicolumn{4}{|l|}{ Technologien } \\
\hline Kooperationssysteme & 20 & 9 & $45 \%$ \\
\hline Multiagentensysteme & 9 & 7 & $77,78 \%$ \\
\hline \multicolumn{4}{|l|}{ Anwendungen } \\
\hline Automotive Services 2010 & 12 & 10 & $83,33 \%$ \\
\hline Betriebliches Umwelt- und Nachhaltigkeitsmanagement & 7 & 4 & $57,15 \%$ \\
\hline Business Intelligence & 24 & 9 & $37,50 \%$ \\
\hline E-Commerce und E-Business & 26 & 12 & $46,15 \%$ \\
\hline E-Government & 17 & 7 & $41,18 \%$ \\
\hline E-Learning, M-Learning und Wissensmanagement (ELMW) & 13 & 7 & $53,85 \%$ \\
\hline Enterprise Resource Planning und Transformation von ERP-Systemen & 17 & 9 & $52,94 \%$ \\
\hline ERP-Software in der Lehre & 21 & 9 & $42,86 \%$ \\
\hline IKT-gestützte Unternehmenskommunikation & 11 & 9 & $81,82 \%$ \\
\hline Informationssysteme in der Finanzwirtschaft & 13 & 6 & $46,15 \%$ \\
\hline Informationssysteme in Transport und Verkehr & 9 & 6 & $66,67 \%$ \\
\hline Integration von Produkt und Dienstleistung - Hybride Wertschöpfung & 12 & 9 & $75 \%$ \\
\hline $\begin{array}{l}\text { Integriertes Ertrags-/Risikomanagement in automatisierten Geschäfts- } \\
\text { prozessen }\end{array}$ & 11 & 5 & $45,45 \%$ \\
\hline IT in der Energiewirtschaft & 7 & 3 & $42,85 \%$ \\
\hline $\begin{array}{l}\text { PuK-Workshop: Planung/Scheduling und } \\
\text { Konfigurieren/Entwerfen }\end{array}$ & 10 & 9 & $90 \%$ \\
\hline RFID, NFC, AdHoc Netze und Web 2.0 & 6 & 5 & $83,33 \%$ \\
\hline Summe / Gesamtannahmequote & 333 & 183 & $54,95 \%$ \\
\hline
\end{tabular}

${ }^{1}$ Im Track „Lern-Service-Engineering“ sind sieben Beiträge abgedruckt. Vier dieser Beiträge wurden auf der MKWI 2010 aufgezeichnet und präsentiert. 
Für die Organisation der Teilkonferenzen, ihre inhaltliche Verantwortung und die gute kooperative Zusammenarbeit - die auch in dem Einhalten der knapp gesetzten Fristen zum Ausdruck kommt - dankt das Organisationskomitee der MKWI ganz herzlich den Teilkonferenzleitungen. Ebenso gebührt der Dank den Mitgliedern der Programmkomitees, die teilweise sehr umfangreich in die Begutachtung von Beiträgen eingebunden waren.

Den zahlreichen Mitarbeiterinnen und Mitarbeitern, die bei der Erstellung dieses Bandes und der operativen Organisation der MKWI mitgewirkt haben, gebührt ebenfalls unser Dank.

Göttingen, im Februar 2010

Matthias Schumann Lutz M. Kolbe Michael H. Breitner Arne Frerichs 



\section{Inhaltsverzeichnis}

\section{Management und Methoden}

\section{Conceptual Analysis and Ontological Modelling in Information Systems}

Vorwort

Heinz Dreher, Torsten Reiners, Stefan Voß, Robert Williams.... .5

Automatic Concept Retrieval with Rubrico

Emanuel Reiterer, Heinz Dreher, Christian Gütl

Ontology-based Product Catalogues: An Example Implementation

Philipp Nowakowski, Heiner Stuckenschmidt

Assisting the Discovery and Reuse of Document-based Knowledge using Semantic Metadata

Hinnerk Brügmann

\section{Enterprise Architecture Management}

Vorwort

Frederik Ahlemann

Utility vs. Efforts of Business Process Modeling

Jörg Becker, Burkhard Weiß, Axel Winkelmann.

Prinzipien für Unternehmensarchitekturen

Dirk Stelzer.

From EA management patterns towards a prescriptive theory for designing enterprise-specific EA management functions

Sabine Buckl, Florian Matthes, Christian M. Schweda....

MCP-Portfolio - eine EAM-orientierte Methode zur fachlichen

Konsolidierung von Geschäftsanwendungs-Portfolios

Stephan Gieffers-Ankel, Gerold Riempp 
Entwicklung eines Metamodells zur Modellierung der fachlichen Fähigkeiten der Softwarearchitektur in Krankenhäusern auf Grundlage von Stakeholder Concerns

René Fitterer, Peter Rohner. 25

Integration von automatisch generierten und manuell konstruierten Prozessmodellen als Grundlage für den Aufbau einer Prozessarchitektur Susanne Leist, Wolfgang Lichtenegger . 27

Zeitbezogene Abhängigkeitsanalysen der Unternehmensarchitektur Jan Saat

A Component-Oriented Tool-Approach to Enterprise Architecture Management

Jan Stefan Addicks, H.-Jürgen Appelrath, Philipp Gringel, Matthias Postina, Ulrike Steffen, Christian Zillmann 31

Konzeption eines generischen Geschäftsprozessmodells für Medizinische Versorgungszentren

Corinna Pütz, Daniel Wagner, Otto K. Ferstl, Elmar J. Sinz 33

\section{IT- und Geschäftsstrategien in der Globalisierung}

Vorwort

Michael Amberg, Karl Kurbel, Peter Schumacher 37

Offshore Software Development (OSD)

Martin Wiener 39

Compliance im IT-Outsourcing

Kian Mossanen, Johannes C. Panitz, Michael Amberg.... 41

\section{IT-Performance Management / IT-Controlling}

\section{Vorwort}

Ulrike Baumöl, Walter Brenner, Falk Übernickel 45 
An economic decision model for business software application deployment on hybrid Cloud environments

Jörg Strebel, Alexander Stage

Understanding IT Governance

Andreas Roland Schwertsik, Petra Wolf, Helmut Krcmar

PROSA - Process-Oriented Service Level Agreements for Providing a Single Point of Contract

Rico Knapper, Tobias Conte, Benjamin Blau, Hans Richter.

Ein konzeptionelles Framework zum Verständnis des multidimensionalen Gegenstandes des Wertbeitrags der IT

Stefan Bartsch, Daniel Schlagwein

Unterstützung des Softwarelizenzmanagements durch IT-Frameworks

Jörg Becker, Bernhard Brüse, Jens Pöppelbuß

IT-Projektkostenschätzung - Ein pragmatischer Ansatz

Christian Fischer, Stephan Aier.

Bewertung von Backsourcing-Entscheidungen im Umfeld des

Cloud Computing

Benedikt Martens, Frank Teuteberg.

Providing More than Just Operational Benefits: An Empirical Research

Mauricio Marrone, Lutz M. Kolbe

Web-Controlling

Darius Zumstein, Andreas Meier.

\section{IT-Ressourcenmanagement}

Vorwort

Lutz M. Kolbe, Rüdiger Zarnekow 67

Der Einfluss von ökologischen Produkteigenschaften bei PCs auf die Kaufentscheidung

Timo Schmidtchen, Nils-Holger Schmidt, Lutz M. Kolbe, Jutta Geldermann. 
Software Resource Management Considering the Interrelation between Explicit Cost, Energy Consumption, and Implicit Cost Jörn Altmann, Juthasit Rohitratana. 71

Soziale Dimensionen von Smart Metering am Arbeitsplatz

Matthias Betz, Tobias Schwartz. 73

\section{Lern-Service-Engineering}

\section{Vorwort}

Roland Gabriel, Martin Gersch, Eric Schoop, Peter Weber 77

Towards an Open SOA for Learning Management

Katrina Leyking, Ralf Angeli, Nils Faltin, Fabrizio Giorgini, Gunnar Martin, Wolf Siberski, Volker Zimmermann 79

Semantische Lehr-, Lern- und Qualifizierungs-Services für die Gründungslehre

Malgorzata Mochol, Robert Tolksdorf, Jörg Freiling, Stefanie Demmler 81

Analyse und Optimierung von Lehrdienstleistungen mittels Service Blueprinting

René Wegener, Philipp Menschner, Jan Marco Leimeister 83

Web 2.0 gestützte Blended Learning Szenarien als kritische Masse Systeme

Christian Lehr 85

Neue Möglichkeiten zur technischen Unterstützung bei der Ausbildung an ERP-Systemen

Liane Haak, Dirk Peters, Jan Aalmink 87

E-Learning Aufgaben für Ereignisgesteuerte Prozessketten mit automatischer Korrektur

Markus Siepermann, Chris Börgermann, Richard Lackes 89

Kompetenzorientierte Lehrevaluation

Corinna Fink 


\section{Software-Industrie}

\section{Vorwort}

Peter Buxmann, Manfred Deues, Norbert Gronau, Georg Herzwurm, Thomas Hess, Alfred M. Keseberg, Friedbert Schuh

Softwarebezogene Wertschöpfung im Wertschöpfungsnetzwerk der Informations- und Kommunikationsindustrie

Auf dem Weg zu mehr Arbeitsteilung durch Open-Source?

Christian M. Wolf, Philipp Nguyen Khuong, Alexander Benlian,

Thomas Hess

Industrialisierung von Softwareunternehmen durch Arbeitsteilung:

Einzelfall oder Trend?

Christian M. Wolf, Alexander Benlian, Thomas Hess

Towards a Pricing Method for Software Product Lines

Johannes Müller.

Preisgestaltung für Software-as-a-Service

Sonja Lehmann, Tobias Draisbach, Corina Koll, Peter Buxmann, Heiner Diefenbach

Towards Requirements Engineering for "Software as a Service"

Marina Berkovich, Sebastian Esch, Jan Marco Leimeister, Helmut Krcmar.......... 107

Preisfindung von IT-Produkten durch retrograde Kalkulation

Georg Herzwurm, Benedikt Krams, Wolfram Pietsch

Mobile Application Marketplaces -

an Investigation from Customers' Perspective

Michael Amberg, Inke Thiessen, Michael Lang, Bartosch Belkius

Platform-as-a-Service as an Enabler for Global Software Development and Delivery

Ivo Stankov, Rastsislau Datsenka 


\section{Unternehmensberatung im IT-Umfeld}

Vorwort

Michael H. Breitner, Thomas Deelmann, Peter Loos, Volker Nissen.

VAT Compliance: Einhaltung umsatzsteuerlicher Anforderungen

beim Prozess- und Datenmanagement in ERP-Systemen

Nick Gehrke, Rainer Thams.

Vorläufige und notwendige Formalisierungslücken in der

IT-Beratung

Paul Drews

Entstehung und Handhabung von Vorgehensmodellen zur

Software-Auswahl und Software-Einführung in der IV-Beratung

Volker Nissen

CRM Evaluation

Ina Friedrich, Jon Sprenger, Michael H. Breitner.

Plädoyer für eine operationalisierbare Methode der Prozesserhebung in der Beratung

Silke Balzert, Peter Fettke, Peter Loos

Geschäftsmodelldiversifikation von Unternehmensberatungen

Thomas Deelmann

\section{Technologien}

\section{Kooperationssysteme}

Vorwort

Michael Koch, David Faller, Gerhard Schwabe, Volker Wulf.

Design IT-gestützter kooperativer Bürger-Beratung

Birgit Schenk, Gerhard Schwabe 
Provisioning 2.0: Diffusion kleinteiliger Software in sozialen Netzwerken

Sebastian Draxler, Hendrik Sander, Gunnar Stevens

SocialNetworkingMirror ${ }^{\mathrm{TM}}$

Florian Ott, Alexander Richter, Michael Koch

\section{Open Value Creation}

Daniel Schlagwein, Kai Fischbach, Detlef Schoder, Stefan Bartsch

Qualitätsmessung in der Wikipedia

Marek Opuszko, Thomas Wöhner, Ralf Peters, Johannes Ruhland.

Zur Appropriation sozio-technischer Systeme am Beispiel des

Microblogging Systems Twitter

Nils Jeners, Peter Mambrey

\section{BPM 2.0}

Matthias Kurz

Enterprise 2.0 - Gegenwart und Zukunft

Alexander Richter, Angelika C. Bullinger

Aneignungspraktiken von Software-Entwicklern beim Offshoring

Alexander Boden, Sebastian Draxler, Volker Wulf

\section{Multiagentensysteme}

\section{Vorwort}

Jörg P. Müller, Lars Braubach, Birgit Burmeister, Alexander Pokahr, Ingo Timm

The Notions of Application, Spaces and Agents

Alexander Pokahr, Lars Braubach.

A Generic Strategy Framework for Policy-directed

Autonomous Trading Agents

Steffen Lamparter, Silvio Becher, Michael Pirker 
Ubiquitous Microblogging als dezentrales Entwurfsparadigma für leichtgewichtige Informationssysteme

Martin Böhringer, Peter Gluchowski...

Kriterien für den Einsatz Service-orientierter Architekturen in der Unternehmens-IT

Markus Knöfel, Thomas Barth 165

Einsatz von Agenten für das Selbstmanagement von Automatisierungssystemen

Hisham Mubarak, Peter Göhner. 167

Eine modellbasierte Software-Architektur zur Unterstützung dezentraler Prozesse in der Produktentwicklung Patrick D. Stiefel, Jörg P. Müller. 169

Coping with global Information Systems

Sebastian Hudert, Torsten Eymann 171

\section{Anwendungen}

\section{Automotive Services 2010}

Vorwort

Jan Marco Leimeister, Helmut Krcmar, Klaus Straub 177

Natural speaking (inter)faces for cars

Valentin Nicolescu, Jan Marco Leimeister, Helmut Krcmar

SOA zur Koordination verteilter Geschäftsnetzwerke Jan Löhe, Christine Legner, Stefan Gumbrich

Vehicle-to-Business Communication: The Example of Claims Assistance in Motor Insurance

Oliver Baecker, Markus Miche, Thomas Michael Bohnert 183 
Prototyping komplexer Geschäftsanwendungen im Automobil

Holger Hoffmann, Jan Marco Leimeister, Helmut Krcmar.

Automotive Software and Service Engineering (ASSE) -

an exploration of challenges and trends in an industry experts' view

Andreas Zauner, Holger Hoffmann, Jan Marco Leimeister, Helmut Krcmar

Theoriebasiertes partizipatives Design von Automotive Services

Maximilian Pühler, Michael Schermann, Helmut Krcmar

Akzeptanz von internet-driven After Sales Services in der Automobil-

branche

Carolin Löffler, Lucia Schleifer

Heuristische Ermittlung und Typisierung von Infrastruktur-

anforderungen mobiler Dienste im Fahrzeug

Maja Golcar, Michael Schermann, Maximilian Pühler, Holger Hoffmann,

Helmut Krcmar.

Elektromobilität: Gegenwart oder Zukunft?

Benjamin Wagner vom Berg, Frank Köster, Jorge Marx Gómez

The context sensitive shopping list

Andreas Pirali, Niklas Klein, Sian Lun Lau, Klaus David

\section{Betriebliches Umwelt- und Nachhaltigkeitsmanagement}

Vorwort

Jorge Marx Gómez, Frank Teuteberg, Burkhardt Funk, Peter Niemeyer

A Systematic Review of Sustainable Supply Chain Management

Frank Teuteberg, David Wittstruck.

Konzeption und Entwurf einer Handelsplattform für Sekundärrohstoffe

Stefan Kleine Stegemann, Burkhardt Funk

Sustainable Supply Chain Management in Recyclingnetzwerken der Elektro- und Elektronikindustrie

David Wittstruck, Frank Teuteberg. 
Betriebliche Umweltinformationssysteme (BUIS)

Horst Junker, Jorge Marx Gómez, Corinna V. Lang. 209

\section{Business Intelligence}

Vorwort

Peter Chamoni, Peter Gluchowski, Hans-Georg Kemper, Bodo Rieger

Ansätze für eine differenzierte Business Intelligence Governance

Henning Baars, Tina Müller-Arnold, Hans-Georg Kemper .

Model Driven Architecture für Data Warehouses:

Computer Aided Warehouse Engineering - CAWE

Christian Kurze, Peter Gluchowski.

Business Intelligence zur Unterstützung der Due Diligence

bei Mergers \& Acquisitions

Sandra Seiz, Nicole Jogsch, Bernd Jahnke, Stefan Ruf

Measuring Master Data Quality

Boris Otto, Verena Ebner.

Klassifizierung von Modellierungsfällen in modellgetriebenen

Decision Support Systemen

Christian Schultewolter.

Root causes affecting data quality in CRM

Wolfgang Leußer, Klaus D. Wilde 225

Towards a Service-Oriented Architecture for Operational BI

Josef Schiefer, Andreas Seufert. 227

Towards Knowledge Discovery in the Semantic Web

Thomas Fischer, Johannes Ruhland. 229

Business Intelligence in Collaborative Networks

Heiko Thimm, Karsten Boye Rasmussen 231 


\section{E-Commerce und E-Business}

Vorwort

Christian Huemer, Katja Duckek, Ulrike Lechner,

Katarina Stanoevska-Slabeva

Web 2.0 driven Open Innovation Networks

Michael Reinhardt, René Frieß, Georg Groh, Martin Wiener,

Michael Amberg

Akzeptanzfähigkeit von Tagging-Systemen in benutzergenerierten virtuellen Welten

Danny Pannicke, Michael Hahne, Nicolas Neubauer, Bartek Ochab,

Renate Lieb, Martin Meister, Klaus Obermayer, Werner Rammert,

Rüdiger Zarnekow

Multiple Issue Negotiation Based on Standardized Documents

Christoph Pflügler

Flexible Business Processes - Evaluation of Current Approaches

Thomas Burkhart, Peter Loos.

Normalo? Tüftler? Profi?

Stefan H. Hallerstede, Anne-Katrin Neyer, Angelika C. Bullinger,

Kathrin M. Möslein

e-Success

Olivier Blattmann, Marcel Grüter, Simone von Burg, Thomas Myrach

Elektronische Rechnungen zur Optimierung der Financial Supply

Chain: Status Quo, empirische Ergebnisse und Akzeptanzprobleme

Harald Schömburg, Michael H. Breitner.

Technisches Konzept zur Service Externalisierung virtueller Prototypen in der Modebranche

Christian Zagel, Carolin Löffler

Das Differenzierungspotential eines mehrdimensionalen Kriterienkatalogs zur Bewertung von Onlineshops: Eine empirische Untersuchung in einem Schweizer Portal

Achim Dannecker, Michael Quade 
Dynamically Scalable Architectures for E-Commerce

Georg Lackermair, Susanne Strahringer, Peter Mandl..... 255

Web-Analyse-Dienste als Instrument des Blog-Monitoring Thomas Walter, Andrea Back

Ein konzeptionelles Model auf Basis von Markov-Ketten zur Berechnung und Bewertung von persönlichen Kompetenzen in sozialen Netzwerken

Axel Winkelmann, Till Haselmann 259

\section{E-Government}

Vorwort

Jörg Becker, Helmut Krcmar, Erwin Schwärzer, Maria A. Wimmer. 263

Transferstrategien zur Wiederverwendung und Verbreitung von

E-Government-Lösungen

Moreen Stein

Bürgernahes Regieren

Daniel Veit, Nils Parasie, Florian Schoppé

Understanding the Advisory Needs of Citizens

Birgit Schenk, Gerhard Schwabe

Skizzierung von Front- und Back-Office-Architekturprinzipien des E-Governments

Konrad Walser, Reinhard Riedl 271

Web 2.0 als Potential für die Verwaltungsmodernisierung am Beispiel der Fortschreibung einer Verwaltungsvorschrift mittels Wiki

Tanja Röchert-Voigt, Norbert Gronau.

\section{E-Partizipation}

Daniel Veit, Manuel Trenz.

Elektronische Verfahrensabwicklung von G2B-eServices: Vorgehensmodell und Anwendungsfall zur Umsetzung der Dienstleistungsrichtlinie

Frank Hogrebe, Beate van Kempen, Markus Nüttgens 


\section{E-Learning, M-Learning und Wissensmanagement (ELMW)}

Vorwort

Michael H. Breitner, Christine Voigtländer.

eLectures: Vom Nebenprodukt zum strukturierten Blended Learning Arrangement

Helena Bukvova, Sylvie Meyer, Eric Schoop.

Ein generisches Framework zur Erstellung von argumentationsunterstützenden Systemen

Frank Loll, Niels Pinkwart, Oliver Scheuer, Bruce M. McLaren.

Gestaltung virtueller kollaborativer Lernprozesse in internationalen

Settings

Helena Bukvova, Christian Lehr, Claudia Lieske, Peter Weber, Eric Schoop

Rapid Authoring meets Web 2.0

Stefan Hausmann, Kai-Uwe Götzelt

Entwicklung und Anwendung eines Internetwerkzeugs zur

Generierung von Forschungsportalen

Jörg Becker, Ralf Knackstedt, Lukasz Lis, Armin Stein

'What are you doing' im Elfenbeinturm? - Microblogging im universitären Einsatz

Lutz Gerlach, Stefan Hauptmann, Martin Böhringer.

Verbesserungspotentiale von Groupwareplattformen für Lehrende

Stefan Bitzer, Marco Klein, Bernd Pape, Matthias Schumann

\section{Enterprise Resource Planning und Transformation von ERP-Systemen}

\section{Vorwort}

Sebastian Asendorf, Norbert Gronau, Karl Kurbel,

Peter Loos, Petra Schubert 
An Approach for Real-Time Control of Enterprise Processes in

Manufacturing using a Rule-Based System

Manfred Grauer, Sachin Karadgi, Daniel Metz, Walter Schäfer.

Towards a Model-based Service Integration Framework for

Extensible Enterprise Systems

Matthias Allgaier, Markus Heller, Martin Weidner.

Ein Maßnahmenkatalog für die Datensicherheit in der ERP

Anwendungsentwicklung am Beispiel von SAP ERP

Holger Wittges, Sonja Hecht, Helmut Krcmar 305

Erwartungen und Nutzen beim Einsatz von Business Software

Petra Schubert, Susan P. Williams 307

Flexibilität in ERP-Standardsoftware

Norbert Frick, Petra Schubert.

Collaborative Project Management (CPM)

Heiko Bartlog, Jochen Boy

Value Chain Cockpit auf Basis betrieblicher Anwendungssysteme

Andrej Werner, Christian Hrach, Karen Heyden, Rainer Alt,

Bogdan Franczyk

ERP-Einführung: Wirkung von kritischen Erfolgsfaktoren der

Projektphase auf den Projekterfolg

Alexander Dreiling

Dynamische Gruppenarbeit

Thilo Münstermann, Jens Völzke, Paul Flachskampf

\section{ERP-Software in der Lehre}

Vorwort

Helmut Krcmar, Norbert Gronau

ERP-Systeme in der Lehre - ein vergleichendes, hochschulübergreifendes Seminar mit mittelgroßen ERP-Systemen Axel Winkelmann, Christian Leyh, Norbert Frick 
Eine integrierte betriebswirtschaftliche Fallstudie mit SAP ERP und deren Einbindung in das SAP LEGO Labor

Danny Szendrei, Tobias Teich, Katja Unger, Jörg Militzer.

ERP-Ausbildung in der Wirtschaftsinformatik an der Universität Stuttgart

Georg Herzwurm, Lars Oliver Mautsch, Sixten Schockert.

Teaching the Transformation from Classical On-Premise towards

On-Demand Enterprise Systems

Oliver Gaß, Alexander Mädche

Didaktisches Kernkonzept des SAP ERP-Praktikums in der

Controlling-Lehre der Universität des Saarlandes

Alexander Baumeister, Claudia Harrer, Ulrike Sträßer.

Integration von SAP Customer Relationship Management in der Lehre

Bernd Heesen, Marcus Hilmer, Timo Simon

Praxisnahe und effiziente ERP-Ausbildung am Beispiel der

TU Ilmenau

Sebastian Büsch, Volker Nissen, Lutz Schmidt.

Planspiele in der ERP-Lehre

Christos Konstantinidis, Harald Kienegger, Holger Wittges,

Helmut Krcmar.

Curriculum Development for Teaching Business

Information Systems to Business Administration Students -

An Example from a University of Applied Sciences

Roland Schwald 339

\section{IKT-gestützte Unternehmenskommunikation}

Vorwort

Jorge Marx Gómez, Ralf Isenmann.

Erfolgsdimensionen von Social Software: Eine fallstudienbasierte Untersuchung

Melanie Steinhüser, Philip Räth 345 
Status Quo der internetbasierten Nachhaltigkeitsberichterstattung

Michael Freundlieb, Frank Teuteberg.. 347

„Sags uns“

Gerald Fricke, Yvonne Gaedke, Susanne Robra-Bissantz

Ansatz zur Entwicklung von Referenzprozessen im Bereich des

Enterprise Content Managements unter dem Aspekt der Wandlungs-

fähigkeit

Sandy Eggert 351

Einsatz und Nutzung von Social Software in kooperativen

Forschungsprojekten

Christoph Lattemann, Miriam Kallischnigg, Stefan Stieglitz

Optimierungsansätze für E-Mail-Kampagnen in der

Kundenkommunikation

Robert Hauke, Klaus D. Wilde.

Fernsehsender im Web 2.0

Kirsten Mrkwicka, Matthias Kießling, Lutz M. Kolbe

Einsatz von Recommender-Systemen zum Management von

Unternehmensportalen

Helmuth Elsner, Jan Krämer

Ideenmanagement im Rahmen von organisatorischem Wandel

Daniel Klein, Ulrike Lechner 361

\section{Informationssysteme in der Finanzwirtschaft}

Vorwort

Susanne Leist, Matthias Goeken, Gregor Zellner. 365

Ansatz zur integrierten Gestaltung und Bewertung am Beispiel des Financial Sourcing

Ken Mansfeldt, Rainer Alt, Thomas Puschmann 367

Improving the Support for Investment Decisions in Financial Markets Using the System Dynamics Methodology Björn-Christopher Witte, Christian Suchan. 
A Software Framework for a News Event Driven Simulation of Algorithmic Trading Strategies

Andreas Storkenmaier, Marius Müller, Christof Weinhardt. 371

Technology-Based Industrialization of Claims Management in Motor Insurance

Oliver Baecker, Albrecht Bereuter 373

Opacity and Exclusivity in Electronic Securities Trading:

The Case of Dark Pools

Bartholomäus Ende, Jan Muntermann

Override von Kreditkunden-Ratings

Christian Wunderlin

\section{Informationssysteme in Transport und Verkehr}

Vorwort

Jens Gottlieb, Dirk Chr. Mattfeld, Leena Suhl

Kapazitätssteuerung in der Transportlogistik

Jörn Schönberger, Herbert Kopfer

Bereitstellung von tageszeitabhängigen Fahrzeiten für die

Tourenplanung in Ballungsräumen

Jan Fabian Ehmke, Dirk Christian Mattfeld

Decision Support for Location Routing with Relocation Aspects

Andreas Stenger, Michael Schneider, Michael Schwind

Collaborative Optimization of Last Mile Networks for Courier, Express and Parcel Delivery Services

Michael Schwind, Marcel Kunkel

Ist besser wirklich besser?

Jürgen Müller, Richard Lohwasser, Andreas Lackner, Alexander Zeier, Hasso Plattner

Informationssysteme im Bike-Sharing: Ein System Dynamics Ansatz Patrick Vogel, Dirk Christian Mattfeld 


\section{Integration von Produkt und Dienstleistung - Hybride Wertschöpfung}

Vorwort

Tilo Böhmann, Jan Marco Leimeister. 397

Hybrid Value Creation

Vivek K. Velamuri, Anne-Katrin Neyer, Kathrin M. Möslein

Ein Bezugsrahmen für Requirements Engineering hybrider Produkte

Marina Berkovich, Jan Marco Leimeister, Helmut Krcmar 401

Vorgehensmodelle des Product-Service Systems Engineering

Marc Gräßle, Oliver Thomas, Michael Fellmann, Julian Krumeich

Vorgehensmodelle für die Entwicklung hybrider Produkte eine Vergleichsanalyse

Philipp Langer, Felix Köbler Marina Berkovich, Felix Weyde,

Jan Marco Leimeister, Helmut Krcmar

Produktivitätsmanagement hybrider Leistungsbündel

Jörg Becker, Daniel Beverungen, Nadine Blinn, Michael Fellmann,

Ralf Knackstedt, Markus Nüttgens, Oliver Thomas 407

Grundlagen für hybride Leistungsbündel für den europäischen

Werkzeugbau

Günther Schuh, Wolfgang Boos, Magdalena Völker

Reducing the Complexity of Services - A new

Approach to Service e-Procurement

Maik Herfurth, Peter Weiß, Christian Rudolf, Robert Kern.

Managing Hybrid IT-Products

Philipp Langer, Thomas Winkler, Tilo Böhmann, Helmut Krcmar.

Vergleich von Reifegradmodellen für die hybride Wertschöpfung und Entwicklungsperspektiven

Jörg Becker, Ralf Knackstedt, Jens Pöppelbuß 


\section{Integriertes Ertrags-/Risikomanagement in auto- matisierten Geschäftsprozessen}

Vorwort

Günter Müller, Dennis Kundisch, Stefan Sackmann, Frank Romeike 419

Integrating Compliance into Business Processes

David Schumm, Frank Leymann, Zhilei Ma, Thorsten Scheibler, Steve Strauch

Eine quantitative Perspektive auf die Priorität von Risikofaktoren in IT-Projekten

Stefan Hörmann, Michael Schermann, Helmut Krcmar

Attacks on the Internet of Services

André Miede, Tobias Ackermann, Nicolas Repp, Daniel F. Abawi,

Ralf Steinmetz, Peter Buxmann 425

Managementsysteme sind Balance-Systeme - Diskussion relevanter

Kennzahlen eines ISMS gemäß ISO/IEC 27001:2005

Wolfgang Böhmer

Konzeption eines Models zur Bestimmung des optimalen

Investitionsbeitrags in IT-Sicherheits- bzw. IT-Notfallmaßnahmen unter Berücksichtigung Compliance-bedingter Anforderungen

Stefan Alexander Kronschnabl

\section{IT in der Energiewirtschaft}

Vorwort

Jürgen Appelrath, Carsten Felden

Economic Robustness of Scheduling Algorithms for Distributed Storage Systems

Klaus-Henning Ahlert, Clemens van Dinther....

Business-Intelligence-gestützte Vorauskalkulation in der

Primärgewinnungsindustrie

Johannes Buder, Carsten Felden, Joel Sieber 437 
IT-gestützte Marktforschung

Iris Kaiser

\section{PuK-Workshop: \\ Planung/Scheduling und Konfigurieren/Entwerfen}

Vorwort

Jürgen Sauer, Stefan Edelkamp, Bernd Schattenberg. 443

Construction of Configuration Models

Lothar Hotz....

Personalized Product Configuration

Alexander Felfernig, Monika Mandl, Juha Tiihonen, Monika Schubert

Parameteroptimierung von Materialflusssimulationen durch

Partikelschwarmalgorithmen

Christoph Laroque, Barthold Urban, Markus Eberling

Optimizing the Berth Allocation Problem using a Genetic

Algorithm and Particle Swarm Optimization

Ole Björn Brodersen, Leif Meier, Matthias Schumann

Finding the Needle in the Haystack with Heuristically Guided

Swarm Tree Search

Stefan Edelkamp, Peter Kissmann, Damian Sulewski,

Hartmut Messerschmidt

Verbal Plan Explanations for Hybrid Planning

Julien Bidot, Susanne Biundo, Tobias Heinroth, Wolfgang Minker,

Florian Nothdurft, Bernd Schattenberg

Workforce Management an einem Praxisbeispiel aus der Produktion

Maik Günther.

Swarm Intelligence in der dynamischen Tourenplanung

Menno Heeren.

An Agent-based Approach for Dynamic Combination and

Adaptation of Metaheuristics

Tjorben Bogon, Andreas D. Lattner, Yann Lorion, Ingo J. Timm. 


\section{RFID, NFC, Ad Hoc Netze und Web 2.0: \\ Logistik, mobile Informationsverarbeitung und neue Wertschöpfungsnetzwerke}

Vorwort

Michael H. Breitner, Günter Wohlers 465

Drahtlose Kommunikation in der Flugzeugkabine für effiziente Arbeitsabläufe und Passagierdienstleistungen Ralf God, Hartmut Hintze.

Datengeneratoren für die Unterstützung von Design-ScienceProjekten im Umfeld RFID-basierter Analyseanwendungen

Xuanpu Sun, Henning Baars

Einsatzmöglichkeiten der RFID-Technologie im Technischen Außendienst

Christian Scharfe, Daniel Fischer, Bernd Markscheffel, Dirk Stelzer, Ramón Somoza..

Prozessorientierte RFID-Einsatzplanung

Günther Schuh, Tobias Rhensius .... 473

Modellierung eines Anwendungssystems zur Behälterlokalisation und Behälterreservierung auf Basis des Architekturstils REST Thomas Barton, Harriet Bach 

Management und Methoden 

Conceptual Analysis and Ontological Modelling in Information Systems

Anwendung der Konzeptanalyse und ontologische Modellierung in der Wirtschaftsinformatik 



\title{
Preface to the Track
}

\section{Conceptual Analysis and Ontological Modelling in Information Systems}

\section{Anwendung der Konzeptanalyse und ontologische Model- lierung in der Wirtschaftsinformatik}

\author{
Heinz Dreher', Torsten Reiners'2, Stefan Voß², Robert Williams ${ }^{3}$ \\ ${ }^{1}$ Curtin Business School, Curtin University, Perth \\ 2Institute of Information Systems (IWI), University of Hamburg \\ ${ }^{3}$ Blue Wren Software Pty. Ltd., Perth
}

With Web 2.0, the creation of digital content using all types of media accelerated as a result of increasing Internet accessibility, social communities who share and collect information as well as improved applications allowing everyone to become a producer. The current usage of tags and asynchronous linking provides a means to reveal some basic structural elements within the vast 'repositories of knowledge' and generally in the seemingly endless proliferation of data but ignores the needs of (basic) users with respect to differentiate expert knowledge from 'data' (opinion, unverified reports, and other material disguised amateurish gossip and musings. Users wish to retrieve precise results on questions asked in imprecise natural languages, and to overcome any cultural or language barrier, or to extract additional information such as context, temporal relationships, concept interconnectedness, and alternative media documents representing like content.

The next generation of the Web is called 3.0 and is supposed to be 'intelligent' in that it emphasizes machine-facilitated understanding of information by incorporating, for example, semantic networks, machine learning, autonomous agents, artificial intelligence and distributed databases. Of especial importance is the 'understanding' of content by applications, requiring agents to operate autonomously, and the provision of service oriented architectures where missing components are automatically searched for and integrated and provided via a natural interface to the user.

One popular approach for progressing issues relating to concept 'understanding' being used in recent times is ontology based. Ontologies are an explicit, formal specification of common conceptual classification schemes and generally describe a hierarchy of concepts using a subsumption relation. From a theoretic understanding, ontologies also describe semantic relations between concepts allowing 
the distinguishing of different objects with the same identifier (domain knowledge). Concepts represent the meaning behind 'objects', i.e. to understand and express similarity or differences among objects, provide a classification, and identify objects as belonging. Conceptual analysis is the examination and analysis of texts to identify and mark concepts for 'understanding', detecting the context, or scenario, and other forms of sophisticated information-rich structures.

This track reviews state-of-the-art theories and applications of conceptual analysis in the context of Information Science. The importance of understanding the content rather than manipulating and managing the documents is increasing as the amount of data is no longer manageable by humans, especially considering influences of language, culture and required expert knowledge. The three papers presented in this track explore the semantic aspects of text based documents.

Assisting the Discovery and Reuse of Document-based Knowledge using Semantic Metadata by Hinnerk Brïgmann illustrates the basis for an approach to assist in the enterprise wide lifecycle management of electronic documents by utilizing the context of document access by knowledge workers. From this context data one can deduct semantic relations among documents and business-domain specific entities that can be combined into a semantic network. Querying the resulting network allows for the discovery and reuse of unstructured documents.

Electronic product catalogues are the basis for offering and selling products in online market places. These catalogues have to provide a semantically precise description of product features to allow for effective matchmaking of products and customer requests. At the same time, the description has to follow a common terminology allowing the integration with the catalogues of other providers on the same market place. Pbilipp Nowakowski and Heiner Stuckenscbmidt in their paper Ontology-based Product Catalogues: An Example Implementation argue for the use of ontologies as a basis for defining product catalogues. More precisely, they suggest using an ontological model as a basis for encoding product information as formal class descriptions in the web ontology language OWL.

Emanuel Reiterer, Heinz Dreher, and Christian Gütl present Automatic Concept Retrieval with Rubrico, where the software prototype Rubrico was developed for automated essay grading. For this task concepts are retrieved, which are used to get a summary of the learning subject for the purpose of matching those concepts against a specific essay for use in marking. Rubrico works with electronic textual input in multiple formats such as text, html or pdf. It extracts concepts from these files using several algorithms and provides the option to alter the retrieved concepts (stored as an ontology) through human support.

\section{Acknowledgement}

We greatly appreciate the support that we received from the following members of the program committee of our track: Christian Gütl (Graz, Austria), Felix Mödritscher (Vienna, Austria), Alan Rea (Kalamazoo/Michigan, USA). 


\title{
Automatic Concept Retrieval with Rubrico
}

\author{
Emanuel Reiterer ${ }^{1}$, Heinz Dreher ${ }^{2}$, Christian Gütl1,2 \\ ${ }^{1}$ Graz Technical University of Technology, Graz, \\ ${ }^{2}$ Curtin University, Perth
}

With the vast repositories of knowledge available via computers nowadays there is an expectation that the relevant portion of this knowledge is accessed and used when creating contributions to the world's store of knowledge. Search engines have been popular and useful for some decades but there remains the key problem of narrowing down the search to find precisely what there in a given document of document set. While the Information Retrieval community has developed solutions to increase relevance and precision, the work is based largely on key-word searching (Zhu and Dreher 2009). Clearly, manual search and find options are useful only with very limited data sets and we need computers to help us achieve that final set for manual inspection and processing. Recent developments, especially in ontologies (W3C 2004) have made possible the treatment of text-based datasets in 'intelligent' ways. Our work falls into this category.

Here we report of the outcome of our effort to search for, or find, as we prefer to think of it, concepts rather than key-words. The software embodying the conceptual searching ideas being reported in this article has been named Rubrico and is intended to use for lecturers who can use this program for marking purposes or for getting a better impression of the concepts that are lectured in a specific topic. But it also can be used for any other task where ontologies should be retrieved out of an electronic text or ontologies have to be improved.

This article starts with background information regarding concept analysis, ontologies and available software toolkits. Rubrico our prototype software package to find concepts reposing within a document set, and assist users in structuring them into a rubric or (possibly) hierarchical framework, is explained. There are many applications for concept search and find and one strong motivator in our research group named SATM4BE ${ }^{1}$ was our work in automated essay grading, and plagiarism detection. For this task concepts are retrieved from a reference or model and compared with the retrieved concepts in target documents. This permits forming an idea of the taught subject via its concepts and the possibility to match those concepts against a specific essay for checking purposes.

We describe a manual process of retrieving concepts and then present our automatic approach. An explanation of the algorithms and toolkits that are used

${ }^{1}$ SATM4BE - Semantic Analysis \& Text Mining for Business \& Education www.eaglesemantics.com 
nowadays precedes the real implementation of the software Rubrico, which retrieves an ontology out of a specific corpus, stores and visualizes that ontology, and provides a graphical interface for the human interaction functionality. After that the deviation of the manually and automatically retrieved concepts will be mentioned. Finally we will give a conclusion of our work and state further developments in this field.

\section{References}

W3C (2004) OWL Web Ontology Language Overview.

http://www.w3.org/TR/owl-features/. Last visit 2009-09-13.

Zhu D, Dreher H (2009) Discovering Semantic Aspects of Socially Constructed Knowledge Hierarchy to Boost the Relevance of Web Searching. JUCS

Volume 15 Issue 8. 


\title{
Ontology-based Product Catalogues: An Example Implementation
}

\author{
Philipp Nowakowski, Heiner Stuckenschmidt
}

KR\&KM Research Group, University of Mannheim

One of the basic problems connected to an efficient use of electronic marketplaces is the problem of matchmaking between offers for products or services and requests by potential customers (Veit 2003). Determining which offers are 'good enough' often does not only depend on offer and request itself, but also on the preferences of the customer and the intended use of the product or service. This means that matching has to understand the nature of a product to be able to decide if it is similar to what the customer wants and it has to be customizable towards the specific needs of a customer. Another challenge for successful matchmaking in electronic marketplaces is the need to integrate heterogeneous product and service descriptions from different product catalogues to enable the customer to choose from different providers, as the representations used by different participants for their internal purposes are often geared towards the specific needs of the company and show little standardization across different enterprises.

In previous work, we have argued for the need of providing flexible matchmaking services for finding complex products and services in product catalogues and proposed ontology-based representation and matchmaking as a basis for intelligent access to product information (Stuckenschmidt and Kolb 2008). In this paper, we present a demonstration system implementing the idea of ontology-based representation and matchmaking in product catalogues. In particular, we have implemented the prototype of a web shop that uses the eClassOWL ontology (Hepp 2006) as a basis for representing the product catalogue of Pepperl\&Fuchs, one of the worldwide leading providers of components for process automation.

By using eClassOWL as background ontology, we take advantage of having a product taxonomy present, which we can use for fine tuning our matching tasks. Our system is aware of the taxonomic position of product items, and uses this knowledge during the selection for suitable matching candidates. Moreover we have extended the matchmaking algorithm with several new strategies which expand the subset of relaxed concepts and help finding the most relevant matches as early as possible. For example we have defined strategies that exploit the background ontology as well as strategies that take into account user preferences.

From our experience, we conclude that ontology-based product catalogues are feasible from a technical point of view. Development in the field of semantic web technologies has led to stable ontology language and mature query and reasoning tools. Further, models like eClassOWL are emerging that can provide the termino- 
logical basis for catalogues. The biggest obstacle so far seems to be the link between proprietary catalogue solutions and standard classifications like eCl@ss. Standardization efforts are progressing, but legacy data is still a problem. On the other hand, companies that want to compete on the major markets will have to adhere to the leading standards anyways, making the most critical step towards ontology-based product catalogues.

\section{References}

Hepp M (2006) Products and Services Ontologies: A Methodology for Deriving OWL Ontologies from Industrial Categorization Standards. International Journal on Semantic Web and Information Systems (IJSWIS) 2(1):72-99.

Stuckenschmidt H, Kolb M (2008) Partial Matchmaking for Complex Product and Service Descriptions. Proceedings of Multikonferenz Wirtschaftsinformatik (MKWI 2008), München.

Veit D (2003) Matchmaking in Electronic Markets, Jgg. 2882 of Lecture Notes in Computer, Science. Springer Verlag, Berlin. 


\title{
Assisting the Discovery and Reuse of Document- based Knowledge using Semantic Metadata
}

\author{
Hinnerk Brügmann \\ Department of Information Systems, University of Erlangen-Nurenberg
}

Due to the high importance computer-mediated communication and IT systems have in the execution of today's business processes, enterprises are producing a rapidly growing amount of information as by-product of their business activities.

Only $20 \%$ - $40 \%$ of this information is stored in a structured, semantically described form in electronic databases or structured business applications (e.g. SAP $\mathrm{R} / 3$ ). The other $60 \%-80 \%$ are contained in the unstructured form of electronic documents (e.g. Microsoft Office files, emails, images, multimedia files, or web based content). The unstructured nature of the bulk of existing as well as newly generated information is cause for a whole host of inefficiencies and problems. Especially in multi-user enterprise environments with numerous competing repositories, document management systems, shared network drives and local file systems for each distinctive user knowledge workers spend a substantial part of their working hours looking for the correct and most up-to-date information needed in workflow steps or tasks (Guther 2007, p. 25).

To assist in the enterprise wide lifecycle management and discovery of electronic documents we propose to integrate the different types of available document-related metadata to infer semantic relations among those unstructured documents and correlate them to known business entities (employees, partners, projects, products or processes) in a querieable way. The used metadata is in particular extracted from the multi-informational aspects of unstructured documents (static metadata) and the context in which knowledge workers access and modify those documents (contextual metadata) using semantic network techniques (Barreau 1995, p. 329ff; Shen et al. 2005, p. 2f). To establish a semantic link to the business domain external entity information is initially seeded to act as relationship-anchors.

To aggregate and analyze the assumptions made on semantic relations we implemented a distributed prototypical application "ConSense". The business domain in this scenario is the ecosystem of knowledge workers in the enterprise accessing and modifying electronic documents as well as collaborating in teams and exchanging email messages. Client-side plugins on the knowledge workers workstations extract static metadata from documents as well as detect and record the context of user activities related to document access and modification. Additionally sensors obtain the context of document-related user actions in the enterprises central document management applications and collaborative project workplace solutions. To 
represent the set of concepts specific to the enterprise domain and the possible relationships among those concepts ontology vocabularies are used. The sensor plugins validate gathered context input against the common domain ontologies and persist it in the form of semantic networks in local Resource Description Framework (RDF) triple-stores. In the next step the sensor-plugins add further statements based on the initially gathered static metadata by using heuristics based on typical usage patterns of document access. These statements contain assumptions on the similarity or implicit correlation of either document-pairs or relations of documents to the seeded business entities.

Document-related metadata with relevance beyond that of the local user (that is higher-level metadata relating to the domain-specific business entities) is then submitted to a central semantic information repository which is also implemented as an $\mathrm{RDF}$ triple-store. To reduce the amount of necessary network communication between a client and the central semantic store the transmission of RDF triples is selective. So will the central semantic store only consider aggregated and higher-level predicates which will actually be the target of subsequent discovery queries (regarding domain specific-questions) as input from the sensor plugins. This allows for baseline metadata to remain in the local RDF-stores on the clients while only higher-level metadata (the common ontology-subset) is transferred and made available to other users.

For example a product manager could access the central semantic store to query for and detect documents having a relation to a product line at the end of its lifecycle. The query parameters could then be narrowed to documents having additional relations to the company's sales process to locate documents potentially needing to be updated or reviewed after the product phase-out. Alternatively a rule- or heuristic based electronic service can directly access the semantic aggregation layer and query or manipulate the semantic network.

\section{References}

Barreau D (1995) Context as a factor in personal information management systems. Journal of the American Society for Information Science 5(46), pp. 327-339.

Guther M (2007) Duet - eine Software verknüpft Microsoft Office mit SAPGeschäftsanwendungen. IM - Information Management \& Consulting.

Shen X, Tan B, Zhai C (2005) UCAIR: Capturing and exploiting context for personalized search. Techreport, University of Illinois at Urbana Champaign 
Enterprise Architecture Management 



\title{
Vorwort für den Track
}

\section{Enterprise Architecture Management}

\author{
Frederik Ablemann \\ Lehrstubl für Information Management and Systems, \\ European Business School, Wiesbaden
}

Enterprise Architecture Management (EAM) bezeichnet als Managementdisziplin die Dokumentation, bedarfsorientierte Analyse und zielgerichtete Weiterentwicklung der Unternehmensarchitektur (Enterprise Architecture, EA). Unternehmensarchitekturen beschreiben die grundlegende Strukturierung eines Unternehmens bzw. einer Behörde als Ganzes, in Teilen, oder zusammen mit Kunden, Lieferanten oder anderen Partnern. Die Unternehmensarchitektur umfasst fachliche wie auch IT-Artefakte. Sie stellt damit den Zusammenhang zwischen bestehenden Teilarchitekturen, wie z. B. Geschäfts-, Prozess- und Informationssystemarchitektur, her.

Die Teilkonferenz Enterprise Architecture Management ist die erste ihrer Art, die im Rahmen der MKWI abgehalten wird. Sie hat sich zum Ziel gesetzt, Wissenschaftlern, die sich mit EAM beschäftigen, eine Plattform für den wissenschaftlichen Diskurs zu geben. Dabei sollen - der methodischen Vielfalt der Wirtschaftsinformatik gerecht werdend - sowohl verhaltensorientierte als auch gestaltungsorientierte Forschungsergebnisse diskutiert werden. So geht es zum einen darum, den stark praktisch geprägten Ansatz EAM als empirisches Phänomen besser zu verstehen und erklären zu können. Zum anderen sollen wissenschaftlich fundierte Vorschläge für die Gestaltung von Unternehmensarchitekturen und Unternehmensarchitekturmanagement in Unternehmen und Behörden präsentiert werden.

Mit Freude nimmt das Programmkomitee zur Kenntnis, dass die Teilkonferenz diesem Anspruch gerecht werden kann. Insgesamt 17 Beiträge wurden eingereicht. Diese wurden in einem doppel-blinden Verfahren von mindestens zwei Fachkollegen begutachtet. Bei stark unterschiedlichen Einschätzungen der Qualität der Beiträge wurde ein drittes Gutachten angefertigt. Schlussendlich konnten neun Beiträge angenommen werden, was einer Annahmequote von 53\% entspricht.

Die angenommenen Beiträge repräsentieren das Spektrum EAM-bezogener Forschung in besonders guter Weise. Grundlegend lassen sich die Arbeiten in drei zentrale Bereiche einteilen: (1) Grundlagen des Enterprise Architecture Management, (2) Methoden und Werkzeuge des Enterprise Architecture Management und (3) konkrete Modelle bzw. Artefakte.

Im Bereich der Grundlagen thematisiert Stelzer Prinzipien des EAM, wie sie bei der Beschreibung und Entwicklung der Architektur zur Anwendung kommen 
können. Die Arbeit beruht auf einer systematischen Literaturanalyse und zeigt zukünftige Forschungspotenziale auf. Von gleichfalls elementarer Bedeutung ist die Arbeit von Buckl et al., die sich mit der Konstruktion einer präskriptiven Theorie zur Gestaltung von EAM-Funktionen (im Sinne von Organisationseinheiten) befassen. Becker et al. stellen die Frage der Nützlichkeit von Modellierungsaktivitäten, indem Sie Faktoren auf den Grund gehen, die erklären können, warum und unter welchen Bedingungen Geschäftsprozessmodellierung in Banken nützlich und vorteilhaft ist.

Am breitesten vertreten ist der Themenkomplex Methoden und Tools. Insgesamt vier Beiträge beleuchten verschiedene Facetten der Modellkonstruktion, der Toolunterstützung, der Modellanalyse und dem GeschäftsanwendungsPortfoliomanagement. Leist et al. beschäftigen sich mit dem Problem der Integration von automatisch und manuell konstruierten Prozessmodellen. Addicks et al. reagieren auf die Herausforderungen das EAM in mittelständischen Unternehmen erfolgreich und nutzenstiftend zu implementieren. Sie schlagen hierzu einen komponenten-orientierten Werkzeugansatz vor. Während statische Abhängigkeitsanalysen im Enterprise Architecture Management als Standard zu nennen sind, sind die von Saat vorgeschlagenen dynamischen Abhängigkeitsanalysen als innovativ zu bezeichnen. Saat stellt diesen Analysetyp vor und skizziert seine Anwendungsbereiche. Einen weniger modell-orientierten Ansatz für das Management von Geschäftsanwendungs-Portfolios präsentieren Gieffers-Ankel et al., die eine im Wesentlichen auf Governance-Mechanismen beruhende Methode vorstellt.

Zwei Autorenteams haben erfolgreich Beiträge platzieren können, die sich mit konkreten Modellen für das Enterprise Architecture Management beschäftigen. Fitterer et al. schlagen ein Metamodell zur Modellierung der fachlichen Fähigkeiten der Softwarearchitektur in Krankenhäusern vor und setzen dabei auf die Analyse der Bedenken relevanter Anspruchsgruppen. In Hinblick auf die Problemdomäne verwandt ist der Beitrag von Pütz et al., die ein generisches Geschäftsprozessmodell für medizinische Versorgungszentren konzipieren. Damit beschäftigen sich gleich zwei Forschergruppen mit der spannenden und dynamischen Problemdomäne Gesundheitswesen.

Als Vorsitzender des Programmkomitees möchte ich an dieser Stelle allen Personen danken, die zum Gelingen der Teilkonferenz Enterprise Arcbitecture Management beigetragen haben und noch beitragen. Mein Dank gilt den Mitgliedern des Programmkomitees und den Gutachtern: Prof. Dr. Otto Ferstl, Prof. Dr. Ulrich Frank, Prof. Dr. Helmut Krcmar, Prof. Dr. Ulrike Lechner, Dr. Christine Legner, Prof. Dr. Susanne Leist, Johannes Lux MSc., Prof. Dr. Florian Matthes, Dipl.Wirtsch.-Inform. Frank Radeke, Prof. Dr. Gerold Riempp, Prof. Dr. Elmar J. Sinz, Prof. Dr. Dirk Stelzer, Prof. Dr. Robert Winter. Schließlich danke ich den Autoren sowie den Tagungsorganisatoren der MKWI 2010. 


\title{
Utility vs. Efforts of Business Process Modeling
}

\section{An Exploratory Survey in the Financial Sector}

\author{
Jörg Becker', Burkhard Weiß', Axel Winkelmann² \\ ${ }^{1}$ European Research Center for Information Systems, \\ Lehrstubl für Wirtschaftsinformatik und Informationsmanagement, \\ Westfälische Wilhelms-Universität Münster \\ 2Institut für Wirtschafts- und Verwaltungsinformatik, \\ Professur für Betriebliche Anwendungssysteme, \\ Universität Koblen₹-Landau
}

Process modeling and documentation is a way to capture the implicit process knowledge of an organization and document it explicitly in a (semi-)formal way. Models can be used e.g. as a basis for decisions on IT investments, reorganizations or the selection and implementation of information systems (Becker et al. 2009b). They describe the logical sequence of activities in a business process, the resulting products, required resources and data, as well as organizational units (Lindsay et al. 2003). In organizational practice high efforts are being spent on the creation of business process models for the documentation and analysis of business processes (Mendling et al. 2009). However, little is known about the utility and efforts of process modeling (Indulska et al. 2009).

This is especially true for the financial sector, which currently has a high need for modeling and analyzing business processes for multiple purposes (Cocheo and Harris 2005; Becker et al. 2009b). Although there are studies about the general usage of process models in banks, or the need for reorganization, there is hardly any empirical research on the efforts and utility of business process modeling in the financial sector. With our exploratory study among 60 banks, we aim at providing new knowledge to this open research field by comparing efforts invested during modeling with benefits gained from the analysis of these models.

We found out that a misfit between the effort spent into modeling and the utility derived from analysis exists in many banks. For instance, 64\% of the participating banks spent high efforts in their modeling in terms of addressed modeling purposes but only 15\% also exploited the analysis potential of their process models to a high degree. In total, the banks invested $61 \%$ of the maximum possible efforts, but only received a return of $45 \%$ of the maximum possible benefit. This is 
Jörg Becker, Burkhard Weiß, Axel Winkelmann

rather surprising and we assume that there is much potential for more efficient process modeling projects and better process modeling languages.

Banks seem to be dissatisfied with existing modeling and analysis approaches and are even willing to create their own methods, due to a lack of appropriate methods available. One reason for our assumption is that two thirds of all banks (41 banks) use standard business process modeling languages, whereas one third adapted standard methods or created their own business process modeling specifications (19 banks). Accordingly, only $26 \%$ of the banks, that have developed their own methods (five banks), consecutively wanted to cooperate with us in developing a new business process modeling language specifically designed for banks. However, $29 \%$ of all banks using standardized business process modeling notations (e.g. BPMN, EPC etc.) were interested in a joint development of a new bankspecific process modeling notation with a special focus on the analyzability of process models. As a result, 31\% of all banks participating in our survey have already developed their own modeling methods to meet their requirements (even though $26 \%$ of these banks are still not satisfied with their results) and another $20 \%$ of all banks, that currently use standardized modeling notations, are willing to develop a new business process modeling language just for banks. Thus, more than half of all banks (51\%) are looking for better alternatives for business process modeling and analysis.

\section{References}

Becker J, Mathas C, Winkelmann A (2009) Geschäftsprozessmanagement. Berlin, Heidelberg 2009.

Becker J, Weiß B, Winkelmann A (2009b) Developing a Business Process Modeling Language for the Banking Sector: A Design Science Approach.

Proceedings of the 15th Americas Conference on Information Systems (AMCIS 2009).

Cocheo S, Harris K, (2005) Key Customers Today and Tomorrow ABA BANKING JOURNAL 97 (3): 3-6.

Indulska M, Recker JC, Rosemann M, Green P (2009) Process Modeling: Current Issues and Future Challenges. In: van Eck P, Gordijn J, Wieringa R (eds.): Advanced Information Systems Engineering - CAiSE 2009. LNCS, Vol. 5565. Springer, Amsterdam, The Netherlands, pp. 501-514.

Lindsay A, Downs D, Lunn K (2003) Business processes: attempts to find a definition. INF. AND SOFTW. TECHNOLOGY 45 (15): 1015-1019.

Mendling J, Recker J, Reijers HA (2009) Process Modeling Quality. A Framework and Research Agenda. BPM CENTER REPORT BPM09-02, BPMcenter.org. 


\title{
Prinzipien für Unternehmensarchitekturen
}

\section{Grundlagen und Systematisierung}

\author{
Dirk Stelzer \\ Fachgebiet Informations- und Wissensmanagement, \\ TU Ilmenau
}

Architektur wird in Anlehnung an IEEE 1471 (2000) als grundsätzliche Struktur eines Systems bezeichnet, die sich in den Systemelementen, deren Beziehungen zueinander und zur Systemumwelt widerspiegelt, sowie die Prinzipien, die für den Entwurf und die Entwicklung des Systems maßgeblich sind.

Eine Unternehmensarchitektur stellt die grundlegende Struktur eines Unternehmens dar, wobei die Gesamtheit der Informationssysteme des Unternehmens besondere Beachtung findet (Aier et al. 2008, S. 292). Welche Elemente dabei genau berücksichtigt werden, hängt unter anderem vom Betrachter und dem Zweck der Architekturbetrachtung ab (Esswein und Weller 2008, S. 9).

Das Management von Unternehmensarchitekturen bezeichnet die Planung, Steuerung, Kontrolle und Verbesserung von Unternehmensarchitekturen (Dern 2006). Die Aufgaben des Managements von Unternehmensarchitekturen sind vielfältig und komplex (Lux et al. 2008). Die Verwendung von Prinzipien ermöglicht es, Grundsätze festzulegen und den Akteuren Richtlinien zu geben, anhand derer sie sich orientieren können. Sie entbindet die für die Architektur Verantwortlichen davon, Detailentscheidungen zu treffen, die sinnvoller von anderen Akteuren getroffen werden, und gibt diesen einen Spielraum für die Ausführung ihrer Aufgaben (Malan und Bredemeyer 2002, S. 46-48).

Prinzipien bezeichnen Grundlagen oder Grundsätze, (Natur-)Gesetze, Regeln, Richtlinien oder Postulate. Im Zusammenhang mit Unternehmensarchitekturen sind Prinzipien nicht einheitlich definiert.

Die Literatur zur Unternehmensarchitektur ist umfangreich und vielfältig (Schönherr 2009, 400-413). Im Vergleich dazu sind Publikationen, die sich mit Prinzipien für Unternehmensarchitekturen beschäftigen, selten. Dies ist erstaunlich, da Prinzipien zentrale Bestandteile von Architekturdefinitionen (z. B. IEEE 1471 2000, S. 3 und The Open Group 2009, S. 9) und damit auch von Unternehmensarchitekturen sind (Richardson et al. 1990, S. 389).

Ziel dieses Beitrags ist, publizierte Erkenntnisse zu Prinzipien für Unternehmensarchitekturen zu beschreiben, zu systematisieren und die Grundlagen dafür zu legen, dass diese Erkenntnisse von Forschung und Praxis genutzt werden können. 
Im Einzelnen werden folgende Forschungsfragen beantwortet:

- Wie wird der Begriff „Prinzipien für Unternehmensarchitekturen“ definiert?

- $\quad$ Auf welche Objekte beziehen sich die Prinzipien?

- In welche Kontexte werden die Prinzipien eingebettet?

- Werden die Prinzipien generisch, d.h. unabhängig von den spezifischen Rahmenbedingungen, formuliert?

- Im Zusammenhang mit welchen anderen Prinzipien werden Architekturprinzipien dargestellt?

Antworten auf diese Fragen werden mit Hilfe einer Literaturanalyse erarbeitet.

\section{Literatur}

Aier S, Riege C, Winter R (2008) Unternehmensarchitektur - Literaturüberblick und Stand der Praxis. WIRTSCHAFTSINFORMATIK 50(4):292-304.

Dern G (2006) Management von IT-Architekturen. Leitlinien für die Ausrichtung, Planung und Gestaltung von Informationssystemen. 2. Aufl. Vieweg, Wiesbaden.

Esswein W, Weller J (2008) Unternehmensarchitekturen - Grundlagen, Verwendung und Frameworks. HMD 262:6-18.

IEEE 1471 (2000) IEEE Recommended practice for architectural description of software-intensive systems - Description.

Lux J, Wiedenhöfer J, Ahlemann F (2008) Modellorientierte Einführung von Enterprise Architecture Management. HMD 262:19-28.

Malan R, Bredemeyer D (2002) Less is more with minimalist architecture. IEEE IT Professional 4(5):46-48.

Richardson GL, Jackson BM, Dickson GW (1990) A principles-based enterprise architecture: Lessons from Texaco and Star Enterprise. MISQ 14(4):385-403.

Schönherr M (2009) Towards a common terminology in the discipline of enterprise architecture. In: Feuerlicht G, Lamersdorf W (Hrsg.) Serviceoriented computing. ICSOC 2008 Workshops, Berlin.

The Open Group (2009) TOGAF Version 9. The Open Group architecture framework. http://www.opengroup.org. Abruf am 2009-08-31. 


\title{
From EA management patterns towards a prescriptive theory for designing enterprise-specific EA management functions
}

\author{
Outline of a research stream
}

\author{
Sabine Buckl, Florian Matthes, Christian M. Schweda \\ Chair for Software Engineering of Business Information Systems, \\ Technische Universität München
}

In response to the challenges of ever-changing economic, regulatory, and technical environments, many of today's enterprises have established or are on the way of establishing an enterprise architecture (EA) management function. Thereby, the enterprises go through a situated design process, for which yet only a few prescriptions exist. This may be ascribed to the novelty of the field, which is only recently target to an increasing number of publications that broadened the scope as well as discussed various application scenarios for EA management for which Aier et al. (2008) give the following non-comprehensive list of examples: process optimization, architectural conformity of projects, quality and security management, and strategic planning. Thus, EA management involves different stakeholders with various backgrounds in these application scenarios (van der Raad et al. 2008). In this vein, EA management forms an intricate subject for research, which is typically addressed by an engineering approach, aiming at solving problems relevant in practice. Although we do not doubt the importance of the thereby achieved results, the contributions remain isolated and a comprehensive understanding of the design process for the EA management function has not yet developed.

A promising contribution in this direction is the EAM Pattern Language proposed by Ernst (2008). The pattern language introduces three types of patterns, namely methodology pattern (M-Pattern), viewpoint pattern (V-Pattern), and information model pattern (I-Pattern), that are used to develop an enterprise-specific EA management function. These three types of patterns describe constituents of provenpractice solutions for EA management as found in literature but also in partnering enterprises (sebis 2009), namely:

- $\quad$ M-Patterns describe management step-by-step management methods that solve a specific EA-related problem.

- $\quad V$-Patterns and I-Patterns describe types of visualizations that are employed by M-Patterns and their underlying conceptual models, respectively. Together they form special purpose EA modeling languages. 
In the sense of Schermann et al. (2007) the pattern-language for EA management shows typical characteristics of a design theory for this field. In their work, they discuss the idea of using pattern languages to describe design theories, and provide a structural framework for pattern-based design theories. In the light of the framework, the focus of the EAM pattern language on building blocks for EA management functions becomes apparent. In contrast, guidelines on how to design an EA management function based on patterns are not provided.

A research stream seeking to close the aforementioned gap and to develop the EAM pattern language towards a comprehensive design theory would have to accommodate for the broadness of the subject EA management and decompose it into handily researchable domains, either

- $\quad$ by restricting itself to a limited number of EA-related problems, but targeting them from both a method and a language perspective, or

- $\quad$ by targeting a broad variety of EA-related problems either with management methods or modeling languages.

We propose to apply the latter decomposition, thereby yielding two research streams: Stream one develops a library of management methods together with a set of integration and evolution guidelines. Stream two is concerned with a language toolset for EA management consisting of a meta-language, addressing the challenges of configurable method composition and suitable integration guidelines.

\section{References}

Aier S, Riege C, and Winter R (2008) Unternehmensarchitektur - Literaturüberblick und Stand der Praxis. In WIRTSCHAFTSINFORMATIK 50(4):292-302.

Ernst AM (2008) Enterprise Architecture Management Patterns. In: PLoP08: Proceedings of the Pattern Languages of Programs Conference, Nashville, 2008.

Schermann M, Böhmann T, and Krcmar H (2007) Fostering the evaluation of reference models: Application and extension of the concept of IS design theories. In Oberweis A et al. (edts) eOrganisation: Service-, Prozess-, Marketing Engineering - 8. Internationale Tagung Wirtschaftsinformatik, Band 2.

Sebis (2009) EAM Pattern Catalog Wiki. Chair for Informatics 19 (sebis), Technische Universität München, München, http:// eampcwiki.systemcartography.info (cited 2009-11-25)

Van der Raad B, Schouten S, and van Vliet H (2008) Stakeholder perception of enterprise architecture. In Morrison R, Balasubramaniam D, and Falkner KE (edts) $2^{\text {nd }}$ European Conference on Software Architecture, ECSA2008. 


\title{
MCP-Portfolio - eine EAM-orientierte Methode zur fachlichen Konsolidierung von Geschäftsanwendungs-Portfolios
}

\author{
Stephan Gieffers-Ankel, Gerold Riempp \\ Lehrstubl für Information Management and Systems, \\ European Business School, Wiesbaden
}

Die Konsolidierung komplexer Geschäftsanwendungs-Portfolios ist für große Organisationen eine wichtige Herausforderung. Aufgrund von dezentralen Geschäfts- und IT-Entscheidungen, Reorganisationen, Firmenfusionen und anderen Faktoren werden in vielen großen Unternehmen für die gleichen Geschäftsprozesse in verschiedenen Unternehmensteilen unterschiedliche Geschäftsanwendungen eingesetzt. Diese Redundanzen erzeugen zusätzliche IT-Kosten und erschweren die Harmonisierung von Geschäftsprozessen. Diese allgemeinen Beobachtungen spiegeln sich in besonderem Maße in einer von uns durchgeführten LangzeitFeldstudie (2005-2009) bei der Volkswagen AG wider. Die Identifikation, Bereitstellung und der Rollout von konzernweiten Geschäftsanwendungsstandards ist für die Leiter der Fach-IT-Bereiche auf Konzernebene eine der zentralen Managementherausforderungen, die durch die im Rahmen der Feldstudie entwickelte Master Construction Plan (MCP) Methode unterstützt wird.

Schwerpunkt des Artikels ist die Konstruktion und Validierung des MCPPortfolios, eines wichtigen Ergebnistyps der MCP-Methode. Statt eine allgemeinen Beweisführung über den Nutzen und die Wirksamkeit anzutreten, werden grounded theories über Wirkungsweise sowie Erfolg oder Misserfolg beim wiederholten Einsatz in insgesamt 12 Iterationen der MCP-Methode in den vier Fach-IT Bereichen des Volkswagenkonzerns herausgearbeitet. Die Struktur des MCP-Portfolios ist in Abbildung 1 dargestellt. Auf der horizontalen Achse werden Geschäftsanwendungen obne Zukunft (links) und mit Zukunft (rechts) unterschieden; auf der vertikalen Achse Konzernanwendungen (oben) und „lokale Anwendungen“ (unten). In dieses einfache Schema werden für unterschiedliche Anwendungsdomänen alle Geschäftsanwendungen von Managern aus Konzern, Marken und Region gemeinsam gesetzt.

Das MCP-Portfolio adressiert die fachliche Standardisierung vor allem als Management-Herausforderung. Als „Management Scorecard“ kann das MCPPortfolio „auf einen Blick“ den Stand der Standardisierung zeigen und so diskutierbar machen. Über die geschaffene Transparenz erhöht sich der Druck auf das Management, wichtige Standardisierungsthemen trotz dringender operativer Aufgaben anzugehen. Als Grundvoraussetzung für dieses Anwendungsfeld müssen die Portfolio-Domänen jedoch weitgehend unabhängig voneinander entscheidbar sein, 
damit die zuständigen Manager tatsächlich auch die Entscheidungen ihres Portfolios eigenverantwortlich vorantreiben können. Da das Portfolio keine ,inhaltliche“ Hilfe dabei bietet, welche Anwendungen des Portfolios sich eventuell überschneiden oder miteinander konkurrieren, funktioniert das MCP-Portfolio nur in der Diskussion mit Managern, die diese Anwendungen kennen und inhaltlich beurteilen können.

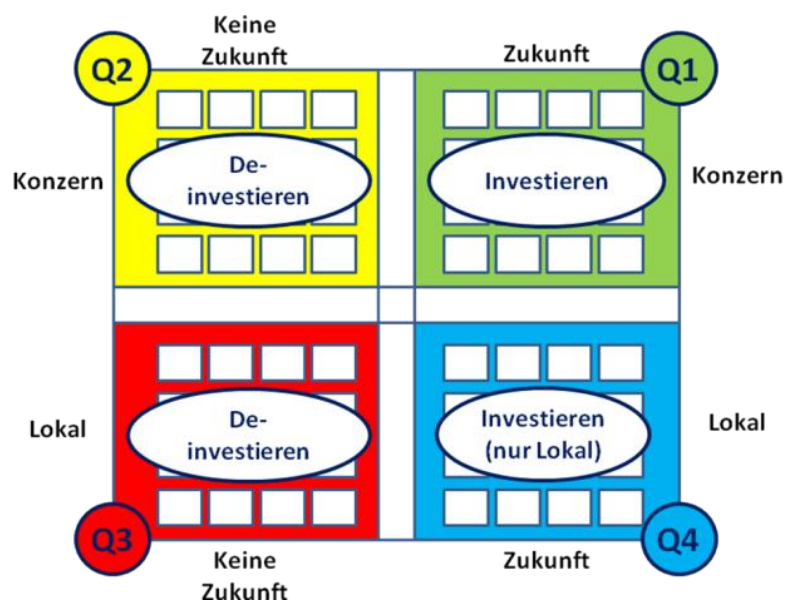

Abbildung 1: Struktur des MCP-Portfolios

MCP-Portfolios erweisen sich ebenfalls als nützlich zur Dokumentation von Standardisierungsentscheidungen. Durch die pointierte Darstellung der strategischen Positionen „Zukunft - Investieren“, „keine Zukunft - Deinvestiern“ und „Konzern - lokal“ ist eine einprägsame und leicht kommunizierbare Darstellung verfügbar, die strategische Einordnung in Einklang mit Investitionsentscheidungen bringt. Maßgeblich für den nachhaltigen Erfolg als „Dokumentationsmodell“ ist natürlich die tatsächliche Berücksichtigung bei Budget- und Investitionsentscheidungen.

Ein weiteres Anwendungsfeld ist die Variante „lokales Portfolio“, die Standardisierungsentscheidungen mit einzelnen Gesellschaften und Werken in einer Darstellung zusammenfasst.

Der Einsatz der Methode basiert auf der impliziten Annahme, dass das eigentliche Problem der fachlichen Standardisierung auf der Ebene des zuständigen fachlichen IT-Managements liegt und durch die komplexen Abhängigkeiten und Interessen unterschiedlicher Stakeholder innerhalb des Gesamtkonzerns bedingt ist. Um die fachliche Standardisierung voranzutreiben, sind deshalb zunächst keine breit angelegten, komplexen technischen Analysen notwendig, sondern organisatorische Maßnahmen, um die Entscheidungsfindung zu katalysieren. 


\title{
Entwicklung eines Metamodells zur Modellierung der fachlichen Fähigkeiten der Softwarearchitektur in Krankenhäusern auf Grundlage von Stakeholder Concerns
}

\author{
René Fitterer, Peter Rohner \\ Institut für Wirtschaftsinformatik, Universität St. Gallen
}

Das Management von Unternehmensarchitekturen (EAM) als ganzheitlicher Ansatz zur Analyse und Gestaltung von Organisationen wird in zunehmendem Maße in Unternehmen eingesetzt. Zur Darstellung und Modellierung der EA werden in verschiedenen Beiträgen von Wissenschaftlern und Praktikern zahlreiche Frameworks definiert. Im Sinne eines ganzheitlichen und systematischen Vorgehens integriert die EA Modelle der Ebenen Geschäftsarchitektur (Strategie, Ziele, etc.), Prozessarchitektur (Ablauf- und Aufbauorganisation, etc.), Integrationsarchitektur (sozio-technische Informationssysteme - IS), Softwarearchitektur (Softwarekomponenten, Datenstrukturen, etc.) und Technologiearchitektur (Hardware, Netzwerk, etc.) (Winter und Fischer 2007), um eine konsistente und zielgerichtete Veränderung der Organisation zu unterstützen. Insbesondere für Organisationen, die eine geringe Reife bezüglich des EAM aufweisen, besteht auf Grund der Komplexität der Artefakte des EAM die Herausforderung, den Betrachtungshorizont (Breite und Tiefe) auf die relevanten Aspekte der Stakeholder (engl. Concerns) (Sutton und Rouvellou 2001) zu reduzieren.

Dieser Artikel beschreibt am Beispiel von Krankenhäusern die Anwendung einer Methodik zur Entwicklung eines Metamodells, welches auf Grundlage einer Analyse der Concerns die relevanten Informationsbedürfnisse abbildet. Das in Abbildung 1 dargestellte Metamodell ermöglicht die Modellierung und Analyse der fachlich funktionalen Fähigkeiten der Softwarearchitektur. Es schränkt den Betrachtungshorizont somit auf die relevante Problemstellung der Analyse und Entwicklung des Beitrags von Softwarearchitekturen zu der an den Berufsgruppen orientierten, funktional strukturierten Aufbau- und Ablauforganisation von Krankenhäusern ein. Auf der Basis eines Referenzkatalogs fachlicher Funktionalitäten (z. B. Department of Medical Informatics - University of Heidelberg 2001) ermöglicht das Metamodell eine einheitliche Dokumentation und somit eine vergleichende Abdeckungs- und Nutzenanalyse der Softwarearchitekturen, die im Rahmen einer Benchmark-Studie mit 13 Schweizer Krankenhäusern realisiert wird. 


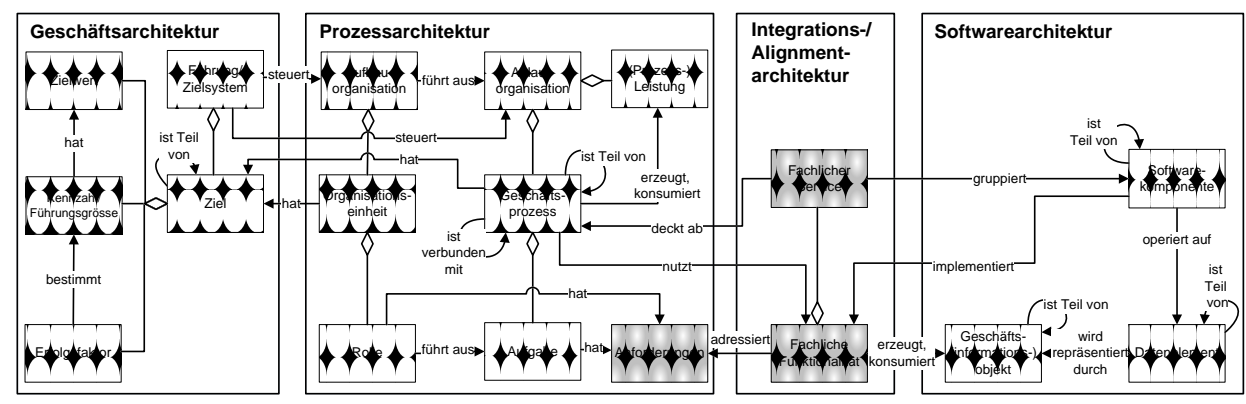

Abbildung 1: Metamodell zur Modellierung der fachlichen, funktionalen Fähigkeiten der Softwarearchitektur in Krankenhäusern, in Anlehnung an (Mettler et al. 2008)

Die fachlich funktionalen Fähigkeiten der Softwarearchitektur werden hierbei zur Krankenhaus-Wertkette (Porter und Olmsted Teisberg 2006, S. 200 ff.) in Bezug gesetzt und somit wird eine Bewertung des Beitrags der Softwarearchitektur zu den primären und unterstützenden Aktivitäten des Krankenhauses ermöglicht. Erste Ergebnisse der Studie zeigen eine umfangreiche Unterstützung der administrativen operativen Aufgaben von Krankenhäusern (Rechnungswesen, Personalwirtschaft, etc.) sowie eine grundsätzliche Unterstützung medizinisch operativer Aufgaben (Behandlungsprozesse, etc.) und administrativer Planungs- und Steuerungsprozesse (Controlling, etc.).

\section{Literatur}

Department of Medical Informatics - University of Heidelberg (2001)

Requirements Index for Information Processing in Hospitals, University of Heidelberg, Heidelberg.

Mettler T, Rohner P und Baacke L (2008) Improving Data Quality in Health Information Systems - A Holistic Design-oriented Approach. In: Golden W, Acton T, Conboy K, van der Heijden H, und Tuuainen V (Hrsg) Proceedings of the 16th European Conference on Information Systems, Galway.

Porter ME und Olmsted Teisberg E (2006) Redefining Health Care: Creating Value-Based Competition on Results. Harvard Business School Press, Boston, MA.

Sutton SM und Rouvellou I (Hrsg) Issues in the Design and Implementation of a Concern-Space Modeling Schema (2001). Toronto, Canada.

Winter R und Fischer R (2007) Essential Layers, Artifacts, and Dependencies of Enterprise Architecture. Journal of Enterprise Architecture 3(2): 7-18. 


\title{
Integration von automatisch generierten und manuell konstruierten Prozessmodellen als Grundlage für den Aufbau einer Prozessarchitektur
}

\author{
Susanne Leist, Wolfgang Lichtenegger \\ Lehrstubl für Wirtschaftsinformatik III, insh. Business Engineering, \\ Universität Regensburg
}

Die systematische Entwicklung und Modellierung der Unternehmensarchitektur wird von der Praxis (Jung 2005, Uhl 2004) wie auch der Wissenschaft (Buhl und Heinrich 2004, Zachman 1987) als ein bedeutendes aber auch umfassendes Aufgabengebiet angesehen. Der vorliegende Beitrag greift die Modellierung der Prozessarchitektur im Ist-Zustand als Themenstellungen heraus und begründet, dass durch Integration von manuell konstruierten und automatisch generierten Modellen die Aktualität, Korrektheit und Vollständigkeit der Architektur erhöht und der Aktualisierungsaufwand reduziert werden kann.

Das Ziel des Beitrags ist es, Herausforderungen der Integration von manuell konstruierten und automatisch generierten Prozessmodellen zu untersuchen sowie Ansätze zur Lösung zu geben. Hierzu werden die beiden Modellierungsansätze (manuelle Konstruktion und automatische Generierung) vorgestellt und die Vorteilhaftigkeit der Integration sowie ihr Beitrag im Rahmen der Unternehmensarchitektur begründet. Darüber hinaus werden Herausforderungen bei der Integration identifiziert und deren Grundlagen in einem Morphologischen Kasten zusammengestellt. Der Morphologische Kasten enthält sowohl Herausforderungen, die Eigenschaften der Ausgangsmodelle als auch Aspekte der Integration umfassen. Beispielsweise ergeben sich Herausforderungen bei der Integration, wenn die Ausgangsmodelle in unterschiedlichen Modellierungssprachen konstruiert oder generiert werden und deshalb die Transformation eines Modells erforderlich wird. Das Vorgehen der Integration wird maßgeblich dadurch beeinflusst, ob die Ausgangmodelle bereits generiert bzw. konstruiert sind oder ob Anforderung der Integration bei der Erstellung der Ausgangsmodelle mit einbezogen werden können. Die Untersuchung zeigt, dass die Integration der Modelle aufgrund der vielen Einflussfaktoren ein komplexer Vorgang ist und dass unterschiedliche Integrationsszenarios unterschieden werden müssen. Der morphologische Kasten bildet eine strukturierte Grundlage, Integrationszenarios abzugrenzen, die durch unterschiedliche Pfade anhand einer Auswahl von Merkmalsausprägungen definiert werden. Die Analyse bestehender Ansätze zeigte, dass keine der untersuchten Methoden die Problemstellung vollständig lösen kann, sich jedoch einzelne Techniken der bestehenden Methoden eigenen, um Teilprobleme zu lösen und damit wiederverwendet 
werden können. Für ein ausgewähltes Integrationsszenario wurde ein erster Ansatz mit den vier Schritten 1) Transformation der Modellierungssprache, 2) Behandlung auftretender Konflikte, 3) Verbinden der Ausgangsmodelle und 4) Qualitätssicherung der Zielmodelle entwickelt und am Beispiel des Lieferantenwechselprozesses eines Unternehmens in der Energiewirtschaft durchgeführt. Der Ansatz wird aktuell an mehreren Anwendungsfällen validiert. Besondere Herausforderungen ergeben sich dabei insbesondere bei der Transformation der Modellierungssprachen und der Konfliktbehandlung bei unterschiedlichen Bezeichnungen.

\section{Literatur}

Buhl HU, Heinrich B (2004) Unternehmensarchitekturen in der Praxis Architekturdesign am Reißbrett vs. situationsbedingte Realisierung von Informationssystemen. Wirtschaftsinformatik 46 (4): 311-322.

Jung E (2005) Das IT-Architekturmodell - Ein Rahmenwerkzeug für die Prozessoptimierung in der HypoVereinsbank. BIT - Banking and Information Technology 6 (2): 62-64.

Leist S, Zellner G (2008) Situational Architecture Engineering (SAE) - Improving Strategic Change Through Architecture, International Conference on Information Systems, ICIS 2008 Paris, France, December 14 to 17, 2008.

Uhl J (2004) "Unternehmensarchitekturen" ist ein Dauerthema - aber die Ziele bzw. Motivation und damit Schwerpunkte ändern sich, vor allem mit wirtschaftlichen Randbedingungen. Wirtschaftsinformatik 46 (4): 317.

Zachman JA (1987) A framework for information systems architecture. IBM Systems Journal Vol. 26, No. 3 276-295. 


\title{
Zeitbezogene Abhängigkeitsanalysen der Unternehmensarchitektur
}

\author{
Jan Saat \\ Institut für Wirtschaftsinformatik, Universität St. Gallen
}

Modelle und Analysen der Unternehmensarchitektur können wichtige Instrumente zur Planung und Durchführung von Veränderungen im Unternehmen sein. Da sich ein Unternehmen - somit auch die Modelle der Unternehmensarchitektur ständig verändern, besteht eine zentrale Herausforderung in dem Management der zeitbezogenen Komplexität. Diese ist z. B. mit unterschiedlichen Lebenszyklen verschiedener Elemente der Unternehmensarchitektur zu begründen. Um Modelle der Unternehmensarchitektur als Planungsinstrument wirksam nutzen zu können, müssen sie eine Analysegrundlage bereitstellen, die die beschriebenen zeitbezogenen Aspekte berücksichtigt und ein konsistentes Gesamtbild der Strukturen ermöglicht.

Der vorliegende Beitrag beschreibt hierzu die Entwicklung und Anwendung eines Analyseansatzes, der Lebenszyklen für zeitbezogene Abhängigkeitsanalysen der Unternehmensarchitektur nutzt. Hierbei wird auf bestehende Arbeiten zu Lifecycle Management (Niemann et al. 2009, S. 8), zeitbezogener Metamodellierung (Buckl et al. 2009, S. 11f.), Gap-Analysen (Postina et al. 2009), Blueprint Management Systemen (Sousa et al. 2009, S. 82) sowie Transformation von Modellen der Unternehmensarchitektur (Aier und Gleichauf 2010) zurückgegriffen und Teilkonzepte hieraus werden wiederverwendet.

Der entwickelte Ansatz beschreibt eine strukturelle Sicht und eine prozedurale Sicht. In der strukturellen Sicht wird eine Attributierung von Ist- und SollModellen und deren Elementen vorgeschlagen, mittels deren die Lebenszyklen und der jeweilige Status erfasst werden können. Die prozedurale Sicht erlaubt die Darstellung von Veränderungsprojekten, die notwendig sind, um ein Ist-Modell in ein Soll-Modell zu überführen. Die Anwendung des Ansatzes wird anhand einer Fallstudie beschrieben. Die Ergebnisse dieser Analysen können zur effizienten Koordination von Veränderungsprojekten eingesetzt werden.

\section{Literatur}

Aier S und Gleichauf B (2010) Towards a Systematic Approach for Capturing Dynamic Transformation in Enterprise Models. In: Proceedings of 43rd Annual Hawaii International Conference on System Sciences (HICSS-43), Hawaii. 
Buckl S, Ernst AM, Matthes F und Schweda CM (2009) An Information Model for Managed Application Landscape Evolution. Journal of Enterprise Architecture 5(1): 12-26.

Niemann M, Appel MA, Repp N und Steinmetz R (2009) Towards a Consistent Service Lifecycle Model in Service Governance. In: Proceedings of 15th Americas Conference on Information Systems (AMCIS 2009), San Francisco.

Postina M, González J und Sechyn I (2009) On the Architecture Development of Utility Enterprises with Special Respect to the Gap Analysis of Application Landscapes. In: Proceedings of Workshop MDD, SOA and IT-Management (MSI 2009), Oldenburg.

Sousa P, Lima J, Sampaio A und Pereira C (2009) An Approach for Creating and Managing Enterprise Blueprints: A Case for IT Blueprints. In: Proceedings of 5th International Workshop on Cooperation \& Interoperability - Architecture \& Ontology (CIAO! 2009) in conjunction with the CAiSE'09 conference, Amsterdam.. 


\title{
A Component-Oriented Tool-Approach to Enterprise Architecture Management
}

\author{
Jan Stefan Addicks', H.-Jürgen Appelrath², Philipp Gringel1, \\ Matthias Postina ${ }^{1}$, Ulrike Steffens ${ }^{1}$, Christian Zillmann ${ }^{1}$ \\ ${ }^{1}$ OFFIS-Institut für Informatik, Oldenburg \\ ${ }^{2}$ Praktische Informatik, Abteilung Informationssysteme, \\ Carl von Ossietzky Universität Oldenburg
}

The discipline of Enterprise Architecture Management (EAM), while becoming firmly established with global enterprises, is often unheard-of by medium-sized enterprises. EAM comes with a holistic view regarding architecture domains such as business, application, data and infrastructure. With respect to the complexity of the different architecture domains and their relations, management tools play an important role to keep track of the architecture development and the Business-ITAlignment.

The necessity to offer independent EAM parts which can be assembled and gradually refined into enterprise-specific solutions has already been recognized by EAM research and resulted in a number of conceptual approaches to structure the introduction of EAM (Winter and Fischer 2006, p. 1-4; Buckl et. al. 2008b, p. 17). Componentization of tools has not yet been considered in EAM research.

There are a number of commercial tools which claim to be extensible by some means or other (e.g. ARIS IT Architect, Telelogic's System Architect). Nevertheless, there are no tools available that provide well-defined logical interfaces for adding new EAM functionality. A modular approach like described in this paper seems to be a promising step towards real tool extensibility and hence towards adaptability of EAM tools with regard to enterprise-specific requirements.

In consulting both large and medium-sized enterprises on a daily basis, we experience a strong demand for solutions to solve enterprise-wide architecture concerns. Such concerns vary along multiple dimensions: (1.) The Complexity of the enterprise model. (2.) The Budget for enterprise architecture development. (3.) The Skills in the domain of Enterprise Architecture. When regarding these dimensions in medium-sized enterprises, we deem the following requirements relevant for adequate tool support: Minimal Costs, Ease of training, Adequate complexity and Adaptability.

When it comes to the acceptance of EAM in medium-sized enterprises, a modular approach by which the EAM tool grows according to the progress of the EA-initiative and embodies a flexible meta model seems to be promising. To this end we present our EAM tool prototype which is a web-based open source enter- 
Addicks, Appelrath, Gringel, Postina, Steffens, Zillmann

prise solution in Java based on OSGi (Equinox Server-Side). In a nutshell, the use of OSGi is aimed to provide our prototype with the flexibility and customization facility for different kinds of enterprises. Our prototype is intended to show how a cost-effective modular EAM tool can be designed. Therefore, besides the open standard (OSGi) we only make use of open source software.

Our prototype provides a core-bundle, bundling mandatory functionality needed by all installations regardless of the actual overall bundle configuration. It provides a meta model as basis for creating enterprise models as one of the core functions as well as the support of user management with corresponding operations like managing rights, roles, groups, the definition of data access rights on attribute level and the creation of views. Bundles offer Java interfaces and/or, XML-based declarative services which can be used by other bundles. Possible bundles' interdependencies defined in the manifest of each bundle are managed by the management agent of Equinox Server-Side.

The modular, component-oriented approach reaches down to the deploymentlevel of our prototype; the application-server (Jetty) itself is provided as a bundle and can easily be replaced. Further technical bundles that come with Equinox are essential to run the environment but are irrelevant from an EA perspective.

To extend our prototype and to demonstrate the potential of our componentoriented approach, we implemented some extension-bundles e.g. an extension bundles a visual reporting facility, configurable to visualize parts of the enterprisearchitecture defined by the enterprise model.

The prototyped bundle functionality has been successfully related to the majority of scenarios described by Technische Universität München/sebis in the Enterprise Architecture Management Tool Survey 2008.

\section{References}

Buckl S, Ernst AM, Lankes J und Matthes F (2008b) Enterprise Architecture Management Pattern Catalog (Version 1.0, February 2008). München.

Winter R and Fischer R (2006). Essential Layers, Artefacts, and Dependencies of Enterprise Architecture. 10th IEEE International Enterprise Distributed Object Computing Conference Workshops (EDOCW'06). 


\title{
Konzeption eines generischen Geschäftsprozessmodells für Medizinische Versorgungszentren
}

\author{
Corinna Pütz, Daniel Wagner, Otto K. Ferstl, Elmar J. Sinz. \\ Forschungsverbund forFLEX, Universität Bamberg
}

Dienstleistungsunternehmen des medizinischen Bereichs wie z. B. Medizinische Versorgungszentren (MVZ) stehen wie andere Unternehmen vor den Herausforderungen turbulenter Märkte, sich schnell ändernder Leistungen und dynamischer Wertschöpfungsnetze. Zielsysteme, Strategien, Geschäftsprozesse und Anwendungssysteme dieser Unternehmen sind zeitnah anzupassen und aufeinander abzustimmen.

Im Zusammenhang mit Unternehmensarchitekturen wurden MVZ bislang nur wenig betrachtet. MVZ tragen seit 2004 neben bestehenden Leistungserbringern zur ambulanten vertragsärztlichen Versorgung von Patienten bei $(\$ 95$ Abs. 1 S. 1 SGB V) und stellen eine neue Art von Dienstleistungsunternehmen dar, die eine Lücke zwischen ambulanter und stationärer Versorgung schließen (Pütz et al. 2009). MVZ erfahren im Gesundheitswesen eine zunehmende Verbreitung, was sich in Deutschland in einer rasch steigenden Anzahl von MVZ widerspiegelt (KBV 2009). Charakteristisch für MVZ sind eher kurzlebige Strukturen, rasch veränderliche Rahmenbedingungen sowie hohe Flexibilitätsanforderungen an die Geschäftsprozesse. Weitere charakteristische Merkmale, deren Ausprägungen die Struktur eines MVZ beeinflussen, werden im Beitrag anhand eines generischen Leistungsnetzes, des Kontextes von MVZ, sowie von Flexibilitätsanforderungen an MVZ beschrieben.

Um trotz der hohen Volatilität der Geschäftsprozesse von MVZ eine weitgehend stabile Basis für die Unternehmensarchitektur zu schaffen, wird im vorliegenden Beitrag ein generisches Geschäftsprozessmodell (gGPM) entwickelt. Durch dessen Parametrisierung lassen sich konkrete Ausprägungen einzelner MVZ abbilden. Das gGPM bildet zusätzlich einen Bezugsrahmen für die Flexibilisierung der Gestaltung und des Betriebs von MVZ sowie eine fachliche Grundlage für die Gestaltung von Anwendungssystemen zur Unterstützung der Geschäftsprozesse.

Zur Erstellung des gGPM wird eine Top-Down-Vorgehensweise (Literaturanalyse, Auswertung einschlägiger gesetzlicher Regelungen) und eine Bottom-UpVorgehensweise (Fallstudie mit mehreren realen MVZ, Funktionsanalyse von Standardsoftware für Arztpraxen) kombiniert.

Das entwickelte gGPM bildet aufgrund seines modularen Aufbaus die Grundlage einer flexiblen Unternehmensarchitektur sowohl auf der Typ- (gGPM) als auch auf der Instanzebene von MVZ. 
Das gGPM und dessen Parameter spannen einen strukturellen Lösungsraum auf, aus dem Implementierungsmodelle für konkrete MVZ abgeleitet werden können. Die volatilen Strukturen hinsichtlich Organisation und Geschäftsprozesse von MVZ werden durch die im gGPM berücksichtigten Parameter modellierbar. Während das gGPM einen längerfristig stabilen Bezugsrahmen für konkrete MVZInstanzen bildet, kann sich das jeweilige Implementierungsmodell einer konkreten MVZ-Instanz permanent ändern.

Dem gGPM liegt eine agentenorientierte Sichtweise zugrunde, um die weitgehend nicht-hierarchischen Koordinationsmechanismen innerhalb eines MVZ zu erfassen. Damit wird sichtbar, welch hohe Flexibilität in MVZ benötigt wird. Die untersuchten konkreten MVZ weisen eine entsprechende Struktur tatsächlich auf.

\section{Literatur}

KBV (2009) Entwicklung der Medizinischen Versorgungszentren.

http://daris.kbv.de/daris/link.asp?ID=1003758994, Abruf am 2009-08-21.

Pütz C, Wagner D, Ferstl OK, Sinz EJ (2009) Geschäftsprozesse in Medizinischen Versorgungszentren und ihre Flexibilitätsanforderungen - ein fallstudienbasiertes Szenario. http://www.forflex.de/uploads/AB/forflex2009-001.pdf, Abruf am 2009-09-01.

Sozialgesetzbuch (SGB) Fünftes Buch (V), Gesetzliche Krankenversicherung (Artikel 1 des Gesetzes v. 20. Dezember 1988, BGBl. I S. 2477). http://bundesrecht.juris.de/sgb_5/, Abruf am 2009-09-07. 
IT- und Geschäftsstrategien in der Globalisierung 



\title{
Vorwort zum Track
}

\section{IT- und Geschäftsstrategien in der Globalisierung}

\author{
Michael Amberg', Karl Kurbel2, Peter Schumacher ${ }^{3}$ \\ ${ }^{1}$ Lehrstubl für BWL, insh. Wirtschaftsinformatik III, \\ Universität Erlangen-Nürnberg \\ ${ }^{2}$ Lebrstubl für Wirtschaftsinformatik, \\ Europa-Universität Viadrina Frankfurt (Oder) \\ ${ }^{3}$ Value Leadership Group, Frankfurt (Main)
}

Themen des Tracks

Das Rahmenthema des Tracks umfasst neue Strategien, Geschäftskonzepte und Geschäftsmodelle auf allen Management- und Fachebenen. Innovative Unternehmen erzielen Wettbewerbsvorteile und realisieren die Chancen der Globalisierung, indem sie die Verteilbarkeit digitalisierbarer Aufgaben und Prozesse gezielt ausnutzen, um ihr Kundenangebot zu verbessern und zu differenzieren und effizienter zu arbeiten.

Global aufgestellte IT-Dienstleistungsunternehmen übernehmen bereits große Teile der weltweiten Informationsverarbeitung, unabhängig vom Standort ihrer Kunden. Auch Anwenderunternehmen schaffen neue arbeitsteilige globale Geschäftsmodelle, wobei sie ihre Informationssysteme und Geschäftsprozesse in Tochtergesellschaften und Joint Ventures in Indien, China oder Osteuropa betreiben. Die Globalisierung verändert die ökonomischen Rahmenbedingungen und das operative Projektgeschäft in der Softwareentwicklung und -wartung nachhaltig, wobei inzwischen auch die "frühen" Phasen im Software Life Cycle betroffen sind.

Über das reine Offshoring hinaus zeigen zahlreiche Beispiele aus der Praxis, dass kooperative Aufgabenerfüllung durch vernetzte, über die Welt verteilte Unternehmen, Organisationseinheiten und/oder Individuen zu qualitativ und kostenmäßig besseren Lösungen führt. Das Konzept der virtuellen Unternehmen erfährt einen starken Aufschwung, da es strategisch orientierten Unternehmen zahlreiche Vorteile bietet. Auch mittelständige Unternehmen nutzen die Möglichkeiten der globalen Geschäftsmodellinnovation, um ihre Marktposition nachhaltig zu verbessern.

Die Beiträge in dem Track diskutieren insbesondere die Themen Offshoring im Bereich der Softwareentwicklung und Compliance bei Outsourcing-Entscheidungen im IT-Bereich. Die Ausführungen der Autoren wie auch die überschaubare 
Zahl der einschlägigen Einreichungen in dem Track machen deutlich, dass auf dem Gebiet der IT- und Geschäftsstrategien, das mit Blick auf die Globalisierung für die zukünftige Entwicklung der Unternehmen von enormer Bedeutung ist, noch erheblicher Forschungsbedarf besteht.

Programmkomitee

Prof. Dr. Michael Amberg, Universität Erlangen-Nürnberg

Dieter Berz, Cognizant Technologies Solutions, Frankfurt (Main)

Prof. Dr. Peter Buxmann, TU Darmstadt

Prof. Dr. Dimitris Karagiannis, Universität Wien

Prof. Dr. Karl Kurbel, Europa-Universität Frankfurt (Oder)

Prof. Dr. Ronald Maier, Universität Innsbruck

Prof. Dr. Markus Nüttgens, Universität Hamburg

Peter Schumacher, Value Leadership Group Inc., Frankfurt (Main)

Prof. Dr. Rolf Wigand, University of Arkansas at Little Rock 


\title{
Offshore Software Development (OSD)
}

\section{A Multi-Perspective Research Framework and Agenda}

\author{
Martin Wiener \\ Department for Business Information Systems III, \\ Friedrich-Alexander University Erlangen-Nurenberg
}

In recent years, IS offshoring has become one of the most discussed phenomena in IS research and practice (King and Torkzadeh 2008). Offshore software development is a specific form of IS offshoring (e.g., Krishna et al. 2004) and represents a relatively new phenomenon (Delmonte and McCarthy 2003). Probably due to its rapid evolvement, current research on OSD lacks a consolidated view on existing results (Mertens et al. 2005). The paper at hands seeks to meet this need by systematically reviewing and analyzing prior academic OSD literature. Based on a review of top-ranked IS and management journals as well as IS conference proceedings, we compiled an exhaustive bibliography of 73 publications solely focusing on OSD. To adequately address the immense diversity of these publications, we developed a multi-perspective research framework in the style of Dibbern et al. 2004), consisting of three perspectives (research focus, reference theory, and research approach) and eight associated dimensions. By organizing relevant concepts, the framework enabled a common understanding of basic terms and formed the basis for our literature analysis.

The analysis results confirmed the appropriateness of our framework and revealed directions for future research along the framework perspectives:

- Research focus: Future research should pay particular attention to the preparatory stages of an OSD initiative. In particular, relevant research questions might deal with decision-related aspects. Concerning the later OSD stages, the design and the development of project management tools and outcome measurement systems would be desirable. Across all stages, future OSD research should not only concentrate on the client point of view, but incorporate multiple points of view. The integration of different stakeholder perspectives might also enhance the robustness of research results. Moreover, future research should examine the special nature of nearshoring and captive offshoring. Based on such research, comparisons between different OSD variations could be drawn.

- Reference theory: OSD research papers frequently lack any theoretical foundation. Therefore, first of all, researchers should aim at building their OSD research studies on some kind of reference theory. In this context, particularly popular economic theories like agency theory (e.g., in terms of OSD project control) and 
transaction cost theory (e.g., in terms of OSD project decision) still offer a high potential for future research.

- Research approach: Due to the current focus on positivist research, a stronger emphasis on (qualitative) interpretive research would be eligible. This might also contribute to an increasing diffusion of OSD articles in European journals. Here, beside case study research, the application of other qualitative methods (e.g., action research or grounded theory) as well as design research approaches should be taken into account.

In summary, the paper has significant implications for researchers. Most importantly, it clearly points to the need for further research on OSD. For instance, from the perspective of German client companies, a significant research backlog exists. By providing respective research results, researchers can support German firms in their efforts to effectively leverage OSD services.

When designing, implementing, and writing down an OSD research project, researchers should make sure that they provide an accurate description of the unit under study. Here, researchers may use our proposed OSD research framework to systematically classify and describe their study. This might contribute to significantly increase the trustworthiness of their research results (Guba and Lincoln 1985). Only by precisely understanding the research context, both other researchers and practitioners can correctly interpret and build on existing results.

\section{References}

Delmonte AJ, McCarthy RV (2003) Offshore software development: is the benefit worth the risk? In: Proc. of the 9th Am. Conf. on Inf. Sys. (AMCIS), Tampa.

Dibbern J, Goles T, Hirschheim R, Jayatilaka B (2004) Information systems outsourcing: a survey and analysis of the literature. The Data Base for Advances in Inf. Sys. 35(4):6-102.

Guba E, Lincoln Y (1985) Naturalistic inquiry. Sage, Beverly Hills.

King WR, Torkzadeh G (2008) Information systems offshoring: research status and issues. MIS Quarterly 32(2):205-225.

Krishna S, Sahay S, Walsham G (2004) Managing cross-cultural issues in global software outsourcing. Communications of the ACM 47(4):62-66.

Mertens P, Große-Wilde J, Wilkens I (2005) Die (Aus-)Wanderung der Softwareproduktion: Eine Zwischenbilanz. Friedrich-Alexander-Universität Erlangen-Nürnberg, Arbeitsberichte des Instituts für Informatik 38(3). 


\title{
Compliance im IT-Outsourcing
}

\section{Ermittlung von Einflussfaktoren und Entwicklung von Gestaltungsempfehlungen}

\author{
Kian Mossanen, Johannes C. Panitr, Michael Amberg \\ Lebrstubl für Wirtschaftsinformatik III, \\ Friedrich-Alexander-Universität Erlangen-Nürnberg
}

Dieser Artikel analysiert den Einfluss von Compliance auf das IT-Outsourcing und gibt Gestaltungsempfehlungen, wie dieser in IT-Outsourcing-Entscheidungsmodellen berücksichtigt werden kann. Die Ausführungen verstehen sich handlungsleitend für eine pragmatische Organisation von Compliance im ITOutsourcing.

Das explorative Vorgehen ist auf qualitative und quantitative empirische Erhebungen gestützt und mündet in einem Bezugsrahmen. Dieser in Abbildung 1 dargestellte Bezugsrahmen bildet durch die Angabe von Zielgrößen, Bedingungen und Aktionsparametern eine Gestaltungsgrundlage für die Berücksichtigung von Compliance im IT-Outsourcing.

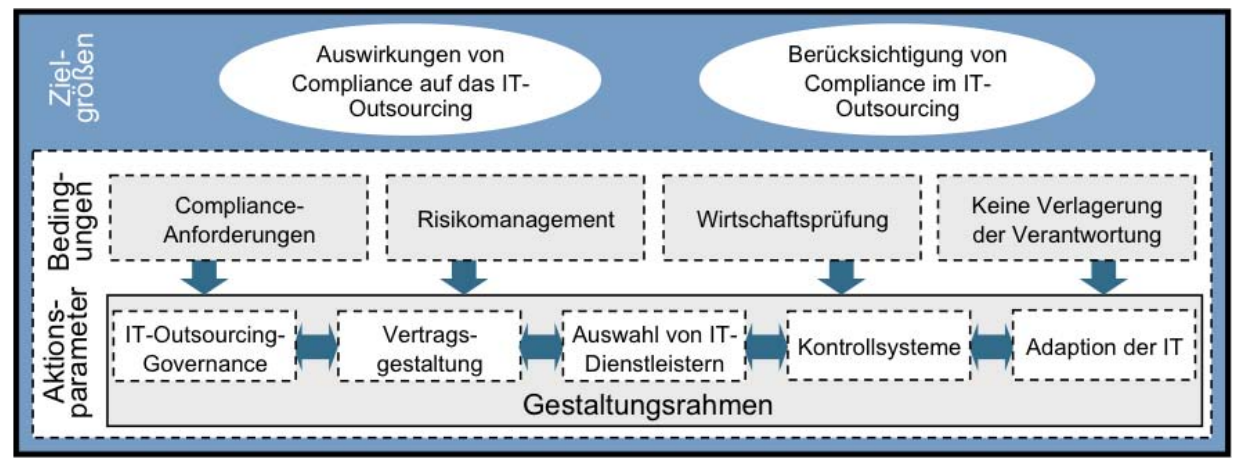

Abbildung 1: Bezugsrahmen zur Ermittlung der Auswirkungen von Compliance und Berücksichtigung von Compliance im IT-Outsourcing

Für die Aktionsparameter des Bezugsrahmens wurden Mechanismen entwickelt, welche für die Berücksichtigung von Compliance im IT-Outsourcing genutzt werden können. Abbildung 2 fasst diese in einem Gestaltungsrahmen zusammen. 


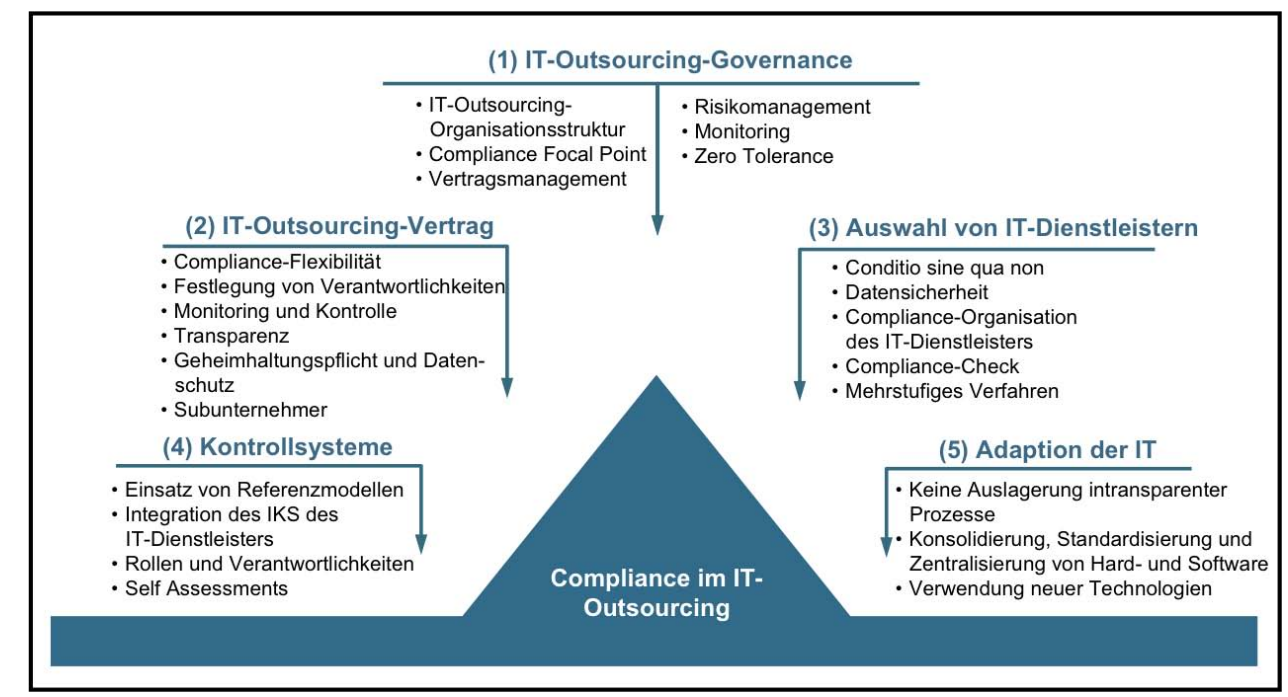

\section{Abbildung 2: Aktionsparameter und Mechanismen zur Berücksichtigung von Compliance im IT-Outsourcing.}

Durch die literaturbasierten Recherchen und die empirischen Erhebungen konnten für das IT-Outsourcing relevante Compliance-Anforderungen und entsprechende Gestaltungsempfehlungen identifiziert, bestätigt und besser eingeordnet werden. Diese Erkenntnisse sind Teil der Untersuchungen des Bezugsrahmens und lassen einen zusätzlichen Handlungsbedarf bei der Durchführung von IT-Outsourcing erkennen.

Die Ergebnisse zeigen, dass auch zukünftig eine intensive Auseinandersetzung in Wissenschaft und Praxis mit der Berücksichtigung von Compliance im ITOutsourcing, der Ermittlung der diskriminierenden Compliance-Faktoren, der Entwicklung von Gestaltungsempfehlungen sowie deren praktische Umsetzung erforderlich sein wird. 
IT Performance Management / IT-Controlling 



\title{
Vorwort zur Teilkonferenz
}

\section{IT Performance Management/IT-Controlling}

\author{
Ulrike Baumöl1', Walter Brenner², Falk Übernickel² \\ ${ }^{1}$ Lehrstubl für BWL, insh. Informationsmanagement, FernUniversität in Hagen \\ 2Institut für Wirtschaftsinformatik, Universität St. Gallen
}

\author{
Wir danken unserem Programmkomitee: \\ Gernot Dern, SEB AG \\ Ines Flügge, Airbus AG \\ Norbert Hoffmann, Swiss Life AG \\ Prof. Dr. Reinhard Jung, Universität St. Gallen \\ Prof. Dr. Lutz Kolbe, Georg-August-Universität Göttingen \\ Prof. Dr. Susanne Leist, Universität Regensburg \\ Thomas Nestke, gkv Informatik \\ Dr. Stefan Strecker, Universität Duisburg-Essen \\ Prof. Dr. Jan vom Brocke, Hochschule Liechtenstein \\ Prof. Dr. Rüdiger Zarnekow, Technische Universität Berlin
}

Unter dem Eindruck der Finanz- und Wirtschaftskrise und eines weithin erhofften baldigen Aufschwungs wächst erneut der Druck auf die Unternehmen, durch eine konsequente „Industrialisierung“ der Informationstechnologie (IT) weitere Kostensenkungspotenziale freizusetzen und gleichzeitig flexibler zu werden. Dazu kommen Methoden und Ansätze in verschiedenen Bereichen zum Einsatz, die inzwischen eine gewisse Reife erreicht haben, aber oftmals noch einen effizienten Steuerungsansatz vermissen lassen. Zu den Effizienztreibern gehören Architekturkonzepte, die Entwicklung und Integration von Software, der standardisierte Betrieb bzw. neue Betriebskonzepte, das Angebot von IT-Dienstleistungen, aber auch das Projektmanagement.

Inzwischen haben viele mittlere und große Unternehmen z. B. Serviceorientierte Architekturen (SOA) eingeführt. Dadurch stehen neben umfangreichen Erfahrungen zur Entwurfs- und Implementierungsphase nun auch Erkenntnisse aus dem Betrieb zur Verfügung. Zum einen wird damit beurteilbar, ob sich die erhoffte Flexibilisierung eingestellt hat, zum anderen sind erste fundierte Total cost of ownership-Betrachtungen (TCO) möglich.

Im Zuge der IT-Industrialisierung gewinnt Software as a Service (SaaS) weiter an Bedeutung, ermöglicht die Flexibilisierung der IT-Produktion und bewirkt das Entstehen von Kostensenkungspotenzialen. SaaS erfordert mit seiner weitreichen- 
den Verlagerung der Informationsverarbeitung nach Außen gleichzeitig aber auch neue Organisations- und Controllingkonzepte.

Ein weiterer prägender Trend im Bereich der IT-Industrialisierung ist die Virtualisierung. Die flexible Zuordnung von Applikationen zu verfügbaren Serverkapazitäten verspricht Kostensenkungen, bedingt aber auch neue Herausforderungen für das IT-Controlling.

Die in dieser Teilkonferenz vertretenen Beiträge spiegeln das Themenspektrum eindrücklich wieder.

Bartsch beschäftigt sich in seinem Beitrag mit einem Gestaltungsrahmen, der es erlaubt, den Wertbeitrag der IT zu erfassen. Aspekte der Steuerung der IT im Rahmen der IT-Governance nehmen zwei Beiträge auf: Marrone/Kolbe diskutieren den strategischen Beitrag von ITIL zum Business/IT-Alignment und Schwertsik/Wolf/Krcmar kümmern sich um die Prozesse und Strukturen für ein erfolgreiches Business/IT-Alignment. Die Leistungssteuerung der IT ist Thema bei Knapper/Conte/Blau; sie präsentieren einen Ansatz für die Definition von prozessorientierten Service Level Agreements. Drei Beiträge thematisieren zentrale Problemstellungen im IT-Controlling: Fischer/Aier entwickeln einen pragmatischen Ansatz für die IT-Projektkostenschätzung, Becker/Brüse/Pöppelbuß untersuchen die Anwendbarkeit von IT-Frameworks, wie z. B. ITIL, für das Softwarelizenzmanagement und Zumstein/Meier stellen einen Ansatz für ein Controlling von Websites vor, um das Marketing und Kundenbeziehungsmanagement im digitalen Umfeld zu unterstützen. Schließlich beschäftigen sich zwei Beiträge mit dem noch relativ neuen Betriebskonzept „Cloud Computing“: Strebel/Stage diskutieren ein Entscheidungsmodell für die Auswahl einer kostenoptimierten Versorgung mit Betriebsdienstleistungen. Martens/Teuteberg stellen in ihrem Beitrag Überlegungen zur Bewertung von Backsourcing-Entscheidungen an. 


\title{
An economic decision model for business software application deployment on hybrid Cloud environments
}

\author{
Jörg Strebel1, Alexander Stage ${ }^{2}$ \\ ${ }^{1}$ Institute of Information Systems and Management (IISM), Information \& \\ Market Engineering (IM), Karlsrube Institute of Technology \\ 2Internet-based Information Systems (IBIS), Department of Informatics, \\ Technische Universität München
}

Large-scale, virtualization-based distributed computing environments have emerged during the last couple of years providing scalable computing resources as a service. In the past, a main challenge in operating corporate data centers has been and still are resource demand fluctuations, recurring patterns at various time scales as well as trends and shifts in application resource demands (Urgaonkar et al. 2008; Gmach et al. 2008). In dedicated hosting environments these phenomena often required peak demand oriented capacity planning in order to prevent resource shortages, which in turn lead to low average resource utilization levels. Even in virtualized data centers that allow for workload consolidation, correlated bursts or regular peak demand periods require the acquisition and maintenance of large pools of physical servers. Enterprises that aim at lowering overall data center costs, which include (but are not restricted to) hardware acquisition, opportunity, energy, and labor costs for administration, can utilize this new breed of scalable application hosting platforms in order to achieve cost reductions. In order to compensate for peak demands or whenever the overall data center workload exceeds locally available resource capacities, workloads can be evicted from the local data centers by offloading virtual machines into public IaaS (Infrastructure-as-a-Service) clouds. However, the existence of various pricing and tariff models renders capacity planning and management decisions difficult, even in highly transparent infrastructure markets. On a strategic level, decision makers are confronted with the problem of selecting economically beneficial providers and virtual machine tariffs, while on an operational level the workloads to evict and the assignment of appropriate virtual machine types needs to be done in a way that conforms with the tariff and provider selection outcomes. Both problems are highly interdependent and require sophisticated models for making efficient decisions. Consequently, we propose a TCO-based integrated decision model for selecting virtual machine tariffs, types and workloads to evict from local data centers that aim at economic efficiency. The 
decision model consists of a mixed-integer linear programming formulation for the cost-minimal assignment of virtual machines to computing resources and of a linear regression model to identify the most important decision factors for the selection of suitable workloads.

The model was tested on a real-world business scenario where a number of SAP ERP applications were targeted for outsourcing. Based on experimental results, it can be stated that the number of applications available for Cloud computing positively impacts IaaS usage more than any other variable. Interestingly, the average CPU load across all business applications has a negative effect on IaaS usage. This outcome could be explained with the higher consolidation potential that a growing number of virtualized applications possesses (i.e. the more applications are available for internal server assignment, the better those internal servers can be utilized). The average amount of storage is positively correlated to IaaS usage. This outcome seems sensible, as modern ERP systems usually have rather demanding storage requirements compared to other applications and as the IaaS storage cost is assumed to be smaller than the storage cost in the corporate data center. However, as the scenario shows, using external resources is mainly beneficial for scenarios in which a great number of applications with low computing requirements and high storage requirements need to be outsourced.

Our model can be used to determine, given internal cost structures, an optimal mix of inhouse and external resources for a given planning period. The method is helpful for both optimizing the internal and the external deployment of business applications; it can be set up using data that is readily available in the enterprise (system traces, internal cost figures). A careful analysis of a larger number of business scenarios and a larger number of IT resource providers has to be conducted in order to verify those findings.

\section{References}

Gmach, D, Krompass S, Scholz A, Wimmer, Kemper A (2008) Adaptive quality of service management for enterprise services. ACM Transactions on the Web (TWEB) 2(1):1-46.

Urgaonkar B, Shenoy P, Chandra A, Goyal P, Wood T (2008) Agile dynamic provisioning of multi-tier Internet applications. ACM Trans. Auton. Adapt. Syst. 3(1): 1-39. 


\title{
Understanding IT Governance
}

\section{Towards Dimensions for specifying Decision Rights}

\author{
Andreas Roland Schwertsik, Petra Wolf, Helmut Krmar \\ Lehrstubl für Wirtschaftsinformatik (117), Technische Universität München
}

Typically IT decisions are of enterprise wide importance as IT increasingly supports business processes. On the one hand Information Systems (IS) are part of complex social and organizational processes (Serafeimidis/Smithson 2003), on the other hand process orientation and service orientation (Grohmann 2003; Meyer/Zarnekow/Kolbe 2003) require more and more effort regarding integration and coordination between different parts of an organization. The more complex the coordination setting gets the more problems and challenges regarding interfaces emerge. An overall augmentation regarding the significance of IT governance can be observed as effective IT governance can help to cope with these challenges (Weill/Ross 2004).

Whilst the importance of alignment and the supporting role of IT governance to reach alignment are widely accepted, existing literature focuses rather on the outcome of IT governance - IT/Business alignment - than on the underlying structures and processes. As a consequence, the described types of governance remain rather generic. How exactly they are implemented in practice is not known.

This paper derives dimensions for specifying decision rights from a literature review in order to contribute to a deeper understanding of structures and processes of IT governance. It is guided by the following research question: What dimensions need to be taken into account for specifying decision rights?

Conducting the literature review we built on the notion that previous information systems (IS) research on IT governance can be classified according to two major research streams: IT Governance Forms on the one hand and IT Governance Contingency Analysis on the other hand (Brown/Grant 2005). As a consequence we divided our review in these two streams, too. In addition we searched for literature on business/ IT alignment which we found to be the source of the ongoing centralization-decentralization debate. Building on the results of this literature review we propose a classification of IT governance dimensions in IT/ Business alignment, contingency factors, approach, and structures and processes. The four dimensions must be taken into account and can be considered by the following questions: 
1. IT/business alignment: What culture and what requirements influence the relationship between business and IT?

2. Contingency factors: What surrounding conditions and contingency factors characterize the environment of IT governance?

3. Approach: What concepts build the foundations of IT governance, central, federal, or decentral?

4. Structures and Processes: What governance methods, instruments, and mechanisms are implemented?

\section{References}

Brown, A.E.; Grant, G.G. (2005): Framing the Frameworks: A Review of IT

Governance Research. In: Communications of the Association for Information Systems, Vol. 15 (2005) Nr. 38, p. 696-712.

Grohmann, H.H. (2003): Prinzipien der IT-Governance. In: HMD Praxis der Wirtschaftsinformatik, Vol. 2003 (2003) Nr. 232, p. 17-23.

Meyer, M.; Zarnekow, R.; Kolbe, L. (2003): IT-Governance: Begriff, Status quo und Bedeutung. In: Wirtschaftsinformatik, (2003) Nr. 45, p. 445-448.

Serafeimidis, V.; Smithson, S. (2003): Information systems evaluation as an organizational institution - experience from a case study. In: Information Systems Journal, (2003) Nr. 13, p. 251-274.

Weill, P.; Ross, J.W. (2004): IT governance on one page. In: MIT Sloan

Management CISR, (2004) Nr. Working Paper Nr. 349 and Sloan Nr. 4516-04. 


\title{
PROSA - Process-Oriented Service Level Agreements for Providing a Single Point of Contract $^{1}$
}

\author{
Rico Knapper', Tobias Conte ${ }^{1}$, Benjamin Blau', Hans Richter ${ }^{2}$ \\ ${ }^{1}$ Institute of Information Systems and Management (IISM), \\ KIT Karlsrube Institute of Technology \\ ${ }^{2} \mathrm{EnBW}$ Energie Baden-Württemberg $A G$
}

The fundamental paradigm shift from a product- to a service-oriented economy implies novel technical and organizational challenges. The value generated by a service is mainly represented by intangible elements exposed at execution (Hill 1977). Therefore, a service consumer expects a service to function reliably and to deliver a consistent outcome at a variety of levels, i.e. Quality of Service (QoS). QoS is the key criterion to keep the business side competitive as it has serious implications on the provider and consumer side (Papazoglou 2008).

From a business perspective, QoS characteristics defined based on technical services within the infrastructural layer have to be aggregated to more businessrelevant Key Performance Indicators (KPIs) (Blake and Cummings 2007; Jaeger et al. 2004; Unger et al. 2008). An agreement between service provider and service consumer about the quality to be delivered must be founded on a legal basis, i.e. by specifying a Service Level Agreement (SLA). It defines service characteristics and the quality to be delivered by the provider i.e. Service Level Indicators (SLIs), Service Level Objectives (SLOs) and monetary penalties in case of non-performance. The situation today requires that companies agree on multiple SLAs that specify the SLOs for each business service as depicted in Figure 1.

In this paper we provide a comprehensive methodology for implementing Process-oriented Service Level Agreements (PROSA) which enables a single point of contract for service requesters while significantly reducing the managerial overhead generated from multiple contractual relations.

Consequently, the big picture of PROSA is a two-sided approach with the involvement of the customer as well as the provider of the services. Provider-sided we introduce a methodology to aggregate non-functional aspects of Services as exemplified by the SLI "maximum downtime". On customer-side, we recommend the adoption of service value networks (SVN).

\footnotetext{
${ }^{1}$ We thank Oliver Schäfer and Jürgen Väth from EnBW AG for their significant contribution to the presented methodology.
} 


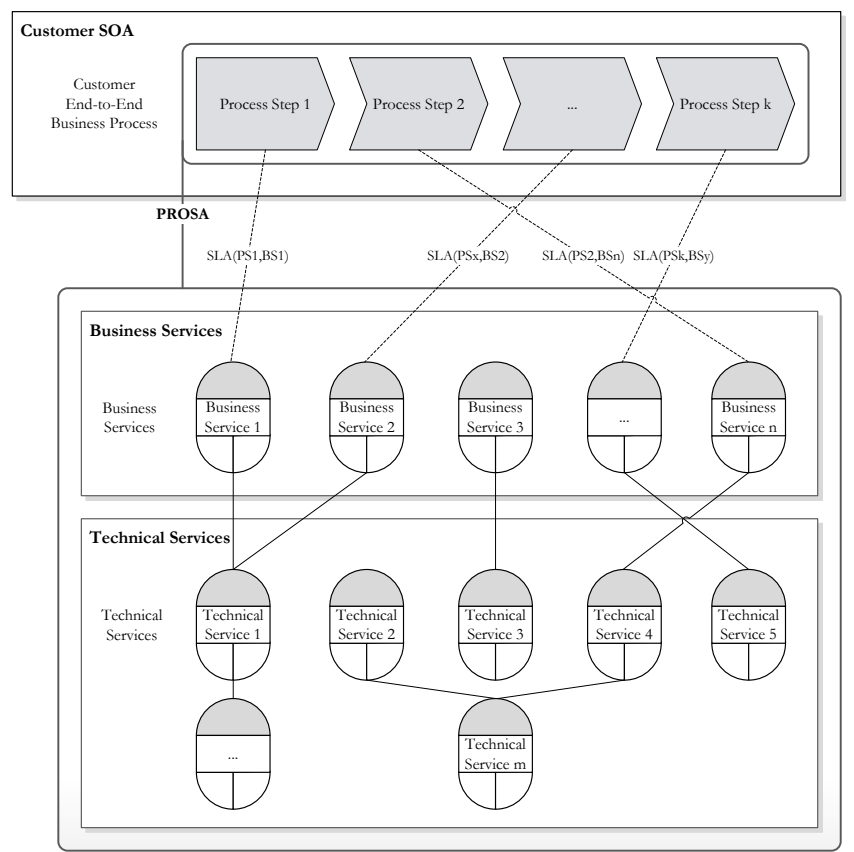

Figure 1: Legal framework for flexible binding of external services.

Our approach enables a single point of contract for service requesters and significantly reduces the managerial overhead generated from multiple SLAs. We evaluated our methodology by providing an industrial case study from the EnBW AG.

\section{References}

Blake, M. B., Cummings, D. J. (2007) Workflow Composition of Service Level

Agreements. IEEE International Conference on Services Computing, 138-145.

Hill, T. P. (1977) On Goods and Services. Review of Income and Wealth. 23 (4): 315-338.

Jaeger, M. C., Rojec-Goldmann, G., Muhl, G. (2004) Qos aggregation for web service composition using workflow patterns. In: Proceedings of the 8th IEEE International Enterprise Distributed Object Computing Conference, 149-159.

Papazoglou, P. (2008) Web Services: Principles and Technologies. Prentice Hall. Unger, T., Leymann, F., Mauchart, S., Scheibler, T. (2008) Aggregation of Service Level Agreements in the Context of Business Processes. In: Proceedings of the Enterprise Distributed Object Computing Conference, 43-52. 


\title{
Ein konzeptionelles Framework zum Verständnis des multidimensionalen Gegenstandes des Wertbeitrags der IT
}

\author{
Stefan Bartsch ${ }^{1}$, Daniel Schlagwein ${ }^{2}$ \\ ${ }^{1}$ Institut für Wirtschaftsinformatik, Philipps-Universität Marburg \\ 2 Seminar für Wirtschaftsinformatik und Informationsmanagement, \\ Universität ₹u Köln
}

Der mit dem Einsatz von Informations- und Kommunikationssystemen zusammenhängende Wertbeitrag wird bereits seit Beginn der Achtziger Jahre diskutiert (Pfeifer 2003, S. 45). Trotz der langjährigen Auseinandersetzung hat sich bisher kein klares Verständnis des Wertbeitrags der IT herausgebildet (Stewart 2003, S. 16). Es zeigt sich, dass sowohl in der Theorie als auch in der Praxis unterschiedliche Annahmen über die Wirkungsweise des IT-Einsatzes existieren, die unter dem Sammelbegriff des Wertbeitrags der IT diskutiert werden. In dem Beitrag wird der Begriff des Wertbeitrags der IT ausgehend von einem abstrakten und einem betriebswirtschaftlichem Wertbegriff hergeleitet und Dimensionen, in denen sich die Sichtweisen des Wertbeitrags der IT unterscheiden, identifiziert und diese in einem Framework integriert.

Auf Basis eines abstrakten Wertbegriffs werden zunächst der enge Zusammenhang von Zielen und die inhärente Subjektivität des Wertbegriffes herausgearbeitet, welche die unterschiedlichen Sichtweisen des Wertbeitrags rechtfertigt.

Im betriebswirtschaftlichen Zusammenhang lässt sich der Wertbegriff als Beitrag zur Erreichung von Unternehmenszielen auffassen, welche durch sämtliche Stakeholder motiviert sein können und sowohl monetäre, als auch qualitative Ziele umfasst. In diesem Zusammenhang stellt Wertbeitrag einen Input-OutputTransformationsprozess dar, dessen Generierung durch die Komponenten (1) Ressource, (2) Leistungserstellungsprozess und (3) Ziel beschrieben werden kann.

Überträgt man den diskutierten betriebswirtschaftlichen Wertbegriff auf die IT, so lässt sich Wertbeitrag als Beitrag zur Erreichung von Unternehmenszielen auffassen, welcher durch den Einsatz von IT Ressourcen in der IT Organisation erreicht wird. Diese Auffassungen ist zwar universell, für ein genaues Verständnis jedoch zu wenig greifbar und bedarf einer Konkretisierung.

Analog zum betriebswirtschaftlichen Wertbegriff lässt sich der Wertbeitrag der IT durch die Komponenten (1) IT Ressourcen, (2) IT Organisation und (3) IT Ziel beschreiben. Die IT Ressourcen und die IT Organisation stellen in dem Zusammenhang Steuerungsobjekte dar, für die eine Strukturierung vorgeschlagen wird. Die IT Organisation wird in Ablauf- und Aufbauorganisation gegliedert. Ziele der 
IT können unmittelbare (IT interne) Ziele sein, die sich aus den Anforderungen des Business ergeben oder mittelbare Geschäftsprozess- und Unternehmens-Ziele. Die identifizierten Aspekte des Wertbeitrags der IT sind zusammengefasst in dem Framework in Abb. 1 dargestellt.

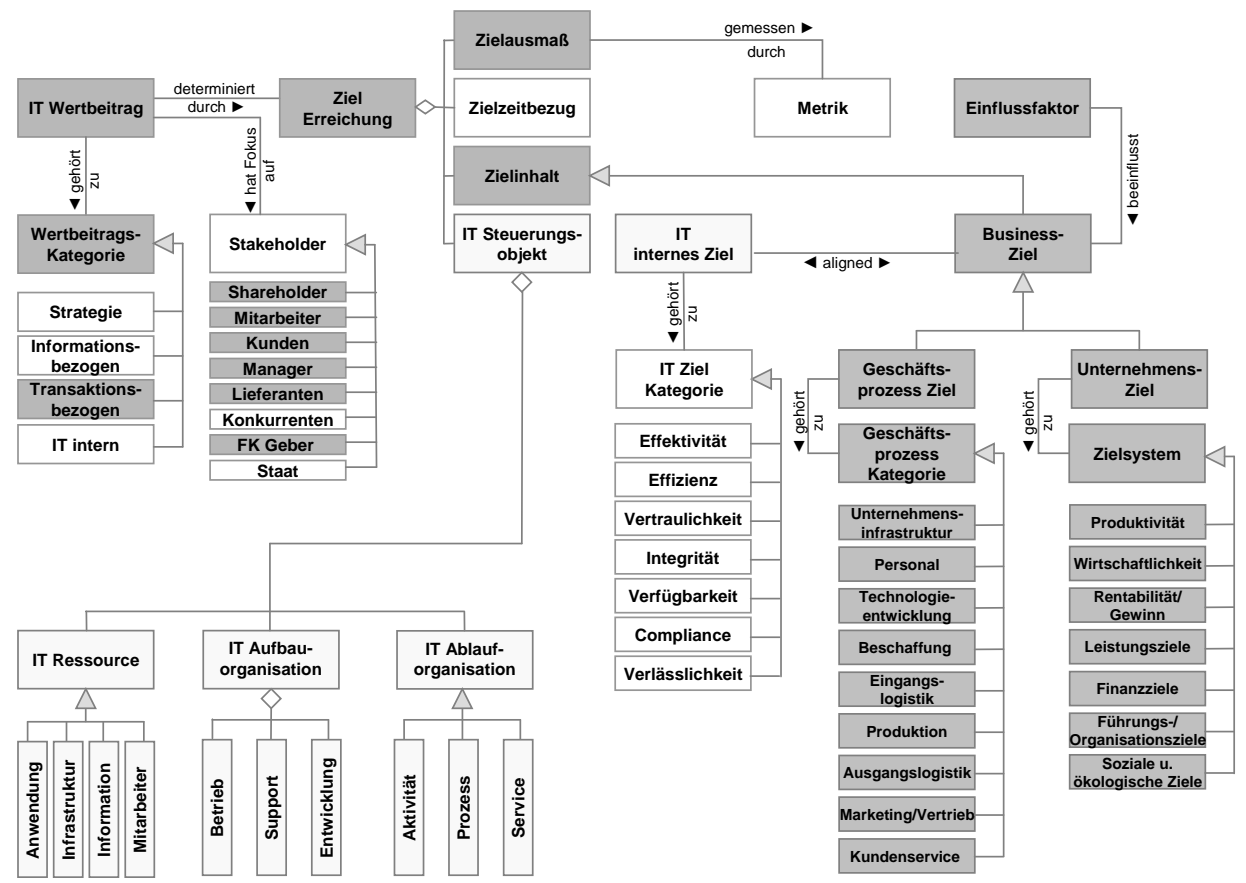

Abbildung 1: Framework zur Klassifikation des Wertbeitrags der IT

\section{Literatur}

Pfeifer A (2003) Zum Wertbeitrag von Informationstechnologie. http://www.opus-bayern.de/uni-passau/volltexte/2004/34/pdf/

PfeiferAndreas.PDF. Abruf am 2006-05-08.

Stewart RA (2003) Lifecycle management of information technology (IT) projects in construction. http:/ /www4.gu.edu.au:8080/adt-root/uploads/approved/ adt-QGU20030423.122317/public/04Chapter5-6.pdf. Abruf am 2009-01-29. 


\title{
Unterstützung des Softwarelizenzmanagements durch IT-Frameworks
}

\author{
Anforderungsanalyse und Status quo \\ Jörg Becker, Bernhard Brüse, Jens Pöppelbuß \\ European Research Center for Information Systems, \\ Westfälische Wilhelms-Universität Münster
}

Unternehmen sind auf die Unterstützung durch eine Vielzahl verschiedener Software angewiesen und verfügen über dementsprechend viele Lizenzen. Damit das IT-Management bei einer komplexen Softwarelandschaft nicht den Überblick über die Masse an vorhandenen Lizenzen verliert, ist ein effektives Softwarelizenzmanagement erforderlich. Dieses beginnt bereits bei der an den Geschäftsbedürfnissen ausgerichteten Bedarfsplanung und endet bei der Verwertung von Lizenzen am Ende der Einsatzdauer im Unternehmen (Bayer 2009, S. 17).

Ziel des Softwarelizenzmanagements in Unternehmen ist es vor allem, für eine rechtliche Absicherung des Unternehmens beim Einsatz von Software sowie für eine kosteneffiziente Ausrichtung der IT zu sorgen. Unternehmen fällt es häufig schwer, die genau richtige Anzahl an Lizenzen zu beschaffen. Laut einer Umfrage unter 239 deutschen Unternehmen sehen sich 18,9\% unterlizensiert und 27,0\% vermuten eine Überlizensierung (Niemann 2009, S. 19). Zur Unterstützung setzen bereits viele Unternehmen Software-Tools und Lizenzmanagement-Suites ein. Technische Unterstützung allein reicht jedoch nicht, sondern es bedarf i. d. R. auch organisatorischer Umstellungen und der Einführung neuer Prozesse. Zur Ausgestaltung der Prozesse im IT-Management erfreuen sich IT-Frameworks wie z. B. die IT Infrastructure Library (ITIL) großer Beliebtheit. Dieser Beitrag untersucht, ob diese Frameworks auch das Softwarelizenzmanagement als eine spezielle Aufgabe des IT-Managements geeignet abbilden.

Um zunächst die Anforderungen von Unternehmen an das Softwarelizenzmanagement zu ermitteln, wurden problemzentrierte Interviews mit Vertretern des IT-Managements geführt. Es erklärten sich insgesamt fünf als Experten anzusehende Personen aus unterschiedlichen Unternehmen für diese Interviews bereit. Als Ergebnis dieser Expertenbefragung lassen sich acht Anforderungen (A1 bis A8; vgl. Tabelle 1) an das Softwarelizenzmanagement ableiten.

Die Experten der befragten Unternehmen unterschiedlicher Branchen sind sich einig, dass eine umfassende Auseinandersetzung mit dem Management von Softwarelizenzen notwendig ist. Dabei ist die Vermeidung von Unterlizenzierung der größte Treiber. Diejenigen Teilnehmer, die bereits ein Softwarelizenzmanage- 
ment eingeführt haben oder derzeitig eine Einführung vornehmen, berichten von der großen Herausforderung der strategischen und organisatorischen Einbettung des Lizenzmanagements im Unternehmen.

Tabelle 1: Gegenüberstellung der untersuchten IT-Frameworks

\begin{tabular}{|l|c|c|c|c|}
\hline Hauptaussagen der Experten & ITIL & CobiT & $\begin{array}{c}\text { CMMI- } \\
\text { ACQ }\end{array}$ & SAM \\
\hline $\begin{array}{c}\text { A1. Vermeidung von Unterlizenzierung } \\
\text { und Reduzierung der Kosten }\end{array}$ & $(\checkmark)$ & $\checkmark$ & $(\checkmark)$ & $\checkmark$ \\
\hline A2. Eigenständige Einführung & & $(\checkmark)$ & $(\checkmark)$ & $\checkmark$ \\
\hline A3. Bildung eines Kompetenzteams & & & & \\
\hline A4. Standardisierter Beschaffungsprozess & $\checkmark$ & $\checkmark$ & $\checkmark$ & $\checkmark$ \\
\hline A5. Akzeptanz bei Personal maximieren & & & & \\
\hline A6. Regelmäßiges Lizenzcontrolling & & $\checkmark$ & $\checkmark$ & $(\checkmark)$ \\
\hline A7. Produktspez. Verantwortlichkeiten & $(\checkmark)$ & $\checkmark$ & $(\checkmark)$ & $\checkmark$ \\
\hline A8. Unterstützung durch ein Werkzeug & $\checkmark$ & $\checkmark$ & & $\checkmark$ \\
\hline
\end{tabular}

Um das Unterstützungspotenzial von IT-Frameworks zur Bewältigung dieser Herausforderungen herauszuarbeiten, werden folgende näher analysiert: ITIL, Control Objectives for Information and Related Technology (CobiT), Capability Maturity Model Integration for Acquisition (CMMI-ACQ) und Software Asset Management (SAM). Der Grad der Erfüllung der acht Anforderungen durch die vier Frameworks fällt unterschiedlich aus. Lediglich das SAM betrachtet das Softwarelizenzmanagement in einem Maße, dass sich dessen Benutzung für diesen speziellen Aspekt uneingeschränkt empfiehlt. Hieraus lässt sich ableiten, dass, trotz der Möglichkeit verschiedene IT-Frameworks (bspw. ITIL und SAM) zu kombinieren, die populären Frameworks das Softwarelizenzmanagement stärker berücksichtigen und diesbezüglich erweitert werden sollten.

\section{Literatur}

Bayer M (2009) Zwischen Diebstahl und Verschwendung. Computerwoche Nr. 34/35: 16-17.

Niemann F (2009) Anwender kritisieren wirre Lizenzbedingungen.

Computerwoche Nr. 34/35: 18-19. 


\title{
IT-Projektkostenschätzung - Ein pragmatischer Ansatz
}

\author{
Christian Fischer, Stephan Aier \\ Institut für Wirtschaftsinformatik, Universität St. Gallen
}

Kostenschätzung für IT-Projekte ist ein vergleichsweise lang behandeltes Thema, das in der Vergangenheit eine breite Spanne an Schätztechniken hervorgebracht hat (Jørgensen und Shepperd 2007). Nichtsdestotrotz zeigen regelmäßig durchgeführte Studien, dass beispielsweise im Jahr $200844 \%$ aller IT-Projekte nicht rechtzeitig, nicht im vorhergesehen Budget und/oder mit geringerem Umfang beendet wurden (Standish Group 2009). Das ist ein Hinweis dafür, dass Schätzungen für IT-Projektkosten und -dauer oft zu niedrig ausfallen. Erklärungshinweise dafür liegen in den Tatsachen, dass in der Praxis kaum modellbasierte Schätztechniken eingesetzt werden, dass andererseits aber $83 \%$ der wissenschaftlichen Publikationen modellbasierte Schätztechniken fokussieren (Jørgensen 2004) und nur $16 \%$ der Publikationen organisatorische Probleme in Zusammenhang mit Kostenschätzungen berücksichtigen (Jørgensen und Shepperd 2007).

Trotz ihres großen Aufwands bei der Parametrisierung haben modellbasierte Schätztechniken im Vergleich zu Expertenschätztechniken den Vorteil, dass der Schätzer bei modellbasierten Techniken nur die Ausprägung der einzelnen Kriterien rechtfertigen muss, bei Expertenschätztechniken hingegen kann er nur auf seine Erfahrung verweisen. Dies betrifft besonders die Einschätzung von Unsicherheiten, für die ein Aufschlag in der Schätzung eingeplant wird.

In diesem Beitrag gehen wird der Frage nach, wie sich die beschriebenen Vorteile einer modellbasierten Kostenschätzung mit denen einer Expertenschätzung kombinieren lassen. Das Ergebnis ist eine Technik die in einer Fallstudienorganisation entwickelt und eingeführt wurde. Aufgrund der Unzufriedenheit mit der bisher angewandten Schätztechnik wurde ein erfahrener IT-Projektleiter aus der Organisation mit der Konstruktion einer präziseren Schätztechnik beauftragt und von den Autoren begleitet. Im Ergebnis hat der Projektleiter der Organisation eine Dokumentenvorlage mit sechs Kriterien entwickelt und in einer Tabellenkalkulation implementiert, aufgrund derer ein Aufschlag auf die Schätzung der Projektkosten (ohne Aufschläge) bestimmt werden soll. Grundsätzlich wird auf jede Schätzung ohne Aufschlag ein Aufschlag von $5 \%$ berechnet. Durch die einzelnen Unsicherheitsfaktoren erhöht sich der Aufschlag jeweils um einen bestimmten Prozentsatz, z. B. bei einem sehr großen Projekt um $50 \%$ auf $7.5 \%$. Wenn für alle Kriterien die Ausprägung mit dem höchsten Aufschlag ausgewählt wird, wird insgesamt ein Aufschlag von $28.4 \%$ auf die Kostenschätzung ohne Aufschlag gemacht. Die konkreten Aufschläge finden sich in Tabelle 1. Die Auswahl der Di- 
mensionen und Dimensionsausprägungen erfolgte aufgrund von Interviews mit Projektleitern. Zur genaueren Bestimmung der Aufschlaghöhe plant die Organisation eine Nachkalkulation von zwei beendeten Projekten.

Tabelle 1: Aufschläge auf IT-Projekte

\begin{tabular}{|l|l|l|}
\hline Dimension & Ausprägung & Aufschlaghöhe \\
\hline Projektgröße & 5 Größen & Bis zu $50 \%$ \\
\hline Anzahl beteiligter Applikationen & Numerisch & Bis zu $50 \%$ \\
\hline Projektkomplexität & 3 Grade & Bis zu $50 \%$ \\
\hline Abhängigkeit zu anderen Projekten & 4 Grade & Bis zu $40 \%$ \\
\hline Erfahrung der Schlüssel-Personen & 4 Grade & Bis zu 20\% \\
\hline Bekanntheitsgrad der Anforderungen & 5 Grade & Bis zu $40 \%$ \\
\hline
\end{tabular}

Ein besonderer Schwerpunkt wurde auf die Technikeinführung in der Organisation gelegt, da hier typischerweise die Wirksamkeit der Schätzung stark beeinflusst wird. Abbildung 1 zeigt das Vorgehensmodell zur Einführung der Technik.

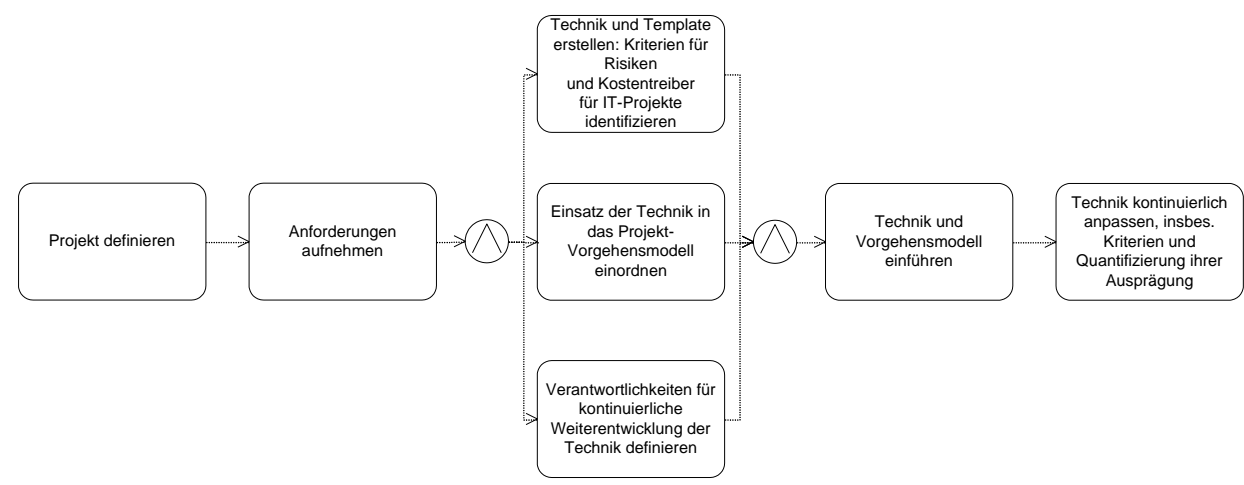

Abbildung 1: Vorgehensmodell der Einführungsmethode

\section{Literatur}

Jørgensen M (2004) A review of studies on expert estimation of software development effort. Journal Of Systems And Software 70(1-2):37-60.

Jørgensen M, Shepperd M (2007) A Systematic Review of Software Development Cost Estimation Studies. IEEE Transactions On Software Engineering 33(1):33-53.

Standish Group (2009) Chaos 2009 Summary and EPPM Study, Standish Group, West Yarmouth MA. 


\title{
Bewertung von Backsourcing-Entscheidungen im Umfeld des Cloud Computing
}

\author{
Ein System Dynamics Ansatz
}

\author{
Benediket Martens, Frank Teuteberg \\ Fachgebiet Unternehmensrechnung und Wirtschaftsinformatik, \\ Universität Osnabrück
}

Im Rahmen des vorliegenden Beitrags wird ein quantitatives Entscheidungsmodell auf Basis eines System Dynamics Ansatzes vorgestellt, welches Entscheider in die Lage versetzt, die Auswirkungen einer Backsourcing-Entscheidung im Umfeld des Cloud Computing im Hinblick auf zu erwartende Kosten und Service-Qualität im Zeitablauf zu simulieren. Auf der Basis von Break-Even- sowie Sensitivitätsanalysen ist es möglich, die Vorteilhaftigkeit von Backsourcing-Entscheidungen in unterschiedlichen Szenarien zu untersuchen. Für die Erstellung eines möglichst realistischen Modells und der Verwendung von realistischen Daten wurden neben einer Literaturanalyse zwei Experteninterviews mit IT-Dienstleistern durchgeführt. Für die Literaturanalyse wurden sogenannte A-Journals (wissenschaftlich hochwertige Journals) des WKWI-Rankings nach Stichwörtern im Bereich des IT-Outsourcings von Januar 2002 bis September 2009 durchsucht. Aus dieser Literaturbasis wurden Arbeiten aus dem Bereich des IT-Backsourcing ausgewählt und bspw. im Hinblick auf identifizierte Einflussfaktoren des Backsourcing und Evaluierung der Ergebnisse analysiert. Die Literaturanalyse zeigt, dass die Untersuchung des Backsourcing bisher auf einer eher qualitativen Ebene durchgeführt wurde. Besonders häufig sind Einflussfaktoren auf das Backsourcing untersucht worden. Es wird deutlich, dass in den identifizierten Beiträgen keine quantitativen Datenanalysen oder Simulationen durchgeführt wurden, um weitere Erkenntnisse über das Backsourcing gewinnen zu können.

Das Ziel der Modellierung und Simulation des Backsourcing-Modells im vorliegenden Beitrag ist die Ermittlung der Vorteilhaftigkeit der internen oder externen Leistungserstellung eines Cloud Computing Services unter Berücksichtigung einer Backsourcing-Entscheidung. Als zu untersuchende Cloud Computing Services werden in dem Modell die Bereitstellung von Speicherplatz und Rechenleistung untersucht. In Abbildung ist die Modellübersicht dargestellt. Zur Veranschaulichung und Vereinfachung wurde in dem Modell mit zentralen Kennzahlen (Backsourcing- und Cloud Computing (CC)-Kennzahl) und Schattenvariablen (grau hinterlegt) gearbeitet. 


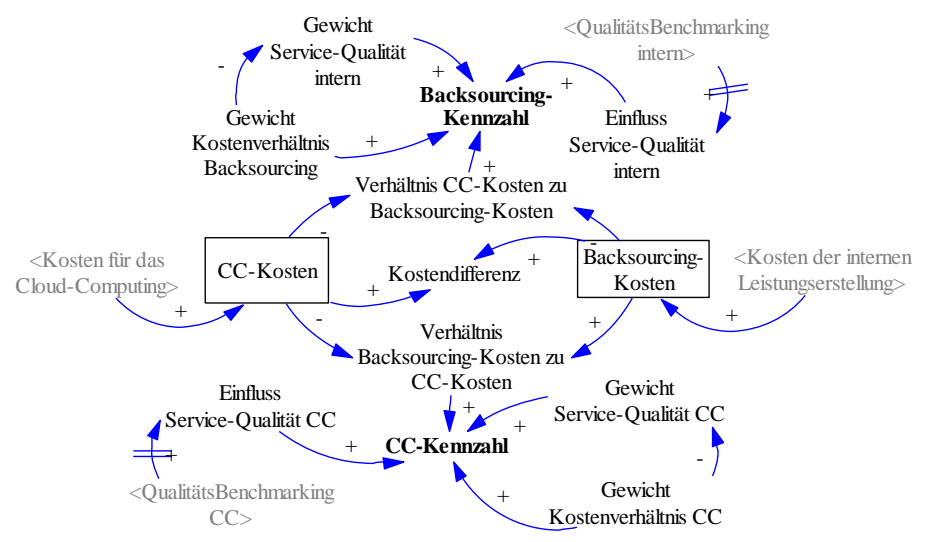

\section{Abbildung 1: Modellübersicht}

Für die Simulation des Modells wurden auf Basis der Experteninterviews vier realistische Szenarien entwickelt, die über einen Zeitraum von 10 Jahren simuliert werden. Mit Hilfe der Szenarien soll ermittelt werden, ob bzw. ab wann Cloud Computing Services bezogen werden sollten oder ob Backsourcing betrieben werden sollte.

In dem vorliegenden Beitrag wurde ein flexibler sytemdynamischer Ansatz für Backsourcing-Entscheidungen im Umfeld des Cloud Computing vorgestellt. Das vorgestellte und mit der Software Vensim implementierte Modell ermöglicht es, Entscheidungsprozesse im Backsourcing und deren Auswirkungen im Zeitablauf für unterschiedliche Szenarien zu prüfen. Es ist anzumerken, dass die Realität im Bereich Backsourcing in Cloud Computing Umgebungen um ein Vielfaches komplexer ist als dies mit dem Modell abgebildet werden konnte. Gemäß des KISS ("keep it simple and stupid")-Ansatzes stand jedoch im Vordergrund, ein erstes System Dynamics Modell als Basis zu entwickeln, welches in weiteren Schritten noch detaillierter ausgestaltet werden kann. In dem hier vorgestellten Modell wurden bestimmte Faktoren und Risiken von Cloud Computing Umgebungen (noch) nicht berücksichtigt, wie z. B. Aspekte des Datenschutzes, der Privatsphäre oder der Datensicherheit. 


\title{
Providing More than Just Operational Benefits: An Empirical Research
}

\author{
Mauricio Marrone, Lutz. M. Kolbe \\ Chair of Information Management, University of Göttingen
}

Academic research on ITIL is still in its early stages despite its numerous appearances in the popular press and practitioners' magazines. Existing academic literature merely presents the description of the areas documented on ITIL (e.g. Cervone 2008) or analyzes adopters of ITIL through case studies (e.g. Hochstein et al. 2005; Kießling et al. 2009). A summary of benefits of ITIL, which have been uncovered by research, is shown in Table 1 .

Table 1: Summary of Benefits of ITIL

\begin{tabular}{l|ccccc}
\hline Improvement of... & $\begin{array}{c}\text { Hochstein et } \\
\text { al., 2005 }\end{array}$ & $\begin{array}{c}\text { Potgieter et } \\
\text { al., 2005 }\end{array}$ & $\begin{array}{c}\text { Kießling et } \\
\text { al., 2009 }\end{array}$ & $\begin{array}{c}\text { Cater-Steel } \\
\text { et al., 2008 }\end{array}$ & $\begin{array}{c}\text { Cervone, } \\
2008\end{array}$ \\
\hline $\begin{array}{l}\text { Service Quality } \\
\text { Standardization of }\end{array}$ & $\mathrm{X}$ & $\mathrm{X}$ & $\mathrm{X}$ & $\mathrm{X}$ & $\mathrm{X}$ \\
Service & & $\mathrm{X}$ & $\mathrm{X}$ & $\mathrm{X}$ & \\
$\begin{array}{l}\text { Customer } \\
\text { Satisfaction }\end{array}$ & & & $\mathrm{X}$ & $\mathrm{X}$ & $\mathrm{X}$ \\
$\begin{array}{l}\text { Return on } \\
\text { Investment }\end{array}$ & & & $\mathrm{X}$ & $\mathrm{X}$ \\
$\begin{array}{l}\text { Reduction of } \\
\text { Downtime } \\
\text { Best Practice }\end{array}$ & & $\mathrm{X}$ & & & \\
Financial & & & $\mathrm{X}$ & \\
Contribution Control & & & & $\mathrm{X}$ & \\
Call Fix Rate & & & $\mathrm{X}$ & \\
Morale of IT & & & & &
\end{tabular}

An increase of benefits at the operational level is necessary to achieve superior profitability, but it is usually not sufficient. The reason for this is that the usage of best practices such as ITIL can be quickly imitated. The higher the usage of best practices, the more similar the companies are. Therefore, as proposed by Porter (1996), improvements created by best practice do not lead to improvements for any organization. Porter argues that a company should focus on both the operational effectiveness and the strategic positioning.

Therefore, in this research we focus on ITIL and the benefits it provides to the IT department, specifically the operational effectiveness and strategic positioning. The research concentrates on the Business-IT alignment because it helps to sup- 
port the services provided by IT to meet the needs of the business. This may provide the organization with a sustainable competitive advantage.

An international survey of 503 firms was conducted to examine operational benefits provided by ITIL, as well as the impact that ITIL may have on the Business-IT interaction. Statistical tests such as Kruskal-Wallis and post hoc MannWhitney $\mathrm{U}$ tests were applied. Further and to understand the underlying trends, the Jonckheere-Terpstra tests were also used.

Four principal observations from the study show that as the maturity of ITIL increases 1) the perception of the level of Business-IT alignment also increases, 2) so does the number of realized benefits, 3) the usage of metrics to measure the benefits of the implementation as well as 4) the number of benefits provided by IT that are acknowledged by the business.

Since ITIL may have a direct impact on the strategic positioning through Business-IT alignment, this results in a possible positive effect on business performance, competitive advantage and increased profitability. As well, there is an indirect positive impact from the usage of metrics and in the business acknowledging the benefits provided by IT on strategic positioning. It can be concluded that ITIL may contribute to both, the operational effectiveness as well as the strategy positioning of the IT department.

\section{References}

Cater-Steel A, Tan WG, Toleman M (2008) itSMF Australia 2007 Conference: summary of ITSM standards and frameworks survey responses.

Cervone F (2008) ITIL: a framework for managing digital library services. OCLC Systems \& Services 24:87-90.

Kießling M, Marrone M, Kolbe LM (2009) Influence of IT Service Management on Innovation Management: First insights from exploratory studies. In: itAIS 2009 VI Conference of the Italian Chapter of AIS.

Hochstein A, Tamm G, Brenner W (2005) Service-Oriented IT Management:

Benefit, Cost and Success Factors. In: Proceedings of the Thirteenth European Conference on Information Systems, Regensburg.

Porter ME (1996) What Is Strategy? Harvard Business Review 74:61-78.

Potgieter BC, Botha JH, Lew C (2005) Evidence that use of the ITIL framework is effective. 18th Annual Conference of the National Advisory Committee on Computing Qualifications, Tauranga, NZ. 


\title{
Web-Controlling
}

\section{Analyse und Optimierung der digitalen Wertschöpfungskette mit Web Analytics}

\author{
Darius Zumstein, Andreas Meier \\ Information Systems Research Group, Departement für Informatik, \\ Universität Fribourg (Schweiz)
}

Seit der Entstehung des World Wide Web vor 20 Jahren ist das Internet für Unternehmen ein unverzichtbares Instrument der Information, Kommunikation und des elektronischen Geschäfts geworden. Mit der zunehmenden Bedeutung des Web wird die Analyse, das Controlling und die Optimierung des Internetauftritts und eMarketings - das Web Analytics - wichtiger Gegenstand für Theorie und Praxis. Web Analytics (WA), i.w.S. auch als Web-Controlling bezeichnet, ermöglicht durch den Einsatz client- und serverseitiger Methoden (Page Tagging und LogfileAnalyse) den Traffic auf der Site anhand verschiedener Metriken zu messen und zu analysieren. Damit lässt sich die Nutzung des Online-Angebotes (Web Content Controlling) und das User-Verhalten (Web User Controlling) besser verstehen und die Seite (z. B. Navigation, Content, Design, Usability) optimieren (Hassler 2008).

Dank Web Analytics können auch Rückschlüsse auf die Effektivität der Online-Kommunikation und der verschiedenen Instrumente des elektronischen Marketings gezogen werden, zum Beispiel, ob und wie sich der Versand eines Newsletters, die Publikation eines Blogeintrages oder die Lancierung einer Online-Kampagne in der Website-Nutzung und im eCommerce niederschlägt. Auch bei der Suchmaschinenoptimierung ist Web Analytics ein wirksames Controlling-Instrument.

Im Rahmen des elektronischen Customer Relationship Managements (eCRM) kann durch die Analyse des User-Verhaltens die Kundennähe bzw. Kundenorientierung erhöht werden. Das Web-Controlling ermöglicht damit, die Gewinnung und Bindung von (potentiellen) Online-Kunden zu messen und besser zu steuern. Zudem dient das Web-Controlling der Überprüfung der strategischen Zielerreichung und der Legitimation und Begründung websitebezogener Management-Entscheidungen.

Das Web-Controlling als Teilbereich des IT-Controlling ist ein unterstützender Prozess beim Management der digitalen Wertschöpfungskette und untersucht vier Stufen, bzw. Reifegrade. Das vorgeschlagene Reifegradmodell (s. Abbildung 1 und Vollbeitrag dieses Papers) erlaubt, das eigene Unternehmen anhand verschiedener Kennzahlen zu bewerten und bei Bedarf ein Benchmarking zu betreiben.

Stufe D - Information: Dazu zählt das Bereitstellen von Unternehmensinformationen oder Produkt- und Dienstleistungskataloge. Wie oft, wann und von 


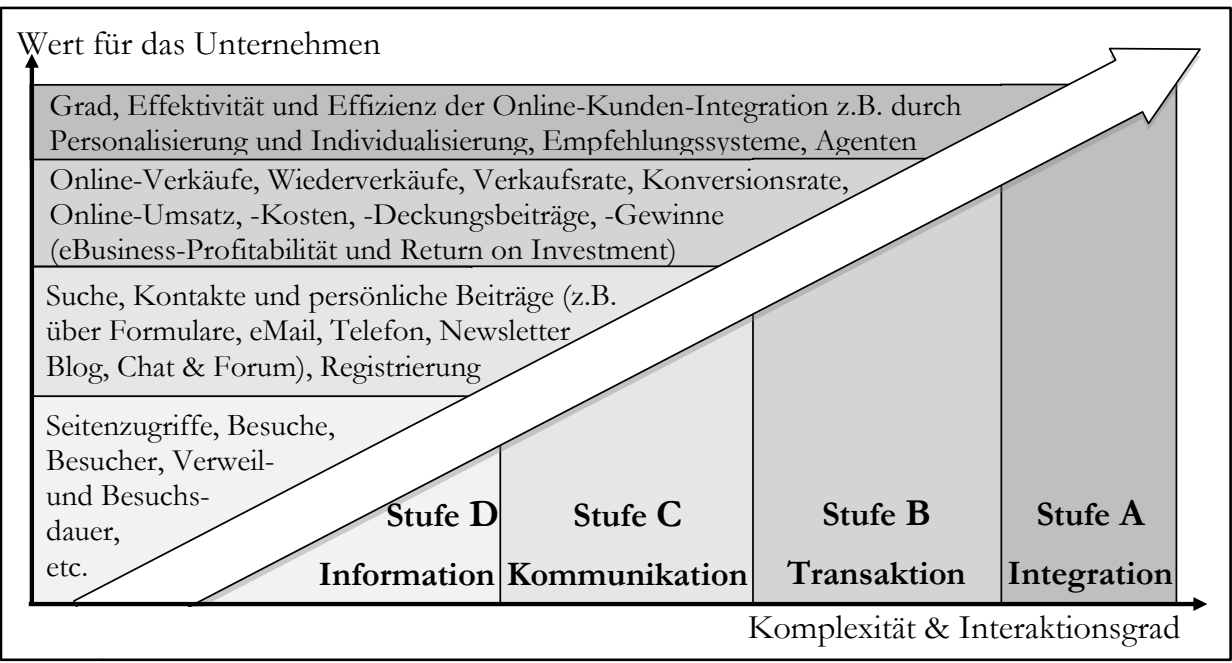

Abbildung 1: Kennzahlen für verschiedene Reifegrade im eBusiness

wem solche Informationen nachgefragt werden, wird im WA anhand der Anzahl Seitenzugriffe, Besuche, Besucher, Verweildauer und mittels weiterer Metriken wie z.B. Absprungraten, Besuchstiefe (Anzahl Seiten pro Besuch) oder Besuchsfrequenz analysiert.

Stufe C - Kommunikation: Hierzu zählen Dienste und Anwendungen wie Suchfunktion, FAQ, eMail, Formulare, Newsletter, Newsgroups, Corporate Blogs, Wikis, Chat oder Diskussionsforen, um die Interaktivität mit den Usern zu fördern. Die Anzahl und Qualität persönlicher Beiträge, Anfragen, Kontakte, Registrierungen und der Grad der Dialogfähigkeit sind dabei wichtige Metriken.

Stufe B - Transaktion: Bei dieser Stufe geht es um die elektronische Geschäftsabwicklung mit Online-Angebotserstellung, Bestellwesen, Bezahlung und Distribution. Das Monitoring von Key Performance Indicators (KPIs) wie z.B. Bestell- und Konversionsraten, Online-Verkäufe, Kaufsaktualität und -frequenz oder OnlineUmsatz ist von besonderer Bedeutung für das eBusiness (Zumstein \& Hugi 2009).

Stufe A - Integration: Die höchste Stufe betrifft Integration resp. Kundenbindung durch Personalisierung und Individualisierung (d.h. Mass Customization), One-to-One-Marketing, Online-Order-Tracking, Empfehlungssysteme oder durch den Einsatz von digitalen Agenten. Der Grad und die Effektivität einer BenutzerIntegration sollte im Web-Controlling grob eingeschätzt werden können.

\section{Literatur}

Hassler M (2008) Web Analytics, Redline, Heidelberg.

Zumstein D, Hugi P (2009) Unscharfe Klassifikation von Webkennzahlen. In: Knoll M, Meier A (Hrsg) Web \& Data Mining. HMD - Praxis der Wirtschaftsinformatik, 265(8):135-146. 
IT-Ressourcenmanagement 



\title{
Vorwort zum Track
}

\section{IT-Ressourcenmanagement}

\author{
Lutz M. Kolbe', Rüdiger Zarnekow², Reinhard Hoebn³, David Murphy ${ }^{4}$ \\ ${ }^{1}$ Professur für Informationsmanagement, Georg-August-Universität Göttingen \\ 2Institut für Technologie und Management, Technische Universität Berlin \\ ${ }^{3}$ IBM Deutschland, Stuttgart \\ 4Siemens AG, München
}

Wir danken unserem Programmkomitee:

Nico Ebert, Universität St. Gallen, Schweiz

Koray Erek, Technische Universität Berlin

Prof. Dr. Dennis Kundisch, Universität Paderborn

Prof. Dr. Norbert Mundorf, University of Rhode Island, USA

Nils-Holger Schmidt, Universität Göttingen

Durch die zunehmende Industrialisierung des Informationsmanagements und den wachsenden Ressourcenbedarf von Informationssystemen rücken neben ökonomischen, verstärkt ökologische und soziale Fragestellungen in den Fokus der ITManager. Der zunehmende Druck von Investoren, Gesetzgebern und Mitarbeitern auf die Unternehmen zu einem verantwortungsvolleren Umgang mit natürlichen und seltenen Ressourcen, überträgt sich auf die IT-Bereiche und macht ein ITRessourcenmanagement notwendig. Während verantwortungsvolles Ressourcenmanagement in anderen industrialisierten Branchen bereits lange verfolgt wird, fehlt es im Informationsmanagement an theoretischen und konzeptionellen Grundlagen. Neue strategische, prozessorientierte und technische Konzepte der effizienteren Bereitstellung, Nutzung und Allokation von IT-Ressourcen sind erforderlich. Das in der Praxis diskutierte „Green IT“ liefert hierzu erste Ansätze. Darüber hinaus bedarf es neuer Ansätze eines IT-Marketing, um durch eingeleitete Initiativen Nutzenpotentiale voll ausschöpfen zu können.

Die in dieser Teilkonferenz vertretenen Beiträge spiegeln das Themenspektrum eindrücklich wieder.

Betz, und Schwartz beschäftigen sich in ihrem Beitrag mit der Messung des Energieverbrauchs am Arbeitsplatz und dessen Beeinflussung durch soziales Verhalten. Dabei kann gezeigt werden, dass Transparenz zeitlich begrenzt zu einem ressourcenschonenden Verhalten führt. 
Die Integration von Green IT Kriterien in ein Entscheidungsmodell der Software Lizenzierung wird durch Altmann und Robitratana veranschaulicht und hilft diesen Aspekt zu berücksichtigen.

Die Bedeutung von ökologischen Eigenschaften eines PCs im Marketing zeigen Schmidtchen/Schmidt/Kolbe und Geldermann. Durch eine Conjoint-Analyse wird offenbart, dass insbesondere ältere, gebildete und weibliche Konsumenten für umweltfreundliche PCs einen höheren Preis zu zahlen bereit sind. 


\title{
Der Einfluss von ökologischen Produkteigenschaften bei PCs auf die Kaufentscheidung
}

\author{
Ergebnisse einer Conjoint-Analyse
}

\section{Timo Schmidtchen ${ }^{1}$, Nils-Holger Schmidt', Lutz M. Kolbe', Jutta Geldermann² \\ ${ }^{1}$ Professur für Informationsmanagement, 2 Professur für Produktion und Logistik, (beide) Georg-August-Universität Göttingen}

Aus der zunehmenden Bedeutung von Informationssystemen für die Wirtschaft resultieren ein steigender Energiebedarf und eine zunehmende Umweltbelastung. Im Jahr 2005 benötigten Server und deren Infrastruktur weltweit 123.000 Gigawattstunden Energie. Von insgesamt 820 Millionen Tonnen $\mathrm{CO}_{2}$-Ausstoß pro Jahr gehen zwei Prozent zu Lasten der IT (Heng 2009, S. 1). Dabei spielt die Diskussion um Green IT eine wesentliche Rolle (Schmidt et al. 2009, S. 1).

Wissenschaftliche Literatur zur Green IT befasst sich überwiegend mit der Situation in Unternehmen. Entscheidend für ein Umdenken in der Gesellschaft kann nicht ausschließlich die Unternehmensseite sein. Aus Sicht der Konsumenten und Nutzer von IT leitet sich die Frage ab, ob ökologische Produkteigenschaften bei PCs (Personal Computer) einen Einfluss auf das Konsumentenverhalten haben. Im Rahmen dieser Arbeit wurden 500 Personen unterschiedliche PC-Konzepte zur Bildung einer Präferenzreihenfolge vorgelegt. Mittels einer Conjoint-Analyse wurden dann die partiellen Teilnutzenwerte von ökologischen Produkteigenschaften bei PCs bestimmt und eine Cluster- und Diskriminanzanalyse durchgeführt.

Für die Erhebung der Daten wurde ein Online-Fragebogen mit zwölf verschieden Produktkonzepte von PCs entwickelt. Drei dieser Produktkonzepte dienten als Prüffälle zur Bestimmung der Validität. Zur Durchführbarkeit der Conjoint-Analyse wurden die PCs durch die vier Eigenschaften Preis, Leistung, Energieverbrauch und Entsorgung mit jeweils drei Eigenschaftsausprägungen beschrieben.

Die Wichtigkeit des Energieverbrauchs bei der Präferenzbildung erreicht 19,2\%. Die Entsorgungseigenschaften liegen mit 21,2\% knapp hinter dem Preis mit 21,6\%. Die Leistung verzeichnet mit einem Anteil von 37,9\% die größte Wichtigkeit. Zusammengefasst nehmen die kumulierten Wichtigkeiten der ökologischen Produkteigenschaften einen Anteil von 40,4\% ein, der damit unter dem Erwartungswert von 50 \% bei zwei von vier Eigenschaften liegt. Ökologische Pro- 
dukteigenschaften können damit als unterdurchschnittlich wichtig bezeichnet werden.

Es zeigt sich durch eine Clusteranalyse, dass jeweils eine Eigenschaft für die Teilnehmer eines Clusters besonders wichtig ist und die verbleibenden drei Eigenschaften im Mittel etwa auf einem Niveau liegen. Die Cluster werden daher als Leistungs-, Preis-, Energie- und Entsorgungsorientierte bezeichnet. Beim Merkmal „Geschlecht" ist auffällig, dass die Entsorgungsorientierten im Vergleich zu den anderen Clustern einen deutlich erhöhten Frauenanteil aufweisen. Die Energieorientierten sind überdurchschnittlich gebildet und verzeichnen den höchsten Altersdurchschnitt. Insgesamt werden ökologische Produkteigenschaften eher von älteren Umfrageteilnehmern bevorzugt. Aufgrund der Signifikanz von 0,156 kann dieser Zusammenhang jedoch zufällig entstanden sein.

Die Ergebnisse bestätigen die These, dass nachhaltige Produkte das Konsumentenverhalten positiv beeinflussen können (Du et al. 2007, S. 224-241; Lichtenstein et al. 2004, S. 16-32; Luo und Bhattacharya 2006, S. 1-18).

Desweiteren lassen sich Handlungsempfehlungen für das MarketingProgramm und die darin enthaltenen absatzpolitischen Instrumente der ITHersteller ableiten. Das Programm sieht vor, einen PC mit ökologischen Produkteigenschaften zu vertreiben. Mit den entwickelten Maßnahmen lässt sich ein Marksegment von bis zu 26,6\% der potenziellen Konsumenten von PCs ansprechen.

\section{Literatur}

Du S, Bhattarchaya CB, Sen S (2007) Reaping relational rewards from corporate social responsibility: The role of competitive positioning. In: International Journal of Research in Marketing 24(3):224-241.

Heng S (2009) Green-IT: IT is not green and never ever will be!. http://www.dbresearch.de/PROD/DBR_INTERNET_DEPROD/PROD0000000000238000.pdf. Abruf am 2009-05-26.

Lichtenstein DR, Drumwright ME, Braig BM (2004) The effect of Corporate Social Responsibility on Customer Donations to Corporate-Supported Nonprofits. In: Journal of Marketing 68(4):16-32.

Luo X, Bhattacharya CB (2006) Corporate Social Responsibility, Customer Satisfaction, and Market Value. In: Journal of Marketing 70(4):1-18.

Schmidt NH, Erek K, Kolbe LM, Zarnekow R (2009) Towards a Procedural Model for Sustainable Information Systems Management. In: Sprague RH (Hrsg) Proceedings of the 42th Hawaii International Conference on System Sciences 2009 (HICSS-42), Hawaii. 


\title{
Software Resource Management Considering the Interrelation between Explicit Cost, Energy Consumption, and Implicit Cost
}

\author{
A Decision Support Model for IT Managers \\ Jörn Altmann, Juthasit Robitratana \\ TEMEP, College of Engineering, Seoul National University
}

Although the adoption of Software-as-a-Service (SaaS) is expanding, the question whether SaaS is the right choice for an organization is still open. On the one hand, according to a 2008 survey by Cutter Consortium and THINKstrategies (2008), the top reason for adopting SaaS is lower IT infrastructure cost. On the other hand, depending on the maturity of the IT infrastructure, Kaplan (2007) pointed out that $\mathrm{SaaS}$ requires significant investments into the existing IT infrastructure. In general, the existing literature shows that organizations need to consider a number of criteria related to their IT situation, in order to make optimal decisions about the type of software licensing model to be used (Choudhary, 2007).

Recently, this decision making process got even more complex by following a "Green IT" policy. Green IT refers to activities concerning sustainable IT resource management from an ecological perspective. IT managers have to optimize the use of IT resources such that fewer resources are wasted, in particular less energy. Since the execution of software determines the demand for computing resources, the software procurement process has to be included in this analysis as well. In turn, the software procurement process has to consider different software licensing models as alternative solutions. If the software is used rarely, the SaaS licensing model allows sharing of computing resources at times of low utilization. By aggregating the reduced demand for servers, it reduces the energy consumption caused by idle computing cycles. However, if the software needs the server continuously, the perpetual licensing model is more efficient. This situation corresponds to the computing resource model analyzed by Risch et. al. (2008). They discussed that Grid, as an on-demand model, is less expensive if the frequency of resource use is low. Based on this, we can state that reducing energy consumption is equivalent to finding the best software licensing model. The economic and Green IT motivations are aligned. In this paper, we show to what extent this is valid.

We use the Analytic Hierarchy Analysis (AHP) approach of Saaty (1980) to compare both software licensing models. The application of AHP for supplier selection has been illustrated in many fields (Lai et al., 1999). The advantage of AHP is that it can cover tangible and intangible criteria. 
Jörn Altmann, Juthasit Rohitratana

The main objective of this work is to propose the Software Licensing Selection Support (SL2S) model for selecting a SaaS Licensing (SaaSL) model or a Perpetual Software Licensing (PSL) model. The SL2S model considers different types of criteria (i.e. explicit and implicit cost-related criteria and energy consumption) under Green IT policies and sustainable IT resource conditions (Jadhav et al., 2009; Xin et al., 2008). Based on the criteria that influence the selection of the software licensing type, we perform a sensitivity analysis, to understand the dependencies between explicit cost, implicit cost, and energy consumption. To validate the model, we constructed a scenario, in which a medium-sized company has to decide on a software licensing model. The scenario and its parameters were populated with data about CRM solutions of Salesforce.com and Microsoft, the SME definition of the European Commission (2003), and case studies (Lai et al., 1999; Chau, 1995; SAManage, 2008). In our analysis, we investigated not only the overall decision outcome under explicit cost but also its relationship to implicit cost criteria and energy consumption, as needed for establishing a Green IT policy. The results show that implicit cost criteria ( $25 \%$ of SaaSLM) are major decision factors. It suggests that a sustainable IT policy cannot only consider lowering cost (as a mean for lowering energy consumption) but also has to re-evaluate its relation to implicit cost criteria.

\section{References}

Chau PYK (1995) Factors used in the selection of packaged software in small businesses: Views of owners and managers. In: Information and Management 29:71-78.

Choudhary V (2007) Comparison of software quality under perpetual licensing and Software as a Service. In: Management Information Systems 24(2):141-165.

Jadhav AS, and Sonar RM (2009) Evaluating and selecting software packages: A review. In: Information and Software Technology 51:555-563.

Kaplan JM (2007) SaaS: Friend or Foe? In: Business Communications Review 2007:48-53.

Lai VS, Trueblood RP, and Wong BK (1999) Software selection: a case study of the application of the analytical hierarchical process to the selection of a multimedia authoring system. In: Information \& Management 36:221-232.

Risch M, and Altmann J (2008) Cost analysis of current Grids and its implications for future Grid markets. In: Lecture Notes in Computer Science 5206:13-27.

Saaty TL (1980) The Analytic Hierarchy Process. McGraw-Hill, New York.

SAManage (2008) SaaS vs. Traditional ITAM Tools: How the Hosted Approach Delivers Greater Value. www.SAManage.com. Fetched on 2008-05-06.

THINKStrategies in conjunction with Cutter Consortium. (2008) What is the greatest benefit of the SaaS offering? www.thinkstrategies.com. Fetched on 2008-10-12.

Xin M, and Levina N (2008) Software-as-a-Service model: elaborating client-side adoption factors. In: Proc. of $29^{\text {th }}$ Intl. Conf. on Information Systems, Paris, France. 


\title{
Soziale Dimensionen von Smart Metering am Arbeitsplatz
}

\author{
Matthias Betz, Tobias Schwartz. \\ Fraunhofer FIT, Sankt Augustin
}

Bei wachsendem globalen Energiebedarf und einer zunehmender Verknappung der natürlichen Ressourcen, ist die Zahl von Unternehmen, die den betrieblichen Energiekonsum auf Einsparungspotenziale prüfen und gleichzeitig die Schaffung von nachhaltig energieeffizienten Arbeitsplätzen vorantreiben verhältnismäßig gering. Hinzu kommt, dass die effiziente Nutzung der Energieressource Elektrizität im betrieblichen Alltag dabei häufig der mangelnden Transparenz des Verbrauchs, der resultierenden Kosten und der Wahrnehmung von Effizienzpotenzialen geschuldet ist. Bleibt der tatsächliche Energiekonsum diffus ist eine Bewertung und Optimierung des Ressourceneinsatzes ein diffiziles Unterfangen. Softwaresysteme zur Steuerung von, intelligenter Messtechnologie am Arbeitsplatz' stellen hierbei einen möglichen Lösungsansatz dar, welcher Verbrauchsinformationen zur Aufdeckung von Einsparpotenzialen für den Nutzer angemessen visualisiert, um neue Kompetenzen und Handlungsspielräume für einen effizienten Umgang mit Elektrizität zu erschließen.

Der Beitrag zeigt anhand einer empirischen Studie über die Anwendung von intelligenter Messtechnologie in einer Organisation, wie in einem GruppenAuswertungsworkshop mittels SmartMetering-Messdaten gemeinschaftlich Möglichkeiten für mehr Energieeffizienz am Arbeitsplatz ausgehandelt werden und welche Effekte sich im Nachgang des Workshops bezüglich des Energieverbrauchs am Arbeitsplatz eingestellt haben. Die Phänomene auf der sozialen Ebene werden dabei mit Messdaten des Energieverbrauchs vor, unmittelbar nach und in einer längerfristigen Perspektive nach dem veranstalteten Workshop in Relation gesetzt, um die entstehenden Effekte hinsichtlich des tatsächlichen Verbrauchs nachvollziehbar zu machen. Untersuchungsinteresse ist es, soziale Aspekte im Zusammenhang mit intelligenter Messtechnologie näher zu beleuchten und zu überprüfen, welche Nebeneffekte die neu gewonnen Transparenz über den Stromverbrauch hat. Das empirische Material wird daraufhin untersucht, welche sozialen Interdependenzen sich innerhalb einer Gruppe von Mitarbeitern durch die Anwendung intelligenter Messtechnologie bei Büroarbeitsplätzen ergeben. Dabei zeigt die Studie, dass in dem untersuchten Fall durch die Verwendung von Messtechnologien in Verbindung mit Interpretationsunterstützung positive Effekte in Bezug auf den Energiekonsum in den gemessenen Büroräumen festellbar sind. Aushandlungsprozesse auf sozialer Ebene ohne technische Intervention führen dabei zu einer Ver- 
ringerung des Stromverbrauchs. Dabei haben die Teilnehmer der Studie im Sinne des „Double-Loop-Learnings“ ihre handlungsleitenden Logik expliziert und in Bezug auf den Umgang mit den vorhandenen elektrischen Geräten kollektiv reflektiert.

Die Auswertung des empirischen Materials zeigt, dass sich das kollektiv gebildete Problembewusstsein als Handlungstreiber besonders wirksam herausgestellt hat, da das Verhalten des Einzelnen stetig von der Gruppe auf die im Workshop diskutierten Zielen mit reflektiert wurde. Es wurde deutlich, dass die Kontrolleffekte der Gruppe ein energieeffizientes Verhalten des einzelnen Mitarbeiters gefördert hat und dass das individuelle Handlungsfeld auf Basis der diskutierten Verbrauchsdaten bestimmt wurde. Aus der durchgeführten Studie geht hervor, dass detaillierte Informationen über den Energiekonsum am Arbeitsplatz zu einem besseren Verständnis über die Verwendung der Ressource Elektrizität beitragen kann und somit ein Schlüssel zur Identifikation von Einsparungspotenzialen sind. Liegen diese Informationen vor, ist es gleichermaßen wichtig, die Messdaten verständlich und bedarfsgerecht aufzubereiten und über angemessene Kanäle an den Nutzer von elektrischen Geräten zurück zu spiegeln. Ansätze zur Schaffung eines energieeffizienten Arbeitsplatzes durch mehr Energietransparenz und die Verwendung von intelligenter Messtechnologie stehen jedoch noch am Anfang. Dabei ist gleichzeitig ein Trend zur Verbreitung von intelligenter Messtechnologie zu beobachten. Möchte man Büroarbeitsplätze nachhaltig energieeffizient gestalten, ist es wichtig, bei der Gestaltung und Konzeption von Feedback-Systemen auch die soziale Dimension von Smart Metering zu berücksichtigen.

Basierend auf den gewonnenen Einsichten und Erkenntnissen müssen in weiterführenden Forschungsarbeiten die Effekte der Sichtbarmachung von Energieverbrauchsdaten am Arbeitsplatz näher untersucht werden. Geplant ist die Granularität der intelligenten Messtechnologie zu erhöhen und die zeitlichen Abstände zwischen Energiekonsum und Feedback zu verringern. Sich einstellende Phänomene sollen dabei in-situ beobachtet werden. 
Lern-Service-Engineering 



\title{
Vorwort zum Track
}

\section{Lern-Service-Engineering}

\section{Herausforderungen bei der systematischen Entwicklung zukünftiger Lehr-/Lernprozesse}

\author{
Roland Gabriel', Martin Gersch², Eric Schoop’3, Peter Weber ${ }^{1}$ \\ ${ }^{1}$ Lehrstuhl für Wirtschaftsinformatik, Competence Center E-Commerce, \\ Rubr-Universität Bochum \\ ${ }^{2}$ Professur für Betriebswirtschaftslehre, Competence Center E-Commerce, \\ Freie Universität Berlin \\ ${ }^{3}$ Lehrstubl für Wirtschaftsinformatik insbesondere Informationsmanagement, \\ Technische Universität Dresden
}

Charakterisierung und Dokumentation der Teilkonferenz

Das Lern-Service-Engineering nimmt Bezug auf Themenfelder, die im Dienstleistungsmanagement als „Service Engineering“ sowie in der Wirtschaftsinformatik als „Software Engineering“ etabliert sind. Es kann dementsprechend charakterisiert werden als interdisziplinäre Bereitstellung und systematische Verwendung von Prinzipien, Methoden und Werkzeugen für die zielorientierte (arbeitsteilige, ingenieurgleiche) Gestaltung und Entwicklung von hybriden Lehr-Lern-Leistungsangeboten, die als Services interpretiert werden können. Die gegenwärtigen Veränderungen im Bildungswesen begründen insbesondere aufgrund der Wettbewerbsintensivierung und der veränderten Rahmenbedingungen die Notwendigkeit einer sowohl didaktisch als auch technisch und ökonomisch überzeugenden, qualitätsorientierten Gestaltung der Leistungserstellungs- sowie zukünftiger Nutzungsprozesse (Lehr-/Lernprozesse). Bisher werden dabei nur unzureichend die Erfahrungen anderer Branchen bei der erfolgreichen Erstellung und Vermarktung von Dienstleistungen berücksichtigt. Daher ist - neben der Erarbeitung spezifischer Konzepte - die Übertragung, Adaption und Integration von Konzepten und Ansätzen aus der Wirtschaftsinformatik und dem Dienstleistungsmanagement ein wichtiger Aspekt des Lern-Service-Engineering, da hier bereits nachweislich erfolgreiche Ansätze für vergleichbare Herausforderungen geschaffen wurden.

Die Präsentationen der Teilkonferenz wurden aufgezeichnet. Im Rahmen des Reviewprozesses war darüber hinaus neben der Präsentationsannahme eine reine Beitragsannahme für als grundsätzlich geeignet erachtete, aber aufgrund be- 
schränkter Slots dennoch nicht zur Präsentation eingeladene Beiträge möglich. Alle akzeptierten Beiträge und Aufzeichnungen werden im Nachgang der Konferenz bei Einwilligung der Autoren auf der Plattform Slidestar (www.slidestar.de) verfügbar gemacht (Autor: Lern-Service-Engineering@MKWI2010).

\section{Beitragsübersicht}

- Analyse und Optimierung von Lehrdienstleistungen mittels Service Blueprinting (René Wegener, Philipp Menschner, Jan Marco Leimeister)

- Web 2.0 gestützte Blended Learning Szenarien als kritische Masse Systeme (Christian Lehr)

- Semantische Lehr-, Lern- und Qualifizierungsservices für Gründungslehre (Malgorzata Mochol, Robert Tolksdorf, Jörg Freiling, Stefanie Demmler)

- Towards an Open SOA for Learning Management (Katrina Leyking, Roy Ackema, Ralf Angeli, Nils Faltin, Fabrizio Giorgini, Gunnar Martin, Wolf Siberski, Volker Zimmermann)

Auf Slidestar werden bei Zustimmung der Autoren/-innen zusätzlich die folgenden Einreichungen verfügbar gemacht:

- E-Learning Aufgaben für Ereignisgesteuerte Prozessketten mit automatischer Korrektur (Markus Siepermann, Richard Lackes, Chris Börgermann)

- Kompetenzorientierte Lehrevaluation - Diskussion neuer Perspektiven für neue Lehr- und Lernformen (Corinna Fink)

- Neue Möglichkeiten zur technischen Unterstützung bei der Ausbildung an ERP-Systemen (Jan Aalmink, Liane Haak, Dirk Peters)

Wir danken den Mitgliedern des Programmkomitees für Ihr Engagement

- Prof. Dr. Theo Bastiaens, FernUniversität Hagen

- Prof. Dr. Peter Chamoni, Universität Duisburg-Essen

- Dr. Lutz Görtz, MMB-Institut für Medien- und Kompetenzforschung

- Prof. Dr. Uwe Hoppe, Universität Osnabrück

- Prof. Dr. Fritz Klauser, Universität Leipzig

- Prof. Dr. Michael Kleinaltenkamp, Freie Universität Berlin

- Prof. Dr. Richard Lackes, Technische Universität Dortmund

- Dr. Burkhard Lehmann, DISC, Technische Universität Kaiserslautern

- Prof. Dr. Peter F. E. Sloane, Universität Paderborn

- Dr. Anne Thillosen, IWM-KMRC Tübingen

- Prof. Dr. Rainer Thome, Universität Würzburg

- Prof. Dr. Udo Winand, Universität Kassel 


\title{
Towards an Open SOA for Learning Management
}

\author{
Katrina Leyking1, Ralf Angeli², Nils Faltin³, Fabrizio Giorginit, \\ Gunnar Martin', Wolf Siberski5, Volker Zimmermann ${ }^{3}$ \\ ${ }^{1}$ Institut für Wirtschaftsinformatik (IWi) im DFKI GmbH, Saarbrücken \\ 2IDS Scheer AG, Saarbrücken \\ ${ }^{3} I M C$ AG, Saarbrücken \\ ${ }^{4}$ Giunti Lab S.r.l., Sestri Levante \\ ${ }^{5}$ Forschungszentrum L3S, Leibniz Universität Hannover
}

Looking at today's technologies that are used to build a learning environment reveals a large variety of different software systems and components depending on the learning processes that organisations aim to support. Learning Management Systems (LMS) have established as the core systems in a modern learning environment (Kuechler et al. 2009; Grohmann et al. 2007). They integrate different technologies into a common learning infrastructure. However, given the myriad of learning applications, tools and technologies, organizations struggle to manage the interdependencies between software-supported activities within a modern learning environment. Software products for activities as diverse as learning need analysis, learning design, content authoring, learning itself as well as learning assessment interdependent both conceptually and technically. In addition, the integration of learning software with HR management systems (in corporations) or campus management systems (in higher education) is critical to avoid the isolation of the learning environment within the organization. Instead, learning solutions shall interact with business solutions e.g. for personnel management. When it comes to the business value of learning inter-departmental integration is even more necessary to facilitate the alignment between business needs and learning needs. Businessdriven learning needs are tightly linked to business performance, business processes, products and markets and emerge in the operational business units. Therefore, most recent initiatives promote the integration of business planning and business intelligence software with learning management systems (Leyking und Angeli 2009).

Service-oriented architectures present a viable approach that enables organizations to create an integrated solution of multiple heterogeneous software applications. Instead of expecting one software solution to provide all functionality, an organization may pick from diverse software offerings - proprietary or open source, off-the-shelf or custom-tailored, online or offline - and integrate them through service-oriented interfaces. This promotes an evolutionary approach of building learning environments, which are most of the times not built in one step 
but in multiple stages according to business sizes and needs. Service-orientation caters to the underlying requirement of exchangeable and interoperable software components. Standardized service-oriented interfaces and data exchange interfaces promote an unprecedented degree of interoperability channelled by web protocols. By using standards, the environment can be opened for a flexible exchange of components without having one integrated system. While the learning domain provides important data standards such as IMS-Learning Design, SCORM, and LOM, web service standards are still under way. The future challenge is to make LMSs more and more open and extensible, allowing developers to integrate new learning functionality and vice versa allowing learning functions to be integrated into employee portal or collaboration systems. This paper proposes a generic open SOA approach for learning that has been developed in the EU-co-funded research project PROLIX.

After this introduction, state-of-the-art concepts of service-oriented architecture will be outlined with a specific focus on related work in the domain of learning management (chapter 2). Based on these findings, chapter 3 will review the requirements of the learning management landscape. It prepares the groundwork for the proposed service-oriented architecture for learning as outlined in chapter 4. The paper closes with a conclusion and sketches future research in chapter 5 .

\section{References}

Grohmann G, Kraemer W, Milius F, Zimmermann V (2007) Modellbasiertes Curriculum-Design für Learning Management Systeme: Ein Integrationsansatz auf Basis von ARIS und IMS Learning Design. In: Oberweis, A. et. al. (Hrsg.): Tagungsband 8. Internationale Konferenz Wirtschaftsinformatik, Karlsruhe.

Kuechler T, Thissen D, Zimmermann V (2009) Into the great wide open:

Responsive Learning Environments for Personalised Learning. In:

O’Donoghue J (Hrsg): Technology Supported Environment for Personalised Learning: Methods and Case Studies. IGI Global, London.

Leyking K, Angeli R (2009) Model-based, Competency-Oriented Business Process Analysis. Enterprise Modelling and Information Systems Architecture Journal, 4(1):14-25. 


\title{
Semantische Lehr-, Lern- und Qualifizierungs- Services für die Gründungslehre
}

\author{
Malgorzata Mochol', Robert Tolksdorf', Jörg Freiling', Stefanie Demmler' \\ ${ }^{1}$ Institut für Informatik, Freie Universität Berlin \\ ${ }^{2}$ Fachbereich Wirtschaftswissenschaft, Universität Bremen
}

Obwohl sich aktuell der Markt der Gründungsqualifizierung und -beratung auf der Anbieter- und Nachfragerseite durch ein kontinuierliches Wachstum auszeichnet, bestehen immer noch erhebliche Probleme: (a) sehr hohe Mortalitätsraten im Bereich wissensintensiver Gründungen (Eurostat 2004); (b) prekäre Qualifikationslücken in der beruflichen Weiterbildung (BMWi 2009); (c) Mangel an gründungsgerechter, zielgruppenorientierter Ausbildung mit raum-/zeitunabhängigem Lernen sowie modularisierten/digitalisierten Lehrinhalten; (d) wenig Rücksicht auf die betriebswirtschaftlichen Defizitbereiche von Gründenden (Freiling 2009); (e) kein definierter Qualitäts-/Leistungsstandard der Gründungsberatung/-qualifizierung. Angesichts der Problematik, sind geeignete Lehr-/Lernangebote notwendig, die durch deutlich flexiblere E-und/oder Blended Learning-1-Lösungen unterstützt werden. Diese sollen zielgruppen- und themenspezifisch traditionelle Lehr/Lernformen (z.B. Lehrmedien wie Bücher sowie Lehrformen wie Kurse und Übungen) mit Online-Elementen (z.B. internetgestützte Fallstudien) im Rahmen eines Lern Service Engineering (Gabriel et al. 2008) für die Gründungsqualifizierung auf Grundlage von Web3.0 Technologien verbinden: Unter Nutzung neuester didaktischer, organisatorischer und technologischer Potenziale aus den Bereichen des Lern Service Engineering sowie des Web3.0 Technologiefelds sollen in traditioneller Form vorliegende Lehrinhalte zur Gründungslehre so aufbereitet werden, dass sie als wieder verwendbare Lehr-/Lernbausteine in verschiedenen konkreten Veranstaltungsangeboten der Gründerqualifizierung genutzt werden können. Im Kontext von Web 3.0 Technologien, die die Interaktionsfähigkeit und Nutzerbezogenheit von Web 2.0-Anwendungen (Anderson 2007) mit den Vorteilen inhaltlicher Beschreibung und Verarbeitung basierend auf dem Semantic Web (Berners-Lee 2001) kombinieren, werden Benutzer zunehmend zu Mitgestaltern von Anwendungen und können die neuartigen Dienste und Schnittstellen an ihre aktuellen Bedarfe anpassen. Die interaktiven Web2.0-Technologien stärken nicht nur die einfache Austauschmöglichkeit und Bildung von Interessen-Communities im Bereich der Existenzgründung, sondern sind vor allem für Gründungsvorhaben die zentrale Basis sozialer Art zur Umsetzung von Geschäftsideen und -modellen. Ferner lässt sich

\footnotetext{
${ }^{1}$ Eine Mischung traditioneller Lehrformen, insb. mit expliziten Präsenz- und direkten Interaktionsphasen mit IT- und mediengestützten Online-Elementen (Graham 2004).
} 
durch Nutzung von semantischen Technologien die Zielgruppenorientierung und Qualitätssicherung verbessern. Durch Entwurf, Implementierung und Evaluation zielgruppenadäquater, moderner, auf Web3.0 Technologien basierender Lehr-, Lern- und Qualifizierungsservices können die vielfältigen Möglichkeiten des lebenslangen Lernens gefördert sowie zeitgemäße und kreative Arbeits- und Kommunikationsansätze im Bereich der Gründungsqualifizierung etabliert werden. Die Hauptidee der Lehr-, Lern- und Qualifizierungsservices (vgl. Abb. 1) liegt in der Umsetzung eines (1) didaktischen Konæepts in Zusammenspiel mit einer (2) Zertifizierungskonzeption und Geschäftsmodellentwicklung, wobei diese auf erprobte (3) Leminhalte und entsprechend aufbereitete Einsatzszenarien zugreifen, die wiederum einem kontinuierlichen (4) Qualitätssicherungs- und Qualitätsverbesserungsprozess unterliegen und durch Einsatz (5) moderner interaktiver Technologien auf eine benutzerfreundliche Art und Weise den (6) Zielgruppen zur Verfügung gestellt werden.

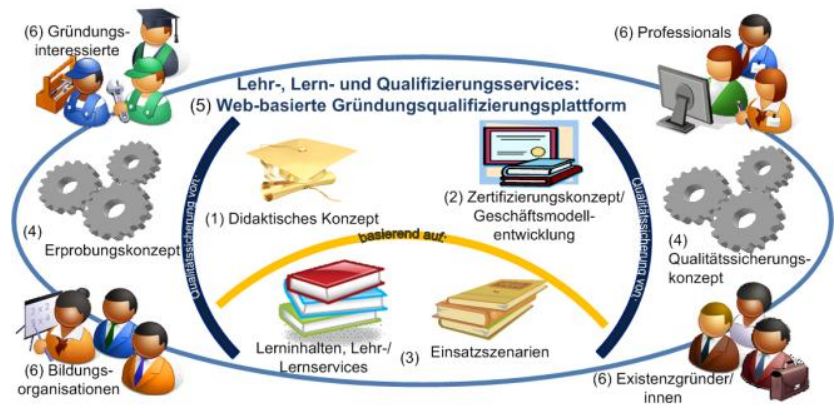

Abbildung 1: Konzept einer modernen Gründungsqualifizierungsumgebung

\section{Literatur}

Berners-Lee T (1998) What the Semantic Web can represent. http://www.w3.org/DesignIssues/RDFnot.html.

Bundesministerium für Wirtschaft und Technologie (BMWi) (2009) Saldo der Existenzgründungen 2008 erstmals seit 30 Jahren negativ, Mitteilung 22.04.09.

Eurostat Jahrbuch (2004) ISBN: 92-894-4962-4

Freiling, J (2009) Entrepreneurial Necessities and Managerial Shortfalls of

Founders: What Does Entrepreneurship Theory Tell Us?, http://ssrn.com/abstract $=1347664$.

Gabriel R et al. (2008) Möglichkeiten und Grenzen von Lern-Services, in:

Wirtschaftswissenschaftliches Studium (WiSt), Heft 10, S. 561-563

Graham CR (2004) Blended learning systems: definition, current trends, and future directions, Handbook of blended learning: global perspectives, local designs, Pfeiffer Publishing, S. 3-21. 


\title{
Analyse und Optimierung von Lehrdienstleistungen mittels Service Blueprinting
}

\author{
René Wegener, Philipp Menschner, Jan Marco Leimeister \\ Fachgebiet Wirtschaftsinformatik, Universität Kassel
}

Das deutsche Hochschulsystem leidet unter anhaltender Unterfinanzierung. Diese Problematik verschärft sich durch die steigenden Studierendenzahlen der nächsten Jahre. Den Lehrenden kommt die Aufgabe zu, einer größeren Anzahl Studierender bei gleichbleibenden Mitteln eine mindestens gleichwertige Lehre anzubieten. Diesem Spannungsverhältnis zwischen individueller Lernerfahrung und Wirtschaftlichkeit wird in diesem Artikel mit Erkenntnissen aus dem Service Engineering begegnet. Dieses liefert Methoden zur systematischen Entwicklung von Dienstleistungen. Zielsetzung ist dabei die Steigerung der Produktivität von Lehrdienstleistungen, womit das Verhältnis von Output (Zufriedenheit, Lernerfolg und Kompetenzerwerb) zu Input (dem Aufwand auf Seiten des Lehrenden) bezeichnet wird.

Die Interaktion zwischen Dozent und Studierendem sowie die Bereithaltung des Erstellungspotenzials (Mitarbeiter, Konzepte, Fachwissen etc.) sind mit hohem Aufwand auf Seiten des Lehranbieters verbunden. Die Gestaltung von Dienstleistungen muss daher Input- und Outputbetrachtungen vereinen. Dies ist mit Methoden der Dienstleistungsentwicklung wie dem Service Blueprinting möglich. Ein Service Blueprint kann als zweidimensionales Bild eines Dienstleistungserstellungsprozesses angesehen werden: Die horizontale Achse stellt die Chronologie der einzelnen Prozessschritte dar, die vom Leistungserbringer und vom Kunden erbracht werden, die vertikale Achse unterteilt die verschiedenen Stufen der Interaktion zwischen diesen beiden (Fließ und Kleinaltenkamp 2004, S. 396).

Zur Identifikation von Produktivitätssteigerungspotenzialen werden die Teilaktivitäten des Blueprints unter verschiedenen Aspekten (bspw. Contact Point Analyse, Standardisierung von Kommunikationsschnittstellen etc.) analysiert. Dies geschah im Rahmen eines Pilotprojekts an der Universität Kassel beispielhaft für eine teilnehmerstarke Lehrveranstaltung. Bei dieser handelt es sich um die Einführung in die Wirtschaftsinformatik (Info1). Diese war ursprünglich eine traditionelle Lehrveranstaltung mit Tutorium, die um E-Learning Methoden ergänzt und so zu einer Blended Learning Veranstaltung ausgebaut werden sollte. Hintergedanke war dabei, durch multimediale Selbstlerneinheiten mehr Freiräume für individuelle Lernprozesse zu schaffen, unterschiedliche Lerntypen besser zu bedienen (Kahiigi et al. 2007, S. 2) und so die Qualität der Veranstaltung deutlich zu steigern. Um diese Neuausrichtung mit vertretbarem Aufwand zu realisieren, wurde das Service Blueprinting angewendet. Die Untersuchung ergab dabei u. a. folgende Einschätzung: 
- Das Kurskonzept als solches existierte bereits und wird regelmäßig angepasst, der Hauptaufwand für die inhaltliche Planung ist somit bereits erledigt.

- Die Lernumgebung wird fachbereichsübergreifend zur Verfügung gestellt und gewartet, sodass an dieser Stelle keine Effizienzsteigerung zu erwarten ist.

- Der gesamte Klausurprozess kostet relativ viel Zeit, was jedoch in Anbetracht der Studierendenzahlen folgerichtig und gegenwärtig nicht vermeidbar ist.

- Die Erstellung der Web Based Trainings (WBTs), also der interaktiven Selbstlerneinheiten, ist aufgrund der Anforderungen (interaktiv, optisch ansprechend, selbsterklärend) als besonders aufwendig einzustufen.

Aufgrund dieser Einschätzung wurde den WBTs die höchste Priorität eingeräumt.

Um diese effizient zu erstellen, wurde eine weitere Lehrveranstaltung (Info 2) einbezogen, in der die Studierenden Grundlagen der Webprogrammierung erlernen. Diese wurde dahingehend modifiziert, dass die Teilnehmer ihre erlernten Kenntnisse nun praktisch nutzen, um selbst kleine Lerneinheiten zu Themen der Info1 zu erstellen. Diese wiederum werden später nach Prüfung durch die Dozenten in der Info1 eingesetzt. Auf diese Weise wird die Individualisierung des Lernangebots durch neue Selbstlerneinheiten mit vertretbarem Aufwand realisiert.

Die Befragung der Studierenden in der Veranstaltung Info 2 zeigt zudem, dass diese das System durchaus schätzen. So zeigt die Befragung hohe Werte für die generelle Zufriedenheit und insbesondere die Einschätzung der Praxisrelevanz der Veranstaltung. Daraus lässt sich schließen, dass die Methodik funktioniert, ohne für die betroffenen Studierenden von Nachteil zu sein. Im Gegenteil profitieren diese eher von dem erläuterten Konzept. Dies betrachten wir als Motivation für weitere Evaluationen und den schrittweisen Ausbau hin zu einem produktivitätsorientierten Kasseler Blended Learning Modell.

\section{Literatur}

Fließ S, Kleinaltenkamp M (2004) Blueprinting the service company Managing service processes efficiently. Journal of Business Research 57 (4): 392-404.

Kahiigi E, Ekenberg L, Hansson M (2007) Exploring the e-Learning State of art. Proceedings of the Conference on E-Learning. 


\title{
Web 2.0 gestützte Blended Learning Szenarien als kritische Masse Systeme
}

\author{
Christian Lebr \\ Fachbereich Wirtschaftswissenschaft, Freie Universität Berlin
}

Die Umstellung auf Bachelor/Master Studiengänge und die im Bologna-Prozess geforderte Kompetenzorientierung der Lehre an europäischen Hochschulen bringen neue Anforderungen an die Hochschullehre mit sich (BMBF 2009, S. 1).

In diesem Zusammenhang scheint der Einsatz von Web 2.0-Anwendungen in der Lehre einen vielversprechenden Mehrwert bieten zu können, der einen Beitrag dazu leisten kann, die geforderte Kompetenzorientierung der Lehre entscheidend zu unterstützen.

Schon Tim O'Reilly (2006, S. 1) stellt in seiner Definition des Web 2.0 die Bedeutung von Netzwerkeffekten in den Vordergrund. Aus dieser Bedeutung lässt sich eine zentrale Determinante der Wettbewerbsfähigkeit und eine spezifische Eigenschaft von (contentorientierten) Web 2.0-Anwendungen erschließen: Sie lassen sich als kritische Masse-Systeme identifizieren, bei denen das Erreichen der kritischen Masse einen entscheidenden Faktor für einen erfolgreichen Diffusionsverlauf darstellt (vgl. Liehr 2005, S. 23).

Beim Einsatz von Web 2.0-Anwendungen in der Lehre wurde diesem Aspekt bisher zu wenig Bedeutung beigemessen. Denn auch bei der Anwendung von Web 2.0 in der Lehre kann das Erreichen einer kritischen Masse und dessen gezielte Förderung einen Mehrwert erbringen, indem es den Lernerfolg und die Motivation der Studierenden nachhaltig steigert. Dabei gilt es, einige Besonderheiten des Diffusionsprozesses von kritischen Masse-Systemen in der Lehre zu beachten:

Die installierte Basis, also die an das System angeschlossenen Nutzer, entspricht im Falle von Lehrveranstaltungen den teilnehmenden Lernenden. Daher kann das Erreichen einer kritischen Masse in Lehrveranstaltungen nicht über die steigende Anzahl von Nutzern erklärt werden. Hier liefert insbesondere die Intensität der Nutzung der eingesetzten Web 2.0 Anwendungen durch die Lernenden den entscheidenden Erklärungsbeitrag.

Eine weitere Besonderheit im Bezug auf das Erreichen einer kritischen Masse stellt die bei handlungsorientierten Blended Learning Szenarien häufig eingesetzte Methode der Gruppenarbeit dar. Dabei kommt eine Besonderheit von kritischen Masse-Systemen zum Tragen, die als gruppenspezifische kritische Masse bekannt ist (vgl. Weiber 1995, S. 54-57).

Das Konzept der gruppenspezifischen kritischen Masse lässt sich auf die Methode der Gruppenarbeit übertragen und liefert einen Erklärungsbeitrag dafür, wie 
die Erreichung einer kritische Masse in einem Web 2.0 gestützten Blended Learning Szenario fokussiert werden kann.

Als konkretisierendes Beispiel dient in diesem Zusammenhang eine BachelorVeranstaltung aus dem Sommersemester 2009 zum Thema „E-Business“, die als Blended Learning Szenario konzipiert wurde.

Während dieser Veranstaltung wurden durch die Teilnehmer Wikibeitäge erarbeitet, in denen verschiedene thematische Inputs anhand konkreter, praxisbezogener Problemstellungen vertieft wurden.

Das Veranstaltungswiki dient dabei als Gegenstand der Untersuchung der zuvor angestellten Überlegungen bezüglich kritischer Masse-Systeme in Web 2.0 gestützten Lehrveranstaltungen.

Die Ergebnisse dieser Untersuchung geben deutliche Hinweise darauf, dass zum einen eine kritische Masse erreicht wurde und zum anderen, dass dies eine direkte Relevanz für den Lernerfolg und das Erreichen der gesteckten Lernziele hatte.

\section{Literatur}

BMBF (2009) Der Bolognaprozess. http://www.bmbf.de/de/3336.php. Abruf am 08.09.2009.

Liehr M (2005) Die Adoption von Kritische-Masse-Systemen : das Problem der individuellen kritischen Masse. Dt. Univ.-Verl, Wiesbaden.

O'Reilly T (2006) Web 2.0 Compact Definition: Trying Again. http://radar.oreilly.com/2006/12/web-20-compact-definition-tryi.html. Abruf am 08.09.2009.

Weiber R (1995) Systemgüter und klassische Diffusionstheorie - Elemente einer Diffusionstheorie für Kritische Masse-Systeme. In: Stoetzer M-W, Mahler A (Hrsg) Die Diffusion von Innovationen in der Telekommunikation. Springer, Berlin. 


\title{
Neue Möglichkeiten zur technischen Unterstützung bei der Ausbildung an ERP-Systemen
}

\section{Adaptive Lernkonzepte durch AUM und Enterprise Tomo- graphie}

\author{
Liane Haak, Dirk Peters, Jan Aalmink \\ Department für Informatik, Abteilung Wirtschaftsinformatik I, \\ Carl von Ossietzky Universität Oldenburg
}

Konfrontiert mit einem internationalen Bildungsmarkt, mit einer konkurrierenden, wachsenden Anzahl von Angeboten, verbunden mit fast unbeschränkter Mobilität Lernender und der Bereitschaft sich ständig weiterzubilden, steht unser Bildungswesen vor großen Herausforderungen. Davon sind besonders Bildungseinrichtungen wie Hochschulen und Universitäten betroffen. Studienabschlüsse sind kein „Selbstzweck“, sondern unterliegen steigenden Anforderungen, wie denen der Industrie nach kürzeren Studienzeiten bei gleichzeitiger Orientierung an den Problemen der Praxis. Dies ist besonders in stark anwendungsorientierten Studiengängen wie der Wirtschaftsinformatik der Fall (Rautenstrauch 2008). In diesem Markt müssen Hochschulen und Universitäten Schwerpunkte in der Ausbildung setzen, um sich auf dem Bildungsmarkt abzuheben und um wettbewerbsfähige Curricula anbieten zu können. Der Lernende soll in den verschiedenen Phasen des Lernprozesses unterstützt und Konzepte mit flexiblem Zugang unter Nutzung modernster Technologien angeboten werden.

Die Ausbildung an großen betrieblichen Anwendungssystemen wie z. B. ERPSystemen ist daher aus dem Lehrangebot der Hochschulen nicht mehr weg zu denken. Die stetig zunehmende Komplexität der Softwareprodukte bereitet aber Probleme, da es für den Anwender immer schwieriger wird, den Umgang mit diesen Systemen zu erlernen und diese Systeme später effizient im täglichen Betrieb einzusetzen. Ein weiteres Problem besteht in dem Mangel an didaktisch aufbereiteten Schulungsmaterialen in Form von Fallstudien u. a.. Zwar existieren theoretische Einführungen in die Benutzung einer Software, diese enthalten aber zu wenig praktische Anteile. Materialien zur Schulung an den Systemen, basieren häufig auf Trainingsmaterialien der Hersteller, die darauf abzielen, den Anwender mit einzelnen Funktionen oder Modulen der Software vertraut zu machen. Durch den primären Fokus auf die Systemfunktionalität fehlt daher oft die Prozesssicht und den Lernenden fällt es schwer, die Hintergründe, warum sie bestimmt Transaktionen ausführen sollen, zu verstehen. Didaktische Herangehensweisen, wie z. B. die Reflektion des Gelernten finden nicht ausreichendes Augenmerk (Hans et al. 2008). 
Der strategische Aspekt hinter vielen Entscheidungen bleibt daher oft unberücksichtigt und für den Lernenden nicht nachvollziehbar.

Dieser Beitrag beschreibt ein Konzept für die Ausbildung an ERP-Systemen auf Grundlage einer adaptiven Lernumgebung unter der Berücksichtigung neuer Technologien für die Auswertung großer betrieblicher Anwendungssysteme. Ziel dieses Ansatzes ist es, den Lernfortschritt am System zu dokumentieren und zu analysieren, um dem Lernenden eine Unterstützung im Ausbildungsprozess zu ermöglichen und dem Lehrenden Rückmeldungen zu dem aktuellen Fortschritt sowie auftretender Probleme zu geben. Vorgestellt wurden zwei Verfahren, das Application Usage Mining (AUM) und die Enterprise Tomographie, die in Kombination dies ermöglichen: das Benutzerverhalten adaptiv zu steuern und dem Lernenden eine adaptive Lernumgebung gemäß seiner individuellen Voraussetzungen bereitzustellen.

\section{Literatur}

Hans D, Marx Gómez J, Peters D, Solsbach A (2008) Case study-design for

Higher Education - a Demonstration in the Data Warehouse Environ-ment.

In: Abramowicz W, Flejter D (Hrsg) Business Information Systems

Workshops (BIS 2009), Poznan, Poland.

Rautenstrauch C (2008) Wirtschaftsinformatik-Ausbildung mit SAP-Software. In:

Bichler M et al (Hrsg) Tagungsband der Multikonferenz Wirtschaftsinformatik (MKWI 2008), München. 


\title{
E-Learning Aufgaben für Ereignisgesteuerte Prozessketten mit automatischer Korrektur
}

\author{
Markus Siepermann, Chris Börgermann, Richard Lackes \\ Lehrstubl für Wirtschaftsinformatik, Technische Universität Dortmund
}

Graphische Modellierungstechniken sind in vielen Fachdisziplinen weit verbreitet und werden deshalb im Rahmen der universitären Lehre häufig eingesetzt. Aufgrund der zahlreichen Möglichkeiten, den gleichen Sachverhalt unterschiedlich abbilden zu können, ist es jedoch meist schwierig, für graphische Modellierungstechniken E-Learning-Aufgaben zu realisieren, bei denen Studierende selbständig ihre Lösung modellieren können, die dann individuell vom System automatisch korrigiert und bewertet wird. Aufgrund ihrer Komplexität und der Schwierigkeit, solche Aufgaben zu realisieren, kommen sie deshalb im praktischen Einsatz kaum vor, obwohl solche auch Intelligent Tutoring Systems genannten E-LearningSysteme bereits seit den 90er Jahren Gegenstand der Forschung sind (vgl. Patel und Kinshuk 1996). Der vorliegende Beitrag stellt einen Ansatz vor, mit dessen Hilfe anspruchsvolle Aufgaben für Ereignisgesteuerte Prozessketten mit Hilfe eines E-Learning-Systems angeboten und automatisch korrigiert werden können.

Bei der Lehre graphischer Modellierungstechniken muss auf drei Fragestellungen geachtet werden: (1) Wird die Modellierungstechnik korrekt genutzt? (Haben Studierende bei der Bearbeitung Modellierungsregeln verletzt?) (2) Genügt die studentische Lösung allen inhaltlichen Anforderungen? (Beschreibt die Lösung alle Informationen, die in einer Aufgabe gegeben worden sind, oder werden einige Informationen vernachlässigt?) (2) Ist die gewählte Lösung elegant? (Gelangt die Lösung lediglich zu einem korrekten Endergebnis, oder genügt sie auch weiteren Qualitätsanforderungen wie z. B. Flexibilität?) Die Syntax der Modellierungstechnik (1), die auf einfachen Regeln basiert, kann über eine einfache Tiefensuche überprüft werden (vgl. Siepermann 2005, S. 1751). Semantik (2) und Eleganz (3) hingegen sind die Ursache für die Schwierigkeit der automatischen Überprüfung: Viele unterschiedliche Lösungen führen zum gleichen Ziel, können dabei gleichwertig sein oder einander in verschiedenen Punkten über- oder unterlegen sein. Z. B. können ein komplett sequentieller Prozess und ein parallel ablaufender Prozess zum gleichen Ergebnis führen, der parallele Prozess ist jedoch wegen seiner Flexibilität vorzuziehen.

Die Überprüfung der Fragestellungen lässt sich mit Hilfe des aus der SoftwareVerifikation bekannten Model Checking realisieren. Zu diesem Zweck werden über temporallogische Formeln inhaltliche Anforderungen an die Lösung einer Aufgabe formuliert. Die Ereignisgesteuerte Prozesskette wird in eine Kripke-Struktur (vgl. Clarke, Grumberg und Peled 2001, S. 35) überführt, anhand der die temporallogi- 
schen Ausdrücke überprüft werden. Wird kein Ausdruck verletzt, genügt die Lösung allen Anforderungen. Tritt eine Verletzung ein, so weist der verletzte Ausdruck auf den Fehler hin, den Studierende bei der Lösung der Aufgabe gemacht haben. Da temporallogische Formeln für Nicht-Mathematiker bzw. NichtInformatiker nur schwer zugänglich sind, wurde für die Formulierung der Anforderungen eine eigene graphische Notation geschaffen. Diese repräsentiert die Temporallogik in einfacher Form. Dadurch lassen sich die Anforderungen auch ohne mathematische Vorkenntnisse formulieren.

Bei der Nutzung des Systems wurde festgestellt, dass eine komplette Anforderungsspezifikation mit Temporallogik trotz der geschaffenen Notation anspruchsvoll und zeitaufwendig ist. Die temporallogischen Regeln, die von Tutoren erstellt werden, sind vielfach sehr einschränkend (z. B. auf ein bestimmtes Ereignis muss eine bestimmte Funktion folgen), da der Umgang mit Temporallogik zunächst nicht intuitiv ist. Allgemeine Regeln, die sich weniger mit bestimmten Abfolgen in der Lösung als vielmehr mit Anforderungen an das Modell befassen, werden nur selten verwendet. Darüber hinaus sind die Anforderungen/Regeln für eine Aufgabe bei der Definition der Referenzlösung vielfach unvollständig und müssen im Laufe der Veranstaltung verfeinert werden. Dennoch stellt das E-Learning-System eine starke Erleichterung dar. Viele Lösungen müssen nicht mehr durch die Tutoren korrigiert werden, sondern werden vom System automatisch korrigiert und bewertet. Dadurch bleibt den Tutoren Zeit, sich um individuelle Probleme der Studierenden zu kümmern.

\section{Literatur}

Clarke E.M., Grumberg O., Peled D.A. (2001). Model Checking. Cambridge London: The MIT Press.

Patel A., Kinshuk (1996). Intelligent Tutoring Tools - A problem solving framework for learning and assessment. In: M. F. Iskander et al. (Eds.): Proceedings of 1996 Frontiers in Education Conference - Technology-Based Re-Engineering Engineering Education. 140-144.

Siepermann M. (2005). Lecture Accompanying E-Learning Exercises with Automatic Marking. In: Richards, G. (Ed.). Proceedings of E-Learn 2005. Chesapeake: Association for the Advancement of Computing in Education, 1750-1755. 


\title{
Kompetenzorientierte Lehrevaluation
}

\section{Diskussion neuer Perspektiven für neue Lehr- und Lern- formen}

\author{
Corinna Fink
}

\section{Fachbereich Wirtschaftswissenschaft, Freie Universität Berlin}

Da im Zuge des Bologna Prozesses von der Kultusministerkonferenz (KMK 2005, S. 3-5) Kompetenzen als Zielkriterien universitärer Lehre verankert wurden, muss sich die künftige Lehrevaluation von der klassischen Form der reinen Erhebung von Input-Faktoren guter Lehre lösen und sich stattdessen Kompetenzen als LehrOutputs zuwenden (Paechter et al. 2007, S. 84).

Verfahren einer kompetenzorientierten Lehrevaluation stehen allerdings noch am Anfang ihrer Entwicklung. Aktuell existieren hierzu lediglich Instrumente, auf denen die Studierenden ihren Zuwachs an Fach-, Methoden-, Personal-, Sozialund ggf. Medienkompetenz in der Lehrveranstaltung selbst einschätzen (vgl. Braun et al. 2008; Paechter et al. 2007). Diese Instrumente wurden testtheoretisch fundiert und erfüllen insbesondere das Kriterium der Testökonomie. Ihre Nachteile liegen v. a. in Testwiederholungseffekten, insbesondere in Anbetracht der Tatsache, dass kaum Möglichkeiten einer Adaption an Veranstaltungsspezifika bestehen.

Entsprechend wird ein alternatives Vorgehen für die kompetenzorientierte Lehrevaluation vorgestellt. Dieses leitet auf Basis des Qualifikationsrahmens der KMK (2005, S. 5) ein universitätsspezifisches Kompetenzprofil ab, welches sich wiederum in fachbereichs-, lehrstuhl- und veranstaltungsspezifische Kompetenzprofile aufgliedern lässt bzw. sich aus diesen zusammensetzt. Das Kompetenzprofil dient der strategischen Positionierung der Universität auf dem Markt und bildet gleichzeitig den Bezugsrahmen der kompetenzorientierten Lehrevaluation.

Diese sollte zweistufig erfolgen. Zum einen mit Skalen, welche eine Adaption an ein lehrveranstaltungsspezifisches Kompetenzprofil ermöglichen. Hierzu eignen sich Zielerreichungsskalen in Anlehnung an Kiresuk und Sherman (1968). Mit diesen können die Lehrveranstaltungsziele von Dozent und Student erhoben und auf ihre subjektive Bedeutsamkeit und Erreichung hin geprüft werden. Da Zielerreichungsskalen jedoch die Schwächen der Selbsteinschätzung mit sich bringen, sollten sie zum anderen um ein objektives Verfahren der Kompetenzmessung ergänzt werden. Namentlich Kompetenzszenarien, welche Kompetenz handlungsbezogen erfassen. Entsprechende Szenarien sind jedoch sehr aufwendig zu konstruieren und sollten aufgrund von Lerneffekten nur jeweils einmal bei derselben Person eingesetzt werden. Deshalb empfiehlt sich für diesen Teil der Lehrevaluation ein Erhebungsrhythmus alle 1-2 Jahre mit Szenarien, die jeweils unterschiedli- 
che Kompetenzstufen erfassen. Die Ergebnisse dieser Erhebungen könnten nicht nur zur Lehrevaluation genutzt werden, sondern auch zur Evaluation ganzer Studiengänge und Fachbereiche. Darüber hinaus können sie den Studierenden dazu dienen, ihre individuelle Kompetenzentwicklung im Studium zu dokumentieren.

Hauptprobleme der Lehrevaluation im Allgemeinen stellen die geringe Akzeptanz derselbigen, Ressourcen-Probleme, Testwiederholungs- sowie Deckeneffekte dar. Hinzu kommen bei der kompetenzorientierten Lehrevaluation Schwierigkeiten mit der lehrveranstaltungsspezifischen Quantifizierung von Kompetenzzuwächsen sowie bei der Modellierung kausaler Beziehungen zwischen Lehrveranstaltung und Kompetenzzuwachs. Da zudem nach Rindermann (1996, S. 136) nur ein indirekter Zusammenhang zwischen der Qualität der Lehre und dem Lehrerfolg besteht, sollte die kompetenzorientierte Lehrevaluation um die Erhebung „klassischer“ Inputfaktoren der Lehre ergänzt werden. Auf diesem Wege kann nicht nur Verbesserungsbedarf aufgezeigt werden, sondern es ergeben sich auch konkrete Anhaltspunkte, welche Aspekte der Lehre verbessert werden müssen. Dies gilt insbesondere im Bezug auf die Evaluation neuer Lehr- und Lernformen. Beispiele für weitere Kriterien bei der Evaluation dieser sowie allgemein bei der Forschung zum Lern Service Engineering sind u. a. Inhaltsanalysen der produzierten Ergebnisse, Usability-Messungen, Klickzahlen, Lernprotokolle etc.

\section{Literatur}

Braun E, Gusy B, Leidner B, Hannover B (2008) Das Berliner

Evaluationsinstrument für selbsteingeschätzte, studentische Kompetenzen (BEvaKomp). DIAGNOSTICA 54(1): 30-42.

Kiresuk TJ, Sherman RE (1968) Goal attainment scaling. COMMUNITY MENTAL HEALTH JOURNAL 4(6): 443-453.

Kultusministerkonferenz (2005) Qualifikationsrahmen für Deutsche Hochschulabschlüsse. Sekretariat der Ständigen Konferenz der Kultusminister der Länder in der Bundesrepublik Deutschland, Bonn.

Paechter M, Maier B, Dorfer Q, Salmhofer G, Sindler A (2007) Kompetenzen als Qualitätskriterien für universitäre Lehre. In: Kluge A (Hrsg) Qualitätssicherung und -entwicklung an Hochschulen. Pabst Science Publishers, Lengerich.

Rindermann H (1996) Zur Qualität studentischer Lehrveranstaltungsevaluationen: Eine Antwort auf Kritik an der Lehrevaluation. ZEITSCHRIFT FÜR PÄDAGOGISCHE PSYCHOLOGIE 10(3/4): 129-145. 
Software-Industrie 



\section{Vorwort zur Teilkonferenz}

\section{Software-Industrie}

Peter Buxmann', Manfred Deues², Norbert Gronau³, Georg Herzwurm 4 , Thomas Hess', Alfred M. Keseberg, Friedbert Schub7

${ }^{1}$ Fachgebiet Information Systems/Wirtschaftsinformatik, TU Darmstadt ${ }^{2}$ ams. binrichs + müller, Kaarst

${ }^{3}$ Lehrstubl für Wirtschaftsinformatik und Electronic Government, Universität Potsdam

${ }^{4}$ Lehrstubl für Allgemeine Betriebswirtschaftslehre und Wirtschaftsinformatik II, Universität Stuttgart

${ }^{5}$ Institut für Wirtschaftsinformatik und neue Medien, LMU München 'PSIPENTA Software Systems, Berlin

${ }^{7}$ Lawson Deutschland, Hilden

Die Informations- und Kommunikationstechnologie hat die Unternehmenswelt weltweit in den vergangenen Jahren nachhaltig verändert. Die Entwicklung sehr leistungsfähiger Hardware und die Möglichkeit in kürzester Zeit mittels Suchmaschinen auf Informationen verschiedenster Anbietern zugreifen zu können, ist inzwischen genauso selbstverständlich geworden wie die immer detailliertere Abbildung inner- und zwischenbetrieblicher Geschäftsprozesse. Alle führenden Softwareunternehmen sind global ausgerichtet und konkurrieren weltweit um Kunden. Neben Sourcing und Shoring wirken sich ebenfalls offene Standards sowie OpenSource-Software auf die zukünftige Struktur und Handlungsmöglichkeiten der Softwareindustrie aus. Diese und weitere Handlungsmöglichkeiten stellen gegenwärtig noch keine stark verbreiteten inhaltlichen Forschungsschwerpunkte der deutschsprachigen Wirtschaftsinformatik dar. Die Heterogenität der deutschsprachigen Softwareindustrie bestehend aus Anbietern wie beispielsweise der SAP AG, Software AG aber auch vieler mittelständischer und Kleinst-Softwareunternehmen, die ebenfalls erfolgreich Umsätze auf den (Welt-)Märkten erzielen, sollte auch zukünftig Gegenstand der wissenschaftlichen Betrachtung sein.

Ziele der Teilkonferenz sind hervorzuheben, dass sich einerseits Software im Hinblick auf die ökonomischen Eigenschaften grundsätzlich von Dienstleistungen oder Industriegütern unterscheidet sowie, wie sich die Softwareindustrie selbst verändert und noch verändern wird. Während es für die wissenschaftliche Ausarbeitung von Inhalten gerichtet an Banken, Versicherungen, Produktionsunterneh- 
men, Dienstleistungsunternehmen spezielle betriebswirtschaftliche Lehrstühle gibt, hat sich etwas Vergleichbares für die Softwarebranche noch nicht entwickelt. Hier könnte und sollte die Wirtschaftsinformatik eine Lücke schließen und mit ihrem eigenen Forschungsprofil über reine BWL-Aspekte zusätzliche Akzente setzen.

In dem folgenden Abschnitt des Tagungsbands werden Sie Beiträge vorfinden, die schwerpunktmäßig Aspekte von Software-as-a-Service, hierbei die Preisfindung sowie die Softwareentwicklung betrachten. Einen weiteren Schwerpunkt stellen die arbeitsteilige Softwareentwicklung sowie die Industrialisierung der Softwareindustrie dar. Die retrograde Preisfindung sowie die Preisfindung von Softwareproduktlinien werden ebenfalls betrachtet.

Die Organisatoren möchten ihren Dank an die Autoren der 17 Einreichungen, welche für das Interesse an dem Thema sprechen richten. Leider konnten auf Basis der Gutachten nur neun Einreichungen berücksichtigt werden.

Die Organisatoren der Teilkonferenz Software-Industrie 


\title{
Softwarebezogene Wertschöpfung im Wertschöpfungsnetzwerk der Informations- und Kommunikationsindustrie
}

\author{
Eine empirische Analyse
}

Jochen Wulf, Rüdiger Zarnekow

\section{Lehrstubl für Informations- und Kommunikationsmanagement, TU Berlin}

Netzwerkphänomene wurden in der ökonomischen Forschung vielfach untersucht. Ein Großteil der Arbeiten fokussiert dabei auf Unternehmensnetzwerke. Diese bestehen aus zwischenbetrieblichen und häufig langfristigen Verbindungen von Unternehmen, die kooperativ Wert schöpfen (Gulati et al. 2000). Hierbei steht im Vordergrund, wie Rollen allokiert und die Zusammenarbeit zwischen Unternehmen gestaltet wird. Grundlegend für die Ausgestaltung von Unternehmensnetzwerken ist das Abhängigkeitsverhältnis von Wertschöpfungsaktivitäten, das in Wertschöpfungsnetzwerken abgebildet wird. In diesen werden die im Unternehmensnetzwerk zu verrichtenden Wertschöpfungsaktivitäten abgebildet und in Verbindung gesetzt (Porter und Millar 1985, Pil and Holweg 2006). Im Vordergrund steht hier also nicht die Rollenverteilung der Unternehmen sondern die zu verrichtenden Aktivitäten und deren Interrelationen.

Insbesondere der Analyse des Wertschöpfungsnetzwerks der Informationsund Kommunikationsindustrie kommt eine besondere Bedeutung zu, da hier aufgrund von technologischen und ökonomischen Einflüssen die Wertschöpfungsstruktur eine starke Dynamik besitzt (Basole 2009, Li und Whalley 2002). Viele wissenschaftliche Arbeiten beschreiben die sich verändernden Wertschöpfungsstrukturen anhand qualitativer Aussagen (Basole 2009, Fransman 2002, Li und Whalley 2002, Peppard and Rylander 2006). Demgegenüber wurden quantitative Analysen zu Wertschöpfungsstrukturen in der Informations- und Kommunikationstechnik (IKT) Industrie, die in dieser Arbeit im Fokus stehen, in der wissenschaftlichen Literatur bisher spärlich publiziert.

Durch Innovationen werden in der IKT Industrie neuartige Wertschöpfungskonstellationen geschaffen und zwingen Unternehmen, aus Wettbewerbsgründen strategische Neupositionierungen im Wertschöpfungsnetz vorzunehmen. Die Analyse und Identifikation von Aktivitätenassoziationen innerhalb von Wertschöpfungsnetzen ist für Unternehmen von strategischem Wert, weil sie den Handlungsraum von Unternehmen definieren, Wertschöpfungsprozesse transparent machen und die Identifikation von Unternehmensstrategien erleichtern. 
So strebt beispielsweise British Telecom in einem aufgrund des Markteintrittes von Internet-Telefonie Anbietern veränderten IKT Wertschöpfungsnetz mit der Bereitstellung einer Entwicklungsplattform für Kommunikationsdienste im Internet und einer Abkehr vom Angebot vertikal integrierter Kommunikationsdienste eine Neupositionierung an. Aus diesem Beispiel lässt sich eine wachsende Bedeutung der softwarebezogenen Wertschöpfung für die Telekommunikationsindustrie ableiten und mit Hilfe einer Wertschöpfungsanalyse genauer untersuchen. So können Rückschlüsse auf Strategien von Telekommunikations- und Softwareunternehmen gezogen werden.

In dieser Arbeit wird basierend auf Daten über Wertschöpfungsaktivitäten von Unternehmen das IKT Wertschöpfungsnetzwerk analysiert. Dabei können die vielfach nur qualitativ vorgenommenen Aussagen über die Existenz eines IKT Wertschöpfungsnetzes bestehend aus Wertschöpfungsaktivitäten der Segmente Hardwarekomponenten, Hardwareequipment, Software, Telekommunikation und Medien empirisch bestätigt werden. Des Weiteren kann gezeigt werden, dass das Wertschöpfungssegment Software eine zentrale Position im IKT Wertschöpfungsnetz einnimmt: insbesondere für Firmen, die Wertschöpfung in den Segmenten Hardwareequipment, Medien und Telekommunikation durchführen, stellen Softwareaktivitäten wichtige Komplementäraktivitäten dar.

\section{Literatur}

Basole RC (2009) Visualization of Interfirm Relations in a Converging Mobile Ecosystem. Journal of Information Technology 24(2):144-159.

Fransman M (2002) Mapping the evolving telecoms industry: the uses and shortcomings of the layer model. Telecommunications Policy 26(9-10):473483.

Gulati R, Nohria N, Zaheer A (2000) Strategic networks. Strategic Management Journal 21(3):203-215.

Li F, Whalley J (2002) Deconstruction of the telecommunications industry: from value chains to value networks. Telecommunications Policy 260-10):451-472.

Peppard J, Rylander A (2006) From Value Chain to Value Network: Insights for Mobile Operators. European Management Journal 24(2-3):128-141.

Pil FK, Holweg M (2006) Evolving from Value Chain to Value Grid. MIT Sloan Management Review 47(4):72-79.

Porter ME, Millar V (1985) How information gives you competitive advantage. Harvard Business Review 63(4):149-160. 


\title{
Auf dem Weg zu mehr Arbeitsteilung durch Open-Source?
}

\author{
Eine fallstudienbasierte Untersuchung \\ am Beispiel von db4objects
}

\author{
Christian M. Wolf', Philipp Nguyen Khuong'2, Alexander Benlian', \\ Thomas Hess ${ }^{1}$ \\ 1 Institut für Wirtschaftsinformatik und Neue Medien, \\ Ludwig-Maximilians-Universität München \\ ${ }^{2} V$ ersant Corporation (db4o)
}

Auf Grund der offenen Struktur von Open-Source-Software und des dazugehörigen Geschäftsmodells liegt die Vermutung nahe, dass die Arbeitsteilung in diesem Bereich noch deutlich stärker ausgeprägt ist als im klassischen Closed-SourceGeschäftsmodell. Daher untersuchten wir mittels einer Fallstudie des Unternehmens $d b 4$ obejcts, ob und in welchen Bereichen der Wertschöpfungskette bei einem typischen Softwareunternehmen mit Open-Source Geschäftsmodell seit der Unternehmensgründung eine zunehmende unternehmensexterne Arbeitsteilung feststellbar ist.

Vergleicht man die Koordinationsformen der Wertschöpfungsaktivitäten von 2004 und 2008 ist auffallend, dass db4objects, gemessen an der Anzahl der Wertschöpfungsaktivitäten im Jahr 2004, eine sehr hohe Leistungstiefe aufwies. Fast alle Aktivitäten wurden intern im Unternehmen durchgeführt - lediglich im Bereich des Marketing und der Distribution wurden Leistungen in Kooperation erbracht oder vom Markt bezogen. Demgegenüber wurden 2008 erheblich mehr Wertschöpfungsteilaktivitäten in Kooperation mit der Nutzer-Community erbracht sowie weitere Leistungen vom Markt bezogen - die Leistungstiefe hat demnach deutlich abgenommen. Am stärksten ist die Veränderung im Bereich der Entwicklung. Hier wurde bei zahlreichen Aktivitäten von der rein hierarchischen Koordinationsform zur Kooperation übergegangen. Darüber hinaus wurde bei der Entwicklung von Plugins und Addons von der Hierarchie sowohl zur Kooperation als auch zum Marktbezug gewechselt. Weitere größere Änderungen haben sich beim Marketing ergeben, auch hier gab es einen Trend hin zur Kooperation. Doch es gab auch eine Änderung der Wertschöpfungsarrangements in die entgegengesetzte Richtung. So wurden alle Aktivitäten der Public Relations 2008 wieder hierarchisch koordiniert, nachdem sie in der Vergangenheit an den Markt ausgelagert waren. 
Der ausschlaggebende Grund für die abgenommene Leistungstiefe in den genannten Bereichen sowie die dort neu hinzugekommenen Aktivitäten war die stark gewachsene Nutzer-Community. Durch den Anstieg der Nutzeranzahl sowie der Nutzer-Expertise konnte die Community im Zeitablauf zunehmend an den einzelnen Aktivitäten beteiligt werden, um von den Vorteilen des Open-Source-Modells zu profitieren. Hierzu zählen im Vergleich zu traditionellen Softwareprodukten beispielsweise die breitere Entwicklerbasis, der höhere Nutzer-Input und die geringeren Marketingkosten (Sharma et al. 2002, S. 8). Diese für die Open-SourceBranche typische Arbeitsteilung zwischen Herstellerunternehmen und Kunden wird auch interaktive Wertschöpfung genannt (Reichwald und Piller 2006). Es gab jedoch auch Entscheidungen, die ohne Bezug zum Open-Source-Modell rein aus Kostengründen getroffen wurden. Dazu zählen die Wiedereingliederung der Public-Relations-Aktivitäten sowie die Auslagerung einer Plugin-Entwicklung an einen indischen IT-Dienstleister.

Ein wie aktuell bei db4objects vorliegendes Wertschöpfungssystem, in dem eine Vielzahl von Akteuren gemeinsam Leistungen erbringen, wird bei traditionellen Unternehmen als Unternehmensnetzwerk bezeichnet (Hensel 2007, S. 23-24; Sydow 1993, S. 79). Bei Open-Source-Software handelt es sich allerdings insbesondere im Bereich der Entwicklung nicht um Unternehmen, die miteinander kooperieren, sondern um Anwender aus der Community, die untereinander und mit dem Projekt-Initiator (hier $d b 4$ objects) - in den meisten Fällen ohne expliziten Kooperationsvertrag - zusammenarbeiten.

Einschränkungen bei der Verallgemeinerung unserer Ergebnisse ergeben sich durch die bekannten Grenzen der qualitativen Betrachtung eines einzelnen Falles, die zwar eine hohe interne, gleichzeitig jedoch eine eingeschränkte externe Validität zur Folge hat. Daher sollte zukünftige Forschung in diesem Bereich unsere Ergebnisse einer quantitativen Kontrolle unterziehen und darauf aufbauend überprüfen, ob die festgestellten Entwicklungen der unternehmensexternen Arbeitsteilung im Spezialfall Open-Source mit den Vorhersagen etablierter Theorien wie der Transaktions- oder Produktionstheorie in Einklang stehen.

\section{Literatur}

Hensel J (2007): Netzwerkmanagement in der Automobilindustrie: Erfolgsfaktoren und Gestaltungsfelder. Gabler, Wiesbaden.

Reichwald R, Piller F (2006) Interaktive Wertschöpfung: Open Innovation, Individualisierung und neue Formen der Arbeitsteilung. Gabler, Wiesbaden.

Sharma S, Sugumaran V, Rajagopalan B (2002) A framework for creating hybrid open source software communities. Inf. Syst. Journal 12(1):7-25. 


\title{
Industrialisierung von Softwareunternehmen durch Arbeitsteilung: Einzelfall oder Trend?
}

\author{
Christian M. Wolf, Alexander Benlian, Thomas Hess \\ Institut für Wirtschaftsinformatik und Neue Medien, \\ Ludwig-Maximilians-Universität München
}

In letzter Zeit ließ sich in verschiedenen Branchen (z. B. Automobil, Chemie) eine zunehmende Auflösung der Wertschöpfungsketten beobachten. Diese manifestiert sich in einer verringerten Bedeutung hierarchischer Koordination zugunsten von marktlichen oder hybriden Koordinationsformen und somit einer Industrialisierung im Sinne einer zunehmenden zwischenbetrieblichen Arbeitsteilung (Picot und Baumann 2009, S. 75).

Da Informations- und Kommunikationstechnologien Treiber bzw. Enabler dieses organisationstheoretischen Phänomens sind (Picot et al. 2008) und diese gleichzeitig einen wichtigen Betrachtungsgegenstand der an der Nahtstelle von Technologie und Wirtschaft angesiedelten Wirtschaftsinformatik darstellen, schlagen Picot und Baumann (2009, S. 78) der Wirtschaftsinformatikforschung vor, organisationsbezogenen Fragestellungen, wie „den Einflussgrößen und Einsichten zu organisatorischen Phänomenen und Beziehungen" (Picot und Baumann 2008, S. 72) eine größere Aufmerksamkeit zu widmen. Diese Anregung wollten wir mit einer Betrachtung der Entwicklung der zwischenbetrieblichen Arbeitsteilung von Softwareunternehmen, die bzw. deren Produkte einen weiteren wichtigen Betrachtungsgegenstand der Wirtschaftsinformatik darstellen, im Rahmen des vorliegenden Beitrags aufgreifen.

Um die damit verbundene konkrete Forschungsfrage zu beantworten, ob und in welchen Bereichen der Wertschöpfungskette sich die zunehmende zwischenbetriebliche Arbeitsteilung bei Softwareunternehmen manifestiert, führten wir eine Multifallstudienuntersuchung bei mittelständischen Herstellern erklärungs- und anpassungsbedürftiger betriebswirtschaftlicher Standardsoftware aus Deutschland durch. Unser erklärtes Ziel war es dabei, die Entwicklung der Arbeitsteilung auf den einzelnen Wertschöpfungsstufen detailliert zu beschreiben, um so die Basis für eine Erklärung der festgestellten Entwicklungen zu liefern.

Die Vorgängerstudie von Wolf et al. (2008) konnte eine zunehmende zwischenbetriebliche Arbeitsteilung vor allem auf den DownstreamWertschöpfungsstufen von SAP feststellen. In der vorliegenden Untersuchung hingegen ergab sich tendenziell eine zunehmende zwischenbetriebliche Arbeitsteilung vor allem auf den Upstream-Wertschöpfungsstufen produktbezogene Forschung (Fälle 1, 2) sowie Produktentwicklung (Fälle 1, 2, 3). 
Ein im Zeitvergleich geringerer Grad an zwischenbetrieblicher Arbeitsteilung ergab sich - wiederum im Gegenteil zur erwähnten Studie - auf den DownstreamWertschöpfungsstufen vertriebliche Beratung (Fälle 1, 3), Implementierung (Fall 3) und Schulung (Fälle 1, 3). Bei Fallstudie 1 lässt sich die Veränderung von 2005 zu 2009 mit der hinzugekommenen Unterstützung der Vertriebspartner durch das Fallstudienunternehmen bei komplexeren und größeren Kundenpräsentationen im Rahmen der vertrieblichen Beratung sowie bei der fachlichen Schulung nach bestimmten neuen Releases erklären. Bei Fallstudie 3 hingegen ist die Reintegration der Wertschöpfungsstufen vertriebliche Beratung, Implementierung und Schulung auf eine strategisch motivierte Fokussierung des Zielmarktes und den damit verbundenen Wechsel zu einem direkten Vertriebsmodell zurückzuführen.

Die festgestellten Unterschiede zur Vorgängerstudie lassen sich dahingehend interpretieren, dass wir mittelständische Softwarehersteller untersucht haben, die einen vergleichsmäßig kleineren Zielmarkt ansprechen. Deshalb mussten diese kein so enormes Wachstum bewältigen wie SAP und sie benötigten in geringerem Maße nicht zu den eigenen Kernkompetenzen zählendes spezifisches Wissen (wie z. B. länderspezifische Rechnungslegungsstandards), was die festgestellten Hauptgründe für die gestiegene zwischenbetriebliche Arbeitsteilung auf den DownstreamWertschöpfungsstufen von SAP war. Eine Industrialisierung durch zunehmende zwischenbetriebliche Arbeitsteilung ist somit zwar grundsätzlich auch in den von uns erhobenen Fallstudien festzustellen, allerdings ausschließlich im Rahmen des seit längerem bekannten Outsourcing der eigentlichen Softwareentwicklung im Sinne der Erstellung von Programmcode sowie der Kooperation mit Partnern oder Forschungseinrichtungen bei der produktberogenen Forschung. Auf den anderen Wertschöpfungsstufen ergaben sich keine oder sogar gegenläufige Entwicklungen.

\section{Literatur}

Picot A, Baumann O (2009) Die Bedeutung der Organisationstheorie für die Entwicklung der Wirtschaftsinformatik. WIRTSCHAFTSINFORMATIK 2009(1): 72-81.

Picot A, Reichwald R, Wigand RT (2008) Information, organization and management. Springer, Heidelberg.

Wolf CM, Geiger K, Benlian A, Hess T, Buxmann P (2008) Spezialisierung als Ausprägungsform einer Industrialisierung der Software-Branche - Eine Analyse am Beispiel der ERP-Software von SAP. In Herzwurm G, Mikusz M (Hrsg) Industrialisierung des Software-Managements. GI, Stuttgart. 


\title{
Towards a Pricing Method for Software Product Lines
}

\author{
First Steps, Problems, Needs, and Research Agenda
}

\author{
Johannes Müller \\ Information Systems Institute, University of Leipzig
}

Software product lines (spls) are an emerging paradigm, which can help to increase the efficiency of software development thereby, decrease the development costs for a single system within a given domain. This is achieved by exploiting similarities between systems of a whole family of systems while managing their variability in a systematic manner. Spl practices comprise three main processes: domain engineering, application engineering, and management (Clements and Northrop 2007). Hence, the orientation towards spls is a strategical decision, which affects the whole organization (v.d. Linden 2007).

However, an issue not addressed yet is: Which prices optimize the profit of a spl vendor? To answer this, one is faced with the special properties of software or digital goods in general (Buxmann et al. 2008, p. 1). A promising approach to price spls is value based pricing (Nagle and Hogan 2006). Whereas the main objective of a pricing method is to cope with the competitive forces (i.e. customers, competitors, suppliers, entrants, substitutes) (Porter 1998).

Nagle and Hogan (2006) propose a framework for value based pricing strategies. It consists of five general levels: value creation, align prices on market segments, communicate value, define price policies, and set price level. However, the framework is to general and needs adaption to spl practices. According to that task, the activities of fig. 1 have to be mapped to sub processes and activities of spl practices.

Concept creation, value estimation, and market segmentation are accomplished during domain analysis. Prior to the activity domain implementation a price structure needs to be developed as a activity of the management process to give rationals about the importance of the features to implement. The communication strategy is developed by the organizational management and is approached after the development of the price structure. The rules of the pricing policy is defined by the organizational management. Establishing the final price level for the features and feature combinations of a spl is part of the management subprocess by the organizational management. Price optimization is realized by a feedback loop between application engineering and management. The final prices and price policies are communicated to stakeholders by the organizational management due to its authority to enforce the pricing decisions. 


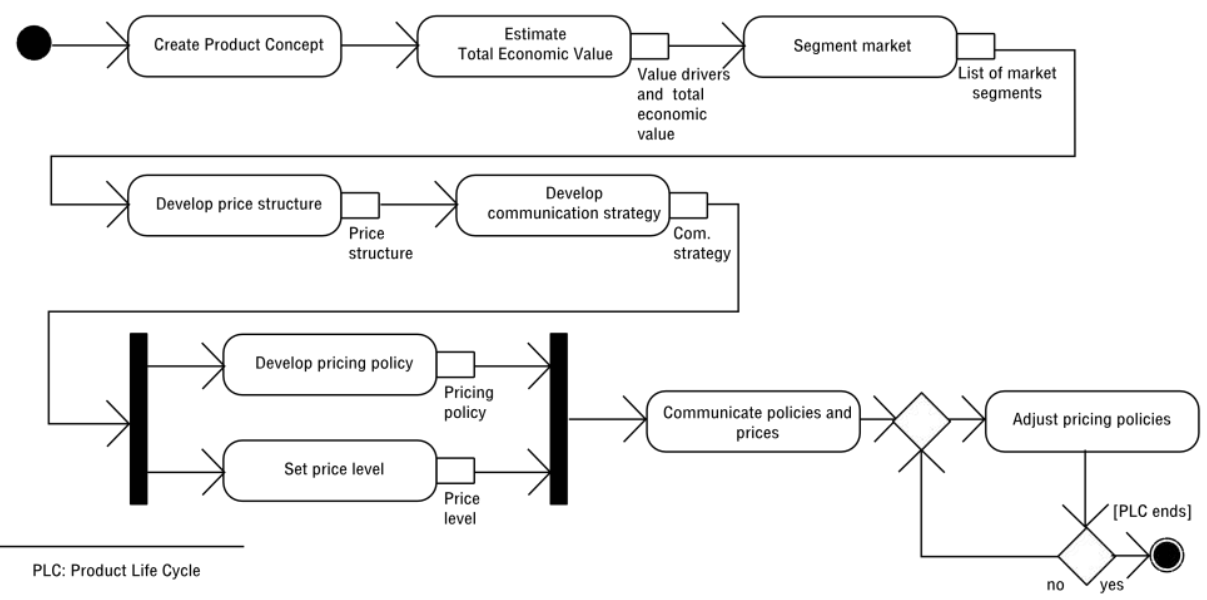

Figure 1: Process to implement a value-based pricing strategy

To summarize, the paper presents a method to price products of spls, which is based on a general pricing method. This method is mapped to processes and activities of spl practices. Further research is needed in order to develop a widely used resilient pricing method for spls.

\section{References}

Buxmann P, Diefenbach H, Hess T (2008) Die Softwareindustrie: Ökonomische Prinzipien, Strategien, Perspektiven. Springer, Berlin.

Clements P, Northrop L (2007) Software product lines: Practices and patterns, 6. Addison-Wesley, Boston.

v.d. Linden FJ, Schmid K, Rommes E (2007) Software product lines in action: The best industrial practice in product line engineering. Springer, New York.

Nagle TT, Hogan JE (2006) The strategy and tactics of pricing: A guide to grow more profitabiliy, 4. Prentice Hall, Upper Saddle River.

Porter ME (1998) Competitive Strategy: Techniques for Analyzing Industries and Competitors, Free Press, New York. 


\title{
Preisgestaltung für Software-as-a-Service
}

\section{Ergebnisse einer empirischen Analyse mit Fokus auf nutzungsabhängige Preismodelle}

\author{
Sonja Lehmann', Tobias Draisbach'1, Corina Koll1, Peter Buxmann', \\ Heiner Diefenbach ${ }^{2}$ \\ ${ }^{1}$ Fachgebiet Information Systems, TU Darmstadt \\ ${ }^{2} T D S$ AG, Neckarsulm
}

Software wird heute zunehmend auch als Dienstleistung angeboten. Hierbei stellen Software- und Serviceanbieter Kunden Softwarelösungen via Internet zur Verfügung (Buxmann et al. 2008a, S. 8). Bei solchen Software-as-a-Service (SaaS)Lösungen liegt die Verantwortung für Betrieb und Wartung der Software beim Anbieter (Cusumano 2007, S. 20; Buxmann et al. 2008b, S. 500). Kunden verbinden mit SaaS häufig den Wunsch nach flexiblen, ihren Bedürfnissen angepassten Preismodellen. Daher stehen SaaS-Anbieter vor der Herausforderung, neue geeignete Preiskonzepte für diesen Markt zu entwickeln. Dabei besteht eine Grundsatzentscheidung darin, ob die Bepreisung nutzungsabhängig oder -unabhängig durchgeführt werden soll.

Vor diesem Hintergrund soll im vorliegenden Beitrag ein Überblick über derzeitig verwendete Preismodelle von SaaS-Anbietern gegeben werden. Basierend auf der Gliederung der Softwarepreisgestaltung (Lehmann und Buxmann 2009) wurden die Parameter „Struktur des Zahlungsstroms“, „Preisdifferenzierung“ und „Bemessungsgrundlage“ untersucht. Hierzu wurden 114 SaaS-Anwendungen von 80 Anbietern anhand der Informationen ihrer Webseiten analysiert.

Außerdem soll die häufig in der Literatur vertretene These, dass nutzungsabhängige Preismodelle für SaaS grundsätzlich gut geeignet sind (siehe z. B. Kittlaus und Clough 2009, S. 59), näher untersucht werden. Hierzu haben wir mit einem Anbieter statistischer Anwendungssoftware für den B2B-Bereich eine Fallstudie durchgeführt. Dabei wurde im Rahmen einer telefonischen Kundenbefragung mit 28 Teilnehmern die van-Westendorp-Methode zur Ermittlung der Zahlungsbereitschaften für nutzungsabhängige und nutzungsunabhängige SaaS-Angebote eingesetzt.

Derzeitige Diskussionen über die nutzungsabhängige Preisgestaltung von SaaSLösungen konnten durch unsere Ergebnisse nicht bestätigt werden. Bei den Bemessungsgrundlagen kommen überwiegend nutzungsunabhängige Einheiten zum Einsatz. Nutzungsabhängige Bemessungsgrundlagen wie Preise je durchgeführte Transaktion hingegen sind für Kunden meist optional. 
Die Analyse der Zahlungsbereitschaften für nutzungsabhängige Bemessungsgrundlagen mit Hilfe der van Westendorp-Methode im Rahmen einer Fallstudie hat ergeben, dass die Mehrheit der Kunden ihre Nutzungsintensität nicht kennt und daher keine Aussage treffen kann. Unter den Kunden, die eine konkrete Zahlungsbereitschaft nannten, variierte die Zahlungsbereitschaft um den Faktor 1000. Aus Anbieterperspektive ist diese ausgeprägte Heterogenität der Zahlungsbereitschaften besonders ungünstig. Um einerseits viele Kunden zu erreichen und andererseits deren Zahlungsbereitschaft bestmöglich abzuschöpfen, ist eine homogene Struktur der Zahlungsbereitschaften mit einer geringen Varianz erforderlich.

Generell bietet sich die Preisdifferenzierung zum Abschöpfen unterschiedlicher Zahlungsbereitschaften an. Dies dürfte jedoch in diesen Dimensionen schwierig umzusetzen sein. Weiterhin kommt hinzu, dass die Preisgestaltung für SaaSProdukte vermutlich aufgrund des Vertriebs über Internet tendenziell transparenter sein wird als beispielsweise bei on-Premise-Software. Dies erschwert insbesondere Preisdifferenzierung dritten Grades.

Die Ergebnisse der Untersuchung zeigen, dass eine nutzungsabhängige Bemessungsgrundlage - auch wenn sie für SaaS als prädestiniert angesehen wird - ungeeignet sein kann, wenn die ungeschickte Wahl der Bemessungsgrundlage zu einer stark heterogenen Struktur der Zahlungsbereitschaften führt.

\section{Literatur}

Buxmann P, Diefenbach H, Hess T (2008a) Die Softwareindustrie: Ökonomische Prinzipien, Strategien, Perspektiven. Springer, Berlin.

Buxmann P, Hess T, Lehmann S (2008b) Software as a Service. WIRTSCHAFTSINFORMATIK 50(6):500-503.

Cusumano MA (2007) The changing labyrinth of software pricing. Communications of the ACM 50(7):19-22.

Kittlaus HB, Clough PN (2009) Software product management and pricing. Key success factors for software organizations. Springer, Berlin.

Lehmann S, Buxmann P (2009) Preisstrategien von Softwareanbietern. WIRTSCHAFTSINFORMATIK 51(6), im Druck.. 


\title{
Towards Requirements Engineering for "Software as a Service"*
}

\author{
Marina Berkovich', Sebastian Esch', Jan Marco Leimeister ${ }^{2}$, Helmut Krcmar ${ }^{1}$ \\ ${ }^{1}$ Lehrstubl für Wirtschaftsinformatik, Technische Universität München \\ ${ }^{2}$ Fachgebiet Wirtschaftsinformatik, Universität Kassel
}

Software as a Service $(\mathrm{SaaS})$ is defined as a software offered as a service that can be accessed via the Internet (Buxmann et al. 2008). A main characteristic of SaaS is that it separates the owner and the user of software. SaaS can be seen as a complex solution, aiming at solving customers' problems and providing advantages for him (Böhmann und Krcmar 2007; Berkovich et al. 2009). Such a complex solution is also known as a hybrid product (Leimeister und Glauner 2008), which is a combination of physical products and services offered on the market as integrated service bundle. The success of a solution depends on how good it satisfies the wishes and requirements of the customer. To know these requirements it is important to carry out requirements engineering (RE) accurately. Especially interesting is how $\mathrm{RE}$ for $\mathrm{SaaS}$ has to be done. In this paper the RE for SaaS is discussed from the perspective of hybrid products. The main contribution of it is a list of requirements on the RE of SaaS as a hybrid product.

The first step in the research was to understand the characteristics of SaaS. Thus, different scenarios for delivering SaaS were formulated and analyzed. In the second step of the research, the definition of RE in literature has been reviewed. Then, by confronting the characteristics of SaaS and RE, requirements for the RE for SaaS have been derived.

In the context of SaaS and hybrid products two scenarios arise. In the first scenario $\mathrm{SaaS}$ itself can be seen as a hybrid product, a bundle of software and services. The software is often especially developed to be offered as a service, considering different requirements concerning data-handling or multitenancy. In the second scenario, SaaS is used to enable face-to-face services. In this scenario additional requirements are derived from the service provider, who needs specific functionality to support his service processes. In contrast to the first scenario the coupling between service processes and software functionality is tighter.

The review of the RE literature revealed that up to now there exists no dedicated RE for hybrid Products. In literature all involved domains have their

\footnotetext{
* Dieser Beitrag ist im Rahmen des Sonderforschungsbereiches 768 „Zyklenmanagement von Innovationsprozessen - Verzahnte Entwicklung von Leistungsbündeln auf Basis technischer Produkte“, gefördert von der Deutschen Forschungsgemeinschaft (DFG), sowie des Forschungsprojekts SPRINT entstanden, gefördert durch das Bundesministerium für Bildung und Forschung unter dem Förderungskennzeichen 01FD0609.
} 
own RE models, but there is no integrated one. Based on our review of SaaS as a hybrid product and of RE in general, we derived seven requirements for the RE of hybrid products. Each of these requirements is justified by at least one characteristic of SaaS. The requirements are:

1. Coordinated and integrated RE for all single components of SaaS

2. Selection of the right stakeholders

3. Better customer integration into the RE-process

4. Consideration of changes of requirements after delivery

5. Thorough and continuous documentation of requirements

6. Consideration of the source of requirements during elicitation

7. Consideration of the source of requirements during change-management

Through the analysis of different scenarios for SaaS, a deeper understanding of SaaS could be gained. Also, by the viewpoint of hybrid products, SaaS were analyzed without focusing on one special issue. The main contribution of this paper is the list of requirements on the RE for SaaS. These requirements summarize important topics that are relevant for a successful RE. For research this enables the possibility of a systematic integration of existing RE methods and techniques in the development of SaaS. In practice, through a comprehensive RE model, the collaboration between different domains involved in the development is enhanced. The first step in future research will therefore be to develop a RE model for SaaS based on the requirements presented in this paper.

\section{References}

Berkncesovich M, Esch S, Leimeister JM, Krcmar H (2009) Requirements engineering for hybrid products as bundles of hardware, software and service elements - a literature review. 9. Internationale Tagung Wirtschaftsinformatik. Wien, Österreich.

Böhmann T, Krcmar H (2007) Hybride Produkte: Merkmale und Herausforderungen. In: Wertschöpfungsprozesse bei Dienstleistungen: Forum Dienstleistungsmanagement. Hrsg.: Bruhn, M.; Stauss, B. Gabler, p. 240-255.

Buxmann P, Lehmann S, Hess T (2008) Software as a Service. In: Wirtschaftsinformatik, 50(6): 500-503.

Leimeister JM, Glauner C (2008) Hybride Produkte - Einordnung und Herausforderungen für die Wirtschaftsinformatik. In: Wirtschaftsinformatik, 3. 


\title{
Preisfindung von IT-Produkten durch retrograde Kalkulation
}

\section{Einsatz von Quality Function Deployment und modifizier- tem Target Costing für die Preisfindung bei Neu- und Wei- terentwicklung von IT-Produkten}

\author{
Georg Herzwurm ${ }^{1}$, Benedikt Krams², Wolfram Pietsch² \\ ${ }^{1}$ Lehrstubl für ABWL und Wirtschaftsinformatik II, \\ Betriebswirtschaftliches Institut, Universität Stuttgart \\ ${ }^{2}$ Fachbereich Wirtschaftswissenschaften, Fachbochschule Aachen
}

Bei der Bestimmung des Preises von IT-Produkten kommt der Identifikation und Priorisierung der relevanten Kundenanforderungen eine Schlüsselrolle zu, und zwar sowohl bei deren Neu- als auch Weiterentwicklung. Quality Function Deployment (QFD) hat sich als verlässliches Instrument für die Identifikation originärer Kundenanforderungen an IT-Produkte bewährt. Der Beitrag diskutiert, ob QFD in Kombination mit retrograder Kalkulation im Sinne des Target Costing für die Preisfindung neuer und weiterzuentwickelnder IT-Produkte eingesetzt werden kann.Klassische Ansätze zur Preisfindung wie die kosten-, wettbewerbs- und marktorientierte Preispolitik können für IT-Produkte und angrenzende Services übertragen werden; die Zielführung für eine adäquate Bedienung der Kundenbedürfnisse und insbesondere der Preisfindung durch kostenorientierte Preispolitik ist jedoch begrenzt (Buxmann et al. 2008, S. 78).

QFD und Target Costing unterstützen die anforderungs- und marktgerechte sowie zielkostenkonforme Entwicklung von IT-Produkten. Die retrograde Kalkulation durch Kombination von QFD und Target Costing unterstützt die wettbewerbs- und nutzenorientierten Preispolitik.

Bei Neuentwicklungen von IT-Produkten kommt es aufgrund geringer Erfahrung der Entwickler bezüglich des neuen Produkts dazu, dass eine Vielzahl der übertragenen Funktionen außerhalb der Zielkostenzone des Target Costings liegen. Dies gilt insbesondere für Neuentwicklungen von Individualsoftware: Durch die Einmaligkeit der Entwicklung kommt es in der Regel nicht zu einer Nachkalkulation des Produkts (Ehrlenspiel 2003, S. 592)1, auch deshalb, weil das Produkt dem

\footnotetext{
${ }^{1}$ Ehrlenspiel differenziert im Kontext des zielkostengesteuerten Konstruierens zwischen Serien-, Einzel- und Kleinserienfertigung. Seine Aussage bzgl. Einzelfertigung wurde auf Individualsoftwareentwicklungen übertragen.
} 
Kunden bereits übergeben wurde. Eine Weiterentwicklung des Produkts zur Senkung der Kosten findet nicht statt.

Bei der Preisfindung von IT-Produkten für Neuentwicklungen tragen QFD und modifiziertes Target Costing dazu bei, für priorisierte Funktionen die Zielkosten zu bestimmen. Deren Einhaltung wird jedoch in laufenden Entwicklungsprojekten gesteuert und ggf. zu Lasten der geforderten Qualität, Funktionalität oder Zeit erreicht. Die Findung eines „,zweiten“ Preises (nach Durchführung von QFD und modifiziertem Target Costing) findet bei Neuentwicklungen nicht statt.

Für die Preisfindung bei Weiterentwicklungen von IT-Produkten sind die Ergebnisse der bereits für die Neuentwicklung angewendeten Methoden QFD und modifiziertes Target Costing Ausgangslage. Kosten werden erneut gesenkt, bspw. durch schnellere Entwicklungsverfahren oder Anpassungen derart, dass OverEngineering vermieden wird.

Solche Kostensenkungspotentiale ermöglichen die Findung eines „zweiten“ Preises. Die gesunkenen Entwicklungskosten können entweder an Kunden weitergegeben werden, um einen Vorteil gegenüber dem Wettbewerb zu erzielen (die Gewinnmarge bleibt gegenüber der Neuentwicklung identisch), oder aber gesunkene Entwicklungskosten werden bei gleichem Preis der ursprünglichen Neuentwicklung nicht in den Markt kommuniziert (Erhöhung der Gewinnmarge).

Darüber hinaus kann bei entsprechender Wettbewerbslage ein höherer Preis veranschlagt werden, der darin begründet ist, dass die Funktionalität des ITProdukts verbessert wurde.

Für Standard- als auch Individualsoftwareentwicklungen ist die Kombination aus QFD und modifiziertem Target Costing für IT-Produkt-Weiterentwicklungen anwendbar. Für IT-Produkt-Neuentwicklungen ist die dargestellte Vorgehensweise weniger geeignet, da sie nicht zu einer Preisfindung führt. Das bedeutet nicht, dass die Kombination von QFD und Target Costing unzweckmäßig ist; vielmehr können die Ergebnisse der Neuentwicklungen für Nachkalkulationen genutzt werden.

\section{Literatur}

Buxmann P, Lehmann S, Hess T, Staritz M (2008) Entwicklung und Implementierung von Preisstrategien für die Softwareindustrie. In: Gronau N, Eggert S (Hrsg.) Beratung, Service und Vertrieb für ERP-Anbieter. Gito, Berlin.

Ehrlenspiel K (2003) Integrierte Produktentwicklung. Denkabläufe Methodeneinsatz Zusammenarbeit. Hanser, München. 


\title{
Mobile Application Marketplaces - an Investigation from Customers' Perspective
}

\author{
Michael Amberg, Inke Thiessen, Michael Lang, Bartosch Belkius \\ School of Business Economics and Information Systems, \\ Friedrich-Alexander University Erlangen-Nurenberg
}

Since the opening of the AppStore on the $11^{\text {th }}$ of July 2008 more than one billion downloads of iPhone Apps have proceeded and generate revenues of thirty million dollar each month. In the meantime similar concepts emerge, e.g. the android market of Google and the Blackberry AppWorld of RIM. Are these efforts rewarded by the customers? Is the existence of a mobile application marketplace crucial in their choice for a new mobile device? This question arises because the choice directly ties the customer down to the use of one specific marketplace.

In this paper the impact fields on the choice for a mobile device are explored with an exploratory case study on young German speaking interviewees. The young age participants are expected to have a relatively high technical affinity and interest. Due to the low level of previous research in the field of mobile application marketplaces, an iterative research approach is used. The first interview phase is coded and results in core and second level categories concerning the choice for a mobile device. The second and third interview sample tightens these factors and provides each with a statistical weight. The weight of a second level category represents the impact of this category on the choice for a mobile device.

Table 1: Given impact on $2^{\text {nd }}$ level categories in the choice for a mobile device at nonobservance of monetary influences

\begin{tabular}{|c|c|c|c|c|c|c|c|c|}
\hline $\begin{array}{c}\text { Core } \\
\text { category }\end{array}$ & \multicolumn{2}{|c|}{ Design } & \multicolumn{2}{|c|}{ Brand } & \multicolumn{4}{|c|}{ Function } \\
\hline $\begin{array}{c}\text { Second } \\
\text { level } \\
\text { category }\end{array}$ & E્ઞિ & 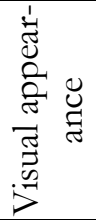 & 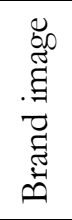 & 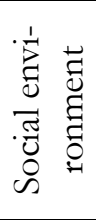 & 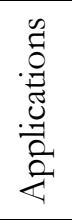 & 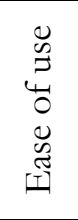 & 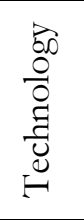 & 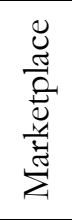 \\
\hline Median & 4 & 4 & 3 & 2 & 3 & 4 & 3 & 2 \\
\hline Variance & 0,97 & 1,05 & 1,28 & 0,92 & 1,54 & 0,95 & 1,65 & 1,67 \\
\hline
\end{tabular}

The interview results show that the existence and character of a mobile application marketplace only has a subordinate role during the choice for a specific device and contract. Coherent aspects of the device to the existence of an application market- 
place like design, technology and diversity of functions affect the choice to a great extent. The relevant aspects for mobile customers during their decision are the visual appearance, form and ease of use of the smartphone itself. Based on the second level categories and their given weights, a scoring model for mobile devices is derived for a customer focused rating. An exemplary showcase indicates the model usage possibilities with three actual devices.

Due to the high sales rates and the increased competitive pressure regarding the mobile business, new alliances between previous cooperators as well as competitors emerge weakly. Combining this evolution and analysts' prospects, the research concerning mobile application marketplaces and the innovative value chain they represent ought to follow the rapid growth. The ability of a transmittance of this new form of value chain including the innovative potential of free and open development communities from the field of mobile business to e.g. home computers and the software industry, can be an emerging research field. Parallel the mobile business has still evolution brakes like the platform dependency. As one application needs to be built in a new programming language, programming environment and published on a new marketplace for each operating system and/or screen size, the innovation potential on application diversity has still growth opportunities. 


\title{
Platform-as-a-Service as an Enabler for Global Software Development and Delivery
}

\author{
Ivo Stankov, Rastsislau Datsenka \\ Lehrstubl für Wirtschaftsinformatik, \\ Europa-Universität Viadrina Frankfurt (Oder)
}

The convergence of several technological, organizational and political trends such as outsourcing, distributed software work, the emergence of internet-based collaborative environments, and the expansion of the global delivery of IT services, made a new offshoring strategy possible. This is described in this paper as Global Software Development and Delivery (GSDD).

At the same time, the emergence of the Platform-as-a-Service (PaaS) concept in the last couple of years has opened new horizons for software outsourcing and offshoring in general and for GSDD in particular. This is a result of the establishment and proliferation of the Software-as-a-Service delivery paradigm and the expansion of web-based development and collaboration.

This paper focuses on the Platform-as-a-Service concept and aims to explore its current and potential technological capabilities as an enabler for innovative service-based global software development and delivery strategies.

\begin{tabular}{|c|c|}
\hline Global Software Development and Delivery \\
\hline \multicolumn{2}{|c|}{ Collaborative environment } \\
\hline Software development & Service delivery \\
\hline $\begin{array}{c}\text { Requirements } \\
\text { Design } \\
\text { Implementation } \\
\text { Operation } \\
\text { Optimization }\end{array}$ & $\begin{array}{c}\text { Capacity management } \\
\text { Availability management } \\
\text { Continuity management } \\
\text { Financial management }\end{array}$ \\
\hline
\end{tabular}

Figure 1: GSDD methodology based on IT infrastructure library

The term Global Software Development and Delivery describes the work-flow and governance of distributed IT offshoring projects. The three major elements of GSDD are geographically shared software development, global delivery, and seamless collaboration between production teams. This paper focuses mainly on its delivery component and references the ITIL V2 as a sample model of investigation. Figure 1 illustrates this -methodological framework. 
Furthermore, the term Platform-as-a-Service generally refers to internet-based software delivery platforms on and for which third-party independent software vendors or custom application developers can create multi-tenant, web-based applications that are hosted on the provider's infrastructure and are offered as a service to customers. Based on the technical and organizational considerations of GSDD, a number of recommendations for an on-demand platform are proposed as shown in Table 1.

Table 1: Major characteristics of PaaS-based GSDD

\begin{tabular}{|l|l|}
\hline \multicolumn{1}{|c|}{ GSDD element } & \multicolumn{1}{c|}{ PaaS characteristics } \\
\hline Development & $\begin{array}{l}\text { Web-based IDE, common programming languages, industry standards, } \\
\text { code repositories, easy/automatic updates }\end{array}$ \\
\hline Delivery & Scalable hosting, easy expansion of teams and computing power \\
\hline Capacity management & $\begin{array}{l}\text { Hosting by large trustworthy providers, computing power available on- } \\
\text { demand }\end{array}$ \\
\hline IT continuity management \\
\hline Availability management & $\begin{array}{l}24 / 7 \text { delivery of product to the customer, diminishing risks of unavaila- } \\
\text { bility due to limited infrastructure on vendor's side }\end{array}$ \\
\hline Financial management & $\begin{array}{l}\text { On-demand scalable billing systems, automatic charging, and transpa- } \\
\text { rent financial management accessible per web-interface }\end{array}$ \\
\hline $\begin{array}{l}\text { Collaborative } \\
\text { environment }\end{array}$ & $\begin{array}{l}\text { Social networking, shared code development, project management, } \\
\text { tracking systems, web dashboards. }\end{array}$ \\
\hline
\end{tabular}

Finally, the current PaaS market is briefly examined with the aim to estimate its suitability to support GSDD activities. To summarize, currently there is a lack of viable solutions that support high-end, standard language, web-based development.

Being mainly a delivery mechanism, most of ITILs service delivery management processes are covered by a typical PaaS, but there are still differences in the approaches of the different providers. At the current stage, most PaaS solutions do not offer collaboration tools as a core component of their architecture.

It can be concluded that PaaS and GSDD are definitely compatible, and a suitably designed platform can theoretically support all required service delivery management processes even at the current technological level.

The current PaaS market does not necessarily support the needed functionalities and workflows that would make it viable for a broad-scale global software development and delivery adoption. It should be noted that this area of computing is, while very new, also very dynamic and open for innovation, changes and research. 
Unternehmensberatung im IT-Umfeld 



\title{
Vorwort zur Teilkonferenz
}

\section{Unternehmensberatung im IT-Umfeld}

\author{
Michael H. Breitner', Thomas Deelmann², Peter Loos³, Volker Nissen ${ }^{4}$ \\ ${ }^{1}$ Institut für Wirtschaftsinformatik, Leibniz Universität Hannover \\ 2Strategy Development, T-Systems International GmbH, Bonn \\ ${ }^{3}$ Institut für Wirtschaftsinformatik im DFKI, Universität des Saarlandes \\ ${ }^{4}$ Institut für Wirtschaftsinformatik, Technische Universität Ilmenau
}

Wie kaum eine andere Branche verzeichnete die Unternehmensberatung in den letzten Dekaden ein überaus dynamisches Wachstum. Sie durchdringt viele Themenbereiche im Umfeld der betrieblichen Informationsverarbeitung (IV). Dort übernimmt die Unternehmensberatung eine Mittlerfunktion zwischen Forschung und Praxis, bei der sich die Möglichkeit ergibt, die Umsetzung aktueller Forschungsergebnisse in der Praxis zu beobachten oder voranzutreiben und die wissenschaftliche Aufbereitung von in der Praxis aufgetretenen Frage und Problemstellungen anzustoßen. Die angesprochene Mittlerfunktion äußert sich nicht zuletzt in der Attraktivität der Beratung als Arbeitgeber für Hochschulabsolventen.

Zwischen der Beratungspraxis und den Managementwissenschaften gibt es viele Berührungspunkte. So haben viele Managementkonzepte ihren Ursprung in der Beratungspraxis und wurden dann von der Wissenschaft aufgegriffen. Die Managementwissenschaften definieren sich selbst als angewandte Forschung, welche Angebote für die Lösung praktischer Probleme bereit stellen. Diese Nähe legt eine fruchtbare wechselseitige Auseinandersetzung zwischen der Beratungspraxis und einer wissenschaftlichen Forschungsdisziplin, die sich mit der Unternehmensberatung befasst, nahe.

Trotz der großen praktischen Bedeutung von Unternehmensberatung wird der Branche in der deutschsprachigen Forschung und Lehre noch zu wenig Aufmerksamkeit zuteil. Im Rahmen dieses Workshops werden daher IV-bezogene Themen im Umfeld der Unternehmensberatung aus wissenschaftlicher Perspektive aufgearbeitet. Die vorliegenden Beiträge liefern vielfältige Erkenntnisse zur Forschungsdomäne Consulting Research. Sie lassen sich auf einem thematischen Bogen von Consulting as a Business bis zu Consulting Business anordnen. So beschäftigen sich die Aufsätze von Drews sowie von Deelmann eher mit den internen Strukturen von Beratungsunternehmen und ihren grundsätzlichen Handlungsmöglichkeiten. Die Beiträge von Friedrich, Sprenger und Breitner sowie von Gehrke und Thams setzen sich demgegenüber mit konkreten Projektsituationen und Anforderungen auseinander. Die Aufsätze von Balzert, Fettke und Loos sowie von Nissen lassen sich zwischen beiden Polen platzieren. 
Dieser Workshop wird von dem Arbeitskreis IV-Beratung der Gesellschaft für Informatik e.V. in Kooperation mit der Gesellschaft für Consulting Research (GCR) e.V. veranstaltet. Er richtet sich in gleicher Weise an Forscher unterschiedlicher Disziplinen im Umfeld der IV-Beratung wie auch an Vertreter aus der Praxis, die in der IV-Beratung aktiv sind oder als Kunden IV-Berater einsetzen. Studenten beratungsaffiner Fachrichtungen sind ebenfalls angesprochen.

Die Organisatoren bedanken sich bei allen Autoren, die das Themengebiet durch die Einreichung ihrer Forschungsergebnisse unterstützt haben. Ebenfalls gedankt sei den Mitgliedern des Programmkomitees, die durch ihre Gutachten die Qualität der Teilkonferenz sicherstellen: Ulrich Bode (Sprecher des Beirats der Selbständigen in der GI e.V.), Prof. Dr. Georg Rainer Hofmann (Hochschule Aschaffenburg), Dr. Nick Gehrke (Universität Hamburg), Prof. Dr. Norbert Gronau (Universität Potsdam), Dr. Claudia M. Koenig (Managementtrainerin, Aachen und Hannover), Prof. Dr. Franz Lehner (Universität Passau), Dr. Ulrich Müller (TSystems International GmbH), Dr. Dr. Björn Niehaves (ERCIS - Universität Münster), Prof. Dr. Jochen Prümper (HTW Berlin), Dr. Jochen Sprengart (DHC GmbH), Karin Thelemann (Ernst \& Young AG) und Frank Thiele (Deloitte Consulting $\mathrm{GmbH})$.

Unser Dank gilt ebenso den Organisatoren und Veranstaltern der Multikonferenz Wirtschaftsinformatik 2010 für die gute Zusammenarbeit.

Hannover, Bonn, Saarbrücken, Ilmenau, im November 2009

Michael H. Breitner, Thomas Deelmann, Peter Loos, Volker Nissen 


\title{
VAT Compliance: Einhaltung umsatzsteuerlicher Anforderungen beim Prozess- und Datenmanagement in ERP-Systemen
}

\author{
Nick Gebrke1, Rainer Thams² \\ ${ }^{1}$ Arbeitsbereich Wirtschaftsinformatik, Universität Hamburg \\ ${ }^{2}$ PricewaterhouseCoopers AG, Hamburg
}

Der vorliegende Beitrag beschäftigt sich mit dem Thema, wie umsatzsteuerliche Anforderungen, vorgegeben vom Umsatzsteuergesetz, bei der Abwicklung von Geschäftsvorfällen in ERP-Systemen ordnungsgemäß berücksichtigt werden können. Es handelt sich um ein Compliance Thema, da diese spezifischen Anforderungen der Gesetzgebung entspringen. Der Beitrag fokussiert dabei zwei Szenarien: erstens die Sicherstellung der umsatzsteuerlichen Ordnungsmäßigkeit bei der Einführung von ERP-Systemen und zweitens eine Betrachtung im Regelbetrieb mit Hilfe eines sogenannten VAT-Scans. Der Beitrag ist wie folgt gegliedert. Zunächst wird der Stand der Forschung dargelegt. Dabei stellt sich heraus, dass bisher wenig wissenschaftliche Literatur zu diesem Thema vorliegt, jedoch bereits Arbeiten dazu existieren, wie rechtliche Bestimmungen in Anforderungen an IT-Systeme überführt werden können. Anschließend folgt die Darlegung der Problemrelevanz. Da das Thema Bezüge zum Recht als auch zur IT hat, werden folgend Grundsätze des Umsatzsteuerrechts erläutert. Aufgrund der Rechtskomplexität kann dies nur oberflächlich geschehen. Danach werden Vorgehensweisen dargestellt, wie Anforderungen des Umsatzsteuerrechts bei der Konzeption der Einführung, sowie des Regelbetriebs eines ERP-Systems berücksichtigt werden können. Der Beitrag schließt mit einem kurzen Fazit.

Die Problemrelevanz von VAT Compliance im Zusammenhang mit Geschäfts-prozessen in ERP-Systemen ergibt sich hauptsächlich aus der Komplexität des europäischen Umsatzsteurrechts und den damit verbundenen Problemen, die steuerliche Handhabung der Geschäftsprozesse richtig im ERP-System abzubilden. Aus organisatorischer Sicht kann die Problemrelevanz wie folgt begründet werden:

- Die Arbeitsteilung in Organisationen, in denen ERP-Systeme eingesetzt werden, kann als hoch eingeschätzt werden. Dies bedeutet, dass einzelne Personen nicht mehr den vollständigen Überblick über den umsatzsteuerlich relevanten Geschäftsvorfall haben. Im Rahmen dieser Arbeitsteilung müssen sich die beteiligten Personen insofern auf das System und dessen Einstellungen bezüglich der umsatzsteuerlichen Einordnung von Geschäftsvorfällen verlassen.

- Expandiert eine Unternehmung, so werden ggfs. neue Geschäftsvorfälle auftreten, deren umsatzsteuerliche Behandlung im System nicht anfangs vorgese- 
hen war. Dies ist insbesondere der Fall, wenn grenzüberschreitende Geschäftsvorfälle relevant werden.

Aus rechtlicher Sicht besteht folgende Problemrelevanz:

- Wird durch eine Betriebsprüfung festgestellt, dass Geschäftsvorfälle umsatzsteuerfrei behandelt wurden, obwohl diese steuerpflichtig sind, so rechnet der Fiskus den Steuerbetrag heraus, sodass Umsatzsteuer nachbezahlt werden muss. Hieraus ergeben sich erhebliche Liquiditätsrisiken.

- Wird vom Fiskus festgestellt, dass Geschäftsvorfälle umsatzsteuerlich falsch behandelt wurden und entsteht daraus ein höherer Steueranspruch, so muss zusätzlich zur Nachzahlung mit einer Verzinsung des höheren Steueranspruchs gerechnet werden. Die Zinsbeträge können sehr hoch sein, da Betriebsprüfungen erst Jahre nach der Steuerentstehung stattfinden können.

- Die Finanzverwaltung hat besonderes Augenmerk auf die Umsatzsteuer, da durch Vorsteuerbetrug dem Staat erhebliche Einkommenseinbußen entstehen. Insbesondere sog. Karussellgeschäfte haben zu dieser Aufmerksamkeit geführt.

- Die Finanzverwaltung kann die Umsatzteuer - im Gegensatz zu den anderen Steuerarten - spontan und ohne Anmeldung außerhalb einer Außenprüfung prüfen (sog. Umsatzsteuernachschau).

Im Rahmen der Einführung eines ERP-Systems werden zwei Methoden vorgestellt, die lineare Methode und das Entscheidungsbaumverfahren. Da das Entscheidungsbaumverfahren isoliert an einzelne Tatbestandsmerkmale anknüpft, kann vermutet werden, dass dieses für Nicht-Steuerexperten die einfachere Methode ist, da die lineare Methode die Kenntnis einer ganzen Rechtsnorm bereits voraussetzt. Für den Regelbetrieb wird die VAT-Scan Vorgehensweise vorgestellt, die keine exakte Vorgehensweise ist, jedoch einen Einblick in die umsatzsteuerlichen Verhältnisse innerhalb eines ERP-Systems in relativ kurzer Zeit erlauben soll. Als weiterer Forschungsbedarf wird insbesondere die Anwendung der beschriebenen Vorgehensweisen gesehen. Für den VAT-Scan liegen bereits Ergebnisse aus einer Anwendung vor, die jedoch noch nicht verallgemeinert werden können. Zukünftige Anwendungsfälle werden die Vorgehensweise weiter verfeinern. 


\title{
Vorläufige und notwendige Formalisierungslücken in der IT-Beratung
}

\author{
Paul Drews \\ Department Informatik, Universität Hamburg
}

Die Einführung von Informationstechnik (IT) hat in den vergangenen Jahren zu erheblichen Veränderungen in Unternehmen geführt. Unternehmensberater spielen in der Wechselwirkung zwischen Entwicklungs- und Aneignungsprozessen eine bedeutende Rolle, dennoch steht die Forschung zur IT-Beratung noch am Anfang (Nissen 2007). Neben empirischen Arbeiten kann auch die Nutzbarmachung von Theorien und Modellen aus unterschiedlichen Disziplinen einen Beitrag zum wissenschaftlichen Fortschritt leisten (Drews 2009). Ein Ausgangspunkt für die Forschung sind Probleme, die in der Praxis auftreten. Zu diesen Problemen gehört, dass IT-Einführungsprojekte, ein wesentlicher Tätigkeitsbereich der IT-Beratung, regelmäßig scheitern oder Zeit- und Kostenbudgets überschreiten (Brödner et al. 2009).

Diese Probleme sind der Anlass für eine Betrachtung der Frage, wie die in ITProjekten stattfindende Formalisierung an die Grenzen des von Mertens formulierten Langfristziels der Wirtschaftsinformatik - sinnhafte Vollautomation (Mertens 1995) - gerät. Mögliche Gründe für das Scheitern von IT-Projekten sind die unzureichende Berücksichtigung von Formalisierungslücken und das Schließen notwendiger Formalisierungslücken (Rolf 1998, 1999, 2008). In diesem Artikel werden die Konzepte der vorläufigen und notwendigen Formalisierungslücke dargestellt und ihre Nützlichkeit für die IT-Beratung aufgezeigt. Die adressierte Fragestellung lautet: Welchen Beitrag können die Erkenntnisse über Grenzen der Formalisierung für die IT-Beratung leisten?

Der Artikel gliedert sich wie folgt: Zunächst werden die theoretischen Hintergründe des Konzeptes dargestellt, bevor die Differenzierung in vorläufige und notwendige Formalisierungslücken eingeführt wird. Es folgt eine Übertragung des Konzeptes auf die IT-Beratung sowie eine Diskussion aktueller Entwicklungen Enterprise 2.0 und Open Innovation - in Hinblick auf die dabei gewonnenen Erkenntnisse. Als weiteres Ergebnis werden Handlungsempfehlungen für die ITBeratung abgeleitet.

IT-Beratung wird sich zukünftig stärker mit weniger stark strukturierten Handlungszusammenhängen in Organisationen beschäftigen, wie am Beispiel von Enterprise 2.0 und Open Innovation deutlich wird. Die wissenschaftlichen Methoden und die dafür relevanten IT-Verfahren sind gänzlich anderer Natur, als die bisher primär verwendeten. Zukünftige Forschung zur IT-Beratung sollte einer- 
seits reflektieren, wie IT-Berater in der Praxis mit Formalisierungslücken umgehen. Zusätzlich wäre zu prüfen, ob und in welchen Fällen das Schließen notwendiger Formalisierungslücken zum Scheitern von IT-Projekten bzw. zu erheblichen Nebenfolgen geführt hat.

\section{Literatur}

Brödner P et al. (2009) Skizze einer Theorie der Informatik-Anwendungen. International Journal of Sustainability Communication 5(2009): 118-140.

Drews P (2009) IT-Beratung zwischen transdisziplinärer Forschung und Praxis Ein Orientierungsrahmen. In: Fischer S et al. (Hrsg) INFORMATIK 2009 Proceedings.

Mertens P (1995) Wirtschaftsinformatik: von den Moden zum Trend. In: König W (Hrsg) Wirtschaftsinformatik '95: Wettbewerbsfähigkeit, Innovation, Wirtschaftlichkeit. Physica, Heidelberg.

Nissen V (2007) Consulting Research - Eine Einführung. In: Nissen V (Hrsg)

Consulting Research - Unternehmensberatung aus wissenschaftlicher Perspektive. GWV, Wiesbaden.

Rolf A (1998) Grundlagen der Organisations- und Wirtschaftsinformatik. Springer, Berlin.

Rolf A (1999) Von Leitbildern, Moden und Langfristzielen der Wirtschaftsinformatik. Wirtschaftsinformatik 41(1999): 184-186.

Rolf A (2008) Mikropolis 2010 - Menschen, Computer, Internet in der globalen Gesellschaft. Metropolis, Marburg. 


\title{
Entstehung und Handhabung von Vorgehensmodellen zur Software-Auswahl und Software-Einführung in der IV-Beratung
}

\author{
Volker Nissen \\ Institut für Wirtschaftsinformatik, FG WI-2, \\ Technische Universität Ilmenau
}

Da die Gewährleistung einer qualitativ hochwertigen Dienstleistung ein zentraler Wettbewerbsfaktor ist, sollten laut Mieschke (IV)Beratungsunternehmen Vorgehensmodelle für einzelne Projekttypen bzw. Beratungsthemen mit dem Ziel der Qualitätssteigerung anwenden (Mieschke 2004, S.105). Für viele Tätigkeitsbereiche der IV-Beratung existieren in der Literatur empfohlene Vorgehensmodelle oder Beschreibungen von Arbeitsfolgen. Die Fragen der Entstehung und tatsächlichen Nutzung von Vorgehensmodellen in Beratungshäusern scheinen jedoch bislang noch nicht untersucht worden zu sein. Dies soll empirisch für den deutschen Beratungsmarkt geklärt werden. Hierzu werden nachfolgend die Ergebnisse einer im Umfang beschränkten qualitativen Fallstudienanalyse vorgestellt (Nissen und Simon 2009). Deren Ergebnisse sollen im zweiten Schritt durch eine breitere, quantitative Erhebung abgesichert werden, die derzeit vorbereitet wird.

Tabelle 1: Einordnung von Aufgaben der IV-Beratung in Komplexitätskategorien

\begin{tabular}{|l|c|c|c|}
\hline $\begin{array}{l}\text { Beratungsfeld innerhalb der IV- } \\
\text { Beratung }\end{array}$ & $\begin{array}{l}\text { Brain } \\
\text { Projekte }\end{array}$ & $\begin{array}{l}\text { Grey Hair } \\
\text { Projekte }\end{array}$ & $\begin{array}{l}\text { Procedure } \\
\text { Projekte }\end{array}$ \\
\hline Software-Auswahl & & $\mathrm{X}$ & $\underline{\mathrm{X}}$ \\
\hline Software-Einführung & $\mathrm{X}$ & $\underline{\mathrm{X}}$ & \\
\hline Informationsarchitektur & & $\underline{\mathrm{X}}$ & $\mathrm{X}$ \\
\hline IT-Sicherheit & & $\mathrm{X}$ & \\
\hline IT-Integration & $\mathrm{X}$ & $\underline{\mathrm{X}}$ & $\mathrm{X}$ \\
\hline IT-Strategie & & $\mathrm{X}$ & \\
\hline IT-Organisation & & & \\
\hline IT-Outsourcing & & & \\
\hline
\end{tabular}


Anhand einer umfassenden Analyse der Internetseiten von 30 etablierten IV-Beratungsunternehmen konnte ein vielfältiges Angebot an Beratungsleistungen identifiziert werden. Ein Vorschlag, wie die so ermittelten Kernaufgaben der IVBeratung anhand von Komplexität und Wiederholungscharakter den Kategorien von Maister (2003) zugeordnet werden können, ist in Tabelle 1 dargestellt. Aufgabenstellungen aus der Kategorie „Procedure Projekt“ erscheinen besonders geeignet, um durch weitgehend standardisierte Vorgehensmodelle die Projektarbeit effizienter zu gestalten.

Die hier vorgestellte Untersuchung zeigt für die beiden klassischen IVBeratungsfelder der Auswahl und Einführung von Unternehmenssoftware eine überwiegend bereits langjährige Verwendung von Vorgehensmodellen in der Beratungspraxis auf. Diese Modelle entstammen wesentlich eigenen Projekterfahrungen der jeweiligen Beratungshäuser, wobei oft Anregungen aus externen Quellen, wie beispielsweise Industriestandards und Literaturquellen einfließen. Qualitäts- und Effizienzziele sind die dominierenden Gründe für eine Modellentwicklung.

Hinsichtlich der Modellhandhabung ist festzuhalten, dass gerade größere Firmen stark auf die Anwendung der Modelle in Projekten drängen, diese vollständig intern, jedoch nicht nach außen explizieren und dann im Rahmen des Wissensmanagements breit nutzen. Die Modelle sind nur selten Teil einer formalen Zertifizierung gewesen, werden aber regelmäßig intern evaluiert und weiterentwickelt. Ihre Relevanz für die Projektakquisition wird überwiegend als mäßig eingeschätzt, denn sie erfüllen eher eine Erwartung der Klienten hinsichtlich professioneller Beratung als das sie ein wettbewerbsdifferenzierender Faktor wären.

\section{Literatur}

Maister, DH (2003) Managing the Professional Service Firm. Schuster \& Schuster, London.

Mieschke L (2004) Strategisches Geschäftsmodell der Informationstechnologieberatung. DUV, Wiesbaden.

Nissen V, Simon C (2009) Kernaufgaben und Vorgehensmodelle in der IVBeratung. TU Ilmenau, FG Wirtschaftsinformatik für Dienstleistungen. Forschungsberichte zur Unternehmensberatung Nr. 2009-02. 


\title{
CRM Evaluation
}

\section{An Approach for Selecting Suitable Software Packages}

\author{
Ina Friedrich'1, Jon Sprenger ${ }^{2}$, Michael H. Breitner ${ }^{2}$ \\ ${ }^{1}$ Accenture GmbH, Kronberg \\ 2Institut für Wirtschaftsinformatik, Leibniz Universität Hannover
}

Customer Relationship Management (CRM) has been discussed in the literature since the nineties. As reported by earlier works, the success rate of CRM implementation projects is up to today still not satisfactory (Becker et al. 2009; Finnegan and Currie 2009). Evaluating software solutions before making an investment decision is crucial - considering the current economic climate as well as the success that the implementation projects have up until today. A methodological approach is necessary in order to make efficient decisions in this area. This paper systematically researched and reviewed articles which discuss the topic of CRM evaluation, or IT evaluation in general, and which have been published in science journals and conference proceedings. The goal is to evaluate the current status of methods and tools for CRM evaluation and finally to develop an approach for selecting suitable CRM software packages.

To get an overview on the currently available information, a content analysis was performed. As a result, a deficiency in the area of CRM evaluation has been identified. In total, 137 papers were reviewed. The literature verification to the individual statements could not be fitted into the given paper for spatial reasons; a table with supporting documents concerning the mentioned statements can be sent by the authors on request.

Table 1 summarizes the results of analyzing the selected relevant publications. 77 papers were excluded as they did not relate to the predefined criteria. As some papers discussed more than one category then the total number does not equal the sum of all categories. 
Table 1: Overview of the results of the literature search

\begin{tabular}{|l|c|c|}
\hline & IT & CRM \\
\hline Method & 15 & 9 \\
\hline Evaluation technique & 14 & 1 \\
\hline Criteria & 13 & 21 \\
\hline Tool & 2 & 0 \\
\hline \hline Total & 36 & 24 \\
\hline
\end{tabular}

Based on the results of the structured analysis of the identified literature, an approach that tries to address the categories Method $^{1}$, Criteria ${ }^{2}$, and Technique ${ }^{3}$ has been developed - adopted from Jadhav and Sonar (2009).

Finally, the results of each category have been combined to develop the CRM Evaluation Approach. Some limitations remain and are mentioned in the article. Furthermore, the individual results of our research for individual assessment categories are not considered to be new overall. Combining these, however, as well as the CRM-specific results cumulating in the CRM Evaluation Approach proposed in the full text article, adds a new contribution to the field of CRM evaluation. The approach is grounded and pragmatic and can be used for selecting the best CRM package according to the company-specific weighting of CRM specific criteria.

All in all, this paper presents an overview on the latest research on CRM software selection for tender evaluation. It provides an impression of published papers, specifically regarding CRM evaluation, and might therefore serve as a basis for other researchers in this field. Verification of the approach as a further course of action is being proposed.

\section{References}

Becker JU, Greve G, Albers S (2009) The impact of technological and organizational implementation of CRM on customer acquisition, maintenance, and retention. To appear in: International Journal of Research in Marketing.

Finnegan DJ and Currie WL (2009) A multi-layered approach to CRM implementation: An integration perspective. To appear in: European Management Journal.

Jadhav AS and Sonar RM (2009) Evaluating and selecting software packages: A review. In: Information and Software Technology 51(3): 555-563.

\footnotetext{
${ }^{1}$ Methodology to perform the actual evaluation, including the steps and scope to be considered.

2 Criteria or areas supporting the comparison of CRM software.

3 Approach to apply the identified criteria. It supports the decision-making process over the available alternatives, and is aimed at selecting one CRM package that is superior to the researched alternatives.
} 


\title{
Plädoyer für eine operationalisierbare Methode der Prozesserhebung in der Beratung
}

\section{Klassifikation bestehender Ansätze und Implikationen für die Beratungspraxis}

\author{
Silke Balzert, Peter Fettke, Peter Loos \\ Institut für Wirtschaftsinformatik \\ im Deutschen Forschungszentrum für Künstliche Intelligenz, \\ Universität des Saarlandes
}

Mit einem Umsatz von 18,2 Milliarden Euro und mehr als 115.000 Beschäftigen (Stand: 2008) stellt die Beratungsbranche einen bedeutenden Wirtschaftszweig in Deutschland dar. Gleichwohl steht die wissenschaftliche Auseinandersetzung mit dem Thema Unternehmensberatung erst am Anfang. So wird aufgrund der weitgehenden Isolation der bisherigen Forschungsbemühungen in der Literatur die Institutionalisierung eines entsprechenden Forschungsfeldes Consulting Research gefordert (Nissen 2007, S. 14; sowie die dort genannten Quellen), um sowohl die wissenschaftliche Durchdringung des Themas Unternehmensberatung zu forcieren als auch wissenschaftliche Methoden und Erkenntnisse in die Beratungspraxis zu transferieren.

Der Ergebnistransfer in die Praxis ist auch Gegenstand des vorliegenden Beitrags, wobei aus zwei Gründen speziell das Geschäftsprozessmanagement und die Geschäftsprozessreorganisation fokussiert werden. Zum einen entfallen in Deutschland rund 8 Milliarden Euro Umsatz allein auf den Bereich der Organisations- und Prozessberatung und Untersuchungen zeigen, dass dieser Markt für Beratungsunternehmen hoch lukrativ ist (Hess und Schuller 2005, S. 370-371). Zum anderen scheint sich in der Literatur bisher kein Vorgehensmodell zur Prozessmodellierung im Allgemeinen und zur Prozesserhebung im Speziellen etabliert zu haben, welches allgemein akzeptiert wird (Eggert 2009, S. 2; sowie die dort zitierte Quelle), und auch zur Geschäftsprozessreorganisation hat sich offenbar noch keine standardisierte Methode durchgesetzt (Hess und Schuller 2005, S. 355-373).

Ziel des vorliegenden Beitrags ist es daher, den Durchdringungsgrad der Literatur mit Vorgehensmodellen zum Geschäftsprozessmanagement anhand einer Auswahl von etablierten Lehrbüchern zu untersuchen und daraus Implikationen für die Beratungspraxis abzuleiten. Der Betrachtungsschwerpunkt liegt dabei auf der Phase der Prozesserhebung, da ihr eine hohe Bedeutung zur Identifikation von Schwachstellen und Verbesserungspotenzialen zugesprochen wird (Schwegmann und Laske 2005, S. 155-157). Darüber hinaus ist eine solche Prozesserhebung 
meist mit erheblichem Aufwand verbunden und kann daher in besonderem Maße von einer strukturierten und effizienten Vorgehensweise profitieren, die theoretisch fundiert und in die Praxis umsetzbar - also operationalisierbar - ist.

Als Ergebnis der durchgeführten Literaturanalyse kann festgehalten werden, dass kaum konkrete Ansatzpunkte zur Umsetzung bzw. Durchführung einer Geschäftsprozesserhebung vorhanden sind. Für die Beratungspraxis implizieren diese Ergebnisse, dass zurzeit keine einheitlichen, theoretisch fundierten Handlungsempfehlungen zur durchgängigen Unterstützung aller Tätigkeiten im Rahmen der Geschäftsprozesserhebung vorliegen. Vor dem Hintergrund der Wertschöpfungspotenziale in der Prozessberatung ist diese Erkenntnis paradox. Zwar steht zu vermuten, dass etablierte Beratungsunternehmen entsprechende Vorgehensmodelle entwickelt haben und einsetzen, sie jedoch als schutzwürdiges Wissen einstufen und daher nicht veröffentlichen. Einer wissenschaftlichen Diskussion der Prozesserhebung aus praktischer Sicht fehlt damit die Grundlage. Gleichwohl könnte die Beratungspraxis von einer Öffnung hin zur Wissenschaft profitieren.

Die in diesem Beitrag vorgestellten Ergebnisse basieren auf der Untersuchung einer limitierten Anzahl von etablierten Standardwerken zum Geschäftsprozessmanagement. Gleichwohl erfüllt diese Literaturauswahl ihren Zweck, auf das vermutete Erkenntnisdefizit aufmerksam zu machen. Darüber hinaus ist es auf Basis dieser Literaturquellen möglich, einen ersten Systematisierungsansatz für die Vorgehensmodelle zu entwickeln. Diese Systematisierung kann zukünftigen Arbeiten als Ausgangsbasis dienen und weiter ausdifferenziert werden mit dem Ziel, perspektivisch ein Vorgehensmodell mit konkreten Hinweisen zur operativen Umsetzung der einzelnen Modellphasen zu entwickeln und dieses in die Unternehmenspraxis zu transferieren.

\section{Literatur}

Eggert S (2009) Das aktuelle Stichwort: Prozessmanagement. ERP Management 5:16.

Hess T, Schuller D (2005) Business Process Reengineering als nachhaltiger Trend? Eine Analyse der Praxis in deutschen Großunternehmen nach einer Dekade. ZFBF 57:355-373.

Nissen V (2007) Consulting Research - Eine Einführung. In: Nissen V (Hrsg)

Consulting Research - Unternehmensberatung aus wissenschaftlicher Perspektive. Gabler, Wiesbaden.

Schwegmann A, Laske M (2005) Istmodellierung und Istanalyse. In: Becker J, Kugeler M, Rosemann M (Hrsg.) Prozessmanagement - Ein Leitfaden zur prozessorientierten Organisationsgestaltung. Springer, Berlin. 


\section{Geschäftsmodelldiversifikation von Unternehmensberatungen}

\section{Einige Überlegungen zu den Optionen für Beratungs- und Service-Unternehmen im IT-Umfeld}

Thomas Deelmann

Strategy Development, T-Systems International GmbH, Bonn

Die Branche der Unternehmensberatung (Beratung) ist in ihrem Umfang, ausgedrückt z. B. im Marktvolumen, in den letzten Jahren mit einigen kurzfristigen Unterbrechungen stetig gewachsen. Dies gilt ebenso für viele individuelle Beratungsunternehmen. Regelmäßig müssen sie neben z. B. inhaltlichen, qualitativen und personellen Zielen, ein Wachstumsziel für Umsatz und Finanzergebnis verfolgen. Hierdurch können z. B. partnerschaftliche organisierte Beratungen den im Turniermodus neu bestimmten Partnern Aufgaben (z. B. durch Kunden- oder Themenverantwortung), Ressourcen und Entlohnung zukommen zu lassen.

Ein solches Wachstumsziel kann horizontal, d. h. durch die Erschließung neuer Kunden im bestehenden Kompetenzumfeld sowie stärkere Durchdringung vorhandener Kunden (Erweiterung des ,Share of Wallets') oder vertikal erfolgen. Bei einem vertikalen Vorgehen versucht das Beratungsunternehmen andere Beratungsprodukte anzubieten und andere Beratungsarten zu erschließen. Auch bei ITService-Unternehmen, die teilweise Beratungs- und teilweise Implementierungsaufgaben für ihre Kunden übernehmen, können solche Versuche beobachtet werden. Exemplarisch seien hier Anbieter von sogenannten Gesamtdienstleistungen oder Business Innovation / Transformation Partner genannt.

Auf Basis von drei idealtypischen Beratungsarten (Strategie-, Fach- und Implementierungsberatung) will der vorliegende Beitrag einen Diversifikationsrahmen für Unternehmensberatungen von einem Ausgangs- zu einem angrenzenden Beratungsgeschäftsmodell konzipieren und die Erfolgsaussichten einer Diversifikation auf Basis von antizipierten Markterwiderungen bewerten. Im Fokus stehen die Möglichkeiten von Beratungen und Dienstleistungsunternehmen im IT-Umfeld. Damit wird zum einen das Verhältnis von IT- zu Strategieberatung weiter geschärft und zum anderen aktuelle Strukturen und Trends des Beratungsmarktes aufgegriffen.

Anschließend wird der Versuch unternommen, übergreifenden Gemeinsamkeiten für die Erfolgsaussichten und antizipierten Markterwiderungen zu identifizieren. Vier Ergebnisse lassen sich auf Basis der getätigten Überlegungen festhalten: 
Aufwendige Neupositionierung beacbten: In allen diskutierten Fällen scheint es grundsätzliche Schwierigkeiten bei der Geschäftsmodelldiversifikation zu geben, keine Entwicklung bietet sich vorbehaltlos an. Auch wenn alle Schritte grundsätzlich durchführbar erscheinen, ist jeweils eine Neupositionierung der Beratung notwendig, welche zum Ziel hat, den Kunden und potentiellen Kunden die Diversifikation zu erläutern. Diese Neupositionierung kann als aufwendig und risikobehaftet angesehen werden, da vielen Beratungen die Erfahrung in der Marketingkommunikation des eigenen Unternehmens fehlt.

Vorsichtige Diversifikationsentwicklung: Im Falle einer Geschäftsmodelldiversifikation erscheinen die Möglichkeiten, welche sich nicht zu weit vom Ausgangsgeschäftsmodell entfernen (Strategie- zu Fachberatung, Fach- zu Strategieberatung, Fach- zu Implementierungsberatung, Implementierungs- zu Fachberatung) erfolgversprechender gegenüber den Möglichkeiten, welche größere Diversifikationsanstrengungen umfassen (Strategie- zu Implementierungsberatung, Implementierungs- zu Strategieberatung).

Gefahr von Preisverfall einkalkulieren: Downstream-Geschäftsmodelldiversifikationen, also eine Diversifikation von höhermargigen Geschäftsmodellen zu solchen mit niedrigeren Margen und entsprechenden Stundensätzen, beinhalten die Gefahr, dass die niedrigeren Vergütungen für z. B. Implementierungsberatung auch für Fachberatungsleistungen von den Kunden gefordert und von den Beratungen gewährt werden. Diese Preiserosion wirkt sich schließlich auf die operativen Gewinnmargen der Beratungen aus, soweit sie an ihrer Personalkostenstruktur festhalten.

Akzeptanzproblemen begegnen: Bei einer Upstream-Geschäftsmodelldiversifikation, also einer Diversifikation von Geschäftsmodellen mit niedrigeren Margen (z. B. Implementierungs- oder Fachberatung) zu solchen mit höheren Margen (entsprechend z. B. Fach- oder Strategieberatung), ist zu befürchten, dass die neue Leistung bei Kunden auf Akzeptanzprobleme stößt.

Als Fazit lässt sich festhalten, dass im aufgezeigten Diversifikationsrahmen eine Geschäftsmodellerweiterung grundsätzlich vorstellbar ist, hierbei jedoch der Aufwand einer Neupositionierung zu berücksichtigen ist, eine Diversifikation mit großer Vorsicht erfolgen sollte, bei einer Downstream-Diversifikation die Gefahr einer Preiserosion vorhanden ist und Beratungen insbesondere bei UpstreamDiversifikationen mit Akzeptanzproblemen bei Kunden gegenüber ihrer Entwicklung rechnen müssen. Diesen Gefahren kann zumindest in Teilen durch ein vorsichtiges, unter Umständen mehrstufiges Vorgehen und eine Mehrmarkenstrategie begegnet werden.

Zur weiteren Konkretisierung der getroffenen Aussagen erscheint es hilfreich, erfolgte Diversifikationsversuche, unbeschadet vom jeweiligen Erfolg, zu untersuchen sowie die getroffenen Handlungsempfehlungen weiter zu konkretisieren. 


\section{Technologien}



Kooperationssysteme 



\title{
Vorwort zur Teilkonferenz
}

\section{Kooperationssysteme}

\author{
Michael Koch', David Faller², Gerhard Schwabe3, Volker Wulf \\ ${ }^{1}$ Institut für Softwaretechnologie, Universität der Bundeswehr München \\ 2IBM Deutschland Research \& Development GmbH, Böblingen \\ 3 Abteilung für Informationsmanagement, Universität Zürich \\ ${ }^{4}$ Lehrstubl für Wirtschaftsinformatik und Neue Medien, Universität Siegen
}

Durch die Globalisierung von Unternehmen und das Aufbrechen der Wertschöpfungskette in immer mehr Branchen gewinnt die verteilte Zusammenarbeit weiter an Bedeutung. Eine solche Zusammenarbeit ist nur über elektronische Medien bewältigbar. Organisationen führen deshalb zunehmend weitere Kooperationsmedien ein. Das Spektrum reicht von Klassikern (z. B. Videokonfernzsystemen) über schwergewichtigen Anwendungen wie kooperativen Produktentwicklungssystemen hin zu neuen leichtgewichtigen Lösungen wie Social Software. Im Gegensatz zu vielen anderen Bereichen der IT beobachten wir dabei das spannende Phänomen, dass viele erfolgreiche Systeme aus dem Privatbereich in die Unternehmen gelangen (und nicht umgekehrt).

Der Track ,Kooperationssysteme' beschäftigt sich mit der Unterstützung kooperativer Arbeit vermittelt durch I\&K-Technologien. Das Paradigma der Unterstützung menschlicher Arbeit setzt ein hinreichendes Verständnis von kooperativer Arbeitspraxis voraus, um diese dann durch Entwicklung und Anwendung von I\&K-Technologien produktiver, flexibler, ökologischer oder humaner werden zu lassen. Für die sich dabei manifestierenden Veränderungen der Arbeits- und Lebenswelten müssen in integrierter Weise organisatorische, qualifikatorische und technische Konzepte entwickelt werden. Der Terminus ,Kooperationssysteme' ist also im sozio-technischen Sinne zu verstehen.

Ziel des Tracks ist es, die verschiedenen Facetten der Veränderung des traditionellen Arbeitens in Organisationen und der technologischen Unterstützung dieser Veränderung zu thematisieren und dazu interessierte Forscher-, Entwickler- und Anwendergruppen zusammenzubringen. Es sollen dabei sowohl Formen neuer Arbeit als auch neue Technologien und deren Unterstützungspotentiale thematisiert werden.

Dieser Track knüpft an die Teilkonferenzen und Tracks zum selben Thema auf den Tagungen WI 2007 und 2009 sowie MKWI 2006 und 2008 sowie an frühere Veranstaltungen der Fachgruppe CSCW in der Gesellschaft für Informatik (www.fgcscw.de) an. 


\section{Themen}

Im Track sind Fallstudien, empirische Arbeiten (qualitative Feldstudien, Surveys, Experimente), Designorientierte Arbeiten (Prototypen, Konzeptionen, Architekturen), theoretische Arbeiten und konzeptuelle Arbeiten aus Forschung und Praxis $z u$ verschiedenen Themen zu finden.

Das Spektrum reicht dabei vom Design IT-gestützter kooperativer BürgerBeratung über die Qualitätsmessung in Wikipedia bis hin zum Vorschlag einer Forschungsagenda für Enterprise 2.0.

Programmkomitee und Annahmequote

Bei der Auswahl der neun Beiträge in diesem Track aus den zwanæig Einreichungen haben uns folgende Personen im Programmkomitee und als zusätzliche Gutachter tatkräftig unterstützt: Robinson Aschoff, Jean Paul Buchwald, Steffen Budweg, Angelika Bullinger, Tom Gross, Jan Marco Leimeister, Stephan Lukosch, Philipp Nussbaumer, Tom Phillip, Niels Pinkwart, Volkmar Pipek, Wolfgang Prinz, Alexander Richter, Kai Riemer, Ansgar Schmidt, Susanne Schmidt-Rauch und Gunnar Stevens. 


\title{
Design IT-gestützter kooperativer Bürger-Beratung
}

\author{
Birgit Schenk1, Gerhard Schwabe $e^{*}$ \\ ${ }^{1}$ Fachbereich Verwaltungsinformatik, Hochschule für öffentliche Verwaltung Kehl \\ 2Institut für Informatik, Universität Zürich
}

In komplexen neuartigen Lebenslagen benötigen Bürger selbst in Zeiten des Internets persönliche Beratung, denn das Internet setzt voraus, dass sie wissen, wonach sie suchen (Belkin 1982). Im Projekt Bürgerberatung 2.0 wurde ein neuartiger Beratungsprozess und ein dazu passendes Bürgerberatungssystem entwickelt, welche die Informationsversorgung des Bürgers verbessern, die Effizienz des Prozesses erhöhen und den Bürger handlungsfähig machen (vgl. Bretscher (2009), Schwabe et al. (2010)). Ausgangspunkt war die bisher wenig zufriedenstellende Bürgerberatung, auf die Daten aus Workshops und "Mystery Shoppings" hindeuten (Schenk und Schwabe 2010). In diesem Beitrag diskutieren wir drei wesentliche Designaspekte einer kooperativen Bürgerberatung: "Externalisierung von Wissen", "Dialoggestaltung" und "Beraterarbeitsplatz".

Externalisierung von Wissen - Ein gutes Beratungsgespräch umfasst drei Phasen. In der ersten geht es um das gemeinsame Verständnis der Ausgangssituation mit den dazugehörigen Anliegen, es folgt das Erarbeiten von Lösungen und der Gesprächsabschluss. In den Mysteryshoppings fehlte ein strukturierter Prozess, und es wurde nur mündlich kommuniziert ohne Hilfsmittel hinzuziehen, um Besprochenes sichtbar zu machen und ein gemeinsames Verständnis zu entwickeln. Anliegen können somit nicht priorisiert und/oder strukturiert bearbeitet werden und werden teilweise im Gesprächsverlauf vergessen. Aus den beobachteten Problemen und dem natürlichen Verlauf des Beratungsgesprächs ergeben sich folgende Anforderungen: Gemeinsames digitales Material (Schwabe 1995) muss in den natürlichen Gesprächsverlauf integriert werden können und damit flexibel erweiterbar und veränderbar sein sowie Sortieren, Priorisieren und Strukturieren erlauben. Mit in strukturierte oder semi-strukturierte Fragebögen erhobenen Daten ist dies nicht möglich; sie sind deshalb für Beratungsgespräche in komplexen Lebenslagen ungeeignet. Gut geeignet sind Moderationskärtchen, wie wir in der Evaluation mit Nutzern zeigen können.

Leitbilder der Dialoggestaltung - Im öffentlichen Sektor wird kooperative Bürgerberatung angestrebt (Mutzeck 2005, Hielscher und Ochs 2009). Aus der Analyse traditioneller Beratungen lassen sich zwei Barrieren für eine gute Gestaltung des Dialogs erkennen: (1) Die Unterlagen (Broschüren, Formularen, Hinweisblätter)

\footnotetext{
* Autoren erscheinen in alphabetischer Reihenfolge; beide haben gleich viel zu diesem Paper beigetragen
} 
werden als Ersatz für einen echte Dialog verwendet anstatt sie in die Beratung einzubeziehen. (2) Die Berater gestalten keinen echten Dialog: Weder stellen sie Fragen, noch strukturieren sie den Beratungsprozess. Wir nennen dieses vorgefundene Leitbild der Dialoggestaltung "Berater als Ergebnispräsentator". Mit diesem sind die Kunden der Verwaltung nicht zufrieden. Neben offensichtlich Schulungsmängeln sind auch fehlende Werkzeuge für das Vorherrschen dieser Praxis verantwortlich. In Pilottests wurde das gemeinsame Material Beratern und Kunden auf einem Touchscreen präsentiert. Zwar wurden dadurch Berater und Kunden noch nicht echte "Kooperationspartner", aber der Berater wurde zu einem "Chauffeur" in einem gemeinsam gestalteten Prozess. Dadurch erhöhte sich die Beteiligung und die Zufriedenheit der Testkunden.

Beratungsarbeitsplatz und Raumdesign - Bürgerberatung muss in eine konkrete Büroumgebung situiert werden. Technik darf dabei nicht zur Barriere werden; so sind Augenkontakt und gemeinsame Sicht die Hauptherausforderungen. Da Themen nicht immer konfliktfrei sind, muss insgesamt eine angemessene Privatsphäre und entsprechend Abstand zwischen den Beteiligten möglich sein. Positive Bewertungen erhielten in der Evaluation die Beratungsgespräche, die in Einzelbüros an runden und 135 Grad Winkel-Tischen statt fanden. Der Großbildschirm wurde dabei so angeordnet, dass er für beide Beteiligte gut einzusehen war, doch nicht den Blickkontakt verhinderte.

\section{Literatur}

Belkin NJ, Oddy R., Brooks H (1982): Ask for information retrieval. Part 1: Background and theory. Journal of Documentation, 38. p. 61-71.

Bretscher C (2009): Design und Implementation Bürgerberatungstools im Rahmen des E-Government. Diplomarbeit, Universität Zürich, Institut für Informatik.

Hielscher V, Ochs P (2009): Arbeitslose als Kunden? Beratungsgespräche in der Arbeitsvermittlung zwischen Druck und Dialog. Reihe „Modernisierung des öffentlichen Sektors. Band 12. Edition sigma, Berlin.

Mutzeck W (2005): Kooperative Beratung. Grundlagen und Methoden der Beratung und Supervision im Berufsalltag. 5. Akt. Auflage. Beltz, Weinheim und Basel

Schenk, B. Schwabe (2010): Understanding the advisory needs of citizens. Erscheint in: Multikonferenz Wirtschaftsinformatik 2010.

Schwabe G (1995): Objekte der Gruppenarbeit, Gabler 1995.

Schwabe, G; Schenk, B; Bretscher C (2010): Bedarfsermittlung und Informationsaggregation bei der Bürgerberatung 2.0. Eingereicht bei Verwaltungsinformatik 2010. 


\title{
Provisioning 2.0: Diffusion kleinteiliger Software in sozialen Netzwerken
}

\author{
Sebastian Draxler, Hendrik Sander, Gunnar Stevens \\ Lehrstubl für Wirtschaftsinformatike und Neue Medien, \\ Universität Siegen
}

Der Trend zu verteilter Projektarbeit, als auch der Trend zu kleinteiliger, kontinuierlich weiterentwickelter Software ${ }^{1}$ verstärken die Notwendigkeit Softwarewerkzeuge schnell und flexibel in den lokalen Kontext von Arbeitsgruppen zu integrieren. Dies stellt neue Anforderungen an Provisioning-Werkzeuge, welche die Bereitstellung, Konfiguration, Wartung und Administration von IT-Systemen in Unternehmen unterstützen sollen.

In Unternehmen ist die Diffusion und Aneignung von IT-Systemen oft durch formale, hierarchische Strukturen geprägt. Die vergleichende Betrachtung der Diffusion of Innovations Theorie (DOI) (Rogers 2003), sowie der CSCW Forschung (Gantt und Nardi 1992) zeigt jedoch, dass auch in solchen Fällen lokale Experten (bzw. Lead User, Translator oder Local Developer) für die effektive Nutzung von für den Arbeitskontext angepassten Werkzeugen eine wichtige Rolle spielen und dass die Diffusion von Werkzeug und Werkzeug-Expertise miteinander verkoppelt ist. Trotzdem haben die Erkenntnisse zur informellen, selbst-organisierten Arbeitsplatzgestaltung nur vereinzelt Eingang in die Gestaltung technischer Unterstützungssysteme gefunden. Insbesondere existierende Provisioning-Ansätze konzentrieren sich allein auf formal erfassbare Aspekte der Bereitstellung von ITSystemen. Demgegenüber werden die informellen Formen der Aneignung in lokalen Netzwerken zu wenig unterstützt. Die Provisioning Lösungen der Zukunft sollten daher um diese Aspekte ergänzt werden.

Darüber hinaus hat der Markt an frei verfügbarer, kleinteiliger Software in den letzten Jahren stark zugenommen, so dass der Nutzer sich häufig entscheiden muss, ob es günstiger ist eine passende Lösung im Netz zu suchen, oder eine bestehende Anwendung anzupassen, d. h. er steht immer häufiger vor der Frage: adopt or adapt?

In diesem Beitrag stellen wir deshalb basierend auf den Erkenntnissen der DOI und der CSCW Forschung, sowie verschiedenen eigenen empirischen Studien zur Aneignung von Eclipse (Stevens und Draxler 2009) einen neuen Ansatz vor. Dieser unterstützt die Rolle der Kollegen als „Empfehlungssystem“ für die Aus-

\footnotetext{
${ }^{1}$ Beispiele solcher kleinteiliger Software stellen z.B. Microsoft Gadgets, iGoogle oder Widgets für das Apple Dashboard dar. Eine andere Klasse stellen die Erweiterungen (Plug-ins) für StandardProdukte, wie z.B. Word, Skype, Firefox oder Eclipse, dar.
} 
wahl und Aneignung geeigneter Tools und fördert das Gruppenbewusstsein über Werkzeugnutzung im Team. Unsere empirischen Arbeiten zeigten, dass im Anwendungsfeld von Eclipse eine Vielzahl von zusätzlichen Werkzeugen als Komponenten existiert. Häufig werden diese von den Endanwendern zu funktionsfähigen Arbeitsumgebungen zusammengesetzt. Hierbei konnten wir konkrete Strategien identifizieren, wie das Kopieren ganzer Umgebungen und die Weitergabe von Expertise im Umgang mit neuen Tools. Insbesondere konnten wir das lokale soziale Netzwerk als Quelle für Innovation und Diffusion von Werkzeugen bestätigen.

Der Peerclipse Prototyp wurde selbst als Eclipse Komponente umgesetzt und integriert sich damit direkt in die von uns erforschte Anwendung. Er unterstützt die Forderung, nach einem dezentralen Ansatz um das Aneignen neuer Komponenten im Team zu erleichtern. Mit Hilfe der Peer-to-Peer Netzwerktechnologie JXTA kommunizieren die Peerclipse Prototypen miteinander und visualisieren die im Team eingesetzten Komponenten um einen Überblick über eingesetzte Software und evtl. vorhandene Expertise zu bieten. Die Visualisierung wird dabei durch einen auf Gruppen zugeschnittenen Recommender Algorithmus gefiltert. Das (Ver-)Teilen von Komponenten im Team wird vereinfacht indem Benutzer mit Peerclipse das Teamrepository durchsuchen und gegebenenfalls interessante Komponenten/Tools per Mausklick von ihren Kollegen laden und installieren können. So wird der Aufbau virtueller Gruppen-Repositories von Tools etabliert. Zudem wird die Verständigung über Gruppenbedarfe vereinfacht und das Bewusstsein für lokal vorhandene Werkzeug-Expertise gesteigert.

Im Weiteren wollen wir Peerclipse im größeren Kontext erproben und die eingesetzten Recommender Algorithmen verfeinern. Dabei gilt es insbesondere lokale und globale Netzwerke der Anwender-Community Eclipse besser miteinander zu verzahnen.

\section{Literatur}

Gantt, M. and B. A. Nardi (1992). Gardeners and gurus: patterns of cooperation among CAD users. Proceedings of the SIGCHI conference on Human factors in computing systems. Monterey, California, United States, ACM.

Rogers, E. (2003). Diffusion of Innovations. New York, Free Press.

Stevens, G. and S. Draxler (2009). Appropriation of the Eclipse Ecosystem: Local Integration of Global Network Production. COOP. Aix-en-Provence, Springer. 


\title{
SocialNetworkingMirror ${ }^{\mathrm{TM}^{1}}$
}

\section{Einsatz halböffentlicher Touchscreens als ubiquitäre Benutzerschnittstellen für Social Networking Services}

\author{
Florian Ott, Alexander Richter, Michael Koch \\ Forschungsgruppe Kooperationssysteme, \\ Universität der Bundeswehr München
}

Die folgenden Ausführungen stützen sich auf zwei unabhängige Entwicklungen:

Erstens erfreuen sich internetbasierte Social Networking Services (SNS), wie beispielsweise Facebook oder XING, steigenden Zuspruchs und ziehen vermehrt das Interesse vieler Unternehmen auf sich. Vor allem große Organisationen überlegen, wie sie das Wissens- und Innovationsmanagement ihrer Mitarbeiter durch den Einsatz von SNS im Intranet besser unterstützen können.

Zweitens sind viele Unternehmen durch stetig sinkende Preise von LCDHardware inzwischen im Besitz großer, z. T. interaktiver Wandbildschirme, die an verschiedenen (halb-)öffentlichen Orten, wie Empfangshallen, Kaffee-Ecken oder Konferenzräumen innerhalb des Unternehmens prinzipiell zur Verfügung stehen.

Im vorliegenden Beitrag führen wir diese vorwiegend getrennt betrachteten Entwicklungen in einem Konzept für ubiquitäre Benutzerschnittstellen für SNS, sog. SocialNetworkingMirrors ${ }^{\mathrm{TM}}$, zusammen.

Um eine möglichst nahtlose Integration in den sozialen Kontext zu ermöglichen, schlagen wir vor, große Touchscreens als zusätzliche interaktive „Fenster“ in SNS zu nutzen. Die erforderlichen Bildschirme sind oftmals bereits in Unternehmen verfügbar und können mit geringem finanziellem Aufwand mit TouchOverlays ausgestattet werden, um sie zu intuitiv-freudvoll bedienbaren, ubiquitären Benutzerschnittstellen umzufunktionieren.
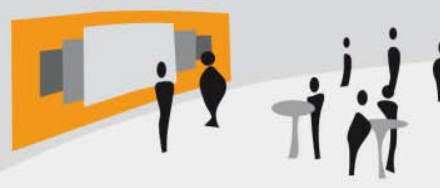

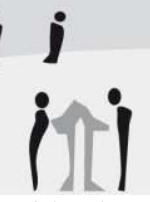

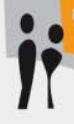

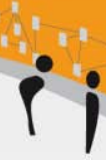

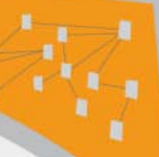

Abbildung 1: Große Touchscreens als ubiquitäre Benutzerschnittstellen

Die Grundidee dieser von uns als CommunityMirrors ${ }^{\mathrm{TM}}$ bezeichneten Touchscreens besteht darin, in IT-Systemen enthaltene Information jenseits klassischer

${ }^{1}$ Trademark der Forschungsgruppe Kooperationssysteme, Universität der Bundeswehr 
Desktoprechner sichtbar, greifbar und erlebbar zu machen und so eine in den sozialen Kontext eingebettete und omnipräsent-sichtbare ubiquitäre Benutzerschnittstelle als „Informationsstrahler“ bereitzustellen. Mithilfe dieser können in KaffeEcken, Gruppenräumen oder beim Warten auf den Fahrstuhl ohne definiertes Ziel in den dargestellten Profilen gestöbert und neue Kontakte geknüpft werden (Abbildung 1). Im Gegensatz zu Desktop-Systemen wird zudem der aktive und gemeinschaftliche Austausch der Personen vor dem Bildschirm und so auch eine natürlich-zwischenmenschliche Kontaktaufnahme gefördert

Der Beitrag gliedert sich in sechs Abschnitte und beginnt mit einer Einführung in den Prozess des IT-gestützten Social Networking sowie den damit verbundenen Herausforderungen (Kapitel 2). Anschließend erläutern wir den Lösungsansatz sowie konkrete Einsatzmöglichkeiten (Kapitel 3). Im vierten Kapitel erfolgt die Vorstellung einer ersten prototypischen Umsetzung. Der Prototyp wird in Abbildung 2 dargestellt. Kapitel 5 fasst schließlich die Erkenntnisse eines Feldtests auf einer großen Webkonferenz zusammen, bevor in Kapitel 6 ein Ausblick auf zukünftige Forschungsaktivitäten gegeben wird.

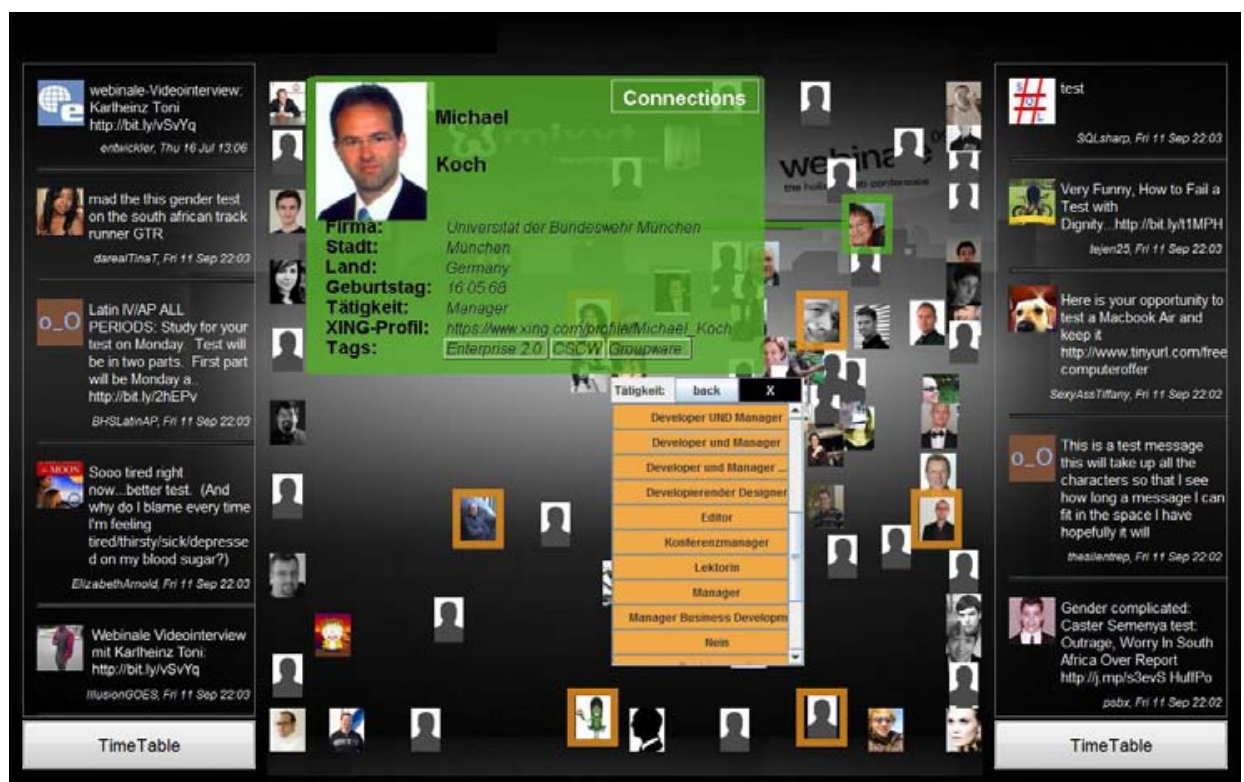

Abbildung 2: Screenshot des SocialNetworkingMirrors ${ }^{\text {TM }}$ 


\title{
Open Value Creation
}

\section{A Framework for Open and Collaborative Value Creation Concepts}

\author{
Daniel Schlagwein', Kai Fischbach', Detlef Schoder', Stefan Bartsch² \\ 1 Seminar für Wirtschaftsinformatik und Informationsmanagement, \\ Universität ₹u Köln \\ 2Institut für Wirtschaftsinformatik, Philipps-Universität Marburg
}

In medieval times, experts in alchemy and other disciplines believed in secrecy being the key element of their scientific success and in hiding their results from curious eyes (paradigm of closedness). This principle changed with the advent of the European Renaissance around 1600, when the modern universities got established: Scientists published their findings for others to draw on them in an open science landscape (paradigm of openness) (David 2005). While openness describes the academic work of today, the classical assumption in the business world is still that internal resources and knowledge should be protected to serve as the foundation for the firms to create added value (Schumpeter 1942).

In recent years, we see more and more exceptions from the paradigm of closedness in the business world: Alternative concepts of what we label "open value creation" are arising. We thereby define 'openness' as the concept of promoting agents with open access to information resources to enable contributions from a wide range of entities in a flat organizational structure; and 'open value creation' as value creation based on the principle of openness.

Many cases illustrate, how valuable those open contributions are: Facebook, Google (Open Social \& Android), Apple (App Store), IBM (open source development of Linux), and Wikipedia are just the most prominent of many more examples (Tapscott and Williams 2006). Hence, open value creation describes value creating processes that are enabled or enhanced by integrating explicit information (information resources) or tacit information (know-how) from external sources, formal partners, and independent contributors like end-users, through open, collaborative IS platforms.

While there is a lot of research on specific open phenomena and concepts, we could not find a framework or meta-term that integrates all of these concepts. The first aim of this paper is to define "open value creation", and propose it as such a meta-term. The second aim is to identify open value creation concepts and connect them in a conceptual framework, based on an extensive literature review and following taxonomy building guidelines (Doty and Glick 1994, Gregor 2006, Schwarz 
et al. 2007). The objective of creating such a taxonomy of open value creation is to identify diversely labelled but substantially identical concepts, to group similar concepts to classes, to enable a comprehensive understanding of "open value creation" and thereby to provide a conceptual framework to make existing and future research in this area more integrative and accessible. By doing so, we hope to better integrate open value creation concepts and make them more accessible for meta-research.

\section{References}

David P (2005) From Keeping 'Nature's Secrets' to the Institutionalization of 'Open Science'. In: Ghosh Ra (Hrsg) Code. Collaborative Ownership and the Digital Economy. The MIT Press, Cambridge.

Doty DH, Glick WH (1994) Typologies as a Unique Form of Theory Building: Toward Improved Understanding and Modeling. Acad. Manage. Rev. 19 (2):230-251.

Gregor S (2006) The Nature of Theory in Information Systems. MISQ 30 (3):611642.

Schumpeter JA (1942) Capitalism, Socialism, and Democracy. Harper, New York.

Schwarz A, Mehta M, Johnson N, Chin WW (2007) Understanding Frameworks and Reviews. DATABASE 38 (3):29-34.

Tapscott D, Williams AD (2006) Wikinomics. Penguin, New York.

Journal codes according to AIS; journal abbreviations according to WoS. 


\title{
Qualitätsmessung in der Wikipedia
}

\section{Ein Ansatz auf Basis von Markov-Modellen}

\author{
Marek Opuszkeo', Thomas Wöhner², Ralf Peters², Johannes Rubland' \\ ${ }^{1}$ Lehrstubl für Wirtschaftsinformatik, Friedrich-Schiller-Universität Jena \\ ${ }^{2}$ Lehrstubl für Wirtschaftsinformatik, insb. E-Business, \\ Martin-Luther Universität Halle-Wittenberg
}

Der offene Zugang der freien Online-Enzyklopädie Wikipedia führte zu einer hohen Beteiligung der Nutzer, sodass die Wikipedia seit einiger Zeit zu den erfolgreichsten Anwendungen im Web 2.0 zählt. Jedoch lassen sich als Nachteil der offenen Kollaboration beispielsweise mutwillige Manipulationen nicht ausschließen, sodass die Qualität eines Artikels nicht garantiert ist. In den vergangenen Jahren sind deshalb Verfahren zur automatischen Qualitätsmessung in den Fokus der Forschung gerückt (Blumenstock 2008). Besonders die Analyse des Lebenszyklus eines Artikels scheint laut Studien ein lohnender Ansatz zu sein. Der Lebenszyklus beschreibt dabei die Entwicklung der Bearbeitungsintensität eines Artikels und stellt so eine Zeitreihe dar (Wöhner und Peters 2009).

In diesem Beitrag wird ein neuer Ansatz zur Zeitreihenanalyse im Kontext der Wikipedia vorgestellt. Wie in Abbildung 1dargestellt wird eine Zeitreihe zunächst auf Basis des Anstiegs der Bearbeitungsintensität in eine symbolische Repräsentation transformiert. Anschließend wird die Repräsentation anhand der Häufigkeiten der Zustandsübergänge in ein Markov-Modell überführt. Zur Qualitätsmessung wird ein Markov-Modell für qualitativ minderwertige bzw. hochwertige Artikel berechnet. Für einen beliebigen Artikel kann nun die Wahrscheinlichkeit des Auftretens des jeweiligen Lebenszyklus in den beiden Modellen als Qualitätsmetrik genutzt werden.

Zur Evaluierung der Metrik wurden auf Basis der Wikipedia-internen Bewertungen qualitativ minderwertige (Löschkandidaten) und hochwertige Artikel (exzellente und lesenswerte Artikel) aus dem Datenbestand der Wikipedia selektiert und anhand des vorgestellten Verfahrens klassifiziert. In mehreren Durchläufen konnte eine mittlere Klassifikationsgüte von $87,6 \%$ erreicht werden. Dies zeigt, dass der vorgestellte Ansatz die Ergebnisse bisheriger Messgrößen erreicht bzw. verbessern kann. In einer Diskriminanzanalyse wird die Messgröße mit bestehenden MessgröBen anderer Studien kombiniert. 


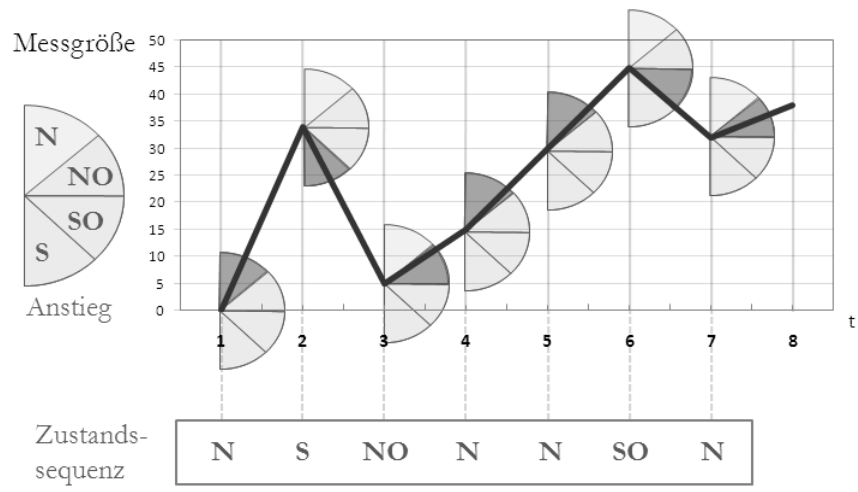

\section{Abbildung 1: Anstiegsbasierte Repräsentation einer Zeitreihe}

Die Klassifikationsgüte kann somit auf $90 \%$ gesteigert werden. Auch zeigt sich in dieser Analyse, dass die hier vorgestellte auf Markov-Modellen basierende Messgröße den größten Anteil an der Erklärung der Gruppenunterschiede leistet.

Der beschriebene Ansatz bietet die Möglichkeit, Zeitreihen jeglicher Art in kompakter und vielseitiger Form zu operationalisieren. Deutlich wurde weiterhin, dass innerhalb der Änderungshistorie von Wikipedia-Artikeln eine hohe Menge an Informationen enthalten ist, die es in Zukunft zu untersuchen gilt.

\section{Literatur}

Blumenstock JE (2008) Size matters: word count as a measure of quality on Wikipedia. In: Proceedings of the $17^{\text {th }}$ international conference on World Wide Web. Beijing.

Wöhner T, Peters R (2009) Assessing the Quality of Wikipedia Articles with Lifecycle Based Metrics. In: Proceedings of the $5^{\text {th }}$ International Symposium on Wikis and Open Collaboration. Orlando. 


\title{
Zur Appropriation sozio-technischer Systeme am Beispiel des Microblogging Systems Twitter
}

\author{
Nils Jeners, Peter Mambrey \\ Fraunhofer FIT, Sankt Augustin
}

Technische Systeme werden gesellschaftlich produziert, das heißt durch Menschen auf Basis kultureller Erfahrungen, Werte und bestimmter Ziele. Das beginnt zuerst mit der Intention und ersten Realisierung vor dem kulturellen Hintergrund der Systementwickler und Auftraggeber. Der Kreis der Handelnden erweitert sich dann schnell durch die Aneignung und allägliche Nutzung, also dem praktischen Einsatz durch Nutzer. Dies ist kein linearer, kausaler und zielgesteuerter Prozess, sondern er ist vielfältigen - aus Sicht der Entwickler - externen Einflüssen unterlegen. Diese sind oft eher zufällig, nicht kalkulierbar, kreativ chaotisch und spiegeln das soziale Lernen in der Auseinandersetzung Mensch-Aufgabe-Werkzeug wider: neue Anwendungen, neue Bedarfe, neue Vorstellungen von Unterstützungen etc. Technikentwicklungen sind häufig innovativ und emergent, d.h. aus intentionalem Handeln entsteht etwas qualitativ Neues, das so nicht intendiert war. Wir bezeichnen diesen Vorgang als Appropriation: Die Aneignung eines schon bestehenden Systems, dessen Nutzung und die Weiterentwicklung zu etwas Neuem. Das technikzentrierte Angebot des Microblogging Systems Twitter trifft in der alltäglichen Praxis auf die Aneignung und Nutzung durch eine Vielzahl von Anwendern. Wir zeigen in dem Aufsatz auf, dass sich Twitter durch die Nutzungspraxis zu niedrigschwelligen kollaborativen Anwendungen hin öffnet und insbesondere durch den nebenläufigen Aufbau von Metawissen zukünftige Kollaborationen fördert. Nimmt man die Ausgangsfrage von Twitter „What Are You Doing“ ernst, müsste sich bei korrekter Antwort der Twitter-Anwender eine kollaborative Funktionsweise und Nutzung verschließen. Da geht es um persönliches Befinden und Empfinden. Die kollaborative Welt, die immer einen Bezug auf einen Anderen oder eine Gruppe darstellt, wird so nicht erreicht. Doch abweichend von der Ausgangsfrage werden vielfältige Informationen in die Twitter-Welt gepostet, die weitere Inhalte transportieren. Im Gegensatz zu den Nutzungserwartungen der Urheber findet so eine funktionale Erweiterung von Twitter statt. Dies erfolgt durch die Art und Weise der Nutzung durch die Anwender als Appropriation der Anwendung Twitter. Es steht zu erwarten, dass sich dieser funktionalen Erweiterung eine Anpassung der technischen Realisierung anschließt. So ließe sich dann wie bei anderen sozio-technischen Entwicklungen z. B. der SMS Funktion des Mobiltelefons eine ko-evolutionäre, sozio-technische Innovation beobachten. Die Ergebnisse der Untersuchung zeigen, dass Kollaboration durch Twitter technisch unterstützt wird: Es gibt die direkte Frage, die direkte Ansprache einer oder mehrerer Personen 
bzw. Organisationen, die direkte Aufforderung etwas gemeinsam zu tun und das gemeinsame Arbeiten an einem Produkt. Es finden also Funktionserweiterungen statt, die wir auf alltägliche Nutzungspraktiken und soziales Lernen zurückführen können. Aber zusätzlich zum Einsatz als Informations- und Organisationsmedium werden Voraussetzungen für eine gelingende zukünftige Kollaboration geschaffen: Durch die Nutzung erfolgt ein nebenläufiger Aufbau von Metawissen, welches zukünftige Kollaboration erleichtert. Ganz im Sinne des partizipativen, peripheren Lernens wird ohne konkretes Ziel durch Lesen von Tweets wahrgenommen, was anderen wichtig ist und wie sie es beurteilen. Hier findet ein Agenda Setting und Framing durch jeden einzelnen Twitter-Anwender statt, welches der Leser der Tweets wahrnimmt und auf Basis seiner Erfahrungen interpretiert. Tweets sind damit Teil der virtuellen Außenwelt jedes einzelnen Twitter-Anwenders. Sie unterstützen ihn bei seiner interpersonellen Koorientierung innerhalb seiner Referenzgruppe. Ob diese Informationen in seine Sicht der Außenwelt eingehen, ist eher wahrscheinlich, da er ja bewusst und freiwillig den Tweets folgt. Ebenso wird wahrgenommen, wie Andere sich selbst zum Thema machen und darstellen. Dadurch entsteht die „negotiated experience of self“ die durch Reflexion der Darstellung Anderer oder durch direkte Teilnahme in einer virtuellen Gemeinschaft entsteht. Metawissen ist für die Kontrolle und Steuerung der eigenen Handlungen nötig und fördert so das Gelingen von zukünftigen Kollaborationen. Es reduziert Unsicherheit und bietet eine Wissensbasis über Personen, die im Bedarfsfall abgerufen werden kann. Wenn wir unsere Erfahrungen mit dem Wissen über Appropriation und Technikentwicklung vergleichen, bestätigen sich viele Erkenntnisse: Es lässt sich keine Zukunft der sozio-technischen Anwendung prognostizieren, zu viele Faktoren gerade auf der Nutzerseite bestimmen über Annahme und Nutzung des Angebots. Lineare Pfade diffundieren zu einem dynamischen Wechselspiel aus technology-push, social-call, technology-push etc.; Die Offenheit der Systementwickler für Neuerungen scheint den dynamischen Appropriationsprozess zu unterstützen und zu einem auf die Nutzerbedarfe angepassten Produkt zu machen. Dabei wechselt die Initiative von Entwicklern zu Nutzern aber auch wieder zurück; Das augenscheinliche Fehlen eines Geschäftsmodells und die Kostenfreiheit suggeriert das Vorhandensein eines öffentlichen, gemeinsamen Guts, das frei genutzt und gestaltet werden kann. Dies scheint die Kreativität bei der Aneignung zu unterstützen; Das Entwicklungsprinzip der „Leichtgewichtigkeit“ trifft auf die Vorstellungen und Nutzungsgewohnheiten der Nutzer und fördert diese. Geringe Voraussetzungen, geringe Bindungen sowie ein nutzerautonome Vernetzung suggerieren dem Nutzer, dass er Herr des sozio-technischen Systems ist. 


\title{
BPM 2.0
}

\section{Kollaborative Gestaltung von Geschäftsprozessen}

\author{
Matthias Kurz \\ Lebrstubl Wirtschaftsinformatik II, \\ Friedrich-Alexander-Universität Erlangen-Nürnberg
}

Geschäftsprozesse sind immer höheren Flexibilitätsanforderungen ausgesetzt, da sich Unternehmen immer schneller auf veränderte Umweltbedingungen einstellen müssen. Schreyögg (2008, S. 97) zufolge sind Unternehmen mit einer zunehmenden Umweltdynamik und einer steigenden Umweltkomplexität konfrontiert.

Bisher wird im Geschäftsprozessmanagement auf die von Taylor (1998, S. 16) propagierte Trennung von Planung und Ausführung zurückgegriffen. Es zeichnet sich jedoch inzwischen $a b$, dass die für dieses Vorgehen notwendige Kontinuität der Rahmenbedingungen zusehends nicht mehr gegeben ist (Picot et al. 2001, S. 9). Stattdessen führen die zunehmende Variabilität der Geschäftsprozesse sowie Umweltdynamik und -komplexität zu einer abnehmenden Planbarkeit von Geschäftsprozessen.

Die Nutzung von Elementen der Selbstorganisation im Geschäftsprozessmanagement bietet einen Ausweg aus diesem Dilemma: Da Mitarbeiter aus den operativen Fachabteilungen ohnehin häufig zuerst mit neuen Herausforderungen des Marktes konfrontiert werden, ist es plausibel, diese Mitarbeiter und ihr operatives Know-how auch zur Reaktion auf diese Herausforderungen heranzuziehen.

Mit Empowerment und Enterprise 2.0 gibt es bereits Organisationstheorien, die Mitarbeitern einen deutlich größeren Einfluss auf die Unternehmensstrukturen einräumen. Diese Form der Selbstorganisation birgt mehrere Potenziale: (1) Entscheidungen, die von einer Vielzahl von Menschen getroffen werden, erweisen sich Surowiecki (2005, S. 271) zufolge häufig als besser als die einer kleinen Gruppe von Experten. (2) Die Verlagerung von Verantwortung an die Mitarbeiter bewirkt eine höhere Motivation und damit quantitativ bessere Arbeitsergebnisse. (3) Eine frühzeitige Einbindung von Mitarbeitern in die Gestaltung von Veränderungen wirkt Widerständen gegen diese Veränderungen entgegen und erhöht somit die Aussicht auf eine erfolgreiche Umsetzung. (4) Die Einbindung der relevanten Stakeholder stellt sicher, dass die geplanten Veränderungen tatsächlich umsetzbar sind. 


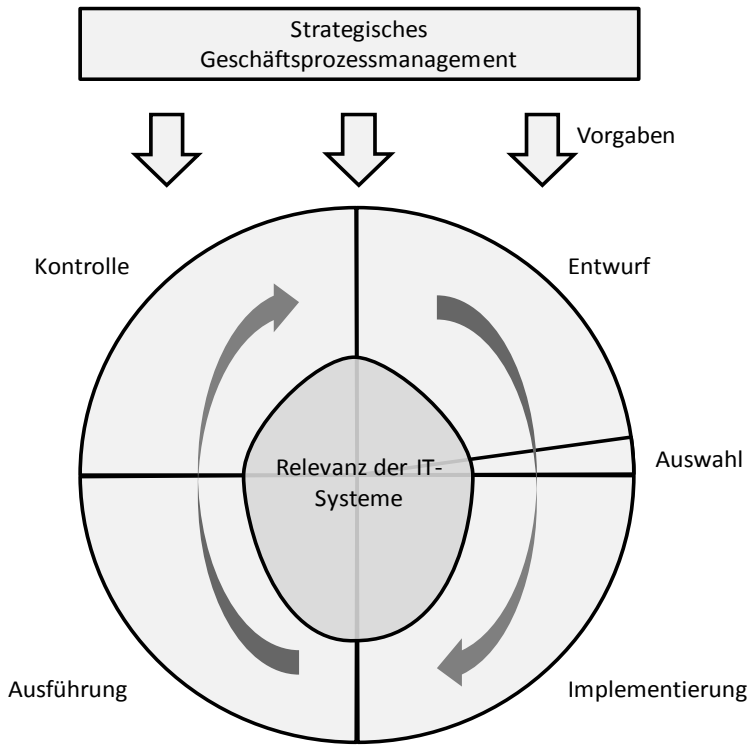

Abbildung 1: Der Managementlebenszyklus von BPM 2.0

Diese Arbeit untersucht anhand einer Fallstudie bei zwei Unternehmen die Grenzen des klassischen Geschäftsprozessmanagements und leitet daraus das in Abbildung 1 dargestellte Vorgehensmodell für die Nutzung der Selbstorganisation im Geschäftsprozessmanagement (BPM 2.0) sowie eine entsprechende SoftwarePlattform ab, mit deren Hilfe Geschäftsprozesse kollaborativ an neue Herausforderungen angepasst und kontinuierlich weiterentwickelt werden können. Ganz im Sinne des Web 2.0 werden Mitarbeiter durch geringe Hemmschwellen dazu ermuntert, mit gelegentlichen Beiträgen zur Verbesserung ,ihrer“ Geschäftsprozesse beizutragen.

\section{Literatur}

Picot A, Reichwald R, Wigand RT (2001) Die grenzenlose Unternehmung.

Information, Organisation und Management. Gabler, Wiesbaden.

Schreyögg G (2008) Organisation. Grundlagen moderner Organisationsgestaltung.

Mit Fallstudien. Gabler, Wiesbaden.

Surowiecki J (2005) The wisdom of crowds. Anchor Books, New York, NY.

Taylor FW (1998) The principles of scientific management. Dover, Mineola, NY. 


\title{
Enterprise 2.0 - Gegenwart und Zukunft
}

\section{Vorschlag einer Forschungsagenda}

\author{
Alexander Richter', Angelika C. Bullinger² \\ ${ }^{1}$ Forschungsgruppe Kooperationssysteme, Universität der Bundeswehr München \\ ${ }^{2}$ Lehrstubl für Wirtschaftsinformatik I, Universität Erlangen-Nürnberg
}

Im Angesicht einer stark im Wandel begriffenen Unternehmenswelt - Globalisierung, verkürzte Produktlebenszyklen, stark steigende Anforderungen an Mobilität und Flexibilität der Mitarbeiter - gewinnt die IT-gestützte Zusammenarbeit weiter an Bedeutung. In diesem Zusammenhang erscheint zwar eine "Groupware Suite", welche die notwendige Sicherheit und Orientierung zu bieten in der Lage wäre, oberflächlich als Lösung. Allerdings zeigt sich, dass die Entwicklung eines umfassenden Instrumentenkastens zur Unterstützung von Koordination, Kommunikation und Kollaboration nicht ohne weiteres möglich ist. Stattdessen fanden in den letzten Jahren insbesondere verschiedene modulare Instrumente, die im World Wide Web privat außergewöhnlich stark genutzt wurden, Social Software, unter dem Schlagwort Enterprise 2.0 auch in der Unternehmenspraxis zunehmend Anwendung.

In den vergangenen Jahren wurde der Einsatz von Social Software in Unternehmen deswegen in vielen Forschungsprojekten untersucht und es findet sich ein großer Forschungskörper. Dieser reicht von Einzel- und Mehrfachfallstudien über Aktionsforschung zu quantitativen Umfragen. Dabei finden sich Erkenntnisse zur Verbreitung einzelner Anwendungsklassen und -beispiele, Nutzenanalysen, oder auch die Identifikation von Barrieren und Erfolgsfaktoren von Enterprise 2.0.

Angesichts der facettenreichen Entwicklung in der Forschung scheint Orientierung notwendig geworden zu sein. Neben einer Aufbereitung des aktuellen Stands der Forschung schlägt der vorliegende Beitrag sechs Metathemata vor, welche Querverbindungen über die einzelnen Forschungsansätze herzustellen in der Lage sind. Er zielt auf die Identifikation zukünftig relevanter Forschungsfelder im Bereich Enterprise 2.0 und zeigt somit eine Forschungsagenda für das Feld auf.

Wir beginnen mit einer Aufarbeitung der deutschsprachigen Literatur im Feld (Abschnitt 2), danach werden die Ergebnisse eines eintägigen Workshops zur Zukunft von Enterprise 2.0 mit 19 Experten aus Wissenschaft und Praxis vorgestellt (Abschnitte 3 und 4). Zusammenfassung und Diskussion runden den Beitrag ab (Abschnitt 5).

Das Ergebnis des Workshops ist eine Forschungsagenda mit sechs Metathemata, welche in der nachfolgenden Abbildung dargestellt sind: Zielsetzung 
\& Definition, Enterprise 2.0 in der Organisation, Funktionalitäten, Motivation, Nutzung und Daten.

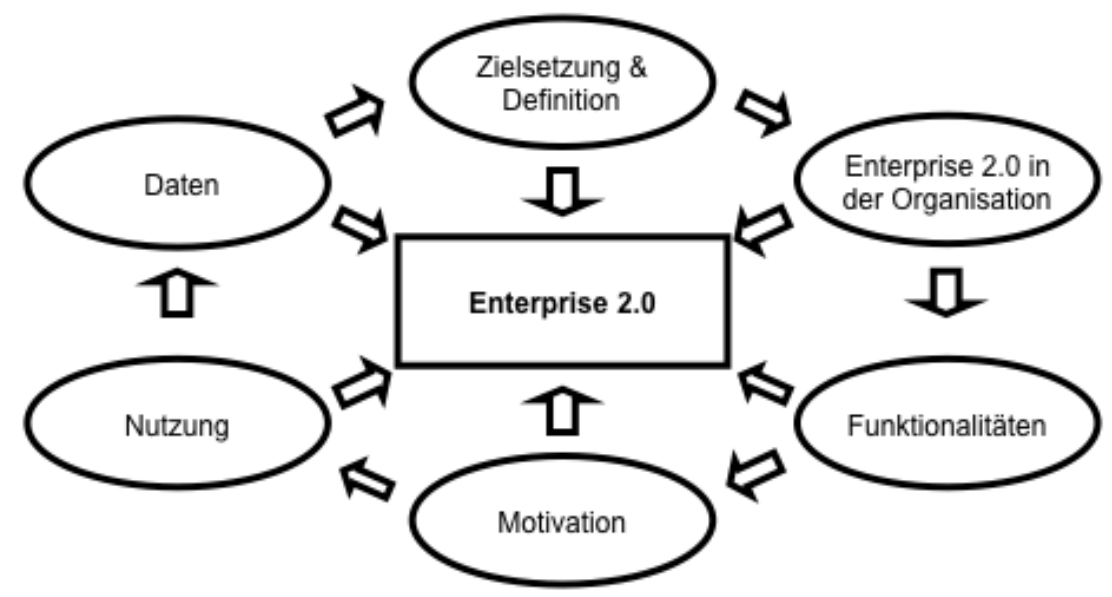

Abbildung 1: Metathemata im Bereich Enterprise 2.0

Unser Beitrag zeigt, basierend auf der Literaturanalyse und der Fokusgruppe mit Experten, die Vielseitigkeit der Themenfelder im Bereich Enterprise 2.0 auf und systematisiert den aktuellen Stand der Erkenntnis. Wir zeigen die identifizierten sechs Metathemen, in welchen Forschungsbedarf für Wissenschaft und Praxis im Feld Enterprise 2.0 besteht. Jedes dieser Metathemata verdient für sich genommen die Aufmerksamkeit von Forschung und Praxis.

Allerdings erachten wir auch die Verbindung verschiedener Metathemata für relevant, da diese miteinander in Beziehung stehen und sich gegenseitig beeinflussen (siehe Abbildung 1). Beispielsweise sind der Zusammenhang zwischen Enterprise 2.0 in der Organisation und der Umgang mit Daten eng verbunden. Wie im Beitrag dargestellt, ist die einzelne Betrachtung eines Metathemas in der bestehenden Forschung eher die Ausnahme, sondern es werden zumeist mehrere Metathemata beobachtet. 


\title{
Aneignungspraktiken von Software-Entwicklern beim Offshoring
}

\section{Fallstudie eines kleinen deutschen Softwareunternehmens}

\author{
Alexander Boden, Sebastian Draxler, Volker Wulf \\ Institut für Wirtschaftsinformatik und Neue Medien, Universität Siegen
}

Die Auslagerung von Teilen der Softwareentwicklung im Rahmen von SoftwareOffshoring ist eine zunehmend verbreitete Geschäftsstrategie in der deutschen ITBranche. Während die Anwendung sowie das Design von Offshoring-Werkzeugen bereits Gegenstand zahlreicher Studien ist, beschäftigen sich wenige Forschungsarbeiten mit dem Problem der Diffusion und Aneignung neuer Softwarewerkzeuge durch die, in diesem Fall verteilten, Projektmitarbeiter.

Um Aneignungspraktiken in verteilt arbeitenden Teams besser zu verstehen, haben wir eine Fallstudie in einem kleinen deutschen Softwareunternehmen durchgeführt, dass eng mit einem Partnerunternehmen in Tomsk, Sibirien kooperiert. Insbesondere gehen wir den Fragen nach, wie sich Entwickler in kleinen Softwareunternehmen neue Werkzeuge und Technologien aneignen, wie entsprechendes Wissen zwischen den Mitarbeitern ausgetauscht wird und in welchem Wechselspiel die firmenspezifischen Praktiken des Unternehmens mit den Rahmenbedingungen verteilter Zusammenarbeit stehen.

Dabei fokussieren wir einerseits auf die Rolle von Artikulationsarbeit beim Offshoring (vgl. Boden et al. 2009) für die Aneignung von neuen Werkzeugen und Technologien; andererseits insbesondere auf die Aneignungsforschung und den Aneignungsbegriff nach Pipek (2005). Aneignung steht dabei in Relation zu Konzepten aus dem Bereich der Forschung zu Anpassbarkeit (Tailorability), aber auch des Bereichs der Einführung von Software in Organisationen in Bezug auf die dabei relevanten Veränderungen der Arbeitspraxis von Organisationen.

Im Rahmen unserer Studie konnten wir zwei unterschiedliche Strategien der Aneignung von Software und der Diffusion von Praktiken innerhalb der Organisation im Zusammenhang mit Artikulationsarbeit beobachten. Einerseits bezogen sich diese auf geplante Änderungen, wie den Umstieg auf die Eclipse Plattform, die im Rahmen formaler Meetings von den Beteiligten diskutiert wurden. Andererseits stießen wir auf ungeplante, ad hoc auftretende und informelle Prozesse der Aneignung von neuen Funktionen und Tools. Als Beispiel behandeln wir das Einrichten einer gemeinsamen Entwicklungsumgebung für ein neues Software Projekt im Rahmen der alltäglichen Zusammenarbeit. Unsere Studie zeigte dabei, dass Aneignungspraktiken im Rahmen informeller Artikulationsarbeit eine wichtige Rolle 
spielen können, da auf diese Weise z. B. Grassroots-Innovationen für die Kooperation nutzbar gemacht werden können. Es zeigte sich auch, dass die Aneignung von Tools über Teamgrenzen hinweg in erster Linie im Rahmen formaler und geplanter Maßnahmen stattfindet. Der Prozess wurde hier jeweils auf persönlichen Treffen (beider Unternehmensteile) an einem der Standorte angestoßen. Dies bringt allerdings erhebliche Einschränkungen mit sich, da nur bestimmte Personen (oftmals Projektleiter statt Early Adopters) regelmäßig das andere Team besuchen konnten. Weiterhin waren persönliche Treffen nur selten möglich und fanden häufig unter starkem Zeit- und Arbeitsdruck statt (z. B. kurz vor Projektabschluss, wenn dringend Ergebnisse benötigt werden).

Dementsprechende konnten in der verteilten Softwareentwicklung gegenüber lokalen Teams ein Missverhältnis von formaler zu informeller Aneignung feststellen. Um die informellen Aneignungsprozesse auch beim Offshoring unterstützen zu können, ziehen wir Erkenntnisse von Pipek (2005) heran. Dabei empfehlen wir einerseits, Mitarbeiter mit Schlüsselrollen an den beiden Standorten zu identifizieren, die durch ihre Expertise lokale, informelle Aneignungsprozesse anstoßen können und diese als Mediatoren stärker zu vernetzen. Als zusätzliche Möglichkeit bietet sich andererseits softwaretechnische Unterstützung an. So kann beispielsweise visualisiert werden, wer welche Werkzeuge einsetzt (und evtl. ein Experte im Umgang ist). Mit diesen Herangehensweisen soll eine bessere Wahrnehmung über die entfernten Standorte hinweg entstehen, die ähnlich wie in lokalen Teams Aneignungsprozesse anstoßen kann.

Die Unterstützung von auch informellen Aneignungspraktiken beim SoftwareOffshoring ist ein wichtiges Thema, das bisher wenig Beachtung erfahren hat. Dabei werden die von uns fokussierten Aspekte in der Zukunft eher zunehmen, da es einen deutlichen Trend hin zu agilen Entwicklungsmethoden sowie zu modularen, flexibel konfigurierbaren Softwarewerkzeugen zu verzeichnen gibt. Gleichzeitig müssen die Akteure immer mehr Konfigurations- und Anpassungsaufgaben selbst bewältigen, was eine entsprechende Unterstützung erfordert. Ein detailliertes Verständnis von Artikulationsarbeit in verteilten Teams ist daher erforderlich, um verteilte Aneignungsprozesse gezielt und kontextgerecht zu unterstützen.

\section{Literatur}

Boden, A., Nett, B. \& Wulf, V., 2009. Offshoring in kleinen und mittleren Unternehmen der Softwareindustrie. HMD. Praxis der Wirtschaftsinformatik, $265,92-100$.

Pipek, V., 2005. From tailoring to appropriation support: Negotiating groupware usage, Oulu: University of Oulu. 
Multiagentensysteme 



\title{
Vorwort zur Teilkonferenz
}

\section{Multiagentensysteme: Dezentralität als Entwurfs-, Organisations- und Betriebsprinzip für Informationssysteme}

\author{
Jörg P. Müller', Lars Braubach², Birgit Burmeister', \\ Alexander Pokahr², Ingo Timm ${ }^{4}$ \\ ${ }^{1}$ Institut für Informatik, TU Clausthal \\ ${ }^{2} V$ erteilte Systeme und Informationssysteme, Universität Hamburg \\ ${ }^{3}$ Daimler AG, Stuttgart \\ ${ }^{4}$ Professur für Wirtschaftsinformatik und Simulation, \\ Universität Frankfurt
}

Seit der Entstehung des Internet gilt Dezentralisierung als grundlegendes Prinzip für den Entwurf, die Organisation und den Betrieb komplexer verteilter Systeme und für das Management globaler Netzwerke. Spätestens seit der Entstehung des Internet gilt Dezentralität als wesentliches Prinzip für den Entwurf komplexer verteilter Systeme und für das Management globaler und dynamischer Netzwerke. Systeme und Netzwerke, die auf dezentralen Konzepten beruhen, haben viele Vorteile: Sie sind robuster, wandlungsfähiger und weniger leicht durch unbeabsichtigte oder beabsichtige Angriffe zu stören; sie ermöglichen eine natürliche Modellierung inhärent dezentraler, autonomer Systeme; sie ermöglichen durch die gemeinsame Verwendung teurer Ressourcen flexible und potentiell kostengünstigere ITSysteme, und sie ermöglichen effektiveres Finden, Zugreifen und Verwalten verteilter Ressourcen, speziell in Umgebungen, in denen autonome Akteure und Gruppen lokal handeln und Ressourcen lokal entstehen, lokal verwaltet werden und sich dynamisch ändern.

Gegenwärtig stellen aktuelle Markttrends wie die Globalisierung von Märkten und Unternehmen, Global Outsourcing und das Entstehen Globaler Partnerschaften als virtuelle Netzwerke die Wirtschaftsinformatik vor große Herausforderungen: Wie können solche komplexen, adaptiven, oft temporären und per se unternehmensübergreifenden und dezentral organisierten Systeme und Unternehmensnetzwerke durch entsprechende Informations $\neg$ systeme effizient und effektiv unterstützt werden? Ähnliche Herausforderungen entstehen durch technologische Entwicklungen wie dem „Internet der Dinge“ und Gemeinschaften und Marktplätzen des „Web 2.0“, die Nutzungsszenarios implizieren, die mit zentralen Architekturen und Entwurfsansätzen nur schwer handhabbar wären. 
Prinzipiell spricht vieles dafür, dass Dezentralität - das Entwurfsprinzip des Internets - auch ein geeignetes Prinzip für die Entwurf, Organisation und Betrieb zukünftiger Informationssysteme sein kann. Dennoch basiert die überwältigende Mehrheit heutiger Informationssysteme auf zentralen Architekturen. In Rechenzentren und IT-Abteilungen ist derzeit sogar häufig zu beobachten, dass dezentrale Strukturen im Rahmen einer Konsolidierung der IT-Landschaft zentralisiert werden. Wo dezentrale Systeme eingesetzt werden, führen grundlegende Anforderungen und Fragestellungen wie Konsistenz und Effizienz der Datenverwaltung, aber auch Sicherheit sowie das Management von Rollen, Rechten und Nutzern zu Problemen und weiterem Forschungsbedarf.

Die genannten Fragen und Aspekte waren Gegenstand der Teilkonferenz „Multiagentensysteme: Dezentralität als Entwurfs-, Organisations- und Betriebsprinzip für Informationssysteme“, deren akzeptierte Papiere im folgenden Teil dieses Konferenzbandes enthalten sind.

Neun wissenschaftliche Papiere wurden für die Teilkonferenz eingereicht; jedes Papier wurde von mindestens drei Mitgliedern des Programmkomitees der Teilkonferenz begutachtet. Fünf Papiere wurden zur Präsentation und Veröffentlichung in diesem Tagungsband ausgewählt; weiterhin wird der Track durch zwei eingeladene Vorträge von Dr. Michael Pirker, Siemens AG, sowie Prof. Stefan Kirn, Universität Hohenheim, aufgewertet. Dr. Pirkers Vortrag gibt einen Überblick über aktuelle Themen und Projekte im Bereich der Agententechnologie bei der Siemens AG; Prof. Kirn beleuchtet in seinem Vortrag Stand und Perspektiven der Multiagenten-Forschung in der Wirtschaftsinformatik.

Es ist den Veranstaltern der Teilkonferenz ein Bedürfnis, den Mitgliedern des Programmkomitees, für deren Namen wir auf die Website der Konferenz unter http://winf.in.tu-clausthal.de/mkwi10 verweisen, recht herzlich für Ihre Mitwirkung zu danken. Wir hoffen, dem geneigten Leser mit den folgenden Beiträgen ein repräsentatives Spektrum an Aktivitäten und Forschungsergebnissen aus diesem spannenden Teilgebiet der Wirtschaftsinformatik präsentieren zu können. Es ist weiterhin unsere Hoffnung, dass die Teilkonferenz Wissenschaftler und Anwender zusammenbringen möge, um Potenziale, Anwendungen und zu lösende Probleme bei der Gestaltung und Nutzung dezentraler Informationssysteme zu erörtern und Lösungsansätze dafür zu finden.

Clausthal, im Februar 2010

Jörg Müller, Lars Braubach, Birgit Burmeister, Alexander Pokahr, Ingo Timm 


\title{
The Notions of Application, Spaces and Agents
}

\section{New Concepts for Constructing Agent Applications}

\author{
Alexander Pokahr, Lars Braubach \\ Distributed Systems and Information Systems, \\ Computer Science Department, University of Hamburg
}

Agent-based approaches offer promising solutions for recent demands in enterprise software like adaptability cooperation and coordination. While current systems consider agents as first-class entities, they currently offer only limited conceptual and technological support for 1) dealing with the agent application as a whole and 2) coping with other non-agent components of an application (i.e. the agent environment). As a result, today's agent-based software systems often employ adhoc solutions for these issues, suffering from a lack of tool support as well as poor maintainability.

This paper tackles the topic of agent applications and concepts for supporting the developer in constructing multi-agent systems. The first proposition is that an explicit description of a multi-agent system is necessary to enable a developer thinking not only in terms of agents but also in terms of all other involved components. Building on previous and related work it is highlighted that an agent application often consists of agents and some kind of environment, whereby support for building both is advantageous. As environments may be quite different, ranging from simulated $2 \mathrm{~d}$ or $3 \mathrm{~d}$ worlds to real application contexts, application descriptions should reflect these requirements and offer a flexible way for defining constituting parts of the application.

The concepts and tools presented in this paper allow for defining an agent application as a conceptual entity, which is composed of agents as well as additional non-agent components. A pluggable architecture is proposed that allows defining common types of environment models, thereby facilitating seamless integration of the corresponding environment components into an agent application. The architecture is implemented as part of the Jadex agent framework. ${ }^{1}$

As an answer to the aforementioned demands based on previous work (Braubach et al. 2005) an extensible XML agent application descriptor is presented (Figure 1). It cleanly separates the model from the runtime level and introduces, besides agents, spaces as a new core concept for specifying applications. Each application can own as many (same or different) spaces as necessary. A space can be

${ }^{1}$ http://jadex.informatik.uni-hamburg.de 
interpreted in a very broad sense and can represent an environment artifact, which can be accessed from all agents of the application. As an example the environment space is introduced. It allows for defining virtual $2 \mathrm{~d}$ environments and takes over many aspects of simulated worlds, e.g. the domain model, the agent environment interaction and also the visualization.

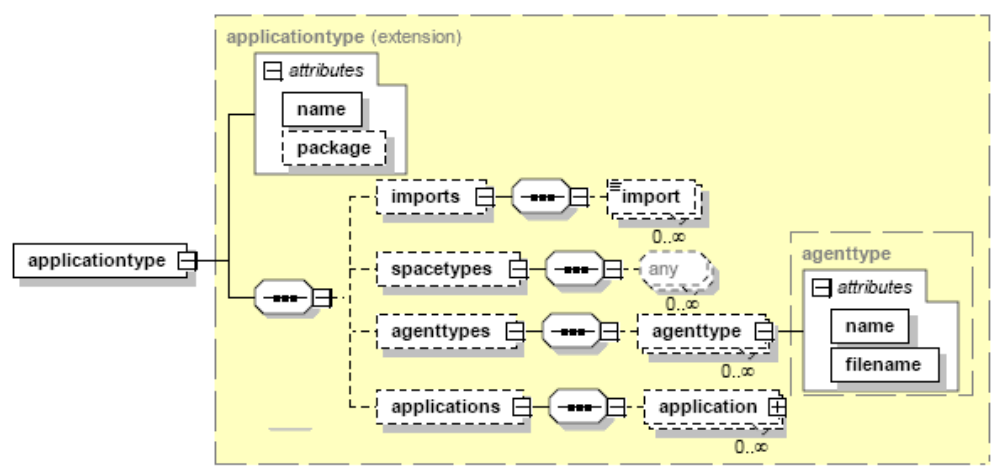

Figure 1: XML-schema for application descriptors

At runtime an application represents an entity in its own right, which mainly acts as a container for agents and spaces. Agents that are part of an application can access the spaces via their application instance. In this way the access to spaces is restricted to agents from the same application context. Representing applications as entities also allows for handling them at the tool level, i.e. instead of starting or stopping single agents, whole applications can be managed.

Finally, as an example application, the MedPAge application (Paulussen et al. 2006) is shown. It demonstrates the usage of the application and environment descriptor for a resource planning problem in hospitals. One main benefit that can be achieved using the presented techniques is a clear separation of concerns between agent and environment responsibilities. Hence, it was possible to reuse the same system for simulating the scheduling algorithms as well as for real field tests.

\section{References}

Braubach L, Pokahr A, Bade D, Krempels KH, Lamersdorf W (2005) Deployment of Distributed Multi-Agent Systems. Proceedings of ESAW, Springer, Berlin.

Paulussen TO, Zöller A, Rothlauf F, Heinzl A, Braubach L, Pokahr A, Lamersdorf W (2006) Agent-based Patient Scheduling in Hospitals. Multiagent Engineering - Theory and Applications in Enterprises, Springer, Berlin. 


\title{
A Generic Strategy Framework for Policy-directed Autonomous Trading Agents
}

\author{
Steffen Lamparter, Silvio Becher, Michael Pirker \\ Corporate Technology, Siemens AG, Munich
}

One of the fundamental questions within multi-agent systems research is how autonomous, self-interested agents can be coordinated in a way that the global performance of the system is maximized. In recent years, market mechanisms have become popular as efficient means for coordinating self-interesting agents competing with each other about scarce resources/tasks. A wide range of different negotiation or auction mechanisms have been proposed in this context - many of them exhibiting favourable properties such as efficiency or/and incentive compatibility.

The paper extends the generic strategy framework presented in Vytelingum (2005) to support developers in specifying device specific agent strategies. It can be used to implement widely autonomous bidding agents that are able to coordinate a wide range of different resources and interact with different market mechanisms. The architecture leverages the idea of policies (Kephart, 2004) for realizing a highdegree of autonomy while making sure that the agents behave within a predefined action space. As policies are declarative descriptions they can be added and removed at runtime which allows the dynamic adaption of strategies. An energy market scenario is given in the paper, where e.g. new appliances in the household come with their own policies. These policies specify how appliances can be regulated and thereby define the bidding strategy of the agents.

\section{Agent Architecture}

The reasoning process of an autonomous agent is a closed control loop that involves the steps perception, cognition and action, which corresponds to the three layers of agent strategy design as defined in Vytelingum (2005). The process is depicted in Figure 1. 
In the first step of the process, market, environment and agent state information are perceived. Based on this information the agent's strategy space is compiled. The strategy space is then passed to the cognition step. Here the strategy space is filtered with respect to the agent's policies. Policies

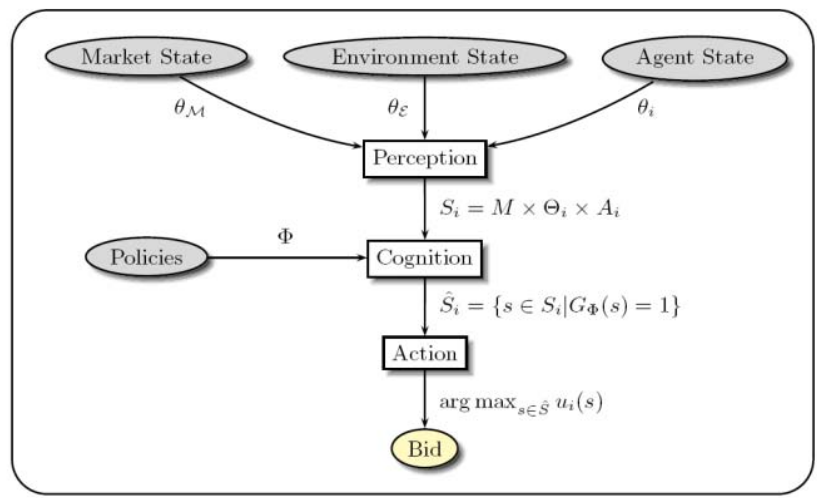

Figure 1: Agent Reasoning Process can be seen as constraints that govern the behaviour of the agent. They capture information about how an agent is able to regulate the underlying technical infrastructure (e.g. appliances or energy generators in a SmartGrid), how an agent has to react on environmental changes (e.g. temperature changes) and how the agents react on new market information. As a result of the cognition step, a set of valid/acceptable agent strategies is passed to the action phase. Here the most promising action is selected based on the user's preferences and auction protocol. An action is connected to a single or a sequence of bids that are finally sent to the market. The consequences are perceived by a changing market state and may trigger new actions.

The goal of the strategy framework is to provide a generic layer that supports different market-based coordination approaches in various domains. As a first application area, the paper proposes an agent-based energy marketplace for balancing energy supply and demand using price incentives. In this context, agents represent appliances or generators connected to the power grid. Each appliance/generator comes with a policy how it can be regulated by the agent. Based on these policies the agent strategy is dynamically adapted and reactions on price signals are executed. The overall goal of the approach is to realize peak load reductions and a higher utilization of the power grid.

\section{References}

Kephart JO, Walsh WE (2004) An artificial intelligence perspective on autonomic computing policies. In: Policies for Distributed Systems and Networks, pages $3-12$.

Vytelingum P, Dash R, He M, Jennings NR (2005) A framework for designing strategies for trading agents. In: IJCAI Workshop on Trading Agent Design and Analysis, pages 7-13. 


\title{
Ubiquitous Microblogging als dezentrales Entwurfsparadigma für leichtgewichtige Informationssysteme
}

\section{Nutzenpotenziale und Forschungsperspektiven}

\author{
Martin Böhringer, Peter Gluchowski \\ Lehrstubl für Wirtschaftsinformatik II, TU Chemnitz.
}

Unternehmen verzeichnen eine stetig steigende Flut von Informationen und Komplexität. Unterschiedlichste Datenquellen sind zu verknüpfen und zugänglich zu machen. SOA stellt eine populäre technologische Antwort auf diese Herausforderungen dar. Das Service-orientierte Paradigma akzeptiert, dass heutige Informationssysteme zu komplex sind, als dass sie von einer zentralen Instanz erdacht und entwickelt werden könnten. SOA ermöglicht daher die Erstellung von Einzelkomponenten und deren dynamische Zusammensetzung im Zeitablauf. Die Treiber der Implementierung sind allerdings weiterhin in den IT-Abteilungen der Unternehmen zu verorten. Für die in vielen fachlichen Bereichen vorherrschende Dynamik und die damit einhergehenden ständigen Änderungen des Informationsbedarfs eines jeden Nutzers erweist sich daher selbst der SOA-Ansatz als zu starr (Hoyer und Stanoevska-Slabeva 2009). Mashups sind als eine Art „SOA light“ zur Lösung dieses Problems konzipiert worden. Jedoch erfordern auch sie ein hohes Maß an technologischem Verständnis und entspringen in ihrem Funktionsansatz der datenbasierten Denkweise der Informatik. Als Resultat fristen selbst erfolgreiche Dienste wie Yahoo Pipes ein Nischendasein.

Parallel zu dieser Entwicklung hat sich im Internet die neue Anwendungsklasse des Microbloggings herausgebildet. Millionen Menschen nutzen den populären Dienst Twitter, um Informationen auszutauschen. Während das Bedienkonzept der Microblogging-Anwendungen extrem simpel ist, scheint es den hochkomplexen Informationsaustausch zwischen den zahlreichen Nutzern sehr gut zu unterstützen. Durch diese Eigenschaft hat Microblogging sehr früh das Interesse von Forschern und Softwareanbietern geweckt, die das Prinzip in das Unternehmensumfeld übertragen (Enterprise Microblogging, vgl. Barnes et al. 2010).

Der vorliegende Beitrag argumentiert nun für eine Erweiterung des Verständnisses von Microblogging. Während Twitter-ähnliche Werkzeuge bisher vorwiegend im Sinne der Zusammenarbeits-Unterstützung diskutiert werden, schlägt der hier präsentierte Ansatz des „Ubiquitous Microbloggings“ eine Nutzung der Technologie über reine Kollaborations-Szenarien hinaus vor. Ubiquitous Microblogging beschreibt ein Informationssystem, in welchem neben menschlichen Nutzern auch 
nicht-menschliche Akteure wie Software, Sensoren und Maschinen eingebunden sind. Der Term „Ubiquitous“ wird dabei gemäß seiner ursprünglich Wortherkunft im Sinne von „alles umfassend“ verwendet (vgl. auch „Ubiquitous Computing“). Damit entsteht ein komplexes System, welches den flexiblen Zugriff auf verschiedenste Informationen mit Hilfe des simplen Microblogging-Mechanismus ermöglicht und gleichzeitig die einfache dezentrale Publikation von Inhalten erlaubt. Ubiquitous Microblogging verbirgt die zugrundeliegende informationstechnische Komplexität vor dem Anwender und ordnet sich somit als endnutzerorientierte Ergänzung einer IT-Hierarchie aus SOA (Implementierungsebene) und Mashup (Analystenebene) ein.

Der vorliegende Beitrag präsentiert das Konzept von Ubiquitous Microblogging. Nachdem Kapitel 2 die Anwendungsklasse Microblogging vorstellt und seine Adaption in Unternehmen motiviert, gibt Kapitel 3 einen konzeptionellen Überblick über die mögliche Architektur einer derartigen Lösung. Das anschließende Kapitel 4 diskutiert daraufhin vorhandene Forschungsarbeiten und zukünftige Forschungsfragen zur Erfüllung des aufgezeigten Szenarios, bevor Zusammenfassung und Ausblick die Überlegungen abschließen.

Das Microblogging-Umfeld ist äußerst volatil. Twitter selbst zeigt zunehmende Bestrebungen hinsichtlich einer Funktionserweiterung. Parallel hierzu wurde mit Google Wave ein Anwendungssystem vorgestellt, welches bereits als möglicher Nachfolger von Microblogging diskutiert wird. Aus Sicht der Forschung ist diese starke Aktivität ein großer Vorteil bei der Suche nach geeigneten Mechanismen für den innerbetrieblichen Informationsaustausch. Ubiquitous Microblogging stellt hierbei einen Forschungsansatz dar, der nun in praktischen Projekten erprobt werden muss. Die entsprechenden Aktivitäten werden auf der Community-Seite http://ubimic.org zusammengetragen. Interessierte Forscher sind herzlich zur Teilnahme aufgerufen.

\section{Literatur}

Barnes SJ, Böhringer M, Kurze C, Stietzel J (2010) Towards an understanding of social software: the case of Arinia. In: Proceedings of the 43rd Hawaii International Conference on System Sciences (HICSS).

Hoyer V, Stanoevska-Slabeva K (2009) Towards a reference model for grassroots enterprise mashup environments. In: 17th European Conference on Information Systems (ECIS). 


\title{
Kriterien für den Einsatz Service-orientierter Architekturen in der Unternehmens-IT
}

\author{
Markus Knöfel, Thomas Barth \\ Institut für Wirtschaftsinformatik, Universität Siegen
}

Die Einführung von Konzepten Service-Orientierter Architekturen (SOA) in die IT wird in vielen Unternehmen diskutiert. Wissenschaft und Wirtschaft proklamieren die Notwendigkeit einer SOA für Unternehmen, damit diese in Zukunft hinreichend flexibel sind und in einer globalen Weltwirtschaft konkurrieren können. Die verbreitet angeführten Argumente für eine SOA sind nach Dreifus et al. (2008) eine gesteigerte Flexibilität und Wirtschaftlichkeit. Die Wirtschaftlichkeit lässt sich in Unternehmen kaum im Vorfeld nachweisen; verbreitete Methoden zur Wirtschaftlichkeitsberechnung (ROI, „return on investment") lassen sich vergleichsweise gut zur Analyse der Wirtschaftlichkeit von Prozessautomation verwenden, sind jedoch bei der viel komplexeren Analyse der Wirtschaftlichkeit einer Service-orientierten IT-Infrastruktur nur schwer anwendbar. Eine in (Fiedler und Seufert 2007, S. 32) wiedergegebene Metrik des ROI einer SOA basiert bspw. auf der (monetären) Bewertung von Sachverhalten wie „,benefit of agility“ und „cost of inflexibility“ deren Messung oder a priori-Abschätzung zum Zeitpunkt einer Entscheidung pro/contra einer SOA-Einführung jedoch schwierig ist. (Laures 2006, S. 165) gibt dazu ein Maß für Flexibilität von IT bei sich ändernden Geschäftsprozessen an und gibt einen Überblick über weitere Ansätze. Allerdings basieren diese auf dem Vergleich mit einer existierenden Infrastruktur, sind also ebenfalls für eine Analyse im Vorfeld einer Änderung der Infrastruktur durch SOA-Konzepte nicht anwendbar.

In diesem Beitrag wird dargestellt, welche Kriterien in einer systematischen Vorgehensweise wie untersucht werden können, um das Nutzenpotential einer SOA für ein gegebenes Unternehmen a priori abschätzen zu können. Auf dieser Basis kann eine Entscheidung für oder gegen eine SOA-Einführung unterstützt werden. Die Autoren stützen sich dabei auf eine über einen Zeitraum von mehreren Jahren aufgebaute Basis von Fallbeispielen aus 77 Unternehmen unterschiedlicher Branchen und Größen.

Die prinzipielle Vorgehensweise lässt sich wie folgt darstellen: In einem ersten Schritt wird die Abhängigkeit des Unternehmens von den Prozessen ermittelt. Dazu werden die Prozesse aufgeführt und nach strategischem Nutzen und operationalem Mehrwert gegliedert. Mit Hilfe dieser Einteilung können die zu erwartenden Effekte einer SOA-Einführung bewertet werden. Im darauf folgenden Schritt wird der zukünftige Bedarf der identifizierten Prozesse in punkto Flexibilität festgestellt. In Schritt drei wird die Abhängigkeit der als nicht ausreichend flexibel identifizierten Prozesse von der 
IT-Infrastruktur untersucht. Analog zu Schritt drei muss im folgenden Schritt die Abhängigkeit der IT-Applikationen von der IT-Infrastruktur bezüglich der Veränderbarkeit geprüft werden. Diese Vorgehensweise leitet sich aus der Kategorisierung von ITInfrastrukturen ab, die von Weill und Broadbent (1998) empirisch ermittelt wurde. Daraus lässt sich eine erste Empfehlung für die Einführung von Konzepten der SOA in eine IT-Infrastruktur darstellen: In der Kategorie „None" sind Unternehmen, die durch einen Verzicht an interner Bürokratie hohe Flexibilität aufweisen. Bei den meisten Unternehmen mit einer Infrastruktur vom Typ „Utility“ sind die Geschäftsprozesse stabil oder kaum von der IT abhängig. Unternehmen des Typs „Dependent" richten ihre Infrastruktur nach den Anforderungen der Fachabteilungen unter Berücksichtigung eines ausgewogenen Verhältnisses von Kosten und Nutzen aus. Die Unternehmen mit Infrastrukturen des Typs „Enabled“ haben eine systemische Kompetenz für Flexibilität. Zusammenfassend gilt, dass die Entscheidung für eine SOA primär von den künftigen Anforderungen der Prozesse an die IT und weiterhin dem gegenwärtigen Stand der IT-Infrastruktur abhängt. Ein Ausbau der ITInfrastruktur unter Berücksichtigung der Konzepte einer SOA sollte erfolgen, wenn die künftigen Prozesse aus fachlicher Sicht mit der vorhandenen Infrastruktur nicht hinreichend flexibel sind. In diesem Fall lässt sich der Aufwand wirtschaftlich rechtfertigen. Unternehmen mit IT-Infrastruktur-Rollen „None“ und „Utility“ sollten ihre Infrastruktur für eine Flexibilisierung zunächst ausbauen. Dies hilft den Unternehmen, schneller auf Geschäftsanforderungen zu reagieren und die technischen, prozessorientierten, organisatorischen und kulturellen Voraussetzungen für eine SOA zu schaffen. Unternehmen vom Typ „Dependent“ können mit der Untersuchung der geeigneten Geschäftsprozesse und deren benötigter Flexibilisierung eine Kosten-Nutzen-Analyse zur Rechtfertigung einer SOA begründen. Für Infrastrukturen vom Typ „Enabled“ eröffnet die Einführung einer SOA zur Darstellung künftiger Anforderungen der fachlichen Seite ein erhebliches Potential.

\section{Literatur}

Dreifus F, Leyking K, Loos, P (2008) Systematisierung der Nutzenpotentiale einer SOA. In:, Nissen V, Petsch M, Schorcht H (Hrsg) Service-orientierte Architekturen: Chancen und Herausforderungen bei der Flexibilisierung und Integration von Unternehmensprozessen, Gabler

Fiedler M, Seufert A (2006), Der SOA-Entscheidungsprozess und Ansatzpunkte für ROI-Betrachtungen. HMD 253, S. 28-36, dpunkt

Laures G (2006) Flexibilitätsanalyse serviceorientierter Architekturen zur Realisierung von Geschäftsprozessen. In: Weske M, Nüttgens M (Hrsg.). EMISA 2006. LNI 95, S.163-177, Springer

Weill P, Broadbent M (1998) Leveraging the New Infrastructure: How Market Leaders Capitalize on Information Technology. Harvard Business Scholl Press, Boston 


\title{
Einsatz von Agenten für das Selbstmanagement von Automatisierungssystemen
}

\author{
Hisham Mubarak, Peter Göbner \\ Institut für Automatisierungs- und Softwaretechnik, Universität Stuttgart
}

Die automatisierungstechnischen Entwicklungen und Innovationen der letzten Jahre zeigen einen deutlichen Trend zur Dezentralisierung von Automatisierungssystemen (vgl. Wahlster und Raffler 2008). Zusammen mit der gleichzeitig erfolgenden funktionalen Flexibilisierung von Automatisierungskomponenten führen diese Entwicklungen zu leistungsfähigeren, anpassbareren und wirtschaftlicheren Automatisierungssystemen, die jedoch eine immer höhere Gesamtkomplexität im Betrieb aufweisen und aufgrund der damit einhergehenden Überforderung des Betriebspersonals die Beherrschbarkeit der Systeme gefährden. Es ist daher unabdingbar das Betriebspersonal wo möglich zu entlasten und es bei der Durchführung technischer Betriebsbetreuungsaufgaben in bestmöglicher Weise zu unterstützen. Die verfügbare menschliche Expertise soll dabei in den wichtigen Funktionen effizient eingesetzt werden, während automatisierbare Funktionen durch ein Unterstützungssystem autonom und ohne Zutun des Betriebspersonals durchgeführt werden. Eine Möglichkeit zur Entlastung des Betriebspersonals und zur Beherrschung komplexer Systeme ist das Prinzip der Selbstorganisation (Jelasity et al. 2006, S. 8-9). Im Kontext des Betriebs komplexer Automatisierungssysteme bedeutet dies die autonome Durchführung von technischen Betriebsbetreuungsaufgaben durch Softwareagenten. Der vorliegende Beitrag stellt eine agentenbasierte Erweiterung klassischer Prozessautomatisierungssysteme durch ein Selbstmanagementsystem vor, welches die schrittweise Einführung von Selbst-X-Funktionen (automatisierte Funktionen zur Unterstützung der Betriebsbetreuung, vgl. Kephart und Chess 2003, S. 41-50) in Automatisierungssysteme erlaubt.

Es gilt zu beachten, dass in modernen Automatisierungsanlagen die manuellen Tätigkeiten des Prozesspersonals zum einen der Führung operativen Betriebs dienen und zum anderen sich mit der technischen Betriebsbetreuung (Inbetriebnahme, Instandhaltung, Optimierung und Sicherung des Automatisierungssystems) befassen. Das Ziel des Selbstmanagements ist die Unterstützung des Prozesspersonals durch Automatisierung der Betriebsbetreuungsaufgaben. Betriebsbetreuungsaufgaben lassen sich in die Kategorien Konfiguration, Heilung, Optimierung und Schutz einteilen. Durch ihre Automatisierung entstehen entsprechende Selbst-X-Funktionen (Kephart und Chess 2003, S. 41-50). Grundidee des Ansatzes ist es, basierend auf dem Modell des Selbstmanagementprozesses individuelle Selbstmanagementfunktionen in dedizierte Selbstmanagement-Agenten zu kapseln, welche vollständig autonom handeln und somit den Bediener von der entspre- 
chenden Aufgabe entlasten. Zu diesem Zweck wird das klassische Prozessautomatisierungssystem um ein viertes Teilsystem, das Selbstmanagement-System, erweitert, welches in drei agentenorientierte Ebenen aufgeteilt ist.

Die Ebene „Selbstmanagement-Funktionalität“ ist der Kern des Selbstmanagementsystems und dient der Bereitstellung der eigentlichen SelbstmanagementFunktionalität. Die autonome Durchführung von Selbstmanagementfunktionen erfordert neben deren eigentlicher Bereitstellung auch die Koordination deren Handlung im Automatisierungssystem. Analog zur Abstimmung des Prozesspersonals bei der manuellen Durchführung, muss diese Koordination ebenfalls automatisiert erfolgen können, um einen autonomen Betrieb zu gewährleisten. Ein Selbstmanagementagent (SEMA) stellt genau eine SMF bereit. Aufgrund der Heterogenität der Betriebsbetreuungsaufgaben und den dementsprechend heterogenen Lösungsverfahren erlaubt die individuelle Realisierung durch einen SEMA die höchste Flexibilität. Er wird spezifisch für eine Betriebsbetreuungsaufgabe eines Automatisierungssystems entwickelt und kapselt das funktionsspezifische Wissen zur Identifikation von Handlungsbedarf und zur Ableitung von Maßnahmen zur Behebung funktionalitätsrelevanter Situationen. Hierzu interagiert jeder SEMA mit der darunterliegenden Ebene, der Automatisierungssystemanbindung, um die von ihm zur Erfüllung seiner Aufgaben benötigten Informationen aus dem Automatisierungssystem zu ermitteln. Erfasste Informationen werden vom SEMA analysiert und wissensbasiert auf einen Handlungsbedarf ausgewertet. Ist dies zutreffend erfolgt in einem weiteren wissensbasierten Schritt die Ableitung von Maßnahmen zur Ausführung im Automatisierungssystem. Sowohl die Handlungsbedarfsermittlung, als auch die Maßnahmenauswahl können durch regelbasierte Systeme zur Formalisierung und Nutzung des entsprechenden Wissens unterstützt werden.

\section{Literatur}

Jelasity M, Babaoglu O, Laddaga R (2006) Self-Management through SelfOrganization. IEEE Intelligent Systems 21(2): 8-9.

Kephart JO, Chess DM (2003) The Vision of Autonomic Computing. IEEE Computer 36(1):41-50.

Wahlster W, Raffler H (2008) Studie 2008 des Feldafinger Kreises "Forschen für die Internet-Gesellschaft: Trends, Technologien, Anwendungen" http://www.feldafingerkreis.de/Feldafinger-Kreis_Studie_2008.pdf. Abruf am 2009-09-15. 


\title{
Eine modellbasierte Software-Architektur zur Unterstützung dezentraler Prozesse in der Produktentwicklung
}

\author{
Patrick D. Stiefel, Jörg P. Müller \\ Institut für Informatik, Technische Universität Clausthal
}

Die Zusammenarbeit zwischen einzelnen Bereichen eines Unternehmens und organisationsübergreifende Kollaborationen werden immer mehr zunehmen. In (Krause 2007, S.75ff) werden dazu drei aktuelle Trends aufgezeigt, die dabei helfen sollen, die gegenwärtigen Defizite - zu hohe Komplexität, zu geringe Integrationstiefe und Interoperabilität - zu beseitigen:

1. Ausreifung der existierenden Methoden, Werkzeuge und Systeme,

2. Entwicklung anpassungsfähiger, skalierbarer und offener Integrationsplattformen mit intelligenter Umgebung, sowie

3. Durchsetzung von Standards in Werkzeugen.

Kollaborationsplattformen müssen einen entscheidenden Mehrwert insbesondere bei der Unterstützung der organisationsübergreifenden Zusammenarbeit in den frühen Phasen der Produktentwicklung liefern, da Engineering-Kernkompetenzen im Bereich Entwicklung \& Design, sowie Prozesswissen gebündelt werden müssen (Li and Ong 2006).

Diesen Herausforderungen bei der Entwicklung von Kollaborationsplattformen für die kollaborative Produktentwicklung (engl.: Collaborative Product Development, CPD) stellt sich unsere Forschung. Wir beschäftigen uns mit der Frage, wie mit Hilfe dezentraler Informationstechnologien CPD-Prozesse effizienter unterstützt werden können (Stiefel und Müller 2007; Stiefel und Müller 2008). Ein konkretes Ergebnis ist die prototypische Product Collaboration Platform (PCP) eine peer-to-peer(P2P)-basierte Kollaborationsplattform für die dezentrale Verwaltung von Produktmodellen und die Unterstützung dezentral organisierter Kollaborationsprozesse in der Produktentwicklung.

Dazu verfolgen wir einen modellbasierten Entwicklungsansatz (MBE) für dezentrale Informationssysteme zur Unterstützung der CPD. Auf der Basis von ITunabhängigen Prozess- und Kollaborationsmodellen (Computation Independent Model, CIM) werden in einem iterativen Entwicklungsprozess unterschiedliche Typen IT-basierter Modelle abgeleitet, die sich in ihrem Abstraktionsgrad unter-

1 Siehe auch http://plm.in.tu-clausthal.de/PCP/cebit.php [01.09.2009] 
scheiden: Von IT-Architekturmodellen (Platform Independent Model, PIM) über plattformspezifische Modelle (PSM) hin zu konkreten Software-Artefakten.

Eine offene Frage hierbei ist, wie im Rahmen eines MBE-Ansatzes die Anforderungen dezentraler Entwicklungsprozesse einerseits und die Möglichkeiten dezentraler IT-Plattformen (und im speziellen P2P-Umgebungen) berücksichtigt werden können. In diesem Papier stellen wir Methoden und Modelle vor, die im Rahmen der sonst üblichen Entwicklung von zentralen (Kollaborations-)Systemen keine Rolle spielen und daher im MBE-Kontext bisher kaum Betrachtung finden. Wir schlagen Modellierungsansätze auf den verschiedenen Ebenen der MBE vor, die eingesetzt werden können, um - unterstützt durch teilweise automatisierte Modelltransformationen - dezentrale Architekturmodelle und schließlich konkrete Software-Artefakte zu generieren. Unser Ziel ist es hier, wichtige Grundlagen für die nächste Generation lose gekoppelter Kollaborationssysteme für organisationsgreifende Produktentwicklung zu schaffen (Roser 2008, Stäber 2008).

In Abschnitt 2 dieses Beitrags werden dazu zunächst die Grundlagen der dezentralen, kollaborativen Produktentwicklung diskutiert. Abschnitt 3 stellt den „State-of-the-art" der modellbasierten Entwicklung vor und diskutiert spezielle Anforderungen bei der Anwendung des MBE-Ansatzes zur Entwicklung dezentraler Kooperationssysteme. Im vierten Abschnitt werden Modelle der unterschiedlichen MBE-Ebenen präsentiert, bevor im letzten Abschnitt eine Bewertung des vorgestellten Ansatzes vorgenommen und dessen Eignung hinsichtlich der Praxistauglichkeit beurteilt wird.

\section{Literatur}

Krause, Frank-Lothar (2007) Innovationspotenziale in der Produktentwicklung. Hanser, München.

Li, W.D. and Qiu, Z.M. (2006) State-of-the-Art Technologies and Methodologies for Collaborative Product Development Systems. In: International Journal of Production Research, 44(13): 2525-2559.

Roser (2008) Designing and Enacting Cross-organizational Business Processes.

Dissertation, Uni Augsburg.

Stäber (2008) Service layer components for decentralized applications.

Dissertation, TU Clausthal.

Stiefel, Patrick D.; Müller, J. P. (2007) ICT interoperability challenges in decentral, cross-enterprise product engineering. In: Gonçalves; Ricardo J., S. 171-182. http://dx.doi.org/10.1007/978-1-84628-858-6_18.

Stiefel, Patrick D.; Müller J.P. (2008) Realizing dynamic product collaboration processes in a model-driven framework: Case study and lessons learnt, in K.D. Thoben, K. S. Pawar, \& R. Gonçalves, eds., 14th International Conference on Concurrent Enterprising, , 23-25 June 2008, Lisbon, Portugal. 


\title{
Coping with global Information Systems
}

\section{Requirements for a flexible SLA Discovery and Negotiation Infrastructure for the future Internet of Services}

\author{
Sebastian Hudert, Torsten Eymann \\ Chair of Information Systems Management (BWL VII), \\ University of Bayreuth
}

Visions of 21st century's information systems show highly specialized digital services, which interact on a global scope. Today's internet of mainly human interaction evolves to a distributed information infrastructure, where humans as well as software agents continuously interact to exchange data and computational resources. Such infrastructures will possibly consist of millions of service providers (SP), consumers (SC) and a multitude of possible intermediaries, thus forming a global economic environment and realizing the vision of an open Internet of Services (IoS) (Schroth and Janner, 2007, pp. 36-41).

For a broad adoption of this vision it is important to have a mechanism in place to guarantee quality of service (QoS) for each service invocation, even across enterprise boundaries. This becomes crucial when parts of mission critical workflows will be executed on external services. Since such scenarios inherently lack the applicability of centralized QoS management, guarantees must be obtained in the form of bi- or even multi-lateral service level agreements (SLAs) assuring service quality across individual sites (Ludwig et al., 2003, pp. 43-59).

In order to support a comprehensive SLA-based management for such settings, the main phases of the SLA lifecycle should be directly supported by the underlying infrastructure: service discovery, negotiation, implementation, monitoring and post processing, which, as a set, form a fundamental basis of a comprehensive service management overlay as needed in the envisioned settings.

The contribution of this paper is twofold. First we derive a detailed scenario model for the future IoS as an initial step to understand the challenges of next generation information systems (IS). This model will be deduced from current developments in distributed computing, such as Service Oriented Computing (SOC), Grid Computing (GC) or Cloud Computing (CC). All of the abovementioned developments in today's distributed IS point to the same vision of the future internet based on highly dynamic networks of composable services, offered and consumed on a global scope, ultimately leading to innovative business models and supporting the transition from value chains to value nets. Shortly sketched this scenario exhibits the following characteristics: 
- The IoS focuses on a (potentially huge) set of electronic services, varying in complexity and therefore ranging from raw resources to very complex workflows

- These services will be employed in potentially mission-critical business workflows and thus have to fulfill a (pre-negotiated) set of QoS guarantees

- SPs and SCs communicate over the global Internet to trade these services

- New business models will cope with the possibility of trading even individual services and charging them based solely on their actual usage

Based on this model, we conduct a comprehensive requirement analysis for technical infrastructures supporting the derived IoS vision (due to space reasons we focus on the negotiation and discovery phases). Among others the identified requirements were:

- After the Discovery Phase all parties must have a common understanding of the protocol to be executed in the negotiation phase (Ludwig et al., 2006), which should generated dynamically at runtime (Brandic et al., 2008, p. 4).

- RN21 Different marketplaces and protocols even within one market are needed for different services to be traded (Neumann et al., 2008, pp. 325-347).

- RN31 Software Agents should act as negotiators (Ludwig et al., 2006), and do so on different markets, even simultaneously (Brandic et al., 2008).

Finally we give a short overview on current research projects and show to which extent the identified requirements are fulfilled as of now.

\section{References}

Brandic I, Venugopal S, Mattess M, Buyya R (2008), Towards a Meta-Negotiation Architecture for SLA-aware Grid Services, Technical Report, University of Melbourne

Ludwig H, Keller A, Dan A, King R, Franck R (2003), A Service Level Agreement Language for Dynamic Electronic Services, JECR, Vol. 3

Ludwig A, Braun P, Kowalczyk R, Franczyk B (2006), A Framework for Automated Negotiation of Service Level Agreements in Services Grids LNCS, Proc. of the Workshop on Web Service Choreography and Orchestration for Business Process Management, Vol. 3812/2006

Neumann D, Stoesser J, Weinhardt C, Nimis J (2008), A Framework for Commercial Grids - Economic and Technical Challenges, JOGC, Vol. 6, 325347

Schroth C, Janner T (2007), Web 2.0 and SOA: Converging Concepts Enabling the Internet of Services, IT Professional, IEEE Computer Society, Vol. 9, 36-41 
Anwendungen 

Automotive Services 2010 



\title{
Vorwort zur Teilkonferenz
}

\author{
Automotive Services 2010 \\ Jan Marco Leimeister', Helmut Kromar' ${ }^{2}$, Klaus Straub ${ }^{3}$ \\ ${ }^{1}$ Fachgebiet Wirtschaftsinformatik, Universität Kassel \\ ${ }^{2}$ Lehrstubl für Wirtschaftsinformatik, Technische Universität München \\ ${ }^{3}$ Audi AG, Ingolstadt
}

Die aktuellen ökonomischen und ökologischen Herausforderungen für Akteure in der Automobilbranche verlangen nach neuartigen Services in und um das Fahrzeug im Allgemeinen sowie im Kontext von Individualmobilität im Besonderen. Die Konzeption; Entwicklung und Umsetzung solcher Automotive Services steht dabei im Spannungsfeld eines rasanten Fortschritt im Bereich mobiler Informationstechnik mit kurzen Lebenszyklen und den anderen Lebenszyklen der Komponenten und Nutzungssituationen moderner Fahrzeuge und ihrer Nutzer.

Besonderes Augenmerk muss bei Automotive Services auf die jeweilige Fahrsituation gelegt werden. So spielt die Sicherheit des Fahrers und die Richtung der Aufmerksamkeit bei der Nutzung von Automotive Services im Fahrzeug eine kritische Rolle. Gleichzeitig müssen die im Fahrzeug zur Verfügung stehenden Ressourcen berücksichtigt werden. Die Konzeption, Entwicklung und Umsetzung neuer Automotive Services setzen daher entsprechend fokussierte Werkzeuge, Konzepte und Methoden voraus.

Die in dieser Konferenz vorgestellten Beiträge können in drei Kategorien unterteilt werden. Die erste Kategorie umfasst Beispiele für Automotive Services sowie deren systematische Entwicklung und Evaluation. So stellen Valentin Nicolescu, Jan Marco Leimeister und Helmut Krcmar Grundprinzipien eines avatarbasierten natürlichsprachlichen Interaktionskonzepts vor, das Handbuchinhalte interaktiv und situationsgerecht den Fahrzeuginsassen anbietet. Oliver Baecker, Markus Miche und Thomas Michael Bohnert zeigen die Potenziale von Vehicle-to-Business-Services am Beispiel der Schadensaufnahme und abwicklung nach einem Unfall. Andreas Pirali, Niklas Klein, Sian Lun Lau und Klaus David erläutern die Ergänzung bestehender Entertainmentsysteme durch Web 2.0 Funktionalitäten sowie die daraus entstehenden Potenziale am Beispiel einer kontextsensitiven Einkaufsliste.

Die zweite Kategorie beinhaltet Beiträge, die Methoden für die Konzeption und Entwicklung von Automotive Services vorstellen. Andreas Zauner, Holger Hoffmann, Jan Marco Leimeister und Helmut Krcmar zeigen hierfür zunächst die aktuellen Herausforderungen und Trends auf. Anschließend entwickeln Maja Golcar, Michael Schermann, Maximilian Pühler, Holger Hoffmann und Helmut 
Krcmar Heuristiken für die Analyse von Infrastrukturanforderungen von Automotive Services. Hoffmann, Jan Marco Leimeister und Helmut Krcmar zeigen die Prinzipien einer systematischen Analyse von Anforderungen an Automotive Services anhand einer Rapid Prototyping-Plattform, die die partizipative Entwicklung von Automotive Services ermöglicht. Maximilian Pühler, Michael Schermann und Helmut Krcmar untersuchen anschließend theoretische Grundlagen für eine partizipative Entwicklung von Automotive Services und überführen diese in entsprechende Designprinzipien.

Die dritte Kategorie umfasst Beiträge, die den Fokus auf die Nutzenpotenziale von Informationstechnik in der Automobilbranche. So erläutern Jan Löhe, Christine Legner, und Stefan Gumbrich die Potenziale serviceorientierter Architekturen zur Entwicklung innovativer Services im Bereich des Gebrauchtwagenhandels. Carolin Löffler und Lucia Schleifer hingegen untersuchen die Akzeptanz von internetbasierten After Sales Services sowie deren Nutzen insbesondere für Automobilhersteller. Benjamin Wagner vom Berg und Frank Köster geben einen Überblick über den Stand der Technik im Bereich Elektromobilität und untersuchen mit Blick auf mögliche Geschäftsmodelle Analogien mit Mobilfunkanbietern.

Somit setzt diese Konferenz die erfolgreiche Auftaktkonferenz zu Automotive Services (AS2008) in München fort. Unser Dank gebührt dabei den Mitgliedern des Programmkomitees, die durch kritische, aber konstruktive und umfangreiche Begutachtungen der Beiträge den Autoren wertvolle Hilfestellungen bei der Weiterentwicklung ihrer Ansätze geliefert haben. Dies sind insbesondere Frau Prof. Dr. Legner, Frau Prof. Dr. Robra-Bissantz sowie die Herrn Prof. Dr. Dr. h.c. Manfred Broy, Prof. Dr. Klaus David und Dr. Michael Schermann.

Es bleibt zu hoffen, dass die Chancen die sich durch Automotive Services an der Schnittstelle zwischen IT im Fahrzeug und IT-gestützten Dienstleistungen von der Wirtschaft und Wissenschaft aufgegriffen werden und die Innovationspotenziale in diesem vielversprechenden Feld gemeinsam weiter gehoben werden können. 


\title{
Natural speaking (inter)faces for cars
}

\author{
Valentin Nicolescu', Jan Marco Leimeister², Helmut Krcmar ${ }^{1}$ \\ ${ }^{1}$ Lehrstubl für Wirtschaftsinformatik, Technische Universität München \\ ${ }^{2}$ Fachgebiet Wirtschaftsinformatik, Universität Kassel
}

\section{Introduction}

Modern upper class vehicles provide an increasing number of functions, the purpose of which is to make travelling by car more pleasant and safer for passengers. On the one hand, the quantity of functions require car manufacturers to integrate the corresponding control elements; on the other hand, the driver is expected to become familiar with the use of the new or sometimes even hidden secondary functions of these control elements. These hidden functions are due to the limited space on the dashboard, making the combination of different functions in one control element necessary. Another challenge that car manufacturers and their customers have to face is the increasing number of software based functions, particularly in secondary and tertiary task areas, i.e. the use of comfort or entertainment devices such as air conditioning, media centers or navigation systems (Tönnis et al. 2006). The application of software development concepts for automotive services requires drivers to have full control of the new functions. These new automotive services also need more information about the driver in order to tap full potential. For example, an electric car will need information about the driving behavior of a user to develop its battery loading strategy.

This makes a new approach necessary for the in-vehicle user interface that enables easy access to a large number of existing and/or future functionality, as well as information without distracting the driver from driving.

The optimal solution for this issue would be the permanent presence of a "codriver-in-the-box" who could recite all user manuals of the car. This co-driver would also adapt to the driver's behavior and provide information in crucial situations whenever the driver requested assistance. The information would be suitable to the situation and be provided in the right amount at the right point in time.

This article provides a general outline of this vision and elaborates on the experiences of working with a prototype in a series-production vehicle.

\section{Use Cases of an ECA}

The use of the prototype in the vehicle can be done in two different ways: by first asking for specific information and or by explorative use of control elements in the secondary and tertiary sector. The first use case is designed as a substitute for the user's manual and allows access to information about features and functions of the 
car, also while driving. In addition to the information of the manual, the responses of the Embodied Conversational Agent (ECA) are supported by media that could not be integrated in a print medium. This can happen by displaying a figure or a $3 \mathrm{D}$ object on any topic, or the operation of control elements can be presented by the avatar using animations of the corresponding control elements. We implemented an avatar system and report on experiences and the evaluation results.

\section{Discussion}

The reaction of users has shown that the idea of an ECA in a vehicle is perceived in a positive way even if the prototype is pointing out different areas for improvement. In addition, the question of the appropriateness of an avatar for such a system in a vehicle has to be faced. The avatar is not mandatory for basic information mediation. The avatar primarily has the task of a social actor (Reeves and Nass 1998), giving the driver the sensation that he can speak naturally to the car. Without a figurative illustration, it is expected that the user predominantly uses command words instead of natural language (Cheyer and Julia 1999). Using only command words, the driver holds back information that he would probably share when using natural speech. Due to the lack of information, the ECA can identify only with a low probability what the user wants and thus cannot respond with a reaction which the user may expect. This leads to increased frustration for the user, and thus the loss of acceptance for such systems. However, such ECA systems are necessary to handle a multitude of new functions in a meaningful way.

\section{References}

Cheyer A, Julia L (1999) InfoWiz: An Animated Voice Interactive Information System. In: Proceedings of the Proceedings of the Workshop on Communicative Agents(Agents '99), Seattle.

Reeves B, Nass C (1998) The Media Equation - How People Treat computers, Television, and New media Like Real People and Places. CSLI Publications, Stanford, California.

Tönnis M, Broy V, Klinker G (2006) A Survey of Challenges Related to the Design of 3D User Interfaces for Car Drivers. In: Proceedings of the IEEE Virtual Reality Conference (VR 2006), Alexandria, Virginia, USA. 


\title{
SOA zur Koordination verteilter Geschäftsnetzwerke
}

\section{Anwendungsbeispiel im deutschen Gebrauchtwagenhandel}

\author{
Jan Löhe', Christine Legner', Stefan Gumbrich² \\ IInstitute of Research on Information Systems, \\ European Business School, Oestrich-Winkel \\ ${ }^{2}$ Global Business Services, \\ IBM Deutschland GmbH, Mannheim
}

Das Gebrauchtwagengeschäft stellt mit einem Umsatzvolumen von rund 53 Mrd. Euro mehr als ein Drittel des automobilen Gesamtmarkts in Deutschland dar. Dabei werden mit 6,11 Mio. Gebrauchtwagen etwa doppelt so viele gebrauchte wie neue Fahrzeuge (3,09 Mio.) abgesetzt. Mit dem Aufkommen des Internets hat sich auch der Gebrauchtwagenvertrieb kontinuierlich gewandelt. Mittlerweile sind in Onlinebörsen mehr als 85\% der Gebrauchtfahrzeuge verfügbar. Das so entstandene überregionale Angebot und die damit verbundene Transparenz über Qualität und Preise verändert das Kauf- und Suchverhalten der Kunden nachhaltig.

Ziel des vorliegenden Beitrages ist es, zu untersuchen, inwieweit das Paradigma der service-orientierten Architektur (SOA) neue, elektronische Vernetzungspotentiale in der Gebrauchtwagendistribution ermöglicht. Unsere Forschungsfrage lautet: Wie verbessern SOA und B2B-Services die Koordination zwischen den Akteuren zur Steigerung der Effizienz oder Hebung neuer Geschäftspotentiale im Gebrauchtwagenhandel? Die methodische Grundlage unserer Entwicklung von Serviceideen zur Professionalisierung des Gebrauchtwagengeschäfts bildet eine Geschäftsnetzwerkanalyse, die auf dem von Österle et al. (2001) entwickelten Business Networking Ansatz beruht. In einem ersten Schritt bestimmten wir ausgehend vom generischen Kundenprozess alle Akteure des Geschäftsnetzwerks und identifizierten aus der Sekundärliteratur deren Interessen und Herausforderungen. Im zweiten Schritt befragten wir 14 Gebrauchtwagenhändler mit Hilfe eines strukturierten Interviewleitfadens (je $90 \mathrm{~min}$ ), um das Geschäftsnetzwerk zu validieren sowie aktuelle Problemfelder und Maßnahmen zu bewerten. Auf dieser Grundlage konkretisierten wir organisatorische und technologische Lösungsansätze durch Serviceideen, die auf dem Paradigma service-orientierter Architekturen beruhen. Durch weitere qualitative Experteninterviews validierten wir die Erkenntnisse der ersten Interviewrunde und die entwickelten Serviceideen.

Ergebnis des Beitrags ist zunächst eine Analyse des Gebrauchtwagennetzwerks sowie der aktuellen Herausforderungen, die auf Basis von Sekundärliteratur und 
Händlerinterviews abgeleitet wurden. Neben den Herausforderungen beurteilten die befragten Händler im Rahmen der Interviews insgesamt 56 Einzelmaßnahmen nach ihrer Relevanz zur Behebung der Probleme und dem heutigen Umsetzungsgrad auf einer 5-Punkte Likert-Skala. Folgende fünf zusammengefasste Maßnahmenpakete wurden sowohl von den Händlern als auch später von den Experten als effektiv und effizient zur Behebung der Herausforderungen angesehen: (1) Proaktives GW-Portfoliomanagement, (2) Zwverlässige Fabrzenginformationen, (3) Proaktive Marktbearbeitung, (4) Integrierte Bundlingabwicklung, sowie (5) Integrierte Prozesssteuerung. Diese Maßnahmepakete implizieren organisatorischen Handlungsbedarf, der sein volles Potential erst durch systemtechnische Unterstützung ausschöpft. Während in der Vergangenheit eine individuelle Optimierung erfolgte, identifiziert der Beitrag Serviceideen, die im Verbund von verschiedenen Akteuren zu realisieren sind, und damit zur verbesserten Koordination beitragen. Weiteres Ergebnis ist der konzeptionelle Aufbau einer SOA-basierten Lösung, die den fachlichen Servicebedarf in autonome Domänen Kunden-, Auftrags- und Fahrzengverwaltung sowie Cross-FunctionalServices unterteilt. Grundlegende Überlegung dabei ist, dass innerhalb der Domänen eine hohe Kohäsion besteht und Services die zugehörige, fachliche Funktionalität erbringen. So wird innerhalb der Domäne Fahrzeugverwaltung die übergreifende Sicht auf die Fahrzeuge, vom Zugang, über Bewertung, Instandsetzung, Bestandsverwaltung bis zum Abgang von Gebrauchtwagen, erzeugt. Die Kundenverwaltung stellt Services zum Management von Kundenkontakten und Kundenprofilen bereit. Die Domäne Auftragsmanagement ermöglicht die Erstellung und Individualisierung von Angeboten, die Verteilung von Angeboten, die Integration von Ergänzungsleistungen und die Verwaltung und Nachverfolgung von Auftragsdaten und Kaufabschlüssen. Domänen interagieren untereinander über Services als definierte Schnittstellen. Die Einbindung und Interaktion mit Partnern erfolgt ebenfalls über Services. Voraussetzung ist eine eindeutige Schnittstellenbeschreibung und hohe Interoperabilität der Services, um beispielsweise verschiedene Dienstleister für Finanzprodukte oder die Marktbeobachtung einbinden zu können.

Das geschilderte Vorgehen basiert auf der Erkenntnis, dass innovative Ansätze wie SOA sich nur dann durchsetzen, wenn deren fachliche Wirksamkeit gegeben ist und sie geschäftliche Interessen der betroffenen Akteure unterstützen. Die Analyse und Entwicklung von Serviceideen bilden einen ersten Forschungsschritt, der im Zuge weiterer Arbeiten durch die Implementierung der vorgestellten Lösung und deren Evaluierung fortzuführen ist. 


\title{
Vehicle-to-Business Communication: The Example of Claims Assistance in Motor Insurance
}

\author{
Oliver Baecker1,2, Markus Miche2, Thomas Michael Bohnert² \\ ${ }^{1}$ Institute of Technology Management, University of St. Gallen \\ ${ }^{2} S$ AP Research Switzerland, St. Gallen
}

Due to consequent policy making and research funding in Europe, the US, and Japan, Intelligent Transportation Systems (ITS) are gaining momentum and provide mature concepts for vehicular communications enabling vehicles to communicate among themselves (Vehicle-to-Vehicle, V2V) and with their immediate vicinity (Vehicle-to-Infrastructure, V2I). Amongst others, the European research projects PRE-DRIVE C2X and CVIS as well as the German research project simTD aim at improving road safety and traffic efficiency by facilitating a continuous information exchange between vehicles and their surrounding roadside infrastructure such as traffic lights or roadwork warning signs.

In recent years, the proliferation of powerful Broadband Wireless Access (BWA) technologies such as for instance UMTS, WiMAX, or LTE paves the way for a new generation of telematic services. One of the compelling features of such BWA technologies is the fact that the required infrastructure comes at no additional cost for the automotive sector. So is the number of telematics applications steadily rising in domains such as logistics, fleet management, or the insurance industry. Common to all application domains is the integration of real-world vehicle data into business applications in order to generate additional revenue by means of innovative services or to reduce costs based on business process improvements. As any of them is driven by a certain economic objective, we summarize them under the term Vehicle-to-Business (V2B), which reflects our general vision of integrating vehicles into business operations. As the in-vehicle communication units required for V2B telematic services could be directly equipped with the respective V2V and V2I communication functionalities, the former might be seen as an enabler of V2V and V2I communications, which are subject to large network effects (Matheus et al. 2004, pp. 2-6). As a result, the concept of V2B complements V2V and V2I communications resulting in an all-embracing cooperative intelligent road transport systems.

While V2B applications are emerging, there is still a lack of well-defined and standardized integration platforms that allow for a reliable and scalable interconnection of vehicles and providers of telematic services. This paper showcases the potential of $\mathrm{V} 2 \mathrm{~B}$ communications by presenting an application scenario from the insurance industry ("V2B-based Claims Assistance"). The scenario includes vehicles that submit a loss report after a minor car accident using V2B communications. It 
points out the benefits for both the insurance company due to the integration of comprehensive accident data into the claims management process (Accenture 2002; Bieber and Hoberg 2007) and the drivers that can make use of different value-added services (e.g., navigation to the closest repair shop, requesting a tow truck) provided by the insurance company. In addition, this application scenario serves as the foundation for the investigation and derivation of challenges and requirements for $\mathrm{V} 2 \mathrm{~B}$ communications.

A V2B integration platform tackling these challenges is proposed, which facilitates a reliable, scalable, and secure information exchange between vehicles and business systems. This platform consists of a Vehicle Integration Platform (VIP) that enables a loosely coupled, asynchronous communication between vehicles and business systems plus an in-vehicle communication component that complements the VIP, the Back-end Integration Manager (BIM). In addition, a dedicated invehicle abstraction layer is defined that encapsulated vehicle-specific interfaces and provides a standardized access to both the CAN bus and the in-vehicle HMI. Hence, vehicle applications neither have to deal with the communication functionality nor with any hardware specifics of different vehicle manufacturers. This improves not only the maintenance and further developments of vehicle applications, but allows for a cost-effective deployment of the latter on different vehicles.

Consequently, the proposed V2B integration platform plus the outlined vehicle abstraction layer lowers the barrier for service providers and customers to step into the upcoming V2B market. Both concepts are indispensable to achieve the full potential of $\mathrm{V} 2 \mathrm{~B}$ communications not only as regards the opportunity of novel business models, but also to foster the introduction of V2V and V2I technologies.

\section{References}

Accenture (2002) Unlocking the Value in Claims. Accenture - Insurance Solution Group.

Bieber T, Hoberg S (2007) Aktives K-Schadenmanagement gemessen und bewertet. Versicherungswirtschaft 12:992-995.

Matheus K, Morich R., Luebke A (2004) Economic Background of Car to-Car Communications. In: Proceedings of the 2. Braunschweiger Symposium Informationssysteme für mobile Anwendungen. Braunschweig. 


\title{
Prototyping komplexer Geschäftsanwendungen im Automobil
}

\section{Ein Tag im Lebens eines Handlungsreisenden der Zukunft}

\author{
Holger Hoffmann', Jan Marco Leimeister², Helmut Krcmar ${ }^{1}$ \\ ${ }^{1}$ Lehrstubl für Wirtschaftsinformatik, Technische Universität München \\ ${ }^{2}$ Fachgebiet Wirtschaftsinformatik, Universität Kassel
}

Software wird als Innovationstreiber in der Automobilbranche zunehmend wichtiger, der Anteil softwarebasierte Systeme an der Gesamtwertschöpfung am Fahrzeug wächst stetig. Aus diesem Grund fokussieren Automobilhersteller zunehmend auf die Entwicklung kundennaher softwarebasierter Funktionen im Fahrzeug. Dabei beziehen sich aktuelle Arbeiten und Publikationen hauptsächlich auf die Kundengruppe der Endkunden, die ihr Fahrzeug privat - bzw. auf dem Weg zur Arbeit oder für gelegentliche Dienstfahrten - nutzen, außen vor steht die Gruppe der Kunden, die ihr Fahrzeug im Rahmen ihres täglichen Geschäftes nutzen. Damit fallen in den USA rund 15 Millionen (12\% aller Beschäftigten), in Europa geschätzte 25 Millionen, potentielle Kunden aus dem Raster. Dies ist vor dem Hintergrund, dass diese geschäftlichen Kunden eher bereit sind für Mehrwertdienste zu bezahlen als private Endkunden, besonders negativ zu bewerten.

Um auch für diese Kunden sinnvolle und damit am Markt erfolgreiche Anwendungen im Fahrzeug anbieten zu können, müssen - wie bei anderer Software auch - die speziellen Anforderungen der mobilen Arbeit an solche Anwendungen erfasst und erfüllt werden. Bisher fehlen jedoch in der noch sehr am Produkt „Fahrzeug“ orientierten und hierarchisch strukturierten Automobilbranche geeignete Möglichkeiten, systematisch Kundenanforderungen an Softwarefunktionen zu erfassen und zu analysieren. Dieser Beitrag zeigt daher auf, wie Kunden mit Hilfe eines Prototypingwerkzeugs in die partizipative Gestaltung von Anwendungen integriert werden können, welche besonderen Anforderungen aus der mobilen Arbeit von Außendienstmitarbeitern für ein System im Fahrzeug abgeleitet werden können und wie dieses schließlich in Form eines Prototypen für eine Nutzerevaluation bzw. eine Pilotierungsstudie umgesetzt werden kann. Durch dieses „erlebbar machen“ der Anwendung für Außendienstmitarbeiter ist es einerseits möglich, deren Anforderungen und Bedürfnisse systematisch zu ermitteln, andererseits können aber auch innovative Ideen für Anwendungen sowie konkrete Lösungsinformationen für bestehende Probleme direkt von Experten - den Außendienstmitarbeitern selbst - gesammelt, bewertet und in Unternehmensprodukten zum Einsatz gebracht werden. 
Die im Rahmen dieses Beitrages mit Hilfe der für Anwendungen im Automobil ausgelegten Highly Integrated Modular Embedded Prototyping Platform (HIMEPP) umgesetzte MobileWorker Anwendung adressiert die in der Literatur beschriebenen Probleme mobil Arbeitender und unterstützt diese beim Zugriff auf Unternehmensressourcen und weitere Informationen. Die MobileWorker Anwendung selbst unterstützt die mobile Arbeit im Unternehmen dadurch, dass die in der Literatur beschriebenen wesentlichen Probleme entweder gelöst oder zumindest deren Auswirkungen geschmälert werden. Für den mobilen Mitarbeiter bedeutet dies konkret, dass er einerseits mobil Zugriff auf die Ressourcen seines Unternehmens hat und sich andererseits Medienbrüche durch die Fahrzeugintegration sowie die Möglichkeit zur Synchronisierung mobiler Endgeräte vermeiden lassen. Wesentlicher Vorteil für das Unternehmen ist, dass nun die Ressourcen (in Form von Mitarbeitern oder Fahrzeugen) effizienter eingesetzt werden können, da den Disponenten sowohl aktuelle Aufenthaltsorte wie auch aktuelle Terminplanungen bei dessen Planung zur Verfügung stehen.

Zudem wird mit der MobileWorker Anwendung eine Möglichkeit aufgezeigt, wie sich mit Hilfe der HIMEPP Plattform zukünftig Projekte erstmalig nach dem Paradigma des User-driven Design umsetzen lassen. Die Erstellung von Prototypen mit HIMEPP als Toolkit für Automotive Software und Services, sowie die Möglichkeit, diese Anwendungen evaluieren lassen zu können, ermöglichen die partizipative Gestaltung neuartiger Geschäftsanwendungen gemeinsam mit Vertretern der späteren Nutzergruppe. Durch das User-driven Design von Anwendungen werden explizite und implizite Anforderungen genauer und kompletter erhoben, von den Nutzern als Branchenexperten Lösungsinformationen zu bestimmten Problemen erfasst, die Umsetzung dieser Anforderungen und Lösungsinformationen direkt im späteren Umfeld getestet und damit der wahrgenommene Nutzen der Anwendungen für die späteren Nutzer gesteigert. So ist es Automobilherstellern und Zulieferern möglich, zusammen mit Unternehmen am Markt erfolgreiche Geschäftsanwendungen umzusetzen die auf die IT-Infrastruktur des Unternehmens angepasst sind und den mobilen Arbeitern einen tatsächlichen Mehrwert liefern. 


\title{
Automotive Software and Service Engineering (ASSE) - an exploration of challenges and trends in an industry experts' view
}

\author{
Andreas Zauner', Holger Hoffmann' ${ }^{1}$ Jan Marco Leimeister², Helmut Kromar \\ ${ }^{1}$ Lehrstubl für Wirtschaftsinformatik, Technische Universität München \\ ${ }^{2}$ Fachgebiet Wirtschaftsinformatik, Universität Kassel
}

\section{Introduction and Motivation}

Mobile and software based value-added applications in an automobile, known as Automotive Software and Services (Reichwald et al. 2007), represent a differentiation factor and are currently increasing in importance in a "young generation" (Stolte 2008) of customers(Reichwald et al. 2007)(Reichwald et al. 2007). And hence, Automotive Software and Services are used as means for differentiation and also to increase customers loyalty (Mercer 2004; Reichwald et al. 2007). Instead of only being producers of cars, which loose customer contact after selling the car, OEMs are moving more and more towards providers of holistic solutions which supply the customer with new and different services throughout the automobile's entire lifetime (Mercer 2004).

\section{Basics and Structure of the Expert Survey}

The aim of the survey is to identify the current state of Automotive Software and Services in science and industry to determine challenges and obstacles and to discover trends and strategies for the future. The expert survey method was chosen deliberately because no opinion should be influenced by a discussion between the experts, as it would be using a Delphi method, for example. In the following sections, we present the fundamentals of our expert survey. The design and implementation of the expert survey is based on the model of the phases of data collection by Nieschlag (Nieschlag et al. 2002), a respected model in German social sciences. In this study, the model was adapted and applied in such a manner that it can respond to the research question and the research obstacles very well.

\section{Implications and Future Prospects}

In future, Software and Service Engineering will play a key role. Not only OEMs and suppliers will be highly interested in this topic, but also all the other stakeholders of the automotive industry like telecommunications companies and customers. The very high response rate of the conducted expert survey demonstrates that, too. The scope of our study was focused on Germany and the German market. In the 
future, we would like to extend our study to the international market; including also the view of important pioneers in the field of Automotive Services in Asia (e.g. Japan).

The interviews with the different stakeholders have confirmed the results of some other studies in this topic. Our research shows the importance of mobile value-added applications in the vehicle for the future development. In our study, the following components for Automotive Software and Service Engineering can be derived (Fig. 1). The degree of cooperation, which is necessarily included in the development process, raises more and more in direction of norms and standards. Moreover, customer's visibility of these software or services is actually only possible when killer applications are realized.

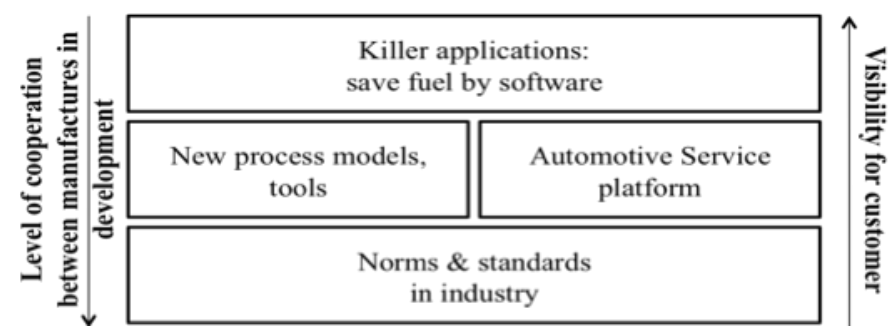

Figure 1: Components of Automotive Software and Service Engineering

\section{References}

Mercer (2004) Future Automotive lndustry Structure (FAST) 2015. Henrich Druck + Medien, Frankfurt a. M.

Nieschlag R, Dichtl E, Hörschgen H (2002) Marketing. 19, Duncker\&Humboldt, Berlin.

Reichwald R, Krcmar H, Reindl S (Hrsg.) (2007) Mobile Dienste im Auto der Zukunft EUL Verlag, Lohmar - Köln 2007.

Stolte P (2008) Das Auto im Connected Life. In: Proceedings of the Das vernetzte Automobil, München. 


\title{
Theoriebasiertes partizipatives Design von Automotive Services
}

\author{
Maximilian Pübler, Michael Schermann, Helmut Krcmar \\ Lehrstubl für Wirtschaftsinformatik, Technische Universität München
}

Die vergangenen Jahre haben gezeigt, dass innovative Automotive Services eine zunehmend wichtige Rolle in der Automobilindustrie spielen. Die Wertschöpfung moderner Systemen basiert zu einem großen Teil auf Software (Liggesmeyer und Rombach 2005) wobei deren Kostenanteil bis zu 50\% der gesamten Entwicklungskosten beträgt (Baskerville 1999). Zusätzlich ermöglicht die steigende Verfügbarkeit von Systemressourcen, wie Rechenkapazität, Speicher oder hochauflösenden Displays, immer komplexere Services und Anwendungen in modernen Fahrzeugen.

Der zunehmende Ausbau breitbandiger mobiler Datenanbindungen wie z. B. UMTS/HSxPA beschleunigt diese Entwicklung noch zusätzlich. Zwei bekannte Beispiele dieses Entwicklungsprozesses sind das aktuelle iDrive von BMW und das Audi Multimedia Interface. Bei der Identifikation von Anforderungen für neuen Automotive Services ist eine frühzeitige Integration der Kunden in den Entwicklungsprozesse sowie das Erlangen eines gemeinsamen Verständnisses allen Beteiligen ein entschiedener Faktor um Akzeptanz und Zufriedenheit mit neuen Service zu gewährleisten. Dies gilt insbesondere für die Entwicklung von Automotive Sevices, bei denen domänenspezifische Aspekte wie z.B. Fahrsicherheit oder Usability besonders berücksichtigt werden müssen. Einer der erfolgreichsten Ansätze um dies zu erreichen, ist der Einsatz von Prototypen. Sie erlauben es dem Kunden, in einer sehr frühen Phase des Entwicklungsprozesses eine konkrete Vorstellung des Automotive Service zu erhalten (Floyd et al. (1989).

Mit existierenden prototyping Frameworks und Tools ist dies allerdings immer noch sehr Zeit- und Kostenintensiv (Grechanik et al. 2007). Obwohl neue Prototypingplatformen wie z.B. HIMEPP ${ }^{1}$ (Hoffmann 2009) einen großen Beitrag zu Erleichterung des Prototypingprozesses für Entwickler leisten, sind sie bislang nur für technisch versierte Entwickler nutzbar. Nach (Breindahl 2008) ist, "es effizienter ein System im Dialog mit dem Nutzer zu entwickeln".

Aus diesem Grund ist die Fragestellung dieses Beitrags, wie partizipative Gestaltung neuer Automotive Services durch die Integration nicht-technischen Stakeholder in den Innovationsprozess realisiert werden kann. Hierfür werden Designrichtlinien für eine Prototyping-Workbench definiert, die eine aktive Beteiligung der Kunden an der Entwicklung von Automotive Service Prototypen erlau-

${ }^{1}$ Highly Integrated Modular Embedded Prototyping Platform 
ben und so die bislang existierenden Ansätze ergänzen. Experten müssen weiterhin die Möglichkeit haben über den Funktionsumfang einer Prototyping-Workbench hinauszugehen und diesen zu erweitern, Desweiteren ist eine Entwicklungsumgebung auf eine darunterliegende technische Realisierung aufgebaut und unterliegt auch deren Beschränkungen.

Das Paper ist wie folgt gegliedert. Zunächst werden die speziellen Herausforderungen im Automotive Services Bereich diskutiert um auf dieser Grundlage anschließend Designrichtlinien für eine Automotive Service Workbench aus grundlegenden IS-Theorien abzuleiten. Der Beitrag schließt mit einer Zusammenfassung und einem Ausblick ab.

\section{References}

Baskerville, Richard (1999): Investigating information systems with action research. In: Communications of the AIS (3), S. 4.

Breindahl (2008): A Scandinavian Approach to Interaction Design.

http://www.dcdr.dk/uk/Menu/Update/Webzine/Articles/A+Scandinavian+ Approach+to+Interaction+Design, Abruf am 2008-11-21.

Floyd, Christine; Mehl, Wolf-Michael; Resin, Fanny-Michaela; Schmidt, Gerhard; Wolf, Gregor (1989): Out of Scandinavia: Alternative Approaches to Software Design and System Development. In: Human-Computer Interaction. 4 (4), S. 253-350. http://www.informaworld.com/10.1207/s15327051hci0404_1, Abruf am 2008-08-29.

Grechanik, M.; Conroy, K. M.; Probst, K. A. (2007): Finding Relevant Applications for Prototyping. In: Mining Software Repositories, 2007. ICSE Workshops MSR '07. Fourth International Workshop on, S. 12-12.

Hoffmann, Holger (2009): Ein Werkzeug zur Entwicklung nutzerorientierter Software- und Service-Prototypen im Fahrzeug. Dissertation, München.

Liggesmeyer, Peter; Rombach, Dieter (2005): Software Engineering eingebetteter Systeme. Grundlagen - Methodik - Anwendungen. 1. Aufl. Elsevier Spektrum Akad. Verl., München. 


\title{
Akzeptanz von internet-driven After Sales Services in der Automobilbranche
}

\section{Eine empirische Untersuchung}

\author{
Carolin Löffler, Lucia Schleifer \\ Lehrstubl für Wirtschaftsinformatik II, Universität Erlangen-Nürnberg
}

Für Automobilhersteller ist es immer schwieriger, sich mit dem eigentlichen Kernprodukt von den Wettbewerbern zu differenzieren. Die Gewinnung von Marktanteilen ist nur durch die Verdrängung bestehender Anbieter möglich (Mattes et. al 2004, S. 15). OEMs sind mit dem Problem konfrontiert, neue Ertragsquellen zu finden. Dienstleistungen, die nach dem Kauf eines Produktes angeboten werden, bieten eine Chance sich von den Wettbewerbern abzusetzen (Mann 1998, S. 74; Meyer 1985, S. 102; Kallenberg und Fischer 1999, S. 15). Über diese so genannten After Sales Services wird in der Automobilwirtschaft bereits ein Großteil der Gewinne generiert (Bullinger und Husen 2006, S. 27; Deraëd 2005), welche jedoch hauptsächlich den Automobilhändlern und -werkstätten zugutekommt. Die Integration des Internets in After Sales Services bietet den OEMs die Möglichkeit, das Service-Angebot durch völlig neue After Sales Services zu erweitern. Zu den Charakteristika des Internet zählen Aktualität, Schnelligkeit, Zeitunabhängigkeit, Simultaneität, Interaktivität, Ortsunabhängigkeit, Datenmächtigkeit und Multimedialität (Hünerberg 1996, S. 108). Die Integration des Internet in die After Sales Services ermöglicht zum einen eine verbesserte Kundenbeziehung, da Kundenprobleme zielgerichtet und schnell gelöst werden können: Unternehmen sind durch interaktive Kommunikation eng mit dem Kunden verbunden (Muther et al. 1998, S. 110). Zum Zweiten führt die Internet-Unterstützung zu Effizienzsteigerungen, da Dienstleistungen mit Internet (häufig) kostengünstiger erbracht werden können (Nippa 2005, S. 7; Benkenstein und Steiner 2004, S. 36).

Internet-driven After Sales Services (IDASS) haben eine strategisch und wirtschaftlich hohe Bedeutung für Automobilhersteller.

Ziel dieser Arbeit ist es, etablierte IDASS neben neuen Service-Ideen zu analysieren. Dabei soll ein Verständnis für die Wünsche und Bedürfnisse der Kunden entwickelt und der Nutzen und die Akzeptanz der Services untersucht werden um kritische Erfolgsfaktoren für IDASS aufzudecken.

Hierfür wird eine empirische Untersuchung der Internet-driven After Sales Services durchgeführt. Für das Vorgehen wird eine Kombination von qualitativer und quantitativer Forschung ausgewählt. Die explorative Stärke der qualitativen Studie dient dazu, die IDASS anzupassen und adäquate Hypothesen zur Akzep- 
tanzmessung zu generieren, welche anschließend in einer quantitativen Untersuchung überprüft werden.

Das Ergebnis weißt Implikationen für das Management bei der Gestaltung von Internet-driven After Sales Services auf. 


\title{
Heuristische Ermittlung und Typisierung von Infrastrukturanforderungen mobiler Dienste im Fahrzeug
}

\author{
Maja Golcar, Michael Schermann, Maximilian Pühler, \\ Holger Hoffmann, Helmut Kromar
}

Lehrstubl für Wirtschaftsinformatik, Technische Universität München

Die Entwicklung mobiler Dienste im Automobilsektor hat vor allem im Hinblick auf den Einsatz onlinebasierter Informationsdienste im Business-to-ConsumerBereich hat in den letzten Jahren einen signifikanten Anstieg erzielt (Viswanathan et al. 2007, S. 92). Trotz der zunehmenden Leistungsfähigkeit moderner Informationssysteme im Fahrzeug werden aber längst nicht alle mobilen Dienste, die für eine Verwendung durch mobile Endgeräte zur Verfügung stehen, im Fahrzeug realisiert.

Ein Grund hierfür ist, dass die Markenloyalität im Automobilsektor in wesentlichem Maße von der Kundenzufriedenheit beeinflusst wird. Ein Mangel, den der Kunde beispielsweise durch eine für die Verwendung eines mobilen Dienstes unzureichende Verfügbarkeit von Mobilfunkressourcen verspürt, kann sich im schlechtesten Falle auf die Kundenzufriedenheit auswirken, welche sich in den Phänomenen des Wiederkaufverhaltens, des Zusatzkaufverhaltens sowie des Weiterempfehlungsverhaltens des Kunden gegenüber anderen widerspiegelt (Ilzarbe 2005, S. 20).

Gleichzeitig werden die Herausforderungen in der Automobilbranche zunehmend verschiedenartiger, und der stetig wachsende globale Kosten- und Konkurrenzdruck stellt die Automobilhersteller vor die Aufgabe einer kontinuierlichen Differenzierung (Zauner et al. 2008, S. 5), welche beispielsweise durch die Integration mobiler Dienste erreicht werden kann. Vor einer Integration dieser in das Fahrzeug gilt es allerdings zu klären, inwieweit die Realisierung unter den Bedingungen der gegebenen Mobilfunkinfrastruktur möglich ist.

Dabei sind Anbieter mobiler Dienste im Fahrzeug einerseits mit einer Vielzahl möglicher Dienste konfrontiert, deren spezifische Untersuchung zu aufwändig ist, andererseits mit ortsabhängigen Verfügbarkeitsprofilen bestimmter Mobilfunktechniken. Es stellt sich somit die Frage, wie eine Typisierung mobiler Dienste sowie deren Plausibilitätsprüfung erlaubt, detaillierte Abschätzungen zur Realisierungsfähigkeit bestimmter mobiler Dienste im Fahrzeug umzusetzen.

In der Literatur wird das Komplexitätsproblem bezüglich der mobilen Dienste gelöst, indem Dienstklassen untersucht werden, die sich aus mobilen Diensten desselben Zwecks zusammensetzen, wobei unterschiedliche Kategorisierungsaus- 
prägungen existieren (Van de Kar und Verbraeck 2007, S. 32). Die Anforderungen mobiler Dienstklassen an ihre Mobilfunkinfrastruktur weisen allerdings eine sehr vielfältige Ausprägung auf, aus denen es gilt, Kriterien zur Typisierung mobiler Dienste hinsichtlich ihrer Anforderungen an die Mobilfunkinfrastruktur zu definieren, die einen Bezug zu den knappen Ressourcen der Mobilfunktechniken aufweisen, damit eine Aussage über die mögliche Realisierung durch eine bestimmte Mobilfunktechnik getroffen werden kann.

Die in diesem Beitrag vorgeschlagene Heuristik zur Typisierung soll letztendlich eine Abschätzung ermöglichen, ob die serienmäßige Verfügbarkeit mobiler Dienste verschiedener Dienstklassen im Fahrzeug für den Kunden zufriedenstellend durchgeführt werden kann, indem die jeweiligen Anforderungen an die Mobilfunkinfrastruktur abgeschätzt werden. Mithilfe eines Ansatzes zur Konsolidierung der Anforderungen soll ein grober Überblick über Anforderungen ganzer Nutzugsszenarios erlaubt werden. Dabei wird zunächst der aktuelle Stand mobiler Dienste und der Mobilfunkinfrastruktur dargestellt werden, um darauf aufbauend Kriterien zur Typisierung mobiler Dienste abzuleiten. Anschließend werden anhand eines beispielhaften Nutzungsszenarios die Grundprinzipien einer Heuristik zur Ermittlung der Infrastrukturanforderungen mobiler Dienste Vorgestellt. Der Beitrag schließt mit einer Zusammenfassung und einem Ausblick ab.

\section{Literatur}

Ilzarbe L (2005): Wirkung von Einflussgrößen - insbesondere der Produktqualität - auf die Kundenzufriedenheit in der Automobilindustrie. Dissertation, Berlin.

Van de Kar E, Verbraeck A (2007): Designing Mobile Service Systems. Wiley \& Sons, Brisbane.

Viswanathan S, Kuruzovich J, Gosain S, Agarwal R (2007): Online Infomediaries and Price Discrimination: Evidence from the Automotive Retailing Sector. In: Journal of Marketing 71 (3) 89-107. doi: 10.1509/jmkg.71.3.89.

Zauner A, Hoffmann H, Leimeister JM, Krcmar H (2008): Automotive Software \& Service Engineering Eine Exploration von Herausforderungen und Trends aus der Sicht von Branchenexperten. Studie des Lehrstuhls für Wirtschaftsinformatik, Technische Universität München 2008. 


\title{
Elektromobilität: Gegenwart oder Zukunft?
}

\section{Förderung der Elektromobilität durch innovative Infra- struktur- und Geschäftsmodelle}

\author{
Benjamin Wagner vom Berg', Frank Köster², Jorge Marx Gómez'? \\ 1 Abteilung Wirtschaftsinformatik I, Carl von Ossietzky Universität Oldenburg \\ 2Institut für Verkehrssystemtechnik, \\ Deutsches Zentrum für Luft- und Raumfahrt e.V., Braunschweig
}

Das Thema Elektromobilität ist kein Zukunftsthema, sondern in der Gegenwart angekommen. Neben der Automobilindustrie haben hier insbesondere auch die Energiekonzerne, neue Akteure wie der Service Provider „Better Place“ und auch die staatlichen Regierungen einen zukunftsträchtigen Markt mit enormem Potential identifiziert. Rolle und Gewichtsverteilung der Akteure aus der Industrie auf diesem Markt ist dabei noch nicht abzusehen. Dieser neue Markt hat auch das Potential für positive Auswirkungen auf den Arbeitsmarkt und den fortschreitenden Klimawandel. Ausgewiesene Fachleute wie Prof. Dr. Ferdinand Dudenhöffer, Inhaber des Lehrstuhls für ABWL und Automobilwirtschaft an der Universität Duisburg-Essen bezeichnen die Entwicklung hin zur Elektromobilität in den nächsten 20 Jahren ,als größte Revolution seit Henry Fords Tin Lizzy“ (Dudenhöffer 2009).

Ein größeres Hindernis für den Durchbruch der Elektromobilität stellen momentan noch technische Limitierungen dar, die insbesondere auf dem derzeitigen Stand der Batterietechnik beruhen.

Betrachtet man aber das Nutzungsverhalten der Kunden und die schon heute existenten Infrastrukturkonzepte wie Ladestrategien mit intelligenten Stromnetzen sog. Smart-Grids (Beyers 2009) oder das Batterieaustauschsystem von Better Place (Graunitz 2009), so lassen sich Lösungsansätze erarbeiten, die diese Defizite ausgleichen können. Im Zusammenspiel mit geeigneten Geschäftsmodellen können hier entsprechende Anreize für den Kunden geschaffen werden, die der Elektromobilität zu einem schnelleren Durchbruch verhelfen.

Dabei ergeben sich gegenüber dem heutigen Markt mit den getrennten Akteuren (Automobilhersteller, Tankstellenbetreiber etc.) ganz neue Möglichkeiten mit der Zusammenstellung von hybriden Leistungsbündeln durch die Integration von Produkt und Dienstleistung zu einem Service Mobilität, der von einem einzelnen Service Provider angeboten wird.

Insbesondere der Mobilfunkmarkt bietet eine brauchbare Vorlage für die Entwicklung eines solchen neuen Geschäftsmodells. So lassen sich für einen Service 
Mobilität Fahrzeug, Strom und Serviceleistungen in einem Vertrag bündeln, wie dies im Mobilfunkbereich für Handy und Netzkapazitäten bereits üblich ist.

Darüber hinaus ist der Kunde innerhalb eines Smart-Grids nicht nur Kunde sondern gleichzeitig auch Lieferant, da er sein Auto in Stillstandzeiten als Stromspeicher für Strom aus regenerativen Energiequellen zur Verfügung stellen kann. Die Kundenbeziehung nimmt also eine ganz neue Form an, die es innerhalb des Geschäftsmodells abzubilden gilt.

Auf dieser Grundlage ist es dann möglich Business-Applikationen insbesondere im Bereich CRM und SCM neu zu entwickeln beziehungsweise umzugestalten und $\mathrm{zu}$ adaptieren. Es ist dabei davon auszugehen, dass eine solche Entwicklung über eine reine Branchenanpassung weit hinausgeht und somit erscheint eine weitere wissenschaftliche Untersuchung des Themas als weiterführend.

\section{Literatur}

Beyers B (2009) Fährt das Auto der Zukunft elektrisch. Aachener Stiftung Kathy Beyes. http://www.faktor-x.info/ressourcen/elektromobilitaetbraugust-2009/ Abruf am 2009-11-24

Dudenhöffer F (2009) Interview Elektromobilität. E-World News Offizielle Messezeitung der E-World energy \& water 2009: 4-5. http:/ /www.e-world2010.com/tools/dateien/download.php?id=1065. Abruf am 2009-11-24

Graunitz B (2009) Batteriewechsel und Geschäftsmodell. Elektroniknet.de. http:/ /www.elektroniknet.de/home/better-place/n/d/die-in-car-softwareautos/. Abruf am 2009-11-24 


\title{
The context sensitive shopping list
}

\author{
Andreas Pirali, Niklas Klein, Sian Lun Lau, Klaus David \\ Chair for Communication Technology (ComTec), Universität Kassel
}

With the introduction of the digital world in all areas of life new appliances were also installed in cars. In the past, the radio and the cassette player were the main means for infotainment in a car. Today, the car navigation systems have taken over the dominant position of modern car infotainment. Current off-the-shelf navigation systems feature a satellite navigation device, media player, radio and TV reception. The idea to connect a car with the web is more than 10 years old (Lind et al. 1999), cars with a web connection are available meanwhile (Gafron 2008).

Another convergence that has taken place is the increasing usage of mobile phones in the car world. Hands-free sets allow mobile phone usage while driving, offering different ways of communication and more.

Nevertheless, all these exciting technologies and available functionalities are only first steps towards what will be possible in the future through the usage of a web connection in the car.

In co-operation with a major OEM (Original Equipment Manufacturer), our goal was to develop a social network application for cars based solely on web services. The application chosen, from a jointly developed overview of possibilities, was a social network enhanced, context sensitive shopping list.

Therefore the research question was how to transfer the concepts of web services and social networks to the automotive context. How could these concepts be integrated into a car that way that the driver has an added value?

We specified a set of requirements for the shopping list regarding accessibility, visualisation and interaction. The shopping list should have the following functionality: add and remove items from a shopping list from home and on the move, find appropriate shops for the items on the shopping list, locate these shops, locate the car and inform about shopping possibilities/needs while driving around.

Also the application should demand minimal attention from the driver. The implementation should focus on web services and re-use as many existing web services as possible to avoid reinventing the wheel.

Context awareness (Schilit 1994) became the key concept to automate functionalities as much as possible. To give location based recommendations while driving around is in principal not a new idea as there have been approaches like hybrid, context sensitive recommender systems (Brocco et al. 2008) and others. Our approach is to use web services which recommend Points of Interest (shops) based on the actual position of the car. The shops that should be searched are specified 
by the items on the list. The shopping list itself is part of a social network where the members manage the items on the list.

The demonstrator was presented to the public at the CeBit 2009 in Hannover and received positive feedback from the technical interested audience. The demonstrator was shown to more than 250 people. Overall the feedback from the polled persons was mostly unanimous (even from the sceptics) that this application gives us an impression of the things to come. So far it is not the case that everybody needs it, but for special user groups like families, it seems to be a good help and many asked persons are open for such an application.

\section{References}

Brocco M, Eigner R, Wörndl W (2008) Ein hybrides, kontextsensitives

Recommender System für mobile Anwendungen in vernetzten Fahrzeugen. In: Tagungsband der Multi-Konferenz Wirtschaftsinformatik 2008 - Teilkonferenz "Automotive Services", München.

Gafron S (2008) Fernsehen und Internet erobern das Auto.

http://www.welt.de/webwelt/article1911322/Fernsehen_und_Internet_erobe rn_das_Auto.html. Last viewed on 2009-07-28.

Lind R, Schumacher R, Reger R, Olney R, Yen H, Laur M, Freeman R (1999) The Network Vehicle-a glimpse into the future of mobile multi-media. In: IEEE Aerospace and Electronic Systems Magazine 14 (9): 27-32.

Schilit B N, Adams N L, Want R (1994) Context-Aware Computing Applications. In: Proceedings of the Workshop on Mobile Computing Systems and Applications. Santa Cruz, CA 
Betriebliches Umwelt- und

Nachhaltigkeitsmanagement 



\title{
Vorwort zur Teilkonferenz
}

\section{Betriebliches Umwelt- und Nachhaltigkeitsmanagement}

\author{
Jorge Marx Gómez', Frank Teuteberg², Burkhardt Funk³, Peter Niemeyer ${ }^{3}$ \\ 1 Abteilung Wirtschaftsinformatik I, Carl von Ossietzky Universität Oldenburg \\ ${ }^{2}$ Fachgebiet Unternehmensrechnung und Wirtschaftsinformatik, \\ Universität Osnabrück \\ 3Institut für elektronische Geschäftsprozesse, \\ Leuphana Universität Lüneburg,
}

Das betriebliche Nachhaltigkeitsmanagement umfasst die ökonomische, soziale und ökologische Dimension unternehmerischen Handelns sowie deren Wechselwirkungen untereinander. Der Einsatz von Informations- und Kommunikationstechnologien (IKT) ist dabei notwendige Voraussetzung für die erfolgreiche Etablierung in Unternehmen. IKT kann einerseits die informatorische Absicherung unternehmerischer Entscheidungen (z. B. durch Betriebliche Umweltinformationssysteme und Nachhaltigkeitsrechnungswesen) unterstützen und andererseits erst die Realisierung von Optimierungspotenzialen im Nachhaltigkeitsmanagement ermöglichen. Eine zentrale Fragestellung ist, wie und welche Erkenntnisse und Konzepte der Wirtschaftsinformatik hierfür genutzt werden können.

Der Track fokussiert die IKT-Unterstützung der ökonomischen, sozialen und ökologischen Dimensionen des betrieblichen Nachhaltigkeitsmanagements und erörtert Forschungsfragen, Perspektiven und State-of-the-art-Anwendungen in diesem Feld.

Das Ziel des Tracks „Betriebliches Umwelt- und Nachhaltigkeitsmanagement“ ist es, innovative Ansätze sowie Erfolg versprechende Vorgehensweisen und Best Practice vorzustellen, die von Experten aus verschiedenen Fachrichtungen entworfen wurden.

Nach einem sorgsam durchgeführten Begutachtungsverfahren haben sich vier Beiträge qualifiziert, die sich thematisch in folgende Schwerpunkte bündeln lassen:

- Den ersten thematischen Schwerpunkt bildet das Sustainable Supply Chain Management mit zwei Beiträgen (Teuteberg; Wittstruck)

- Die Ausgestaltung von Handelsplattformen für Sekundärrohstoffe (Kleine Stegemann) 
- Im dritten Schwerpunkt wird die allgemeine Ausrichtung und Weiterentwicklung von betrieblichen Umweltinformationssystemen untersucht (Junker).

Die hier ausgewählten Beiträge geben einen Einblick in die Vielfalt der Realisierungsmöglichkeiten und Herausforderungen im Bereich des betrieblichen Umweltund Nachhaltigkeitsmanagement, wobei sowohl die Reflexion bestehender Ansätze als auch mögliche zukünftige Entwicklungen berücksichtigt werden. Die einzelnen Beiträge verfolgen dabei verschiedene fachliche Perspektiven und wählen unterschiedliche methodische Zugänge bei der Untersuchung der verschiedenen Bereiche des Betrieblichen Umwelt- und Nachhaltigkeitsmanagement, wie empirischen Studien, systematischer Literaturrecherche oder die Konzeption einer Fallstudie.

Somit bieten die vier Beiträge eine gute Möglichkeit zur Reflexion und Gestaltung eines entsprechenden betrieblicher Umwelt- und Nachhaltigkeitsmanagement, sowohl für Wissenschaftler und Experten an Forschungseinrichtungen als auch für Fach- und Führungskräfte in der Praxis.

Abschließend sei den Mitgliedern im Programmkomitee für ihre engagierte Unterstützung sowie für ihre fundierte und konstruktive fachliche Einschätzung der eingereichten Beiträge herzlich gedankt. 


\title{
A Systematic Review of Sustainable Supply Chain Management
}

\section{What is there and what is missing?}

\author{
Frank Teuteberg, David Wittstruck \\ Accounting and Information Systems, University of Osnabrück
}

In recent years the topic of Sustainable Supply Chain Management (sSCM) has received growing attention and has become an increasingly popular research area. Today, companies must tackle multiple new challenges: they have to address the problem of rapid climate changes, face the negative impact of the financial crisis and the prices, deal with the growing public interest in ecology and ensure environmental sustainability and energy efficiency.

The objective of this paper is to determine the state of the art in sSCM research by using the research method of a systematic literature review. In line with Dibbern et al. (2004, p. 13), we use an analytical framework to analyze articles that deal with sSCM. Table 1 shows the framework of analysis applied in this paper.

Table 1: Framework of Analysis (cf. Dibbern et al. 2004)

\begin{tabular}{|l|l|l|l|}
\hline Phase & Stages & Research Question/Content & Section \\
\hline Motivation & 1. Why? & Why sSCM? & 4.1 \\
\hline $\begin{array}{l}\text { Scope and } \\
\text { Applied } \\
\text { Methods }\end{array}$ & 2. What? & What are the existing research perspectives on sSCM? & 4.2 \\
\cline { 2 - 4 } & 3. How? & Which research methods are applied in sSCM literature? & 4.3 \\
\hline Evaluation & 4. Outcome & Unsolved research problems & 4.4 \\
\cline { 2 - 4 } & 5. Implications & Trends in sSCM and implications for future research & 5 \\
\hline
\end{tabular}

The limitations of a systematic literature review lie in the paper selection process.

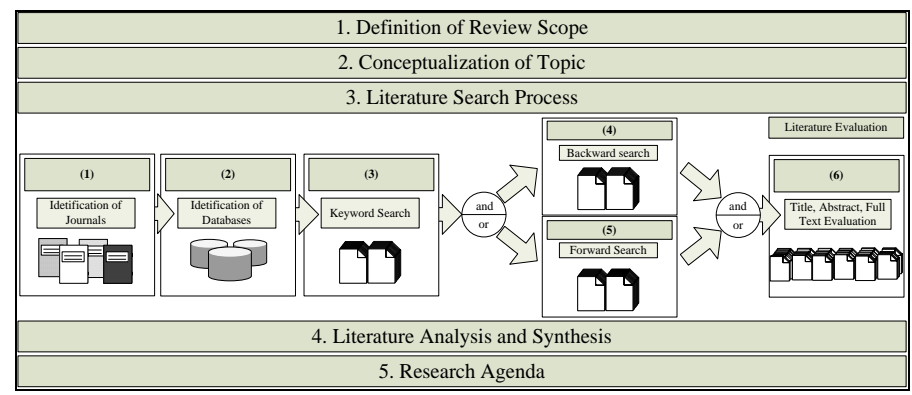

Figure 1: Research Process (vom Brocke et al. 2009, Webster and Watson 2002.) 
However, we tried to minimize this risk by following a proven course of action for the creation of a literature review (Fettke 2006, Webster and Watson 2002). The restriction of the source material to high-quality articles leads to reliable results about the state of the art in sSCM research. Figure 1 illustrates the individual steps of our research process.

The review results of the review show that since $2002 \mathrm{sSCM}$ has been analyzed particularly from an economic perspective. As far as economic aspects are concerned, researchers have to deal with one prime question: Can a sustainable supply chain be profitable? (cf. Heinelt 1999). This crucial problem entails further questions: How and with which key performance indicators can sSCM be measured? Which cause and effect relationships exist between sustainabilility and long-term financial success? The answers to these questions can provide important information about the monitoring and controlling of sSCM. For example, if there is information at hand about a sustainability level that increases the company value, key performance indicators can give important steering impulses to the management (Radke 1996). These complex questions require long-term analysis, since the impact of sSCM on financial key performance indicators may take years to become perceptible.

Acknowledgement: The authors are indebted to Ms Anja Grube and several anonymous reviewers for fruitful discussions and substantive comments relating to this article.

\section{References}

Dibbern J, Goles T, Hirschheim R, Jayatilaka B (2004) Information systems outsourcing: a survey and analysis of the literature. The DATA BASE for Advances in Information Systems 35(4):6-102.

Heinelt, H. (1999) Öko-Audits in deutschen Betrieben. Zeitschrift für Umweltpolitik \& Umweltrecht 22( 4):541-560.

Fettke P (2006) State-of-the-Art des State-of-the-Art - Eine Untersuchung der Forschungsmethode "Review" innerhalb der Wirtschaftsinformatik. Wirtschaftsinformatik 48(4):257-266.

Radke, V. (1996) Ökonomische Aspekte nachhaltiger Technologie. Zeitschrift für Umweltpolitik \& Umweltrecht 19( 1): 109-128.

Webster J, Watson R (2002) Analyzing the past to prepare for the future: writing a literature review. Management Information Systems Quarterly 26(2):13-23. 


\title{
Konzeption und Entwurf einer Handelsplattform für Sekundärrohstoffe
}

\author{
Stefan Kleine Stegemann, Burkhardt Funk \\ Institut für elektronische Geschäftsprozesse, \\ Leuphana Universität Lüneburg
}

Der Einsatz von Sekundärrohstoffen in der Produktion gewinnt angesichts steigender Rohstoffpreise, verschärfter Umweltrichtlinien und des in Deutschland ausgeprägten Umweltbewusstseins der Verbraucher an Bedeutung. Unternehmen verfolgen mit dem Einsatz von Sekundärrohstoffen in der Produktion sowohl die Senkung der Produktionskosten als auch der Umweltwirkung ihrer Produkte und Produktionsprozesse. Altmetalle, -papiere und -kunststoffe gehören dabei zu den am häufigsten recycelten Materialien.

Europaweit sind zahlreiche Unternehmen im Bereich des Recyclings tätig. Neben den produzierenden Unternehmen, die gleichzeitig Rohstoffkonsumenten und damit auch Nachfrager der gereinigten Sekundärrohstoffe sind, spielen Entsorgungsbetriebe, die die Zerlegung von Produkten übernehmen, eine wichtige Rolle. Sie sind meist regional orientiert (Kurbel und Schoof, 1998) und übernehmen die Aufgabe, Sekundärrohstoffe vorab zu sortieren und in größeren Mengen zu paketieren. Endverwerter sorgen schließlich für die Rückgewinnung des originären Rohstoffs oder nutzen die Sekundärrohstoffe direkt für ihre Produkte.

Der Grad der Recycling-Fähigkeit einzelner Materialien ist von verschiedenen Faktoren abhängig. Bellmann und Khare (2000) zählen dazu die Reinheit der zurückgewonnenen Materialien, deren monetären Wert, die Kosten für die Sortierung und Trennung, die Kosten für die Entsorgung verbleibender Abfälle, die Kosten für Sammlung und Transport sowie die Existenz und Funktionsfähigkeit von Sekundärrohstoffmärkten. Die daraus resultierenden Herausforderungen umfassen neben den Anforderungen an ein recyclingorientiertes Design von Produkten die Notwendigkeit zur organisierten Sammlung der zu recycelnden Materialien, um im betriebswirtschaftlichen Sinne kritische Massen zu erreichen und so die Kosten für Sammlung und Transport zu reduzieren. Hinzukommen weitere Transaktionskosten, die durch die Suche nach Abnehmern bzw. Lieferanten, die Verhandlung der Konditionen sowie die Abwicklung der Transaktion zustande kommen.

Intermediäre wie die ISR Interseroh Rohstoff GmbH (im folgenden ISR), die zu den führenden Unternehmen im Recycling von Altpapieren, -kunststoffen und holz gehört, verfolgen das Ziel diese Transaktionskosten zu reduzieren und fungieren so als marktschaffendes Bindeglied zwischen Entsorgungsbetrieben und Endverwertern. Elektronische Recyclingbörsen ermöglichen in diesem Zusammenhang Marktteilnehmern den effizienten Handel auch geringer Menge an Sekundärroh- 
stoffen. Die vorliegende Arbeit beschreibt die Anforderungen und den architekturgeleiteten Entwurf einer solchen elektronischen Recyclingbörse. Das Projekt wurde in Zusammenarbeit der ISR und der Leuphana Universität Lüneburg entwickelt und befindet sich nach einer Testphase nun unter http://www.interserohtrading.com in Produktion.

Die Arbeit bietet sowohl einen fachlichen als auch einen softwaretechnischen Beitrag. Fachlich handelt es sich um die erste Darstellung (in der wissenschaftlichen Literatur) einer Fallstudie zu den Anforderungen und insb. dem Entwurf einer in der Praxis eingesetzten Handelsplattform für Sekundärrohstoffe. Diese Aussage stützt sich auf eine systematische Schlagwortsuche mit Hilfe verschiedener Literaturdatenbanken (Google Scholar, scientificCommons, DBLP). Derzeit existieren in der wissenschaftlichen Literatur lediglich softwaretechnische Entwür$\mathrm{fe}$, die jedoch nicht in realen Systemen evaluiert wurden (siehe beispielsweise Beverungen et al., 2008, Tamura et al., 2008). Softwaretechnisch nutzen wir erstmals in einer in der Praxis eingesetzten Anwendung ein von uns in der Literatur beschriebenes und weiter entwickeltes Architekturmuster, das die Entwicklung von flexiblen Workflows in der Individualsoftwareentwicklung unterstützt (Kleine Stegemann et al., 2007).

Eine Analyse der bestehenden Recyclingbörsen zeigt, dass eine erfolgreiche Marktetablierung dieser bisher fehlgeschlagen ist. Selbst die führende europäische Börse Garwer wickelt mit 5-10 täglichen Transaktion nur einen verschwindend geringen Teil der in Europa anfallenden Recyclingmengen ab. Wir argumentieren, dass dies im Kern nicht in technischen Funktionslücken begründet ist, sondern in den Industriestrukturen und den daraus resultierenden geringen freien Mengen an nachgefragten Sekundärrohstoffen. Dies wiederum bedingt eine nur geringe Liquidität auf den Börsen und damit eine unzureichende Attraktivität für Marktteilnehmer. Im beschriebenen Projekt fungiert ISR als Marktmacher. Die Recyclingbörse konnte 2008 erfolgreich im Markt eingeführt warden. Die Anwendung wurde in 5 Sprachen übersetzt (deutsch, englisch, spanisch, französisch, italienisch) und wird in mehreren europäischen Ländern eingesetzt. Softwaretechnisch wurde das von uns entwickelte Framework, das auf dem Blackboard Architekturmuster beruht, erstmals in einer Praxisstudie eingesetzt. Die technische Umsetzung des Registrierungsprozesses aber insb. auch die nachgelagerte Umsetzung des Verhandlungsprozesses mit Hilfe des Frameworks konnte die in der Theorie erwartete Flexibilität der Architektur bestätigen. Hervorzuheben ist dabei insb. die Möglichkeit den Ablauf von Workflows - also einzelne Instanzen von Geschäftsprozessen - situativ durch den Anwender zu modifizieren.

\section{Literatur:}

Auf eine Angabe der detaillierten Quellen wurde in der Kurzfassung des Beitrages verzichtet, sie sind ausführlich in der Langfassung dargestellt. 


\title{
Sustainable Supply Chain Management in Recyclingnetzwerken der Elektro- und Elektronikindustrie
}

\author{
Eine empirische Studie zum Status Quo
}

\author{
David Wittstruck, Frank Teuteberg \\ Fachgebiet Unternehmensrechnung und Wirtschaftsinformatik, \\ Universität Osnabrück
}

Laut einer Studie von Greenpeace nimmt die Menge von Elektronikschrott Jahr für Jahr zu und beträgt weltweit derzeit zwischen 20 und 50 Millionen Tonnen pro Jahr (Greenpeace 2006, S. 1). Um eine mittel- bis langfristige Entsorgungssicherheit dieser Abfälle zu schaffen, schließen sich Unternehmen zunehmend zu überbetrieblichen Recyclingnetzwerken zusammen, die auf der Grundlage multilateraler Anbieter-Abnehmer-Kontrakte fungieren. Diesen Netzwerken stellen sich zahlreiche Herausforderungen wie z. B. eine Vielzahl von (neuen) regulatorischen und gesetzlichen Anforderungen zum Umweltschutz (WEEE, ElektroG) sowie Normen und Standards (z. B. Energy Star Computer Program). Das Sustainable Supply Chain Management (sSCM) erweitert traditionelle Konzepte des Supply Chain Managements (SCM) um umweltorientierte und soziale Aspekte (vgl. Abb. 1) und trägt damit den Forderungen nach mehr Nachhaltigkeit Rechnung (Carter und Rogers 2008; Seuring und Müller 2007).

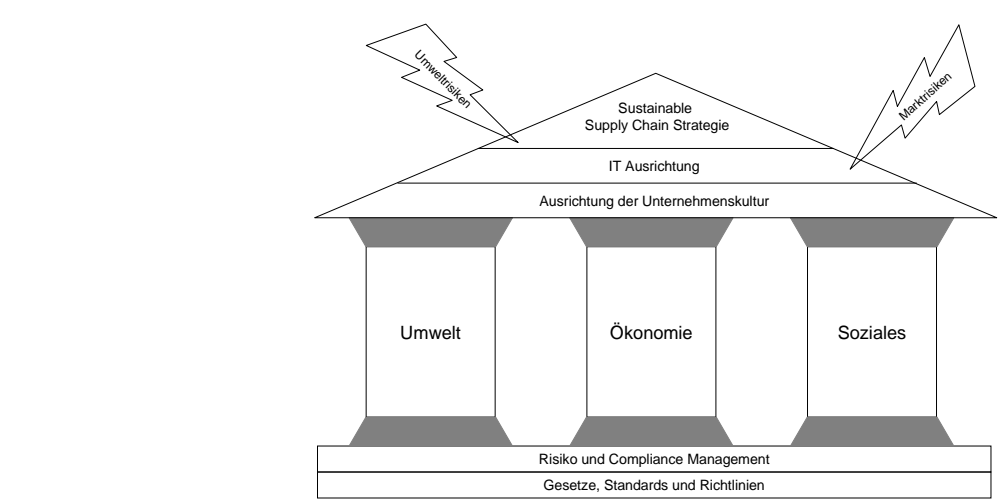

Abbildung 1: "House of sSCM"

Die in diesem Beitrag vorgestellte empirische Studie, die sich auf Recyclingnetzwerke der Elektro- und Elektronikindustrie konzentriert, verfolgt folgende Ziele: 
Erstens wird analysiert, inwieweit „Nachhaltigkeit“ schon fest in der Strategie der Netzwerke verankert ist und ob sich inner- und überbetrieblich bereits entsprechende Organisationseinheiten etabliert haben, die für ein sSCM verantwortlich sind. Zweitens wird erhoben, welche Standards und Referenzmodelle für das sSCM verwendet werden, welcher Nutzen (vgl. Abb. 2) aus dem sSCM gezogen werden kann und welcher sSCM-Reifegrad in den Unternehmen vorliegt. Drittens erhebt die vorliegende Studie, welche spezifischen Softwareprodukte für das sSCM eingesetzt werden und welche Funktionalitäten die Software aus Sicht der befragten Experten zur Verfügung stellen sollte. Ferner werden aus den Ergebnissen Implikationen für Wissenschaft und Praxis abgeleitet.

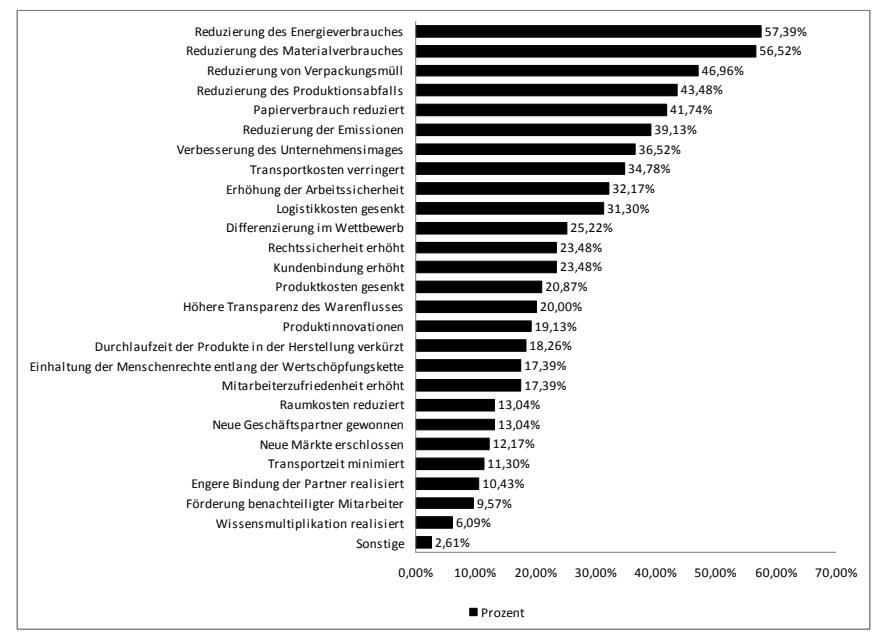

Abbildung 2: Realisierte Nutzeffekte durch das sSCM

Etwa die Hälfte der Befragten gab an, dass durch das sSCM der Verbrauch von Energie und Materialien gesenkt sowie der Produktions- und Verpackungsmüll reduziert werden konnte.

\section{Literatur}

Carter C, Rogers D (2008) A framework of sustainable supply chain management: moving toward new theory. International Journal of Physical Distribution and Logistics Management 38(5):56-68.

Greenpeace (2006) Elektronikschrott in China und Indien.

http://www.greenpeace.de/fileadmin/gpd/user_upload/themen/umweltgifte /greenpeace_studie_elektroschrott_dt_zusammenfassung.pdf. Abruf am 200909-29.

Seuring S, Müller M (2007) Core issues in sustainable supply chain management - a Delphi study. Business Strategy and the Environment 17(8):455-466. 


\title{
Betriebliche Umweltinformationssysteme (BUIS)
}

\author{
Horst Junker', Jorge Marx Gómez², Corinna V. Lang ${ }^{3}$ \\ ${ }^{1} I M B C$ GmbH, Berlin \\ 2 Abteilung Wirtschaftsinformatik I, Carl-von-Ossietzky-Universität Oldenburg \\ ${ }^{3}$ Fachbereich Wirtschaft, Hochschule Anhalt
}

Das Leitbild der nachhaltigen Entwicklung geht über die Betrachtung der umweltpolitischen Komponente hinaus und berührt unmittelbar ökonomische, ökologische und soziale Entwicklungsprozesse. (Deutscher Bundestag 1997, S. 11) Ihr Ziel ist die Realisierung eines Ressourcen sparenden technischen Fortschritts. Insbesondere durch die Realisierung einer Kreislaufwirtschaft (Vermeiden-VerwertenBeseitigen) und eines integrierten Umweltschutzes (Prozess-, Produktions- und Produktintegration) sind erste Möglichkeiten zur Ausgestaltung eines nachhaltigen Wirtschaftens in den Unternehmen gegeben. Dies führt zu einer nachhaltigen Unternehmensführung, was bedeutet, vermehrt auf interdisziplinäre Information und Wissen zurückzugreifen.

Eine bislang unternehmensintern isolierte Betrachtung und Verfolgung umweltrelevanter Ziele hat in der Praxis dazu geführt, dass die Durchführung von Umweltschutzaktivitäten weitgehend der operativen Managementebene zugewiesen wurde, während die ökonomischen Ziele im Interessenfokus des strategischen Managements verblieben. Die im Nachhaltigkeitsbegriff formulierten ökonomische und ökologische Dimensionen machen es erforderlich, dass neben den ökonomischen Kategorien auch der betriebliche Umweltschutz, die Beachtung der schädlichen Auswirkungen, die bei der Produktion von Gütern und Dienstleistungen entstehen, in den Fokus des Managementhandelns zu rücken haben.

Für nachhaltigkeitsorientierte Systeme sind neben den sich aus der Betriebswirtschaftslehre ergebenden Zielvorstellungen zusätzlich diejenigen zu integrieren, die sich aus dem betrieblichen Umweltmanagement ergeben. Somit ist das Zielsystem eines BUIS durch die Integration ökonomischer und ökologischer Teilziele geprägt. (Hilty und Rautenstrauch 1995, S. 296)

Es ist möglich, ein nachhaltigkeitsorientiertes Zielsystem zu konstruieren wie aus Gründen der Übersichtlichkeit und der Komplexitätsreduzierung am Beispiel der innerbetrieblichen Logistik eines Unternehmens gezeigt werden kann. (Lang 2007, 37ff)

Der hohe Abstraktionsgrad eines Gesamtzielsystems macht zwar die Auskunft, ob die genannten Einzelziele in komplementären, neutralen oder konfliktären Beziehungen zueinander stehen, schwierig, dennoch ist es erforderlich, jede einzelne Zielbeziehung innerhalb des Zielsystems zu bewerten und die sich daraus ergebenden Konsequenzen abzuschätzen. 
Eine erste Abschätzung zeigt auf, dass die Anzahl der komplementären und neutralen Zielbeziehungen die der konfliktären deutlich übertrifft - nur ca. 12\% der Zielbeziehungen sind konfliktär. Daraus lässt sich die Annahme ableiten, dass das Gesamtzielsystem einer nachhaltigkeitsorientierten innerbetrieblichen Logistik grundsätzlich realisierbar ist.

Die divergierenden Sichtweisen der ökonomischen und ökologischen Dimensionen führen zu unterschiedlichen Ausprägungen der jeweils realisierbaren Informationssysteme und damit zur Berechtigung der Existenz zweier unterschiedlich ausgeprägter Informationssysteme für die jeweiligen betrieblichen Funktionsbereiche.

Im Lichte der Ergebnisse der Nachhaltigkeitsdebatte zeigt sich, dass die traditionellen Definitionen von BUIS obsolet geworden sind - sie sind nicht länger „Stand der Kunst“. Die überkommenen Definitionen unterstützen die Umsetzung der operativen Zielvorstellungen und - damit verbunden - die Verfolgung isolierter, nicht aufeinander abgestimmter Einzelziele. Wie viele der in der Praxis eingesetzten Anwendungssysteme demonstrieren, führt diese Sichtweise zur Realisierung sog. Insellösungen, somit zu Softwaresystemen, die ohne jegliche Integrationseffekte unverbunden nebeneinander existieren.

Der mit dem Informationsmanagement verbundene ganzheitliche Zugang zur Gestaltung und Realisierung von Informationssystemen ist somit auch innerhalb der Betrieblichen Umweltinformatik zwingend erforderlich, weil es offenbar nur so gelingen kann, die bisherige operative Ausrichtung der BUIS zu überwinden.

\section{Literatur}

Deutscher Bundestag (Hrsg.) (1997): Konzept Nachhaltigkeit. Fundamente für die Gesellschaft von morgen; Zwischenbericht der Enquetekommission „Schutz des Menschen und der Umwelt - Ziele und Rahmenbedingungen einer nachhaltig zukunftsverträglichen Entwicklung“" des 13. Deutschen Bundestages. Bonn

Hilty L, Rautenstrauch C (1995) Betriebliche Umweltinformatik. In: Page, Hilty (Hrsg.): Betriebliche Umweltinformatik. München.

Lang C (2007) Konzeption eines Referenzmodells für betriebliche Umweltinformationssysteme im Bereich der innerbetrieblichen Logistik. Aachen. 
Business Intelligence 



\title{
Vorwort zur Teilkonferenz
}

\section{Business Intelligence}

\author{
Peter Chamoni', Peter Gluchowski², Hans-Georg Kemper³, Bodo Riegert \\ ${ }^{1}$ Mercator School of Management, \\ Lehrstubl für Wirtschaftsinformatik und Operations Research, \\ Universität Duisburg-Essen \\ ${ }^{2}$ Lebrstubl für Wirtschaftsinformatik, \\ insb. Systementwicklung und Anwendungssysteme, \\ Technische Universität Chemnitz. \\ ${ }^{3}$ Lehrstubl für ABWL und Wirtschaftsinformatik 1, \\ Universität Stuttgart
}

\section{${ }^{4}$ Lehrstubl BWL/Management Support und Wirtschaftsinformatik, Universität Osnabrück}

Business Intelligence (BI) beschäftigt sich mit der IT-basierten Entscheidungsunterstützung und fokussiert heute zunehmend prozessorientierte sowie unternehmensumfassende bzw. -übergreifende Ansätze. BI eröffnet somit neue Potenziale im Rahmen der Unternehmenssteuerung, konfrontiert die Wissenschaft und Praxis jedoch auch mit komplexen Herausforderungen bei der Konzeption, dem Aufbau und dem Betrieb anspruchsvoller Lösungen.

Ziel der Teilkonferenz ist es, originäre Arbeiten aus dem Themenfeld Business Intelligence (BI) vorzustellen und zu diskutieren. Hierbei stehen sowohl empirische als auch konstruktivistische Forschungsarbeiten zur BI-Organisation, BIArchitektur, BI-Methodik sowie zu BI-Anwendungsfeldern im Mittelpunkt. Insgesamt werden 9 Beiträge vorgestellt, die nach doppelt blinder Begutachtung aus insgesamt 24 Einreichungen ausgewählt werden konnten.

Das Themenspektrum erweist sich als breit gefächert. So finden sich Beiträge zum Aufbau einer Business Intelligence Governance, zur Mustererkennung im Semantic Web wie auch zu modellgetriebenen Decision Support Systemen. Eher der Systementwicklung sind die Beiträge zuzurechnen, die sich der Nutzung einer Model Driven Architecture oder der Event-orientierten Gestaltung beim Aufbau einer BI-Lösung widmen. Besonderes Gewicht scheint auch dem Datenqualitätsmanagement zuzukommen. Zwei Betrachtungen aus den Themenfeldern Stamm- 
datenmanagement und Customer Relationship Management belegen diese Relevanz. Mit gänzlich neuen Einsatzbereichen beschäftigen sich zwei Einreichungen zur Anwendung von BI im Rahmen der Unterstützung der Due Dilligence bei Mergers and Acquisitions sowie dem Einsatz von BI in kollaborativen Netzwerken. 


\title{
Ansätze für eine differenzierte Business Intelligence Governance
}

\section{Eine Konzeptentwicklung auf Basis einer Exploration}

\author{
Henning Baars, Tina-Müller Arnold, Hans-Georg Kemper \\ Lehrstubl für ABWL und Wirtschaftsinformatik. I, \\ Universität Stuttgart
}

Mit der wachsenden Komplexität, Relevanz und Größe integrierter Infrastrukturen für die Management- und Entscheidungsunterstützung (Business Intelligence, BI) nimmt auch der Bedarf für übergeordnete Leitungs- und Kontrollstrukturen (Governance) zu. Aufgrund der Spezifika von BI (besonders enge Verzahnung von fachlichen und technischen Aspekten, hohe Änderungsdynamik, fließender Übergang zwischen lokalen Änderungen im Systembetrieb und Entwicklungsprojekten mit infrastrukturellem Charakter, vgl. Moss und Shakru, 2005, S. 63-80) können existierende IT-Governance-Ansätze nicht unmittelbar übernommen werden. Es ist vielmehr eine eigene BI-Governance nötig - die auf der übergeordneten ITGovernance aufsetzt und diese zielgerichtet erweitert (Gutierrez 2008; Dinter et al. 2008, S. 253-254; Totok 2006, S. 63). Gegenstände einer solchen BI-Governance sind die Definition BI-spezifischer Rollen, Verantwortlichkeiten und Organisationseinheiten, die Gestaltung eines strategieadäquaten Portfolios von BI-Lösungen sowie BI-spezifische Richtlinien, Regelungen und Empfehlungen (Dinter 2008, S. 257-258; Gutierrez 2008).

Die hiermit verbundenen Herausforderungen und Lösungsansätze wurden im Rahmen einer explorativen Studie bei acht Unternehmen mit langjährigem BIEinsatz eruiert. Die Ergebnisse der Untersuchung verdeutlichen, dass es für größere BI-Installationen nicht sinnvoll ist, undifferenzierte Regelungsstrukturen aufzubauen, die unternehmensweit starr durchgesetzt werden. Zum einen sind ungewollte Regelungen im BI-Bereich politisch schwer durchsetzbar und zum anderen können diese auch besonders leicht umgegangen werden. Sowohl bei der Aufgabenabgrenzung und Rollendefinition, bei der Architekturgestaltung als auch bei der Ausgestaltung der zugehörigen Vorgehensmodelle sind ein Entgegenkommen auf die Fachabteilungen sowie eine flexible Ausgestaltung der GovernanceRahmenbedingungen vonnöten. Gleichzeitig bestätigt sich allerdings, dass auf eine übergreifende Governance keineswegs verzichtet werden kann und ein unkontrollierter Wildwuchs von Lösungen auf jeden Fall vermieden werden muss.

Auf Grundlage dieser Erkenntnisse wird ein Konzept für eine differenzierte BI Governance entwickelt, das die Ausgestaltung lokaler BI-Governance-Umgebungen vor- 
sieht, die jedoch zentral koordiniert sowie aus einer definierten Grundmenge an Regulierungsbausteinen (Governance-Modul-Set) zusammengestellt werden. Darüber hinaus ist die aufbauorganisatorische Trägerschaft für die BI-Governance zu verankern. Eine besondere Rolle kommt hierbei einem nahe an der Leitungsebene aufgehängten BI-Governance-Committee zu, das u.a. die Legitimation und Kommunikation übergreifender BI-Regulierungen fördert. Dieses Committee komplementiert ein zentrales BI Competence Center (BICC) (vgl. hierzu auch Miller et al., 2006 und Baars et al. 2009) sowie bedarfsweise dezentrale BI-Kompetenzteams, die an der Schnittstelle zum Anwender fachliche und technische Kompetenzen zusammenzubringen.

Weitere Forschungsbedarfe bestehen insbesondere noch bei der weiteren Konkretisierung des Ansatzes, bezüglich der Zusammenhänge zum IT- und BIService-Management, einer möglichen IT-Unterstützung sowie hinsichtlich der Entwicklung adäquater Kennzahlenkonzepte.

\section{Literatur}

Baars H, Zimmer M, Kemper HG (2009) The Business Intelligence Competence Centre as an Interface between IT and User Departments in Maintenance and Release Development. roceedings of The 17th European Conference on Information Systems (ECIS 2009), 08.-10.06.2009, Verona

Dinter B, Lahrmann G, Meyer D, Schmaltz M (2008) Governance in der Informationslogistik am Beispiel eines Energieversorgers. In: Dinter B, Winter R, Chamoni P, Gronau N, Turowski K (Hrsg.) Tagungsband der DW 2008: Synergien durch Integration und Informationslogistik. S. 249-266.

Gutierrez N (2008) Business Intelligence (BI) Governance, http:/ /www.businessintelligence.com/article.asp?id=170. Abgerufen am 30.10.2008.

Miller GJ, Bräutigam D, Gerlach SV (2006) Business intelligence competency centers: a team approach to maximizing competitive advantage. Wiley, Hoboken, USA.

Moss, ST, Shakru A (2005) Business Intelligence Roadmap - The Complete Project Lifecycle for Decision Support Applications. Wiley, Boston u.a., USA..

Totok A (2006) Entwicklung einer Business-Intelligence-Stragie. In: Chamoni P, Gluchowski P (Hrsg.) Analytische Informationssysteme, 3. Aufl. Springer, Berlin, Heidelberg, S. 51-70. 


\title{
Model Driven Architecture für Data Warehouses: Computer Aided Warehouse Engineering - CAWE
}

\author{
Christian Kurze, Peter Gluchowski \\ Lehrstubl für Wirtschaftsinformatik, \\ insb. Systementwicklung und Anwendungssysteme, \\ Technische Universität Chemnitr.
}

Die Model Driven Architecture (MDA) ist ein Standard der Object Management Group (2003) zur modellgetriebenen Software-Entwicklung. Modelle auf verschiedenen Abstraktionsebenen und automatisierte Transformationen zwischen Modellen setzen die Idee einer strikten Trennung von Spezifikation und Implementierung um. Als neuerer Ansatz im Kontext der MDA adressiert die Architecture Driven Modernization (ADM) die Modernisierung bzw. das Reengineering von Systemen und stellt formale Modelle für diesen Kontext bereit. ADM umfasst sowohl die fachliche als auch die technische Domäne von Systemen (Khusidman und Ulrich 2007, S. 1).

Data Warehouses stellen insbesondere Informationen zur Unterstützung strategischer und taktischer Entscheidungen bereit und verkörpern dadurch zentrale Komponenten moderner Entscheidungsunterstützungssysteme. In der letzten Dekade hat das Data Warehousing einen hohen Reifegrad erreicht und flächendeckende Akzeptanz gefunden. Datenschutz, Datensicherheit und Compliance stellen zunehmend höhere Anforderungen an die Entwicklung und Dokumentation von Data Warehouses sowie an das Metadatenmanagement. Zusätzlich ist eine Schwerpunkverschiebung der Initiativen auf die fachliche Seite - weg von der IT zu verzeichnen (Kimball et al. 2008, S. xxxi).

Diese Punkte stellen den Mehrwert einer modellgetriebenen Data WarehouseEntwicklung heraus: Eine detaillierte Systembeschreibung auf mehreren Abstraktionsebenen reduziert die Komplexität und dient als Zusammenstellung der Anforderungen an das System und damit einhergehend auch als Dokumentation für Endnutzer und Entwickler; gleichzeitig aber auch als solide Basis für die Implementierung des Zielsystems, da die Befolgung des MDA-Paradigmas die Transformation in ein reales System erlaubt.

Die aktuelle Literatur, die MDA auf die Entwicklung von Data Warehouses anwendet, berücksichtigt den ADM-Ansatz nur unzureichend. Dennoch erweist sich vor allem das Framework von Mazón und Trujillo (2008, S. 45) als ein belastbarer Ausgangspunkt für die Entwicklung von CAWE-Werkzeugen auf Basis des MDA-Paradigmas. Die Autoren zeigen in einer Fallstudie die praktische Umsetzbarkeit ihres Ansatzes, geben allerdings keine konkreten Hinweise zur Implementierung. Ähnlich der genannten Arbeit konzentriert sich der vorliegende Beitrag 
maßgeblich auf die Datenhaltungsschicht einer Data Warehouse-Lösung, speziell auf die Modellierung mehrdimensionaler Datenstrukturen sowie der Generierung von Datenbankschemata aus diesen Modellen. Dabei steht der ADM-Ansatz für ein tragfähiges Fundament zur Durchführung eines Reverse-Engineerings auf bestehenden Data Warehouse-Systemen - daraus resultieren Datenmodelle für bereits existente Systeme.

Das vorgestellte Werkzeug ist sowohl für die Wissenschaft als auch für Praktiker relevant. Es stellt eine Plattform für Forscher bereit, um aktuelle Methoden des Software-Engineerings auf die Spezifika des Data Warehousings anzupassen; Praktikern hilft es bei der Bewältigung von komplexen Fragestellung im Rahmen der (Weiter-) Entwicklung von Data Warehouse-Systemen.

Das vorliegende Paper präsentiert zunächst eine konzeptionelle Architektur für ein CAWE-Werkzeug. Sie integriert sowohl MDA als auch ADM. Bereits existierende Frameworks zum effizienten Umgang mit Modellen werden auf das Problem angewendet, um durch eine prototypische Implementierung die Umsetzbarkeit der Architektur zu zeigen. Als wissenschaftliche Vorgehensweise dient der von Peffers et al. (2008) vorgestellte Design Science-Prozess. Somit sind ein methodisch korrektes Vorgehen sowie eine nachvollziehbare Präsentation der Ergebnisse gewährleistet.

\section{Literatur}

Khusidman V, Ulrich W (2007) Architecture-Driven Modernization: Transforming the enterprise. http://www.omg.org/cgi-bin/doc?admtf/2007-12-01. Abruf am 2009-07-11.

Kimball R, Ross M, Thornthwaite W, Mundy J, Becker B (2008) The data warehouse lifecycle toolkit. Wiley, Indianapolis.

Mazón J, Trujillo J (2008) An MDA approach for the development of data warehouses. Decision Support Systems 45(1):41-58.

Object Management Group (2003) MDA Guide V1.0.1. http://www.omg.org/ cgi-bin/doc?omg/03-06-01. Abruf am 2009-07-24.

Peffers K, Tuunanen T, Rothenberger M, Chatterjee S (2008) A Design Science Research methodology for information systems research. Journal of Management Information Systems 24 (3):45-77. 


\title{
Business Intelligence zur Unterstützung der Due Diligence bei Mergers \& Acquisitions
}

\author{
Sandra Seiz, Nicole Jogsch, Bernd Jahnke, Stefan Ruf \\ Lehrstubl für Wirtschaftsinformatik, \\ Eberhard Karls Universität Tübingen
}

Mergers \& Acquisitions (M\&A), verstanden als Unternehmenskonzentrationen, stellen eine attraktive Option für Unternehmen dar, Wachstum zu generieren und Synergien zu realisieren. M\&A-Projekte zeichnen sich durch ihre große Bedeutung für die beteiligten Unternehmen, die damit verbundenen Risiken und Chancen sowie einen hohen Komplexitätsgrad aus. Empirische Studien gehen von Misserfolgsquoten bis zu 76\% aus. Besonders die Due Diligence (DD) als Prüfung des Zielunternehmens und die anschließende Integration werden als entscheidend für eine erfolgreiche Transaktion hervorgehoben. Ziel ist, die bisher getrennten Themenfelder DD und Business Intelligence (BI) zusammenzuführen, Spannungsfelder zu identifizieren und konzeptionelle Überlegungen zur Gestaltung adäquater BI-Lösungen anzustellen.

Im Rahmen der DD wird das Zielunternehmen intensiven Prüfungen unterzogen, wodurch bestehende Informationsasymmetrien abgebaut werden können. Dabei erheben Teams aus internen und externen Experten in kurzer Zeit eine große Menge qualitativer und quantitativer Daten, die sowohl aus dem Zielunternehmen als auch aus externen Quellen stammen und strukturiert sowie unstrukturiert vorliegen können. Die zügige und adäquate Speicherung, Auswertung, Interpretation und Repräsentation dieser Daten und die daraus resultierenden Ergebnisse sollen zeigen, ob die der Transaktion zugrunde liegenden Ziele erreicht werden können und bilden die Grundlage für die Entscheidung über deren Durchführung, für die Kaufpreisverhandlungen und für die Planung der Integration. Dabei ist die Berücksichtigung der Ergebnisse der individuellen Prüfungen sowie deren Zusammenfassung zu einer Gesamtsicht und der Abgleich mit den Akquisitionszielen von besonderer Bedeutung. BI-Lösungen können dabei helfen diesen Herausforderungen gerecht zu werden. Sie erlauben eine zeitnahe, schnelle, individuelle, auf die spezifische Problematik angepasste Auswertung und ermöglichen einen umfassenden Überblick aus unterschiedlichen Perspektiven.

Die konkrete Ausgestaltung entsprechender BI-Systeme bewegt sich aufgrund der Anforderungen der DD in verschiedenen Spannungsfeldern. Eines beschreibt die Dynamik des Datenbedarfs und die damit verbundene Flexibilität der Auswertung von Daten unterschiedlichen Typs. Durch den Abbau der Informationsasymmetrie bei der DD kann eine Strategieanpassung notwendig werden, die neuen Informationsbedarf generiert. Zu diesem Zweck eignet sich die Einrichtung 
anwendungsorientierter M\&A-Data-Marts, die alle für ein M\&A-Projekt relevanten Daten themenorientiert und bedarfsgerecht enthalten.

Ein weiteres Spannungsfeld wird durch die Detail- bzw. Gesamtsicht der Ergebnisauswertung charakterisiert, bei der sowohl die Auswertung strukturiert und unstrukturiert vorliegender Daten als auch die Berücksichtigung quantitativer und qualitativer Daten gewährleistet sein muss. Um die Review-Ergebnisse detailliert und aggregiert zu analysieren, ist daher neben einem Data Warehouse ein Document Warehouse einzusetzen, in dem qualitative und unstrukturierte Daten aus internen und externen multimedialen Quellen zusammengeführt und mit Text Mining und OLAP multidimensional ausgewertet werden können. Hieran schließt sich das Spannungsfeld der maßgeschneiderten aber zugleich schnellen Auswertung an, dem durch den Einsatz ausgewählter BI-Funktionalitäten wie DD-Reports und DD-Prognosen, Szenarioanalysen und Dashboards begegnet werden kann. So lässt sich die Analyse der von mehreren Personen erhobenen Daten individuell durchführen und Entscheidungsträgern zeitnah in adäquater Form bereitstellen.

Hierbei zeichnet sich das vierte Spannungsfeld der Vertraulichkeit der Daten (bei Nichtzustandekommen der Transaktion ist die Vernichtung bzw. die NichtWeiterverwertung der Daten erforderlich) einerseits und ihre Nutzung zur Integrationsvorbereitung andererseits ab. Diesem Dilemma kann durch Nutzung eines BIPortals mit differenzierten Rechten für diverse Anspruchsgruppen innerhalb des DD-Teams Rechnung getragen werden.

Alle genannten Problembereiche verdeutlichen die Notwendigkeit, die Gestaltung der BI-Unterstützung für die DD sowohl an den bereits vorhandenen Daten und Systemen als auch an der fallspezifischen Zielsetzung auszurichten. Gelingt dies, können BI-Lösungen die DD in der Pre-Merger-Phase erheblich unterstützen, indem sie Entscheidungsträgern einen gezielten Überblick über die Fülle der in den Reviews erhobenen Daten verschaffen. So geartete BI-Anpassungen dienen dann auch der Konkurrenz- und Marktbeobachtung und Sondierung geeigneter Akquisitionsobjekte im Hinblick auf weitere M\&A-Vorhaben. Aus den aufgezeigten Herausforderungen für ein DD-gerechtes BI-Konzept folgt weiterer Forschungsbedarf zur konkreten Gestaltung geeigneter DD-BI-Lösungen.

\section{Literatur}

Berens W, Brauner HU, Strauch J (2008) (Hrsg) Due Diligence bei Unternehmensakquisitionen, 5. Auflage. Schäffer-Poeschel, Stuttgart.

Gluchowski P, Gabriel R, Dittmar C (2008) Management Support Systeme und Business Intelligence. Computergestützte Informationssysteme für Fach- und Führungskräfte, 2., vollst. überarb. Auflage. Springer, Berlin. 


\title{
Measuring Master Data Quality
}

\section{Findings from an Expert Survey}

\author{
Boris Otto, Verena Ebner \\ Institute of Information Management, University of St. Gallen
}

Data quality management (DQM) plays a critical role in all kinds of organizations (Pipino et al. 2002). With data being the foundation for information it is one of the most important criteria for strategic business decisions. For the assessment of a company's data quality and to monitor the effectiveness of data quality initiatives, data quality (DQ) has to be measured. The research question addressed in this paper focuses on the progress of organizations regarding the measurement of data quality. That means: "do organizations already measure data quality?", "what and how do they measure?".

Master data (MD) stores and describes features of a company's core entities (e.g. customers, products) (Loshin 2008). Typically, MD is used across multiple business processes and stored in multiple application systems. Master data management (MDM) aims at creating an unambiguous understanding of a company's core entities (Smith \& McKeen 2003).

Data is defined of high quality if it has the ability to satisfy the requirements for its intended use in a specific situation, referred to as "fitness for use" (English 1999). The intended use is commonly described as a multi-dimensional construct consisting of a set of quality attributes, called data quality dimensions (e.g. accuracy, completeness) which are determined by the data consumer (Wang \& Strong 1996).

Considering data as a product, a distinction between the product with its requirements and the production process with its actors can be made (Wang 1998). Upon this differentiation three points of measurement can be identified: the product, i.e. the data stored within the data base ("measuring at the data base"), the production process ("data lifecycle"), and the connection between these elements ("impact on business processes"). The measuring characteristics applied for the product-part are the data quality dimensions as defined by Wang and Strong (1996). Key performance indicators (KPIs) like cycle time, status of lifecycle (e.g. completed, approved, deactivated) and process costing are used for the production process. The impact of the product onto the business processes can be measured as loss of quality, time and costs.

The purpose of the study at hand is descriptive. The sample size and selection does not support statistical representativeness or exploratory results. The study aims at gaining an overview on the progress of companies in measuring data quali- 
ty in the three components identified. For data collection, an online questionnaire addressed to 300 experts on MDM and DQ was used.

With 41 participants - a response rate of $15 \%$ - the study shows that there are companies already measuring DQ. It also states an unclear understanding of the relevant tasks and a need for a further definition.

Organizations measuring data quality are mainly focusing on the measurement of the data quality within the data base. The data lifecycle KPIs and even more the impact on product quality, costs and execution time are less important to most of the interviewees. The importance of measuring data quality at the origin of its problems, where mistakes can be identified before they arise right away, has to be stated.

Future research will focus on the development of a framework to implement a measurement system. The framework should assess the complete extent of data quality problems from its root causes to its impacts.

\section{References}

English, LP (1999) Improving Data Warehouse and Business Information Quality. John Wiley \& Sons, Inc., New York, NY.

Loshin, D (2008) Master Data Management. Elsevier Science \& Technology Books, Burlington, MA.

Pipino, LL, Lee, YW and Wang, RY (2002) Data Quality Assessment. Communications of the ACM 45 (4), 211-218.

Smith, HA and McKeen, JD (2003) Developments in Practice VIII: Enterprise Content Management. Communications of the Association for Information Systems 11, 647-659.

Wang, RY (1998) A Product Perspective on Total Data Quality Management. Communications of the ACM 41 (2), 58-65.

Wang, RY and Strong, DM (1996) Beyond Accuracy: What Data Quality Means to Data Consumers. Journal of Management Information Systems 12 (4), 5-34. 


\title{
Klassifizierung von Modellierungsfällen in modellgetriebenen Decision Support Systemen
}

\author{
Christian Schultewolter \\ Fachgebiet BWL, Management Support und Wirtschaftsinformatik, \\ Institut für Informationsmanagement und Unternehmensführung, \\ Universität Osnabrück.
}

Dieser Beitrag befasst sich mit modellgetriebenen Decision Support Systemen (MDSS) gemäß der Klassifizierung der Special Interest Group on Decision Support, Knowledge and Data Management Systems der Association for Information Systems (Turban et al. 2007, S. 119), die überwiegend mittels Spreadsheet Paketen umgesetzt und in jüngerer Zeit verstärkt zum Einsatz kommen.

Empirische Studien haben gezeigt, dass Spreadsheet-Modelle oft Fehler enthalten (Panko und Sprague 1998, S. 337-353; Powell et al. 2009, S. 128-138). Das Auftreten von Fehlern ist hierbei häufig auf eine logisch-falsche Modellierung zurückzuführen, die durch eine einschränkende, oft wenig intuitive Modellierungskomponente forciert wird.

Analog zur konzeptuellen Datenmodellierung könnte eine konzeptuelle Modellierungsebene für MDSS nicht nur Unabhängigkeit von Werkzeug-spezifischen Konzepten und Einschränkungen bieten, sondern auch eine natürlichere, fehlerfreiere Modellierung ermöglichen. Diese Ebene muss alle domänenspezifisch benötigten Modellierungsfälle vollständig abbilden können.

Hierzu wird eine generische Spezifikation vorgeschlagen, welche eine Klassifizierung der Beziehungstypen von MDSS ermöglicht. Zentrales Element der vorgeschlagenen Spezifikation ist die (formelmäßige) Beziehung zwischen Modellelementen innerhalb eines diskreten, multidimensionalen Raums. In SpreadsheetSystemen sind dies typischerweise Modellzellen. Zur Klassifizierung der an einer Modellbeziehung beteiligten Dimensionen werden die Kriterien Grad, Homogenität und Simultanität vorgeschlagen (in Anlehnung an Rieger 1993, S. 136). Die Kombination der binär ausgestalteten Kriterien führt zu acht Modellierungsfällen. Abbildung 1 stellt diesen Zusammenhang schematisch dar. 


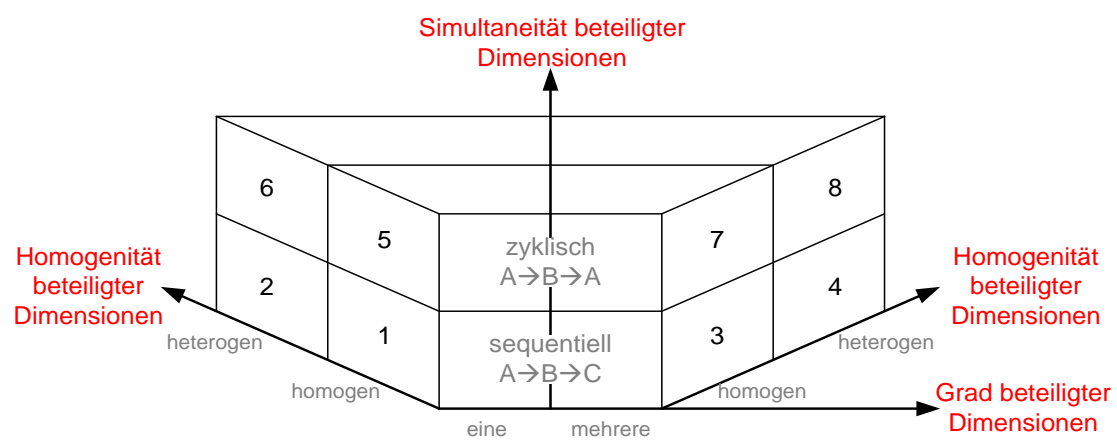

\section{Abbildung 1: Vorgeschlagene Klassifizierungskriterien}

Grad beschreibt die Anzahl der an einer Beziehung beteiligten Dimensionen, Homogenität gibt an, ob Intervalle bzw. Formelalternativen zur Abbildung eines logischen Zusammenhangs notwendig sind und Simultanität kennzeichnet zyklische Formeln, die in einer gesonderten Rechnung aufgelöst werden müssen.

Der Artikel beschreibt jeden Modellierungsfall anhand eines betriebswirtschaftlichen Beispiels und stellt die Beziehungen mittels eines Ursache-WirkungsDiagramms graphisch dar. Ferner wird insbesondere auf die Konsequenzen dieser Art Modellierung für die Gewährleistung einer korrekten prozeduralen Berechnungsreihenfolge der Modellelemente eingegangen.

Aufbauend auf diesen Kriterien ist die Entwicklung einer konzeptuellen Modellierungssprache geplant, mittels derer eine intuitive und nicht-prozedurale Modellierung betriebswirtschaftlicher Zusammenhänge möglich ist.

\section{Literatur}

Panko R, Sprague R Jr (1998) Hitting the wall: errors in developing and code inspecting a 'simple' spreadsheet model. Decision Support Systems 22(4): 337353.

Powell S, Baker K, Lawson B (2008) A critical review of the literature on spreadsheet errors. Decision Support Systems 46(1): 128-138.

Rieger B (1993) Der rechnergestützte Arbeitsplatz für Führungskräfte, Habilitationsschrift. Technische Universität Berlin.

Turban E, Aronson J, Ting-Peng L, Sharda R (2007) Decision Support and Business Intelligence Systems. Pearson, New-Jersey. 


\title{
Root causes affecting data quality in CRM
}

\author{
Wolfgang Leußer, Klaus D. Wilde \\ Chair of Business Informatics, \\ Catholic University of Eichstaett-Ingolstadt
}

An important field of application of business intelligence tools is in Customer Relationship Management (CRM). Especially analyses of a firm's customers' behavior and believes are a prerequisite to develop and realize innovative concepts to initiate and tighten customer relationships. So the quality of available customer data is a decisive factor for companies applying this customer focused strategy. Data quality problems often hinder the implementation and cause major negative consequences (Redman 1996, pp. 6-7). To address this problem from a quality management point of view a systematic management, based on a definition of data quality and specification of requirements for the quality along the different dimensions of data quality, a continuous quality measurement, and processes to find improvement measures for identified and prioritized problems should be implemented (Wang 1998, pp. 61-65). A root cause analysis is necessary to identify useful and effective improvement measures.

In the research area of service management Parasuraman et al. (1985, pp. 4446) conceptualized a model of service quality identifying gaps responsible for the perceived quality, known as gap model. Based on this approach we developed a gap model for data quality (see Figure 1). By applying this model we derived a list of responsible causes for the respective gaps in the context of CRM. The basis for the evaluation constituted a survey, whose respondents were users of data in CRM. Four groups of causes for data quality problems have been identified by applying an exploratory factor analysis: Design and operation of business processes and IT systems, implementation and application of data quality management, adequacy of data capture and obsolescence of data. A $\chi^{2}$ test for independence between the proposed hypotheses and the data quality level lead to the conclusion, that a dependency with the level of data quality as assessed by data users of the first two groups can be assumed, for the last two hypotheses this has been refused.

More advanced sources of DQ problems, like the items of our first two hypotheses, may have more relevance for the companies taking part in our study. Trivial easy-to-cure causes, like data capture and also obsolescence of data may be under control. In addition it can be argued, that the obsolescence of data is an inherent attribute of data. Hence measures can only be taken against the aftermath, but not positioned on the root cause. 


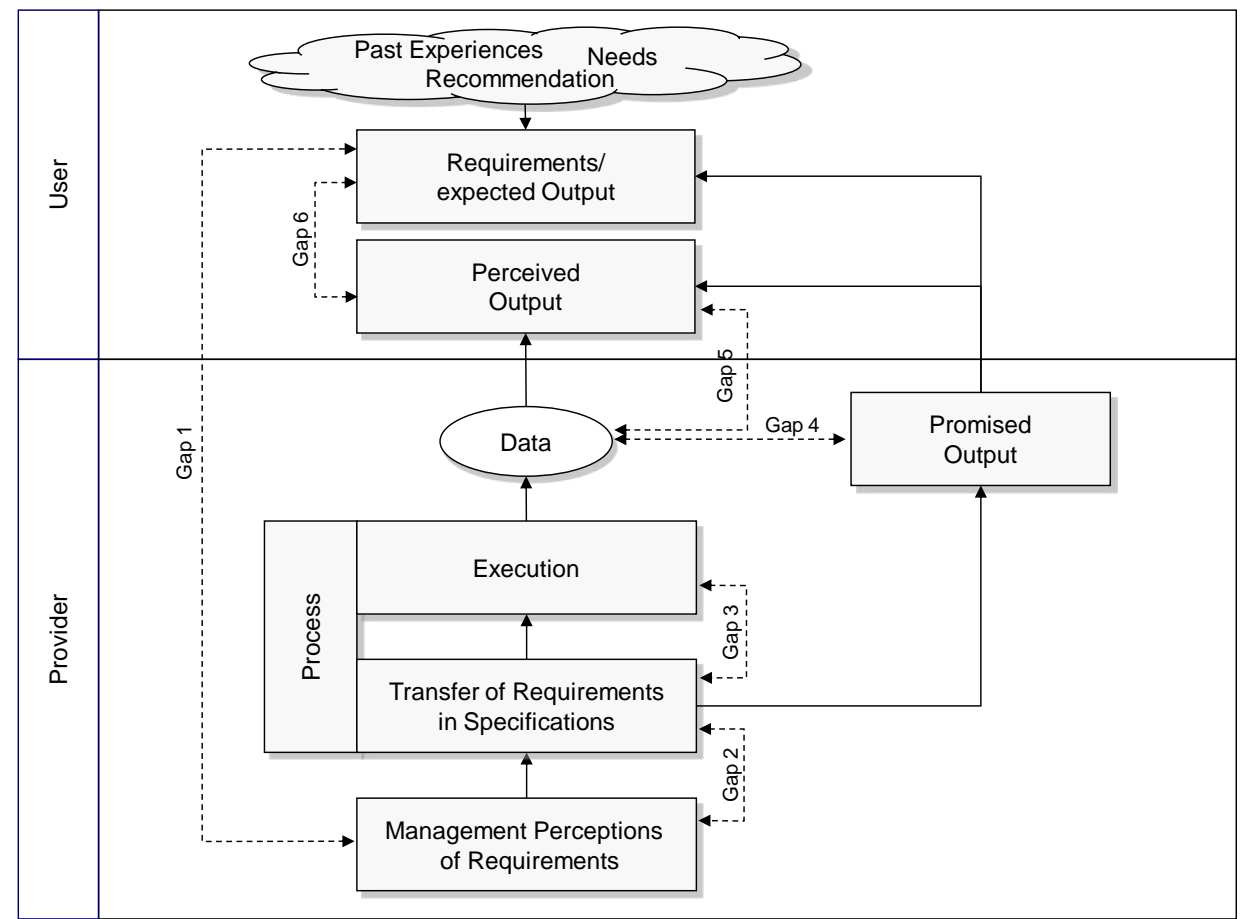

Figure 1: Gap model for Data

The proposed model intends to support practitioners during root cause analysis. An analysis of the reasons along the described gaps for a specific data quality problem can help in specifying efficient and effective improvement measures. The specification of a combined mix of reactive and proactive measures (Heinrich 2007, pp. 546-550) will be supported by the model described beforehand. Consequently, the next step will be to use the model proposed in this article to analyze the existing improvement measures to lever data quality at the identified points.

\section{References}

Heinrich B (2007) Der effiziente Einsatz proaktiver und reaktiver

Datenqualitätsmaßnahmen. Die Betriebswirtschaft 67(76):539-562.

Parasuraman A, Zeithaml VA, Berry LL (1985) A conceptual model of service quality and its implications for future research. J Mark 49(4):41-50.

Redman TC (1996) Data quality for the information age. Artech House, Norwoord.

Wang, RY (1998) A product perspective on total data quality management. Comm ACM 41(2):58-65. 


\title{
Towards a Service-Oriented Architecture for Operational BI-A Framework for Rule-Model Composition
}

\author{
Josef Schiefer ${ }^{1}$, Andreas Seufert ${ }^{2}$ \\ ${ }^{1}$ UC4 Senactive Software GmbH, Wolfsgraben (Österreich) \\ 2Institut für Business Intelligence, Steinbeis Hochschule Berlin
}

The information revolution is sweeping through our economy. Dramatic reductions in the cost of obtaining, processing, and transmitting information are changing the way we do business. Information technology is transforming the nature of products, processes, companies, industries and even the competition itself. As a consequence new approaches have to be put into action for decisionmaking, too (Clark et al. 2007; Davenport and Harris 2007). One of the key challenges is to move decision making applications (Business Intelligence) out of the back room and embeds it into business, intertwining it with operational processes and applications that drive current daily decisions. In essence, Operational Business Intelligence merges analytical and operational processes into a unified whole (Eckerson 2007; Van der Aalst 2004).

This paper introduces the rule management of the CEP system SARI (Sense And Respond Infrastructure). SARI is able to process large amounts of events from a business environment, and provides functions to monitor, steer and optimize business processes in real time. In SARI, business situations and exceptions are modeled with sense and respond rules which have been designed to be created and modified by business users. SARI offers a user-friendly modeling interface for event-triggered rules, which allows to model rules by breaking them down into simple, understandable elements.

The main contribution of this paper lies in a new model for constructing rules with a correlation model and a graph for representing business situations with a combination of event conditions and event patterns. Sense and respond rules offer a new way for business users to define and manage the response to typical patterns of business events. A key advantage of sense and respond rules is that they allow to graphically model a comprehensive set of event conditions and event patterns without using nested or complex expressions. In contrast, SQL-based system for querying event streams require technical knowledge for specifying and changing event queries which makes them difficult to use for a wider range of users.

Sense and respond rules flexibly combine rule triggers which can be used to define IF-THEN-ELSE decisions. Event conditions and event cases can be checked for a true or false evaluation, and facilitates the implementation of "oth- 
erwise" situations. Complex decision scenarios are displayed with a decision graph which can delegate the decision-making to pattern models.

By describing correlation and event pattern aspects in a separate model, the definition of event conditions and patterns are simplified. Correlation sets capture the relationships between events and can also be defined with a graphical model. A separate event pattern model allows capturing the detection of business situations. The advantage of modeling and reusing event patterns are:

Event patterns can be used parameterized and reused in various types of rules

Rule complexity is reduced by breaking down large rule models into smaller rule parts

Clean separation of event pattern matching logic and the binding logic of event actions.

These characteristics of modeling rules are essential when building rule sets for large industry solutions. Rule sets for industry solutions must be highly configurable and reusable for a wide range of business problems.

The service-oriented event processing system of SARI allows to flexibly link a rule service, processing sense and respond rules with other services. Event services are executed in parallel and controlled by the system. Services can be used to prepare the data for the rule processing as well as to process the response events generated by the rule service. The input and output for sense and respond rules are events which are delivered and processed by event services. Sense and respond rules, therefore, allow an easy integration within a service-oriented system environment.

\section{References}

Clark T, Jones M, Armstrong C (2007) The Dynamic Structure of Management Support Systems. Theory, Development, Research Focus and Directions. MIS Quarterly Vol. 31 No. 3, 603-607.

Davenport TH, Harris JG (2007) Competing on Analytics. The New Science of Winning, Boston.

Eckerson W (2007), Best Practices in Operational BI. Converging Analytical and Operational Processes. TDWI Reserarch.

Grigori D, Casati F, Castellanos M, Daysal U, Sayal M, Shan MC (2004) Business Process Intelligence. In: Computers in Industry, 53, S. 321-343.

Van der Aalst W (2004) Business Alignment. Using Process Mining as a Tool for Delta Analysis. In: Proceedings of the 5th Workshop on Business Process Modelling, Development and Support (BPMDS), Riga. 


\title{
Towards Knowledge Discovery in the Semantic Web
}

\author{
Thomas Fischer, Johannes Rubland \\ Department of Information Systems, \\ Friedrich-Schiller-University Jena
}

In the past, data mining and machine learning research has developed various techniques to learn on data and to extract patterns from data to support decision makers in various tasks, such as customer profiling, targeted marketing, store layout, and fraud detection. Furthermore, the Web has the potential for a high impact on competitive actions and competitive dynamics of enterprises. Therefore, enterprises should utilize this information. However, the growing amount of these distributed information leads to increasing information saturation. In this context, it becomes more and more important to detect useful patterns in the Web, thus use it as a rich source for data mining in addition to company internal databases.

The extraction of information and interesting patterns out of the Web is a complex task, because the current Web is mainly utilized for human consumption. Markup languages like XHTML are not sufficient to let software agents"understand" the information they are processing. Due to this, the discovery of useful patterns in such unstructured information is very difficult and has been addressed by research on web mining (Stumme et al., 2006). However, there have been increasing efforts in the research community to realize the vision of the so called Semantic Web: "The Semantic Web is not a separate Web but an extension of the current one, in which information is given well-defined meaning, better enabling computers and people to work in cooperation" (Berners-Lee et al., 2001).

It seems to be valuable to perform data mining on information with a welldefined meaning to improve the knowledge discovery process, as we will motivate in this paper. Unfortunately, the traditional data mining techniques are only able to process data that is in the form of a propositional representation (Raedt, 2008). This means a representation by a single table with an attribute-value structure. This is not suitable for the relational representation of information in the semantic web. The limitations of traditional algorithms are addressed by relational learning and relational data mining algorithms that are based on inductive logic programming techniques (ILP) (Stumme et al., 2006). Such algorithms have been successfully applied in different scenarios such as genetics, molecules, social network analysis, as well as natural language processing (Dzeroski, 2001). 
However, the utilization of relational data mining based on semantic web information for business intelligence has got not much attendance in the research community and there are a lot of open topics that have to be addressed. In context of the semantic web there are important differences to the traditional knowledge discovery process. Especially the selection, modelling and transformation steps are different to the standard approach. Data from data warehouses, databases as well as the Web has to be transformed into the logical formalisms of the semantic web (see Figure 1). Then the knowledge discovery process selects parts of this semantic data to fully utilize information and background information derived from a variety of sources. This relational data can improve the expressivity and amount of patterns that can be found in the subsequent relational data mining step.

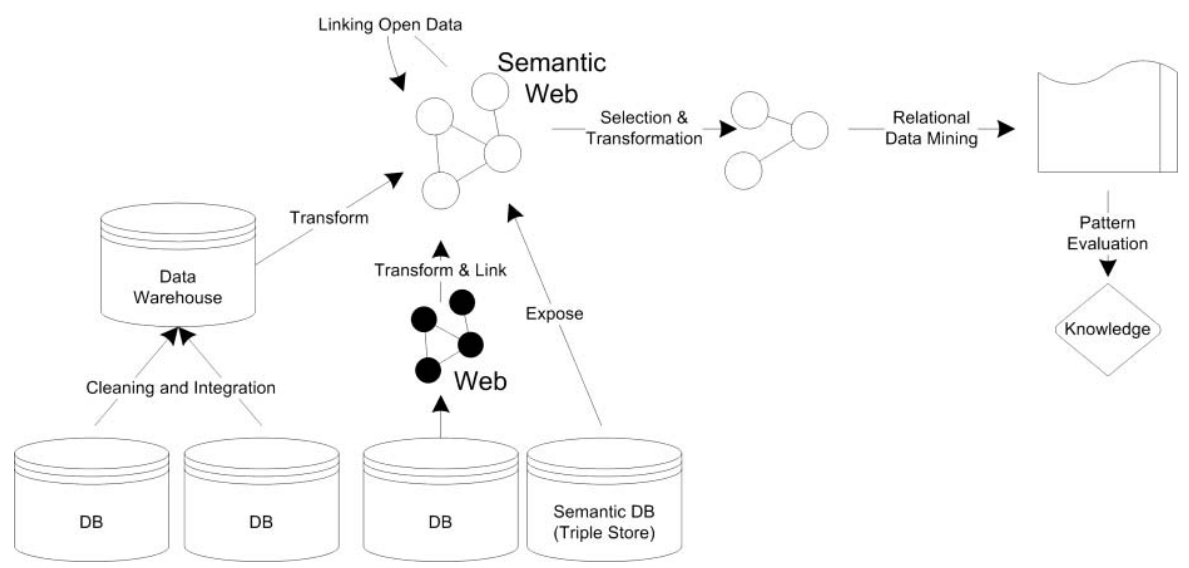

Figure 1: Knowledge Discovery in the Semantic Web

\section{References}

Berners-Lee T, Hendler J, Lassila O (2001) The semantic web. Scientific American: 29-37.

Dzeroski S (2003) Multi-relational Data Mining: An Introduction. SIGKDD Explorations, 5(1):1-16.

Raedt LD (2008) Logical and Relational Learning (Cognitive Technologies). Springer, Berlin.

Stumme G, Hotho A, Berendt B (2006) Semantic Web Mining -State of the Art and Future Directions. Journal of Web Semantics, 4(2):124-143. 


\title{
Business Intelligence in Collaborative Networks
}

\section{Towards New Services for Moderation Management}

\author{
Heiko Thimm¹, Karsten Boye Rasmussen² \\ 1 School of Engineering, Pforz̧heim University \\ ${ }^{2}$ Department Marketing and Management, \\ University of Southern Denmark, Odense
}

Implementation of the concepts of networking and collaboration have been promoted as a successful approach when dealing with the present business challenges especially for small and medium sized enterprises (SMEs). As a result more and more collaborative networks (Camarinha-Matos and Afsarmanesh 2005, pp. 439452; Grandori and Soda 1995, pp. 183-214) are emerging in which companies come together to jointly act on the market. For given business opportunities this means that following a selection process a created subset of the network members forms a temporary virtual enterprise to transform the opportunity into profit which implies a set of collaborative business processes that are to be coordinated. Collaborative networks must be supported by an appropriate IT infrastructure that offers suitable operational services in order to provide value for business partners. These computer-mediated services will include special information and decision support services for effective moderation of the collaboration within the network by a human moderator.

Moderation management in collaborative networks can be broadly described as the set of strategic and operative tasks that are carried out by a human moderator in order to meet the objectives of the network. As management tasks in single companies are supported by dedicated IT applications, also moderation management in collaborative networks requires from the IT infrastructure dedicated services. In our research framework we investigate especially the operative level of the decision making practice in collaborative networks with the goal to invent and experiment with new services for moderators and the network members.

We present two of such new services: 1) decision support of moderators, and 2) transparency support of the moderator and the network members. Both services support the concept of Business Intelligence (BI) in the sense that they are based upon complex data analyses performed on a comprehensive data repository. The processes, however, are not drawing upon the usual BI building blocks such as a data warehouse or a processor for Online Analytical Operations (OLAP).

Our decision support service enables moderators to effectively perform the selection decisions for the forming of virtual enterprises (Thimm et al. 2008). The 
service provides the moderator with the possibility to start from an initial search profile for a virtual enterprise followed by the generation of a ranked set of configuration alternatives. By performing several iterations with modified search profiles the problem space is explored and more and more assurance about the choice of the best fitting virtual enterprise is gained and thus support for the moderator's final decision is accumulated. The search criteria of the search profile apart from company related criteria also contain criteria that refer to the network as a whole. These criteria are especially useful when addressing the more long term strategic goals and policies of the network such as an equal revenue distribution between the participating companies within the network.

Our transparency support service allows for an automated completion of the moderator's communication task. When a proposed virtual enterprise configuration alternative is chosen from the result list of the decision support service the moderator can instruct the transparency support service to generate and distribute the data rich decision explanations within the network. These explanations contain comprehensive background information about the decisions including information about the anticipated impact of the decision on the network and the companies. The explanations not only inform about the rationale for each selection decision they also contain data that is useful for benchmarking as well as for the strategic alignment of the company to the performance of the network.

It is our assumption that the decision support service and transparency support service will through their BI capabilities ease decision making for moderators and lead to better and less subjective decisions regarding the configuration of virtual enterprises. These results will contribute to a good reputation of network moderators and also an open and vital collaboration climate which are both important factors for the long term success of collaborative networks.

\section{References}

Camarinha-Matos LM, Afsarmanesh H (2005) Collaborative networks: a new scientific discipline. Journal of Intelligent Manufacturing (16):439-452.

Grandori A, Soda G (1995) Inter-firm Networks: Antecedents, Mechanisms and Forms. Organization Studies 16(2):183-214.

Thimm H, Thimm K, Rasmussen KB (2008) Supporting Moderators of Company Networks by an Optimization Service for Orchestration. In: Proceedings 16th European Conf. Information Systems (ECIS 2008), Galway, Ireland. 


\section{E-Commerce und E-Business}





\title{
Vorwort zur Teilkonferenz
}

\section{E-Commerce und E-Business}

\author{
Christian Huemer', Katja Duckeke', \\ Ulrike Lechner ${ }^{3}$, Katarina Stanoevska-Slabeva ${ }^{4}$
}

${ }^{1}$ Institut für Softwaretechnik und Interaktive Systeme, TU Wien

${ }^{2}$ Lehrstubl für Wirtschaftsinformatik I, Universität Hohenheim

3Institut für Angewandte Informatik, Universität der Bundeswebr München

${ }^{4}$ Institut für Medien und Kommunikationsmanagement, Universität St. Gallen

Als Leitungsgremium der Fachgruppe 5.5 Electronic Commerce (WI-EC) der Gesellschaft für Informatik heißen wir Sie zu unserem Track e-Commerce und eBusiness der MKWI 2010 herzlich willkommen. In unserem Track zielen wir auf die Unterstützung des elektronischen Geschäftsverkehrs und die Durchführung von Transaktionen über globale Netze über unterschiedliche Endgeräte in einem weltweit wachsenden Markt ab. Dabei sind sowohl ökonomische, technische, rechtliche als auch benutzerorientierte Aspekten des e-Commerce und e-Business von hoher Relevanz.

Obwohl der Zeitpunkt der Einreichung für unseren Track im Jahre 2009 durch eine Konjunkturabschwächung gekennzeichnet war und die Phase des eCommerce Hype auch schon überschritten ist, sind wir froh festzustellen, dass unsere Community durchaus noch sehr aktiv ist und die Forschung im Bereich des e-Commerce und e-Business weiter voran schreitet. Dies wird durch die hohe Anzahl von 26 Einreichungen zu unserem Track untermauert. Jede dieser 26 Einreichungen wurde von mindestens drei Mitgliedern des Programmkomitees begutachtet. Entsprechend den Ergebnissen des Begutachtungsprozesses waren wir als Track Chairs in der Lage 12 Beiträge für die Präsentation in vier Sessions der MKWI zu akzeptieren. Die akzeptierten Beiträge befassen sich mit den folgenden Teilbereichen des e-Commerce und e-Business gemäß dem Call for Papers:

- Kommunikation im e-Commerce und e-Business

- E-Commerce und e-Business Geschäftsmodelle

- Social Networks und Emotions in e-Business und Collaborative Business

- E-Commerce und e-Business Transaktionen

- E-Commerce und e-Business Architekturen

- Geschäftsprozesse im e-Commerce und e-Business

- Business Services 
Der Erfolg jeder wissenschaftlichen Konferenz und ihrer Tracks hängt in hohem Maße von der Einsatzbereitschaft vieler involvierter Freiwilliger ab. Wir sind dankbar, dass wir auf den Einsatz vieler Kollegen und Kolleginnen im Programmkomitee zählen konnten. Da die Anzahl an Einreichungen von Beiträgen in der Verlängerungsfrist unsere Erwartungen bei weitem übertraf, konnten wir uns glücklich schätzen, dass manche Kollegen und Kolleginnen auch kurzfristig bereit waren unserem Programmkomitee beizutreten. Aus der Verlängerung der Einreichfrist und der Neuformation des Programmkomitees resultierte auch eine sehr knapp bemessene Frist für die Begutachtungen. Trotzdem waren - wie in ganz seltenen Fällen - bis zum Ende der Begutachtungsfrist für jeden der Beiträge drei Gutachten eingegangen. Dafür möchten wir uns bei jedem einzelnen Mitglied unseres Programmkomitees herzlich bedanken:

- Prof. Dr. Rainer Alt, Universität Leipzig

- Prof. Dr. Ulrike Baumöl, Fernuniversität Hagen

- Prof. Dr. Martin Bichler, TU München

- Prof. Dr. Jürgen Dorn, TU Wien

- Dr. Hans-Georg Fill, Universität Wien

- Dr. Birgit Hofreiter, Universität Liechtenstein

- Volker Hoyer, SAP AG

- Prof. Dr. Sabine Köszegi, TU Wien

- Dr. Christine Legner, European Business School Wiesbaden

- Prof. Dr. Jan-Marco Leimeister, Universität Kassel

- Prof. Dr. Susanne Leist, Universität Regensburg

- Prof. Dr. Jan Mendling, HU Berlin

- Prof. Dr. Dirk Neumann, Universität Freiburg

- Prof. Dr. Michael Rebstock, Hochschule Darmstadt

- Prof. Dr. Mareike Schoop, Universität Hohenheim

- Prof. Dr. Petra Schubert, Copenhagen Business School

- Prof. Dr. Rudolf Vetschera, Universität Wien

- Prof. Dr. Hannes Werthner, TU Wien

- Dr. Andreas Wombacher, University of Twente

- Prof. Dr. Hans-Dieter Zimmermann, FHS St. Gallen

Abschließend gilt unser Dank auch den Organisatoren der MKWI 2010, die es uns ermöglichen diesen hoffentlich für alle Teilnehmer und Teilnehmerinnen interessanten und herausfordernden e-Commerce und e-Business Track abzuhalten. Ein besonderes Lob möchten wir Arne Frerichs aussprechen, der uns über das gewöhnliche Ausmaß hinaus bei den organisatorischen Belangen unseres Tracks unterstützt hat. 


\title{
Web 2.0 driven Open Innovation Networks
}

\section{A Social Network Approach to Support the Innovation Context within Companies}

\author{
Michael Reinhardt', René Frieß², Georg Groh², Martin Wiener'1, \\ Michael Amberg ${ }^{1}$ \\ ${ }^{1}$ Lebrstubl für BWL, insh. Wirtschaftsinformatik III, \\ Friedrich-Alexander University Erlangen-Nürnberg \\ ${ }^{2}$ Lehrstubl für Angewandte Informatik - Kooperative Systeme, \\ Technische Universität München
}

Nowadays, learning to generate, refine, and develop ideas in an open or semi-open manner towards commercially valuable innovations becomes more and more crucial for companies to succeed in their markets. Hence, trying to raise flexibility and to leverage external know-how, companies increasingly opened their innovation management in recent times, integrating external partners and customers into their innovation networks and value creation (Chesbrough 2003, von Hippel 2005, Reichwald \& Piller 2006). Nevertheless, one important group of potential innovators has been quite neglected in research and practice so far, namely the employees of a company. This paper describes a social network-based platform prototype, which seeks to integrate all employees of a company into an open innovation (OI) network. Given the lack of prior research on IT support for OI within companies and the potentials arising from recent social software concepts and applications, a design science approach is used (Hevner et al. 2004).

This research is targeted towards the development of discontinuous innovations, which show specific characteristics and requirements: There is not only one initial, but rather a recurring idea generation (Rice et al. 1998). There are often "[...] no clear rules [...] high tolerance of ambiguity [...] fuzzy, emergent selection environment $[\ldots]$ operating patterns emergent and "fuzzy" [...] weak ties and peripheral vision important" (Phillips et al. 2006, p.181). All in all, a higher degree of context sensitivity and context dependency can be stated. This context - manifesting in shared stories, experiences, enterprise culture, personalities and informal networks - has high influence on the project success (Rice et al. 1998).

Thus, the consideration of the social context and social software paradigms, especially Social Networking Services (SNS), offer huge potential for open innovation systems, which aim at supporting the development of discontinuous innovations within a company. Richter and Koch (2008) developed a model to illustrate 
six basic functionality groups in SNS-applications, which are also represented in our application: Identity management, contact management, (expert) search, context awareness, network awareness, common exchange. Each functionality group is addressed by customized tools and functionalities, which overall shall contribute to leverage the mutual understanding and collaboration abilities, when co-developing innovations. The described system enables the interactive, collaborative, but geographically distributed cooperation of multidisciplinary actors.

The paper at hands deals especially with the IT-based context support, which proved to be highly relevant for the creativity and development phases of an idea. For these aspects activities and results with regard to the platform development are described. Over the whole project duration of 19 months (state August 2009), several in-depth interviews, project meetings, internal research and concept workshops, tool and training workshops as well as (partially virtual) test and development sessions, were conducted. These helped to increase the reliability of the findings and enhanced the quality and performance of the prototype.

This approach shows how open innovation management within companies can profit from supporting the social context of innovations, using customized Web 2.0 social networking services. Thus the presented work addresses both companies and researchers, as it enables an interactive and open innovation management, offering an integrative and flexible web-based platform.

\section{References}

Chesbrough HW (2003) Open Innovation: The New Imperative for Creating and Profiting from Technology. McGraw-Hill, Boston.

Hevner AR, March ST, Park J (2004) Design Science in Information Systems Research. MIS Quarterly 28(1):75-105.

Phillips W, Noke H, Bessant J, Lamming R (2006) Beyond the Steady State: Managing Discontinuous Product and Process Innovation. International Journal of Innovation Management 10(2):175-196.

Reichwald R, Piller F (2006) Interaktive Wertschöpfung. Open Innovation, Individualisierung und neue Formen der Arbeitsteilung. Gabler, Wiesbaden.

Rice MP, O'Connor GC, Peters LS, Morone JG (1998) Managing discontinuous innovation. Research Technology Management 41(3):52-58.

Richter A, Koch M (2008) Funktionen von Social-Networking-Diensten. In:

Proceedings Multikonferenz Wirtschaftsinformatik 2008, Teilkonferenz

Kooperationssysteme.

von Hippel E (2005) Democratizing Innovation. MIT, Cambridge. 


\title{
Akzeptanzfähigkeit von Tagging-Systemen in benutzergenerierten virtuellen Welten
}

\author{
Danny Pannicke1, Michael Habne $e^{3}$, Nicolas Neubauer², Bartek Ochab², \\ Renate Lieb33, Martin Meister ${ }^{3}$, Klaus Obermayer'2, Werner Rammert ${ }^{3}$, \\ Rüdiger Zarnekow ${ }^{1}$ \\ ${ }^{1}$ Fachgebiet IuK-Management, \\ ${ }^{2}$ Fachgebiet Neuronale Informationsverarbeitung, \\ ${ }^{3}$ Zentrum Technik und Gesellschaft, \\ (alle) Technische Universität Berlin
}

Benutzergenerierte virtuelle Welten haben in den vergangenen Jahren große Aufmerksamkeit erfahren. Dennoch stellt die Navigation und Orientierung gerade für unerfahrene Nutzer eine große Hürde dar. Eine analoge Problematik stand auch am Anfang der Verbreitung des WWW. Die dezentral erschaffenen Inhalte mussten zunächst systematisiert und klassifiziert werden. Diese Aufgabe wurde vor allem von Suchmaschinen und auf dem Prinzip der Sozialen Navigation basierenden Social Bookmarking Diensten übernommen. Folgt man der Analogie zwischen dem WWW und benutzergenerierten virtuellen Welten, stellt sich die Frage: Wie kann dem Benutzer in einer unkoordiniert wachsenden Fülle von Inhalten das Auffinden von für ihn relevanten Inhalten erleichtert werden? Der vorliegende Beitrag geht dabei der Forschungsfrage nach, inwieweit Tagging-Systeme in benutzergenerierten virtuellen Welten akzeptanzfähig sind.

Zur Beantwortung dieser Frage wurde auf Basis strukturationstheoretischer Überlegungen ein methodisches Vorgehen gewählt, das sowohl die Gegebenheiten der benutzergenerierten virtuellen Welt (Implementierungsumgebung „Second Life") als auch die Erfahrungen mit bestehenden Tagging-Systemen (Analogieumgebung) berücksichtigt. Der Grundgedanke besteht dabei darin, dass die Akzeptanzfähigkeit einer neuen Technologie umso größer ist, je mehr Praxiselemente der Analogie- und Implementierungsumgebung übertragbar sind (Abbildung 1). Dem Konzept der Technology-in-Practice nach Orlikowski (2000, S. 409f) folgend werden drei Arten von Praxis-Elementen unterschieden: Fazilitäten (Funktionen und Eigenschaften der Technologie), Normen (nach denen Handlungen von anderen bewertet werden) und Interpretationsschemata (im Sinne von Bedeutungszuschreibungen). In einem weiteren Schritt wurden Second Life Benutzer mit einem funktionsfähigen Prototyp einer Tagging-Anwendung konfrontiert. 


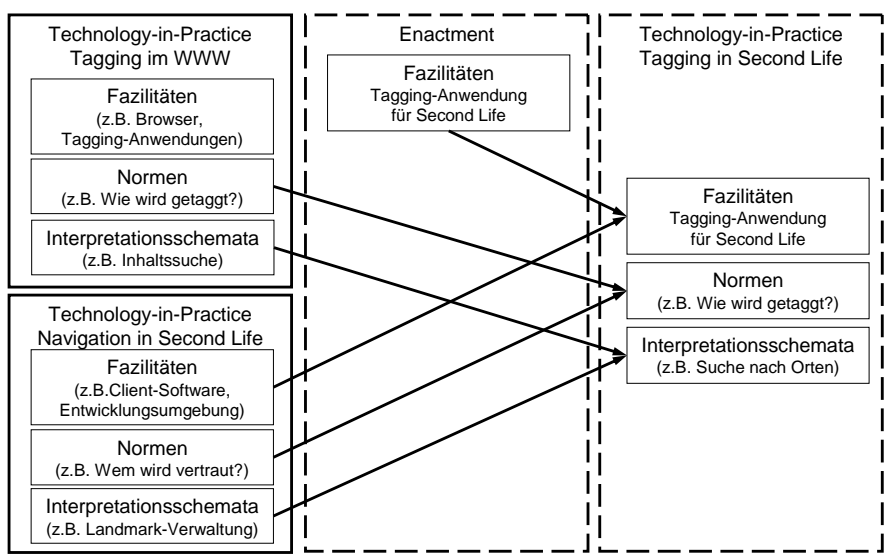

\section{Abbildung 1: Tagging-Systeme in Analogie- und Implementierungsumgebung}

Im Ergebnis zeigen sich sowohl Argumente für eine erfolgreiche Übertragung von Tagging-Systemen als auch Restriktionen und Hürden für eine solche Übertragung. Die Interpretationsschemata erwiesen sich dabei als überwiegend kompatibel zu den Analogieumgebungen. Im Hinblick auf Normen ergab die Untersuchung verschiedene Anforderungen an das Design eines entsprechenden Tagging-Systems. Wichtige Aspekte waren hier die Reputation des taggenden Benutzers und der notwendige Schutz der Privatheit. Hinsichtlich der derzeit vorhandenen Fazilitäten in „Second Life“ konnte festgestellt werden, dass diese aus der Sicht der befragten Probanden keine ausreichende Unterstützung bei der Navigation und Orientierung bieten, so dass durchaus ein technologischer Unterstützungsbedarf besteht. In der Konfrontation potenzieller Benutzer mit dem Prototyp zeigten sich die Grenzen der in „Second Life“ verfügbaren technischen Mittel. Der Grad an Einfachheit und Effizienz der Tagging-Anwendung blieb hinter den Erwartungen der Probanden zurück. Ein Redesign des Prototyps, das die im Rahmen dieser Studie gewonnenen Erkenntnisse umsetzt, könnte weiteren Aufschluss über die Akzeptanzfähigkeit von Tagging-Systemen im Kontext benutzergenerierter virtueller Welten geben.

\section{Literatur}

Orlikowski W J (2000) Using Technology and Constituting Structures: A Practice Lens for Studying Technology in Organizations. Organization Science, 11(4): 404-428. 


\title{
Multiple Issue Negotiation Based on Standardized Documents
}

\author{
A Communication Framework \\ Christoph Pflügler \\ Inter-organizational Systems Group, University of Augsburg
}

An evolving approach to conduct business transactions over B2B electronic marketplaces is to temporarily connect the ERP systems of the involved business partners. For this type of $\mathrm{B} 2 \mathrm{~B}$ integration, standardized processes, interfaces and business documents as provided by, e.g., RosettaNet ${ }^{1}$ or the NES Consortium ${ }^{2}$ constitute an indispensable requirement. However, the respective standards have significant deficits regarding the negotiation of agreements and are often little more than an electronic catalogue from which a customer can select and order goods. This may be appropriate to many scenarios, but other ones certainly require more sophisticated mechanisms to computationally negotiate, e.g., price, quality and delivery terms of a purchase order (Turowski 2002).

The work at hand closes that gap by providing a novel XML communication framework for multiple issue negotiation which incorporates standardized business documents and allows for interaction between human as well as software agents. As Först et al. (2009) have shown in an empirical case study that argumentationbased negotiation produces significantly better results regarding social welfare as well as number of communication units and steps than negotiation based on the exchange of simple proposals, the framework presented by the work at hand also allows for the use of arguments.

After elaborating on requirements for negotiation mechanisms by literature review, the core components of the proposed communication framework are presented: The structure of a so-called negotiation extension for pre-existing business document definitions and a bilateral negotiation process defining required tasks, the control flow of each participant, as well as the resulting message exchanges. An exemplary incorporation of the negotiation extension into a pre-existing XML document definition is depicted. Concrete application scenarios, such as the integration of the framework into the RosettaNet or the NES standards, are outlined.

\footnotetext{
1 http://www.rosettanet.org/

2 http://www.nesubl.eu/
} 


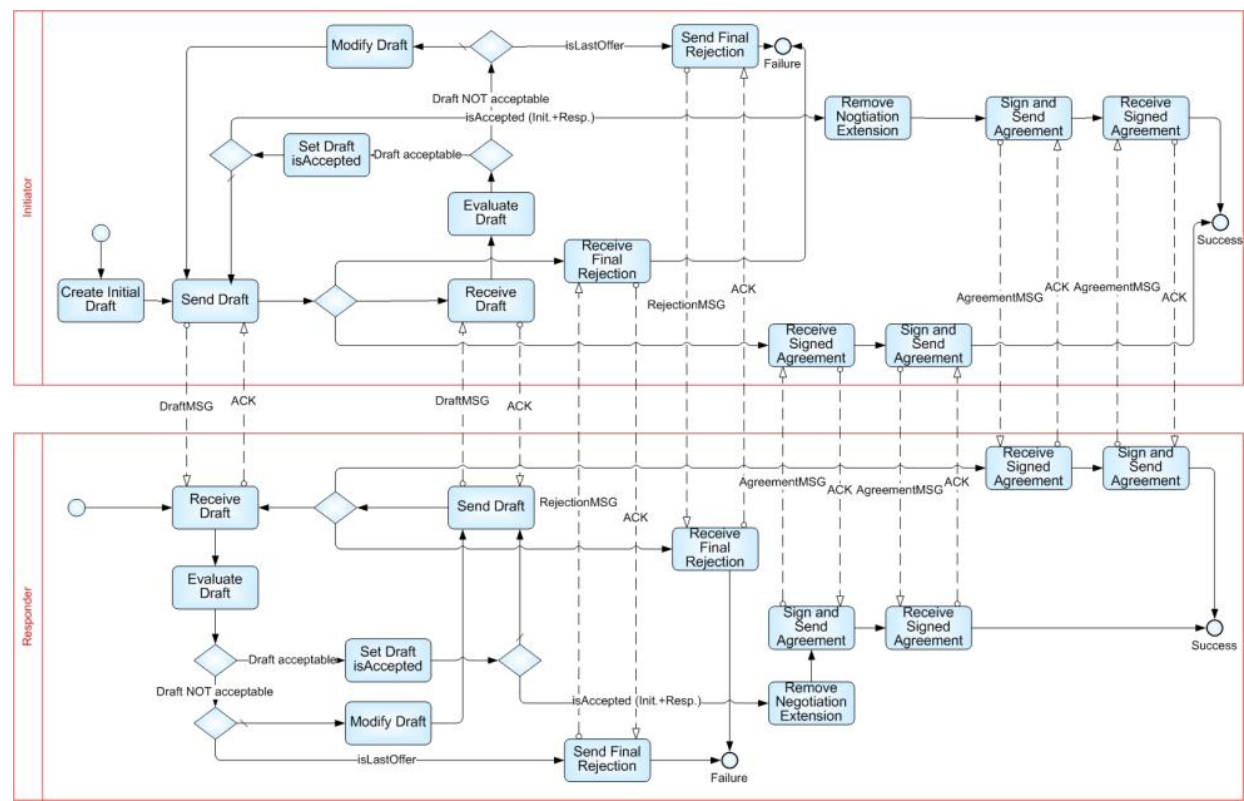

Figure 1: Proposal-counter-proposal negotiation process modelled in BPMN

In a future extension of this work, well-defined sets of arguments for key application domains need to be developed in order to facilitate the development of software agents that incorporate the argumentation-based approach. The design of respective agents that can cope with the complexity of real-world e-commerce scenarios constitutes another crucial aspect that needs further attention.

\section{References}

Först A, Rettinger A, Nickles M (2009) Argumentation- vs. proposal-based negotiation: an empirical case study on the basis of game-theoretic solution concepts. In: Argumentation in multi-agent systems: fifth international workshop. Revised selected and invited papers. Estoril.

Turowski K (2002) Agent-based e-commerce in case of mass customization. International Journal of Production Economics 75(1-2):69-81. 


\title{
Flexible Business Processes - Evaluation of Current Approaches
}

\author{
Thomas Burkhart, Peter Loos \\ Institute for Information Systems (IWi) at the \\ German Research Center for Artificial Intelligence (DFKI), \\ Universität des Saarlandes
}

The way people work, especially in group-based or creative processes, is getting more and more unpredictable. In order to fulfill these flexible business situations, there is a need for the use of support systems. However, classical systems do not offer the necessary agility to support today's rapidly changing or generally unpredictable workflows due to their static runtime execution. Thus, modern systems have the need to provide a flexible, but process aware execution at runtime that do not force users into a pre-defined way of working. Additionally, they must still support users during execution like classical systems already do (Herbst 2004, p. 4).

To be able to compare and classify current approaches in this paper, the background of this field of research has to be defined, with a focus on the term flexibility and business processes. In the sense of business, flexibility could be projected on the ability to change or alter the way things are handled without facing serious complexity issues. In terms of a business process, flexibility consists of an extrinsic trigger for a change process and secondly of an intrinsic process adaption itself (Rosemann and Recker 2006). Thus, business process flexibility is the capability to yield to externally triggered changes by modifying the process accordingly. However, change may only concern actually affected parts of a process, which can only be achieved by an appropriate structure regarding the problems and tasks faced by companies (Regev and Wegmann 2005). The underlying processes can be distinguished in ad-hoc processes (flexible processes), semi-structured processes (somewhere inbetween) and structured processes (classical processes) (Huth and Nastansky 2000).

According to prior research papers (Schwarz et al. 2001; Wargitsch 1998), the examined systems were not appropriate for supporting flexible workflows. Thus, this paper evaluates the state-of-the-art of current systems based on the viewpoint which requirements have already been achieved and for which requirements technical concepts are still to be developed. Therefore, a framework with six criterions is derived that allows a structural evaluation of current approaches and technologies that are selected and reviewed based on an extensive literature research. The first criterion Implicit Flexibility refers to the three process classes defined above: adhoc, semi structured and structured processes. Degree of Freedom measures the rigidity of a solution in leading the user along a pre-defined path. Late Modeling describes 
the extent of late modeling features offered by a system. Late Binding classifies the extent of late binding features offered by a system. Support measures a system's capabilities in supporting the user in his execution of certain processes. Finally, Adaptability evaluates the features of a system to enact changes on predefined process models in case of exceptional or unexpected circumstances.

The comparison of the classified systems clearly shows a polarization of processes with high support and those with a high implicit flexibility. Another extreme point of view is taken by the ad-hoc process support. They naturally have to overcome a lack of support as users might not always know which course of action to choose. However, there are some interesting approaches to overcome this lack of support. As the technology reviews show, there is a wide range of different focuses among the systems; some aim at supporting creative or fastchanging processes in smaller enterprises, whereas others offer a full spectrum of business process management features for larger companies.

According to these observations, one important conclusion is the fact that there is no "perfect" or "almighty" approach for flexible processes. As a result, future researches have to be focused on combining particular functionalities of the different approaches to gain a more widely applicable technology that integrates the features in a seamless and easy-to-use way. Finally, such a system might be able to yield flexible process support in a number of business scenarios.

\section{References}

Herbst J (2004): Ein induktiver Ansatz zur Akquisition und Adaption von Workflow-Modellen. Berlin : Tenea Verlag.

Huth C, Nastansky L (2000): GroupProcess: Partizipatives, verteiltes Design und simultane Ausführung von Ad hoc Geschäftsprozessen.

Regev G, Wegmann A (2005) A regulation-based view on business process and supporting system flexibility. In: Proceedings of the CAiSE'05 workshops.

Rosemann M, Recker J (2006) Context-aware process design: exploring the extrinsic drivers for process flexibility. In: 18th International conference on advanced information systems engineering.

Schwarz S, Abecker A, Maus H, Sintek M (2001) Anforderungen an die WorkflowUnterstützung für wissensintensive Geschäftsprozesse. In: WM'2001 -

Workshop "Geschäftsprozessorientiertes Wissensmanagement", Baden-Baden.

Wargitsch C (1998): Ein Beitrag zur Integration von Workflow- und Wissensmanagement unter besonderer Berücksichtigung komplexer Geschäftsprozesse. 


\title{
Normalo? Tüftler? Profi?
}

\section{Eine Typologisierung von Innovationswettbewerben}

\author{
Stefan H. Hallerstede, Anne-Katrin Neyer, \\ Angelika C. Bullinger, Kathrin M. Möslein \\ Lebrstubl für Wirtschaftsinformatik I, \\ Universität Erlangen-Nürnberg
}

Im vergangenen Jahrzehnt gewannen Online-Innovationswettbewerbe in Forschung und Praxis zunehmend an Beliebtheit. Dennoch fehlt ein tiefgreifendes Verständnis ihrer Funktionsweise, was einen gezielten Einsatz dieses Instruments erschwert (Haller et al. 2009). Ziel dieses Beitrags ist es daher festzustellen, ob sich Innovationswettbewerbe in Abhängigkeit von den jeweilig verwendeten Gestaltungsparametern typologisieren lassen und wenn ja, wie.

Ein Innovationswettbewerb wird als ein internetbasierter Wettbewerb verstanden, bei dem Teilnehmer ihre Fähigkeiten, Erfahrungen und Kreativität nutzen, um ein vorgegebenes Problem zu lösen. Er kann alle Phasen des Innovationsprozesses (Ideengenerierung, Konzeptentwicklung, Auswahl und Implementierung) abdecken. Wir arbeiten mit den zehn Gestaltungsmerkmalen von Innovationswettbewerben, welche Haller et al. (2009) aus der Literatur ableiten, um zehn etablierte Innovationsintermedäre (Atizo, Battle of Concepts, Brainfloor, CrowdSpirit, Fellowforce, Innocentive, Innovation Exchange, NineSigma, TekScout und Yet2) in linearanalytischen Fallstudien zu analysieren.

Insgesamt lassen sich drei Typen von Innovationswettbewerben in den erhobenen Daten identifizieren: Typ 1: Innovationswettbewerbe für Normalos. Die Themenstellungen sind offen und erfordern kein besonderes Fachwissen für die Lösung. Sie können somit von einer breiten Masse an Innovatoren beantwortet werden, da sie sich mit Fragen des täglichen Lebens beschäftigen. Die Teilnehmer sind meist interessierte Laien. Der Ausarbeitungsgrad und somit der benötigte Zeitbedarf für die Erstellung eines Beitrags bleibt gering. Auffällig ist, dass in diesem Typ nur geringe monetäre Anreize geboten werden. Nicht-monetäre Strukturen, die vor allem intrinsische Motive ansprechen, sind hingegen stark vertreten. Typ 2: Innovationswettbewerbe für Tüftler. Die Themenbereiche sind allgemein gehalten, erfordern aber eine genauere Auseinandersetzung mit der Materie als im Typ 1. Der Zeitbedarf für die Erstellung der Konzepte ist mittel, da zwar Überlegungen in die Entwicklung einfließen, aber keine praktische Umsetzung erfolgen muss. Motivationstechnisch werden in diesem Typ zwei verschiedene Modelle eingesetzt: Während sich Innovation Exchange an das Anreizsystem des dritten Typs anlehnt (hohe mone- 
täre Vergütung; wenige nicht-monetäre Anreize; Details siehe unten) und eher professionelle Akteure anspricht, nutzen Battle of Concepts und Fellowforce die Ausprägung des ersten Typs (niedrige monetäre Vergütung; starke nicht-monetäre Anreize) und sprechen eher Innovatoren mit mittlerem Fachwissen an. Typ 3: Innovationswettbewerbe für Profis. Die Themenstellungen sind sehr spezifisch und erfordern ein hohes Fachwissen in dem jeweiligen Bereich. Dementsprechend stammen die Beiträge hier vor allem von Unternehmen oder Fachleuten. Folglich entsteht ein hoher Zeitbedarf, um einen Beitrag auszuarbeiten. Die Anreizstrukturen der hier durchgeführten Innovationswettbewerbe zielen vornehmlich auf extrinsische Motivation ab. Die monetären Vergütungen sind sehr hoch, wohingegen nichtmonetäre Anreize nur vereinzelt vorzufinden sind. Der erforderliche Ausarbeitungsgrad eines Beitrags und das benötigte Facbwissen zur Teilnahme an einem Innovationswettbewerb haben maßgeblichen Einfluss auf die Auswahl des geeigneten Typs (vgl. Abbildung 1).

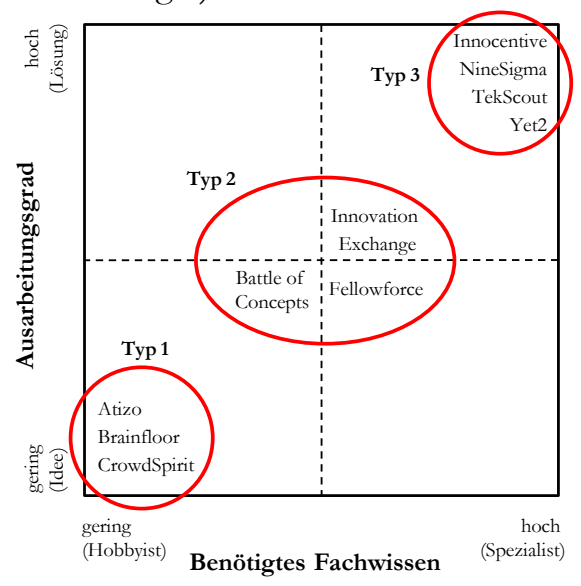

Abbildung 1: Systematisierung von Innovationswettbewerben

Es lassen sich folgende Gestaltungsempfehlungen ableiten: Sollen kreative Ideen zu allgemeinen Fragen generiert werden, die kein Fachwissen oder besondere Ressourcen erfordern, sind Innovationswettbewerbe nach Typ 1 „für Normalos“ am zielführendsten. Werden ausgearbeitete Konzepte zu Themen, die nur ein rudimentäres Fachwissen erfordern, ausgeschrieben, sollten Innovationswettbewerbe des zweiten Typs ,für Tüftler“ eingesetzt werden. Sucht ein Organisator hingegen fertige Lösungen für anspruchsvolle Probleme, die nur von spezialisierten Unternehmen oder Experten gelöst werden können, sollten Innovationswettbewerbe des Typs 3 „für Profis“ eingesetzt werden.

\section{Literatur}

Haller J, Neyer AK, Bullinger AC (2009) Beyond the black box of idea contests. Beitrag präsentiert an der EURAM Annual Conference 2009, Liverpool. 


\title{
e-Success
}

\section{Ein Instrument zur Messung des Erfolgs von Web-Seiten - getestet bei Schweizer Winzern}

\author{
Olivier Blattmann, Marcel Grüter, Simone von Burg, Thomas Myrach \\ Institut für Wirtschaftsinformatik, \\ Abteilung Informationsmanagement, Universität Bern
}

Heutzutage unterhält nahezu jedes Unternehmen einen eigenen Web-Auftritt, vom globalen Konzern bis zum Kleinstbetrieb. In einer pragmatischen, managementorientierten Sichtweise sollte sich die konkrete Ausprägung des Web-Auftritts höchst unmittelbar an den konkreten Zielen ausrichten, die ein Unternehmen mit einem Web-Auftritt erreichen will. Erst durch einen zielgerichteten, auf das Unternehmen angepassten Web-Auftritt lässt sich das Potential des Internets optimal verwirklichen (Merx und Wierl 2001, S. 88-90). Das Instrument e-Success soll mit einem aus der Theorie abgeleiteten Modell, einem umfassenden Zielkatalog sowie einem Mess- und Auswertungsverfahren die Übereinstimmung des Web-Auftritts mit dem Zielsystem eines Unternehmens klären helfen und damit zu einer verbesserten Qualität des Web-Auftritts im Sinne der postulierten Ziele beitragen.

Als theoretische Grundlagen dienen einerseits das bekannte IS Success Model von DeLone/McLean (2003) und andererseits das E-Commerce-Zielsystem von Riemer/Müller-Lankenau (2005). Der Modellansatz von e-Success ist aus der Synthese dieser beiden Ansätze entstanden. Auf dieser Basis wurden für die Kategorien Informations-, System- und Servicequalität, sowie Nutzung(sabsicht), Zufriedenheit der Nutzer, Nettonutzen und Qualitätskontrolle literaturbasiert insgesamt 42 Ziele formuliert, welche Unternehmen mit dem Einsatz der Internetseite verfolgen können. In Anlehnung an Martilla/James (1977) erfolgt die Bewertung der Ziele in Abhängigkeit ihrer Wichtigkeit für ein Unternehmen. Dadurch kann mit eSuccess der Erfolg von Webseiten verschiedenster Unternehmen, unabhängig von Größe und Branche, beurteilt werden. Dies erlaubt die Ableitung von Verbesserungsmaßnahmen und liefert die Grundlage für einen erfolgreichen Interneteinsatz.

Ein erster Pretest bei 30 Schweizer Winzern hat gezeigt, dass sich die Zielbewertung in Abhängigkeit der Wichtigkeit grundsätzlich als sinnvoll erweist. Jedoch wurden sämtliche Ziele jeweils als relativ wichtig beurteilt, was differenzierte Handlungsanweisungen erschwert. Unternehmen müssen sich noch genauer mit der Bewertung der einzelnen Ziele sowie deren relativen Wichtigkeit auseinander setzen. 


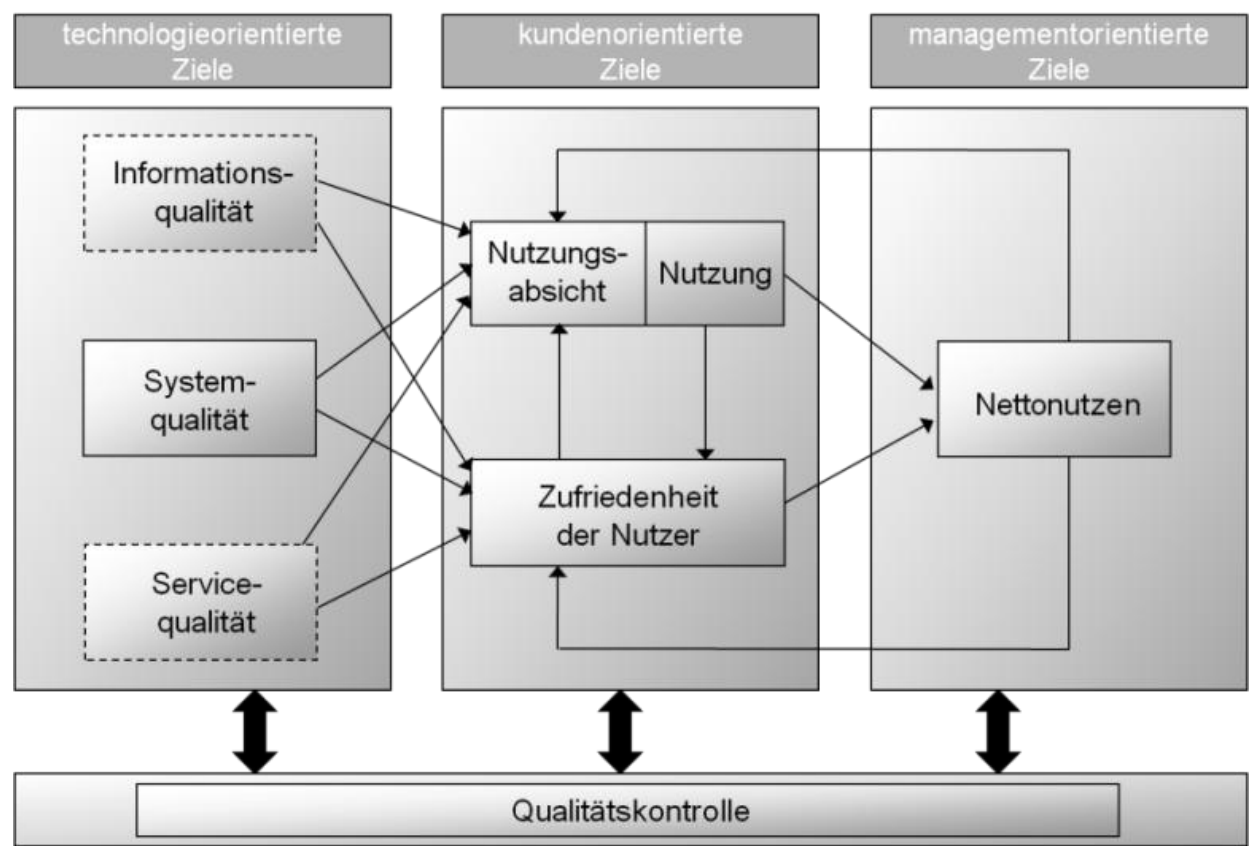

\section{Abbildung 1: Das e-Success Modell}

Aus einer theoretischen Perspektive konnten die postulierten Wirkungszusammenhänge innerhalb des e-Success-Modells mehrheitlich statistisch erhärtet werden. Ausgehend von diesen Ergebnissen können nun weitere Schritte hin zur quantitativen Validierung des Instruments unternommen werden.

\section{Literatur}

DeLone WH, McLean ER (2003) The DeLone and McLean Model of Information Systems Success: A Ten-Year Update. JOURNAL OF MANAGEMENT INFORMATION SYSTEMS 19(4):9-30.

Martilla JA, James JC (1977) Importance-Performance Analysis. JOURNAL OF MARKETING 41(1):77-79.

Merx O, Wierl M (2001) Qualität und Qualitätskriterien im E-Commerce. In:

Hermans A, Sauter M (Hrsg.) Management-Handbuch Electronic Commerce.

Franz Vahlen, München.

Riemer K, Müller-Lankenau C (2005) Web-Evaluation: Einführung in das InternetQualitätsmanagement. Arbeitsbericht Nr. 21, Kompetenzcenter für Internetökonomie und Hybridität, Universität Münster. 


\title{
Elektronische Rechnungen zur Optimierung der Financial Supply Chain: Status Quo, empirische Ergebnisse und Akzeptanzprobleme
}

\author{
Harald Schömburg, Michael H. Breitner \\ Institut für Wirtschaftsinformatik, Leibniz Universität Hannover
}

In den vergangenen Jahren haben Unternehmen durch Konzepte wie „Lean Production“, „Total Quality Management“ oder auch „Business Process Reengineering" vor allem entlang der physischen Supply Chain große Einsparpotentiale erschlossen.

Entlang der Financial Supply Chain haben neue Techniken und kostensparende Verfahren im Gegensatz dazu jedoch nur vergleichsweise langsam Einzug gehalten (Pfaff et al. 2004, S. 107ff). Durchschnittlich 40 Prozent der „Finance \& Accounting Ressourcen“ sind daher auch heute noch durch Geschäftsprozesse ohne strategischen Charakter gebunden. (Langmack und Chalons 2006, S. 17) Rechnungsstellung, Rechnungseingang und Rechnungsbearbeitung gehören zu den Kernaufgaben der Finanzabteilungen. Aber gerade hier sind die Prozesse oft kleinteilig und von manuellen Tätigkeiten bestimmt. (Aleksic o.J., S. 1) Die betriebswirtschaftlichen Kosten einer Rechnung belaufen sich dementsprechend von ca. fünf bis sieben Euro pro versandter und rund zehn bis zwölf Euro pro empfangener Rechnung.

$\mathrm{Da}$ in Deutschland jährlich ca. sechs Milliarden Rechnungen auf dem traditionellen Postweg verschickt werden, liegt hier ein Einsparpotenzial in Milliardenhöhe vor. (Schömburg et al. 2008, S.1ff)

Die elektronische Rechnungsstellung erlaubt eine Optimierung nahezu des kompletten Geschäftsprozesses (siehe Abbildung 1). Insbesondere wird durch die Verarbeitung ausschließlich elektronischer Daten und dem Einsatz automatisierter elektronischer Kontrollverfahren auf beiden Seiten die Transparenz sowie die Qualität der Geschäftsprozesse gesteigert sowie die Geschwindigkeit erhöht. (Frick 2003, S. 322) 


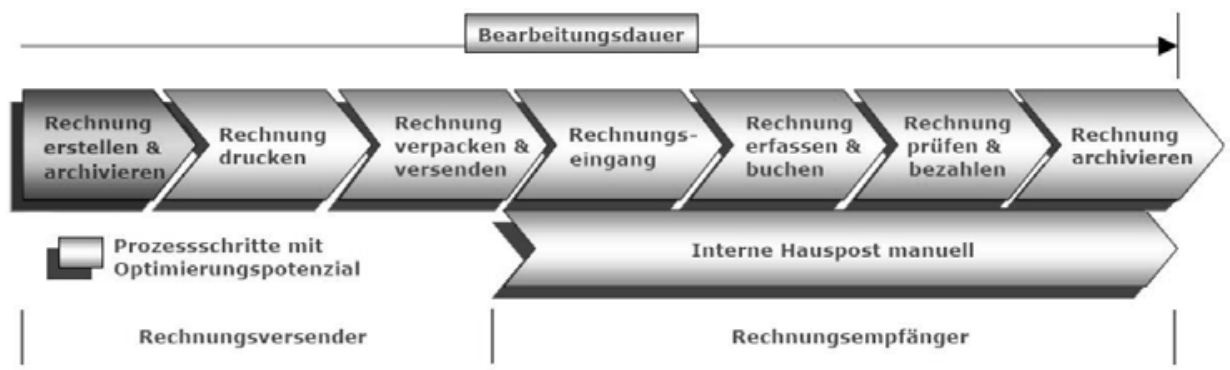

Abbildung 1: Optimierungspotentiale durch elektronische Rechnungsstellung

Dennoch hat die elektronische Rechnungsstellung noch längst nicht die Verbreitung gefunden hat, die ihr praktisches Potential nahelegt.

Methodisch wird in diesem Aufsatz wie folgt vorgegangen: zuerst wird eine umfangreiche, explorative Studie, welche die Ermittlung des Status Quo in der Rechnungseingangsbearbeitung deutscher Unternehmen zum Ziel hat, vorgestellt. Der Fokus wird hierbei insbesondere auf die Verbreitung und Akzeptanz der elektronischen Rechnung gelegt. Die Ergebnisse werden anschließend analysiert und diskutiert.

\section{Literatur}

Aleksic D (o.J.) Damit die Rechnung aufgeht. http://www.fibu markt.de/Fachinfo /ERP-Software/Damit-die-Rechnung-aufgeht.html. Abruf am 2009-29-08.

Frick B (2003) Digitale Signaturen und Zeitstempel im Archiv. In: Digitale

Signaturen in der Praxis - Leitfaden zur Prozessoptimierung und

Kostenreduktion in Unternehmen und Behörden, AWV, Eschborn.

Langmack J, Chalons C (2006) Business Process Outsourcing (BPO) in Deutschland. EDS, Rüsselsheim.

Pfaff D, Skiera B, Weitzel T (2004) Financial-Chain-Management - Ein generisches Modell zur Identifikation von Verbesserungspotenzialen. WIRTSCHAFTSINFORMATIK 46(2):107-118.

Schömburg H, Hoppen G, Breitner MH (2008) Expertenbefragung zur Rechnungseingangsbearbeitung: Status quo und Akzeptanz der elektronischen Rechnung. In: IWI Discussion Paper \# 27, Institut für Wirtschaftsinformatik Leibniz Universität Hannover. 


\title{
Technisches Konzept zur Service Externalisierung von virtuellen Prototypen in der Modebranche
}

\author{
Christian Zagel, Carolin Löffler \\ Lehrstubl für Wirtschaftsinformatik II, \\ Friedrich-Alexander Universität Erlangen-Nürnberg
}

In vielen Unternehmen produzierender Branchen kommen virtuelle Prototypen zum Einsatz. Basierend auf erstellten CAD (Computer Aided Design) -Daten stellen diese ein „komplettes Produkt als verbindliches, dreidimensionales, digitales Modell für alle am Entwicklungsprozess beteiligten Fachbereiche zentral zur Verfügung“ (Schumacher 2004, S.16). Ziele sind neben der kooperativen Produktentwicklung (Seiffert und Gotthard 2008, S. 149) ein schnelleres „Time to Market“ (Handlbauer etc al. 1998, S. 276), die Steigerung der Qualität von Entwicklungsergebnissen (Kaluza und Blecker 2000, S. 368) und das Wegfallen kostenintensiver physischer Prototypen (Behrendt et al. 2007, S. 17).

Neben dem ursprünglichen Einsatz im Produktentwicklungsprozess bergen virtuelle Prototypen großes Potenzial andere Prozesse im Produktlebenszyklus zu unterstützen. Ein Einsatzgebiet ist z. B. die Erstellung virtueller Produktabbildungen. Werden Services, z. B. als E-Commerce Service, über ihren ursprünglichen Einsatzzweck hinaus im Unternehmen eingesetzt, spricht man von Service Externalisierung (Löffler et al. 2009, S. 2). Im hier skizzierten Beispiel besteht der Ursprungsservice im „Einsatz virtueller Prototypen im Produktentwickungsprozess“. Die darüber hinaus identifizierten Einsatzgebiete auf Basis der virtuellen Produktfotografie ordnet man der Service Externalisierung zu. Ziel dieser Arbeit ist es, die technischen Anforderungen für einen breiten Einsatz von Virtual Prototyping Services zu analysieren. Anhand einer Fallstudie in der Modebranche sollen mögliche Optionen zur Prozessautomation als Teil des prototypischen Softwarekonzepts aufgezeigt werden. Abschließend wird geprüft, auf welche Branchen sich das technische Konzept zur Service Externalisierung von virtuellen Prototypen übertragen lässt. Angrenzende Arbeiten beschreiben den Einsatz virtueller Prototypen im E-Business vorwiegend im Sinne der kollaborativen, beschleunigten Produktentwicklung (Zarli et al. 2002, S. 34) oder zur Verifikation von Produktkonzepten (Battarbee und Säde 2002, S. 25; Mak und Huang 2003, S. 25ff). Die vorliegende Arbeit befasst sich dagegen mit dem nachgelagerten Prozess der reinen Produktvisualisierung für den Kunden. 


\section{Literatur}

Battarbee K, Säde S (2002) Emotional Responses to Virtual Prototypes in the Design Evaluation of a Product Concept. In: Green W, Jordan P (Hrsg) Pleasure with Products: Beyond Usability. Routledge Chapman \& Hall, Great Britain.

Behrendt S, Pfitzner R, Kreibich R, Hornschild K (2007) Innovationen zur Nachhaltigkeit: Ökologische Aspekte der Informations- und Kommunikationstechniken. Springer, Berlin.

Handlbauer G, Matzler K, Sauerwein E (1998) Perspektiven im strategischen Management. Gruyter, Berlin.

Kaluza B, Blecker T (2000) Produktions- und Logistikmanagement in virtuellen Unternehmen und Unternehmensnetzwerken. Springer, Heidelberg.

Löffler C, Zagel C, Körber P (2009) Externalisierung von internen Dienstleistungen am Beispiel virtueller Prototypen in der Modebranche. In: 71. Wissenschaftliche Jahrestagung des Verbands der Hochschullehrer für Betriebswirtschaft e. V., Nürnberg.

Schumacher A (2004) Optimierung mechanischer Strukturen: Grundlagen und industrielle Anwendungen. Springer, Heidelberg.

Seiffert U, Gotthard R (2008) Virtuelle Produktentstehung für Fahrzeug und Antrieb im KFZ: Prozesse, Komponenten, Beispiele aus der Praxis. Vieweg \& Teubner, Wiesbaden.

Zarli A, Katranuschkov P, Turk Z, Rezgui Y, Kazi A (2002) Harmonization of the IST research and development for the European construction industry: The ICCI project. In: Turk Z (Hrsg) E-Work and E-Business in Architecture, Engineering and Construction: Proceedings of the $4^{\text {th }}$ European Conference, Portoroz, Slovenia. A A Balkema, Slovenien. 


\title{
Das Differenzierungspotential eines mehrdimensionalen Kriterienkatalogs zur Bewertung von Onlineshops: Eine empirische Untersuchung in einem Schweizer Portal
}

\author{
Achim Dannecker, Michael Quade \\ Competence Center E-Business Basel, \\ Fachbochschule Nordwestschweiz FHNW
}

Im Jahr 2006 sagte Tim O'Reilly auf dem Dresdner Zukunftsforum voraus: „In ein paar Jahren werden die Prinzipien des Web 2.0 absolut Mainstream sein“. Er empfahl demzufolge: „Sie müssen die kollektive Intelligenz der User nutzbar machen“ (Hage 2006). Heute sind viele der Prinzipien des Web 2.0 allgegenwärtig, darunter Bewertungen von Inhalten im Internet.

Internetdienste, die dem Nutzer Bewertungen zu Inhalten ermöglichen und anbieten, können sich einen Vorteil gegenüber Wettbewerbern schaffen.

Die eigentliche Herausforderung besteht in der Definition des Umfangs eines Kriterienkatalogs. Zum einen soll die Bewertung in ihrer Ausprägung dem Nutzer einen einfachen (quasi auf ein Kriterium reduzierten) Eindruck geben, um ihn bei der Transaktionsentscheidung zu unterstützen. Zum anderen soll der Nutzer aufgrund einer möglichst differenzierten Bewertung entscheiden können. Für den Nutzer muss es so einfach wie möglich sein, eine Bewertung abzugeben - und es soll ihm Spaß machen (van der Heijden 2004, S. 1). Die Bewertung soll aber auch so umfangreich sein, dass andere Nutzer ein präzises Bild vom bewerteten Produkte erhalten.

In dieser Arbeit wird ein Portal untersucht, das Onlineshops in der Schweiz auflistet, und diese durch Nutzer des Portals bewerten lässt. Aus dem methodischen Vorgehen der Analyse wird darüber hinaus eine Vorgehensweise entwickelt, die aufzeigt, wie die Qualität (Redundanz vs. Differenzierung) eines Kriterienkatalogs mittels einer explorativen Faktorenanalyse untersucht werden kann.

In einem mehrstufigen Prozess werden Daten vom Portal www.toppreise.ch extrahiert und für SPSS aufbereitet. In SPSS wurde die explorative Faktorenanalyse durchgeführt.

Es wurde aufgezeigt, dass ein Teil der Bewertungskriterien letztlich die gleiche Aussage treffen können. Der Kriterienkatalog differenziert die Onlineshops in vielen Punkten nicht. D.h. es ist nicht klar, ob die unterschiedlichen Bewertungen innerhalb der Kriterien zufällig oder systematischer Natur sind, bzw. der Unterschied der Bewertungen schlicht zu gering sind. Gleichzeitig wurde eine Methode 
aufgezeigt, wie Betreiber von Bewertungsportalen die Kriterien auf Redundanzen hin überprüfen können. Ziel sollte es in der Regel sein, mit möglichst wenigen Kriterien bestmöglich die Inhalte differenzieren zu können, um eine möglichst breite Basis an Nutzern zu erreichen, die eine Bewertung abgeben. Dies wird mit einem umfangreichen - ggf. auch redundanten - Kriterienkatalog nicht unbedingt positiv beeinflusst. Wichtig ist bei diesem Ansatz aber, dass bei einer Reduktion des Kriterienkatalogs, diese immer durch weitere empirische Beobachtungen begleitet werden muss, um sicherzustellen, dass die Interpretation des entstandenen Faktors korrekt ist.

Selbstverständlich können Kriterien vereinzelt auch als Prüfgrößen angedacht bzw. in den Kriterienkatalog eingebaut werden, um die Plausibilität von Bewertungen untermauern zu können. Auch für die Entwicklung von Prüfkriterien kann man die vorgestellte Methodik der Analyse einsetzen. Sie eignet sich um die Validität des Konstrukts zu untermauern. Es gibt mannigfaltige Einsatzmöglichkeiten der Faktorenanalyse im Bereich von Bewertungsportalen. Sie helfen, diese Portale zu verbessern.

Der in diesem Papier beschriebene methodische Ansatz zur Identifikation von differenzierenden Bewertungskriterien wird demnächst im Rahmen weiterer Studien auf andere Bewertungsplattformen wie hrs.de, holidaycheck.de usw. angewendet.

\section{Literatur}

Hage S (2006) Tim O'Reilly - Der Web-Meister. http://www.managermagazin.de/it/artikel/0,2828,449911,00.html. Abruf am: 2009-07-12.

van der Heijden H (2004) User Acceptance of Hedonic Information Systems. MIS Quarterly 28(4): 695-704. 


\title{
Dynamically Scalable Architectures for E-Commerce
}

\section{A Strategy for Partial Integration of Cloud Resources in an E-Commerce System}

\author{
Georg Lackermair ${ }^{1,2}$, Susanne Strahringer', Peter Mandl² \\ ${ }^{1}$ Chair for Business Informatics, Technical University Dresden \\ ${ }^{2}$ Chair for Business Informatics, University of Applied Sciences Munich
}

The paper focuses on the benefits of a partial integration of cloud resources into online shops for small- and medium-sized companies. The conflict between rising energy costs, fluctuating workloads and continuously high availability requirements demand mechanisms that enable automated and dynamic scaling of such an application.

We suggest an architecture for dynamic scaling as depicted in Figure 1 and an architecture for the shop application itself based on our dynamic scaling approach.

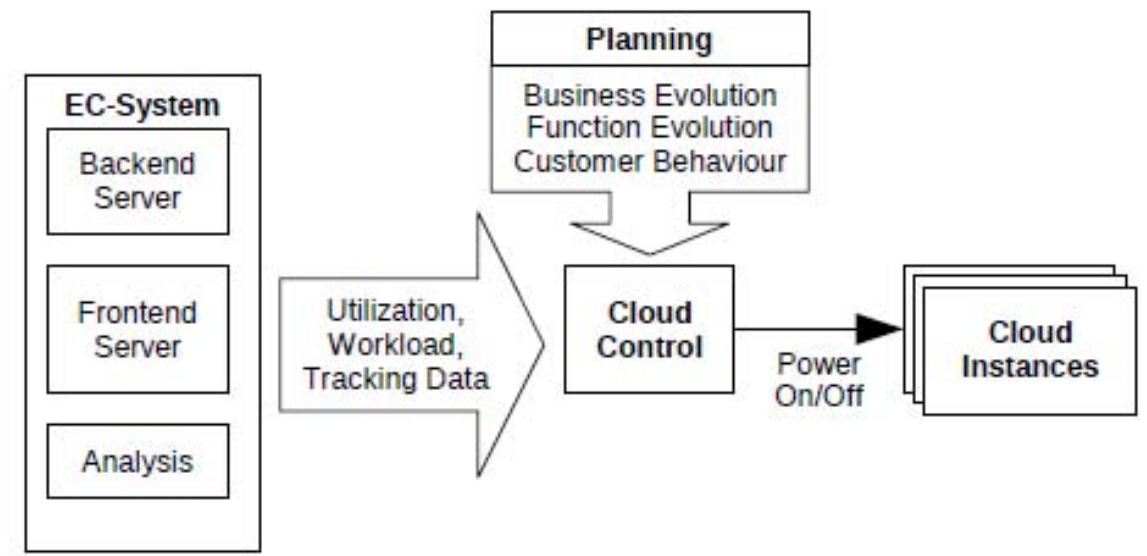

Figure 1: An Architecture for Dynamic Scaling

The application needs to contain a component which controls the cloud instances. Basically, this means to switch on and shut down the instances. An instance has to register as an available node, when the start-up is finished. The cloud control also decides when to add a new node or when to remove a node from the application. 
Therefore, this component must process information from various sources almost in real-time.

In order to estimate the economic benefits of the suggested architecture, we conduct an exemplary calculation of total costs of ownership for a year. The calculation is based on the pricing model for Amazon Elastic Compute Cloud. For sake of simplification the approach of using market prices for renting virtual machines seems to be fairly realistic. For the calculation also workload profiles need to be estimated. First results of an analysis of a B2B e-commerce system indicate that assuming a relative low mean CPU utilization is realistic. Our calculations show that in case of a relative low mean CPU utilization, dynamic application scaling would result in significant cost savings.

Therefore, outsourcing of the infrastructure by using cloud resources seems to be appealing. However, the usage of external service providers has some major drawbacks: A survey among CIOs in Germany pointed out that security concerns and probably evolving dependencies seem to be the most important reasons against the usage of computing capabilities via the internet (TecChannel, 2009). Hayes (2008) emphasizes transparency issues and Quality of Service (QoS) as major concerns when it comes to Cloud Computing. All these concerns need to be addressed. In our paper we especially focus on evolving dependencies, QoSproblems and transparency by deriving respective technical requirements and suggesting how they can be met.

The underlying overall research project is based on a design science approach as suggested by Hevner et al. (2004). The paper at hand focuses on motivating the relevance of the addressed research problem and suggests a first sketch of an architecture that might help solving this problem. As design science "is inherently iterative" (Hevner et al. 2004, S. 87) and is organized along several design and test cycles our first step is to present the results of a first design cycle. In a next step a prototypical implementation will allow effective evaluation.

\section{References}

Hayes B (2008) Cloud Computing. CACM 51(7):9-11.

Hevner A, March S, Park J, Ram, S (2004) Design Science in Information Systems Research. MIS Quarterly 28(1):75-105.

TecChannel (2009) Cloud Computing schürt Angst vor Kontrollverlust. http://www.tecchannel.de/test technik/news/1993816/sicherheitsbedenken bei cloud computing/, visited: 2009/07/23. 


\title{
Web-Analyse-Dienste als Instrument des Blog- Monitoring
}

\author{
Thomas Walter, Andrea Back \\ Institut für Wirtschaftsinformatik, \\ Unviversität St. Gallen
}

Blogs haben in den vergangenen Jahren den Status eines MainstreamKommunikationskanals erreicht. Die Internet-Suchmaschine Technorati gibt im Jahresabschlussbericht 2008133 Millionen indexierte Blogs an. Allein durch diese Blogs entstehen täglich knapp eine Million neue Blog-Posts. Infolgedessen steigt täglich die Zahl von Blogs, die professionell, kommerziell, oder unter der Absicht Gewinn zu erzielen betrieben werden, deren Bedeutung als Werbekanal und letztlich vermehrt das Bedürfnis blogspezifischer Leistungsmessung. Blog-Monitoring, also die Leistungsmessung von Blogs, wird durch die technischen Möglichkeiten von Web-Analyse-Diensten getrieben. Diese Performance-Measurement-Systeme für Webseiten wurden jedoch nicht für Blogs konzipiert, und explizit auf Blogs ausgerichtete Systeme haben bislang keine Berücksichtigung gefunden.

Dieser Beitrag untersucht die Fragen, welche Kennzahlen für das BlogMonitoring von zentralem Interesse sind bzw. inwieweit diese Kennzahlen bereits von bestehenden Web-Analyse-Diensten erhoben und zentral bereitgestellt werden. Als State-of-the-Art des Blog-Monitoring wird zunächst eine Übersicht über Kennzahlen, die aktuell bei verschiedenen Web-Analyse-Diensten erhoben werden, herausgearbeitet. Diese Kennzahlen werden in einer Online-Umfrage unter den ersten 100 Blogs der deutschen Blogcharts in Frage gestellt. Aufbauend auf den erzielten Ergebnissen wird, wie in Abbildung 1 dargestellt, beispielhaft, nach der Vorgehensempfehlung der Balanced Scorecard, ein auf das Blog-Monitoring ausgerichtetes Kennzahlenmodell entworfen.

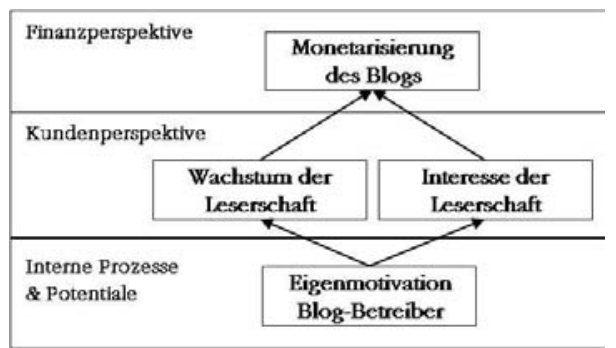

Abbildung 1: Generische Ziele am Beispiel eines Fachblogs in einer Strategy Map in Anlehnung an Kaplan und Norton (2000, S. 170). 
Die Ergebnisse zeigen, dass Blog-Monitoring ein zentrales Thema für BlogBetreiber darstellt. Trotzdem befassen sich diese nicht dediziert mit den Möglichkeiten von Web-Analyse-Diensten. Google Analytics ist der mit Abstand am häufigsten verwendete Dienst, obwohl dort nicht diejenigen Kennzahlen bereitgestellt werden, die von Blog-Betreibern als am wichtigsten eingestuft werden. Für BlogBetreiber sind Kennzahlen essentiell, die die Interaktion mit den Lesern bewerten, also u. a. Länge, Häufigkeit oder Qualität der abgegebenen Kommentare sowie das generelle Kommentierungsbemühen des Lesers. Die Differenz des in Tabelle 1 dargestellten, beispielhaft konzipierten, Kennzahlensystems zu durch WebAnalyse-Dienste bereitgestellten Systemen zeigt den praktischen und theoretischen Handlungsbedarf nach einem speziellen Blog-Monitoring-Dienst auf.

Tabelle 1: Nach Vorgehensempfehlung der Balanced Scorecard entwickeltes beispielhaftes Kennzahlensystem für ein Fachblog

\begin{tabular}{|l|l|l|}
\hline Ziel & Messgrößen & Quelle (Vorschlag) \\
\hline \multirow{2}{*}{$\begin{array}{l}\text { Eigenmotivation des Blog- } \\
\text { Wetreibers }\end{array}$} & Veröffentlichungsfrequenz & Blog-Administration \\
\cline { 2 - 3 } & Länge der Blogbeiträge & Blog-Administration \\
\hline \multirow{4}{*}{ Interesse der Leserschaft } & Unique Visitors & Google Analytics \\
\cline { 2 - 3 } & Visits & Google Analytics \\
\cline { 2 - 3 } & RSS-Abonnenten & Feedburner Stats \\
\cline { 2 - 3 } & Zufriedenheit der Leser & Blog-Administration \\
\cline { 2 - 3 } & Konversationsrate der Leser & Blog-Administration \\
\cline { 2 - 3 } Grundlagen zur Monetarisie- & Feedbackbemühen der Leser & Blog-Administration \\
\cline { 2 - 3 } rung des Blogs schaffen & Zitate und Nennungen & Technorati \\
\cline { 2 - 3 } & Suchmaschinen-Rang & Google Blog-Suche \\
\hline
\end{tabular}

Die Anwendung der Balanced Scorecard zeigt, dass methodisches Vorgehen zu gänzlich anderen Kennzahlensystemen führt. Sie ist jedoch auf Unternehmen und nicht auf Softwaredienste ausgerichtet. Für das Blog-Monitoring fehlt weiterhin ein konzeptioneller Rahmen, genauer eine generische Methode, nach der Kennzahlenmodelle zur Leistungsmessung von Blogs individuell entwickelt werden können.

\section{Literatur}

Bourne M, Mills J, Wilcox M, Neely A, Platts K (2000) Designing, implementing and updating performance measurement systems. International Journal of Operations \& Production Management 20(7):754-771.

Kaplan RS, Norton DP (2000) Having Trouble with Your Strategy? Then Map It. Harvard Business Review 78(5):167-178. 


\title{
Ein konzeptionelles Model auf Basis von Markov- Ketten zur Berechnung und Bewertung von per- sönlichen Kompetenzen in sozialen Netzwerken
}

\author{
Axel Winkelmann', Till Haselmann² \\ ${ }^{1}$ Institut für Wirtschafts- und Verwaltungsinformatik, \\ Professur für Betriebliche Anwendungssysteme, Universität Koblenz-Landau \\ ${ }^{2}$ European Research Center for Information Systems, \\ Lehrstubl für Wirtschaftsinformatik und Informationsmanagement, \\ Westfälische Wilhelms-Universität Münster
}

Bewertungen und Evaluationen sind sehr wichtig für Menschen, besonders um Vertrauen zwischen Geschäftspartnern aufzubauen. Der Vorteil von Onlinebewertungen sind die Skalierbarkeit und Formalisierung von Bewertungen (Bolton u. a. 2004, Dellarocas 2003). Einschätzungen können unabhängig von Zeit und Raum von verschiedenen Parteien gesammelt und an diese kommuniziert werden. Die grundlegende Idee ist dabei, dass Benutzer Entitäten mit Hilfe von WebApplikationen bewerten und damit Bewertungen sammeln, aggregieren und verteilen können (Resnick u. a. 2000). Die aggregierten Bewertungen über eine bestimmte Entität, wie etwa eine Person oder seine oder ihre Eigenschaften, können zur Ableitung eines Wertes, wie z. B. eines virtuellen Vertrauens- oder Reputationswertes, genutzt werden.

Mit dem Aufkommen von sozialen Netzwerken ist es möglich, die Beziehungen zwischen einzelnen Menschen und den Kompetenzen des Einzelnen im Internet zu analysieren. Der Betrieb von Online-Bewertungssystemen setzt jedoch ein passendes Design der zugrunde liegenden Mechanismen voraus. In ihrer State-ofthe-Art-Studie von 102 Bewertungsmechanismen haben Winkelmann u. a. (2009) analysiert, dass existierende Bewertungsmechanismen grundsätzlich sehr einfach auf Basis von Skalen gehalten werden. Es fanden sich bei der Untersuchung aber keine passenden Mechanismen zur Bewertung von persönlichen Kompetenzen im Internet. Dieses deckt sich mit der Forderung von Peters und Reitzenstein (2008), sich intensiv mit der Gestaltung von Formen, Effekten und der Validität von Bewertungsmechanismen auseinander zu setzen.

Mit dem hier vorgestellten Projekt SkillRank wird dieses Problem mit einem Berechnungsalgorithmus zur Bewertung von Fähigkeiten unter Berücksichtigung des sozialen Graphen in Web 2.0- Netzwerken adressiert. Der Bewertungsmechanismus sollte absichtliche Manipulation verhindern oder erschweren (Dellarocas 
2003) und Beleidigungs- und Rachebewertungen, wie von Resnick und Zeckhauser (2001) beobachtet, unterdrücken. Zudem musste der Mechanismus die Bewertungsfähigkeit des Bewertenden (Winkelmann u. a. 2009) und als vierte Anforderung die Nähe des Bewertenden zum bewerteten Objekt beachten (Chen u. a. 2004). Die Anforderungen eins bis vier stellen sicher, dass der Ansatz verlässliche, d. h. „objektive“, Ergebnisse liefert, die für ein seriöses soziales Netzwerk nutzbar sind. Darüber hinaus muss ein derartiger Mechanismus eine unbegrenzte Anzahl von Kompetenzen zur Bewertung verarbeiten. Als sechste Anforderung muss der Ansatz automatisierbar sein, da der Einsatz von Moderatoren in großen Netzwerken mit vielen Millionen Nutzern nicht mehr verlässlich realisierbar ist.

Das theoretische Konzept des an den Anforderungen ausgerichteten Bestätigungsmechanismus baut auf dem von den Google-Gründern zum PageRankPrinzip weiterentwickelten Konzept der Eigenvektor-Zentralität auf und wurde als OpenSocial-Anwendung für einen führenden Anbieter von sozialen Netzwerken als Forschungsprototyp realisiert.

\section{Literatur}

Bolton G, Katok E, Ockenfels A (2004) How Effective are Online Reputation Mechanisms? An experimental investigation. Mgment Science 50(11):1587-1602.

Chen K-Y, Hogg T, Wozny N (2004) Experimental study of market reputation mechanisms. In: Proc. of the 5th ACM conf. on E-Commerce, NY, USA.

Dellarocas C (2003) The Digitization of Word of Mouth: Promise and Challenges of Online Feedback Mechanisms. Mgment Science 49(10):1407-1424.

Peters R, Reitzenstein I (2008) Reputationssysteme im eCommerce - Funktionsweise, Anwendung und Nutzenpotenziale. HMD 45(261):43-50.

Resnick P, Kuwabara K, Zeckhauser R, Friedman E (2000) Reputation systems. Common ACM 43(12):45-48.

Resnick, P. and Zeckhauser, R. (2001). Trust Among Strangers in Internet Transactions: Empirical Analysis of eBay's Reputation System. Working Paper for the NBER workshop on empirical studies of electronic commerce.

Winkelmann, A.; Herwig, S.; Pöppelbuß, J.; Tiebe, D.; Becker, J. (2009):

Discussion of Functional Design Options for Online Rating Systems: A Stateof-the-Art Analysis. In: Proceedings of the European Conference on Information Systems (ECIS). Verona, Italy. 
E-Government 



\title{
Vorwort zur Teilkonferenz
}

\section{Electronic Government}

\author{
Jörg Becker', Helmut Krmar², \\ Erwin Schwärzer ${ }^{3}$, Maria A. Wimmer ${ }^{4}$ \\ ${ }^{1}$ European Research Center for Information Systems, \\ Westfälische Wilhelms-Universität Münster \\ ${ }^{2}$ Lebrstubl für Wirtschaftsinformatik, \\ Technische Universität München \\ ${ }^{3}$ Bundesministerium des Innern, Berlin
${ }^{4}$ Institut für Wirtschafts- und Verwaltungsinformatik, Universität Koblen₹-Landau

In den öffentlichen Verwaltungen ist der Abbau von Bürokratie ein Dauerthema, Kosteneinsparungen werden mehr denn je notwendig. Vor dem Hintergrund der Finanz- und Wirtschaftskrise steigen die Schulden der öffentlichen Hand, diesen können keine vergleichbaren Zusatzeinnahmen gegenüber gestellt werden. Hier bietet E-Government zum einen eine Chance, Effizienz- und Effektivitätsvorteile für die öffentlichen Verwaltungen zu erschließen, gleichzeitig hält E-Government viele und große Herausforderungen für die öffentlichen Verwaltungen in ganz Europa bereit.

Die EU-Dienstleistungsrichtlinie, die E-Procurement-Verordnung der EU und EU-Strategien im Bereich Identitätsmanagement oder im Gesundheitswesen verlangen von Verwaltungen eine gewaltige Umstrukturierung und Öffnung der Dienstleistungslandschaft. Transparenz und Vergleichbarkeit, aber auch das Thema Wiederverwendbarkeit von bestehenden Lösungen sind hier unabdingbar. Neben organisatorischen Veränderungen sind verstärkte organisationsübergreifende Zusammenarbeit, der Austausch von Lösungen und technische Neuerungen notwendig, um die Ziele der Richtlinien und Strategien nicht nur in einer leeren Hülle sondern in der Tiefe umzusetzen. Allein in Deutschland finden wir auf kommunaler Ebene etwa 12.000 Organisationen vor, die viele gleiche Aufgaben zu erfüllen haben. Ein Bewusstsein dafür gilt es zu schaffen und zu schärfen.

Darüber hinaus steigen auch die Erwartungen der Bürger an die Abläufe und Dienste von öffentlichen Verwaltungen. Das Konzept des Behördenrufs 115, der die Ideen der Dienstleistungsrichtlinie mehr oder weniger auf die Bürger in 
Deutschland überträgt, ist eine große Herausforderung. Die Bürger fordern darüber hinaus auch Beteiligungsmöglichkeiten an der Verwaltungssteuerung zwischen den Wahlen ihrer Vertreter vor Ort ein. Sei es die Beteiligung an Haushaltsplänen oder an wohn- und städtebaulichen Konzepten, die Bürger möchten mitreden und dabei möglichst moderne Technologien, z. B. aus dem Themenfeld des web2.0, nutzen, die eine Beteiligung von zuhause ermöglicht, kurz: Instrumente der E-Partizipation nutzen.

Die Teilkonferenz E-Government adressiert diese verschiedenen Themen mit den angenommen Beiträgen und möchte so eine Diskussionsgrundlage für Wissenschaft und Praxis schaffen.

Programmkomitee:

- Dr. Lars Algermissen, PICTURE GmbH

- Prof. Dr. Martin Brüggemeier, Hochschule für Technik und Wirtschaft Berlin

- Prof. Dr.-Ing. Norbert Gronau, Universität Potsdam

- Prof. Dr. Ralf Klischewski, German University in Cairo

- Prof. Dr. Peter Loos, Universität des Saarlands

- Dr. Dr. Björn Niehaves, ERCIS - Universität Münster

- Dr. Daniel Pfeiffer

- Prof. Dr. Alexander Prosser, Wirtschaftsuniversität Wien

- Dr. Sönke E. Schulz, Lorenz-von-Stein-Institut für Verwaltungswissenschaft, Kiel

- Prof. Dr. Tino Schuppan, IfG.CC - The Potsdam eGovernment Competence Center

- Prof. Dr. Gerhard Schwabe, Universität Zürich

- Prof. Dr. Daniel Veit, Universität Mannheim

- Prof. Dr. Jörn von Lucke, Zeppelin University Friedrichshafen

- Dr. Petra Wolf, Technische Universität München 


\title{
Transferstrategien zur Wiederverwendung und Verbreitung von E-Government-Lösungen
}

\author{
Moreen Stein \\ Lehrstubl für Wirtschaftsinformatik und Electronic Government, \\ Universität Potsdam
}

Derzeit existieren zahlreiche E-Government-Lösungen und Lösungsansätze. Dies geht mit redundanten Entwicklungsbemühungen und isolierten Lösungen einher. Problematisch ist weniger die technologische Entwicklung leistungsstarker Informationssysteme, sondern der vertikale wie auch horizontale Transfer bestehender Lösungen. Der Beitrag zeigt auf, wie Transferprozesse gestaltet werden können. Dazu werden mit Bezug zur Diffusionsforschung (Rogers 1962; Holzinger et al. 2007) und auf der Basis einer Befragung von E-Government-Akteuren sechs Transferarten beschrieben. Dies sind die Kategorien

- Leuchtturmwirkung,

- Kooperation,

- Standardisierung,

- Verbindlichkeit,

- Zentralität sowie

- Wettbewerb.

Wird ihre Effektivität hinsichtlich des Transfererfolgs in Fallstudien bestätigt, gehen aus ihnen Transferstrategien hervor, die konkrete Maßnahmen für die Gestaltung von Transferprozessen benennen. Tatsächlich ablaufende Transferprozesse lassen sich kaum einer einzigen Kategorie eindeutig zuordnen. Vielmehr ist es notwendig, auch Mischtypen erfassen zu können. Dies wird über ein Ordnungssystem realisiert, das die vorgestellten Kategorien durch Merkmale unterscheidet. Die einzelnen Merkmale werden jeweils auf einer fünfstufigen Skala bewertet.

Über die zu Grunde liegende Transferkategorie hinaus können Transferinitiativen oder -prozesse weitere Eigenschaften haben, die sich auf den Transfererfolg auswirken können. Daher müssen sie über Attribute genauer beschrieben werden. Nur so kann später ihre Effektivität bezogen auf den Transfererfolg bewertet werden. Ein Attribut bildet zunächst die Transferrichtung. Sie beschreibt, ob der Transferprozess innerhalb einer föderalen Ebene oder ebenenübergreifend abläuft. Zum Beispiel kann ein Land Transfersender und eine oder mehrere Kommunen Transferempfänger sein. Daraus leitet sich ein weiteres Attribut ab. Findet der Transfer zwischen zwei Einheiten statt oder sind auf Seiten des Senders und Empfängers mehrere Einheiten eingeschlossen? Darüber hinaus sind auch rechtliche 
Rahmenbedingungen (z. B. Vergaberecht), akteursbezogene Einflüsse (z. B. fehlende Kompetenzen), politische Faktoren (z. B. Parteipolitik) oder Dritte als mögliche Einflussnehmer (z. B. Softwareanbieter) zu nennen. Diese Attribute beschreiben neben den Transferkategorien weitere Faktoren, die den Transfererfolg maßgeblich beeinflussen können. Diese Einflussfaktoren müssen bei der Formulierung der Transferstrategien als Rahmenbedingungen berücksichtigt werden.

Entscheidend für den Transfererfolg sind auch die Güte und die Übertragbarkeit der zu transferierenden Lösung. Gegenüber dem abzulösenden System muss sich die Vorteilhaftigkeit der Neuerung zeigen (Rogers et al. 2005, S. 4). Dies wird mit dem Begriff Transferwürdigkeit beschrieben. Die Transferfähigkeit bildet den zweiten lösungsbezogenen Faktor. Nur wenn eine Lösung anpassbar ist und somit auch in andere Kontexte übertragen werden kann, ist ein Transfer möglich. Rogers et al. (2005, S. 4) beschreiben diese Eigenschaft mit der Kompatibilität mit Existierendem.

Eine Fallstudie zur Übertragung der Lösung Mobile Bürderdienste zeigt Faktoren auf, die den Transferfolg beeinflussen und macht deutlich, dass die zielgerichtete Gestaltung des Transferprozesses, wie in diesem Beispiel durch Öffentlichkeitsarbeit und die Initiierung von Kooperationen, den Transfer fördern kann. Ziel der Arbeit ist es schließlich, Handlungsempfehlungen für potenzielle Sender oder Intermediäre im Transferprozess abzuleiten, um den breiten Transfer von etablierten E-Government-Lösungen erfolgreich gestalten zu können. Sender im Transferprozess sind öffentliche Verwaltungen, z. B. Bundesbehörden, die eine Lösung verbreiten wollen. Verwaltungen können außerdem als Intermediär fungieren, ebenso Forschungseinrichtungen, Berufskammern oder auch Dachverbände. Intermediäre nehmen auf den Transferprozess Einfluss. Sie vermitteln zwischen Sender und Empfänger im Transferprozess.

\section{Literatur}

Holzinger K, Jörgens H, Knill C (2007) Transfer, Diffusion und Konvergenz. Konzepte und Kausalmechanismen. In: Holzinger K, Jörgens H, Knill C (Hrsg) Transfer, Diffusion und Konvergenz von Politiken, PVS, Sonderheft 38:11-35.

Rogers EM (1962) Diffusion of innovations. The Free Press, New York.

Rogers EM, Medina UE, Rivera MA, Wiley CJ (2005) Complex adaptive systems and the diffusion of innovations. The Innovation Journal 10 (3), Article 30. 


\title{
Bürgernahes Regieren
}

\section{Lässt sich politische Beteiligung durch E-Participation Anwendungen verbessern?}

\author{
Daniel Veit, Nils Parasie, Florian Schoppé \\ Dieter Schwarz. Stiftungslehrstubl für ABWL, \\ E-Business und E-Government, \\ Universität Mannheim
}

„Hi everybody“ - Mit diesen Worten richtete sich der Präsident der Vereinigten Staaten, Barack Obama, zum ersten Mal nach seiner Wahl per Online Videobotschaft direkt an seine Anhänger, ohne den Weg über die traditionellen Medien zu gehen (Moorstedt 2009, S. 15). Dieses Beispiel zeigt, dass eine neue Kultur der Kommunikation mit dem Bürger in den politischen Diskurs Einzug hält.

In diesem Beitrag wird anhand einer quantitativen empirischen Studie untersucht, inwiefern, durch die Bereitstellung elektronischer Beteiligungsmöglichkeiten, eine stärkere Teilnahme der Bürger an politischen Prozessen erreicht werden kann. Untersuchungsgegenstand sind dabei Anwendungen, die der Kommunikationsbeziehung zwischen Repräsentant und Bevölkerung dienen. Als theoretische Grundlage dient das Technology Acceptance Model (Davis et al. 1989, S. 983), welches auf die spezifischen Charakteristika von E-Participation Anwendungen angepasst und erweitert wird. Das Forschungsmodell wird in Abbildung dargestellt.

Die verwendeten Fragen für die Identifikation der Konstrukte im Forschungsmodell orientieren sich an Indikatorvariablen, die bereits in anderen Studien erfolgreich eingesetzt wurden. Dabei wurden jeweils reflektive Messmodelle verwendet, d.h. es wird eine Kausalität unterstellt, die vom untersuchten Konstrukt her auf die Indikatorvariablen zeigt (Chin 1998, S. 35). Die Befragung erfolgte innerhalb von fünf Wochen im Zeitraum vom 09. April bis zum 14. Mai 2009. Insgesamt konnte ein Rücklauf von 151 Teilnehmern verzeichnet werden.

Die Ergebnisse dieser Arbeit bestätigen bisherige Untersuchungen zur Akzeptanz von Technologien und zeigen, dass diese auch in Bezug auf E-Participation Anwendungen gültig sind. Insbesondere wird die Bedeutung eines klaren Nutzenversprechens und einer vertrauenswürdigen Gestaltung elektronischer Beteiligungsmöglichkeiten hervorgehoben. Die Möglichkeit einer einfachen Nutzung von E-Participation Anwendungen konnte als indirekter Einflussfaktor identifiziert werden, der sich positiv auf den erwarteten Nutzen auswirkt. Darüber hinaus weisen die Ergebnisse darauf hin, dass durch die Steigerung des Nutzens einer Anwendung sowohl politisch motivierte als auch politisch weniger interessierte Bürger 
für die Beteiligung am politischen Willensbildungsprozess hinzugewonnen werden können.

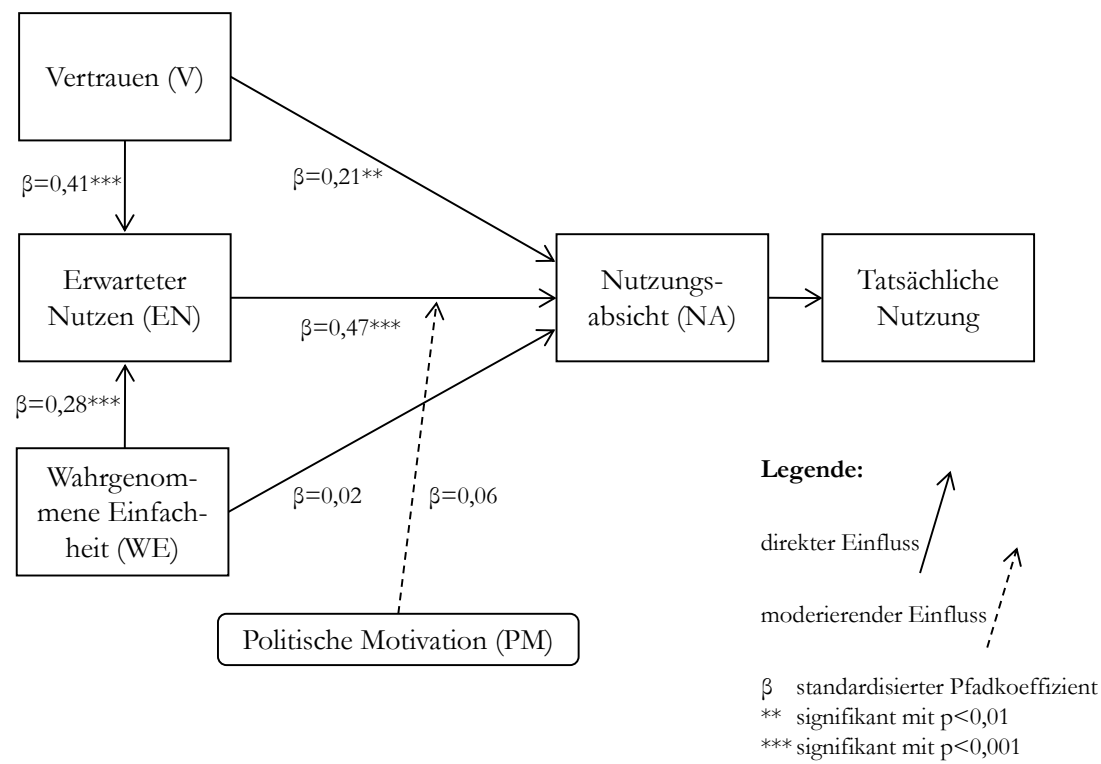

Abbildung 1: Forschungsmodell

Quelle: Eigene Abbildung

\section{Literatur}

Chin, W (1998) Issues and opinion on structural equation modeling. MIS Quarterly 22(I): vii-xvi.

Davis, FD, Bagozzi, RP, Warshaw, PR (1989) User acceptance of computer technology: a comparison of two theoretical models. Management Science 35(8):982-1003.

Moorstedt, T (2009) Ich bin es, Dein Anführer. Süddeutsche Zeitung, Ausgabe vom 18. Februar 2009. 


\title{
Understanding the Advisory Needs of Citizens
}

\author{
Birgit Schenk', Gerhard Schwabe $2^{*}$ \\ ${ }^{1}$ Fachbereich Verwaltungsinformatik, Hochschule für öffentliche Verwaltung Kehl \\ Institut für Informatik, Universität Zürich
}

Most E-Government research has focused on citizens' self-service (Steele 1996). This paper targets citizens' advisory services. Advice is needed in situations where the citizen is unable to make his/her information or service needs explicit and thus cannot search by him/herself (Belkin 1982, Ellis et al. 2002). We use the scenario of the pregnant woman to analyze the current situation and to propose requirements for an IT-based solution. The analysis is based on mystery calling, mystery shopping and on workshops with citizens and advisors from the public administration offices.

The in-depth analysis of problems which pregnant women face when seeking advice in the public administration sector results noting organisational requirements, process requirements and information and IT-requirements (see table 1). These include setting-up of true one-step advisory services, the integration of information from different public sources with extra information from the Internet, the implementation of advisory processes and their support by appropriate IT.

Public administration offices are currently ill-prepared to provide adequate advisory services. The primary reason is the lack of adequate processes. Adequate processes then require the integration of currently disjunct administrative responsibilities with adequate IT support (e.g. Lenk, Klee-Kruse 2000). Public administration has stayed remarkably stable in the face of a changing environment - maybe too stable, if we look at the quality of the service in this area and compare it with other industry sectors (Mogicato und Schwabe 2009). Pilot projects are needed in this area because examples of best practices are particularly powerful mechanisms for change. These pilot projects should develop appropriate processes and ITsystems and demonstrate their benefit to the citizens and the public administration. They would then allow IS-researchers to have a better understanding of how to design socio-technical systems that elicit hidden needs and co-create personalized solutions.

\footnotetext{
* authors appear in alphabetical order; both have contributed much to this paper
} 
Table 1: Requirements

Organizational Requirements

Provide one stop advisory

Separate incidents from problems

Process Requirements

Start advisory process when "being pregnant" instead of after "baby is born"

Provide standardized, but customizable process

Include support to elicit the citizens' implicit needs

Train IT-enable advisory work practice

\section{Information and IT Requirements}

Integrate advisory process with offline and online information resources

Preserve citizens' information, if he/she agrees.

Integrate local, regional, state and federal information

Integrate internal and external information.

Improve comprehensiveness of information

Provide useful, easy to use, transparent and fun support

\section{References}

Belkin, N J, Oddy, R, Brooks, H (1982) Ask for information retrieval. Part 1:

Background and theory. Journal of Documentation, 38, 61-71.

Ellis, D; Wilson TD; Ford, N; Foster, A; Lam HM, Burton, R; Spink, A (2002)

Information Seeking and Mediated Serching. Part 5. User-Intermediary

Interaction. In: Journal of the American Society for Informaton Science and Technology, 11, 883-893.

Lenk, K; Klee-Kruse, G (2000) Multifunktionale Serviceläden. Ein Modellkonzept für die öffentliche Verwaltung im Internet-Zeitalter. Berlin.

Mogicato, R; Schwabe, G (2009) Beratungsqualität in Banken. Was der Kunde erwartet. Was der Kunde erlebt. Solution Providers, Dübendorf, Switzerland.

Steele, J (1996) Information for Citizenship in Europe, Policy Studies Institute, London. 


\title{
Skizzierung von Front- und Back-Office- Architekturprinzipien des E-Governments
}

\author{
Konrad Walser, Reinhard Riedl \\ PEG - Kompetenzzentrum Public Management und E-Government, \\ Berner Fachbochschule
}

Zur Frage der Interoperabilität zwischen Front- und Back-Office-Architekturbereichen existieren bis anhin wenige überzeugende Konzepte im E-GovernmentUmfeld, obwohl dieses Thema heute etwa anlässlich der EU-DienstleistungsRichtlinien-Einführung oder von 1-Stop-Shop-Konzepten von besonderer Bedeutung ist. Im Langbeitrag wird detailliert ein entsprechendes Architekturkonzept entwickelt und dazu gehörige Schnittstellen und wesentliche Architekturmodule oder Domänen erläutert. Das zentrale Interoperabilitäts- und Architekturmodell zur Front- und Back-Office-Integration hat folgende Ausprägung (vgl. Abbildung 1).

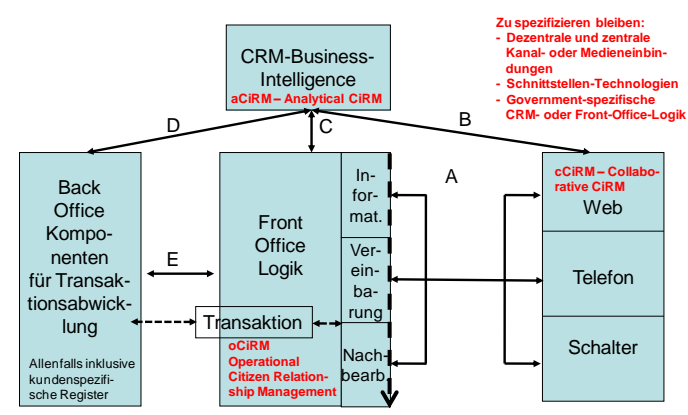

Abbildung 1: CiRM-Komponenten- und -Integrationsmodell.

Es werden darin einerseits die Module für die Kommunikation mit den Endkunden (Bürger, Unternehmen, Verwaltungseinheiten, Stakeholder) unterschieden (kommunikatives CiRM oder Citizen Relationship Management). Zudem kommen operative CiRM-Systeme zum Einsatz, in welchen die für die Kundenbearbeitung relevante Beratungs- und CiRM-Logik enthalten ist, da Front Offices umfassende Bürger-, Unternehmens- und Stakeholderberatungs-Logiken erfordern (da in der Regel das Front Office vom Back Office losgekoppelt ist). Diese sind zudem zu ergänzen um Geschäftsvorfallkontroll-Komponenten und Komponenten zur Kommunikationskanalsteuerung, zur Registereinbindung sowie zur Geschäftsvorfallunterstützung und zur Überwachung derselben. Unter das Back Office sind entsprechend ressortspezifische Informationssysteme zu subsumieren, die kanalunabhängig und geschäftsvorfallspezifisch ans Front Office anzubinden sind. Ak- 
tuelle Interoperabilitätstechnologien ermöglichen hier synchrone, asynchrone sowie Daten-Bulk-Load-Integrationen. Je nach Anzahl der zu integrierenden Informationssysteme und der relevanten Integrationstechnologien kommen wie erwähnt synchrone, asynchrone sowie Bulk-Loaddaten-Integrationstechnologien und -topologien zum Einsatz.

In der entsprechenden Abbildung 1 werden zudem Kommunikationsgeschäftsvorfälle differenzierbar, welche alleine im Front Office verbleiben, sowie Geschäftsvorfälle mit Transaktionsfolgen in den entsprechenden ressortspezifischen Back-Office-Systemen. Nicht zu vergessen sind Geschäftsvorfälle, in welchen Abfragen aus Back Office Systemen erforderlich sind, etwas zu Informationszwecken. Ebenfalls nicht zu vergessen sind außerdem analytische Informationssysteme, über welche etwa Front- und Back Office Geschäftsvorfälle nach erforderlichen Management-orientierten Dimensionen ausgewertet werden können. Dies kann im Rahmen der EU-Dienstleistungsrichtlinie etwa dafür erforderlich sein, um Termineinhaltungen der Geschäftsvorfälle gegenüber Endkunden in Front- und Back Office zu überwachen und dem Verwaltungsmanagement im Front Office Auswertungen zur Performance der Arbeitsleistung zur Verfügung zu stellen.

Im Beitrag erfolgt eine Differenzierung verwaltungsorientierter Anforderungen an Front- und Back Office. Dies lässt eine Konkretisierung von unterschiedlichen Informationssystemtypen zu. Diese bilden die Grundlage für die Gliederung und Bildung von modularen Unternehmensarchitekturen. Dadurch können Interoperabilitätsbedarfe in und zwischen den Modulen konkretisiert werden. Diese ermöglichen wiederum eine Diskussion möglicher Interoperabilitäts-Infrastrukturen, welche zur Befriedigung der Interoperabilitätsbedarfe eingesetzt werden. Im Laufe der Argumentation wurden Prinzipien für die Unternehmensarchitekturgestaltung und die darin relevante Differenzierung in Front- und Back Office Unternehmensarchitekturen konkretisiert. Es wird ferner auf die Mehrdimensionalität des Interoperabilitätsproblems im Front- und Back Office eingegangen, was die entsprechende Komplexität generiert, mit welcher Entscheidungsträger, IT-Architekten sowie Organisatoren im E-Government konfrontiert sind. Der Ausblick auf weitere Forschungsbedarfe im Themenbereich lautet: (1) Weitergehende Konkretisierung von Geschäftsvorfallkategorien und Use Cases; (2) Ableitung von Integrationsmustern aus Geschäftsvorfalltypen und Use Cases; (3) Differenzierung von internen gegenüber externen Interoperabilitätsbedarfen; (4) Weitergehende Konkretisierung und Differenzierung von Front- und Back Office Architekturgestaltungsprinzipien; (5) Entwicklung und Darstellung eines Vorgehensmodells zur Front- und Back Office Integrationsproblematik; (6) Empirische Studien im thematisierten Bereich zur Erkenntnisvalidierung und dem Design von Front- und Back Office Architekturen in der Praxis. 


\title{
Web 2.0 als Potential für die Verwaltungs- modernisierung am Beispiel der Fortschreibung einer Verwaltungsvorschrift mittels Wiki
}

\author{
Tanja Röchert-Voigt, Norbert Gronau \\ Lehrstubl für Wirtschaftsinformatik und Electronic Government, \\ Universität Potsdam
}

Die Kommunalverwaltung besteht zu $75 \%$ aus Binnenprozessen (Jakobs-Woltering 2008, S. 123). Interne Prozesse bergen daher rein prozentual bereits viel mehr Potential als externe, wenn es darum geht, Verwaltungsprozesse zu modernisieren. Im erfolgreichen Fallbeispiel konnten Potentiale von Web 2.0 im Hinblick auf die Rechtsentwicklung innerdienstlicher Verwaltungsvorschriften und damit einhergehender Entbürokratisierung und Transparenz interner Verwaltungsabläufe aufgezeigt werden.

Die innerdienstliche Geschäftsordnung der Landeshauptstadt Potsdam ist ein Regelwerk und Instrument der Verwaltungsführung, das Regeln für Bearbeitungsprozesse und Verantwortlichkeiten festgelegt und die hierarchische Organisation der Verwaltung dadurch nachprüfbar und berechenbar macht (Bogumil und Jann 2009, S. 141). Das hauptsächliche Problem solcher innerorganisatorischer Regelwerke liegt aber darin, dass sie - einmal beschlossen - solange gültig sind, bis sie explizit widerrufen oder durch neue Regelungen ersetzt werden. Änderungen technologischer, rechtlicher oder organisatorischer Art können im Lauf der Zeit dazu führen, dass sich Abläufe behindern oder schlimmstenfalls widersprechen, so dass eine effiziente Abwicklung der Verwaltungsabläufe nicht mehr möglich ist.

Der herkömmliche Ablauf zur Fortschreibung der Dienstordnung, der im Rahmen der Anforderungsanalyse aufgenommen worden ist, ist nicht gesetzlich statuiert, sondern lediglich innerdienstlich festgelegt. Die zuständige Stelle erstellt einen Entwurf, zu dem dann die Fachbereichs- und Bereichsleiter, sowie der Personalrat im innerbehördlichen Beteiligungsverfahren Stellung nehmen können. Eine direkte Kommunikation der Führungskräfte in diesem Verfahren ist aufgrund der hierarchischen Organisation der Verwaltung (Dienstweg) nicht vorgesehen. Bevor die abgestimmte Beschlussvorlage dem Oberbürgermeister zur Ausfertigung vorgelegt werden kann, sind gegebenenfalls mehrere Abstimmungsrunden im Beteiligungsverfahren notwendig. Dieses Verfahren wird von den Beteiligten als langwierig und arbeitsintensiv bezeichnet.

Der von O’Reilly (2005, S. 1-16) geprägte Begriff Web 2.0 steht einerseits für ein offenes Technologiekonzept, im Rahmen dessen Personen, Daten und Dinge verknüpft werden können und andererseits für ein Organisationskonzept, das 
hauptsächlich auf Vernetzung von Personen, Organisationen und Wissen basiert. Für die Unterstützung der Zusammenarbeit im innerbehördlichen Beteiligungsverfahrens der Verwaltung wurde das Kollaborations- und Wissensmanagementwerkzeug Wiki in Form einer OpenSource-Lösung - MediaWiki-Software mit MySQLDatenbank - ausgewählt. Die technischen Anforderungen, die im Rahmen eines Workshops erarbeitet worden sind, mündeten in einen umfangreichen Kriterienkatalog, der Basis für ein intensives Auswahl- und Testverfahren war.

Die praktische Erprobung erfolgte in zwei Stufen, an denen jeweils zuvor geschulte Mitarbeiter auf Grundlage eines speziellen Rechtskonzepts Änderungswünsche und Stellungnahmen zum Entwurf der Dienstordnung einbringen konnten. Im Rahmen der Evaluierung wurden als wesentliche Ergebnisse festgehalten, dass alle Mitarbeiter aufgeschlossen der neuen Verfahrensweise gegenüber standen und das Wiki aktiv zur Fortschreibung genutzt haben. Durch die Volldigitalisierung des Verfahrens wurde sowohl die Auswertung als auch die abschließende Erstellung einer abgestimmten Beschlussvorlage wesentlich erleichtert und verkürzt. Ferner wurde durch die digitale Zusammenarbeit eine Unmittelbarkeit der Kommunikation gleichrangiger Stellen erreicht, die in der hierarchischen Struktur der Verwaltung aufgrund des Dienstweges innerhalb des Beteiligungsverfahrens sonst nicht möglich gewesen wäre. Die bewusste Rechtevergabe im Wiki hatte darüber hinaus Einfluss auf die konkrete Umsetzung festgeschriebener Aufgabenzuständigkeiten und mithin auf die gelebte Verwaltungskultur. Eine Aufgabendelegation war nicht mehr möglich.

Der Einsatz eines Wikis zur Fortschreibung einer innerdienstlichen Verwaltungsvorschrift ist daher ein neuer, innovativer Ansatz in Richtung digitale Verwaltung, Abbau innerbehördlicher Bürokratie und Schaffung von innerbehördlicher Transparenz.

\section{Literatur}

Bogumil J, Jann W (2009): Verwaltung und Verwaltungswissenschaft in Deutschland. Verlag für Sozialwissenschaften, Wiesbaden.

Jakobs-Woltering P (2008) Web 2.0-Anwendungen und ihre Einsatzmöglichkeiten. In: Habbel FR, Huber A (Hrsg.) Web 2.0 für Kommunen und Kommunalpolitik - Neue Formen der Öffentlichkeit und der Zusammenarbeit von Politik, Wirtschaft, Verwaltung und Bürger. VWH, Boizenburg.

O’Reilly T (2005) What is Web 2.0. Design Patterns and Business Models for the next Generation Software. Http://oreilly.com/web2/archive/what-is-web20.html. Abruf am 2009-09-16 


\title{
E-Partizipation
}

\section{Was bringt das „E“ der Partizipation?}

\author{
Daniel Veit, Manuel Trenz. \\ Dieter Schwarz. Stiftungslehrstubl für ABWL, \\ E-Business und E-Government, \\ Universität Mannheim
}

Im Grundgesetz der Bundesrepublik Deutschland ist verankert, dass alle Gewalt vom Volk ausgeht (Art. 20, GG). Zudem hat ,jedermann hat das Recht, sich einzeln oder in der Gemeinschaft mit anderen schriftlich mit Bitten oder Beschwerden an die zuständigen Stellen oder an die Volksvertretung zu wenden“ (Art. 17, GG). Dies ist nicht allein als problemorientierte Beschwerdefunktion zu verstehen, sondern schließt auch eine Beteiligung des Bürgers an den politischen Prozessen mit ein. Die Festlegung von Umfang und Ausgestaltung dieser Partizipation ist die Herausforderung, die politische Entscheidungsträger überwinden müssen.

Diese Arbeit nähert sich dem Thema in zwei Schritten. Zunächst wird Partizipation generell behandelt, um darauf aufbauend anschließend den Fokus auf Partizipation unter Zuhilfenahme von Informations- und Kommunikationstechnologien zu legen.

Von diesen Erkenntnissen ausgehend wird, basierend auf einer Kosten/Nutzenrechnung, eine Balanced Scorecard für die Bewertung und Gestaltung der Einführung von elektronischer Partizipation vorgeschlagen.

Arnstein (1969) unterscheidet acht Stufen der Partizipation, aufgeteilt in drei Klassen (vgl. Abb. 1). Am unteren Ende seines Kontinuums stehen die Stufen ,Manipulation' und ,Therapie‘.

Wirkliche Partizipation findet erst auf den obersten drei Stufen, ,Partnerschaft', ,Ermächtigung ${ }^{\natural}$ und ,Bürgerherrschaft ${ }^{\star}$ statt. Am Ende des Spektrums befindet sich schließlich die Stufe der ,Bürgerherrschaft'.

Informations- und Kommunikationstechnologien (IKT) haben das Potential, das Verhältnis zwischen Bürgern und Staat zu verändern. Der Begriff E-Partizipation „bezieht sich auf Bemühungen, eine breitere und tiefer gehende politische Beteiligung zu erwirken, indem Bürger sich mithilfe von IKT untereinander, mit Beamten sowie mit gewählten Repräsentanten vernetzen“ (O'Donnell et al. 2007). 


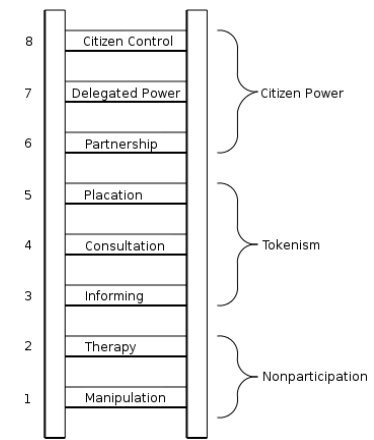

Abbildung 1: Die acht Stufen der Bürgerbeteiligung (Arnstein 1969)

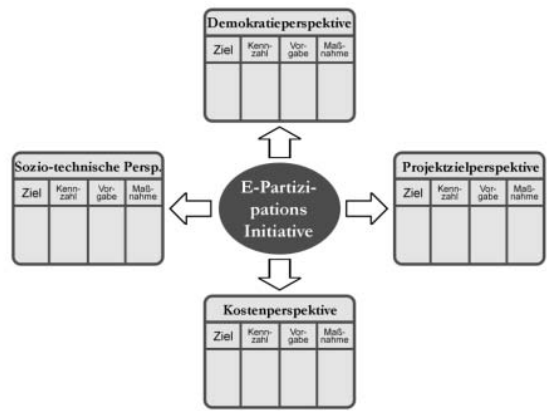

Abbildung 2: E-Partizipations Balanced Scorecard

Im Sinne einer vollständigen Evaluation dürfen allerdings Kostenaspekte nicht außer acht gelassen werden. Ein nutzenstiftender Einsatz von E-PartizipationsWerkzeugen ist nicht in jedem Fall kosteneffizient. Wir schlagen daher vor, bestehende Ansätze zur Bewertung von E-Partizipationsprojekten zu erweitern und mit dem Konzept der Balanced Scorecard (BSC) (Kaplan und Norton 1992) zu verknüpfen (vgl. Abb. 2).

Die Hoffnungen, dass die Nutzung von Informations- und Kommunikationstechnologie die Probleme klassischer Partizipation auslöschen und die Bürgerbeteiligung auf ein neues Niveau bringen könnten, haben sich bisher nicht bestätigt. Dennoch existieren vielfältige Werkzeuge, mit denen eine effizientere Beteiligung einer größeren Anzahl von Bürgern ermöglicht werden kann.

Das vorgestellte Modell der E-Partizipations Balanced Scorecard setzt bei diesem Problem an und fördert eine strukturiertere Planung und Kontrolle von EPartizipations-Initiativen.

In einer weiteren Arbeit muss die Anwendbarkeit der E-Partizipations Balanced Scorecard in Form von Fallstudien überprüft werden.

\section{Literatur}

Arnstein SR (1969) A Ladder of Citizen Participation. JAIP 35(4):216-224.

Kaplan RS, Norton DP (1992) The Balanced Scorecard - Measures that Drive Performance. Harvard Business Review:71-79.

O'Donnell D, McCusker P, Fagan GH, Newman D, Stephens S, Murray M (2007)

Navigating between Utopia and Dystopia in the Public Sphere through

eParticipation: Where is the Value? 


\title{
Elektronische Verfahrensabwicklung von G2B-eServices: Vorgehensmodell und Anwendungs- fall zur Umsetzung der Dienstleistungsrichtlinie
}

\author{
Frank Hogrebe', Beate van Kempen ${ }^{1}$, Markus Nüttgens ${ }^{2}$ \\ ${ }^{1}$ Dezernat für Organisation, Personal, IT und Wirtschaftsförderung, Düsseldorf \\ ${ }^{2}$ Forschungsschwerpunk.t Wirtschaftsinformatik, Universität Hamburg,
}

Vorgehensmodelle in der (Wirtschafts-)Informatik zielen auf eine durchgängige Beschreibung der Entwicklungs- und Einsatzphasen von Informationssystemen in Wirtschaft und Verwaltung. Sie bilden damit auch die Grundlage zur systematischen Realisierung elektronischer Verfahrensabwicklungen von unternehmensbezogenen Dienstleistungen in öffentlichen Verwaltungen (G2B-eServices).

Der Beitrag beschreibt ein domänespezifisches Vorgehensmodell zur Umsetzung der elektronischen Verfahrensabwicklung zur EU-Dienstleistungsrichtlinie (EU-DLR). Nach Diskussion der Anforderungsbereiche für öffentliche Verwaltungen zur Umsetzung der EU-DLR werden verwandte Arbeiten unter diesem Fokus untersucht. Das im Beitrag vorgeschlagene Vorgehensmodell basiert dabei auf Konzepten der integrierten Produkt- und Prozessmodellierung (Hogrebe und Nüttgens 2008). Ansätze domänespezifischer Vorgehensmodelle zu Teilbereichen der EU-Dienstleistungsrichtlinie finden sich bei (DIN FB 158 2009; DOL 2008).

Der aus Kundensicht relevante Phasenschwerpunkt zur technischen Realisierung der elektronischen Verfahrensabwicklung von G2B-eServices (EUKommission 2006, Art. 8) ist in Anwendungsfällen projektbezogen zu konkretisieren und zu validieren; solche Arbeiten sind bisher nicht publiziert. Der vorliegende Beitrag legt hierauf seinen Schwerpunkt. Abbildung 1 zeigt das erarbeitete Vorgehensmodell im Überblick. Es umfasst vier Phasen mit jeweils korrespondierenden Teilaktivitäten. Am Anwendungsfall einer deutschen Großstadt wird das Modell im Rahmen der Umsetzung der EU-DLR angewandt. 


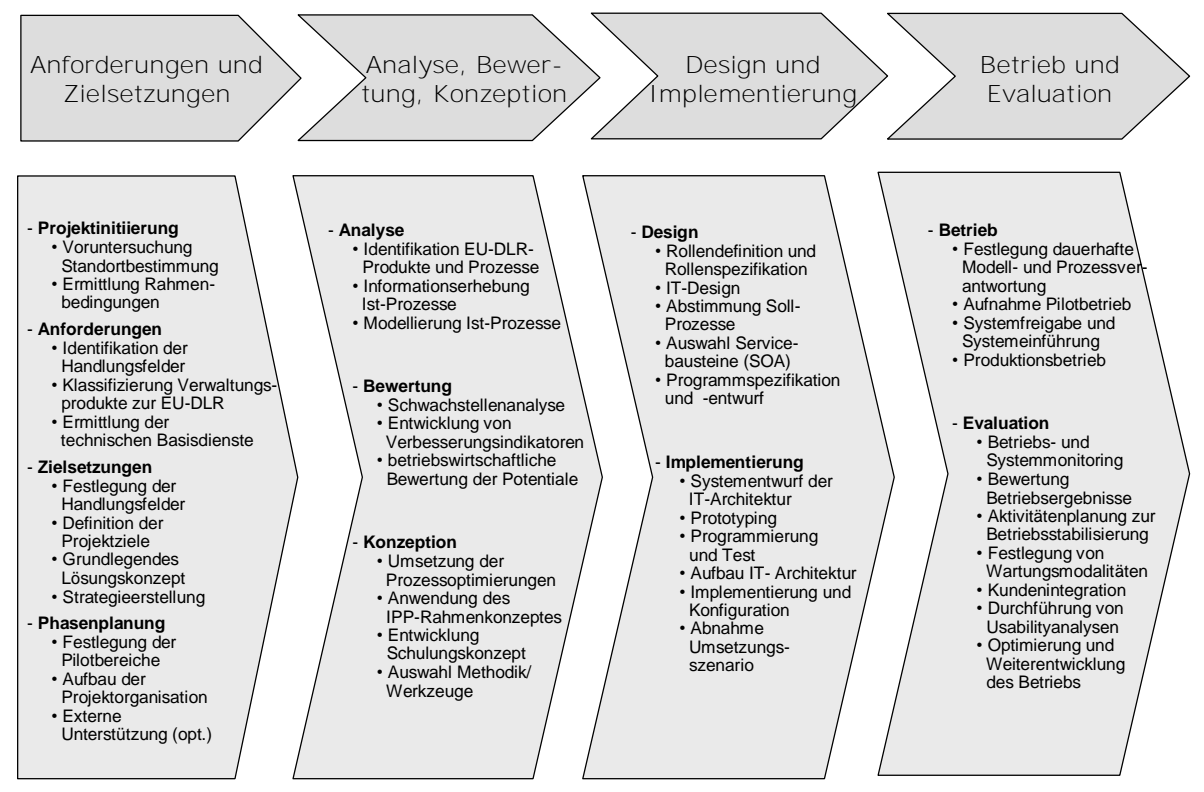

durchgehende Dokumentation und Projektmanagement-Aktivitäten

Abbildung 1: EU-DLR-Vorgehensmodell zur elektr. Verfahrensabwicklung

\section{Literatur}

Deutschland-Online-Vorhaben. IT-Umsetzung der Europäischen

Dienstleistungsrichtlinie. Projektbericht (Stand: 24.09.2008).

http:/ /www.deutschland-online.de/DOL_Internet/broker.jsp?uMen=

90040b06-56c2-3021-688d-941ac0c2f214. Abruf am 23.11.2009.

DIN FB 158:2009-09. DIN-Fachbericht 158. Modell zum prozessorientierten

Vorgehen in der öffentlichen Verwaltung. DIN Deutsches Institut für

Normung, Beuth Verlag, Berlin, 2009.

EU-Dienstleistungsrichtlinie: Europäisches Parlament und Europäischer Rat,

Richtlinie 2006/123/EG über Dienstleistungen im Binnenmarkt vom

12.12.2006, in: Amtsblatt der Europäischen Union, L 376/36, Brüssel, 2006.

Hogrebe, F.; Nüttgens, M.: Integrierte Produkt- und Prozessmodellierung:

Rahmenkonzept und Anwendungsfall zur EU-Dienstleistungsrichtlinie, in:

Loos, P.; Nüttgens, M.; Turowski, K.; Werth, D. (Hrsg.): MobIs 2008 -

Modellierung betrieblicher Informationssysteme: Modellierung zwischen SOA und Compliance Management, Proceedings GI-Tagung, Saarbrücken, 2008. 


\section{E-Learning, M-Learning und Wissensmanagement (ELMW)}





\title{
Vorwort zur Teilkonferenz
}

\section{E-Learning, M-Learning und Wissensmanagement (ELMW)}

\author{
Michael H. Breitner', Christine Voigtländer² \\ ${ }^{1}$ Institut für Wirtschaftsinformatik, Leibniz, Universität Hannover \\ ${ }^{2} \mathrm{HR}$ Transformation and Enablement, SAP AG, Niederkassl-Rheidt
}

Technologiebasiertes, teils mobiles Lehren und Lernen (anglizistisch E(lectronic)bzw. M(obile)-Learning) hat in den letzten Jahren einen deutlich höheren Reifegrad erreicht. Die Diskussion um den nachweisbaren Mehrwert des Technologieeinsatzes sowie die Forderung nach ganzheitlichen Ansätzen prägen dabei den aktuellen Diskurs. So wird zunehmend auch an den Hochschulen die Diskussion einer Professionalisierung im Kontext des gesamt Sinnvollen geführt. Lehrangebote und Lernszenarien werden verstärkt als Mehrwert-Szenarien begriffen. Diese müssen einerseits ein gutes Kosten-/Nutzenverhältnis aufzuweisen und gemäß der Forderung nach Nachhaltigkeit mittel- bis langfristig betriebswirtschaftlich erfolgreich sein. Andererseits steht zunehmend die Erhöhung der Attraktivität der eigenen Hochschule im internationalen Wettbewerb im Vordergrund. So bewerten Studierende das Angebot an innovativen IT-unterstützten Vorlesungen durchweg als positiv und sehen vermehrt den portalbasierten Zugriff auf das Informations- und Serviceportfolio einer Hochschulen als wichtiges Argument für die Studienplatzwahl, gerade im europäischen Kontext. So werden auch E-Learning, M-Learning oder Wissensmanagement in vielen Fällen nicht mehr als separate Themenfelder behandelt. Einen immer wichtigeren Stellenwert nimmt so das Zusammenwachsen des Lernmanagements mit dem Wissensmanagement, der Personalentwicklung und dem Staffing ein.

Diese ganzheitliche, mehrwertorientierte Entwicklung nehmen auch die eingereichten Beiträge auf. Sie dokumentieren, dass Hochschulen sich zunehmend mit einer ganzheitlichen, prozessorientierten (IT-)Unterstützung ihrer Organisation auseinander. So dient die Diskussion um eine solche ganzheitliche Hochschul-IT nicht selten dazu, die eigene Organisation einem kritischen Prozess-Reengineering $\mathrm{zu}$ unterwerfen, die Prozesse u.a. an den Anforderungen der BolognaImplementation auszurichten und auf dieser Grundlage den Mehrwert einer integrierten IT-Unterstützung zu realisieren. Ein weiterer wichtiger Impuls in den angenommenen Artikeln liegt in der verstärkten Bemühung um Internationalisierung im europäischen Kontext. Eine ganzheitliche, strategisch ausgerichtete HochschulIT kann so bei der politisch geforderten Umsetzung eines EU-weiten Hochschulraums als Chance nicht zuletzt für die deutschsprachigen Hochschulen zur Steige- 
rung von Bekanntheitsgrad und Attraktivität genutzt werden. Beispielhaft genannt seien dabei die Vereinfachung des Zugangs für internationale Studierende über ein zentrales Hochschulportal oder das Engagement in einem Hochschulnetzwerk zur Schärfung des eigenen Profils mittels übergreifender, E(lectronic)-Learningunterstützter Lehr- und Lernangebote. Die Rahmenbedingungen für die Realisierung solcher Mehrwert-orientierter Szenarien vereinen aktuell eine Vielzahl von begünstigenden Faktoren. So ist der politischer Wille auf EU- bzw. nationaler Ebene vorhanden, einen Europäischen Hochschulraum auf- und auszubauen. Studierende weisen eine hohe Mobilität und IT-Affinität auf. Sie präferieren u.a. internetbasierte Informations- und Recherchemöglichkeiten und stehen Lernkonzepten wie Ubiquitious Learning, positiv gegenüber. Die angenommenen Beiträge nehmen diese Tendenzen auf und verdeutlichen anschaulich, dass gerade Einrichtungen im deutschsprachigen Raum hier aktiv die Entwicklung treiben, um innovative, zukunftsorientierte Konzepte im europäischen Kontext unter Schaffung eines professionellen Umfelds zu realisieren.

\section{Einreichungen}

In Fortschreibung der vorherigen MKWI-Teilkonferenzen E(lectronic)-Learning in den Jahren 2006 und 2008 nehmen die angenommenen Beiträge wieder wichtige Trends und zukunftsorientierte Themen des E(lectronic)-/M(obile)-Learning sowie Wissensmanagements auf und diskutieren diese auf hohem aktuellen wissenschaftlichen Niveau. Aus den bis September 2009 eingereichten 13 Artikeln wurden sieben herausragende und sehr gute Beiträge aus Wissenschaft und Praxis durch einen kritischen, doppelt blinden Begutachtungsprozess ausgewählt.

Track Chairs:

Auch in diesem Jahr konnten wieder hochrangige Experten zur Leitung der Teilkonferenz gewonnen werden:

- Prof. Dr. Andrea Back, Universität St. Gallen

- Prof. Dr. Michael H. Breitner, Universität Hannover (Koordinator)

- Sünne Eichler, WEBACAD, Eschborn

- Dr. Mathias Kose, Mobilinga GmbH, Bremen

- Prof. Dr. Franz Lehner, Universität Passau

- Prof. Dr. Matthias Schumann, Universität Göttingen

- Dr. Christine Voigtländer, SAP AG, Walldorf (Co-Koordinator) 


\title{
eLectures: Vom Nebenprodukt zum strukturierten Blended Learning Arrangement
}

\author{
Helena Bukvova, Sylvie Meyer, Eric Schoop \\ Lehrstubl für Wirtschaftsinformatik, insbes. Informationsmanagement, \\ Technische Universität Dresden
}

Der Begriff „eBologna“ steht nicht nur für die Hochschulstrukturreform, sondern fokussiert auch die Nutzung der neuen Medien zur Schaffung eines zukünftigen virtuellen europäischen Bildungsraums (Vögele et al. 2005, S. 18). Der Einsatz von eLectures an Hochschulen ist nicht neu und wurde bereits in der Forschung thematisiert. In diesem Beitrag werden die videobasierten Aufnahmen als Bestandteil eines komplexen Blended Learning (bLearning) Arrangements vorgestellt.

$\mathrm{Da}$ die Hochschulbildung vor allem im Bereich der Bachelor-Studiengänge einen berufsqualifizierenden Abschluss fokussiert, ist folglich auch eine curriculare Ausrichtung auf berufliche Tätigkeiten essentiell. Aufgrund der strukturellen Ähnlichkeiten sowie dem gemeinsamen Ziel der Handlungsfähigkeit für zukünftige berufliche Tätigkeiten kann eine Orientierung der Bachelor-Studiengänge an den Konzepten der dualen Berufsausbildung befürwortet werden (Gerholz und Sloane 2008, S. 1-2). Da sowohl hinsichtlich der Inhalte und didaktischen Methoden bei bLearning-Arrangements Forschungsbedarf besteht (Mandl und Kopp 2006, S. 17), wird im Rahmen dieses Beitrags die Untersuchung zur Förderung von Handlungskompetenz für ein bLearning-Einsatzszenario am Lehrstuhl für Informationsmanagement vorgenommen.

Innerhalb des konzipierten bLearning-Arrangements werden die Studierenden in Gruppen eingeteilt. Die Teams erhalten bestimmte Themen, welche sie sich auf Basis bereitgestellter eLectures im Selbststudium aneignen. Damit aber dem viel kritisierten Entstehen trägen Wissens durch die bloße Aufnahme der digitalisierten Vorlesungsinhalte entgegen gewirkt werden kann, ist die sinnvolle Einbindung in einen Anwendungskontext notwendig. Deshalb bekommt jede Gruppe eine authentische Fallstudie, die sie analysieren müssen. Die Teammitglieder präsentieren und verteidigen ihre Ergebnisse im Plenum während den Präsenzveranstaltungen. Dadurch wird einerseits ihre Sozialkompetenz gefördert und andererseits die Generierung von neuem Wissen durch anschließende Diskussionen unterstützt. Da jedoch beim Einsatz solcher komplexen Praxisfälle die Studierenden schnell überfordert oder ineffektiv sein können, ist eine tutorielle Begleitung sowie eine instruktionale Anleitung bzw. Unterstützung durch den Dozenten notwendig. Da dieses Modul ebenfalls die Bearbeitung eines komplexen Lehr-/Lernarrangements in Form eines Projektes vorsieht, kann somit erneut eine 
Förderung beruflicher Handlungskompetenz nach konstruktivistischem Prinzip realisiert werden. Damit die Studierenden aber nicht nur Experten in ihrem zuvor bearbeiteten Themengebiet bleiben, wird in diesem Projekt die Methode des Gruppenpuzzles angewandt. Folglich bestehen die Projektgruppen aus jeweils einem Experten der vorherigen Stammteams um ein kollaboratives Lernen zu gewährleisten (siehe Abbildung 1).

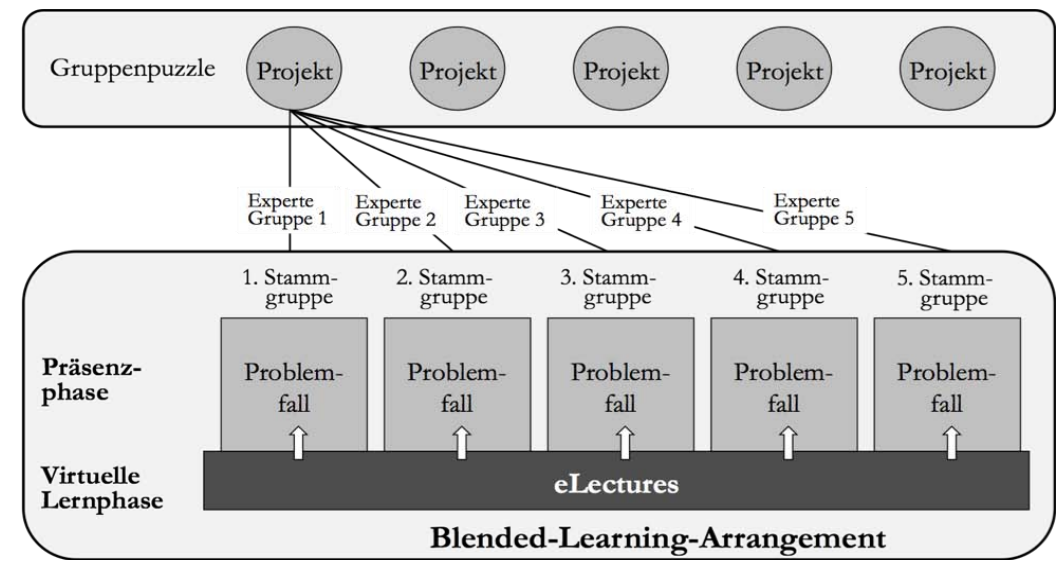

\section{Abbildung 1: Aufbau des bLearning-Arrangements am Lehrstuhl für Informations- management}

In seiner aktuellen Form ermöglicht uns das Lernarrangement die Wandlung einer klassischen lehrerzentrierten Präsenzvorlesung in eine interaktive von Lernenden mitgestaltete Veranstaltung. Unser Ziel ist es, solche Lernarrangements in der universitären Lehre zu etablieren um die Handlungskompetenz der Studierenden aktiv zu stärken.

\section{Literatur}

Gerholz KH, Sloane PFE (2008) Der Bolognaprozess aus curricularer und hochschuldidaktischer Perspektive - Eine Kontrastierung von beruflicher Bildung und Hochschulbildung auf der Bachelor-Stufe. www.bwpat.de/ausgabe14/gerholz_sloane_bwpat14.pdf. Abruf am 10.08.2009.

Mandl H, Kopp B (2006) Blended Learning: Forschungsfragen und Perspektiven. http://epub.ub.uni-muenchen.de/905/. Abruf am 2009-08-10.

Vögele E, Mohnike T, Trahasch S (2005) EUCOR VIRTUALE Herausforderungen und Lösungen von e-Bologna im Kontext einer transnationalen Hochschulkooperation. Zeitschrift für Hochschuldidaktik 5:18-27. 


\title{
Ein generisches Framework zur Erstellung von argumentationsunterstützenden Systemen
}

\author{
Frank Loll', Niels Pinkwart', Oliver Scheuer², Bruce M. McLaren² \\ ${ }^{1}$ Institut für Informatik, Technische Universität Clausthal \\ ${ }^{2}$ Deutsches Forschungszentrum für Künstliche Intelligenz, \\ Universität des Saarlands
}

Argumentationsfähigkeiten spielen in vielen beruflichen Kontexten eine zentrale Rolle - z. B. bei der Arbeit und Entscheidungsfindung in Teams, der Leitung von Arbeitsgruppen, der Kommunikation von Entscheidungen oder bei Verhandlungen mit Geschäftspartnern. Dennoch sind die Argumentationsfähigkeiten vieler Menschen nur schwach ausgeprägt. Studien zeigen, dass viele Personen (auch aus gebildeten Schichten) Probleme dabei haben, Behauptungen von Argumenten zu unterscheiden, Angriffe auf ihre eigenen Argumente zu erkennen und angemessen darauf zu reagieren (Tannen 1998, Easterday et al. 2009). Folgerichtig nimmt die Ausbildung von Argumentationsfähigkeiten eine zentrale Rolle in der Lehre ein (Andriessen 2006) - sowohl im schulischen Klassenzimmer und der Berufsbildung als auch bei der professionellen Weiterbildung, z.B. im Bereich der Verhandlungsführung und Teamleitung. Klassische face-to-face basierte Lehransätze, insbesondere in einem 1:1 Verhältnis zwischen Lehrendem und Lernendem, haben sich hierbei zwar als effektiv erwiesen (Bloom 1984; Kulik \& Kulik 1991), sind jedoch nicht ohne weiteres auf größere Gruppen übertragbar.

Um dieses Defizit auszugleichen, wurden in den letzten Jahren verstärkt argumentationsunterstützende eLearning-Systeme eingesetzt. Hierbei wurden unterschiedliche Ansätze verfolgt: Einerseits wurden Programme entwickelt, die lediglich dazu dienen, Argumentationsstrukturen unterschiedlich zu visualisieren (Kirschner, Buckingham Shum \& Carr 2003). Andererseits wurden Applikationen entwickelt, die Lernende mittels intelligenter Analysemethoden durch geeignetes Feedback in der Akquisition von Argumentationsfähigkeiten unterstützen. Nichtsdestotrotz sind die bestehenden Systeme in ihren Möglichkeiten beschränkt. Zu diesen Einschränkungen gehören unter anderem die mangelnde Generalisierbarkeit bzw. Überspezialisierung einzelner Systeme, die in Teilen mangelhafte Unterstützung für kooperatives Arbeiten mehrerer Nutzer, die fehlende Flexibilität mit Blick auf die zugrunde liegende Ontologie, die eingeschränkte Interoperabilität zwischen einzelnen Systemen sowie das Fehlen einer allgemeinen Methodik zur Entwicklung von argumentationsunterstützenden Systemen.

Ausgehend von diesen Schwachstellen und einer Anforderungsanalyse für ein generisches Framework zur Erstellung von argumentationsunterstützenden Syste- 
men liegt der Schwerpunkt dieses Papers auf der Vorstellung einer Systemarchitektur, die sich den bestehenden Schwächen annimmt. Die Architektur ermöglicht es, mit minimalem Konfigurationsaufwand neue Argumentationssysteme zu erstellen, die einerseits domänenspezifischen Anforderungen genügen, andererseits jedoch flexibel genug sind, in mehreren Domänen eingesetzt werden zu können und Unterstützung für größere Gruppen von Lernenden bieten.

Der flexible Konfigurationsmechanismus wird mit Hilfe eines Beispiels veranschaulicht und ein Ausblick auf die weitere Entwicklung des vorgestellten LASAD (Learning to Argue: Generalized Support Across Domains ${ }^{1}$ ) Frameworks gegeben.

\section{Literatur}

Andriessen J (2006). Arguing to learn. Sawyer RK (Ed.) The Cambridge Handbook of the Learning Sciences, pp. 443-460. Cambridge.

Bloom BS (1984). The 2 sigma problem: The search for methods of group instruction as effective as one-to-one tutoring. Educational Researcher, 13:316.

Easterday MW, Aleven V, Scheines R, Carver SM (2009). Will Google destroy western democracy? Bias in policy problem solving. In Proceedings of the Intl. Conf. on Artificial Intelligence in Education, pp. 249-256. IOS Press, Amsterdam.

Kirschner PA, Buckingham Shum SJ, Carr CS (2003). Visualizing argumentation. Software tools for collaborative and educational sense-making. Springer, London.

Kulik CC, Kulik JA (1991). Effectiveness of computer-based instruction: An updated analysis. Computers in Human Behavior, 7:75-95.

Tannen D (1998). The Argument Culture: Moving from Debate to Dialogue. New York: Random House Trade.

${ }^{1}$ Weitere Informationen unter: http://lasad.dfki.de 


\title{
Gestaltung virtueller kollaborativer Lernprozesse in internationalen Settings
}

\author{
Helena Bukvova3, Christian Lehr', Claudia Lieske3, \\ Peter Weber ${ }^{2}$, Eric Schoop ${ }^{3}$
}

${ }^{1}$ Lehrstuhls für Betriebswirtschaftslehre, insh. Allgemeine Berufsvorbereitung im Kompetenzbereich „Organisation und Management“, Freie Universität Berlin

\section{${ }^{2}$ Lehrstubl für Wirtschaftsinformatik, Rubr-Universität Bochum}

\section{${ }^{3}$ Lehrstubl für Wirtschaftsinformatik, insbes. Informationsmanagement, Technische Universität Dresden}

Die Möglichkeiten zur netzbasierten Zusammenarbeit stellen einen in Theorie und Praxis zunehmend wichtigen Aspekt dar und werden entsprechend auch in der Lehre und speziell in E-Learning-Settings immer häufiger thematisiert (vgl. Bodemer et al. 2009; Ehsan et al. 2008; Hasan und Ali 2007; Stahl et al. 2006). Neben einer zeitlich und räumlich flexibleren sowie einer stärker an individuellen Ansprüchen ausgerichteten Auseinandersetzung mit den Inhalten lassen sich in virtuellen kollaborativen Settings u.a. insbesondere Medienkompetenzen fördern und motivationale Impulse setzen. Darüber hinaus wird ein Lernraum für die Förderung kommunikativer und sozialer Kompetenzen geschaffen und eine Ausrichtung der Lemprozesse auf die im späteren Berufsleben essenzielle Zusammenarbeit in heterogenen und räumlich verteilten Teams gefördert.

In unserem Beitrag werden zwei Beispiele für internationale kollaborative Lernarrangements dargestellt. Bereits in früheren Semestern konnten die betrachteten Veranstaltungen eine erkennbare Motivationssteigerung und eine aktivere Auseinandersetzung mit den Lerninhalten aufseiten der Teilnehmer/-innen im Vergleich zu klassischen Lehrveranstaltungen bewirken (Bukvova et al. 2006a; Bukvova et al. 2006b, Gabriel et al. 2009; Liske et al. 2009). Der internationale Charakter sowie der gezielte Einsatz neuer IuK-Technologien bedingen einen deutlichen Mehrwert sowohl für die Studierende als auch für die Lehrenden.

Dennoch zeigten die ersten Durchläufe, dass virtuelle kollaborative Lernszenarien auch neue Herausforderungen mit sich bringen, denen es sich zu stellen gilt und denen mit den vorgestellten Anpassungen und Neuerungen der Veranstaltungskonzeption begegnet werden soll.

Unter Bezugnahme auf die Literatur wird daher eine Auswahl an typischen Herausforderungen bei der Gestaltung entsprechender Lernprozesse abgeleitet. 
Diesen werden konkrete Lösungsansätze gegenübergestellt, die in Vorbereitung einer erweiterten und kombinierten Fassung der Settings für das Wintersemester 2009/2010 entwickelt wurden. Der Beitrag verfolgt damit das Ziel, Erfahrungen sichtbar zu machen und darauf aufbauend Lösungsansätze für typische Problembereiche und Gestaltungsnotwendigkeiten aufzuzeigen.

\section{Literatur}

Bodemer D, Gaiser B, Hesse FW (2009) Kooperatives netzbasiertes Lernen. In: Issing LJ, Klimsa P (Hrsg) Online-Lernen. Handbuch für Wissenschaft und Praxis. Oldenbourg, München. S. 151-158.

Bukvova H, Gilge S, and Schoop E (2006a) Enhancing the framework for virtual collaborative learning - comparison of two case studies. In: 4th EDEN Research Workshop 2006, Castelldefels.

Bukvova H, Gilge S, Schoop E (2006b) Virtual Collaboration in Higher Education Blended Learning Arrangements. In: Virtuelle Organisationen und Neue Medien GeNeMe 2006, Dresden.

Ehsan N, Mirza E, Ahmad M (2008) Impact of Computer-Mediated Communication on Virtual Teams' Performance: An Empirical Study. World Academy of Science, Engineering and Technology 42:694-703.

Gabriel R, Gersch M, Weber P, Le S, Lehr C (2009) Enhancing Professional, Media, and Social Competencies through International Collaborative ELearning. In: Proceedings of E-LEARN 2009 - World Conference on ELearning in Corporate, Government, Healthcare \& Higher Education (accepted and forthcoming).

Hasan B, Ali J (2007) An Empirical Examination of Factors Affecting Group Effectiveness in Information Systems Projects. Decision Sci J Innovat Educ 5(2):229-243.

Lieske C, Bukvova H, Schoop E (2009) Virtual Collaborative Learning: Auf der Suche nach den Digital Natives. In: Schwill A, Apostolopoulos N (Hrsg.) Lernen im Digitalen Zeitalter,Workshop-Band, Dokumentation der PreConference zur DeLFI2009.Logos, Berlin. S. 61-68.

Stahl G, Koschmann T, Suthers D (2006) Computer-supported collaborative learning: An historical perspective. In: Sawyer RK (Hrsg): The Cambridge handbook of the learning sciences. Cambridge University Press, Cambridge. S. 409-426. 


\title{
Rapid Authoring meets Web 2.0
}

\section{Persönliche Lern- und Präsentationsumgebungen auf Basis lehr-/lernstromgesteuerter Mashups}

\author{
Stefan Hausmann, Kai-Uwe Götrelt \\ Lehrstubl Wirtschaftsinformatik II, \\ Universität Erlangen-Nürnberg
}

Im Bereich der Produktion von Lerninhalten stehen inzwischen eine Reihe sogenannter Rapid-Authoring-Werkzeuge zu Verfügung, die jedoch auf vordefinierte Präsentations- und Anordnungsräume begrenzt sind und durch ihre festgelegte Ablaufsteuerung sowie die vorgegebenen Inhalte und Werkzeuge meist nicht im Hinblick auf persönliche Bedürfnisse angepasst oder erweitert werden können.

Im Gegenzug hierzu eröffnen sich durch die Nutzung Web 2.0-basierter Inhalte und Werkzeuge neue Möglichkeiten für die Gestaltung von E-LearningAngeboten (Kerres 2006, S. 5-8). Die flexible Integration von externen Inhalten und Werkzeugen des Web 2.0 in die Lernumgebung wird dabei als eine der wesentlichen aktuellen Aufgaben angesehen (Agee und Yang 2009).

Persönliche Lernumgebungen können so konzipiert werden, dass sie sowohl traditionelle, durch Rapid-Authoring erstellte Lehr-/Lernelemente als auch externe webbasierte Ressourcen zusammenführen und so die jeweiligen Vorteile beider Welten für die Unterstützung von Lehr-/Lernprozessen nutzen (Kerres 2006, S. 7). Die hier vorgestellte und in Abbildung 1 gezeigte persönliche Lern- und Präsentationsumgebung Castible realisiert eine solche Zusammenführung wie folgt:

- Eine Integrierte Oberfläche ermöglicht, Inhalte und Dienste aus unterschiedlichen Quellen unter einer grafischen Benutzerschnittstelle zusammenzuführen (Schaffert und Kalz 2009, S. 9). Hierzu setzt das System Castible auf bestehende Webtop-Lösungen auf und bietet über ein fensterähnliches Castlet-Konzept die Möglichkeit, beliebige interne und externe Ressourcen einzubinden.

- Durch zeitlich angeordnete Befehlsobjekte wird eine funktionale Integration erzielt. Commandlets erlauben die Steuerung sämtlicher Aspekte des Lerninhalts, z. B. einer Vorlesungsaufzeichnung, sowie die Nutzung von (Teil-)Funktionalitäten eingebundener Web 2.0-Dienste, z. B. zur Manipulation und Erstellung von Inhalten.

- Lehr-/Lernströme gestatten die (An-)Steuerung der erstellten Mashups in einer gemeinsamen Zeitleiste. Abfolgen von Lernaktivitäten können so festgehalten, veröffentlicht und mit anderen Nutzern geteilt werden (Casquero 2009). 


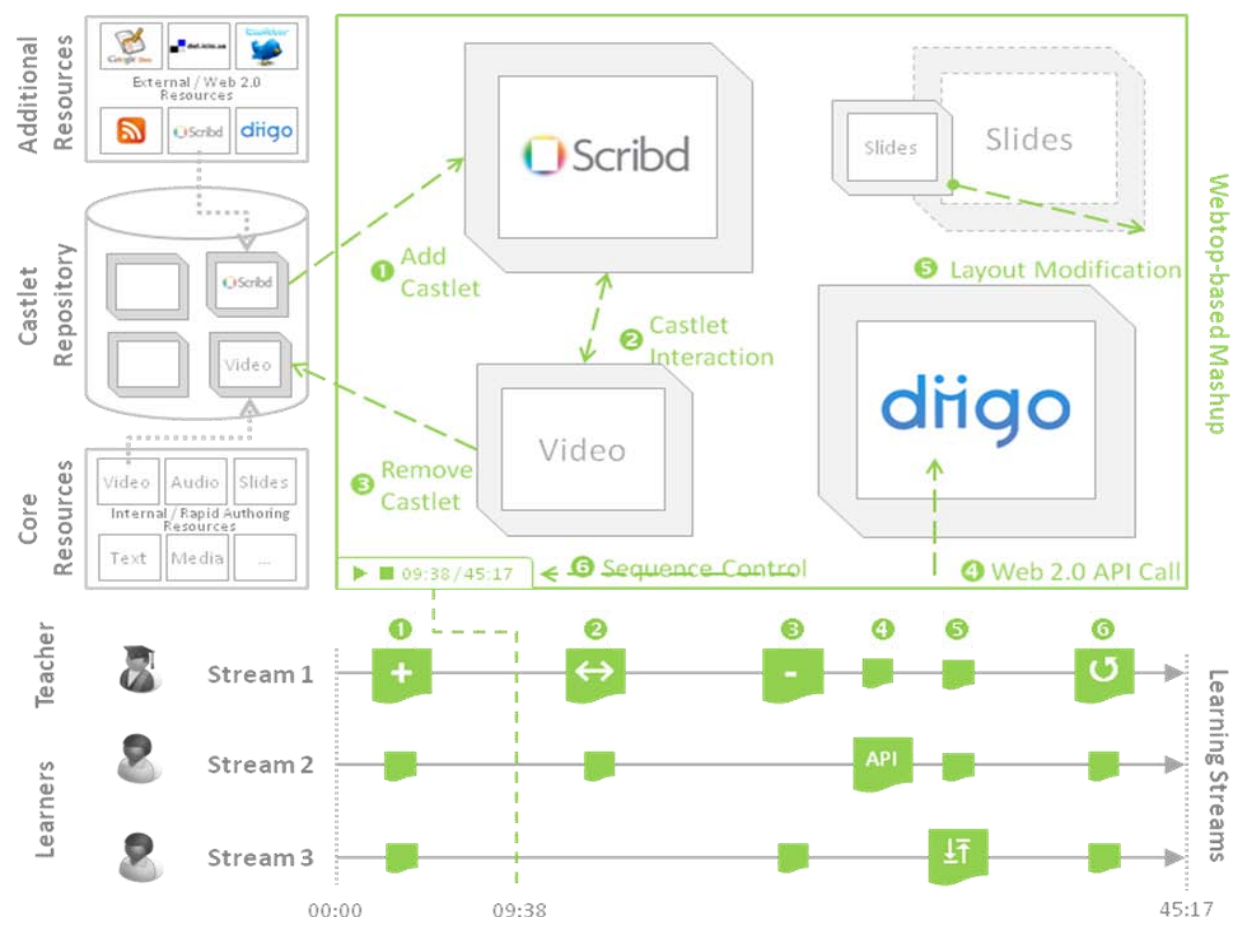

Abbildung 1: Persönliche Lern- und Präsentationsumgebung Castible

\section{Literatur}

Agee AS, Yang C (2009) Top-Ten IT Issues, 2009. EDUCAUSE Review 44(9): 44-59.

Casquero O (2009) Learn-Streaming support for Personal Learning Networks. http:/ / personallearningenvironments.wordpress.com/2009/01/24/learnstreaming-support-for-personal-learning-networks. Abruf am 2009-11-24.

Kerres M (2006) Potenziale von Web 2.0 nutzen. In: Hohenstein A, Wilbers K (Hrsg.) Handbuch E-Learning. Deutscher Wirtschaftsdienst, München.

Schaffert S, Kalz M (2009) Persönliche Lernumgebungen: Grundlagen, Möglichkeiten und Herausforderungen eines neuen Konzepts. In: Hohenstein A, Wilbers K (Hrsg.) Handbuch E-Learning. Deutscher Wirtschaftsdienst, München. 


\title{
Entwicklung und Anwendung eines Internetwerkzeugs zur Generierung von Forschungsportalen
}

\author{
Jörg Becker, Ralf Knackstedt, Lukasz Lis, Armin Stein \\ European Research Center for Information Systems (ERCIS), \\ Westfälische Wilhelms-Universität Münster
}

Unter Forschungsportalen werden themenspezifische, internetbasierte Verzeichnisse verstanden, die bereits vorhandene Informationen strukturiert zugänglich machen (Sandkuhl 2005). Die in das Portal eingestellten Beschreibungen verweisen auf originäre Quellen, in denen Forschungsergebnisse ausführlich präsentiert werden. Durch die Vorgabe von Formularen, von Kriterien zur inhaltlichen Klassifizierung sowie auf diesen Kriterien basierenden Auswertungen greifen Forschungsportale im Wissensmanagement verbreitete Konzepte wie z. B. Wissensträgerkarten, Wissensstrukturkarten, Wissensanwendungskarten und Wissensbestandskarten auf (Kilian et al. 2006, S. 222). Der Aufbau von Forschungsportalen zielt insbesondere darauf ab, zu vermeiden, dass bereits erzielte Arbeitsergebnisse unerkannt bleiben und unnötigerweise ähnliche Forschungsprojekte parallel bzw. zeitversetzt durchgeführt werden und dass Synergiepotenziale von Arbeitsgruppen unterschiedlicher Disziplinen ungenutzt bleiben, wenn potenzielle Kooperationspartner nicht voneinander erfahren.

Die Einrichtung und der Betrieb eines Forschungsportals setzt nicht nur Wissen über das adressierte Themengebiet voraus, sondern erfordert auch erhebliche informationstechnische Kompetenzen. Insbesondere beim Aufbau von Forschungsportalen für informationstechnologieferne Themenbereiche bildet das Fehlen von Kompetenzen zum Aufbau anspruchsvoller Internetportale ein bedeutendes Hindernis. Mit yourResearchPortal.com wurde eine softwaretechnische Infrastruktur entwickelt, welche die Hürden zur Implementierung und zum Betrieb von Forschungsportalen reduziert, um diesem Wissensmanagementinstrument eine größere Verbreitung zu ermöglichen.

Die Architektur von yourResearchPortal.com basiert auf zwei Hauptkomponenten. Die Datenpflegekomponente umfasst Funktionalitäten, die für das Erstellen, Betrachten, Aktualisieren und Löschen von konkreten Daten gebraucht werden. Die Analysekomponente ermöglicht einen nur lesenden Zugriff auf die gesamte Wissensbasis eines Portals. Als Datenpflegekomponente fungiert das Open Source Content Management System Drupal (http://www.drupal.org). Die Analysekomponente wird mit Hilfe des Open Source OLAP-Servers Mondrian (http://mondrian.pentaho.org/) realisiert. Beide Komponenten greifen auf die 
gleiche relationale MySQL-Datenbank (http://www.mysql.com) zu. Als Grundlage für das eingesetzte Datenmodell dienen das Referenzmodell für Forschungslandkarten von Knackstedt et al. (2009), die Ontologie für Forschungscommunities von Sure et al. (2005) sowie der europäische Datenaustauschstandard für Forschungsinformationen CERIF (http://www.eurocris.org).

Das Werkzeug yourResearchPortal.com wurde genutzt, um die „Forschungslandkarte zur hybriden Wertschöpfung", welche unter der Adresse http://www.forschungslandkarte-hybridewertschoepfung.de erreichbar ist, aufzubauen. Die Anwendung hat gezeigt, dass sich die geschaffene Infrastruktur zur Generierung themenspezifischer Forschungsportale eignet. Insbesondere mit ihren flexiblen quantitativen Analysemöglichkeiten weist die „Forschungslandkarte zur hybriden Wertschöpfung" deutliche Vorteile gegenüber anderen Forschungsportalen auf. Weitere Anwendungen sind derzeit in einem prototypischen Stadium (z. B. die Forschungslandkarte der Fachgruppe Management Support Systems der Gesellschaft für Informatik unter http://bi.yourresearchportal.com). In zukünftigen Forschungsarbeiten soll die Evaluationsbasis weiter ausgebaut werden. Hierbei sollen die Akzeptanz der Softwareinfrastruktur und einzelner ihrer Teile untersucht werden, ein Vergleich mit herkömmlich entwickelten Forschungsportalen vorgenommen werden und versucht werden, den Nutzen der Anwendung von yourResearchPortal.com zu messen.

Dieser Beitrag wurde durch die Förderung des BMBF Projektes „FlexNet“ (Flexible Informationssystemarchitekturen für hybride Wertschöpfungsnetzwerke; Förderkennzeichen 01FD0629) im Rahmen des Förderprogramms „Innovationen mit Dienstleistungen" ermöglicht. Wir danken an dieser Stelle dem Projektträger Deutsches Zentrum für Luft- und Raumfahrt (DLR) für die Unterstützung.

\section{Literatur}

Kilian D, Krismer R, Loreck S, Sagmeister A (2006) Wissensmanagement:

Werkzeuge für Praktiker. 2. Aufl. Innsbruck.

Knackstedt R, Lis L, Stein A, Becker J, Barth I (2009) Towards a Reference Model for Online Research Maps. Proceedings of the European Conference on Information Systems (ECIS 2009). Verona.

Sandkuhl K (2005) Wissensportale. Merkmale, Architekturen und Perspektiven. Informatik Spektrum 28(2):193-201.

Sure Y, Bloehdorn S, Haase P, Hartmann J, Oberle D (2005) The SWRC Ontology - Semantic Web for Research Communities. LNCS 3808:218-231. doi: 10.1007/11595014_22. 


\title{
'What are you doing' im Elfenbeinturm?' - Microblogging im universitären Einsatz
}

\section{Erfahrungen aus zwei Pilotprojekten}

\author{
Lutz Gerlach',2, Stefan Hauptmann'1,2, Martin Böhringer \\ ${ }^{1}$ Lehrstubl für Organisation und Arbeitswissenschaften, \\ Technische Universität Chemnitr. \\ ${ }^{2} \mathrm{~cm} \mid d$ - corporate mind development, Chemnitz. \\ ${ }^{3}$ Professur für Wirtschaftsinformatik II, \\ Technische Universität Chemnitz.
}

Die Microblogging-Plattform Twitter, anfangs lediglich als Selbstdarstellungsinstrument wahrgenommen, hält als Kommunikationsinstrument vermehrt auch Einzug in professionelle Arbeitskontexte. Mit der simplen, von Twitter gestellten Initialfrage „Was tust du gerade?", sowie mit einem einfachen Publikations-/ Subskriptionssystem, ermöglicht es diese Plattform, professionelle Kooperations- und Koordinationsleistungen zur Verfügung zu stellen. Denn die Initialfrage lässt sich durch beliebig konkrete Fragen ergänzen, die an ein existierendes Netzwerk gestellt werden können, z. B.: „,Wie funktioniert ...?“; „,Wo kann ich ... finden?“; etc.

Neben Twitter gibt es mittlerweile zahlreiche anderer Tools, welche die Grundprinzipien des Microblogging verwirklichen: Einfachheit, Schnelligkeit, Unverbindlichkeit, Pub-/ Sub-Vernetzung. Die Nutzung dieser Plattformen ist grundsätzlich sehr einfach; durch Methoden des Weiterleitens lassen sich Nachrichten auch in großen Netzwerken sehr schnell verbreiten; auf komplexe Kommunikationsformen, zu denen etwa auch Anreden gehören, wird explizit verzichtet.

Ein anderes herausragendes Merkmal von Microblogging ist, dass durch das Public/ Subscribe-System - im Gegensatz zur E-Mail - ein Großteil der kommunikativen Initiative dem Subskribierenden überlassen wird. Gesendet wird eine Nachricht grundsätzlich „to whom it may concern“. Nützliche Antworten kommen in einem solchen Netzwerk oftmals von unerwarteter Seite (Böhringer 2009).

Solche Erfahrungen lassen sich nach erster Evaluation auch aus zwei Pilotprojekten an der Technischen Universität Chemnitz berichten, in welchen Microblogging-Tools zum Einsatz kommen. Zwar gibt es hier - zum Einsatz kommt Communote - keine Pub/Sub-Funktionen wie bei Twitter. Allerdings gibt es vordefinierte Projekte und Mitgliedschaften. 
Im ersten Projekt vernetzen sich mehrere, aus den Bereichen Maschinenbau und Betriebswirtschaft interdisziplinär agierende Forschergruppen. Mit dem Einsatz von Microblogging soll eine höhere Transparenz geschaffen werden, als es mit EMail und Telefonkonferenzen möglich ist. Gegenseitiges Feedback wird erleuchtert, und somit Diskussionsverläufe initiiert und aufrecht erhalten. Organisatorisches sowie auch Ankündigungen werden über diese Plattform ebenso kommuniziert wie die gesamte Planung von Meetings und Konferenzen Diese Inhalte sollen auch zur Nachnutzung verwendet werden können und werden dementsprechend archiviert.

Die kommunikative Leichtigkeit des Microblogging soll es ermöglichen, eine sog. unwahrscheinliche Kommunikation wahrscheinlich zu machen (Hauptmann 2009), d. h. kommunikative Akte zu ermöglichen, wie sie als Ganggespräche bzw. als Plaudern in der Kaffeeküche bekannt, und deren Effizient in Bezug auf Informationsaustausch mittlerweile anerkannt ist - auch im Virtuellen.

Im zweiten Projekt vernetzen sich Mitarbeiter und Studierende der Professur Wirtschaftsinformatik II (Systementwicklung/ Anwendungssysteme) an der Technischen Universität Chemnitz. Für die Forschung soll somit ein kontinuierlich sich anreichernder Wissensspeicher entstehen, in welchem beispielsweise Gesprächsund Handlungsprotokolle, Meinungen, Diskussionen, Informationsschnipsel und Link-Empfehlungen abgebildet und in ihrer Historie nachvollziehbar sind. Microblogging dient darüber hinaus der Koordination der Lehre, so etwa der Betreuung von Diplom-, Seminar- und Projektarbeiten.

Erste Erfahrungen aus beiden Pilotprojekten zeigen, dass die Anzahl kommunikativer Akte im Vergleich zu trägeren und schwerer zu bedienenden Systemen als sehr hoch einzuschätzen sind. Aber auch erste wichtige Forschungsfragen hinsichtlich der Governance solcher Plattformen lassen sich bereits formulieren - so etwa bezüglich erwünschter vs. unerwünschter Transparenz.

\section{Literatur}

Böhringer M (2009): Fallstudie Enterprise Microblogging bei der Communardo Software GmbH. In: Koch M, Richter A (Hrsg.) Enterprise 2.0, Oldenbourg, München.

Hauptmann S (2009) Microblogging: Unwahrscheinliche Kommunikation wahrscheinlich machen. http://blog.cm-development.de/2009/05/18/ microblogging-unwahrscheinliche-kommunikation/ Abruf am 09.09.2009. 


\title{
Verbesserungspotentiale von Groupwareplattformen für Lehrende
}

\author{
Stefan Bitzer, Marco Klein, Bernd Pape, Matthias Schumann \\ Professur für Anwendungssysteme und E-Business, \\ Georg-August-Universität Göttingen
}

Groupwareplattformen sind im schulischen Umfeld seit einigen Jahren fest in den Schulalltag integriert (Stahl 2004, S. 163). Die Notwendigkeit hierfür liegt vor allem in drei Faktoren begründet. Zum einen wird Groupware über alle Unternehmensbranchen hinweg eingesetzt, so dass die Vermittlung von Groupware-Fähigkeiten eine Schlüsselqualifikation für erfolgreiches berufliches Handeln darstellt (Prinz 2005, S. 286). Diese Qualifikationen können nur vermittelt werden, wenn sich der Lehrende mit den entsprechenden Werkzeugen auskennt. Weiterhin wird Groupware im Rabmen von Weiterbildungen verwendet, um Schulungsmaterialien zentral zur Verfügung zu stellen und schnell distribuieren zu können. Ein dritter wichtiger Einsatz ist Nutzung von Groupware in Bildungsnetzwerken. Hier ermöglicht die Software neben dem Austausch auch einen Diskurs über Lehrmaterialien. Zusammenfassend kann festgehalten werden, dass die Nutzung einer Groupwareplattform eine fortlaufende Qualifizierung von Lehrkräften und eine Verbesserung von Lehrmaterialien ermöglicht (Morgan und Lydon 2009, S. 71). Die Akzeptanz vieler Systeme leidet aber noch an einer umständlichen Bedienung und einem nicht bedürfnisgerechten Funktionsangebot (Jiang et al. 2005, S. 570). Auch die organisatorische Einbettung der Groupware beim Einsatz in Schulen ist eine Herausforderung, der begegnet werden muss.

Der vorliegende Artikel erarbeitet Lösungsvorschläge für die oben beschriebenen Probleme, indem Verbesserungspotentiale von Groupware im schulischen Umfeld aufgezeigt werden. Das Forschungsziel ist es, Defizite entsprechender Groupware zu identifizieren und Ansätze zu deren Minderung in den Bereichen schulinterne Rahmenbedingungen, Usability und Utility zu erarbeiten. Dabei resultieren die Ansätze aus einer empirischen Studie, in der Nutzer des niedersächsischen BBS-BSCW-Groupware-Servers, einer Groupwareplattform für Lehrkörper der niedersächsischen Berufsschulen mit ca. 4.500 Benutzern, befragt wurden.

Bislang ist die Verbesserung des Groupwareeinsatzes im schulischen Umfeld aus Sicht von Lehrkräften wenig erforscht. Erkenntnisse über Anforderungen an Benutzerfreundlichkeit und Funktionalität von Groupware existieren ausschließlich aus benutzerrollenunabhängiger (bspw. Gutwin et al. 1996) oder eingeschränkten benutzerrollenbezogenen Perspektiven (bspw. Wessner et al. 1999). 
Die Studienergebnisse offenbarten interessante Ergebnisse. So zeigte sich, dass die Groupware im vorliegenden Fall hauptsächlich zur innerschulischen Gruppenarbeit eingesetzt wird und dort vorwiegend zum Dokumentenmanagement. Vielfältige andere angebotene Funktionen werden weder verwendet noch gewünscht. Als wichtige Faktoren für einen erfolgreichen Einsatz von Groupware für Lehrkräfte konnten die schulinternen Rahmenbedingungen, die Usability und die Utility identifiziert werden. Bei ersterem ist vor allem der Abbau mentaler Barrieren von hoher Bedeutung. Auch der Usability, insbesondere der Gestaltung der Interaktionsmöglichkeiten, sollte für Groupwareplattformen ein hoher Stellenwert eingeräumt werden. Des Weiteren sollte die Utility den Austausch sowie die Verwaltung von Dokumenten umfassen und diesbezüglich angepasst werden.

Weitere Verbesserungsansätze könnte eine Untersuchung der Schüler-LehrerKooperation über Groupwareplattformen ergeben, die nicht Bestandteil der vorliegenden Studie ist.

\section{Literatur}

Gutwin C, Roseman M, Greenberg S (1996) A Usability Study of Awareness Widgets in a Shared Workspace Groupware System. In: Proceedings of CSCW conference, Boston.

Jiang J, Zhang S, Li Y, Shi M (2005) CoFrame: A Framework for CSCW Applications Based on Grid and Web Services. In: Proceedings of the IEEE International Conference on Web Services (IEEE ICWS), Orlando, Florida, USA.

Morgan B, Lydon J (2009) Bologna: some thoughts on its effect on the internationalisation of higher education. Journal of Applied Research in Higher Education 1(1):63-72.

Prinz U (2005) Netzgestütztes kooperatives Lernen und Lehren in der schulischen Berufsbildung. Erziehungswissenschaft und Beruf 53(2):281-291.

Stahl G (2004) Groupware goes to school: adapting BSCW to the classroom. International Journal of Computer Applications in Technology 19(3):162-174.

Wessner M, Pfister HR, Miao Y (1999) Umgebungen für computerunterstütztes kooperatives Lernen in der Schule. In: 8. GI-Fachtagung Informatik\&Schule, Potsdam. 
Enterprise Resource Planning und Transformation von ERP-Systemen 



\title{
Vorwort zur Teilkonferenz
}

\section{Enterprise Resource Planning und Transformation von ERP-Systemen}

\author{
Sebastian Asendorf', Norbert Gronau², Karl Kurbel', \\ Peter Loos', Petra Schubert ${ }^{5}$ \\ 1 Avinway Consultants, Bad Homburg \\ ${ }^{2}$ Lehrstubl für Wirtschaftsinformatike und Electronic Government, \\ Universität Potsdam \\ ${ }^{3}$ Lehrstuhl für Wirtschaftsinformatik, \\ Europa-Universität Viadrina Frankfurt (Oder) \\ ${ }^{4}$ Institut für Wirtschaftsinformatik, Universität des Saarlandes \\ ${ }^{5}$ Professur für Betriebliche Anwendungssysteme, Universität Koblenz.
}

Themen der Teilkonferenz

Der Begriff Enterprise Resource Planning (ERP) steht heute synonym für integrierte betriebswirtschaftliche Standardsoftware. In der Teilkonferenz werden inhaltliche, methodische und technologische Probleme diskutiert, die sich auf den Gegenstand des Enterprise Resource Planning, auf die ERP-Systeme sowie auf deren Implikationen für die Prozesse und die technologische Infrastruktur des Unternehmens beziehen.

Bestehende ERP-Systeme werden eine Vielzahl von Transformationen durchlaufen müssen, um den Anforderungen durch die Globalisierung von Unternehmen und dem Aufbrechen von Wertschöpfungsketten zur verteilten Wertschöpfung hin gerecht werden zu können.

Die Einbindung neuer Technologien in Produktions- und Logistikprozesse birgt weitere Herausforderungen. So sind elektronische, kontaktlos auslesbare Produktkennzeichnungen mittels RFID-Technologie auf dem Vormarsch. Smart Items und intelligente Produkte, die drahtlos untereinander und mit Anwendungssystemen kommunizieren können, ermöglichen ein besseres Abbild der realen 
Prozesse in diesen Systemen. Da durch intelligente und vernetzte Güter EchtzeitDaten übermittelt werden, muss das ERP-System zudem in der Lage sein, kontextund situationsbewusst zu handeln.

Ziel der Teilkonferenz ist es, sowohl die Grundlagen des Enterprise Resource Planning als auch die verschiedenen Facetten der Veränderung der Wertschöpfungsketten und der technologischen Weiterentwicklung zu thematisieren. Die Teilkonferenz soll interessierte Forscher-, Entwickler- und Anwendergruppen zusammenbringen und den Weg zur Weiterentwicklung des Enterprise Resource Planning aufzeigen.

Programmkomitee

Sebastian Asendorf, Avinway Consultants, Bad Homburg

Prof. Dr. Jörg Becker, Universität Münster

Prof. Dr. Jorge Marx Gómez, Universität Oldenburg

Prof. Dr. Norbert Gronau, Universität Potsdam

Prof. Dr. Axel Hahn, Universität Oldenburg

Prof. Dr. Karl Kurbel, Europa-Universität Frankfurt (Oder)

Prof. Dr. Peter Loos, Universität Saarbrücken

Prof. Dr. Petra Schubert, Universität Koblenz 


\title{
An Approach for Real-Time Control of Enterprise Processes in Manufacturing using a Rule-Based System
}

\author{
Manfred Grauer, Sachin Karadgi, Daniel Metz, Walter Schäfer \\ Information Systems Institute, University of Siegen
}

Today's enterprise business environment is complex, volatile and driven by uncertainties. To address this situation, enterprises strive for flexibility and adaptability. The integration of business functions into a single system utilizing information technology can be seen as a prerequisite for establishing a Real-Time Enterprise (RTE). The horizontal integration of enterprise resource planning (ERP) systems with other business applications leads to Real-Time Business (RTB). Further, the realization of RTE requires the vertical integration of ERP systems with the manufacturing execution. Available Enterprise Application Integration (EAI) systems can be used to horizontally integrate existing business applications, but EAI lacks in incorporating manufacturing execution. Hence, Manufacturing Execution Systems (MES) were introduced. However with MES, major problems still remain open with respect to the interface between ERP system and the manufacturing execution. Different time granularities associated with various enterprise levels of an enterprise result in asynchronization of enterprise planning and manufacturing execution. As a consequence, actual achieved performance values (AS-IS) from manufacturing execution together with the planned performance values (TO-BE) from ERP system are not used for enhancing the performance of manufacturing activities and identifying new knowledge for control of enterprise processes (i.e., business and engineering processes).

Grauer et al. (2009) have articulated an IT-framework for vertical integration of different enterprise control levels. This IT-framework comprises a methodology for monitoring and control of enterprise processes in real-time using Workflow Management Systems (WMS) coupled with Rule-Based System (RBS). Further, offline Knowledge Discovery in Databases (KDD) can be utilized to identify new knowledge from integrated data. In the current contribution the KDD process in manufacturing enterprises is been presented and real-time control of enterprise processes based on the IT-framework articulated by Grauer et al. (2009) is been elaborated.

Knowledge is crucial for an enterprise to remain innovative and competitive. Enterprise members' tacit knowledge is embedded into enterprise processes. These knowledge needs to be identified and utilized to monitor and control enterprise processes. During process execution, AS-IS values together with TO-BE values are 
integrated according to an enterprise data model and stored in relational databases. These stored values are periodically utilized in an offline KDD process to identify tacit knowledge and externalize it as rules to be managed in RBS. Based on the aforementioned IT-framework by Grauer et al. (2009), real-time control of enterprise processes is elaborated in the current contribution and divided into three layers: control execution layer, control configuration layer and graphical user interface (GUI). In control execution layer, traceable objects are created from AS-IS and TO-BE values to be evaluated in RBS. In addition, the whereabouts of traceable objects in certain enterprise processes can be tracked utilizing WMS. Based on the aggregated context information of traceable objects, rules are fired in real-time to align the processes according to business objectives.

Traceable objects and rules are configured interdependently in the control configuration layer incorporating externalized knowledge from KDD process. The traceable objects represent parts, orders and resources that are relevant for enterprise process control. A part is a physical output of the manufacturing process and the corresponding traceable object contains AS-IS and TO-BE values referring to the manipulation through various resources. An order usually comprises several parts to be manufactured and corresponding part traceable objects can be aggregated to derive an order status. Similarly, manufacturing resources can be tracked independently from orders and parts. The status of enterprise processes and details of entities within these processes (i.e., parts, orders, and resources) are presented using GUI. In addition, a domain expert can accomplish configuration and query the relational databases of the integration layer as well as the RBS for resolving process control problems and examining actual process status expressed by means of Key Performance Indicators (KPIs) and Overall Equipment Efficiency (OEE).

Current contribution focuses on intra-enterprise integration in the vertical direction. RBS and WMS enable to establish enterprise-wide monitoring and control. In addition, stored AS-IS and TO-BE values facilitate enterprise-wide knowledge and learning cycle. Already existing functionalities from third party IT-systems (e.g., MES systems) can be integrated into the IT-framework by means of Serviceoriented architecture (SOA). The presented IT-framework is based on several standards which will facilitate to establish inter-enterprise integration.

\section{References}

Grauer M, Metz D, Karadgi SS, Schäfer W, Reichwald JW (2009) Towards an ITframework for digital enterprise integration. In: Proc. of 6th Int. Conf. on Digital Enterprise Technology (DET 2009), Hong Kong. 


\title{
Towards a Model-based Service Integration Framework for Extensible Enterprise Systems
}

\author{
Matthias Allgaier*, Markus Heller*, Martin Weidner \\ SAP Research, Karlsrube
}

In the vision of an Internet of Services (Janiesch et al. 2008, S. 71-75), services will become tradable similar to manufactured goods. Organizations dynamically interact as service consumers and service providers leveraging service marketplaces to design, offer and consume services. With the rise of such service ecosystems (Barros and Dumas 2006, S. 31-37), the simplified consumption of services becomes a key challenge for enterprise organizations. Beside the consumption in composite- or mash-up applications, the integration of services into standard business applications running within enterprise systems (e.g. ERP systems) is most promising as these systems typically implement the core business processes of an organization and can be flexibly enriched with complementary services.

While integrating a service into an enterprise system, two different integration scenarios can be distinguished: In the first scenario, the enterprise system is extended with a service using a pre-defined service interface that has explicitly been foreseen by the enterprise system provider when the core system was shipped. In the second scenario the enterprise system is extended with a service that has not explicitly been foreseen by the enterprise system provider when the core system was shipped (see Figure). To fully integrate the service into the target environment, the core business application needs to be extended or adapted, possibly on different application layers. In this paper we tackle the integration of unforeseen services (scenario 2).

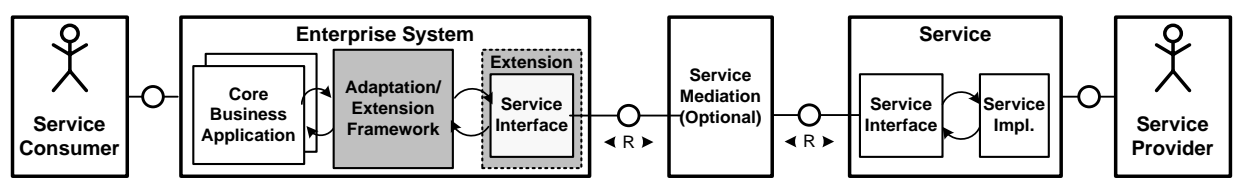

Figure 1: Unforeseen Service Integration using Extensibility Features

Today, three main deficits can be observed when integrating unforeseen services into core business applications: (i) Often much manual programming effort is needed to adapt or extend an enterprise system. (ii) Typically enterprise systems only provide

\footnotetext{
* The work presented in this paper is embedded into THESEUS/TEXO which is funded by means of the German Federal Ministry of Economy and Technology under the promotional reference 01MQ07012. The authors take the responsibility of the contents.
} 
proprietary adaptation and extension techniques with a low level of abstraction (e.g. proprietary code-level interfaces). (iii) It is in the responsibility of the buman integration expert to identify the affected parts of the core business application(s) that need to be adapted for service integration. This again requires deep domain- as well as technical knowledge of the enterprise system. A less-complex integration of services becomes particular relevant for service/partner ecosystems in upcoming ondemand enterprise software environments, cf. (Lo et al. 2009, S. 10).

In order to address these deficits, we propose a Service Integration Framework that allows enterprise system providers to ship standard business applications and at the same time offers dedicated extensibility features that allows partners in a service ecosystem to seamlessly integrate new services at a later stage in the softwarelifecycle. The Service Integration Framework is based on following characteristics:

(1) A model-based integration approach is introduced to enable service integrators to model (or design) the relevant integration aspects on a higher abstraction level.

(2) A pattern-based modelling approach is defined that covers typical adaptation- and extension tasks. It allows controlled extensibility of the core business applications, insofar, as only a proven set of adaptation operations can be performed.

(3) A uniform modelling approach is investigated to enable service integrators to design the adaptation and extension of a core business application on multiple layers (e.g. UI- and business process layer).

Within this paper we present the conceptualization of the Service Integration Framework. First, a motivating scenario from the automotive industry is introduced and the framework is embedded into the context of a service ecosystem. As the main contribution of the paper, a layered modelling approach is presented that allows the explicit linking of (i) the adaptable parts of the core business application with (ii) elements of the service description to be integrated making use of (iii) adaptation patterns. Finally, a prototype is described that demonstrates the integration of a service into the existing user interface of a core business application.

\section{References}

Barros A, Dumas M (2006) The Rise of Web Service Ecosystems. IT Professional 8 (5): 31-37.

Janiesch C, Ruggaber R, Sure Y (2008) Eine Infrastruktur für das Internet der Dienste. HMD - Praxis der Wirtschaftsinformatik 45 (261): 71-79.

Lo H, Wang R, Garbini, JP (2009) The State of Enterprise Software 2009. Forrester Research, Cambridge. 


\title{
Ein Maßnahmenkatalog für die Datensicherheit in der ERP Anwendungsentwicklung am Beispiel von SAP ERP
}

\author{
Holger Wittges, Sonja Hecht, Helmut Kromar \\ Lehrstubl für Wirtschaftsinformatik, \\ Technische Universität München
}

Durch die Etablierung von Enterprise Resource Planning (ERP) Systemen können Unternehmensdaten aus verschiedenen internen und externen Quellen integriert, korreliert und ausgewertet werden. Dadurch steigen die Möglichkeiten zur Nutzung der Daten, zugleich aber auch die Gefahr des Datenmissbrauchs. Vor diesem Hintergrund ist das Management von Unternehmen gefordert, Sicherheitskonzepte zu entwickeln und umzusetzen, um sensible Unternehmensdaten vor einem unberechtigten Zugriff durch Dritte zu schützen. Hierbei sollten insbesondere auch die Gefahren aus dem Inneren des Unternehmens berücksichtigt werden. Gemessen an der Anzahl der Angriffe überwiegen in den letzten Jahren zwar die Angriffe von außen, jedoch entsteht durch Angriffe aus dem Inneren ein wesentlich größerer Schaden (Baker et al. 2008, S. 9-10; Richardson 2008, S. 14). In einer von Verisign durchgeführten Studie (Baker et al. 2008, S. 10-13) waren in $73 \%$ der Fälle Angreifer außerhalb des Unternehmens beteiligt, gemessen an der Anzahl der entwendeten Datensätze war der Schaden durch Angriffe aus dem Inneren der Organisation sowie durch Partner aber fast 19 mal größer. Bei den Angriffen aus dem Inneren oder durch Partner ging ein wesentlicher Anteil der Angriffe direkt von Personen aus, die mit der Administration von IT-Lösungen betraut waren. Ein Angriff von dieser Personengruppe kann aufgrund deren Zugriffsmöglichkeiten und Wissen über die Schwachstellen der Informationssysteme zu schwerwiegenden Schäden führen (Magklaras und Furnell 2002, S. 64-65).

Ziel dieses Beitrags ist, Schwachstellen in der Datensicherheit für die ERP Anwendungsentwicklung zu identifizieren und einen umfassenden Maßnahmenkatalog zu entwickeln, um einen unberechtigten Datenzugriff durch die Personengruppe der Anwendungsentwickler so weit wie möglich zu unterbinden. In einem ersten Schritt werden geeignete Maßnahmen aus dem IT-Grundschutz Maßnahmenkatalog des Bundesamt für Sicherheit in der Informationstechnik (BSI 2008) identifiziert und anhand eines Fallbeispiels auf deren Anwendbarkeit im praktischen Einsatz untersucht. Ergebnisse dieser Untersuchung zeigen, dass eine Umsetzung aller Maßnahmen aus dem IT-Grundschutz Maßnahmenkatalog häufig nicht praktikabel ist. Wie hoch die dadurch entstehende Gefahr für die Datensicherheit tatsächlich ist, hängt sowohl vom Systemtyp (z. B. Qualitätssicherungssys- 
tem oder Produktivsystem) als auch von der Existenz weiterer Sicherheitsmaßnahmen ab. Hieraus wird der Bedarf nach einem Maßnahmenkatalog deutlich, der bei der Wahl alternativer Maßnahmen unterstützt und Unternehmen ein breites Maßnahmenspektrum aufzeigt. In einem nächsten Schritt wird ein Maßnahmenkatalog für die Datensicherheit in der ERP Anwendungsentwicklung vorgeschlagen, der Maßnahmen in den folgenden Kategorien enthält: Abschreckende Maßnahmen, präventive Maßnahmen, Maßnahmen zur Aufdeckung von Angriffen, Maßnahmen zur Behebung und Bestrafung, sowie Maßnahmen in Personalmanagement und -führung. Die Maßnahmen aus dem IT-Grundschutz werden hierfür aufgegriffen, strukturiert, weiter detailliert, ergänzt und bezüglich ihrer Umsetzbarkeit in ERP Systemen bewertet. Der vorgeschlagene Maßnahmenkatalog kann als Grundlage für die Entwicklung und die Analyse von Sicherheitskonzepten im Kontext der ERP Anwendungsentwicklung herangezogen werden.

Neben der Entwicklung eines breitgefächerten Maßnahmenkatalogs zielt dieser Beitrag auch darauf ab, weiteren Forschungsbedarf in diesem Kontext zu identifizieren. Forschungsbedarf besteht z. B. in Bezug auf verbesserte Audit- und Logging-Funktionalitäten, um unberechtigte Datenzugriffe schnell und pro-aktiv erkennen zu können. Ein weiteres Beispiel stellt die Anonymisierung von Testdaten dar. Trotz verschiedener am Markt verfügbarer Lösungen für die Anonymisierung von Daten verwenden viele Unternehmen für Testzwecke unveränderte $\mathrm{Da}$ ten aus dem Produktivsystem. Eine detaillierte Betrachtung der Gründe hierfür, sowie die Ermittlung des Verbesserungspotentials, könnten einen wesentlichen Beitrag für die Datensicherheit in der ERP Anwendungsentwicklung liefern.

\section{Literatur}

Baker WH, Hylender CD, Valentine JA (2008) Data Breach Investigations Report. http://www.verizonbusiness.com/resources/security/databreachreport.pdf. Abruf am 2009-11-25.

BSI (2008) IT Grundschutz-Kataloge - 10. Ergänzungslieferung. https://www.bsi.bund.de/cln_174/ContentBSI/grundschutz/kataloge/katalo ge.html. Abruf am 2009-11-25.

Magklaras GB, Furnell SM (2002) Insider threat prediction tool: Evaluating the probability of IT misuse. Computer \& Security 21(1):62-73.

Richardson R (2008) CSI Computer Crime and Security Survey. http://www.gocsi.com/forms/csi_survey.jhtml. Abruf am 2009-11-25. 


\title{
Erwartungen und Nutzen beim Einsatz von Business Software
}

\section{Empirische Ergebnisse einer Analyse von Fallstudien}

\author{
Petra Schubert', Susan P. Williams ${ }^{2}$ \\ ${ }^{1}$ Centre for Applied Information and Communication Technologies - CAICT, \\ Copenhagen Business School \\ ${ }^{2}$ Discipline of Business Information Systems, \\ University of Sydney
}

\begin{abstract}
In den letzten zehn Jahren wurden über 140 Fallstudien nach der eXperienceMethodik geschrieben (Schubert und Wölfle 2007). Über die Zeit wurde damit eine umfassende Wissensbasis zu Business-Software-Projekten angelegt. Die darin enthaltenen Projektbeschreibungen dokumentieren das Wissen und die Erfahrung von Projektverantwortlichen und geben einen wertvollen Einblick in die Praxis. Aufgrund der einheitlichen Struktur der Projektbeschreibungen nach der eXperience-Systematik ist es möglich, diese gezielt nach bestimmten Fragestellungen zu untersuchen. Der Beitrag widmet sich dem Thema des Nutzens, der aus dem Einsatz von Business Software gewonnen wird. Basierend auf den Inhalten der gesammelten Fallstudien entwickelten Schubert und Williams ein Modell (expectations-benefits framework), das verschiedene Nutzenarten klassifiziert und beschreibt (Schubert und Williams 2009a+b). Das Modell wird in diesem Beitrag als Basis für die strukturierte Auswertung von 14 Fallstudien zum Thema „Erfolgsfaktoren beim Einsatz von Business Software" angewendet, die im eXperienceZyklus des Jahres 2009 erhoben wurden. Der folgende Beitrag stellt die Ergebnisse dieser Studie vor.
\end{abstract}

\section{Forschungsfragen}

Der Beitrag stellt zunächst das eingesetzte Modell für die Identifikation von Nutzen aus dem Einsatz von Business Software vor. Auf der Basis des Modells werden im Anschluss 14 eXperience-Fallstudien (www.experience-online.ch) vergleichend analysiert und Schlussfolgerungen für die Nutzenbestimmung gezogen. Die beiden Forschungsfragen, die diesen Beitrag motiviert haben, sind die folgenden: 
Forschungsfrage 1: Welchen konkreten Nutzen erzielen Unternehmen aus dem Einsatz von Business Software?

Forschungsfrage 2: Gibt es Kriterien für einen ,dauerhaften Erfolg“beim Einsatz von Business Software?

\section{Schlussbemerkungen}

Es ist interessant zu sehen, welche Faktoren von den Autoren für dauerhaften Erfolg identifiziert werden. Deren Charakter ist nur in der Minderheit der Fälle harter Natur, z. B. zeitnaher Informationszugriff. Häufiger genannt werden weiche, nicht eindeutig messbare Kriterien wie Zukunftsfähigkeit, Agilität, Know-how sowie Vertrauensbeziehungen.

Die Fallstudien zeigen, dass sowohl der erzielte Nutzen als auch die Erfolgsfaktoren für jedes Unternehmen unterschiedlich sind. Genauso unterschiedlich sind auch die Anforderungen, mit denen ERP-Anbieter bei der Implementation ihrer Software konfrontiert sind. Dass ERP-Projekte nicht immer einfach sind und auch zu Misserfolgen und Frustrationen führen können, ist vor diesem Hintergrund nicht weiter erstaunlich.

\section{Literatur}

Schubert P, Williams SP (2009a): An Extended Framework for Comparing Expectations and Realized Benefits of Enterprise Systems Implementations, in: Proceedings of the Fifteenth Americas Conference on Information Systems, San Francisco, California, August 6th-9th, 2009.

Schubert P, Williams SP (2009b): Constructing a Framework for Investigating and Visualizing ERP Benefits and Business Change, in: Proceedings of the 22nd International Bled eConference, Bled, Slovenia, June 14-17, 2009.

Schubert P, Wölfle R (2007): The eXperience Methodology for Writing IS Case Studies, in: Thirteenth Americas Conference on Information Systems (AMCIS), 2007. 


\title{
Flexibilität in ERP-Standardsoftware
}

\section{Eine Studie über die zukünftigen Anforderungen}

\author{
Norbert Frick, Petra Schubert \\ Institut für Wirtschafts- und V erwaltungsinformatik, \\ Professur für Betriebliche Anwendungssysteme, \\ Universität Koblen₹-Landau
}

\begin{abstract}
CEOs und CIOs sind mit der Entscheidung konfrontiert, das richtige ERP-System für ihr Unternehmen auszuwählen. Der Evaluationsprozess nimmt dabei eine kritische Funktion ein, denn das Ergebnis der Einführung entscheidet nicht selten über die Wettbewerbsfähigkeit des Unternehmens und somit über dessen Zukunft. ERP-Standardsoftwaresysteme sind leistungsfähige Werkzeuge, die nach einem (oft anspruchsvollen) Customizing-Prozess die Anforderungen von unterschiedlichen Unternehmen in unterschiedlichen Branchen erfüllen können. Trotzdem haben Fallstudien immer wieder gezeigt, dass Einführungsprojekte sehr aufwändig sind und zu hohen Belastungen im Unternehmen führen können. Dies führt zu der Frage, ob und wie ERP-Anbieter die Probleme von ERP-Anwendern wahrnehmen und auf diese eingehen. Diese Frage stimulierte eine tiefgehende, qualitative Studie des deutschsprachigen ERP-Marktes. In über 30 Interviews mit ERP-Anbietern wurde versucht, ein Bild von den Wertversprechen zukünftiger ERPStandardsoftwaresysteme zu zeichnen. Dabei hat sich gezeigt, dass die Abstimmung von Prozess- und Service-Orientierung die Flexibilität der Systeme signifikant erhöhen kann.
\end{abstract}

\section{Motivation und Literatur}

Somit es ist für die Systemauswahl entscheidend, die genauen Leistungsmerkmale und deren technischen Grundlagen präzise einzuschätzen (Akkermans und van Helden 2002, S. 45; Wei et al. 2005; Holland und Light 1999, S. 35). Eine Studie von Dalal et al. (2004, S. 84) unterstreicht, dass Unternehmen oft vor der Entscheidung stehen, ob sie sich der Software anpassen und so ihre Geschäftsprozesse grundlegend ändern müssen, oder ob die Software flexibel genug ist, die Bedürfnisse des Unternehmens erfüllen zu können. Dabei wurden service-orientierte Architekturen (Liebhart 2007) und Business Process Modelling (BPM) als vielversprechende Konzepte für flexible Systeme postuliert, die sich den Bedürfnissen der Anwender anpassen (Newcomer und Lomow 2005). 


\section{Fazit}

Basierend auf den Ergebnissen einer empirischen Studie des deutschsprachigen ERP-Marktes wurden die Aspekte der Prozess-Orientierung, Service-Orientierung und Flexibilität von ERP-Standardsoftwaresystemen diskutiert. Es hat sich gezeigt, dass Anbieter, die dem service-orientierten Paradigma bzw. dem serviceorientierten Gedanken folgen, bereits technologische wie auch organisatorische Anforderungen an ein flexibles System im Ansatz erfüllen können. Allerdings gibt es in einigen Bereichen noch erheblichen Bedarf für Weiterentwicklungen.

\section{Literatur}

Akkermans H, Helden K (2002) Vicious and virtuous cycles in ERP implementation: a case study of interrelations between critical success factors. European Journal of Information Systems 11: 35-46.

Dalal NP, Kamath M, Kolarik WJ, Sivaraman E (2004) Toward an Integrated Framework for Modeling Enterprise Processes. Communications of the ACM 47 (3): 83-87.

Holland C, Light B (1999) A Critical Success Factors Model For ERP Implementation. IEEE Software, May/June: 30-36.

Liebhart D (2007) SOA goes real. Hanser, München.

Newcomer E, Lomow G (2005) Understanding SOA with Web Services. AddisonWesley, Upper Saddle River.

Wei HL, Wang ETG, Ju PH (2005) Understanding misalignment and cascading change of ERP implementation: a stage view of process analysis. European Journal of Information Systems 14: 324-334. 


\title{
Collaborative Project Management (CPM)
}

\section{Ein offener Standard zur unternehmensübergreifenden $\mathrm{Zu}-$ sammenarbeit}

\author{
Heiko Bartlog', Jochen Boy ${ }^{2}$ \\ ${ }^{1}$ Campana \& Schott, Frankfurt (Main) \\ 2ProSTEP iV iP Verein, Darmstadt
}

In der Automobilindustrie verändert sich die Zusammenarbeit zwischen Herstellern und den Zulieferern - bereits die Entwicklung findet unternehmensübergreifend statt. Dies führt zu neuen Herausforderungen im Projektmanagement.

Wesentlicher Ansatzpunkt bei der Erarbeitung des CPM Standards war die Beibehaltung der eigenen etablierten und eingespielten ProjektmanagementStandards bei den beteiligten Partnern, sodass Effizienzverluste durch Umstellungen vermieden werden. Das CPM Modell konzentriert sich auf die Definition gemeinsamer Rollen, Begriffe und Kommunikationsregeln für die Zusammenarbeit in gemeinsamen Projekten.

Die CPM Recommendation wurde vom ProSTEP iViP Verein Ende 2007 veröffentlicht.

Das fachliche Referenzmodell ist das Kernelement der CPM Recommendation. Ziel der Empfehlung ist, die Zusammenarbeit in unternehmensübergreifenden Projekten zu verbessern. Der Fokus

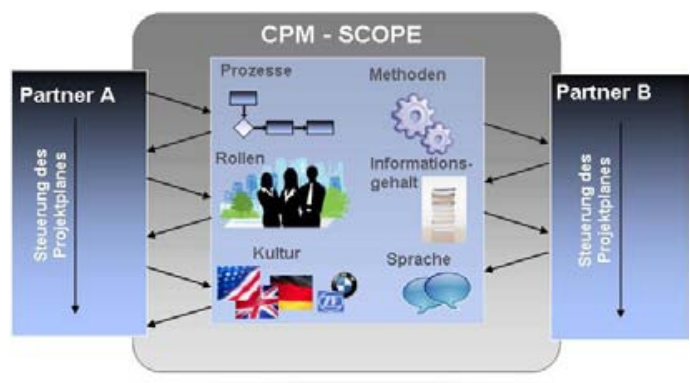

Abbildung 1: CPM Leitbild liegt dabei auf den Bereichen Zeit- Aufgaben- und Kommunikationsmanagement. Der Nutzen ergibt sich aus einer höheren Transparenz und Verbindlichkeit der Kommunikation im Projekt. Als Grundprinzip trennt das Referenzmodell Fachund Projektmanagementprozesse, liefert eine reine Schnittstellenbetrachtung und lässt dabei die Ausgestaltung innerhalb der Partner völlig offen.

Das „Handshake-Prinzip“ ist elementar für die Erhöhung der Transparenz und Verbindlichkeit zwischen den Projektpartnern. Dieses Prinzip beinhaltet, dass eine einseitige Übermittlung von relevanten Projektinformationen für sich nicht ausreicht, sondern dass vom jeweiligen Partner eine aktive Rückmeldung (Annahme, Ablehnung oder Änderung) notwendig ist, um eine Übermittlung erfolgreich durchzuführen. Die hierdurch entstandenen gemeinsamen Vereinbarungen bilden eine verlässliche Basis für weitergehende Entscheidungen. 
Ein gemeinsames Verständnis über die Rollen der beteiligten Personen im Projekt ist elementar. Das CPM Referenzmodell liefert eine standardisierte Definition von vier generischen Projektmanagement-Rollen mit den jeweiligen Merkmalen bezüglich Aufgaben, Befugnissen, Verantwortlichkeiten und Kompetenzen.

Das Referenzmodell definiert zudem drei Werkzenge: Der Interaktionsplan stellt die relevanten Interaktionspunkte der Partner dar. Insbesondere wird die Abstimmung der gemeinsamen Interaktionspunkte zwischen den Beteiligten festgehalten. In der Kommunikationsmatrix werden sämtliche abstimmungsrelevanten Themen mit den jeweils verantwortlichen Ansprechpartnern auf beiden Seiten sowie Eskalationsregeln festgelegt. In der Issue Liste werden alle partnerrelevanten offenen Punkte festgehalten, die im Projektverlauf auftreten und dient der Dokumentation der Bearbeitung und der zwischen den Partnern abgestimmten Lösung.

Das Datenaustauschmodell wurde entworfen, um den Datenaustausch der abstimmungsrelevanten Informationen zwischen den bei den beteiligten Partnern jeweils eingesetzten IT-Systeme zu vereinheitlichen. Es ist unterteilt in einen normativen Teil, im dem die für CPM relevanten Informationsobjekte definiert sind und in einen Refereņ̃teil, der Datenklassen für Informationen beschreibt, die i. d. R. bei einem Partner verbleiben.

CPM ist aktuell der einzige Projektmanagementstandard, der ein implementierungsbereites Datenmodell bereitstellt. Schnittstellen zwischen unterschiedlichen IT-Systemen lassen sich auf Basis dieses einheitlichen Standards kostengünstiger implementieren. Ergänzend beschreibt ein Austauschmodell den Mechanismus zum Austausch der CPM Daten zwischen den Systemen auf Basis von Web-Services.

Das Potential von CPM kann weit über die Automobilbranche hinaus genutzt werden, dabei werden multilaterale Informationsflüsse von Netzwerken auf bilaterale Beziehungen fokussiert. Die Bereitstellung eines „CPM Moduls“ für unterschiedliche IT-Systeme ermöglicht dabei einen standardisierter Datenaustausch.

\section{Literatur}

ProSTEP iViP Association (2007) Recommendation "Collaborative Project Management (CPM) - Reference Model” (PSI 1-1)

ProSTEP iViP Association (2007) Recommendation "Collaborative Project Management (CPM) - Data Exchange Model” (PSI 1-2)

ProSTEP iViP Association (2008) OMG Product Lifecycle Management Services V2.0

Boy J, Plischke D (2009) CPM: Der neue Weg zu erfolgreichen Partnerprojekten. ProduktDatenJournal 1/2008:15-19

VDA (2003) HAWK 2015 - Wissensbasierte Veränderung der automobilen Wertschöpfungskette. Band 30. 


\title{
Value Chain Cockpit auf Basis betrieblicher Anwendungssysteme
}

\author{
Andrej Werner', Christian Hrach'1, Karen Heyden', \\ Rainer Alt'2, Bogdan Franczylk ${ }^{2}$ \\ 'Institut für Angewandte Informatike. e. ., Universität Leipzig
2Institut für Wirtschaftsinformatik, Universität Leipzig
}

Die Vernetzung von Unternehmen mit Kunden und Lieferanten (überbetriebliche Integration) ist eines der wichtigsten Elemente in betrieblichen Wettbewerbsstrategien. Eine in vielen Branchen stark zurückgegangene Fertigungstiefe geht gleichzeitig mit erhöhten Transaktionskosten und Anforderungen an Mechanismen zur Koordination der überbetrieblich verteilten Wertschöpfung einher. Diese informationsbasierten Tätigkeiten können die in den vergangenen Jahrzehnten eingeführten und schrittweise erweiterten integrierten betrieblichen Anwendungssysteme (AS) des Enterprise Resource Planning nur unzureichend übernehmen.

Die Supply Chain (SC) wie auch die Demand Chain (DC) bündeln als unternehmensübergreifende Wertschöpfungssysteme (WS) Kompetenzen verschiedener Unternehmen in Netzwerken. Deren Integration ist mittels einer Value Chain (VC) umsetzbar, wobei die VC als ganzheitliches Prozessmodell die Prozesse von DC und SC basierend auf einem gemeinsamen Informationsfluss mit hoher Transparenz und Verfügbarkeit von Planungs- und Steuerungsdaten der Netzwerkpartner beinhaltet. Zum Nutzen dieser Integration schreiben Duffy et al. (2004, S.79): “... consumer businesses that have integrated their CRM and SCM capabilities have dramatically and measurably outperformed their competition in virtually every critical financial and operating category ...". Auf AS-Ebene erfolgt die Integration der beteiligten CRM- und SCM-Systeme über die Value Chain Integration.

In der Literatur wird die Integration von CRM und SCM bereits thematisiert, wobei aktuelle Systemlösungen für die technische Umsetzung durch das SCM dominiert werden. Der Beitrag schlägt eine Systemlösung vor, die als Value Chain Cockpit (VCC) nicht auf der Dominanz des SCM aufbaut und WS-Informationen in Form von Kennzahlen aus CRM- und SCM-Systemen unternehmensübergreifend integriert. Das VCC ermöglicht die geforderte Transparenz und Verfügbarkeit rollen- und entscheidungsrelevanter Planungs- und Steuerungsdaten mittels grafischer Visualisierungselemente und unterstützt durch Rückkopplung von CRM und SCM den iterativen Prozess, mit dem sich das WS in den Bereichen Produktherstellung, Absatz und zusätzliche Dienstleitungen immer wieder an die Kundenbedürfnisse anpassen und modifizieren lässt. Dies erfordert unter anderem die Nutzung kollaborativer Techniken in Bezug auf Business Intelligence und WS- 
Konfiguration, die Bereitstellung einer individualisierbaren visuellen Nutzerschnittstelle sowie die Integration heterogener Datenquellen der Netzwerkakteure.

Das VCC fasst CRM- und SCM-Aufgaben auf den Ebenen Konfiguration, Planung und Ausführung zusammen. Der Beitrag stellt anhand von Beispielen die einzelnen Ebenen mit ihren Funktionalitäten vor, wobei auch auf die rollenbasierte Informationsverfügbarkeit eingegangen wird. Zur Umsetzung eines Value Chain Cockpits werden Konzepte aus der Literatur wie z.B. Business Process Management Systeme, Enterprise Mashup und Portale diskutiert und Ansatzpunkte für die zukünftige Entwicklung eines Prototypen, der an praxisorientierten Anwendungsszenarien getestet werden soll, aufgezeigt

Zusammenfassend kann gesagt werden, dass die verteilte Wertschöpfung in Unternehmensnetzwerken Mechanismen und Technologien zur überbetrieblichen Koordination und Kommunikation erfordert, die bisherige integrierte (inner-) betriebliche AS allein nicht leisten können. Der in diesem Beitrag thematisierte Ansatz eines Value Chain Cockpits als Erweiterung bestehender AS unterstützt die Transparenz und Verfügbarkeit prozessorientierter Planungs- und Steuerungsdaten und ermöglicht bereichs- und unternehmensübergreifende Sichten auf WS auf Basis einer kennzahlenbasierten Datenintegration von CRM- und SCM-Systemen.

\section{Literatur}

Duffy, J.; Koudal, P.; Pratt, St. (2004): The Future of Collaborative Customer Relationship Management. In: Kracklauer, A. H. et al. (Hg.): Collaborative customer relationship management. Springer, S. 77-106. 


\title{
ERP-Einführung: Wirkung von kritischen Erfolgs- faktoren der Projektphase auf den Projekterfolg
}

\author{
Alexander Dreiling \\ Institut für Technische und Betriebliche Informationssysteme, \\ Otto-von-Guericke-Universität Magdeburg
}

Enterprise Resource Planning (ERP) - Systeme automatisieren Aktivitäten und integrieren Daten, Informationen und Prozesse innerhalb des gesamten Unternehmens. Zwangsläufig muss während der Einführung nicht nur die Lauffähigkeit des Systems sichergestellt, sondern vor allem auch das komplette Unternehmensmodell angepasst werden - eine hochkomplexe und riskante Herausforderung. Esteves und Bohorquez (2007, S. 19-20) stellen fest, dass obwohl bislang die meisten Forschungsanstrengungen zu dem Einführungsprozess unternommen wurden, es gerade in diesem Bereich für viele zentrale Probleme noch keine allgemeinen Lösungen gibt. Nach wie vor scheint zu gelten, dass in ca. 70-90\% aller Fälle das Budget der Projektphase überschritten, die Implementierung verspätet abgeschlossen oder die vereinbarte Funktionalität nicht umgesetzt wird. Den erhofften Grad des (Business-)Erfolgs, nach der Einführung, erreichen weniger als $40 \%$ aller Unternehmen (Wong et al. 2005, S. 2-3).

Begründete Verbesserungsvorschläge können Praktikern allerdings nicht unterbreitet werden, da bislang aus theoretischer Sicht nicht eindeutig geklärt ist, aus welchen Gründen sich Erfolg in und nach einem ERP-Einführungsprojekt einstellt (Robey et al. 2002, S. 20). Berücksichtigt man die Bedeutung des ERP-Marktes, die extremen Einführungskosten sowie die Gefahr der nachhaltigen Schädigung des Unternehmen, bei einer gescheiterten Einführung, erkennt man schnell, dass Forschungsergebnisse in diesem Gebiet keineswegs einem Selbstzweck dienen, sondern dringend gebraucht werden (Esteves und Bohorquez 2007, S. 19).

Vor dem Hintergrund dieser Motivation schlägt diese Arbeit ein Modell vor, welches die Realisierung von Erfolg in dem Kontext einer ERP-Einführung erklärt. Einleitend werden zunächst der ERP-Einführungs- und Einsatzlebenszyklus, Sichtweisen des Erfolgs sowie kritische Erfolgfaktoren besprochen. Jene Ausführungen ermöglichen einen Zugang zu der Thematik und dienen als Grundlagen für die Diskussion des Modells. Folgend wird in erster Annäherung zur Validierung der getroffenen Annahmen, der Wirkungszusammenhang zwischen kritischen Erfolgsfaktoren der Projektphase (KEF) und dem Projekterfolg (PE) näher untersucht. Hierdurch wird nicht nur die notwendige Basis für die Überprüfung der übrigen Annahmen geliefert, sondern vielmehr eine Möglichkeit geboten erste praxisrelevante Schlüsse, für die Planung und Bewertung der Projektphase, zu 
ziehen. In der ERP-Forschungsgemeinde wurde bislang die Wirkung der qualitativ ermittelten kritischen Erfolgsfaktoren auf den Projekterfolg nur angenommen. Der quantitative empirische Nachweis wurde nach Yang und Seddon (2004, S. 9-10) und dem derzeitigen Wissensstand des Autors noch nicht erbracht.

Für die Schließung jener Lücke wurden im Rahmen dieser Studie repräsentative Schlüsselinformanten, von zehn standardisierten SAP Roll-out Projekten an kleinen und mittelgroßen internationalen Standorten eines DAX-Notierten Konzerns, hinsichtlich der Qualität und Beachtung kritischer Erfolgsfaktoren anonym befragt. Hierzu wurde der jeweils tatsächlich erzielte Projekterfolg, welcher durch das Roll-out Programm-Management angegeben wurde, in Beziehung gesetzt. Die statistische Auswertung der erhobenen Daten zeigt, dass eine besondere Beachtung KEF den PE signifikant negativ beeinflusst. In der Ergebnisdiskussion wird festgestellt, dass sich dieses zunächst unerwartete Ergebnis auf die in der Regel verwendeten Metriken zur (Projekt-)Erfolgsbestimmung zurückführen lässt. Demnach kann eine Optimierung über Budget-, Zeitbeschränkungen sowie den gelieferten Systemfunktionsumfang zur Vernachlässigung der langfristigen Einführungsqualität führen. Zur Lösung dieses Problems wird die Erweiterung der Projekterfolgsmetriken, um frühe Systemleistungsparameter, vorgeschlagen. Es bleibt zu prüfen, ob jene Anpassung tatsächlich die verbreitete Annahme, der positiven Korrelation zwischen PE und dem Ausmaß der Beachtung KEF, stützt.

Hilfreiche Verbesserungsvorschläge, um die Scheiterungsrate von Einführungsprojekten zu senken, können den Praktikern erst dann gegeben werden, wenn alle prognostizierten Wirkungszusammenhänge auch tatsächlich empirisch belegt werden. An dieser Stelle müssen zukünftige Forschungsanstrengungen ansetzten.

\section{Literatur}

Esteves J, Bohorquez V (2007) An updated ERP systems annotated bibliography: 2001-2005. Communication of the Association for Information Systems 19: article 18.

Robey D, Ross JW, Boudreau MC (2002) Learning to implement enterprise systems: an exploratory study of the dialectics of change. Journal of Manangement Information Systems 19(1):17-46.

Wong A, Scarbrough H, Chau PYK, Davison R (2005) Critical failure factors in ERP implementation. In: Proceedings of 9th Pacific Asia Conference on Information Systems, Bangkok.

Yang S, Seddon P (2004) Benefits and key project success factors from enterprise systems implementations: lessons from Sapphire 2003. In: Proceedings of the 15th Australasian Conference on Information Systems, Hobart. 


\title{
Dynamische Gruppenarbeit
}

\section{Dezentrale Feinplanungsunterstützung durch einen virtuel- len Marktplatz}

\author{
Thilo Münstermann, Jens Völqke, Paul Flachskampf \\ Institut für Unternehmenskybernetike e.V., \\ RWTH Aachen
}

Im Rahmen eines von der Arbeitsgemeinschaft industrieller Forschungsvereinigungen (AiF) aus Mitteln des BMWi geförderten Forschungsvorhabens entstand in Kooperation des Instituts für Unternehmenskybernetik e.V. und des Instituts für Integrierte Produktion Hannover gGmbH ein Konzept für ein Softwaretool zur Unterstützung von Gruppenarbeit in mittelständischen Metallbetrieben, im folgenden Arbeitsgruppenassistent (AGA) genannt.

Der AGA ist als Erweiterung zentraler Produktionsplanungssysteme konzipiert und entweder als eigenständige Software über Schnittstellen mit diesen koppelbar oder als Modul in bestehende Systeme zu integrieren. Eine Grundidee des AGA besteht in der Dezentralisierung und damit der Dynamisierung der Feinplanungsprozesse. Gruppen sollen eigenverantwortlich innerhalb bestimmter Grenzen ihre Planung optimieren können und aufgrund der höheren Übersichtlichkeit einer Gruppenplanung schnell und flexibel auf Änderungen reagieren können. Wie jede Dezentralisierung bringt dies aber auch den Nachteil mit sich, dass betriebsweite Potenziale der Zusammenarbeit zunächst nicht genutzt werden, da keine zentrale Planungsstelle die innerhalb der Feinplanung benötigten oder frei werdenden Ressourcen gruppenübergreifend zuweist (Flachskampf et al. 2008). Eine solche zentrale Zuweisung wäre auf dem Detaillierungsgrad einer Feinplanung mit enormem Arbeitsaufwand verbunden und würde gleichzeitig dem Prinzip der Gruppenautonomie widersprechen (Antoni et al. 2003).

Um dennoch Möglichkeiten zur Planungsoptimierung (z. B. Verkürzung der Durchlaufzeit oder Einhalten von Lieferterminen) nutzen zu können wird das Konzept des virtuellen Marktplatzes eingeführt, auf dem Gruppen benötigte und frei gewordene Ressourcen sowie Aufträge tauschen, verschieben und handeln können.

Das Konzept beruht auf der Idee, dass aus der Grobplanung eine vorläufige Zuweisung der Aufträge oder Arbeitsschritte auf die einzelnen Gruppen in der Fertigung vorgenommen wurde und diese Gruppen in ihrer Zusammenstellung und Personalstärke für die durchschnittliche Arbeitsbelastung optimiert sind. Aufgrund von Krankheiten, Maschinenausfällen, unvorhergesehenen Problemen oder 
zwischengeschobenen Aufträgen hoher Priorität kann es jedoch immer wieder vorkommen, dass von dieser Planung abgewichen werden muss.

So können bei den einzelnen Gruppen Kapazitätsengpässe und -überschüsse auftreten, die weiteres Optimierungspotenzial bieten. Um dieses Potenzial zu nutzen soll Gruppen die Möglichkeit geboten werden, zusätzliche Mitarbeiter oder Maschinen für bestimmte Zeiträume anzufragen oder Arbeitsschritte zur Bearbeitung anderen Gruppen anbieten und an diese abgeben zu können. Nach der Grundidee des AGA werden diese Abweichungen in der dezentralen Feinplanung der Gruppen behandelt (Henning et al. 2008).

Diese Verhandlungen können je nach Bedarf mehr oder weniger vollständig über den virtuellen Marktplatz vollzogen werden. In einer relativ kleinen Produktion mit wenigen Gruppen und Verantwortlichen wird die Nachfrage, ob man einen Mitarbeiter aus einer Gruppe für ein paar Stunden ausgeliehen haben kann, meist persönlich erfolgen. Bei einer größeren Produktion, besonders wenn diese über mehrere Hallen verteilt ist, kann die Anfrage nach einer benötigen Qualifikation für einen Zeitraum über den virtuellen Marktplatz gestellt, von anderen Gruppen beantwortet und schließlich beidseitig bestätigt werden.

Durch die Anwendung eines solchen Softwaretools kann die Gruppenarbeit in den Unternehmen koordiniert und gestärkt werden. Das Tool kann so nicht nur dazu beitragen die Gruppenarbeitsprozesse transparent und aufwendungsarm zu gestalten, sondern auch dazu die Gruppenarbeit als solche aufrecht zu erhalten und somit einen größeren Nutzen zu generieren (Fecht und Unbehend 2003).

\section{Literatur}

Antoni C, Hofmann K, Bungard W (2003) Gruppen- und Teamarbeit: Neue Organisationsformen in Unternehmen. Ein Handbuch für das moderne Management. 2. Aufl. Springer Verlag, Berlin.

Flachskampf P, Münstermann T, Michulitz C, Askri A, Overmeyer L (2008) Einführung eines Softwaretools zur Unterstützung der Gruppenarbeit. Teil 5., In: Management-Kompetenz in kleinen und mittleren Unternehmen Jahrbuch der KMU Forschung und -Praxis. Hrsg. v. Meyer, Jörn-Axel: Lohmar - Köln: EUL Verlag, 319-329.

Fecht M, Unbehend M (2003) Gruppenarbeit in Produktionsbetrieben. Tectum Verlag, Marburg

Henning K, Flachskampf P, Nickel R, Münstermann T (2009) Konzept für ein Informationssystem zur Unterstützung der Gruppenarbeit in mittelständischen Industriebetrieben der Metallverarbeitung. Books on Demand Verlag, Norderstedt. 
ERP-Software in der Lehre 



\title{
Vorwort zur Teilkonferenz
}

\section{ERP-Software in der Lehre}

\author{
Helmut Krrmar', Norbert Gronau ${ }^{2}$ \\ ${ }^{1}$ Lehrstubl für Wirtschaftsinformatike (117), \\ Technische Universität München
}

\section{${ }^{2}$ Lehrstubl für Wirtschaftsinformatike und Electronic Government, Universität Potsdam}

Der Wandel und Fortschritt im Umfeld betrieblicher Informationssysteme stellen sowohl Unternehmen als auch Bildungseinrichtungen vor neue Herausforderungen. Unter den betrieblichen Informationssystemen kommt den Enterprise Resource Planning (ERP) Systemen eine besondere Bedeutung zu. Sie bilden in vielen Unternehmen das Rückgrat der Informationsverarbeitung und gehören zu den wesentlichen Erfolgsfaktoren für Unternehmen. Daher ist es erforderlich, die Verfügbarkeit von qualifiziertem Personal sicherzustellen und frühzeitig ein Augenmerk auf dessen Ausbildung zu legen. Wurden diese Tätigkeitsfelder vormals oft dem Bereich der unternehmerischen Fort- und Weiterbildung zugeordnet, zeigen Entwicklungen der letzten Jahre, dass es zunehmend zu einer Verschiebung in Richtung der Hochschulen kommt. Die traditionelle Trennung zwischen der Vermittlung theoretischer Inhalte als Domäne des tertiären Bildungssektors, und das Erlangen praktischer Erfahrungen im Unternehmensumfeld vermischen sich mehr und mehr.

Dies alles erfordert, insbesondere im Kontext der Wirtschaftsinformatik, eine sowohl praxisnahe als auch theoretisch fundierte Lehre, die den Absolventen einerseits konzeptuelles Wissen vermittelt und andererseits den praktischen Anforderungen entspricht. Dabei kommt es nicht nur auf den Transfer von Faktenwissen an, sondern auch auf die Vermittlung von Wissen mit Bezug zu realen betrieblichen Prozessen und deren Unterstützung durch ERP-Systeme. Hieraus resultiert die Frage, wie die Ausbildung der ERP-Sperialisten von morgen gestaltet werden soll.

Der Track ERP-Software in der Lehre der Multikonferenz für Wirtschaftsinformatik 2010 widmet sich dieser Fragestellung. Dazu werden sowohl Weiterentwicklungen bereits bestehender als auch Entwürfe und Umsetzungen neuer Lehrkonzepte und -methoden im Umfeld von ERP-Systemen dargestellt. Die einzelnen Beiträge können für die Lehre an Universitäten und Fachhochschulen weiter ent- 
wickelt werden, um die Studierenden noch besser auf ihre zukünftigen Aufgaben in Unternehmen und Hochschulen vorzubereiten.

Für den Track wurden 21 Beiträge eingereicht und doppelt blind begutachtet. Neun Beiträge wurden schließlich ausgewählt.

Die vorliegenden Beiträge befassen sich mit der Fragestellung, wie sich das Thema ERP in die Hochschulausbildung integrieren läßt. Die Schwerpunkte sind: (1) Verknüpfung mit einer BWL-Kern-Vorlesung (wie z.B. Controlling und Marketing), (2) Einbindung externer Experten, (3) Nutzung verschiedener ERP Systeme in einer Vorlesung, (3) Weitgehend freie Arbeit der Studierenden am ERP-System, (4) Weitgehend geführte Arbeit der Studierenden am ERP-System, (5) Verbindung mit einem praktischen Projekt (z.B. im Bereich Maschinenbau), (6) Hochschulübergreifende Lehre und (7) der Einsatz von ERP-Planspielen. Auch wenn sich derartige Konzepte in anderen Bereichen bewährt haben, so ist es notwendig zu untersuchen, inwieweit sie sich auf den Themenbereich der ERP Ausbildung übertragen lassen.

Sowohl die Einreichungen auf diesen Track als auch die ausgewählten Beiträge machen deutlich, dass aktuell an vielen Hochschulen an der Entwicklung geeigneter Curricula gearbeitet wird. Wir hoffen, dass Dozenten durch diese Arbeiten wertvolle Anregungen für ihre eigene ERP-Vorlesung bekommen.

Programmkomitee:

- Prof. Dr. Helmut Krcmar, TU München

- Prof. Dr.-Ing. Norbert Gronau, Universität Potsdam

- Heino Schrader, SAP AG

- Stefan Weidner, Universität Magdeburg

- Dr. Holger Wittges, TU München 


\title{
ERP-Systeme in der Lehre - ein vergleichendes, hochschulübergreifendes Seminar mit mittelgroßen ERP-Systemen
}

\author{
Axel Winkelmann', Christian Leyh², Norbert Frick ${ }^{1}$ \\ ${ }^{1}$ Institut für Wirtschafts- und Verwaltungsinformatik, \\ Professur für Betriebliche Anwendungssysteme, \\ Universität Koblen₹-Landau \\ ${ }^{2}$ Lehrstuhl für Wirtschaftsinformatik, \\ insh. Informationssysteme in Industrie und Handel, \\ Technische Universität Dresden
}

\section{Motivation}

Standardisierte Enterprise Resource Planning (ERP) - Systeme sind heutzutage in einem Großteil der Unternehmen im Einsatz. Daher ist der Markt vor allem im Bereich der klein- und mittelständischen Unternehmen (KMU) sehr stark fragmentiert (Winkelmann et al. 2007). Dies erschwert es den Anwender-Unternehmen, die „richtige“ Software zu finden und dafür entsprechende Fachkräfte an sich zu binden. Dies ist essentiell, da Fehler im Bereich der Auswahl, der Einführung oder des Betriebes von ERP-Systemen finanzielle Nachteile nach sich ziehen können. Um dem vorzubeugen, ergibt sich die Notwendigkeit für Hochschulen, ihren Studierenden das geforderte Fachwissen, insbesondere in informationssystembezogenen Studiengängen zu vermitteln und somit entsprechende Systeme, Prozesse und passende Kurse anzubieten (Fedorowicz et al. 2004, S. 235-236; Venkatesh 2008, S. 141-142). Jedoch gibt es dafür keinen standardisierten Ansatz. Die Systemwahl und die Anzahl der Systeme sowie der Aufbau und die Anzahl der ERP-Kurse divergieren von Hochschule zu Hochschule (Seethamraju 2007, S. 69), wobei in diesem Punkt eine Dominanz großer ERP-System Hersteller zu verzeichnen ist. Allerdings ist eine diversifiziertere Einbindung von ERP-Systemen vor allem aus dem Bereich der KMU ratsam. An diesem Punkt knüpft dieser Artikel an und greift das von Winkelmann und Matzner (2009) beschriebene Seminarmodell - die problemorientierte Bearbeitung einer Fallstudie in Kleingruppen unter Nutzung verschiedener mittelgroßer ERP-Systeme - auf und erweitert das Konzept, um es parallel an drei verschiedenen Universitätsstandorten anwenden zu können. Durch sehr heterogene Studierendengruppen konnte dabei beleuchtet werden, welchen Einfluss die 
Anzahl der Teammitglieder auf den Erfolg des Seminars hat und wie sich die Anzahl der absolvierten Fachsemester der Teilnehmer auswirkt.

\section{Fazit}

Die Evaluation des Seminars zeigte, dass eine zu geringe Gruppengröße für dieses Seminar nicht förderlich ist, da somit der Aufwand von den Studierenden als sehr hoch eingestuft und das Seminar eher als Belastung anstatt als Wissensbereicherung angesehen wird. Somit ist eine deutliche Abhängigkeit zwischen Seminarerfolg, -aufwand und Anzahl der Teammitglieder festzustellen. Auch die Art der Studiengänge, oder genauer gesagt, die Anzahl der von den Studierenden absolvierten Fachsemester haben einen Einfluss auf das Seminar. In den Feedbackgesprächen konnte ermittelt werden, dass vor allem Studierende höherer Semester das Seminar als „passend“ ansahen, während Studierende niedrigerer Semester erheblich mehr Aufwand investieren mussten und daher das Seminar im Vergleich zum erlangten Wissenszuwachs als zu aufwandsintensiv ansahen.

\section{Literatur}

Fedorowicz J, Gelinas UJJ, Usoff C, Hachey G (2004) Twelve tips for successfully integrating enterprise systems across the curriculum. Journal of Information Systems Education 15(3):235-244.

Seethamraju R (2007) Enterprise systems software in business school curriculum Evaluation of design and delivery. Journal of Information Systems Education 18(1):69-83.

Winkelmann A, Knackstedt R, Vering O (2007) Anpassung und Entwicklung von Warenwirtschaftssystemen - eine explorative Untersuchung. Handelsstudie Nr. 3, Münster. http://www.wi.unimuenster.de/udoo/downloads/publications/1975.pdf. Abruf am 2009-09-10.

Winkelmann A, Matzner M (2009) Teaching medium sized ERP systems - a problem-based learning approach. In: Papadopoulos GA, Wojtkowski W, Wojtkowski WG, Wrycza S, Zupancic, J. (Hrsg) Information Systems Development: Towards a Service Provision Society. Springer, New York.

Venkatesh V (2008) “One-Size-Does-Not-Fit-All”: Teaching MBA students different ERP implementation strategies. Journal of Information Systems Education 19(2):141-146. 


\title{
Eine integrierte betriebswirtschaftliche Fallstudie mit SAP ERP und deren Einbindung in das SAP LEGO Labor
}

\author{
Danny Szendrei, Tobias Teich, Katja Unger, Jörg Militzer \\ Fachbereich Wirtschaftswissenschaften, \\ Westsächsische Hochschule Zwickau
}

Der folgende Beitrag stellt ein mehrgliedriges Pädagogikkonzept zum Einsatz des ERP-Systems SAP in der akademischen Lehre an der Westsächsischen Hochschule Zwickau, Fakultät Wirtschaftswissenschaften dar. Inhalt des Lehrkonzeptes ist eine komplexe, integrative betriebswirtschaftliche Fallstudie mit Lehrinhalten aus diversen Studienschwerpunkten.

Zielstellung der Fallstudie ist es, modul- bzw. studiengangsspezifische Lehrangebote in zusammenhängender Form am Lernträger SAP ERP/SCM darzustellen. Auf diese Weise soll bei den Studierenden das Verständnis von interdisziplinären Zusammenhängen und Schnittstellen diverser Geschäftsprozesse ausgebaut werden. Durch die Anbindung der Fallstudie an ein Labor (LEGO-Elemente), können insbesondere Logistikprozesse visualisiert und somit aktiv von Studierenden mitgestaltet werden. Mit der Fallstudie wird einerseits eine moderne, prozessorientierte Lehr- und Ausbildungsplattform geschaffen. Andererseits integriert die Fachgruppe Wirtschaftsinformatik die Bedeutung von aktuell geforderten Kompetenzprofilen (z. B. Teamfähigkeit, Kommunikationsfähigkeit) seitens der Industrie in die akademische Lehre.

Inhaltlich werden in der Fallstudie Geschäftsprozesse aus den Unternehmensbereichen Human Ressources, Logistik, Rechnungswesen, Controlling und Business Intelligence mit der Software SAP ERP und SAP SCM bearbeitet. Daneben wird das Customizing der Prozesse und Unternehmensstrukturen im System den jeweiligen Einheiten vorangestellt. Das Lehrkonzept setzt dabei auf Lerneffekte durch: 
- Interaktive Lerneinheiten (Lehrvorträge und Erörterung von Fachinhalten),

- Begleitaufgaben (eigenständige Berechnungen von Geschäftsvorfällen nach akademischen Mustern),

- Angeleitetes Selbststudium (Bearbeiten von Geschäftsvorfällen in SAP ERP / SCM)
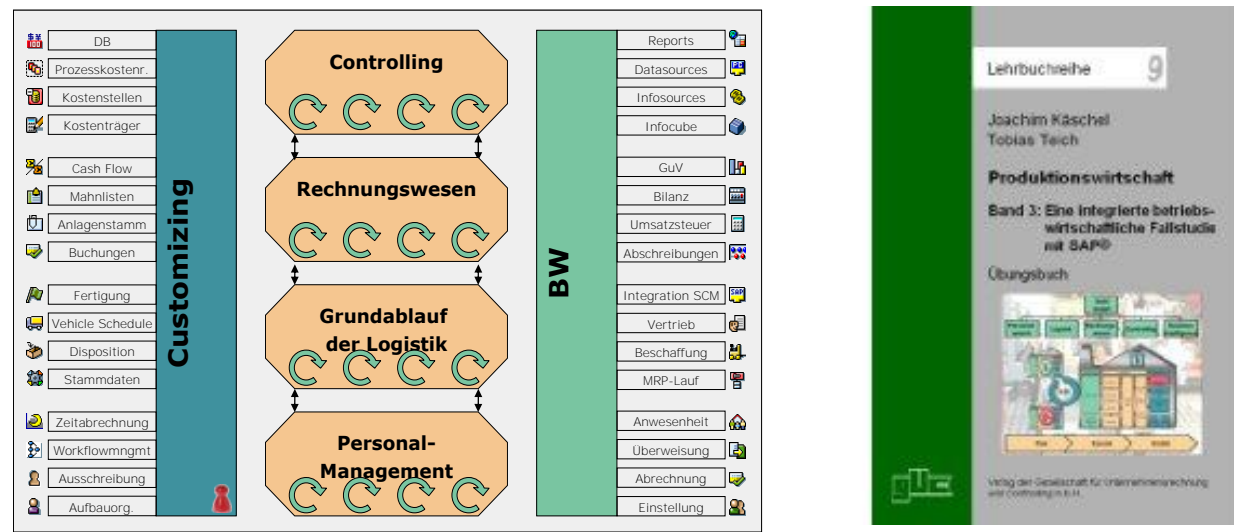

\section{Abbildung 1: Ablaufstruktur der betriebswirtschaftlichen Kernfelder und Lehrbuch}

Die Aufgabenstellungen der Fallstudie sind als Buch erschienen. Dieses beinhaltet die praktischen Einheiten sowie die Übungsaufgaben und ist somit ideales Begleitmaterial. Aufgrund der Dynamik der Inhalte ist geplant, jährlich eine neue Auflage zu erstellen. Ebenfalls in Abbildung 1 ist eine Übersicht der wichtigsten Inhalte dargestellt.

Durch die Verbindung der ERP/SCM Komponenten mit dem SAP LEGO Labor konnten bisweilen interessante Verbindungen $\mathrm{zu}$ Industrie- und Forschungspartnern der Hochschule auf- bzw. ausgebaut werden. Die Visualisierung von Geschäftsprozessen sowie der Modellanlage mit der Software visTABLE ermöglicht kostengünstige und raumsparende Planungsprozesse zukünftiger Szenarien. Das Labor dient ferner der Präsentation aktueller Forschungsarbeiten für Schüler im Rahmen der First Lego League. Somit bedient es die (vor-)akademische Ausbildung im Sinne der Zukunftssicherung der Hochschule. 


\title{
ERP-Ausbildung in der Wirtschaftsinformatik an der Universität Stuttgart
}

\author{
Systematische Ableitung eines Lehrkonzepts \\ auf Basis von Lehrzielen
}

\author{
Georg Herzwurm, Lars Oliver Mautsch, Sixten Schockert \\ Lehrstubl für ABWL und Wirtschaftsinformatik II (Unternehmenssoftware), \\ Betriebswirtschaftliches Institut, Universität Stuttgart
}

Ein erfolgreicher Einsatz von ERP Software in der Lehre ist nicht selbstverständlich und benötigt eine sorgfältige Planung. Dabei sollte die Gestaltung eines Lehrkonzepts zur ERP-Ausbildung - neben Randbedingungen wie die Rahmenempfehlungen zur WI-Ausbildung - auch die Bedürfnisse der beteiligten Personengruppen, im Kern Lehrende und Lernende, berücksichtigen. Um dies in systematischer Weise vorzunehmen, wird in diesem Beitrag eine Methode zur Ableitung eines ERP-Curriculums vorgestellt, die an der Universität Stuttgart eingesetzt wird.

Das Vorgehen basiert auf der sehr flexibel einsetzbaren, ursprünglich aus dem Qualitätsmanagement kommenden Planungsmethode Quality Function Deployment (QFD, siehe z. B. Herzwurm u. a. 2000). Das wesentliche Kennzeichen von QFD ist die konsequente Orientierung an und Umsetzung von Kundenanforderungen. Im Beispiel der Planung eines Curriculums nehmen Lehrziele diese Rolle der Anforderungen an das Lehrkonzept ein. Diesen Herausforderungen für die Lehre kann mit potentiellen Lerneinheiten eines ERP-Curriculums begegnet werden. Für jede mögliche Kombination von Lerneinheit und Lehrziel wird die Stärke der Wirkung einer umfangreichen Umsetzung der Lerneinheit auf die Erfüllung des Lehrziels beurteilt. Auf Basis dieses Abdeckungsgrades von Lehrzielen durch Lerneinheiten und einer Gewichtung der Lehrziele aus Dozentensicht wird das ERP-Curriculum der Universität Stuttgart abgeleitet. Tabelle 1 zeigt die Ergebnisse der Gegenüberstellung von Lehrzielen (Zeilen) und Lerneinheiten (Spalten) in Form einer Korrelationsmatrix. Die Bedeutung einer Lerneinheit ergibt sich dabei aus der Spaltensumme der Multiplikationen der einzelnen Korrelationswerte in den Matrixzellen mit den zugehörigen Gewichten der Lehrziele. 
Tabelle 1: Korrelationen zwischen Lehrzielen und Lerneinheiten

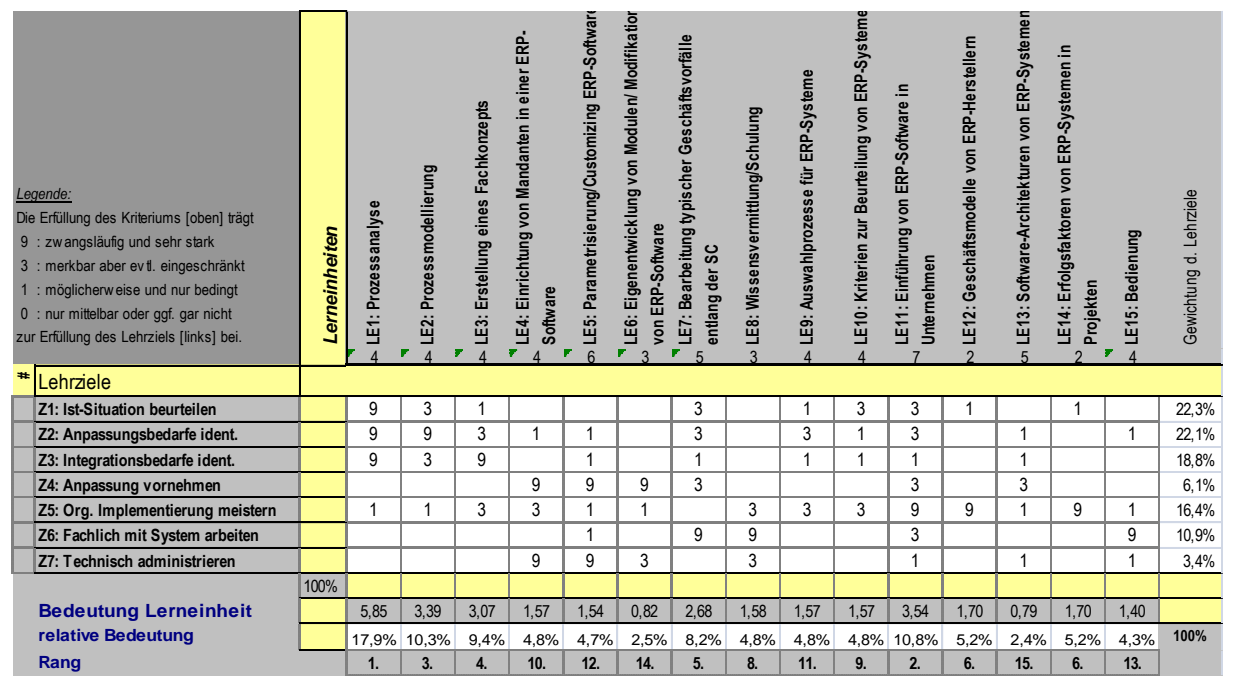

Die im Hochschulbereich in aller Regel knappen Ressourcen (Personal, Budget, Räume, Rechner etc.) können nun gezielt zugewiesen werden. Es kann eine Fokussierung auf die wichtigsten Lerneinheiten erfolgen, die im Sinne von Modulen zu einzelnen Lehrveranstaltungen kombiniert werden. Im Rahmen der praktischen Nutzung des neu eingerichteten ERP-BI-Labors (www.wi.uni-stuttgart.de/erp-bilab) der Universität Stuttgart ergaben sich dabei drei verschiedene FallstudienVeranstaltungen, die insbesondere die wichtigsten fünf Lerneinheiten (Prozessanalyse, ERP-Einführung, Prozessmodellierung, Fachkonzepterstellung, Bearbeitung typischer Geschäftsvorfälle) umfassen. Ebenfalls ist es möglich, ein bestehendes Curriculum zu bewerten und Verbesserungspotentiale aufzudecken.

Durch ein solches, systematisches Vorgehen zur Planung eines Lehrkonzepts ist sichergestellt, dass definierte Lehrziele durch den verfügbaren Fächerkanon abgedeckt werden. Gleichfalls wird vermieden, dass Veranstaltungen ohne Kundennutzen (insb. für Dozenten und Studierende) als Selbstzweck angeboten werden und knappe Ressourcen binden. Die vorgeschlagene Planungsmethode kann auf andere Bildungseinrichtungen und andere Lehrgegenstände übertragen werden. Allerdings empfiehlt es sich, Lehrziele und Lerneinheiten an die eigenen Rahmenbedingungen anzupassen und die Bewertungen der Lehrziele aus verschiedenen Perspektiven (Dozenten, Studierende, Arbeitgeber etc.) vorzunehmen, um möglichen Überbewertungen durch subjektive Einschätzungen entgegen zu wirken.

\section{Literatur}

Herzwurm G, Schockert S, Mellis W (2000) Joint Requirements Engineering. QFD for Rapid Customer-Focused Software Development. Vieweg, Braunschweig. 


\title{
Teaching the Transformation from Classical On- Premise towards On-Demand Enterprise Systems
}

\author{
Oliver Gaß, Alexander Mädche \\ Chair of Information Systems IV, \\ ERIS (Enterprise Information Systems), \\ University of Mannheim
}

Enterprise Systems, specifically integrated information systems such as Enterprise Resource Planning, are heavily used in practice. However, teaching concepts for these system areas haven't been established and standardized yet. When it comes to the practical perspective of enterprise systems, there is a lack of adequate business scenarios and detailed use cases.

Furthermore, enterprise information systems, and in particular ERP systems, are in an ongoing transformation process. One important example is the rise of Software-as-a-Service based solutions in the enterprise systems area. The innovative technology concept and delivery model Software-as-a-Service (SaaS) is expected to have a major impact on enterprise systems from a technological and business perspective. Successful enterprise applications have already been provided, for example, in the CRM area. However, from a research and business perspective it is still unclear if it makes sense to provide full-fledged ERP capabilities as SaaS, or if one should instead focus on light-weight satellite processes. Yet, in a time when buzz words like cloud computing can be found almost everywhere in the media and the scientific literature, this manifesting paradigm shift towards internet based software should not be ignored in teachings of enterprise systems anymore. Our teaching concept wants to fill this gap in teachings and help our students to grasp the impact of these ongoing changes in detail.

We implemented our teaching concept in the Enterprise Systems Course which targets Master of Science in Business Informatics students. As part of the Bachelor education in information systems, the students have already learned the basics of integrated systems, specifically ERP. The master level course now aims on extending this knowledge: It is designed to provide the participants with a comprehensive insight into concepts, methods, tools and current practice of information systems used within enterprises.

The teaching concept followed in the course consists of literature analysis, a foundational lecture and a case study. The literature analysis is carried out by the participants As a pre-assignment to the course. The actual teaching contents are broken down into five modules which are taught in six sessions of 4 hours. Module 1 provides a general introduction into the wide field of enterprise systems. It is followed by module 2 which covers operational systems, especially Enterprise 
Resource Planning (ERP) systems, in detail. Module 3 and module 4 examine information centric systems (module 3), such as software for Business Intelligence, and also people-centric software (module 4), such as groupware and enterprise portals.

Module 5 provides a deep investigation into interdependencies and synergies of current enterprise systems, but also addresses new trends like the shift towards internet based enterprise solutions often referred to as summarized under the term cloud computing or Software-as-a-Service. Every module is complemented with a presentation by a guest speaker which lends valuable insights into how specific enterprise systems are actually used in practice to support specific business functions or which impact current trends in theory actually cause in practice.

Thirdly, a case study is included in the teaching concept. The goal of the study is to understand enterprise systems in detail and get a basic impression of how to design and implement business cases. At the same time we want to make students familiar with the new concept of SaaS. Therefore, we have chosen not just a traditional application server infrastructure but instead the popular on-demand platform force.com for the implementation assignment. The main task here is to analyze the implementation of a common HCM business process in a SAP on-premise solution and migrate it to the force.com platform. During this process the students are confronted with several obstacles like the transformation of the SAP relational data model and the design of a new html based web interface in force.com. The implementation has to be complemented with a detailed comparison of the two solutions and is supposed to address especially the differences which arise due to specific characteristics of the force.com platform and the on-demand delivery model itself.

In the future we are planning on including multiple activities: First, we are currently running the presented teaching concept the first time in this winter semester 2009. Initial feedback of our students is positive and the already handed in deliverables exceed our expectations so far. Second, the presented case study currently focuses on re-implementing the talent management process on an on-demand platform. A more realistic approach would be to follow a hybrid on-premise/ondemand approach, where the major business objects are stored in the on-premise system (e.g. SAP ERP) and only specific processes with the associated data are carried out in the on-demand environment.

Third, we are looking into opportunities to broaden the presented approach and case study to other enterprise system classes (e.g. involving business intelligence or groupware systems). 


\title{
Didaktisches Kernkonzept des SAP ERP-Praktikums in der Controlling-Lehre der Universität des Saarlandes
}

\author{
Alexander Baumeister, Claudia Harrer, Ulrike Sträßer \\ Lehrstubl für Controlling, \\ Universität des Saarlandes
}

Die mit der bundesweiten Einführung berufsqualifizierender Bachelor- und Masterstudiengänge einhergehende zentrale Zielsetzung, durch die Entwicklung von Schlüsselkompetenzen die Praxis- und Berufsfeldorientierung der universitären Ausbildung nachhaltig zu stärken (Nünning 2008, S. 2), erfordert die Einführung neuer handlungsorientierter Lehrkonzepte in die traditionell bislang eher auf die Vermittlung von fachbezogenem Wissen und methodischen Fähigkeiten fokussierende universitäre Management-Lehre. Eine derartige Schlüsselkompetenz stellt für das Berufsfeld des Controllers der Umgang mit dem SAP-System dar, weshalb die SAP-Ausbildung heute mehrheitlich fester Bestandteil der universitären Lehreprogramme ist, so auch in der Controlling-Lehre der Universität des Saarlandes.

Eines der Kernziele des Lehrstuhls für Controlling liegt dabei in der Förderung der typischen beruflichen Handlungskompetenzen des Controllers mit einer fundierten Methodenbasis. In der SAP-Ausbildung soll ebendies durch die Vermittlung von Anwendungskompetenz im Umgang mit SAP ERP bei gleichzeitiger Vertiefung und Erweiterung controllingspezifischer Fachkompetenz gewährleistet werden. Da es zur erfolgreichen Stärkung der beruflichen Handlungskompetenz von Studierenden des Zusammenspiels von Fach-, Methoden-, Sozial- und Wertekompetenzen bedarf (Gnahs 2007, S. 28; Schweitzer 2004, S. 76-77), erscheint jedoch die in der SAP-Ausbildung regelmäßig eingesetzte Dozentenvorführung als alleiniges Lehrkonzept nur bedingt geeignet. So muss beim schwerpunktmäßigen Einsatz derartiger dozentenzentrierter Lehrmethoden generell davon ausgegangen werden, dass aufgrund der eher passiv-rezeptiven Rolle der Studierenden kein optimaler Lernerfolg erzielt werden kann (Hubwieser 2007, S. 36). Auch spiegelt das sachlogisch aufbereitete Wissen der Lernsituation nur sehr eingeschränkt die komplexen und wenig strukturierten Anforderungen des Berufsalltags wider (Humbert 2006, S. 34). Dies gilt insbesondere für die Arbeit mit SAP in der Unternehmenspraxis, die vielmehr durch die Konfrontation mit unbekannten Sachverhalten und deren Umsetzung im SAP-System gekennzeichnet ist.

Im Rahmen des SAP ERP-Praktikums des Lehrstuhls für Controlling kommt daher seit dem Wintersemester 2008/09 ein handlungsorientiertes Lehrkonzept zum Einsatz, welches durch die Kombination von integriertem Frontalunterricht mit 
der Bearbeitung von Transferaufgaben Elemente klassischer und innovativer Lehrformen miteinander vereint. Dabei wechseln sich bei dem nach dem aktuellen Stand der Didaktik empfohlenen integrierten Frontalunterricht Phasen des klassischen Frontalunterrichts, in denen der Dozent ein grundlegendes Instrumentarium zur SAP-gestützten Lösung von Controlling-Aufgaben vermittelt, mit Phasen studentischer Arbeit ab, in denen eine selbstständige Wiederholung der zuvor unter Anleitung durchgeführten Arbeitsschritte erfolgt (Gudjons 2007, S. 24). Der zweite didaktisch zentrale Bestandteil des Lehrkonzepts besteht in der Anfertigung von schriftlichen Ausarbeitungen, in denen über die in der Präsensveranstaltung behandelten Inhalte hinausgehende Transferaufgaben mit Fallstudiencharakter in Gruppenarbeit von den Studierenden eigenständig im SAP-System umzusetzen sind. Somit wird durch das zugrundeliegende Lehrkonzept der Schritt vom Kennen fachlicher Methoden hin zum Umsetzungs- und Anwendungskönnen im SAPSystem unterstützt und durch die Bearbeitung von Transferaufgaben in Gruppenarbeit sowohl die eigenständige Auseinandersetzung mit dem SAP-System als auch die Teamfähigkeit fördert. Dies bestätigten auch die Ergebnisse der zur Überprüfung der praktischen Relevanz der Lehrveranstaltung regelmäßig durchgeführten internen Evaluation eindrucksvoll, wonach die Studierenden die Teamarbeit als gute Vorbereitung auf ihren späteren Berufsalltag sahen, das eigenständige Arbeiten am SAP-System insgesamt hohen Anklang fand und die durchgängige Meinung herrschte, dadurch viel gelernt zu haben.

\section{Literatur}

Gnahs D (2007) Kompetenzen - Erwerb, Erfassung, Instrumente. Bertelsmann, Bielefeld.

Gudjons H (2007) Frontalunterricht - neu entdeckt. Integration in offene Unterrichtsformen. Klinkhardt, Bad Heilbrunn.

Hubwieser P (2007) Didaktik der Informatik. Grundlagen, Konzepte, Beispiele. Springer, Berlin.

Humbert L (2006) Didaktik der Informatik. Mit praxiserprobtem Unterrichtsmaterial. Teubner, Wiesbaden.

Nünning V (2008) Einleitung: Qualifikationen für Studium und Beruf. In: Nünning V (Hrsg) Schlüsselkompetenzen: Qualifikationen für Studium und Beruf. Metzler, Stuttgart.

Schweitzer M (2004) Gegenstand und Methoden der Betriebswirtschaftslehre. In:

Bea FX, Friedl B, Schweitzer M (Hrsg) Allgemeine Betriebswirtschaftslehre.

Bd. 1: Grundfragen. Lucius, Stuttgart. 


\title{
Integration von SAP Customer Relationship Management in der Lehre
}

\section{Praxisnahe Ausbildung anhand einer Fallstudie}

\author{
Bernd Heesen ${ }^{1}$, Marcus Hilmer², Timo Simon² \\ ${ }^{1}$ Fachgebiet Betriebswirtschaftliche Anwendungen, Hochschule Ansbach \\ 2 maibiro GmbH, Ismaning bei München
}

Der vorliegende Beitrag stellt eine Fallstudie auf Basis des SAP CRM-Systems vor, welche in das Curriculum der Lehrveranstaltung „Customer Relationship Management" im Studiengang Wirtschaftsinformatik an der Hochschule Ansbach (2009) integriert wurde. Die Fallstudie wurde von der Hochschule Ansbach in Zusammenarbeit mit der Unternehmensberatung maihiro (2009) erstellt.

In Zeiten der Wirtschaftskrise und des zunehmenden internationalen Wettbewerbs um Absatzmärkte steigt auch die Bedeutung von Systemen, die sowohl Marketing und Vertrieb als auch Servicefunktionen unterstützen. Betriebliche Anwendungen können dort auch zu Wettbewerbsvorteilen führen (Wölfle 2008, S. 9-16). Die Umsetzung dieses neuen Ansatzes erfordert, dass alle Informationen über die Beziehung mit den Kunden festgehalten werden, um einerseits den Kunden bestmöglich betreuen zu können (Operationales CRM) und andererseits auch via Data Mining unausgeschöpfte Potenziale zu erkennen und die jeweils für die Kundengewinnung, Kundenbindung und -Rückgewinnung effizientesten Marketinginstrumente auszuwählen (Analytisches CRM) (Bruhn 2007, S. 8).

Es bot sich an, im Rahmen der Vorlesung eine der führenden CRMSoftwarelösungen einzusetzen. Die Wahl fiel auf die Lösung der SAP AG (2009), deren CRM-System entsprechend der Studie von Gartner (2009) zu den führenden Systemen zählt. Für die hier vorgestellte Fallstudie kam folgende Software zum Einsatz, die von dem SAP University Competence Center in Magdeburg (SAP UCC Magdeburg 2009) zur Verfügung gestellt wurde: SAP ECC 6.0, SAP CRM 5.2, SAP Enterprise Portal, SAP GUI 7.1.

Der Kurs beinhaltete einen Überblick über CRM inklusive Marketing, Vertrieb, Service und Analytisches CRM aus Sicht des Endbenutzers. Darüber hinaus wurde den Studierenden die Konfiguration des Systems vermittelt. Eine Fallstudie, mit der die Studierenden in Teams je 3 Teilnehmern betraut wurden, stellte abschlieBend ein realistisches Beispielszenario einer CRM-Implementierung dar, bei dem das Gelernte angewendet werden konnte. Die Aufgaben der Fallstudie beinhalteten die Oberflächen-Konfiguration (u.a. Definition einer eigenen Businessrolle für Marketing und Service, Zuordnung der Rolle zu einem Mitarbeiter im Organisati- 
onsmodell), Konfiguration und Ausführung von Marketing-Funktionen (u.a. Kampagne einrichten und ausführen) als auch die Konfiguration und Ausführung von Service-Funktionen (u.a. Kundenumfrage einrichten und ausführen).

Das Feedback der mündlich befragten Studierenden war positiv. Besonders die Reflektion der unterschiedlichen Implementierungserfolge der sieben mit der gleichen Aufgabe betrauten Teams wurde positiv wahrgenommen. Eine Weiterentwicklung der Fallstudie ist im Jahr 2010 vorgesehen und soll neben der Bereitstellung der Kursunterlagen auf Englisch auch die Integration des CRM-Systems mit dem BI- und dem ERP-System im Rahmen der Fallstudie erreichen. Gleichzeitig soll auf die aktuellste Softwareversionen, z.B. SAP CRM 7.0, der verwendeten Systeme zurückgegriffen werden.

\section{Literatur}

Bruhn M (2007) Kundenorientierung: Bausteine für ein exzellentes Customer Relationship Management (CRM). Beck, München.

Gartner. (2009) Magic Quadrant for CRM Customer Service Contact Centers. http:/ / mediaproducts.gartner.com/reprints/microsoft/vol9/article3/article3.h tml. Abruf am 2009-10-04

Hochschule Ansbach. (2009) Willkommen beim Bachelor of Arts in

Wirtschaftsinformatik. http:/ /www.wif.fh-ansbach.de. Abruf am 2009-10-04

Maihiro. (2009) Über uns. http://www.maihiro.com/maihiro/de/home.php. Abruf am 2009-10-04

SAP AG. (2009) SAP Customer Relationship Management Solution Map. http://www.sap.com. Abruf am 2009-05-20.

SAP UCC Magdeburg. (2009) SAP Hochschulkompetenzzentrum Otto-vonGuericke-Universität Magdeburg. http://www.hcc.uni-magdeburg.de. Abruf am 2009-10-04.

Wölfle R (2008) Wettbewerbsvorteile in der Kundenbeziehung mit Business

Software. In: Wölfle R, Schubert P (Hrsg) Wettbewerbsvorteile in der

Kundenbeziehung durch Business Software: Praxislösungen im Detail. Hanser, München. 


\title{
Praxisnahe und effiziente ERP-Ausbildung am Beispiel der TU Ilmenau
}

\author{
Sebastian Büsch, Volker Nissen, Lutz, Schmidt \\ Fachgebiet Wirtschaftsinformatike für Dienstleistungen, \\ Technische Universität Ilmenau
}

Mit dieser Arbeit werden Möglichkeiten aufgezeigt, eine universitäre Lehrveranstaltung so zu gestalten, dass sie einen hohen didaktischen, fachlichen und praktischen Anspruch erhält und den teilnehmenden Studenten mehr als nur ERP-spezifisches Fachwissen vermittelt. Um diesem Anspruch gerecht zu werden, wird die Lehrveranstaltung PROBAS an der TU Ilmenau als die universitäre Lehre ergänzendes SAP-Projekt organisiert. Der Beitrag zeigt auf, wie diese ERP-Ausbildung effizient aufgebaut, gemanagt und das Projektmanagement laufend verbessert wird, um kommenden Herausforderungen in der WI-Lehre zu begegnen. Es werden innovative Lehrkonzepte und fachliche Lehrinhalte an erfolgreichen, praktischen Beispielen vorgestellt, die sich im Zusammenhang mit einer universitären Ausbildung im ERP-Bereich über Jahre bewährt haben.

PROBAS (Projektseminar betriebswirtschaftliche Anwendungssysteme) ist eine praxisorientierte Lehrveranstaltung der TU Ilmenau. Durch die langjährige Erfahrung bei der kontinuierlichen Betreuung der Lehrveranstaltung kann die Zielsetzung, bei der Ausbildung von Studenten an der TU Ilmenau, neben fachlichem ERP-Wissen auch weitere, praxisbezogene Fähigkeiten zu vermitteln, erreicht werden. Dazu zählen insbesondere das Entwickeln von Problemlösefähigkeiten, der Erwerb von Soft Skills und Erfahrungen im Projektmanagement sowie der Aufbau von Fähigkeiten zur Bewältigung realer Probleme. Das Erlernen von Kernkompetenzen im ERP-Bereich am Beispiel SAP sowie von fachbezogenem Wissen angrenzender Themen wie Business Intelligence (SAP-BI), Systemintegration (SAPPI) oder Anwendungsentwicklung (ABAP, SAP-EP) auf Basis der SAPNetweaver-Technologieplattform stehen ebenfalls im Mittelpunkt.

Um den hohen Praxisbezug der vermittelten Inhalte zu erreichen, wird PROBAS als Projekt in Form einer praktischen Vertiefung theoretischer Inhalte angeboten und ergänzt darin bestehende ERP-Vorlesungen am Fachgebiet Wirtschaftsinformatik für Dienstleistungen der TU Ilmenau. Dadurch lässt sich ein in sich geschlossenes, aber weitestgehend selbstorganisierendes LehrLern-Arrangement verwirklichen. Durch den Einsatz eigener Funktionsträger im studentischen Projektteam, die für Bereiche des Wissens- und Marketingmanagements der Lehrveranstaltung zuständig sind, kann bei gleichbleibendem Betreuungsaufwand durch Lehrstuhl und Praxispartner eine kontinuierliche Weiterentwicklung des Projektes erfolgen. Insbesondere bei der Vorbereitung auf die Praxis durch Projektstruktur, 
Soft Skills und Problemlösefähigkeiten ergeben sich dadurch Vorteile gegenüber statisch-theoretischen Lehrveranstaltungen in Form von Vorlesungen. Durch die langjährige Erfahrung bei der Durchführung der Veranstaltung seitens des Lehrstuhls und der Praxispartner kann so ein geeignetes Maß an studentischem Engagement flexibel durch die Variation der Aufgabenstellungen oder den Einbezug der Studenten in die Projektorganisation erreicht werden.

Das Ziel dieses Beitrages ist es, Wege aufzuzeigen, ERP-Lehre so zu gestalten, dass sie einem universitären Anspruch genügt und gleichzeitig unmittelbar berufsrelevante Kenntnisse vermittelt. Es wird aufgezeigt, welche Konzepte wie in die Lehre mit welchen Vorteilen eingebunden werden können, welche Ergebnisse sich auf diese Art an praktischen Beispielen ergeben können oder welche Lehrformen und Lehrinhalte sich für eine SAP-Ausbildung anbieten. Dabei werden auch die Vorteile realer Problemstellungen aufgezeigt und dargelegt, wie durch praktische Aufgaben die Tauglichkeit der theoretischen Lehre für die Praxis evaluiert werden kann. Insbesondere das Zusammenspiel zwischen Universität und Praxis ermöglicht hierbei große Synergien, wodurch beide Seiten profitieren.

Im Rahmen dieses Beitrages wird auch dargestellt, wie durch die Verwendung quantitativer Maße zur Steuerung einer Lehrveranstaltung am Beispiel PROBAS ein effizient arbeitendes Projektmanagement etabliert werden kann. Mittels des CMMI-Reifegradmodells des Software Engineering Institute (2006) wird eine Methode der Evaluation der Lehrveranstaltung vorgestellt. Um eine Verbesserung der Qualität des Projektmanagements zu erreichen, schlagen wir eine Balanced Scorecard (BSC) nach Kaplan und Norton (1996) für das Projekt PROBAS vor.

\section{Literatur}

Kaplan RS, Norton DP (1996) The Balanced Scorecard: Translating Strategy Into Action. Harvard, Boston.

Software Engineering Institute (2006) CMMI for Development, Version 1.2. Pittsburgh. 


\section{Planspiele in der ERP-Lehre}

\section{Eine empirische Untersuchung deutscher Bildungseinrichtungen}

\section{Christos Konstantinidis, Harald Kienegger, Holger Wittges, Helmut Kromar \\ Lehrstubl für Wirtschaftsinformatik, Technische Universität München}

Der stetige Wandel und Fortschritt im Umfeld betrieblicher Informationssysteme stellen sowohl Unternehmen als auch Bildungseinrichtungen vor neue Herausforderungen. Dabei kommt den Enterprise Resource Planning (ERP) Systemen eine besondere Bedeutung zu. Sie stellen nach Gronau (2004) einen wesentlichen Erfolgsfaktor für Unternehmen dar. Daher ist es erforderlich, die Verfügbarkeit qualifizierten Personals sicherzustellen und ein besonderes Augenmerk auf dessen Ausbildung zu legen (Mohr 2009). Um die Bewältigung täglicher Aufgaben und das Verständnis für grundlegende Konzepte und Verfahren zu gewährleisten, ist es notwendig, ein weiterführendes, über klassische Schulungen hinausgehendes Lehrmodell zu etablieren.

Die Komplexität von ERP-Lösungen erfordert, dass sich die Qualifizierung nicht nur auf konzeptuelles und Handhabungswissen beschränkt, sondern auch den Prozesskontext aufzeigt (Eggert 2005, S. 19). Handlungskompetenz umfasst dabei Fähigkeiten, die es Individuen erlauben, sich in beruflichen und anderen Lagen, situationsspezifisch, qualifiziert und sozial verantwortlich zu verhalten. Sie kombiniert Fakten- (know what), Anwendungs- (know how), Zusammenhangs- und Transfer- (know why) sowie Orientierungswissen (care why) (Kern 2003, S. 18-23). Für die drei zuletzt genannten Wissensformen eignen sich Planspiele besonders. Ein Planspiel stellt „,...] eine konstruierte Situation, in der sich eine oder mehrere Person(en) in oder an einem [...] Modell nach vorgegebenen Regeln verhalten [...]“ dar (Geilhardt und Mühlbradt 1995, S. 49). Unternehmen setzen sie bereits seit vielen Jahren zur Fort- und Weiterbildung ein. Auch der tertiäre Bildungssektor fokussiert Planspiele zunehmend. Um zu ermitteln, wie viele Planspiele in welchem Umfang in der Lehre eingesetzt werden, wurde im September 2009 eine empirische Untersuchung durchgeführt. Ihr Gegenstand waren deutsche Bildungseinrichtungen (BE), die am SAP University Alliances Program teilnehmen und ERP-Systeme in der Lehre einsetzen. Von den 254 untersuchten BE wurden in den letzten fünf Jahren 254 Planspiele angeboten. Eine innerhalb dieses Zeitraums mehrfach angebotene Veranstaltung wird nur einmal gewertet. Wie in Abbildung 1 dargestellt, bieten 53,94\% (Summe der BE mit einem Planspielangebot $>0$ ) der untersuchten BE Planspiele in der Lehre an. 


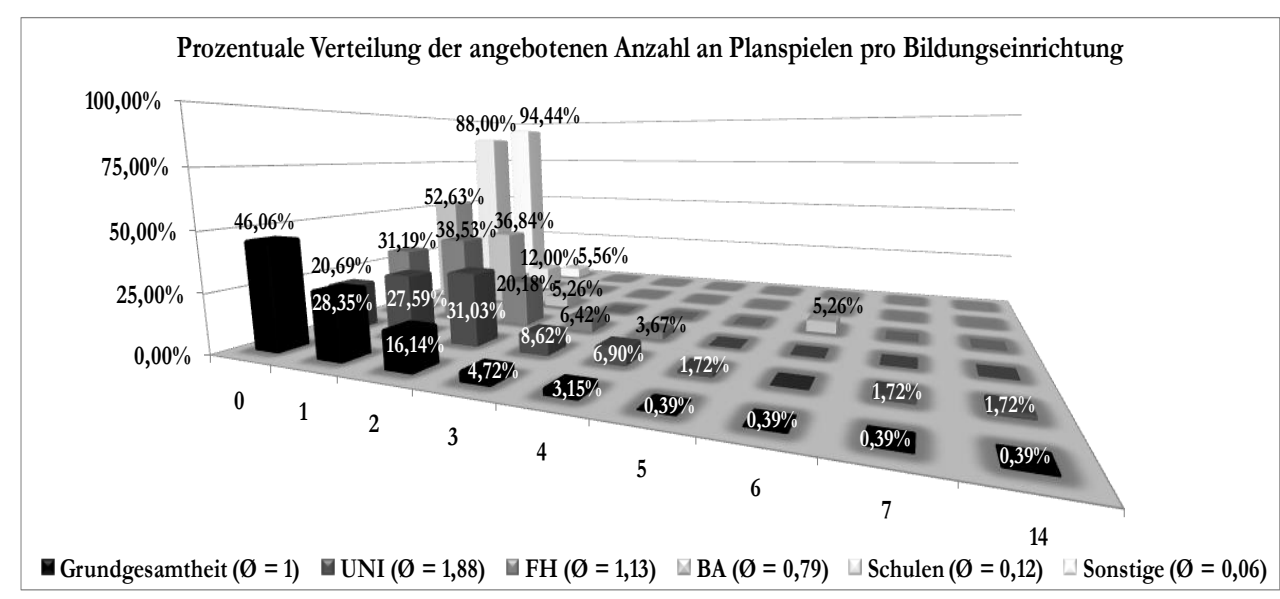

Abbildung 1: Prozentuale Verteilung angebotener Planspiele je BE

Die Untersuchung hat gezeigt, dass Planspiele bereits eine hohe Durchdringung in der Lehre tertiärer Bildungseinrichtungen erreicht haben. Jedoch wurde deutlich, dass der Begriff Planspiel für unterschiedliche Lehrmethoden steht. Besonders in der ERP-Lehre lassen sich hier Fallstudien einordnen, die den Teilnehmern die Möglichkeit geben, mit ERP-Lösungen zu arbeiten und somit Anwendungswissen zu erlernen. Der zunehmende Einsatz von Planspielen in der (ERP-)Lehre führte zu einer Entwicklung von umfangreichen Spielen. ERPsim wurde in diesem Beitrag exemplarisch betrachtet und als Planspiel identifiziert, das den Ansprüchen einer praxisnahen und nachhaltigen Ausbildung gerecht wird und Lernenden ermöglicht, den Umgang mit ERP-Software effizient zu gestalten.

\section{Literatur}

Eggert S (2005) ERP-Qualifizierung. ERP Management 2:19.

Geilhardt T, Mühlbradt T (1995) Planspiele im Personalmanagement und Organisationsmanagement. Hogrefe, Göttingen.

Gronau N (2004) Enterprise Resource Planning und Supply Chain Management. Oldenbourg, München.

Kern M (2003) Planspiele im Internet - Netzbasierte Lernarrangements zur Vermittlung betriebswirtschaftlicher Kompetenz. DUV, Wiesbaden.

Mohr M (2009) Qualifizierungsstrategien für betriebswirtschaftliche Unternehmenssoftware. Gabler, Wiesbaden. 


\title{
Curriculum Development for Teaching Business Information Systems to Business Administration Students - An Example from a University of Applied Sciences
}

\author{
Roland Schwald \\ Department of Business and Computer Science, \\ Albstadt-Sigmaringen University
}

This paper explains a possible curriculum concept for teaching information system content to business administration students at a university of applied sciences in Germany, based on an example from Albstadt-Sigmaringen University. Though this concept theoretically could be adopted by similar universities, the curriculum design should take into account particularities of individual institutions.

Universities of applied sciences were established between 1969 and 1971 in western Germany to offer students a university education, but - compared to scientific universities - with a clearer focus on the application of the major course concepts in organizations such as enterprises. While in recent years it has become a matter of discussion as to how far information technology (IT) itself really creates value and adds to the competitiveness of a business (Carr, 2003), many business administration programs of universities of applied sciences, including those at Albstadt-Sigmaringen University, have braced the idea of teaching business information systems to their students.

Starting point for the development of a curriculum are the relevant qualifications and skills the students should have at the time he or she graduates from university, such as method competences, in-depth knowledge of relevant systems, social skills and decision-making skills.

A practical approach to teaching business information systems is to accompany lectures with realistic exercises on major information systems. Among the IT systems used for this purpose are SAP ERP and Microsoft Dynamics (AX). The SAP system was chosen amongst other things because of its market leader position and Microsoft Dynamics (AX) was chosen because of its relevance to small and medium sized companies. It is also important for the design of a curriculum that the course contents in the various semesters build on each other.

A rough overlook of the sequence of the major contents of the mandatory IT courses offered at Albstadt-Sigmaringen is given in table 1. 
Table 1: IT curriculum for Business Administration students

\begin{tabular}{|c|c|c|c|}
\hline & Semester & IT content & $\begin{array}{l}\text { IT tools I } \\
\text { systems used }\end{array}$ \\
\hline \multirow{7}{*}{ 㟧 } & 1st & Introduction to Business Information Systems & MS Excel \\
\hline & 2nd & -- & \\
\hline & 3rd & $\begin{array}{l}\text { Intermediate Business Information Systems: } \\
\text { Database Management } \\
\text { Data Modeling } \\
\text { Data Warehouse }\end{array}$ & $\begin{array}{l}\text { Microsoft Approach } \\
\text { Microsoft Visio } \\
\text { SAP BI }\end{array}$ \\
\hline & 4th & $\begin{array}{l}\text { Advanced Business Information Systems: } \\
\text { ERP systems }\end{array}$ & $\begin{array}{l}\text { SAP ERP } \\
\text { MS Dynamics (AX) } \\
\text { ARIS Toolset }\end{array}$ \\
\hline & 5th & Internship & \\
\hline & 6th & Project: Standard Business Software & Microsoft Project \\
\hline & 7 th & --- & \\
\hline \multirow{3}{*}{ 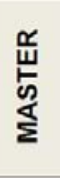 } & $1 \mathrm{st}$ & $\begin{array}{l}\text { Data Warehouse Systems } \\
\text { Data Analysis }\end{array}$ & $\begin{array}{l}\text { SAP BI } \\
\text { SPSS } \\
\text { Microsoft SQL Server }\end{array}$ \\
\hline & 2nd & E-Business Management & \\
\hline & $3 \mathrm{rd}$ & -- & \\
\hline
\end{tabular}

\section{References}

Carr, Nicholas G. (2003) IT Doesn't Matter. Harvard Business Review, May 2003: 41-49.

N.U.: Module descriptions (in German) for the Bachelor of Science in Business Administration program at Albstadt-Sigmaringen University. http://www.fhalbsig.de/ bwl/verweise/bachelor_module/modulbeschreibungen.htm, accessed on 2009-11-24.

N.U.: Module descriptions (in German) for the Master of Science in Business Administration and Management program at Albstadt-Sigmaringen University. http://www.fh-

albsig.de/ bwl/verweise/master_module/modulbeschreibungen.htm, accessed on 2009-11-24.

N.U. (2008) SAP Holds Top Rankings in Worldwide Market Share for SAP® Business Suite Applications. http:/ / www.sap.com/about/newsroom/press.epx?pressid=9913, accessed on 2009-11-24.

N.U. (2003) Universities of Applied Sciences in Germany. Published by Federal Ministry of Education and Research (BMBF), Bonn, Germany. http://www.bmbf.de/pub/universities_of_applied_sciences_in_germany.pdf, accessed on 2009-11-27. 
IKT-gestützte Unternehmenskommunikation 



\title{
Vorwort zur Teilkonferenz
}

\section{IKT-gestützte Unternehmenskommunikation}

\author{
Jorge Marx Gómez', Ralf Isenmann² \\ ${ }^{1}$ Abteilung Wirtschaftsinformatik I, Carl von Ossietzkey Universität Oldenburg \\ ${ }^{2}$ Forschungsgruppe Innovation und Kompeten₹transfer, Universität Bremen
}

Unternehmen stehen heute in einem vielschichtigen Wettbewerb um die Aufmerksamkeit ihrer Zielgruppen. Die traditionelle Konkurrenz auf den Märkten um Kapital, Arbeitskräfte und andere Produktionsfaktoren hat sich erweitert und wird überlagert von einem kommunikativen Spannungsfeld, in dem Unternehmen um die knappe Aufmerksamkeit ringen, z. B. von engagierten Mitarbeitern, aufgeschlossenen Kunden, zuverlässigen Lieferanten, finanzkräftigen Investoren sowie kooperativen Behörden, fairen Journalisten und unvoreingenommenen RatingAgenturen.

Im Wettbewerb um die Aufmerksamkeit ihrer Zielgruppen kommt der Unternehmenskommunikation eine Schlüsselrolle zu, sowohl aus einer Innenperspektive als auch im Zusammenspiel mit externen Zielgruppen.

Bei der Unternehmenskommunikation vollzieht sich derzeit ein tiefgreifender Wandel, vorangetrieben durch die Gestaltungschancen moderner Informationsund Kommunikationstechnologien (IKT). IKT-gestützte Unternehmenskommunikation prägt die Arbeits- und Berufswelt der Mitarbeiter sowie die Art und Weise der betrieblichen Leistungserstellung - und verwertung. Insbesondere durchdringt sie Organisationsstrukturen und Geschäftsprozesse sowie die ökonomischen Aktivitäten in Wertschöpfungsketten auf den verschiedenen Märkten und in vielschichtigen sozio-politischen Beziehungen. Die Gestaltungschancen der Internettechnologien und der dadurch verfügbaren -dienste verändern insgesamt die organisatorische Landschaft.

Im Zeitalter der Internet-Ökonomie und des E-Business erlangt die Gestaltungsaufgabe eines stimmigen Bildes des Unternehmens nach Innen und Außen unternehmenspolitische Relevanz sowie wettbewerbsstrategische Bedeutung und wird damit zur Führungsaufgabe. Informatisierung, Automatisierung und Personalisierung entwickeln sich zu Leitvorstellungen in der Unternehmenskommunikation, nicht nur aus Sicht der Wirtschaftsinformatik. Die aktuellen Entwicklungen in der Unternehmenskommunikation weisen neben anderen Tendenzen in Richtung maßgeschneiderte Unternehmenskommunikation für spezifische Zielgruppen, Einsatz ausgeklügelter IKT-Systeme und Software-Werkzeuge, Einsatz des Inter- 
net, mobile Endgeräte, cross-mediale Verfügbarkeit von Kommunikationsinstrumenten sowie dialogische und interaktive Formen der Online-Kommunikation.

Das Ziel des Tracks „IKT-gestützte Unternehmenskommunikation“ ist es, innovative Ansätze sowie Erfolg versprechende Vorgehensweisen und Best Practice vorzustellen, die von Experten aus verschiedenen Fachrichtungen entworfen wurden.

Nach einem sorgsam durchgeführten Begutachtungsverfahren haben sich neun Beiträge qualifiziert, die sich thematisch in folgende Schwerpunkte bündeln lassen:

- Einsatz von Web-2.0-Technologien zur Unternehmenskommunikation (Elsner; Fricke; Lattemann; Mrkwicka; Steinhüser)

- IKT-Systeme und Software-Werkzeuge zur Unterstützung der Unternehmenskommunikation (Hauke; Eggert)

- Managementansätze (z.B. Organisational Intelligence als Management von Information, Wissen und Meinung, Wissensmanagement, Organizational Memory) (Klein)

- Trends in der inhaltlichen Ausrichtung der Unternehmenskommunikation (Freundlieb)

Insgesamt bieten die neun Beiträge anregende Impulse zur Reflexion und Gestaltung einer IKT-gestützten Unternehmenskommunikation, sowohl für Wissenschaftler aus der Forschung als auch für Fach- und Führungskräfte aus der Praxis.

Abschließend sei den Mitgliedern im Programmkomitee für ihre engagierte Unterstützung sowie für ihre fundierte und konstruktive fachliche Einschätzung der eingereichten Beiträge herzlich gedankt. 


\title{
Erfolgsdimensionen von Social Software: Eine fallstudienbasierte Untersuchung
}

\author{
Melanie Steinbüser' ${ }^{1}$ Philip Räth² \\ ${ }^{1}$ Lehrstubl für BWL/Organisation und Wirtschaftsinformatik, \\ Universität Osnabrück. \\ 2Supply Chain Management Institute, \\ European Business School (ebs), Oestrich-Winkel
}

In den vergangenen zehn Jahren hat sich Social Software von einer neuen Technologie hin zu einem Massenphänomen entwickelt. Inzwischen setzen Unternehmen Tools wie Wikis und Weblogs in unterschiedlichen Bereichen ein. Trotz der steigenden Popularität berichten Unternehmen aber über Probleme bei der Einführung von Social Software. Bisher haben sich nur wenige empirische Studien mit dem Einsatz und Erfolg von Social Software in Unternehmen befasst, die entweder sehr spezifische Facetten beleuchten oder einer theoretischen Grundlage entbehren. Ein fundierter Beitrag zur Theoriebildung fehlt bisher.

Um ein geeignetes Instrument zur Identifikation und Messung von Erfolgsfaktoren von Social Software zu entwickeln, ziehen wir das IS-Impact Measurement Model von Gable et al. (2008) als Rahmen und Ausgangspunkt unserer empirischen Forschung heran. Dieses basiert auf der Arbeit von DeLone und McLean (2003), versucht aber, einigen der Kritikpunkte entgegenzuwirken. Aufgrund der schwachen empirischen Grundlage im Bereich Social Software, wählen wir eine explorative Forschungsmethodik. Diese ermöglicht, Dimensionen und Faktoren zu berücksichtigen, die in dem Modell bisher nicht erfasst wurden. Besonders die Fallstudienforschung eignet sich, Bereiche zu untersuchen, zu denen kein umfangreiches empirisches Material vorliegt. Bzgl. des Forschungsprozesses haben wir uns an dem von Eisenhardt (1989) vorgeschlagenen Vorgehen zur Erhebung und Auswertung von Fallstudien orientiert, drei Unternehmen unterschiedlicher Branchen wurden dabei in die Studie einbezogen. In Interviews, die mit Mitarbeitern unterschiedlicher Positionen geführt wurden, haben wir nach erfolgsrelevanten Faktoren der Social Software gefragt. Dies umfasste die Thematisierung der Definition von Erfolg sowie der Faktoren, die diesen Erfolg beeinflussen und anhand derer sich ein Erfolg messen lassen kann.

Die Ergebnisse unserer Studie liefern wichtige Hinweise auf Erfolgsfaktoren, die bei der Implementierung und Nutzung von Social Software eine entscheidende Rolle spielen. Im Zuge der Analyse wurden einerseits neue Faktoren identifiziert, die für den Erfolg von Social Software relevant sind, aber durch das IS-Impact Measurement Model nicht erklärt werden. Auf der anderen Seite erscheinen einige 
Maße des Modells in einem Social Software Kontext nicht relevant und können bei der Erfolgsmessung außen vor bleiben.

Von den neu identifizierten Faktoren lassen sich einige, wie Spaß und Serendipity oder Mitarbeiterbindung und Vernetzung, in die bestehenden Dimensionen des IS-Impact Measurement Model einordnen. Andere Faktoren wurden aus den Daten gewonnen, die als (sehr) erfolgsrelevant bewertet wurden, sich aber nicht in die vier Dimensionen eingliedern lassen. Sie betreffen zwar durchweg die Qualität des Informationssystems, fallen aber weder unter die technisch orientierte Dimension System Quality noch unter Information Quality. Dies sind: Unternehmensund Kommunikationskultur, verfügbare Ressourcen, Bereitschaft, Wissen zu teilen, Extravertiertheit der Nutzer, Literacy sowie Verantwortung.

Es wird dabei deutlich, dass das Modell ohne weitere Modifikationen nicht zur Erfolgsmessung von Social Software herangezogen werden kann. Vielmehr muss eine neue Social Software - spezifische Dimension hinzugefügt werden, da gerade hier Interaktionen wie Kommunikation und Kollaboration den Erfolg positiv beeinflussen können (Burton-Jones und Gallivan 2007). Das IS-Impact Measurement Model hat zwar den Anspruch, auf unterschiedliche Systeme anwendbar zu sein, wurde aber (wie auch das DeLone \& McLean IS Success Model) unter Annahmen klassischer Informationssysteme (wie ERP) entwickelt und validiert. Es spiegelt somit eine eher technisch orientierte Perspektive wider und vernachlässigt soziale Aspekte, die sich auf die Nutzung und den Erfolg auswirken. Wie wir gezeigt haben, ist eine soziotechnische Betrachtung in einem Social Software Kontext aber nicht zu vernachlässigen. Das heißt, dass Dimensionen etablierter Modell wie System- und Informationsqualität zwar weiterhin wichtig sind, aber nicht ausreichen, um den Erfolg von Social Software umfassend und zuverlässig zu bemessen.

\section{Literatur}

DeLone WH, McLean ER (2003) The DeLone and McLean model of information systems success: A ten-year update. Journal of Management Information Systems 19(4): 9-30.

Gable GG, Sedera D, Chan T (2008) Re-conceptualizing information system success: The IS-impact measurement model. Journal of the AIS 9(7): 377-408.

Eisenhardt KM (1989) Building theories from case study research. Academy of Management Review 14(4): 532-550.

Burton-Jones A, Gallivan MJ (2007) Toward a Deeper Understanding of System Usage in Organizations. MIS Quarterly. 31 (4): 657-679 


\title{
Status Quo der internetbasierten Nachhaltigkeitsberichterstattung
}

\section{Eine länderübergreifende Analyse der Nachhaltigkeits- berichte börsennotierter Unternehmen}

\author{
Michael Freundlieb, Frank Teuteberg \\ Fachgebiet Unternehmensrechnung und Wirtschaftsinformatik, \\ Universität Osnabrück
}

Nachhaltigkeitsberichterstattung (NBE) zielt darauf ab, gegenüber internen und externen Stakeholdern Rechenschaft darüber abzulegen, inwiefern das berichtende Unternehmen ökonomische, ökologische und soziale Nachhaltigkeitsziele miteinander in Einklang bringt, und welche Maßnahmen zur Verbesserung der Umweltleistung des Unternehmens ergriffen werden. Nachhaltigkeitsberichte (NB) sind somit ein wichtiger Bestandteil der Unternehmenskommunikation, der durch den Einsatz von Informations- und Kommunikationstechnologie wirkungsvoll unterstützt werden kann.

Im Rahmen dieses Beitrags wurden die veröffentlichten NB von 97 Unternehmen aus dem DAX (Deutschland), EuroStoxx (Europa) und dem Dow Jones Aktienindex (USA) systematisch quantitativ untersucht und verglichen.

Kurz-Zusammenfassung der wesentlichen Ergebnisse:

Der Großteil der untersuchten Unternehmen veröffentlicht unabhängig von der Region einen aktuellen NB. Deutschland und die USA waren bisher Vorreiter in der NBE, Europa hat diesen Vorsprung jedoch zwischenzeitlich aufgeholt. Insgesamt besteht eine hohe Durchdringung der NBE.

I.d.R. werden in den NB alle drei Säulen der Nachhaltigkeit abgedeckt. Im Bereich der Verbräuche und Abfälle (insb. Abwasser) bestehen in den USA und Europa im Vergleich zu Deutschland jedoch noch Berichtslücken. Deutsche NB sind oft detaillierter als in den übrigen Regionen: Es werden häufiger konkrete Zahlen genannt, und die Kennzahlen werden seltener zu Kennzahlengruppen zusammengefasst. Im Bereich Gesellschaft/Soziales gehören eine Mitarbeiter- sowie eine Sicherheits- bzw. Gesundheitsstatistik zum Standard. In Deutschland werden deutlich häufiger Angaben zur Korruptionsbekämpfung gemacht als im Rest der Welt. Umweltzertifizierungen und -standards spielen in Deutschland und Europa eine deutlich größere Rolle als in den USA. Als wichtigste Instanz für Richtlinien ist die 
Global Reporting Initiative (GRI 2006) zu nennen. Stark umweltverschmutzende Branchen veröffentlichen i. d. R. besonders umfassende NB.

Länderübergreifend sind NB im drucknahen PDF-Format am beliebtesten. Technisch aufwändige Online-Portale haben in den USA 3 von 4 Unternehmen bereits umgesetzt, hier haben Europa und insbesondere Deutschland noch Aufholbedarf. Kommunikation mit den Stakeholdern findet überwiegend per E-Mail statt. Umfragen sind in den USA und Deutschland ebenfalls ein relativ beliebtes Kommunikationsmittel. Kein Unternehmen ermöglicht es den Benutzern, öffentlich sichtbare Kommentare oder Trackbacks zum Nachhaltigkeitsbericht abzugeben.

Neben der Umweltabteilung sind häufig auch die Abteilung für Öffentlichkeitsarbeit oder externe Dienstleister und Beratungsunternehmen in den NBE-Prozess eingebunden.

Forschungsbedarf besteht hinsichtlich einer flexiblen Anpassung der NB an die Informationsbedürfnisse der Stakeholder (Isenmann und Gómez 2008) sowie der bidirektionalen Kommunikation zwischen Unternehmen und Stakeholdern (Süpke et al. 2009). Eine stärkere Ausrichtung der Untersuchung auf qualitative Aspekte sowie andere Unternehmensgruppen wie KMU bieten Ansätze für weitere Forschung.

\section{Literatur}

GRI (2006) Sustainability Reporting Guidelines. http://www.globalreporting.org/. Abruf am 2009-10-04.

Isenmann R, Gómez JM (2008) Internetbasierte Nachhaltigkeitsberichterstattung Maßgeschneiderte Stakeholder-Kommunikation mit IT. Schmidt, Berlin.

Süpke D, Gómez JM, Isenmann R (2009) Web 2.0 for Sustainability Reporting In: Wohlgemuth V, Page B, Voigt K (Hrsg) Proceedings of the 23 ${ }^{\text {rd }}$ International Conference on Informatics for Environmental Protection, Berlin: 219-228. 


\title{
„Sags uns“
}

\section{Von der Beschwerde zur Lösung - das Ideenblog der TU Braunschweig als Ausdruck direkter Demokratie}

\author{
Gerald Fricke, Yvonne Gaedke, Susanne Robra-Bissantz. \\ Institut für Wirtschaftsinformatik, \\ Abteilung Informationsmanagement (wi2), \\ TU Braunschweig
}

Das 2009 gestartete Weblog Sags uns (www.tu-braunschweig.de/sagsuns) der TU Braunschweig versucht, Ideen, Lob, Kritik und Beschwerden aller Studentinnen und Studenten, Mitarbeiterinnen und Mitarbeiter der Universität auf einer InternetPlattform zentral zu bündeln. Alle Beteiligten können mitschreiben, diskutieren und gemeinsam Verbesserungen für Studium und Lehre an der TU Braunschweig initiieren, wie die ersten Erfahrungen zeigen. In diesem Beitrag soll die Begründung und Umsetzung dieses Blogs kurz skizziert und gefragt werden, ob sich an dieser Entwicklung ein Wandel zu mehr direkter Demokratie ablesen lässt.

Eine Möglichkeit wäre es, das Web 2.0 nicht unbedingt und vorbehaltlos als Ausbund der Weisheit zum Umgang mit allgemeinen Gütern zu interpretieren, sondern als ein Instrument, um das Wissen und die Fähigkeiten der Massen zu erfassen und anderen Nutzern zur Verfügung zu stellen. Nach diesem Verständnis könnten Ideenblogs das Wissen von Amateuren und Experten zusammenführen und die Akteure vernetzen. Am besten über die engen Grenzen der eigenen Universität hinaus.

Eine andere normative Sicht auf das Web ergibt sich, wenn man ein Ideenblog nicht als kosmopolitische Graswurzeldemokratie oder als allgemeines Wiki versteht, sondern als ein Medium zur zielgerichteten Auswahl von Inhalten und Experten. Demokratie im Web 2.0 heißt nicht, dass dabei wirklich alle mitmachen müssen, sondern nur die Nutzerinnen und Nutzer, die sich tatsächlich engagieren wollen - und die von anderen Nutzern als wertvolle und relevante Mitdiskutanten oder sogar als Meinungsführer akzeptiert werden. 
Zukünftig wird es um die Frage gehen, so die normative Conclusio, wie neben der indirekten Mitbestimmung über einen Ideenmarkt eine direkt-demokratische Einflussnahme der Studentinnen und Studenten auf die repräsentativ verfasste Institution Universität aussehen könnte. Und damit allgemein um die Frage, ob und wie das Web 2.0 die repräsentative Demokratie ergänzen und stärken könnte. 


\title{
Ansatz zur Entwicklung von Referenzprozessen im Bereich des Enterprise Content Managements unter dem Aspekt der Wandlungsfähigkeit
}

\author{
Sandy Eggert \\ Lehrstubl für Wirtschaftsinformatik und Electronic Government, \\ Universität Potsdam
}

Das Thema Wandlungsfähigkeit gelang über die Fabrikplanung in den Fokus von Organisationen und Unternehmen. Dabei wurde festgestellt, dass vor allem große Unternehmen von ihrer IT-Infrastruktur abhängig sind und der Forderung nach Wandlungsfähigkeit nachkommen müssen (Overby et al. 2006, S. 120). Das Konzept Wandlungsfähigkeit soll nun aufgrund der steigenden Bedeutung des Themas Enterprise Content Management (ECM) auf Prozesse des ECM übertragen werden. Mit Hilfe eines methodischen Ansatzes werden unter Anwendung von Indikatoren zur Erhöhung der Wandungsfähigkeit, wandlungsfähige Referenzprozesse, sog. Kartenmuster entwickelt. Die Methode zur Entwicklung wandlungsfähiger Kartenmuster verwendet die Indikatoren zur Förderung der Wandlungsfähigkeit von Anwendungssystemen mit einer ECM-spezifischen Attributierung. Im Hinblick auf die Erstellung von Kartenmustern werden zuvor einzelne Prozessbausteine zur Betrachtungsgrundlage entwickelt. Ziel ist es, alle möglichen und relevanten Prozessbausteine in den Kartenmustern abzudecken, um schließlich auf Basis der Indikatoren Empfehlungen zur Verbesserung der Wandlungsfähigkeit für einen ECM-Gesamtprozess ableiten zu können. Die zu ermittelnden Prozessbausteine werden als Fragmente bezeichnet, da sie einzelne, spezifische Teile eines ECM-Prozesses verkörpern. Zur Bildung von Fragmenten werden die Lebenszyklusphasen von Dokumenten und Content herangezogen. Es gilt hierbei die Annahme, dass mit Hilfe der Lebenszyklusphasen alle Prozessschritte in allen möglichen ECM-Prozessen abgebildet werden können. Entlang der einzelnen Bestandteile der Fragmente (z. B. Prozesschritt, System) werden anhand der Indikatoren Gestaltungsempfehlungen zur Erhöhung der Wandlungsfähigkeit abgeleitet. Basierend auf den Fragmenten wurden Kartenmustern entwickelt, die im Sinne einer Referenzmodellbildung typische ECM-Prozessbausteine unter dem Aspekt der Wiederverwendung darstellen. Im Bereich des ECM besteht hinsichtlich der Referenzmodellierung enormer Handlungsbedarf. Derzeit existieren nur Hinweise zur Prozessgestaltung im DOMEA ${ }^{\circledR}$-Organisationskonzept (teilweise auch im MoReq2-Standard) sowie in einigen Spezifikationen konkreter Anwendungssysteme. Die grundlegende Idee der Kartenmuster ist die Beschreibung eines ECMProzessschrittes in wandlungsfähiger Form. Die Gestaltung eines Prozessschrittes 
basiert dabei auf einem standardisierten Prozess, welcher einen typischen ECMProzess abbildet. Die Beschreibung eines Kartenmusters besteht aus einem initialen Kontext, der einen allgemeinen, wiederkehrenden Ablauf innerhalb der Dokumentenbearbeitung beschreibt, einem Vorschlag einer wandlungsfähigeren Gestaltung sowie der visualisierten Abbildung in Form einer Prozesskarte. Die entwickelten Kartenmuster sollen als Referenzprozesse im Sinne einer wandlungsfähigen Gestaltung von ECM-Prozessen eingesetzt werden können. Ein sinnvoller Einsatzbereich stellt hierbei die Potenzialanalyse im Rahmen der Aufdeckung von Prozessverbesserungen dar. Die Ableitung von Kartenmustern erfolgt entlang der Gestaltungsempfehlungen der erarbeiteten Fragmente (siehe Abbildung 1).

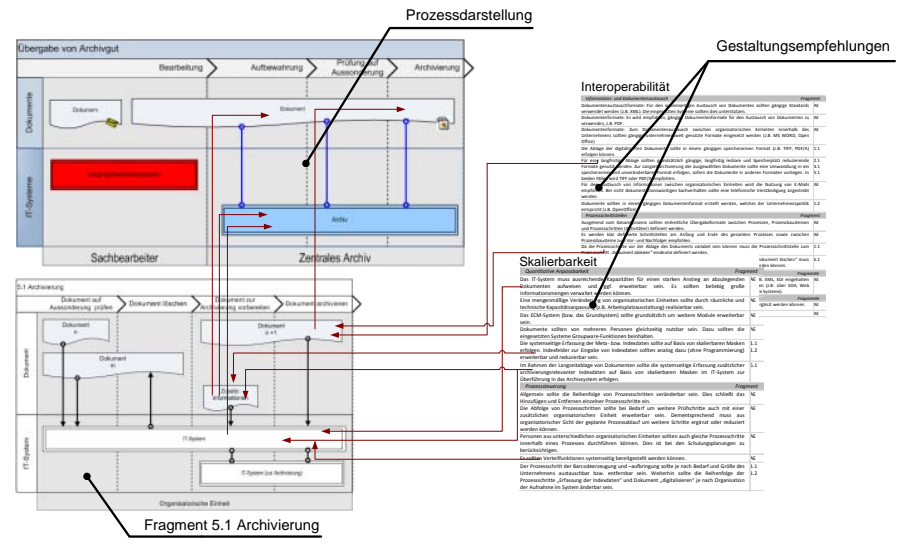

Abbildung 1: Ausschnitt der Ableitung von Potenzialen

Die erarbeiteten Kartenmuster wurden in zwei Fallstudien evaluiert. Entlang der Bewertung der SOLL-Prozesse beider Fallstudien konnte eine Erhöhung der Wandlungsfähigkeit gegenüber den IST-Prozessen festgestellt werden.

\section{Literatur}

Eggert S, Gronau N (2008) Erhöhung der Wandlungsfähigkeit von ECMLösungen unter Verwendung kartographischer Gestaltungsmittel. In: Proceedings of the MKWI. M. Bichler et al.: 433-446.

Overby E, Bharadwaj A, Sambamurthy V (2006) Enterprise agility and the enabling role of information technology. In: European Journal of Information Systems, 15: 120-131. 


\title{
Einsatz und Nutzung von Social Software in kooperativen Forschungsprojekten
}

\author{
Christoph Lattemann, Miriam Kallischnigg, Stefan Stieglitz. \\ Corporate Governance und E-Commerce, \\ Universität Potsdam
}

Social Softwaretechnologien und -verfahren wie Weblogs, Wikis und Soziale Netzwerke bilden neue Möglichkeiten zum Informations- und Wissensaustausch und zur Zusammenarbeit in und zwischen Organisationen (McAfee 2009, Stieglitz 2008). Obgleich einige dieser technologischen Ansätze nicht völlig neu sind, hat der Trend des Web 2.0 den Begriff der Social Software mit erfasst und popularisiert. O’Reilly beschreibt Web 2.0 als Plattform, die auf einer Architektur der Beteiligung basiert, die Services im Paket anbietet, auf der Datenquellen vermischbar sind und dadurch Datentransformationen möglich werden (O'Reilly 2005, Szugat et al. 2006). Social Software stellt heute wesentliche Inhalte dessen dar, was Web 2.0 ausmacht und fasst die „Webanwendungen zusammen, die dazu dienen, die Kommunikation innerhalb menschlicher Netzwerke zu unterstützen. “(Szugat et al. 2006, S. 13). Allen (2004) vereint all solche Anwendungen unter dem Begriff Social Software, die die Interaktion innerhalb einer Gruppe stärkt. Damit forciert Social Software Beziehungen, die innerhalb von bereits formierten Gruppen bestehen.

Eine Reihe von Studien (vgl. Metastudie von Lattemann et al. 2009) belegen die erfolgreiche Implementierung von Web 2.0-Technologien in Unternehmen weltweit. Bislang unbeachtet blieben jedoch die Verwendung und Potenziale dieser Technologien in (Forschungs-)Verbundprojekten. Eine vergleichende Analyse zum Einsatz und Mehrwert von Social Software in (Forschungs-) Verbundprojekten mit der Nutzung in Unternehmen soll dazu beitragen, Best Practices zu identifizieren und Projektleitern einen Soll-Ist-Vergleich für die eignen Projekte zu ermöglichen.

Social Software kann Projekte primär hinsichtlich zweier Aspekte unterstützen. Erstens in der Dokumentation von Projektergebnissen und Datensammlung über Foren und zum zweiten in der kollaborativen Arbeit über Wikis (Koch und Richter 2009). Die Ergebnisse zeigen, dass primär diese beiden Web 2.0-Werkzeuge in Projekten eingesetzt werden. Unternehmen wie auch Forschungsverbundprojekte haben die Bedeutung von Social Software erkannt: Wurde Social Software in Projekten eingesetzt, waren die Nutzer überwiegend „sehr zufrieden“ oder „eher zufrieden".

Gleichzeitig ist der Einsatz von Social Software stark von dem im Forscherteam zur Verfügung stehenden Wissen und der Ausrichtung des Projekts abhängig (bspw. IT-Projekte). Demnach besteht Aufholbedarf in der Erklärung zum Einsatz. Um diese Lücke zu schließen und um die Potenziale für ihre Projekte auszu- 
nutzen, könnte die DFG pro-aktive die Nutzung von Social Software empfehlen oder Partner aus der Wirtschaft bewusst in die Projekte integrieren, so dass Erfahrungen mit der Nutzung der Social Software aktiv ausgetauscht werden können. Hierzu ist zunächst die Entwicklung von Instrumenten notwendig, die speziell darauf ausgerichtet sind, die in Forschungsprojekten herrschenden Bedürfnisse zur erfüllen. So hat die Untersuchung gezeigt, dass eine leichte Bedienbarkeit und unproblematische Implementierung hierfür notwendige Kriterien darstellen.

Diese Untersuchung bzgl. des Einsatzes von Social Software in kooperativen Forschungsprojekten diente unter anderem dazu, Projektleitern einen Status Quo über die Nutzung von Social Software in Forschungsverbundprojekten zu geben.

\section{Literatur}

Allen C (2004) Tracing the evolution of Social Software.

http://www.lifewithalacrity.com/2004/10/tracing_the_evo.html, Zugriff am 28.09.2009.

Koch M, Richter A (2009) Enterprise 2.0: Planung, Einführung und erfolgreicher Einsatz von Social Software in Unternehmen. Oldenbourg Wissenschaftsverlag, München.

Lattemann C; Kupke S; Stieglitz S (2009) Deutsche Unternehmen auf dem Weg zum Web 2.0? - Eine Bestandsaufnahme, in: HMD - Praxis der Wirtschaftsinformatik, Special Issue „E-Collaboration“, Heft Nr. 267, S.18-26.

McAfee A (2009) Enterprise 2.0: New collaborative Tools for your Organization's Toughest Challenges. Harvard Business Press, Boston.

O'Reilly T (2005) What is Web 2.0 - Design Patterns and Business Models for the Next Generation of Software. http://www.oreillynet.com/lpt/a/6228.

Stieglitz S (2008) Steuerung Virtueller Communities: Instrumente, Mechanismen, Wirkungszusammenhänge. Gabler, Wiesbaden.

Szugat M, Gewehr JE, Lochmann C (2006) Social Software. Entwickler.press, Imprint der Software \& Support Verlag GmbH. 


\title{
Optimierungsansätze für E-Mail-Kampagnen in der Kundenkommunikation
}

\author{
Robert Hauke, Klaus D. Wilde \\ Lehrstubl für Allgemeine Betriebswirtschaftslehre und Wirtschaftsinformatik, \\ Katholische Universität Eichstätt-Ingolstadt
}

In den letzten Jahren haben sich innerhalb der Kommunikation mit Kunden zahlreiche Änderungen vollzogen. Diese führten zu einem verstärkten Wettbewerb der Unternehmen um die Aufmerksamkeit ihrer Zielgruppe. Im folgenden Beitrag wird die E-Mail als Kommunikationskanal der Kundenkommunikation näher untersucht.

Der vorliegende Beitrag strebt eine umfassende Analyse zur Identifikation von Optimierungsansätzen in der Kundenkommunikation per E-Mail an. Dafür wurden im Rahmen einer mehrstufigen Delphi-Befragung die Faktoren identifiziert, welche die Wirkung einer E-Mail-Kampagne beeinflussen können.

Zur Strukturierung der Kommunikation sowie der Darstellung der Einflussfaktoren wird das Kommunikationsmodell von Lasswell (1964, S. 37) verwendet. Dabei übermittelt ein Absender (z. B. Unternehmen) eine Kommunikationsbotschaft über einen Kanal (z. B. E-Mail) einem oder mehreren Empfängern (z. B. Kunden). Die Kommunikationsbotschaft wird abhängig vom jeweiligen Übertragungskanal in Wort, Bild, Zeichen und/oder Musik kodiert. Dieser Kernprozess kann von situativen Störfaktoren beeinflusst werden (Bruhn 2007, S. 38-39). Die in der Delphi-Befragung identifizierten Einflussfaktoren können den einzelnen Elementen des Kommunikationsprozesses zugeordnet werden (siehe Abbildung 1). 


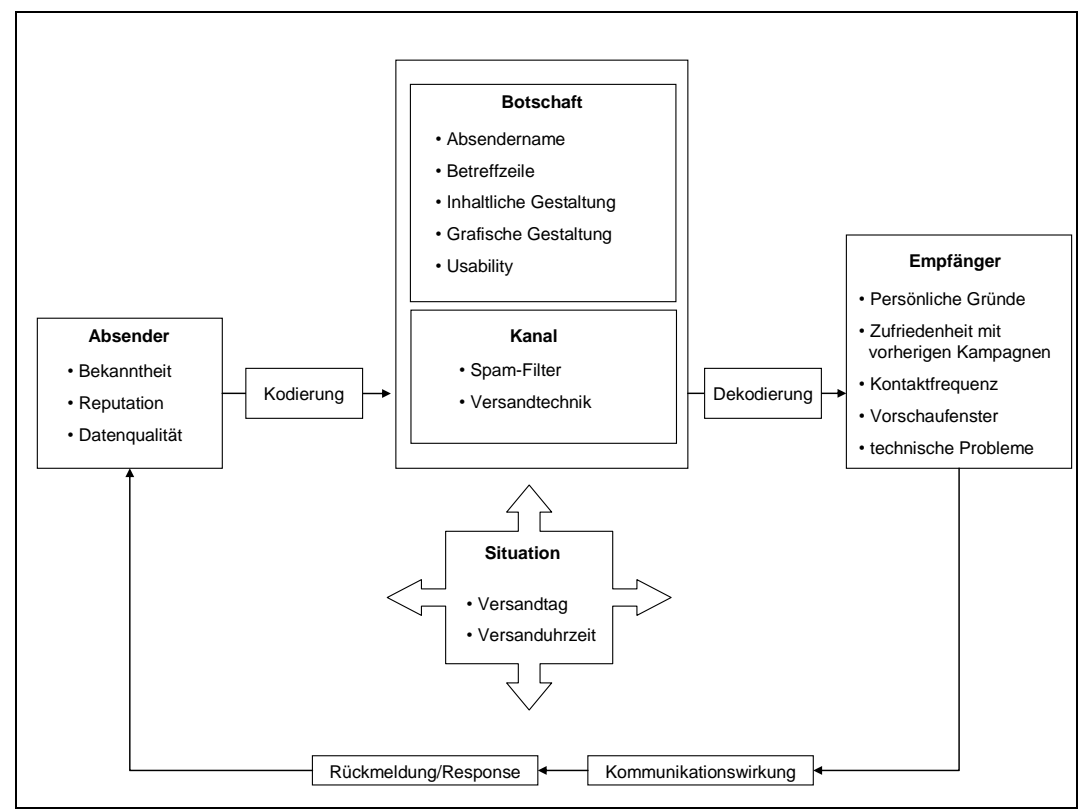

Abbildung 1: Ablauf der Kommunikation und Zuordnung der Einflussfaktoren (in Anlehnung an Bruhn 2007, S. 39)

Im Rahmen zukünftiger Untersuchungen können die identifizierten Einflussfaktoren durch eine branchen- bzw. unternehmensspezifische Betrachtung weiter verfeinert werden. Insbesondere die Überprüfung der Einflussfaktoren durch experimentelle Feldbeobachtungen (z. B. Testkampagnen), kann die tatsächliche Relevanz offenlegen. Somit können Unternehmen die zentralen Einflussfaktoren identifizieren und die Wirkung zukünftiger E-Mail-Kampagnen steigern.

\section{Literatur}

Bruhn M (2007) Kommunikationspolitik. Vahlen, München.

Lasswell HD (1964) The Structure and Function of Communication in Society. In: Bryson L (Hrsg) Communication of Ideas, New York, S. 37-51. 


\title{
Fernsehsender im Web 2.0
}

\section{Potenzial moderner Web-Anwendungen für das Zuschauerbindungsmanagement}

\author{
Kirsten Mrkwicka, Matthias Kießling, Lutz, M. Kolbe \\ Professur für Informationsmanagement, \\ Georg-August-Universität Göttingen
}

Bedingt durch das wachsende Programmangebot und den Erfolgszug der neuen Medien befinden sich Fernsehsender in einem permanenten Konkurrenzkampf um Rezipienten. Um die Marktanteile und Werbeeinnahmen trotz des intensiven Wettbewerbs zu sichern, stellt der Grad der Zuschauerbindung einen kritischen Erfolgsfaktor dar. Nach Diller (1995, S. 443-444) steigern Dialog-, Integrations- und individualisierte Betreuungsangebote abgestuft nach der Attraktivität der Zielgruppen die Loyalität. Während die TV-Sender über das Fernsehen nur einseitig ein anonymes Publikum ansprechen können, ermöglicht das Internet eine Personalisierung der Beziehung und den Einsatz von Maßnahmen des Beziehungsmarketings. Seit seinem Start hat sich das Web von einem starren Informations- zu einem interaktiven Medium weiterentwickelt, bekannt unter dem Schlagwort „Web 2.0“ (O'Reilly 2005, S. 1). Mit den Kernprinzipien der Interaktivität und Nutzerintegration scheinen die modernen Webanwendungen prädestiniert für den Einsatz im Bindungsmanagement. Zwar verwandelt das Web 2.0 die Mehrheit der Fernsehzuschauer und Internetnutzer nicht automatisch in aktive Produzenten, aber für einen wachsenden Teil der Internetnutzer gehören die Anwendungen bereits zum Alltag (Busemann und Gscheidle 2009, S. 358). Das Web 2.0 eröffnet Fernsehsendern zusätzliche Distributions- und Kommunikationskanäle sowie die Möglichkeit neben TV-Inhalten auch crossmediale Dienste anzubieten.

Mit dem Ziel, strategische Handlungsempfehlungen für TV-Anbieter zu entwickeln, versucht der vorliegende Artikel zunächst eine theoretische Untersuchungsgrundlage zu schaffen. Sowohl zum Zuschauerbindungsmanagement als auch zum Beziehungsmarketing im Web 2.0 existieren bislang nur wenige wissenschaftliche Erkenntnisse. Der empirische Teil beschreibt aufbauend auf einer Inhaltsanalyse der Web-2.0-Aktivitäten und Experteninterviews mit TV-Marketingverantwortlichen das gegenwärtige Einsatzniveau führender deutscher Fernsehsender und bewertet das Potenzial der einzelnen Anwendungen hinsichtlich ihres Beitrags zum Zuschauerbindungsmanagement. Als Web-2.0-Applikationen berücksichtigt die Analyse Weblogs, Podcasts, Soziale Netzwerke, Content Sharing Portale, Wikis und Soziale Lesezeichensammlungen. 
Die Inhaltsanalyse zeigt, dass sich der Web-2.0-Einsatz in einer Experimentierphase befindet: Die untersuchten Fernsehsender haben die Relevanz des Onlinemarketings erkannt, testen aber noch die technischen und inhaltlichen Möglichkeiten der Applikationen und ihre Nutzerakzeptanz. Während zum Zeitpunkt der Datenerfassung beispielsweise alle Fernsehsender Weblogs und Video- bzw. FotoPlattformen auf ihren Websites anbieten, bindet bislang keiner Wikis ein. Als externe Plattformen genießen Videoportale die größte Beachtung, während in Sozialen Netzwerken trotz der hohen Popularität bei jungen Zielgruppen lediglich ein Sender regelmäßig aktualisierte Informationen anbietet. Abhängig von ihren Merkmalen eignen sich die Web-2.0-Anwendungen für unterschiedliche Bereiche des Zuschauerbindungsmanagements. Content-Sharing-Portale bieten beispielsweise zusätzliche Distributionskanäle und unterstützen eine individuelle TVNutzung des Publikums. Soziale Netzwerke fördern dagegen den Austausch der Zuschauer mit dem TV-Anbieter und anderen Fans, sodass die Fernsehsender auf Plattformen wie Facebook die Publikumsbedürfnisse besser kennen lernen.

Eine Bewertung der einzelnen Einsatzmöglichkeiten erlauben die Experteneinschätzungen. Das Potenzial der einzelnen Applikationen für das Zuschauerbindungsmanagement variiert mit der Nutzerakzeptanz, den notwendigen Ressourcen sowie rechtlichen Restriktionen. Im Vergleich weisen Soziale Netzwerke durch den hohen Verbreitungsgrad das größte Potenzial auf, das lediglich der Betreuungsaufwand einschränkt. Content-Sharing-Portalen und Podcasts zeigen aufgrund der rechtlichen Einschränkungen nur ein mittleres Potenzial. Soziale Lesezeichensammlungen und Wikis sind aufgrund der aktuell niedrigen Zahl aktiver Nutzer für das Zuschauerbindungsmanagement kaum geeignet.

Zusammengefasst bietet der Artikel Handlungsempfehlungen für Fernsehsender zur Steigerung der Zuschauerbindung im zunehmend wettbewerbsintensiven TV-Umfeld mit Web-2.0-Maßnahmen. Gleichzeitig ermöglichen die deskriptiven Methoden die Ableitung eines Hypothesenmodells als Grundlage für weitere wissenschaftliche Untersuchungen in den bislang weitgehend unerforschten Bereichen des Zuschauerbindungsmanagement und Beziehungsmarketing im Web 2.0.

\section{Literatur}

Busemann K, Gscheidle C (2009) Web 2.0: Communitys bei jungen Nutzern beliebt. ARD/ZDF-Onlinestudie 2009. Media Perspektiven 41(7):356-361.

Diller H (1995) Beziehungs-Marketing. WiSt Wirtschaftswissenschaftliches Studium 24(9):442-447.

O'Reilly T (2005) What is Web 2.0? http://www.oreilly.de/artikel/web20.html. Abruf am 2008-07-31. 


\title{
Einsatz von Recommender-Systemen zum Management von Unternehmensportalen
}

\author{
Helmuth Elsner, Jan Krämer \\ Institute of Information Systems and Management, \\ Karlsrube Institute of Technology (KIT)
}

Unternehmensportale stellen eine weltweit zugängliche Plattform zur Unternehmenskommunikation bereit. Portale der vierten Generation bieten heute nicht nur Wissensmanagement- und Kollaborationsfunktionalitäten an (Terra und Gorden, S. 1-419; Benbya et al. 2004, S. 201-22), sondern können durch intelligente Dienste auch komplexe Geschäftsprozesse unterstützen und koordinieren (Dias 2001, S. 274-279; Murray 1999). White spricht in diesem Zusammenhang von einer Entwicklung hin zu „Enterprise Application Suites“ (2003, S. 2). Die Möglichkeiten, die ein Portal in Bezug auf die Unterstützung verschiedenster Unternehmensprozesse bieten kann, sind also sehr vielfältig und führen in der Folge zu einem stetigen Wachstum des Portals in Bezug auf die Komplexität und Anzahl der Dienste, sowie der Nutzer. Das Management des Portalwachstums und seiner Folgen stellt daher eine zentrale Herausforderung beim Einsatz von Unternehmensportalen der vierten Generation dar. Explizite Lösungsansätze zur Bewältigung dieser Problematik finden sich bisher nicht in der Literatur, die lediglich die Möglichkeiten des Portalkonzepts in Unternehmen hervorhebt. Im Gegensatz zu Internetportalen verfolgen Unternehmensportale kein eigenes Geschäftsmodell, sondern unterstützten Unternehmen in der Verfolgung ihres Geschäftsmodells. Dieser fundamentale Unterschied führt auch zu anderen Anforderungen an das jeweilige Portalmanagement. Die zentrale Herausforderung des Portalmanagements liegt darin, Mitarbeiter effektiv bei der Nutzung des Portals zu unterstützen, wobei das stetige Wachstum besonders berücksichtigt werden muss. Einerseits sollen Mitarbeiter auf die ihnen bekannten Dienste weiter zugreifen können. Andererseits kann das Potenzial des Portals aber nur entfaltet werden wenn andere relevante Dienste auch wahrgenommen werden und zu deren Nutzung angeregt wird.

Um dieses Ziel zu erreichen wird der Einsatz eines Recommender-Systems vorgeschlagen, welches speziell auf den Einsatz in Unternehmensportalen zugeschnitten ist. Wichtige Voraussetzungen für die Wahl eines geeigneten Ansatzes sind insbesondere, dass es impraktikabel oder unerwünscht ist (i) alle Dienste des Portals vollständig semantisch zu beschreiben, (ii) das Nutzungsverhalten und Arbeitsprofil jedes Nutzers vollständig zu erfassen. Daher können nur solche Recommender angewendet werden, die das Nutzungsverhalten der Mitarbeiter analysieren und daraus Empfehlungen ableiten. Diese Eigenschaften befürworten die Wahl des Collaborative Filtering Ansatzes (Goldberg 1992; Resnick et al. 1994) 
gegenüber dem des Content-Based Filtering (Adomavicius und Tuzhilin 2005). Bei den zugehörigen Algorithmen sind wiederum die memory-based- gegenüber den model-based Algorithmen vorzuziehen, da letztere nur auf Grund ihrer Skalierbarkeit Vorteile bringen (Breese et al. 1998; Herlocker et al. 2004). Diese spielt jedoch bei Unternehmensportalen auf Grund der verhältnismäßig geringen Zahl von Nutzern und Objekten eine untergeordnete Rolle. Im Weiteren wird zudem diskutiert, welche weiteren Designentscheidungen des Recommender-Systems im konkreten Unternehmenskontext getroffen werden müssen und wie diese evaluiert werden können. Hierzu gehört unter anderem die Wahl der geeigneten Methodik zur Berechnung der Ähnlichkeit zwischen Nutzern, das Verfahren zur impliziten Berechnung der Ratings für Dienste und die Art der Anzeige der Empfehlungen.

\section{Literatur}

Adomavicius G, Tuzhilin A (2005) Toward the next generation of recommender systems: a survey of the state-of-the-art and possible extensions. IEEE Transactions on Knowledge and Data Engineering 17(6): 734-749.

Benbya H, Passiante G, Belbaly N (2004) Corporate portal: a tool for knowledge management synchronization. International Journal of Information Management 24(3): 201-220.

Breese J, Heckerman D, Kadie C (1998) Empirical analysis of predictive algorithms for collaborative filtering. In: Proceedings of the UAI-98: 43-52.

Dias C (2001) Corporate portals: a literature review of a new concept in Information Management. International Journal of Information Management 21(4): 269-287.

Goldberg D, Nichols D, Oki B, Terry D (1992) Using collaborative filtering to weave an information tapestry. Communications of the ACM 35(12): 61-70.

Herlocker J, Konstan J, Terveen L, Riedl J (2004) Evaluating collaborative filtering recommender systems. ACM Trans. on Inf. Sys. 22(1): 5-53.

Murray G (1999) The portal is the desktop.

Terra J, Gordon C (2003) Realizing the promise of corporate portals: Leveraging knowledge for business success. Butterworth-Heinemann, Amsterdam.

White C (2003) Is the portal dead? Information Management Magazine: 1-3. 


\title{
Ideenmanagement im Rahmen von organisatorischem Wandel
}

\section{Eine Triangulation der Ergebnisse der Aktionsforschung zum Ideenwettbewerb bei BMW Financial Services}

\author{
Daniel Klein, Ulrike Lechner \\ Institut für Angewandte Informatik, \\ Universität der Bundeswehr München
}

Hintergrund

Den Begriffen organisatorischer Wandel und Veränderungsmanagement (Change Management) haftet eher eine negative Note an, als dass sie von vorneherein mit vielversprechenden Aussichten hinsichtlich der zukünftigen Unternehmensentwicklung in Verbindung gebracht werden. Das gilt vor allem für den Standpunkt der Mitarbeiter. Innovationsmanagement und artverwandte Konzepte und Instrumente wie Ideenmanagement oder Ideenwettbewerbe rufen in Wirtschaft und Gesellschaft dagegen überwiegend positive Assoziationen hervor.

In unserer Forschung geht es um die gezielte Verwendung von Ideenmanagement im Rahmen von organisatorischem Wandel. Dabei konzentrieren wir uns auf die Nutzung eines unternehmensinternen Ideenwettbewerbs zur Unterstützung der Ziele von Change Management. In unserer vorangehenden Arbeit analysierten wir die Fähigkeiten des Ideenwettbewerbs, den Wandel auf der kulturellen Seite positiv zu beeinflussen. Zudem untersuchten wir verschiedene Aspekte bezüglich der Schaffung von Ideen seitens der Mitarbeiter und betrachteten zudem die Motivation der Teilnahme. Diese Ergebnisse setzen wir in diesem Papier in einen neuen Bezug und triangulieren Sie mit Hilfe zweier weiterer, unterschiedlicher Datenquellen.

Methodik

In Übereinstimmung mit dem Bedarf an spezifischem Wissen, tiefen Einblicken in die Unternehmung und mit unserem Bestreben, die Vorgänge selbst maßgeblich beeinflussen zu können, war Aktionsforschung der geeignete Ansatz für unsere Untersuchung. Die Problemsituation bei BMW Financial Services bestand darin, innerhalb kurzer Zeit einen Ideenwettbewerb auf die Beine zu stellen, um Inhalte für das Veränderungsprojekt zu produzieren, die Kommunikation des Wandels zu ermöglichen und die Mitarbeiter in das Unternehmensgeschehen zu involvieren. 
Der wissenschaftliche Beitrag unserer Forschung ist ein besseres Verständnis davon, wie sich ein Ideenwettbewerb - und im weiteren Sinne Ideenmanagement im Allgemeinen - im Rahmen eines organisatorischen Wandels auf Unternehmenskultur, Mitarbeiter und deren Kreativität auswirkt.

Die kulturelle Unterstützung des organisatorischen Wandels durch den Ideenwettbewerb und dessen Wahrnehmung durch die Mitarbeiter wurde innerhalb unserer bisherigen Forschung maßgeblich durch eine Mitarbeiterumfrage im Anschluss an den Wettbewerb analysiert und diskutiert. Zusätzlich wurden Mitglieder des Change Management Teams als Experten befragt. Diese Experteninterviews ziehen wir zusammen mit einer durch das Change Management selbst über den hierarchischen Querschnitt des Unternehmens gehaltenen Umfrage heran, um ausgewählte Thesen unserer bisherigen Forschungsresultate zu triangulieren.

\section{Ergebnisse}

Wir sehen die Aussagen zur positiven Wahrnehmung des Ideenwettbewerbs trotz direkter Einbindung in den organisatorischen Wandel bestätigt. Ebenso können wir die Thesen zur gefühlten Mehreinbindung der Mitarbeiter in das Unternehmensgeschehen bekräftigen. Bezüglich der Motivation der Teilnahme der Mitarbeiter am Ideenwettbewerb und der Funktion der Prämien ist es uns möglich, die Besonderheiten unserer aufgestellten Thesen hinsichtlich des Willens der Mitarbeiter, das Unternehmen zu unterstützen, zu betonen. 
Informationssysteme in der Finanzwirtschaft 



\title{
Vorwort zur Teilkonferenz
}

\section{Informationssysteme in der Finanzwirtschaft}

\author{
Susanne Leist', Matthias Goeken², Gregor Zellner' \\ 1 Institut für Wirtschaftsinformatik, Universität Regensburg \\ ${ }^{2}$ Management Research Centre, Frankfurt School of Finance \& Management
}

Die Teilkonferenz befasst sich mit Informationssystemen in der Finanzwirtschaft denen nicht nur vor dem Hintergrund der aktuellen Entwicklungen stetig steigende Bedeutung zugemessen wird. Dabei wird sowohl die Sicht der Finanzdienstleister als auch die der Kunden und Technologielieferanten berücksichtigt.

Die vorübergehende Reduzierung des IT-Projektgeschäfts der Banken und das Sparen bei der eingesetzten IT als Konsequenz der Finanzkrise bremst die Bankmodernisierung. Zudem sind die Ausmaße der Finanzkrise noch nicht vollständig einschätzbar, so dass sich die Unternehmen weiterhin auf Strukturveränderungen einstellen müssen. Dabei kann gerade die IT einen wesentlichen Beitrag bei der Umsetzung regulatorischer Rahmenbedingungen liefern. Auch sind Informationssysteme ein notwendiges Instrument, um eine Vielzahl an Daten zu erfassen und Hinweise auf Risiken auswerten zu können. Die Beobachtung von systemischen Risiken fordert zudem die Zusammenarbeit der Unternehmen, die wiederum vor allem mit Hilfe der IT gestützt wird.

Das Themenspektrum ist somit breit und die Beiträge in dieser Teilkonferenz beschäftigen sich daher mit einer Auswahl an unterschiedlichen Herausforderungen zu diesem Komplex.

- Mansfeldt, Alt und Puschmann stellen in ihrem Beitrag einen Ansatz zur integrierten Gestaltung und Bewertung von unregelmäßig anfallenden, strategischen Entscheidungen wie beispielsweise Reorganisationsentscheidungen vor. Der Ansatz kombiniert Ansätze zur Unternehmensarchitektur mit Elementen der Entscheidungstheorie.

-Witte und Suchan entwickeln in ihrem Beitrag unter Verwendung der SystemDynamics-Methode ein Finanzmarktmodell zur Beschreibung, Erklärung und Prognose, das darüber hinaus die Qualität von Investmententscheidungen verbessern kann. Die Untersuchung wird zudem exemplarisch an den beiden Szenarien „Rally“ und „Crash" durchgeführt.

- Ein Java-basiertes Software-Framework, mit dem sich der Erfolg news-basierter algorithmischer Handelsstrategien simulieren lässt, stellen Storkenmaier, Müller und Weinhardt in ihrem Beitrag vor. Basierend auf historischen Daten von DAX-30Unternehmen werden Ergebnisse von ersten Simulationsläufen vorgestellt. 
- Baecker und Bereuter untersuchen den Einsatz moderner IT-Technologien zur Verbesserung der Schadensbearbeitung in der Kfz-Versicherung. Am Beispiel des Beschwerdemanagementprozess entwickeln die Autoren mehrere technologiebasierte Verbesserungsvorschläge unter Verwendung von serviceorientierten Architekturen.

- Ende und Muntermann behandeln die Vorteilhaftigkeit des Handels von Blocktrades über Liquiditätspools in Relation zur börslichen Ausführung solcher Transaktionen auf Basis einer empirischen Studie.

- Im Beitrag von Wunderlin wird die Qualität von Kreditkundenratings durch ein IT-System oder durch den Kundenberater betrachtet. Die Untersuchung basiert auf einer Auswertung von empirischen Daten.

Wir möchten uns bei allen Autoren sämtlicher eingereichter Beiträge herzlich bedanken. Sie haben uns viele interessante Forschungsergebnisse vorgestellt und uns in die angenehme Lage versetzt, die besten sechs Beiträge auswählen zu können. Ein weiterer großer Dank gilt auch allen Organisatoren der Multikonferenz Wirtschaftsinformatik 2010, die zum Gelingen der Teilkonferenz maßgeblich beigetragen haben.

Für die Bereitschaft zur Mitarbeit im Programmkomitee und die Übernahme der Begutachtung gilt der herzliche Dank Prof. Dr. Dieter Bartmann (Universität Regensburg), Prof. Dr. H. U. Buhl (Universität Augsburg), Dr. M. Foit (ibi research GmbH, Regensburg), Prof. Dr. Matthias Goeken (Frankfurt School of Finance and Management), Thomas Klein (sd\&m AG, München), Prof. Dr. Susanne Leist (Universität Regensburg), Prof. Dr. H.-G. Penzel (Europäische Zentralbank, Frankfurt am Main), Dr. Erhard Petzel, Prof. Dr. P. Roßbach (Frankfurt School of Finance \& Management) und Dr. Gregor Zellner (Universität Regensburg). 


\title{
Ansatz zur integrierten Gestaltung und Bewertung am Beispiel des Financial Sourcing
}

\author{
Ken Mansfeldt ${ }^{1}$, Rainer Alt², Thomas Puschmann ${ }^{1}$ \\ ${ }^{1}$ Institut für Wirtschaftsinformatik, Universität St. Gallen \\ 2Institut für Wirtschaftsinformatik, Universität Leipzig
}

Strategische Entscheidungen zur Konfiguration von Geschäftsmodellen, die Gestaltung von Aufbau- und Ablauforganisation oder der Architektur von Anwendungssystemen benötigen trotz ihrer Unregelmäßigkeit eine Methoden- und Werkzeugunterstützung. Hierzu gehören einerseits die Erfassung, Strukturierung und Visualisierung von Gestaltungsmodellen und anderseits die Beurteilung von Produktkosten, Unternehmenseinheiten, geographischen Märkten etc. im Rahmen von Bewertungsmodellen. Diese integrierte Sichtweise berücksichtigen heutige Ansätze nur teilweise. Obgleich sich Gestaltungs- und Bewertungsmodelle aus Entscheidersicht gegenseitig bedingen, erfolgt ihre Anwendung weitgehend losgelöst voneinander. Fragen nach einer einheitlichen Gestaltungs- und Bewertungsbasis oder den durch ein Outsourcing betroffenen Architekturschichten und ihren Abhängigkeiten bleiben folglich unbeantwortet. Der Beitrag unternimmt einen ersten Versuch zur Überwindung dieser Dichotomie von Gestaltungs- und Bewertungsmodell und entwickelt einen Ansatz für die Einbindung von Architekturmodellen als Entscheidungsgrundlage (Aier et al. 2008, S. 294 ff.) anhand strategischer Gestaltungsoptionen in der Finanzindustrie. Er richtet sich an Geschäftsleitungen und/oder Planungs- und Fachabteilungen sowie Beratungsunternehmen und bietet eine effiziente Möglichkeit zur Evaluation von Reorganisationsoptionen.

Der Mehrwert des integrierten Ansatzes ergibt sich aus der Möglichkeit der Anreicherung von Architekturmodellen mit notwendigen Informationen für quantitative Untersuchungen (Lankhorst 2005, S. 192), der intersubjektiven Bewertbarkeit der Modelle (Leist 2002, S. 5 ff.), der Überprüfbarkeit der Erreichung der Gestaltungsziele einer Architektur (Alt 2008, S. 125) sowie einer einheitliche Semantik und Verständnisgrundlage und der daraus resultierenden Zeit- und Kostenersparnis. Den Kern des Ansatzes bildet ein Metamodell, das als gemeinsamer, übergeordneter und strukturierender Rahmen (Supersystem) eine aufeinander abgestimmte Verwendung der Komponenten Gestaltungs- und Bewertungsmodell (eigenständige Systeme) gewährleistet. Die Elemente des Metamodells (MME) definieren den inhaltlichen Rahmen für die Gestaltungs- und Bewertungsmodellobjekte (GMO und BMO). Abbildung 1 verdeutlicht die Struktur des Metamodells über drei Ebenen. Jedem MME sind GMO hinterlegt, für die jeweils quantitative und qualitative BMO definiert sind. 


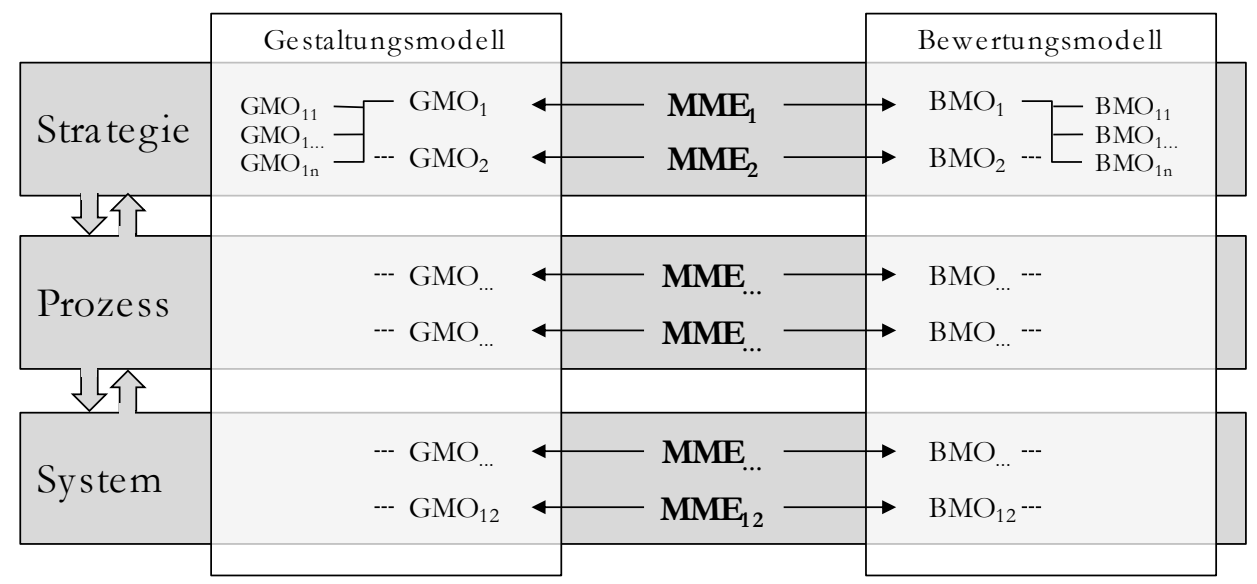

Abbildung 1: Metamodell als strukturierender Integrationsrahmen

Die hinter dem Ansatz stehende Forschungsmethodik der partizipativen Forschung drückt sich neben dem Aufzeigen eines Beispiels in Überlegungen zur technischen Umsetzung des Ansatzes aus. Sowohl eine in einem Gestaltungswerkzeug durchgeführte Bewertung als auch eine in einem Bewertungswerkzeug erfolgte Gestaltung zeigen sich als limitierte und damit unbefriedigende Möglichkeiten. Für die vollumfängliche Umsetzung des Konzepts ist eine Business IntelligenceLösung zielführend, die Gestaltungs- und Bewertungsmodell in einem Werkzeug vereint.

\section{Literatur}

Aier S, Riege C, Winter R (2008) Unternehmensarchitektur - Literaturüberblick und Stand der Praxis. WIRTSCHAFTSINFORMATIK 50(4):292-304.

Alt R (2008) Überbetriebliches Prozessmanagement. Logos, Berlin.

Lankhorst M (2005) Enterprise Architecture at work: modeling, communication and analysis. Springer, Berlin.

Leist S (2002) Bankenarchitektur des Informationszeitalters. In: Leist S, Winter R (Hrsg) Retail Banking im Informationszeitalter. Springer, Berlin. 


\title{
Improving the Support for Investment Decisions in Financial Markets Using the System Dynamics Methodology
}

\author{
Björn-Christopher Witte', Christian Suchan ${ }^{2}$ \\ ${ }^{1}$ Lehrstubl für Volkswirtschaftslebre, insb. Wirtschaftspolitik, \\ Otto-Friedrich-Universität Bamberg \\ ${ }^{2}$ Lehrstubl für Wirtschaftsinformatik, insb. Industrielle Anwendungssysteme, \\ Otto-Friedrich-Universität Bamberg
}

In this paper, the methodology of System Dynamics (SDM) (e.g. Sterman 2000) is used to construct a model of a financial market (FiMa) and to derive insights to support investment decisions. The paper is motivated by problems of "traditional" FiMa-models (survey by Hommes 2006), which base on purely formal representations. These models hardly support to explain system behavior from system structure. In contrast, the SDM implies to construct semiformal models, a Causal Loop Model (CLM) and a Flow Model (FM), which illustrate the system structure and reveal insights for system behavior. Based on these considerations we follow investigations objectives and evaluate the objective achievement of modeling approaches (cf. Table 1).

Table 1: Problem Setting

\begin{tabular}{|l|c|l|l|}
\hline \multicolumn{2}{|c|}{ Investigation Objectives } & \multicolumn{2}{c|}{$\begin{array}{c}\text { Objective Achievement of } \\
\text { Modelling Approaches }\end{array}$} \\
\hline $\begin{array}{c}\text { Financial market } \\
\text { motivated }\end{array}$ & $\begin{array}{c}\text { System theoretic } \\
\text { motivated }\end{array}$ & $\begin{array}{c}\text { Traditional } \\
\text { approach }\end{array}$ & \multicolumn{1}{c|}{ System Dynamics } \\
\hline $\begin{array}{l}\text { What elements constitute } \\
\text { the financial system? }\end{array}$ & Structure & $\begin{array}{l}\text { Informal } \\
\text { descriptions }\end{array}$ & $\begin{array}{l}\text { Informal descriptions, } \\
\text { Semiformal models } \\
\text { (CLM, FM) }\end{array}$ \\
\hline $\begin{array}{l}\text { Why do financial markets } \\
\text { behave in a certain way? }\end{array}$ & $\begin{array}{c}\text { Structure (with } \\
\text { aspects of behavior) }\end{array}$ & not supported & $\begin{array}{l}\text { Semiformal models } \\
\text { (CLM, FM) }\end{array}$ \\
\hline $\begin{array}{l}\text { How will security prices } \\
\text { develop? }\end{array}$ & Behavior & Formal model & Formal model \\
\hline
\end{tabular}

As problem approach we, firstly, develop a FiMa-model following the SDM. The model follows the chartist-fundamentalist approach, i.e. traders fall into two groups, depending if they rely on technical or fundamental strategy. A CLM and an FM illustrate the model structure, which are then quantified formally to simulate the model behavior. Secondly, we test the model for accuracy of behavior. We show that the model replicates eight important stylized facts of FiMa (survey by Cont 2001), 
e.g. volatility clustering. In contrast, Chen et al. (2008) surveys fifty traditional models, of which the most accurate was tested positively for seven facts only. To evaluate the model's validity for application, i.e. in particular the support for investment decisions, we finally simulate two characteristic scenarios, a speculative rally and a market crash, and deduce interpretations from CLM and FM. To give an example, Figure 1 depicts the feedback loop responsible for a speculative rally.

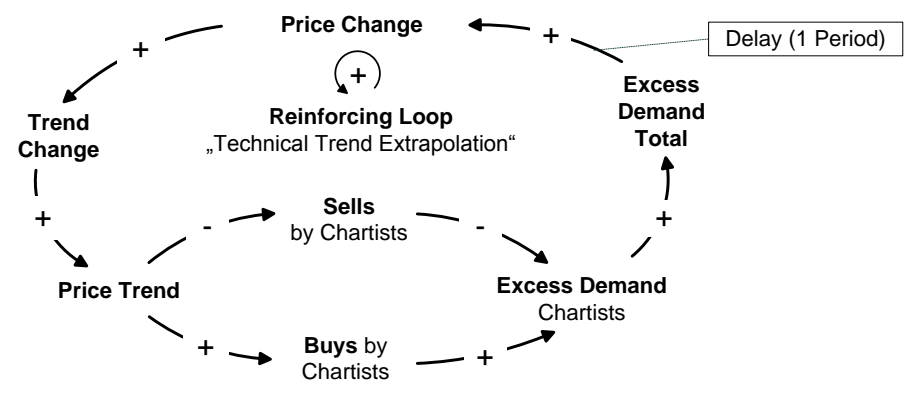

Figure 1: CLM of the reinforcing loop technical trend extrapolation

The loop demonstrates that speculative rallies are not necessarily driven by fundamental events, because the positive feedback runs independently of the fundamental value of the security traded. Furthermore, price dynamics show certain momentum stored in the price trend. It follows that assuming rallies to continue, at least in the short-term, is rational. Hence, the SDM-model suggests that betting on trends to continue is a profitable short-term strategy.

\section{References}

Chen S-H, Chang C-L, Du, Y-R (2008): Agent-based economic models and econometrics. KNOWLEDGE ENGINEERING REVIEW, forthcoming.

Cont R (2001) Empirical properties of asset returns: stylized facts and statistical issues. QUANTITATIVE FINANCE 1(2):223-236.

Hommes CH (2006) Heterogeneous agent models in economics and finance. In: Tesfatsion L, Judd K (eds) Handbook of Computational Economics 2: Agentbased Computational Economics. North-Holland, Amsterdam.

Sterman JD (2000) Business dynamics: system thinking and modeling for a complex world. McGraw-Hill, Boston. 


\title{
A Software Framework for a News Event Driven Simulation of Algorithmic Trading Strategies
}

\section{Architecture and Simulation Tests}

\author{
Andreas Storkenmaier, Marius Müller, Christof Weinhardt \\ Institute of Information Systems and Management, \\ Information and Market Engineering, \\ Karlsrube Institute of Technology
}

The fast development of information systems during the last decades has had a significant impact on the financial services industry and the securities trading industry in particular (Jain 2005, Stoll 2006). Automated trading systems have become standard on major exchanges world-wide and algorithmic trading has overtaken human trading in some markets in terms of generated trading volume. However, little academic evidence exists on the effects and profitability of algorithmic trading.

A single accepted definition of algorithmic trading (AT) does not exist but a commonly used definition in the academic world is that AT is "the use of computer algorithms to manage the trading process" (Hendershott et al. 2008). AT can basically be separated into agency algorithmic trading and proprietary algorithmic trading. This separation can also be found in the New York Stock Exchange (NYSE) program trading descriptions. Agency AT essentially comprises the automation of broker tasks which includes the automation of order generation and submission through monitoring of market conditions and splitting of large orders over time and markets to minimize the impact of orders. Proprietary AT comprises of statistical arbitrage, automated market making, and high frequency trading using high speed recognition of specific market patterns and situations.

A relatively new form of proprietary AT is news based proprietary AT which automatically analyzes stock specific and general economic news to trade profitably. Major providers of financial news like Thomson Reuters or Dow Jones have developed machine readable news products that already offer electronically preinterpreted news. One example is the Reuters NewsScope Sentiment Engine (RNSE) system ${ }^{1}$. Also regulators like the U.S. Securities and Exchange Commission (SEC) increasingly demand from companies to provide regulatory information electronically. SEC regulation requires large companies to submit their annual fi-

1 For more information on the Reuters NewsScope Sentiment Engine system see http://www.thomsonreuters.com/content/financial/pdf/enterprise/NewsScopeSentimentEngine.p df 
nancial statements in XML based XBRL (eXtensible Business Reporting Language) which allows for an easy dissemination and analysis of financial information.

We present in our paper a Java based software framework that supports the agent based simulation of news based proprietary algorithmic trading strategies and provide first simulation test results. We do not model an artificial stock market but base our analysis on historical trade and quote data and model our agents as price takers in highly liquid markets. In liquid markets orders up to a considerable size trade usually at the best bid and best ask which justifies the price taking assumption. With our framework we are trying to address questions between financial econometrics and pure artificial stock market simulations as we enable researchers to analyze dynamic strategies on real world data. Additionally we are able to model explicit exchange system effects like system latency. The main contribution of the paper is the presentation of the software framework.

Although the major goal of the paper is to present the framework, we also include tests based on existing non-artificial data to show that our framework is implemented and running stable. To test our framework we use data from Thomson Reuters that is also used by practitioners in the financial services industry.

\section{References}

Hendershott T, Jones CM, Menkveld AJ (2008) Does algorithmic trading improve liquidity. WFA 2008 Paper. http://ssrn.com/abstract=1100635. Last access 2009-08-24.

Jain P (2005) Financial market design and the equity premium: Electronic versus floor trading. Journal of Finance 60(6): 2955-2985. 


\title{
Technology-Based Industrialization of Claims Management in Motor Insurance
}

\author{
Oliver Baecker, Albrecht Bereuter \\ Institute of Technology Management, University of St. Gallen
}

Over the past years, German motor insurers have been facing growing competition from in- and outside the industry (e.g., car manufacturers, automobile clubs) (Kaeslin and El Hage 2008). Together with a growing pricing pressure on insurance premiums, their combined ratio increased from $94.5 \%$ in 2004 to $101.6 \%$ in 2008 (GDV 2009). At the same time, motor insurance is the class of business with by far the largest volumes within property and casualty insurance with a total of 20.4 billion Euros in premiums (German market in 2008). This indicates that cost savings and efficient process improvements in claims management business processes have a great impact on the key performance indicators of insurers. However, over the last years, earned premiums were decreasing while motor insurers were unable to decrease costs to the same degree. The main reasons why cost savings are hard to achieve include the late involvement of insurers after car accidents, the limited integration of business partners such as repair shops, and improper information about the case circumstances (Kaeslin and El Hage 2008). According to several studies (Accenture 2002; Bieber and Hoberg 2007; Mueller and Kuefner 2003), earlier and more detailed information about motor insurance claims could enable pro-active claims management and shorten the average claim life-cycle from set-up to close as well as decrease the average costs per case. In addition, an early notification simplifies the integration of business partners for settling a claim and enables the insurance company to benefit from volume discounts, for example, by routing their customers to authorized repair shops. However, according to a study by Kaeslin and El Hage (2008), at most $7.5 \%$ of all motor insurance claims are managed pro-actively. The untapped potential was investigated by Accenture (2002) during an analysis of 12,000 settled claims and led to the estimate that cost savings of up to $15 \%$ are possible, while further studies indicate similar results (Bieber and Hoberg 2007; Mueller and Kuefner 2003). This cost saving potential equals a double-digit million Euros saving per year for a mid-size insurer.

While insurance companies are under pressure to decrease costs, customers lack adequate assistance when it comes to an insurance claim, the so-called "moment of truth" where an insurer has to put his value proposition into practice. In such unpleasant and emotionally stressful situations, customers require both immediate services and support with the submission of a loss report to their insurance carrier. Against this background, the usage of mobile phones can address the outlined problems of insurance carriers and the needs of their customers. From a 
customer perspective, mobile phones are ubiquitously available, e.g., the average mobile phone penetration rate in the European Union was $119 \%$ in 2008. At the same time, the mobile phone has emerged as the most dominant mediation device in safety-critical situations. Qualitative interview data as well as quantitative analysis show that people's sense about mobile phones is to never be alone in emergencies. Accordingly, mobile technology can play an important role in the aftermath of car accidents to report an insurance claim and to benefit from value-added services. While this approach provides customers with adequate assistance, it can also lead to more efficient claims management business processes for insurers.

Against this background, we present an analysis of today's claims management process in motor insurance and describe four technology-based process improvements. In order to realize the process improvements, a service-oriented architecture is presented and the implementation of a demonstrator illustrates our solution. Finally, we propose business value metrics to measure the effects linked with each process improvement. Our paper demonstrates the benefits of a mobile claims assistance application for customers involved in a car accident as well as for insurance companies. In addition, we demonstrate that mobile phones are technically capable of reporting claims into commercial claims management enterprise systems. Based on this work, it will be possible to transfer the concepts to related classes within property and casualty insurance, such as household contents insurance or building insurance. As future work, we will conduct a user study with insurance customers to evaluate the solution. As part of the study, participants will use the "Mobile Claims Assistance" solution to re-enact a recent personal loss report. The goal is to investigate whether customers are really willing to use the application in case of an insurance claim and to compare the mobile application with the experienced loss report via phone or internet.

\section{References}

Accenture (2002) Unlocking the Value in Claims, Accenture - Insurance Solution Group.

Bieber T, Hoberg S (2007) Aktives K-Schadenmanagement gemessen und bewertet. Versicherungswirtschaft 12:992-995.

GDV: Gesamtverband der deutschen Versicherungswirtschaft e.V. (2009)

Statistisches Taschenbuch der Versicherungswirtschaft 2009.

Kaeslin B, El Hage B (2008) Kosteneinsparung durch aktives Kfz-

Reparaturmanagement. Versicherungsbetriebe 1:8-10.

Mueller K-W, Kuefner R (2003) Schadenmanagement in der Versicherungswirtschaft. BearingPoint $\mathrm{GmbH}$, Frankfurt. 


\title{
Opacity and Exclusivity in Electronic Securities Trading: The Case of Dark Pools
}

\section{A Comparison of Liquidnet with Electronic Exchanges}

\author{
Bartholomäus Ende', Jan Muntermann ${ }^{2}$ \\ ${ }^{1}$ E-Finance Lab, House of Finance, Frankfurt \\ ${ }^{2}$ House of Finance, Goethe University Frankfurt
}

Today, electronic securities exchanges in Europe are characterized by transparency and worldwide access: they can be accessed by both retail and institutional investors and prices and liquidity are openly advertised. While these characteristics are favorable to many investors, they become a problem for institutional investors which trade large volumes. There exist approaches to address these issues by adapting institutional orders to the requirements of electronic exchanges. In contrast, so-called dark pools provide a market mechanism for large trading volumes. Here, access might be limited to certain organizations, e.g. large in terms of assets under management, and liquidity is not displayed. In this paper, we investigate a major agency broker dark pool (Liquidnet) and explore whether this approach can provide significant benefits to investors. Therefore, we have analyzed Liquidnet trades and compare execution prices with benchmark prices.

First, we have analyzed Liquidnet trades in terms of prices and trading volumes. The descriptive statistics provide evidence that finding counterparties for a given investment appears difficult: during the 57 trading days we have analyzed, 198 trades of EURO STOXX 50 constituents have been observed only. However, since $74.24 \%$ of the Liquidnet executions are being priced inside market (i.e. between best bid/ask limit prices) and even $43.43 \%$ correspond to the home market's midpoint, Liquidnet executions appear beneficial.

Second, we have introduced a benchmark for Liquidnet trades, based on optimally achievable limit prices at the instruments' primary markets. Based on this benchmark, we calculated price improvements (see Figure 1) that have been realized when trading via Liquidnet. 


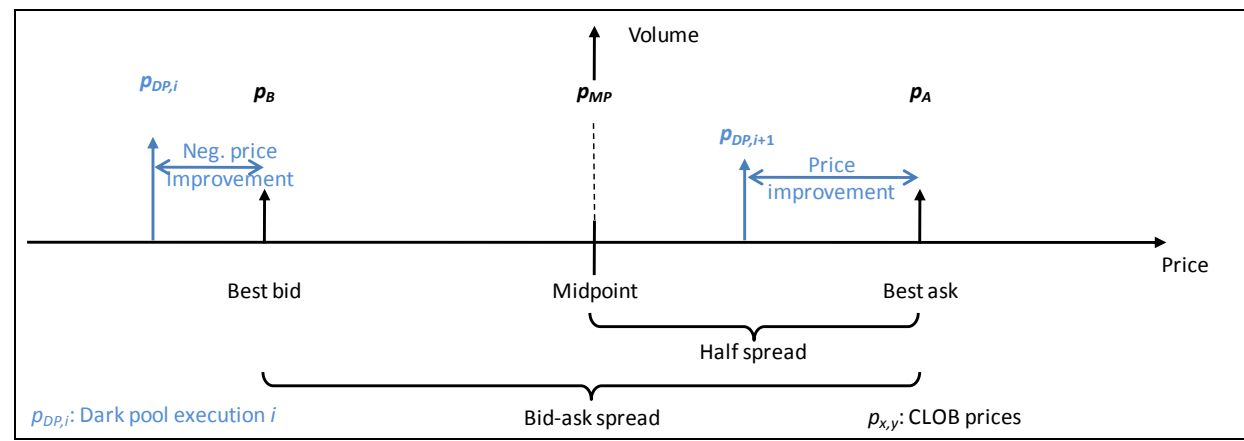

Figure 1: Modeling price improvement

Our major findings were twofold: given the limited number of Liquidnet trades we have observed during the observation period it becomes obvious that finding counterparties within the dark pool is not very likely. However, if a counterparty is found, execution prices are significantly better than the available best bid/ask limit prices at the corresponding primary market. We furthermore have shown that within the dark pool, investors search for trading partners desiring high-volume trades, which would have resulted in significant market impact at markets exhibiting an open order book approach. Since the market mechanism of Liquidnet avoids any market impact for these block trades, the benefits are even more significant for these trading scenarios.

For a detailed quantitative assessment of alternative trading systems such as dark pools, the introduced benchmark approach needs further refinements: therefore, we aim at incorporating reference price corridors including a theoretically derived optimum, an indication for an average trader based on volume weighted average trade prices as well as a worst case scenario. Furthermore, the modeling of execution probabilities is subject to further research. In order to gain deeper insights to the benefits of dark pools, we aim at including further dark pools such as Posit and NYFIX Euromillennium to reveal individual similarities and differences. Another aspect which requires further investigations is the risk of unexecuted trade intentions within dark pools.

\section{Acknowledgment}

We thankfully acknowledge the support of the E-Finance Lab, Frankfurt for this work. 


\title{
Override von Kreditkunden-Ratings
}

\section{Einordnung der manuellen Übersteuerung systemberechneter Ratings}

\author{
Christian Wunderlin \\ Institut für Wirtschaftsinformatik, Universität Bern
}

Für diese Vergabe von Krediten muss jeder Kreditnehmer einer Art Gefahrenklasse, der Ratingklasse, zugeordnet werden. Diese Zuordnung erfolgt, indem die kreditgebenden Banken den einzelnen Kunden anhand von quantitativen und qualitativen Faktoren beurteilen, sprich: raten. Das Ergebnis dieser durch IT-Systeme unterstützten Ratingvorgänge, das gerechnete Rating, wird teilweise durch den Kundenberater als falsch taxiert und mit Freigabe durch Kreditspezialisten geändert. Bei gewissen Banken macht dieser menschliche Einfluss, Override genannt, bis zu $70 \%$ aller schlussendlich bewilligten Ratings aus.

Im Rahmen der Arbeit wurde überprüft, ob dieser menschliche Einfluss positive oder negative Auswirkung auf die Prognosefähigkeit hat. Anhand der statistischen Testmethode der ROC-Kurve bzw. der Fläche darunter (AUROC) wurden über 150‘000 durch IT-Systeme berechnete Ratingvorgänge der Jahre 2004 - 2007 mit den schlussendlich bewilligten Ratings von Kreditkunden in der Schweiz verglichen. Die Untersuchung hat ergeben, dass bei sämtlichen acht teilnehmenden Instituten die Kraft, ausfallende von überlebenden Kunden zu trennen (Trennkraft), zugenommen hat.

Diese Ergebnisse wurden in das aktuelle Zeitgeschehen eingereiht: Viele Banken wollen trotz des positiven Einflusses von Overrides diese reduzieren. Die Gründe für diese Reduktion sind im Bereich Regulator (Verhinderung von Verletzung der Anforderungen) und der Kosten zu sehen: Jedes Override bedingt im Kreditprozess eine spezielle Überprüfung durch teure Spezialisten. Es gibt Banken, die als Ziel eine Overridequote von 10\% nennen, was gemäss Martin (2007, S. 103) im „akzeptablen Bereich“ liegen würde. Die Erwartung liegt bei weniger, besseren begründeten, einheitlicheren, transparenteren und vor allem stärkeren und fokussierteren Overrides. Dieses Ziel soll mit besseren Ratingmodellen erreicht werden. Eine der teilnehmenden Banken hat ihre Ratingmodelle nach dem Beobachtungszeitraum grundlegend überarbeitet und kommt so in den Zielbereich der von der aktuellen Forschung genannten 10\% Grenze.

Neben der Verbesserung der Ratingmodelle ist die Einschränkung ein mögliches Mittel zur Reduktion von Overrides: Bei den Ratinganalysen wurde festgestellt, dass viele Overrides nur um eine Klasse abweichen (Fein-Tuning). Daraus hat sich die Frage gestellt, ob diese minimale Abweichung die damit verbundenen 
Transaktionskosten rechtfertigt. Das Ergebnis zeigt, dass - wenn auf die feintunenden Overrides verzichtet würde - nur bei zwei der acht teilnehmenden Banken eine nennenswerte Verschlechterung der Trennkraft (negative Differenz ROC) resultierte:

Tabelle 1: Effekt fein-tunende Overrides

$\begin{array}{lrrrrrrrr}\text { Bank } & 1 & 2 & 3 & 4 & 5 & 6 & 7 & 8 \\ \text { Anteil } & 56.4 \% & 5.9 \% & 31.7 \% & 39.6 \% & 25.9 \% & 32.1 \% & 42.2 \% & 35.1 \% \\ \text { Anteil o. } \pm 1 & 26.1 \% & 3.4 \% & 24.2 \% & 16.7 \% & 10.6 \% & 20.7 \% & 21.2 \% & 17.3 \% \\ & & & & & & & & -0.34 \% \\ \text { Diff ROC } & 0.11 \% & -0.67 \% & 0.48 \% & 0.09 \% & -0.17 \% & -3.90 \% & -2.00 \% & -0.3 \% \\ \text { Reduktion } & 54 \% & 42 \% & 24 \% & 58 \% & 59 \% & 35 \% & 50 \% & 51 \%\end{array}$

Quelle: eigene Darstellung aufgrund eigener Erhebungen

Damit wäre die Reduktion fein-tunender Overrides ein prüfenswertes Mittel zur Senkung des Override-Anteils ohne Aufgabe des positiven menschlichen Einflusses. Betrachtet man Tabelle 1, dann können mit dieser Massnahme rund 50\% der Overrides reduziert werden. Dieser hohe Anteil rechtfertigt es, innerhalb der einzelnen Banken eine vertiefte Prüfung solcher Massnahme anzuregen.

\section{Literatur}

Martin M. (2007) Regulatorische Aspekte der Prüfung von Risikoklassifizierungsverfahren, in Becker A., / Förschler D., Klein J. (Hrsg): Rating-Systeme und Prozesse, 2007, Frankfurt: Bankakademie-Verlag 
Informationssysteme in Transport und Verkehr 



\title{
Vorwort zum Track
}

\section{Informationssysteme in Transport und Verkehr}

\author{
Jens Gottlieb', Dirk Chr. Mattfeld², Leena Subl3 \\ ${ }^{1} \mathrm{~S} A P$ AG, Walldorf \\ 2Institut für Wirtschaftsinformatik, Technische Universität Braunschweig \\ ${ }^{3}$ Lehrstubl Decision Support \& Operations Research Lab, \\ Universität Paderborn
}

Durch die zunehmende Verlagerung von Fertigungsaktivitäten sowie die zunehmende Mobilität von Menschen wächst das Volumen von Transport- und Verkehrsleistungen kontinuierlich. Für die Planung und Steuerung der vielfältigen Prozesse in Transport und Verkehr sind in der Regel große Mengen an Informationen notwendig, die nur durch den Einsatz spezifischer Informationssysteme beherrscht werden können. In dem Track „Informationssysteme in Transport und Verkehr" der Multikonferenz Wirtschaftsinformatik 2010 werden innovative Informations- und Kommunikationssysteme in den genannten Bereichen vorgestellt und diskutiert.

Die Themen diese Tracks spannen einen weiten Bogen von dynamischen Tourenplanungsproblemen hin zu einem Lokations-Routing-Problem und von mathematischen Formulierungen hin zu heuristischen Optimierungsverfahren. Die Anwendungsszenarien reichen von Bike-Sharing, City-Logistik, Straßengütertransport, Paket- und Express-Distribution bis zur Restrukturierung von Distributionsnetzen.

Wir bedanken uns bei den Autoren der neun eingereichten Artikel, von denen wir sechs Beiträge für den Track akzeptiert haben, die die folgenden Themen behandeln:

- Ehmke und Mattfeld diskutieren die Verarbeitung und Anwendung empirischer Verkehrsdaten für die Tourenplanung in Ballungsräumen und evaluieren den Datenverarbeitungsprozess und die betrachteten Informationsmodelle anhand eines Anwendungsbeispiels aus der City-Logistik.

- Müller vergleicht verschiedene Konstruktions-, Einfüge- und Optimierungsverfahren für dynamische Tourenplanungsprobleme mit Zeitfensterrestriktionen und untersucht den Zusammenhang zwischen Zwischenund Endergebnissen von Optimierungsläufen. 
- Schönberger und Kopfer entwickeln einen Kapazitätssteuerungsansatz zur Allokation von Ressourcen im Straßengütertransport und evaluieren ihn in Computer-basierten Simulationsexperimenten.

- Schwind und Kunkel beschreiben im Forschungsprojekt KolOptNet entwickelte Algorithmen zur Tourenplanung in Transportnetzen für Paketund Expresslieferungen.

- Stenger, Schneider und Schwind betrachten die Restrukturierung von Distributionsnetzen durch eine Kostenanalyse, eine Beschreibung von Reorganisationsmöglichkeiten sowie eine mathematische Formulierung für Lokations-Routing-Probleme.

- Vogel und Mattfeld stellen ein Informationssystem zum Bike-Sharing vor und betrachten Verbesserungsmöglichkeiten für die Leistungsbereitstellung.

Jeder eingereichte Artikel wurde dreifach-blind begutachtet. Wir bedanken uns bei den folgenden Gutachtern, die durch ihre sorgfältige Arbeit eine fundierte Entscheidung bei der Auswahl der akzeptierten Beiträge ermöglicht haben:

- Prof. Dr. Christian Bierwirth, Universität Halle-Wittenberg

- Prof. Dr. Stefan Bock, Universität Wuppertal

- Prof. Dr. Andreas Fink, Helmut-Schmidt-Universität Hamburg

- Dr. Tore Grünert, GTS Systems and Consulting

- Prof. Dr. Hans-Otto Günther, TU Berlin

- Prof. Dr. Richard Hartl, Universität Wien

- Prof. Dr. Natalia Kliewer, Freie Universität Berlin

- Peter Korevaar, IBM Deutschland

- Prof. Dr. Taïeb Mellouli, Universität Halle-Wittenberg

- Peter Möhl, PTV AG

- Prof. Dr. Lars Mönch, FernUniversität Hagen

- Prof. Dr. Volker Nissen, TU Ilmenau

- Dr. Giselher Pankratz, FernUniversität Hagen

Weiterhin bedanken wir uns bei Jan Fabian Ehmke und Arne Frerichs für die Unterstützung mit dem Konferenzverwaltungssystem.

Wir wünschen allen Teilnehmern eine erfolgreiche Konferenz und interessante Vorträge und Diskussionen über Informationssysteme in Transport und Verkehr. 


\title{
Kapazitätssteuerung in der Transportlogistik
}

\section{Ein Ansatz zur Ertrags-maximierenden Ressourcen- Allokation im Straßengüterverkehr}

\author{
Jörn Schönberger, Herbert Kopfer \\ Lehrstubl für Logistik, Universität Bremen
}

Durch die Harmonisierung und Liberalisierung der EU-Binnenmärkte sind Transportunternehmen im Straßengüterverkehr in die Lage versetzt worden, Marktbzw. Nachfrage-orientiert zu agieren. Allerdings sind geeignete Werkzeuge, um vorhandene Transport-Ressourcen wie Laderaum, Container oder Komplettladungen erfolgsmaximal zu allokieren und insbesondere adäquate (Mindest-)Preise für angebotene Transportleistungen festzulegen bisher nur im Einzelfall verfügbar.

Für einen profit-orientiert agierenden Transport-Dienstleister stellt die Selektion von profitablen Transportaufträgen ein zentrales Entscheidungsproblem in der operativen Transportprozess-Planung dar. Die Trennung von profitablen und unprofitablen Aufträgen für eine bekannte Grundgesamtheit von Aufträgen ist umfassend untersucht. Dabei werden oftmals auch Fremdvergabe-Möglichkeiten ausgenutzt. Für den Fall einer zunächst nur partiell bekannten Grundgesamtheit von Anfragen liegen keine umfassenden Ergebnisse vor.

In dieser Arbeit stellen wir einen Kapazitätssteuerungsansatz für TransportRessourcen vor und berichten über die Ergebnisse von Simulationsexperimenten zur Evaluierung des vorgeschlagenen Ansatzes für ein partiell unbekanntes Auftragsportfolio.

Kapazitätssteuerung ist ein Bestandteil des operativen Revenue Managements und wird zur Entscheidung über die Annahme oder Ablehnung von ggf. sukzessiv eintreffenden Reservierungsanfragen für vorgehaltene Ressourcen genutzt. Ursprünglich für die Absatzkontrolle im Passagierluftverkehr konzipiert, werden heute Ansätze der Kapazitätssteuerung in vielen Service-Industrien und im produzierenden Gewerbe angewendet, um eine möglichst profitable Zuteilungen von knappen Ressourcen (z.B. Maschinen oder Dienstleistungen) zu Aufträgen vorzunehmen. Insbesondere Transport-Dienstleister, die eine relativ hohe Grundauslastungsquote durch langfristige Kundenkontrakte besitzen, versuchen vorhandene Restkapazitäten auf dem Spotmarkt Erlös-maximierend zu verkaufen. Dadurch können die insgesamt steigenden Transportnachfragen aufgrund einer effizienteren Fahrzeugauslastung mit einer unterproportionalen Zunahme von Fahrleistungen bewältigt werden. 
Das Annahmeproblem des Transport-Dienstleisters wird als OnlineOptimierungsproblem dargestellt. Wir schlagen zunächst einen Modellierungsansatz vor, bei dem ausschließlich auf Basis der tatsächlich vorliegenden Anfragen die Entscheidung über Annahme oder Zurückweisung der Anfragen getroffen wird. Dieser Ansatz entspricht einer First-Come / First-Serve - Entscheidungsstrategie (FCFS). In einem zweiten Ansatz schlagen wir vor, die Annahme-Entscheidung auf Basis der noch zu erwartenden Nachfrage zu treffen. Aus der Lösung des vorgeschlagenen Optimierungsmodells können wir sog. Bid-Prices ablesen, die eine Untergrenze für die Erlöse darstellen, die mit einem Auftrag erzielt werden müssen, um seine Annahme zu rechtfertigen.

Zur Evaluierung des FCFS-Ansatzes und des Bid-Price Steuerungskonzepts haben wir Testszenarien generiert. Jede Testinstanz umfasst eine vorgegebene Route eines LKWs sowie einen Strom eintreffender Reservierungsanfragen. Wir möchten überprüfen, ob die Qualität der Vorhersage bzw. der Prognose der noch zu erwartenden Reservierungsanfragen einen Einfluss auf die Ziel-Angemessenheit der zu einem Zeitpunkt getroffenen Annahmeentscheidungen hat. Daher kombinieren wir jede der erzeugten Anfragesequenzen mit unterschiedlichen PrognoseGenauigkeiten.

Aufgrund der sukzessiv eintreffenden Reservierungsanfragen ist eine Sequenz von Entscheidungsproblemen zu bearbeiten. Dafür haben wir ein rollierendplanendes Ressourcen-Allokations-System entwickelt. Mit dem Eintreffen einer einzelnen oder mehrerer gemeinsam eintreffender/eintreffenden Reservierungsanfrage(n) wird ein neuer Allokationszyklus gestartet. Die getroffenen Entscheidungen sind nicht revidierbar, d. h. eine einmal akzeptierte Anfrage kann zu einem späteren Zeitraum nicht wieder zurückgegeben werden.

In den Simulationsexperimenten wird gezeigt, dass bei Anwendungen des BidPrice-Ansatzes selbst bei einer ungenauen Prognose der noch zu erwartenden Kapazitäts-Nachfrage die Erträge um 2\% bis 3\% gesteigert werden können gegenüber dem Referenzverfahren FCFS. Eine Steigerung in gleicher Größenordnung ist für die Kapazitätsauslastung beobachtet worden.

Danksagung: Dieser Beitrag entstand im Rahmen des Sonderforschungsbereichs 637 „Selbststeuerung logistischer Prozesse - Ein Paradigmenwechsel und seine Grenzen“ (Teilprojekt B7), gefördert durch die Deutsche Forschungsgemeinschaft (DFG). 


\title{
Bereitstellung von tageszeitabhängigen Fahrzeiten für die Tourenplanung in Ballungsräumen
}

\author{
Jan Fabian Ehmke, Dirk Christian Matteld \\ Institut für Wirtschaftsinformatik, \\ Lehrstubl für Decision Support, \\ Technische Universität Braunschweig
}

Dieser Beitrag thematisiert den Prozess der Erfassung, Verarbeitung, Bereitstellung und Anwendung empirischer Verkehrsdaten für die Tourenplanung in Ballungsräumen. Es wird ein Informationssystem konzipiert, welches empirische Verkehrsdaten in tageszeitabhängige Fahrzeiten für die Tourenplanung überführt. Ziel ist die Unterstützung einer schnellen, zuverlässigen und umweltverträglichen Auslieferung und Abholung von Gütern in der City-Logistik (Taniguchi et al. 2000).

Während sich die überregionale Tourenplanung zumeist auf Distanzen oder statische Fahrzeiten stützt, erfordert die zeitfenstergenaue Tourenplanung in Ballungsräumen die Berücksichtigung tageszeitabhängiger Fahrzeiten für jeden Streckenabschnitt. Grundlage für die Bereitstellung der tageszeitabhängigen Fahrzeiten sind große Mengen empirischer Verkehrsdaten. In Zeiten moderner telematikbasierter Verkehrsdatenerfassung besteht die Möglichkeit, solche Daten in ausreichendem Maße zu erheben und automatisiert zu verarbeiten (Ehmke und Meisel, 2008).

Um von der umfangreichen Datenmenge bei der tageszeitabhängigen Tourenplanung zu profitieren, werden empirische Verkehrsdaten in tageszeitabhängige Fahrzeiten überführt und in erweiterte Verfahren der Tourenplanung integriert. Der Beitrag skizziert die verschiedenen Phasen dieses Datenverarbeitungsprozesses (vgl. Abbildung 1) und beschreibt die erforderlichen Komponenten des Informationssystems.

Zur Rohdatenerhebung wird die Floating-Car-Data-Technologie eingesetzt, bei der Taxis als im Verkehr mitschwimmende Sensoren großflächig empirische Verkehrsdaten sammeln. Diese werden im ersten Aggregationsschritt zu Planungsdaten zusammengeführt. Ergebnis ist ein komplexes, zeitabhängiges Informationsmodell. Im zweiten Aggregationsschritt wird dieses zu einem kompakten, speichereffizienten Informationsmodell verdichtet. Zum Einsatz kommt eine wochentagsabhängige Clusteranalyse, die tageszeitabhängige Gewichtungsfaktoren für Gruppen von Links bereit stellt. 


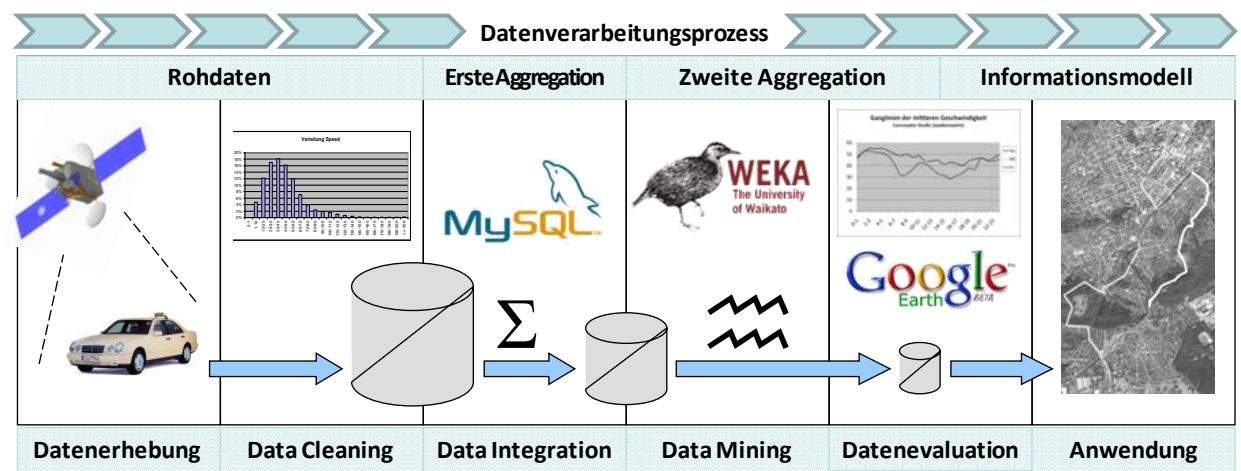

Abbildung 1: Datenverarbeitung von telematikbasierten Rohdaten zur Bereitstellung von Fahrzeiten für die tageszeitabhängige Tourenplanung.

Die bereit gestellten Informationsmodelle werden in erweiterte Tourenplanungsverfahren integriert. Dazu ist eine tageszeitabhängige Modellierung des Planungshorizonts erforderlich. Ferner werden stückweise-lineare Fahrzeitfunktionen abgeleitet, die als Eingangsparameter für die Berechnung kürzester zeitabhängiger Wege dienen. Da die Fahrzeitfunktionen aus empirischen Verkehrsdaten resultieren, erfüllen sie nicht notwendigerweise die FIFO-Bedingung. Wir linearisieren die Fahrzeitfunktionen an ihren Sprungstellen, um eine FIFO-konforme Wegeberechnung zu gewährleisten.

Anhand einer Case-Study für den Großraum Stuttgart wird der Datenverarbeitungsprozess instanziiert und implementiert. Unter Einsatz der verschiedenen Informationsmodelle werden Touren für ein fiktives City-Logstik-Szenario geplant und simuliert. Das durch Data-Mining-Verfahren bereit gestellte Informationsmodell erlaubt eine verlässliche, tageszeitabhängige Tourenplanung bei nahezu identischem Aufwand im Vergleich zum statischen, nicht-zeitabhängigen Fall.

\section{Literatur}

Ehmke JF, Meisel S (2008) Ermittlung dynamischer Fahrzeiten für die CityLogistik. In: Mattfeld DC, Günther H-O, Suhl L, Voß S (Hrsg.) Informationsund Kommunikationssysteme in Supply Chain Management, Logistik und Transport. DSOR Beiträge zur Wirtschaftsinformatik Bd. 5, Books on Demand GmbH, 107-122.

Taniguchi E, Thompson RG, Yamada T, van Duin R (2000) City Logistics. Network Modelling and Intelligent Transport Systems. Emerald Group. 


\title{
Decision Support for Location Routing with Relocation Aspects
}

\author{
Andreas Stenger', Michael Schneider ${ }^{2}$, Michael Schwind ${ }^{1}$ \\ ${ }^{1}$ Chair of IT-based Logistics, Goethe University Frankfurt \\ ${ }^{2}$ Chair of Business Information Systems and Operations Research, \\ Technical University Kaiserslautern
}

Due to drops in orders and decreasing production rates, demand is declining seriously in many logistic sectors like e.g. air cargo, ship and less-than-truckload transports. Hence, small package shippers are looking for ways to improve their transportation network efficiency. In our current research project $K o l \mathrm{O} p t \mathrm{~N} e t$, we focus mainly determining optimal delivery tours on a daily basis considering driver learning effects (Schwind and Kunkel 2009). However, the operational tour efficiency strongly depends on depot locations. This interdependency has to be taken into account in a company's depot location decision (Salhi and Rand 1989). In this context, location-routing problems (LRP) have attracted a great deal of attention among researchers and practitioners over the last two decades. An LRP simultaneously solves three interdependent tasks with the objective of cost minimization (cf. Chien 1993; Perl and Daskin 1985): depot location decisions, customer allocation to opened depots and vehicle routing.

The existing literature on LRP models used for determining the optimal depot locations is extensive (see Nagy and Salhi 2007). Practically all models are based on the assumption that the optimization task is to plan an entirely new delivery network, neglecting the currently established network. However, courier, express and parcel (CEP) service providers cannot ignore existing networks due to the costs that occur when making relocation decisions. Hence, greenfield LRP approaches lead to suboptimal solutions in real-world applications.

To take into account established network structures, planning models have to include the following cost factors:

- Transaction costs for collecting information about potential buyers/potential locations, negotiating with potential buyers/landowners and closing a contract with the buyer/landowner

- Establisbing costs include all costs that arise when a new depot opens. Besides the construction and installation costs, delivery processes between hubs and depots have to be designed. Additionally, training courses for the employees might be necessary.

\footnotetext{
${ }^{1}$ www.koloptnet.de
} 
- Opportunity costs: e.g. changing a depot location seriously influences the costs of the delivery tours as driver learning is neutralized.

These costs are integrated into the mathematical formulation of our relocationoriented location routing problem (RLRP). Furthermore, we give a detailed qualitative description of potential structural problems of delivery networks and suggest reasonable reorganizational moves for each case. For example, consider the case of depots whose capacity utilization depends strongly on the volume of a few large customers. Once too many of them cease doing business, it might be favorable to close the depot and serve the previously allocated customers from remaining depot sites.

In order to provide decision support for real-world applications, we develop a relocation decision support system (RDSS) on the basis of our RLRP model. This tool analyzes the existing depot structure, i.e. location of depots and allocation of customers to depots, and checks if a relocation of existing depots would enhance the cost-efficiency of the delivery network. Besides this one-time determination of optimal depot locations, the relocation tool is coupled with our operational routing module to allow continuous verification of the network efficiency. As soon as changes in demand make modifications of the network structure reasonable, the system will suggest beneficial location changes. We will test the applicability of our RDSS on business data provided by a mid-sized French delivery company.

\section{References}

Chien TW (1993) Heuristic procedures for practical-sized uncapacitated locationcapacitated routing problems. Decision Sci. 24(5):995-1021.

Nagy G, Salhi S (2007) Location-routing: Issues, models and methods. Eur. J. Oper. Res. 177(2):649-672.

Perl J, Daskin MS (1984) A warehouse location-routing problem. Transport. Res. B-Meth. 19B(5):381-396.

Salhi S, Rand GK (1989) The effect of ignoring routes when locating depots. Eur. J. Oper. Res. 39(2):150-156.

Schwind M, Kunkel M (2009) Simulationsbasierte Optimierung kollaborativer Transportlösungen in Transportnetzwerken. In: 39. Jahrestagung der Gesellschaft für Informatik, 2. Workshop Planung und Simulation in logistischen Anwendungen auf der Informatik, Lübeck. 


\title{
Collaborative Optimization of Last Mile Networks for Courier, Express and Parcel Delivery Services
}

\author{
Michael Schwind', Marcel Kunkel2 \\ ${ }^{1}$ Chair of IT-based Logistics, Goethe University Frank.furt \\ 2PickPoint AG, Hofheim-Wallau
}

Rising energy costs and fierce competition force carriers to increase transportation network efficiency. The research project KolOptNet focuses on the creation of sustainable, energy efficient logistics by designing algorithms and implementing methods for optimized network and tour planning within courier, express and parcel service (CEP) networks. Kol-OptNet integrates dynamic tour planning heuristics and combinatorial auctions to ensure the long-term participation of the service providers by using incentive compatible distribution mechanisms to produce cost savings. This article presents KolOptNet's objectives and solution approaches.

Current optimization approaches for CEP networks rely on the near optimal location of the service provider's hubs and depots in connection with tour planning in predefined fixed delivery areas. The KolOptNet approach, however, uses flexible delivery areas in order to enable a more efficient tour planning process that depends on the day-to-day demand situation. It simultaneously trades off between driver learning exploitation and tour planning flexibility. Additionally an exchange mechanism based on a combinatorial auction is used for the collaborative dispatching of transportation tasks between independent last-mile operators that may act as subcontractors for the CEP service provider. This way unutilized synergy potential resulting from overlapping delivery areas of the subcontractors will be raised by the auction mechanism (Zäpfel and Wasner 2002, Schwind et al. 2009).

In order to directly combine the construction of the delivery areas with the tour planning process including driver learning, our CEP optimization approach is designed to be able to deduce the shape of the delivery areas from the iterated calculation of the optimal tours over a time span. Fig. 1 shows two adjacent tours of last-mile operators while the shaded areas depict the core areas around a master tour (called corridor) where the drivers collect knowledge of area's local specifics. Broader corridors will produce less 'dispositive' delivery points (DP) that can be exchanged between the last-mile operators via a combinatorial auction which should result in a stable tour optimization process. By contrast, smaller corridors lead to a higher number of dispositive DPs causing higher flexibility of the tour planning process and should lead to an optimization result closer to the global optimum. This, however, will reduce the positive impact of the diver learning process. A shift of the corridors caused by the multi-period optimization process, 
e.g. due to demand fluctuations, is called drift in the KolOptNet model. Both, corridor size and drift can be used to find the optimal flexibility/learning trade-off.
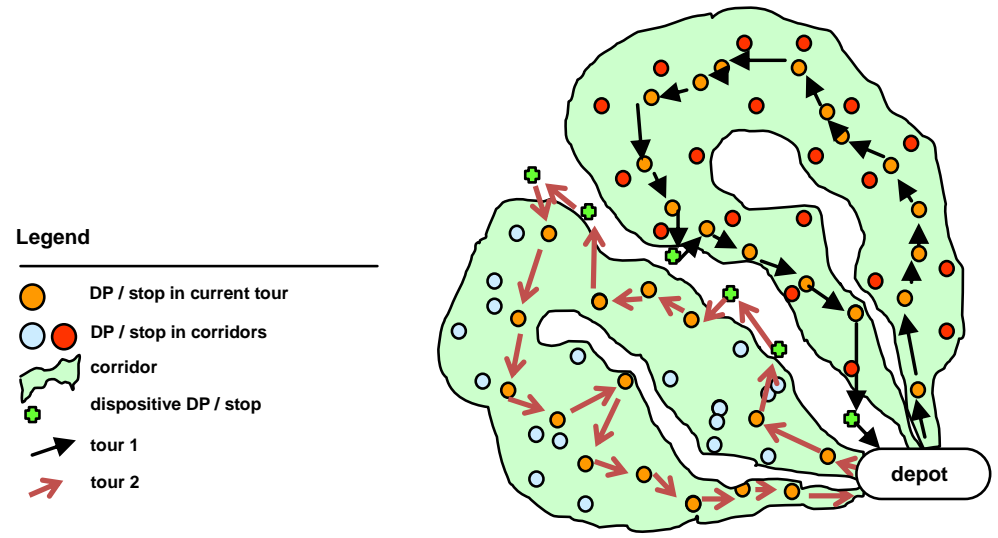

Figure 1: Formation of delivery corridors in the master tour optimization process

In addition to our optimization approach, we present a service-oriented architecture (SOA) that supports the deployment of the KolOptNet system at several CEP service providers (Kunkel et al. 2009). The SOA system makes use of static master data such as delivery area information and dynamic temporal data like the current movement of the vehicles and packages. The output data of the KolOptNet SOA system is sorting plans for the packages and tour plans for the vehicles. This data is transmitted to the wireless mobile package delivery scanner devices that interactively control the delivery process. Furthermore, the devices collect delivery data concerning driver learning and service quality achieved in the optimization process in order to provide a data base for an empirical evaluation of our approach.

\section{References}

Kunkel M, Doppstadt C, Schwind M (2009): Open Service-Oriented Computing for Logistics: A Case in Courier, Express and Parcel Networks; In: 7th Int. Joint Conference on Service Oriented Computing. Stockholm, Sweden.

Schwind M, Gujo O, Vykoukal J (2009): A Combinatorial Intra-Enterprise Exchange for Logistics Services. In: Information Systems and eBusiness Management 7(4):447-471.

Zäpfel G, Wasner M (2002): Planning and optimization of hub-and-spoke transportation networks of cooperative third-party logistics providers. In: Int. J. Prod. Economics 78(2):207-220. 


\title{
Ist besser wirklich besser?
}

\section{Über das Einfügeglück und die Pfadabhängigkeit im Kontext des DVRPTW}

\author{
Jürgen Müller', Richard Lohwasser², Andreas Lackner', \\ Alexander Zeier', Hasso Plattner' \\ ${ }^{1}$ Hasso Plattner Institut für IT Systems Engineering, Potsdam \\ ${ }^{2}$ E.ON ERC, WTH Aachen \\ ${ }^{3} \mathrm{DB}$ Mobility Logistics AG, Frankfurt am Main
}

Einen Problembereich im Verkehrssektor stellen Tourenplanungsprobleme dar. Diese entstehen, wenn Personen oder Güter von einem Transportmittel zu verschiedenen Orten gebracht und/oder abgeholt werden müssen. Im Allgemeinen geht es darum Aufträge zu erfüllen und die Anzahl der nötigen Touren sowie die Gesamtstrecke zu minimieren (Christofides et al. (1979)).

Das Dynamic Vebicle Routing Problem with Time Windows (DVRPWT) beschreibt die Problemstellung, dass verschiedene Kunden mit definierten Bedienfenstern mit einer maximalen Anzahl von Touren bedient werden müssen. Zu Beginn der Tourenplanung sind dabei nicht alle $N$ Kunden, sondern nur $n$ Kunden bekannt. $N$ - $n$ definiert den Dynamikgrad der Problemstellung. Wenn im Verlauf eines DVRPTW-Durchlaufes ein Kunde nachfragt, so wird dieser an der von einem Einfügeverfahren ermittelten Stelle in den Tourenplan integriert. Anschließend werden die Verbesserungsverfahren auf den Tourenplan angewendet, um die Zwischenlösung des Tourenplans zu optimieren.

Das Optimierungsproblem für das DVRPTW mit lexikographischer Zielfunktionsordnung ist wie folgt definiert:

- ZF I: Anzahl abgewiesener Kunden $\rightarrow$ min,

- ZF II: Summe der Länge aller Touren + Summe aller Verspätungen $\rightarrow$ min unter den Nebenbedingungen:

- NB I: Start- und Endpunkt einer jeden Tour ist das Depot,

- NB II: Jede Tour darf frühestens beim Zeitpunkt 0 anfangen und muss bis zum Depotzeitfensterende beendet sein,

- NB III: Die Anzahl der verwendeten Touren muss kleiner oder gleich der verfügbaren Touren der Problemstellung sein,

- NB IV: Kein Kunde darf vor seinem Zeitfensterbeginn bedient werden und 
- NB V: Die Ladekapazität je Tour darf nicht überschritten werden.

Zur Evaluierung der Algorithmen werden die 56 Solomon-Benchmark-Probleme (Solomon (1987) verwendet. Ein im Rahmen dieser Arbeit entwickeltes Computerprogramm wird verwendet um Konstruktions-, Einfüge- und Verbesserungsverfahren in 9.856 unterschiedlichen Tourenplanungskonstellationen $\mathrm{zu}$ analysieren. Überraschenderweise zeigt sich, dass eine bessere Zwischenlösung oftmals zu einem schlechteren Gesamtergebnis führt. Eine gute Bewertung für eine Zwischenlösung entsteht immer dann, wenn die Gesamtstrecke möglichst klein ist. Das bedeutet gleichzeitig, dass möglichst wenige Umwege vorhanden sind. Diese Umwege sind es aber letztendlich, die bei dem DVRPTW die Integration neuer Kunden erleichtern. Es gibt also eine Art Einfügeglück. Es zeigt sich also, dass für einen Tourenplan über seinen Lebenszyklus von der Depoteröffnung bis zum Depotzeitfensterende eine Pfadabhängigkeit besteht, da Entscheidungen nicht revidierbar sind und die zukünftige Güte des Tourenplans maßgeblich beeinflussen.

Wir schlagen vor, dass bei der Bewertung der Zwischenlösung nicht mehr nur die Anzahl der Touren und die zurück gelegte Strecke berücksichtigt werden. Es sollten zum Einen die Flexibilität, mit der neue Kunden in den Tourenplan integriert werden können und zum anderen eine Zeitkomponente mit aufgenommen werden. Diese Zeitkomponente sorgt dafür, dass suboptimale, aber flexible Tourenpläne am Anfang des Planungszeitraums bevorzugt und erst zum Ende des Planungszeitraumes die Flexibilität des Tourenplanes zugunsten der Bewertung verringert wird.

\section{References}

Christofides, N.; Mingozzi, A.; Toth P. (1979) The Vehicle Routing Problem. In: Christofides, N.; Mingozzi, A.; Toth, P.; Sandi, C. (Hrsg.): Combinatorial Optimizations, S. 315-338, New York: John Wiley \& Sons.

Solomon, M. M. (1987) Algorithms for the Vehicle Routing and Scheduling Problems with Time Window Constraints. In: Operations Research, 35(2), S.254 - 265. 


\title{
Informationssysteme im Bike-Sharing: Ein System Dynamics Ansatz
}

\author{
Patrick Vogel, Dirk Christian Mattfeld \\ Institut für Wirtschaftsinformatik, Lehrstubl für Decision Support, \\ Technische Universität Braunschweig
}

Klimaveränderungen, Begrenztheit fossiler Energieträger, hoher Platzbedarf und Lärmemissionen sind nur einige Faktoren dafür, dass das Auto als individuelles Verkehrsmittel in einer Krise steckt. Dem Fahrrad kommt im Stadtverkehr immer mehr Bedeutung zu. Fahrräder tragen nicht zu Stau bei und verursachen keine Umweltverschmutzung. Weiterhin können damit Gebiete erreicht werden, die keine Anbindung an das ÖV-Netz haben. Nachteilig ist, dass die Benutzung des Fahrrads stark vom Wetter und der Topographie abhängig sowie eher für Kurzstrecken geeignet ist (DeMaio 2004). Eine umweltfreundliche, individuelle Mobilität im Stadtverkehr wird in den letzten Jahren verstärkt durch moderne Fahrradverleihsysteme, auch Bike-Sharing Systeme genannt, ermöglicht. Ein flächendeckender Zugang mit teils automatisierten Ausleihprozessen wird durch Informations- und Kommunikationstechnologie unterstützt (Bührmann 2008). Mehr und mehr Bike-Sharing Systeme werden in Großstädten der ganzen Welt eröffnet.

Wissenschaftliche Literatur zu Bike-Sharing Systemen ist rar. Kürzlich erschienene Artikel haben einen starken Praxisbezug. Durch die Einwegnutzung und kurzen Ausleihzeiten kommt es in Bike-Sharing-Systemen zu einem Ungleichgewicht in der räumlichen Verteilung der Fahrräder über die Zeit. Im Rahmen der Leistungsbereitstellung muss informationssystemseitig mittels der Berechnung von Repositionierungen zur Steigerung der Servicequalität auf das Ungleichgewicht reagiert werden. Deshalb beschreibt der vorliegende Beitrag Bike-Sharing Systeme und deren Informationssysteme anhand des Dienstleistungsprozesses nach Mertens et al. (2005), wobei der Fokus auf der Komponente der Leistungsbereitstellung und deren Wirkungszusammenhängen liegt.

Wir übertragen die von Karmarkar (1989) gefundenen Zusammenhänge aus der kapazitierten Produktionsplanung auf Bike-Sharing Systeme. Karmarkar verwendet eine nichtlineare „clearing function“, die den Output eines Produktionssystems als Funktion des durchschnittlichen work-in-process modelliert. Wir nehmen an, dass die Lieferfähigkeit, also die Wahrscheinlichkeit für eine erfolgreiche Entleihung, nichtlinear mit der Anzahl der anfragenden Nutzer im System abnimmt. Die abgeleitete Funktion hält einen Parameter für den Repositionierungsaufwand.

Zur Modellierung der dynamischen Komponenten des Informationssystems wird ein System Dynamics Ansatz (Sterman 2000) verfolgt, der den Zusammen- 
hang zwischen Servicequalität und Lieferfähigkeit eines Bike-Sharing Systems abbildet. Um Aussagen über das Systemverhalten treffen zu können, wird die abgewandelte Funktion der Lieferfähigkeit in ein Lager- und Flussmodell integriert. Eine Simulation mit automatisch generierten Daten zeigt die sich einstellende Servicequalität unter verschiedenem Repositionierungsaufwand. Mehr Repositionierungen führen zu einer höheren Anzahl zufriedener Kunden und tragen somit positiv zum Unternehmenserfolg bei. Daraus lässt sich ableiten, dass eine Erweiterung des Informationssystems um eine Komponente zur automatisierten Repositionierung von Rädern sinnvoll ist.

Als nächster Schritt ist ein Transportmodell zur effizienten Integration der Repositionierung in das Bike-Sharing-Informationssystem zu konzipieren. Ferner sind die getroffenen Annahmen über Lieferfähigkeit und Servicequalität zu verifizieren. Dazu sind die räumlichen und zeitlichen Fahrradflüsse in Bike-SharingSystemen anhand der Entleihungen und Rückgaben zu untersuchen.

\section{Literatur}

Bührmann S (2007) New Seamless Mobility Services: Public Bicycles (NICHES Policy Note 4). Rupprecht Consult Forschung und Beratung GmbH, Köln.

DeMaio P und Gifford J (2004): Will Smart Bikes Succeed as Public Transportation in the United States? In: Journal of Public Transportation, Vol. 7(2):1-15.

Karmarkar US (1989): Capacity loading and release planning with work-in-process (WIP) and lead times. In: Journal of Manufacturing and Operations Management, 2: 105-123.

Mertens P, Bodendorf F, König W, Picot A, Schumann M (2005) Grundzüge der Wirschaftsinformatik. Springer, Berlin Heidelberg New York.

Sterman JD (2006): Business Dynamics: Systems Thinking and Modeling for a Complex World Har/Cdr., Mcgraw-Hill Higher Education. 
Integration von Produkt und Dienstleistung Hybride Wertschöpfung 



\title{
Vorwort zur Teilkonferenz
}

\section{Integration von Produkt und Dienstleistung - Hybride Wertschöpfung}

\author{
Tilo Böhmann', Jan Marco Leimeister² \\ ${ }^{1}$ International Business School of Service Management, Hamburg \\ ${ }^{2}$ Fachgebiet Wirtschaftsinformatik, Universität Kassel
}

Der Wettbewerb für Unternehmen des produzierenden Gewerbes gestaltet sich in zunehmendem Maße schwierig. Eine Differenzierung im Wettbewerb allein durch die Ausgestaltung des physischen Gutes fällt schwer. Die Folge ist (zum Teil ruinöser) Wettbewerb über die Preise für Produkte, so dass die Produzentenmargen auf vielen Märkten in den vergangenen Jahren deutlich schrumpften. Ein möglicher Ausweg für Unternehmen ist die hybride Wertschöpfung. Dabei zielen die Anbieter auf die Bereitstellung von Leistungsbündeln aus Sachgütern und Dienstleistungen, die zu kundenspezifischen Problemlösungen integriert werden. Der Fokus liegt auf der gesamtheitlichen Nutzenerbringung beim Kunden. Ansätze für die Integration von Produkten und Dienstleistungen finden sich schon in vielen Branchen - und auch nicht nur in Investitionsgütermärkten. Die Dynamik, mit der Unternehmen solche neuen Wege zu beschreiten versuchen, wird zunehmend auch in der breiteren Öffentlichkeit wahrgenommen.

Eine solche Integration von Produkten und Dienstleistungen stellt die Unternehmen vor zahlreiche Herausforderungen, die auch in der Forschung in Wirtschaftsinformatik und Betriebswirtschaftslehre noch unzureichend untersucht sind. So vollzieht sich bei produzierenden Unternehmen ein tiefgreifender Wandel, wenn diese konsequent hybride Wertschöpfung umsetzen. Diese Unternehmen verändern ihr Geschäftsmodell, wie sich das z. B. in Ansätzen des Performance Contracting zeigt, bei denen Anbieter integrierte Leistungsbündel nach definierten Qualitäts- und Kostenzielen kontinuierlich bereitstellen (Leistungsgarantie) oder sogar die mit diesen Leistungsbündeln unterstützten Primärprozesse des Kunden übernehmen (Ergebnisgarantie). Dieser Wandel unterstreicht die zunehmende Verantwortung der Anbieter für die Nutzenstiftung in der Verwendung der Leistungen (value-in-use). Dies erfordert bei Anbietern und Kunden nicht nur die Neuausrichtung von Geschäftsprozessen und Informationssystemen, sondern auch die Entwicklung und Anpassung der Qualifikationen der Mitarbeiter.

Aktuelle Forschungsergebnisse zeigen erste Modelle, Methoden und Werkzeuge für die integrierte Entwicklung von Produkt-Dienstleistungssystemen, die fort- 
schreitende Standardisierung von kundenspezifischen Produkt- und Dienstleistungselementen kundenspezifischer Lösungen, die Veränderungen von KundenAnbieter-Beziehungen sowie für die Informationslogistik bei hybrider Wertschöpfung auf. Deshalb zielt die Teilkonferenz auf die Weiterentwicklung dieser Ergebnisse sowie die Untersuchung bislang unberücksichtigter Aspekte hybrider Wertschöpfung.

Zentrale Themen sind daher die Grundlagen hybrider Wertschöpfung, insbesondere zum Wandel von Geschäftsmodellen, die Weiterentwicklung und Erprobung von Methoden und Werkzeuge für die Entwicklung integrierter ProduktDienstleistungsbündel, die Gestaltung der Aufbau- und Ablauforganisation bei hybrider Wertschöpfung in Unternehmen und Unternehmensnetzwerken, die Unterstützung der Auftragsabwicklung bei hybrider Wertschöpfung, die Erfolgs- und Misserfolgsfaktoren von Produkt-Dienstleistungsbündeln, die Über- und innerbetriebliche Standardisierung für hybride Wertschöpfung, das Skill-Management und Kompetenzentwicklung für hybride Wertschöpfung, die Erfolgsfaktoren und Anwenderakzeptanz integrierter Leistungsbündel in unterschiedlichen Branchen sowie die Anforderungen an IT-Architekturen und IT-Governance bei hybrider Wertschöpfung.

\section{Programmkomitee}

- Prof. Dr. Jörg Becker, ERCIS - Universität Münster

- Prof. Dr. Tilo Böhmann, International Business School of Service Management, Hamburg

- Dr. Ralf Knackstedt, ERCIS - Universität Münster

- Prof. Dr. Helmut Krcmar, Technische Universität München

- Prof. Dr. Jan Marco Leimeister, Universität Kassel

- Prof. Dr. Peter Loos, DKFI - Universität Saarbrücken

- Prof. Dr. Markus Nüttgens, Universität Hamburg

- Jürgen Robisch, Siemens AG

- Prof. Dr. Gertrud Schmitz, Universität Duisburg-Essen

- Prof. Dr. Oliver Thomas, Universität Osnabrück 


\title{
Hybrid Value Creation
}

\section{Understanding the Value Creating Attributes}

\author{
Vivek K. Velamuri, Anne-Katrin Neyer, Kathrin M. Möslein \\ Lehrstubl für Wirtschaftsinformatik I, \\ Universität Erlangen-Nürnberg
}

More and more firms shift their focus from offering standalone products or services towards integrated offerings of products and services to meet specific customer demands, thus generating additional value (Tukker 2004). This is known as hybrid value creation. There are many arguments for this increasing popularity of such hybrid offerings in today's business environment. These arguments can broadly be classified under (1) economic as substantial revenue can be generated, (2) strategic as firms can gain a competitive advantage that is difficult to imitate and (3) environmental as the same economic function can be served with a reduction in the quantity of materials required to do so (Oliva and Kallenberg 2003). In addition, hybrid value creation strategy bears the potential to not only generate additional value for customers incrementally, but also to create radically new offerings and solutions even for unknown problems and demands (Möslein and Kölling 2007).

Even though firms have identified the potential of hybrid value creation, they are still not sure what really creates value in various hybrid value creation business models. To gain a deeper understanding of this phenomenon, this paper investigates 52 successful offerings of hybrid value creation and provides an organizational perspective on this challenge.

Using a deductive case study analysis, four distinctive clusters of hybrid value creation are identified. These four cluster can be described as follows: (1) embedded products: contains case studies where the primary goal is to protect a product from competing products by enhancing it with embedded services using digital technologies (e.g., ergo meters embedded with fitness services), (2) leasing/renting/sharing/pooling (from now on termed as "leasing \& co."): contains case studies where the primary goal is to expand the market for capital intensive products by providing them to customers on rental- or use-basis (e.g., car sharing), (3) mass customization: contains case studies where the primary goal is to provide individualized products to end customers at a reasonable premium (e.g., customized shoes), (4) solutions: contains case studies where the primary goal is to help customers focus on their core competencies instead of worrying about aspects such as asset availability (e.g., facility management). Next, the role and impact of four value creating attributes (individualization, marketing-integration, operational- 
integration and firm-customer interaction), identified from the literature in the field of hybrid value creation, for each cluster is systematically derived.

In sum, it was found that there is no standard formula for success in hybrid value creation. Different attributes were crucial for the success of different clusters of hybrid value creation. As a result, a two-step strategy for the successful design of hybrid offerings is suggested: When implementing hybrid value creation, first, the type of hybrid value creation has to be determined. Second, depending on the corresponding cluster the key value creating attribute(s) need to be carefully designed. The paper concludes by indentifying further areas for research.

\section{Acknowledgements}

This research has been funded by project grants from the German Ministry of Research (BMBF). Project: SPRINT, FKZ 01FD0610. The authors would like to their interview partners for their willingness to participate in this research.

\section{References}

Möslein KM, Kölling M (2007) Interaktive hybride Wertschöpfung als Innovationsstrategie. In: Proceedings of the BMBF Conference, 'Innovationsfähigkeit in einer modernen Arbeitswelt' Innovative ability in a modern world of work], March 29-30 2007, Berlin.

Oliva R, Kallenberg R (2003) Managing the transition from products to services. International Journal of Service Industry Management 14(2):160-172.

Tukker A (2004) Eight Types of product-service system: eight ways to sustainability? Experiences from Suspronet. Business Strategy and the Environment (John Wiley \& Sons, Ltd and ERP Environment) 13(4):246-260. 


\title{
Ein Bezugsrahmen für Requirements Engineering hybrider Produkte*
}

\author{
Marina Berkovich'1, Jan Marco Leimeister², Helmut Kromar' \\ ${ }^{1}$ Lehrstuhl für Wirtschaftsinformatik, \\ Technische Universität München \\ ${ }^{2}$ Fachgebiet Wirtschaftsinformatik, \\ Universität Kassel
}

Viele Unternehmen ändern ihre Strategie von „product-centric“ in „customercentric“" und entwickeln sich dadurch zu Lösungsanbietern, die Bündel von Sachund Dienstleistungen anbieten. Solche integrierte Lösungen werden als hybride Produkte (Product Service Systems - PSS) bezeichnet (Leimeister und Glauner 2008). In ihrem Entwicklungsprozess spielt Requirements Engineering (RE), das sich mit der Erfassung und Verwaltung von Anforderungen beschäftigt, eine wichtige Rolle (Sommerville und Kotonya 1998). In diesem Beitrag wird ein Bezugsrahmen für das RE hybrider Produkte vorgestellt. Dieser Bezugsrahmen definiert Begriffe und Konzepte des RE für hybride Produkte.

Um den Bezugsrahmen zu erarbeiten, wurde eine Literaturrecherche über die Eigenschaften hybrider Produkte durchgeführt, um die Rolle des RE während der Entwicklung zu verstehen. Darauf basierend wurden Anforderungen an das RE für hybride Produkte aufgestellt. Anschließend wurde der Bezugsrahmen erstellt.

Wie in Abbildung $1 \mathrm{zu}$ sehen ist, besteht der Bezugsrahmen aus drei Teilen. Ein Artefaktmodell beschreibt die im Rahmen des RE hybrider Produkte zu erarbeitenden Modellierungs- und Spezifikationsergebnisse und deren Beziehungen untereinander. Ein Artefakt kann als eine Klasse von Anforderungen, die ähnliche Eigenschaften aufweisen, definiert werden. Unterschiedliche Lebensdauer von Anforderungen sowie mehrere Iterationen im Entwicklungsprozess führen $\mathrm{zu}$ teilweise neuen oder aktualisierten Anforderungen. Das Artefaktmodell kann dynamisch mit solchen Änderungen umgehen, indem es einen Abgleich zwischen den in den Artefakten enthaltenen und den geänderten oder neuen Anforderungen ermöglicht, die neuen Anforderungen analysiert und in das Artefaktmodell aufnimmt. Auch alle in Verbindung mit der geänderten oder neuen Anforderung stehenden Artefakte werden auf diese Weise aktualisiert. Das Prozessmodell beschreibt die Aktivitäten des RE für hybride Produkte, die an das Artefaktmodell angelehnt

\footnotetext{
* Wir danken der Deutschen Forschungsgemeinschaft (DFG) für die Förderung dieses Projektes als Teil des Sonderforschungsbereiches 768 ,Zyklenmanagement von Innovationsprozessen - Verzahnte Entwicklung von Leistungsbündeln auf Basis technischer Produkte“.
} 
sind, indem eine Aktivität Artefakte als Eingaben erhält und Artefakte erstellt, aktualisiert und ergänzt. Der Methodenbankasten stellt konkrete Methoden zur Verfügung, die zur Durchführung von Aktivitäten im Rahmen des RE eingesetzt werden können. Der Methodenbaukasten ist eine systematisch geordnete Sammlung von Methoden die entlang des Prozessmodells einzelne Methoden für einzelne Aktivitäten zur Verfügung stellt.

Ein solcher Bezugsrahmen ist von großer Bedeutung für die Praxis, da dadurch eine ganzheitliche und domänenübergreifende Sicht auf das RE gewährleistet werden kann. Der Bezugsrahmen bietet die Möglichkeit bekannte Modelle und Methoden aus der Literatur und Praxis zu integrieren und einen Rahmen für neu entwickelte Ansätze für die speziellen Bedürfnisse von hybriden Produkten zu geben.

Der nächste Schritt im Rahmen unserer Forschung besteht darin, die Modelle des Bezugsrahmens genau zu definieren und zu vertiefen. Zur Validierung des Bezugsrahmens werden parallel vertiefende Fallstudien in der Praxis durchgeführt.

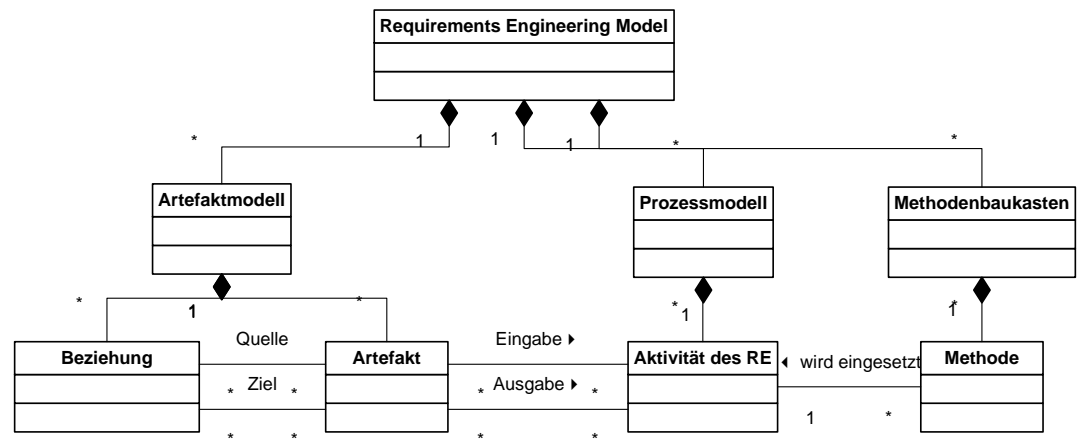

Abbildung 1: Bezugsrahmen für das RE hybrider Produkte

Auf diese Weise kann gezeigt werden, dass unser Ansatz ein domänenübergreifendes Requirements Engineering für hybride Produkte leisten kann.

\section{Literatur}

Leimeister JM, Glauner C (2008) Hybride Produkte - Einordnung und Herausforderungen für die Wirtschaftsinformatik. Wirtschaftsinformatik, 3.

Sommerville I, Kotonya G. (1998) Requirements Engineering: Processes and Techniques (1 Aufl.), Wiley \& Sons. 


\title{
Vorgehensmodelle des Product-Service Systems Engineering
}

\section{Überblick, Klassifikation und Vergleich}

\author{
Marc Gräßle1, Oliver Thomas², Michael Fellmann², Julian Krumeich'1 \\ ${ }^{1}$ Institut für Wirtschaftsinformatike (IWi) im \\ Deutschen Forschungszentrum für Künstliche Intelligen₹. (DFKI), \\ Universität des Saarlands
}
2Institut für Informationsmanagement und Unternehmensfübrung (IMU), Lehrstubl für Informationsmanagement und Wirtschaftsinformatik, Universität Osnabrücke

Knapp vier fünftel der im Rahmen einer Untersuchung befragten Unternehmen planen den Umsatzanteil der Dienstleistungen in Zukunft zu erhöhen und auf kundenindividuelle Anforderungen durch integrierte Sach- und Dienstleistungen zu reagieren (Sturm et al. 2007). Trotz der Bedeutung dieser als Hybriden Produkte oder Product-Service Systems (PSS) bezeichneten Leistungen stimmen nur etwa die Hälfte aller Unternehmen Sach- und Dienstleistungen bereits in der Entwicklungsphase aufeinander ab. Grundsätzlich wurden entsprechende Vorgehensmodelle bereits entwickelt, es existieren aber bisher kaum Untersuchungen, die den Nachweis und die Dokumentation der Vorgehensmodelle im State-of-the-Art zum Gegenstand haben. Ebenso mangelt es darauf aufbauend an Arbeiten zur Behandlung der Frage, welche Vorgehensmodelle in welchen Anwendungssituationen zu nutzen sind. In Anlehnung an vergleichende Übersichten über Konstruktionsmethodiken der Produktentwicklung und Vorgehensmodellen des Service Engineering, Vorarbeiten zur Katalogisierung von Vorgehensmodellen (Höhn 2007) sowie die Typologisierung von PSS (Burianek et al. 2007) wird in diesem Beitrag ein vergleichender Überblick über ausgewählte Vorgehensmodelle des Product-Service Systems Engineering (PSSE) gegeben.

Traditionelle Vorgehensmodelle der Produktentwicklung und des Service Engineering können PSS-spezifischen Anforderungen häufig nicht genügen, was die Notwendigkeit neuartiger PSSE-Vorgehensmodelle begründet. Um die wesentlichen Aspekte zur Beschreibung von PSSE-Vorgehensmodellen herauszuarbeiten, wird eine Klassifikation entworfen. Das in dieser Untersuchung verwendete Klassifikationssystem für PSSE-Vorgehensmodelle besteht aus 24 Merkmalen. Um die Übersichtlichkeit des Klassifikationssystems zu wahren, sind die Merkmale zu fünf 
Merkmalsklassen zusammengefasst worden. Diese Klassen berücksichtigen einerseits, dass Vorgehensmodelle nicht nur entwickelt, sondern auch angewendet werden. Andererseits wird zwischen dem Vorgehen zur Vorgehensmodellkonstruktion und dessen Resultat getrennt. Die Klassifikation orientiert sich ergänzend an den wesentlichen in der Literatur zu Vorgehensmodellen aufgeführten generellen Strukturmerkmalen und -ausprägungen, die um PSS-spezifische Aspekte erweitert sind.

Gemeinsam ist vielen Vorgehensmodellen, dass sie eine stärkere Integration des Kunden in den Entwicklungs- und Leistungserstellungsprozess betonen um eine höhere Individualität der Problemlösungen zu ermöglichen. Es lassen sich jedoch kaum Schlüsse ableiten, inwiefern die anhand von überwiegend theoretischen Beispielen erläuterten Konzepte wirkungsvoll praktisch einsetzbar sind, wenn Kunden anstelle von einzelnen Sach- und Dienstleistungen nach komplexen Problemlösungen fragen. Obwohl die Aufteilung der Wertschöpfung auf mehrere Unternehmen gemäß deren Kernkompetenzen gerade bei komplexeren Anforderungen eine größere Flexibilität verspricht, berücksichtigen nur wenige Vorgehensmodelle explizit Wertschöpfungsnetzwerke.

Insgesamt kann das Profil der aufgezeigten PSSE-Vorgehensmodelle - nicht zuletzt aufgrund der vielfach fehlenden Evaluation - kaum zufrieden stellen. Vor allem scheint ein allgemein anerkanntes, standardisiertes und normiertes Vorgehensmodell für das PSSE noch in weiter Ferne zu liegen. Die in diesem Beitrag durchgeführte Untersuchung liefert eine umfassende Bewertung von PSSEVorgehensmodellen für die Neugestaltung von PSS-Entwicklungsprozessen und der Neuausrichtung bestehender Geschäftsmodelle.

\section{Literatur}

Burianek F, Ihl C, Bonnemeier S, Reichwald R (2007) Typologisierung hybrider Produkte: Ein Ansatz basierend auf der Komplexität der Leistungserbringung. Arbeitsbericht Nr. 01/2007, Lehrstuhl für BWL - Information, Organisation und Management, TU München.

Sturm F, Bading A, Schubert M (2007) Investitionsgüterhersteller auf dem Weg zum Lösungsanbieter: Eine empirische Studie. http://www.fit2solve.de/?download=fit2solve_studie_iat-uni-stuttgart_2007.pdf. Abruf am 2009-02-05.

Höhn R (2007) Beschreibungskriterien für Vorgehensmodelle. http://www1.gi-ev.de/fileadmin/gliederungen/fg-wi-vm/AK-VMK/AKVMK-Beschreibungskriterien.doc. Abruf am 2009-02-05. 


\title{
Vorgehensmodelle für die Entwicklung hybrider Produkte - eine Vergleichsanalyse ${ }^{1}$
}

\author{
Philipp Langer' ${ }^{1}$ Felix Köbler' ${ }^{1}$ Marina Berkovich'1 ${ }^{1}$ Felix Weyde', \\ Jan Marco Leimeister ${ }^{2}$, Helmut Krcmar' \\ ${ }^{1}$ Lehrstuhl für Wirtschaftsinformatik, \\ Technische Universität München \\ ${ }^{2}$ Fachgebiet Wirtschaftsinformatik, \\ Universität Kassel
}

Der Markterfolg eines Unternehmens steht in einem engen Zusammenhang mit dem Aspekt, ob angebotene Sach-oder Dienstleistungen die Anforderungen der Kunden treffen (Leimeister und Glauner 2008; Lindemann 2009). Deswegen orientieren sich viele Unternehmen neu und bieten integrierte Bündel von Sach- und Dienstleistungen an - sog. hybride Produkte - die die Probleme der Kunden lösen sollen (Lönngren et al. 2008; Böhmann et al. 2008; Leimeister und Glauner 2008). Um in domänenübergreifenden Entwicklungsteams produktiv arbeiten zu können, ist ein Vorgehensmodell notwendig, welches sowohl die Art des zu lösenden Problems als auch die Zusammensetzung der Lösung in geeigneter Form berücksichtigt. Bestehende Vorgehensmodelle besitzen meist einen domänenspezifischen Fokus auf bestimmte Komponenten der Lösung oder fokussieren sich auf die technisch begründeten Herausforderungen bei der Umsetzung der Lösung. Solche Modelle leisten nur einen kleinen Beitrag zur Abstraktion von Lösungsteilen und der Verknüpfung von verschiedenen domänenspezifischen Entwicklungsprozessen zu einem integrierten Entwicklungsprozess. Während sich bestehende Vergleichsanalysen meist ausschließlich auf eine Domäne fokussieren, ist es das Ziel dieser Analyse, Modelle aus unterschiedlichen Domänen miteinander in Bezug zu setzen und bezüglich ihrer Eignung für hybride Produkte zu vergleichen.

Nach einer kurzen Einführung zu den Eigenschaften hybrider Produkte vermittelt der Beitrag einen Überblick über den aktuellen Stand der Forschung zu Vorgehensmodellen in der hybriden Leistungserstellung. Um eine Vergleichbarkeit zwischen den Vorgehensmodellen herzustellen, werden Kriterien basierend auf den Eigenschaften hybrider Produkte und ihrer Entwicklung zur Einordnung und Bewertung vorgestellt und auf die identifizierten 18 Vorgehensmodelle aus Pro-

\footnotetext{
1 Wir danken der Deutschen Forschungsgemeinschaft (DFG) für die Förderung dieses Projektes als Teil des Sonderforschungsbereiches 768 ,Zyklenmanagement von Innovationsprozessen - Verzahnte Entwicklung von Leistungsbündeln auf Basis technischer Produkte“.
} 
dukt- Software-, Dienstleistung- und der übergreifenden hybriden Produktentwicklung, angewandt.

Aus der identifizierten Lücke ergibt sich die Notwendigkeit der Entwicklung eines Lebenszyklusmodells hybrider Produkte, das bestimmte Informationen in die kontinuierliche Verbesserung des hybriden Produkts einfließen lässt. Da die Herstellungsprozesse eines produzierenden Unternehmens bis zur Nutzungsphase ausgerichtet sind, müssen folglich alle Prozesse, von der Entwicklung, über die eigentliche Erbringung bis hin zur Abschaffung, erweitert werden. Aus der Produktion von Industriegütern ist bekannt, dass die Ausrichtung an kundenindividuellen Bedürfnissen zu komplexen Varianten eines Gutes führen kann. Somit unterscheiden sich Anbieter hybrider Produkte zu Industriegüter produzierenden Unternehmen, da bei der Entwicklung und Erbringung hybrider Produkte, die Anzahl möglicher Varianten praktisch unbeschränkt ist. Diese Varianten werden durch die Geschäftsprozessausrichtung von Kunden und nicht von Anbietern bestimmt. Anbieter hybrider Produkte können sich vor einer solchen Variantenvielfalt schützen, indem sie versuchen den in Kundenlösungen immer wieder auftretenden Teil eines Leistungsportfolios zu standardisieren und/oder zu modularisieren. Die erfolgreiche Etablierung kundenindividueller Produkte gelingt daher nur mit einem hinreichend komplexen Informationsmodell, das die Kombination eines standardisierten Leistungsportfolios mit einem kundenindividuellen Projektteil erlaubt.

\section{Literatur}

Böhmann, T.; Langer, P.; Schermann, M. (2008) Systematische Überführung von kundenspezifischen IT-Lösungen in integrierte Produkt-

Dienstleistungsbausteine mit der SCORE-Methode.

WIRTSCHAFTSINFORMATIK, Vol. 50(Nr. 3), S. 196-207.

Leimeister, J.M.; Glauner, C. (2008) Hybride Produkte - Einordnung und Herausforderungen für die Wirtschaftsinformatik. Wirtschaftsinformatik, Vol. 50(Nr. 3), S. 248 - 251.

Lindemann, U. (2009) Methodische Entwicklung technischer Produkte: Methoden flexibel und situationsgerecht anwenden, Springer.

Lönngren, H.-M.; Kolbe, H.; Rosenkranz, C. (2008) Erfolgsfaktoren für hybride Wertschöpfungsnetze - Eine Fallstudienanalyse. In: Proceedings of MKWI, München. 


\title{
Produktivitätsmanagement hybrider Leistungsbündel
}

\section{Auf dem Weg zu einer Produktivitätsmanagement- systematik für effiziente Wertschöpfungspartnerschaften}

\author{
Jörg Becker', Daniel Beverungen', Nadine Blinn², Michael Fellmann³, Ralf \\ Knackstedt', Markus Nüttgens ${ }^{2}$, Oliver Thomas ${ }^{3}$
}

\section{${ }^{1}$ European Research Center for Information Systems (ERCIS), WWU Münster ${ }^{2}$ Fakultät Wirtschafts- und Sorialwissenschaften, Universität Hamburg IInstitut für Informationsmanagement und Unternebmensfübrung, Universität Osnabrïck}

Die Betrachtung der betrieblichen Wertschöpfung wandelt sich von einem produktdominierten zu einem dienstleistungsdominierten Paradigma. (Vargo und Lusch, 2008) Entsprechend werden Produkte und Dienstleistungen nicht mehr als dichotom betrachtet (Engelhardt et al. 1993), sondern vielmehr als komplementäre Komponenten verstanden (Vargo und Lusch, 2008), die nach Maßgabe eines Kundenproblems zu sogenannte hybriden Leistungsbündeln kombiniert werden. (Deutsches Institut für Normung 2009)

Jüngste Aktivitäten im Bereich der Dienstleistungsforschung haben die neue interdisziplinäre Forschungsdisziplin Service Science Management and Engineering (SSME, oder Service Science) hervorgebracht. Auch aus der Sicht von Unternehmen ist der Bedarf nach neuen Theorien und Artefakten als Beitrag zum Verständnis und der systematischen Entwickelung von Dienstleistungen vorhanden. So beklagen zahlreiche Unternehmen etwa die mangelnde Bereitschaft ihrer Kunden, ein monetäres Entgelt Beitrag für traditionelle Dienstleistungen (wie bspw. Wartung oder Montagearbeiten) zu entrichten. Hinzu kommt, dass Portfolios aus physischen Produkten und Dienstleistungen in den seltensten Fällen effektiv gemanagt werden. Vielmehr scheinen viele Unternehmen Kundenprobleme nach dedizierten Kundenforderungen reaktiv zu lösen. Um hybride Leistungsbündel effizient und effektiv anbieten zu können, sind jedoch neue Produktivitätskonzepte unerlässlich, die auf die Besonderheiten hybrider Leistungsbündel berücksichtigen. (Oliva und Kallenberg 2003)

Der vorliegende Artikel leistet einen Verständnisbeitrag zum Produktivitätsmanagement hybrider Leistungsbündel und stellt ein Vorgehensmodell zur Entwicklung eines Kennzahlensystems für das Produktivitätsmanagement hybrider Wertschöpfung vor. Der Beitrag ist wie folgt aufgebaut: Zunächst werden beste- 
hende Produktivitätsmanagementansätze aufgezeigt und im Hinblick auf die hybride Wertschöpfung diskutiert. Darauf anbauend wird ein Vorgehensmodell zur Entwicklung des Kennzahlensystems vorgestellt. Das Vorgehensmodell ist im Sinne eines gestaltungswissenschaftlichen Forschungsansatzes als IT-Artefakt anzusehen (vgl. Hevner et al. 2004), das im Zuge seiner Anwendung zu evaluieren und zu verbessern ist. Daher wird die Anwendbarkeit des Vorgehensmodells anhand eines Szenarios der hybriden Wertschöpfung aufgezeigt sowie der Nutzen der Entwicklung eines umfassenden Produktivitätsmanagements für die hybride Wertschöpfung herausgestellt. Die jeweils gemessenen Kennzahlen können im Rahmen von Lebenszyklusrechnungen, die im Artikel anhand idealtypischer Geschäftsmodelle vorgestellt werden, Handlungsfelder zur Produktivitätssteigerung aufzeigen. Der Beitrag schließt mit einem Ausblick auf die kommende Forschung im Bereich des Produktivitätsmanagements für Dienstleistungen.

\section{Literatur}

Deutsches Institut für Normung e.V. (2009) PAS 1094: Hybride Wertschöpfung Integration von Sach- und Dienstleistung. Beuth Verlag, Berlin.

Engelhardt W, Kleinaltenkamp M, Reckenfelderbäumer M (1993) Leistungsbündel als Absatzobjekte : Ein Ansatz zur Überwindung der Dichotomie von Sachund Dienstleistungen. Zeitschrift für betriebswirtschaftliche Forschung, 45, 5, S. 395-426.

Hevner AR, March ST, Park J, Ram S. (2004) Design Science in Information Systems Research. MIS Quarterly, 28, 1, S. 75-105.

Oliva R., Kallenberg R (2003) Managing the transition form products to services. International Journal of Service Industry Management, 14, 2, S. 160-172.

Vargo SL, Lusch RF (2008) Service-dominant logic: continuing the evolution. Journal of the Academy of Marketing Science, 36, 1, S. 1-10. 


\title{
Grundlagen für hybride Leistungsbündel für den europäischen Werkzeugbau
}

\author{
Günther Schuh, Wolfgang Boos, Magdalena Völker \\ Werkzengmaschinenlabor WZL, \\ Rheinisch-Westfälische Technische Hochschule Aachen
}

Der Werkzeugbau hält eine wichtige Position innerhalb der produzierenden Industrien der großen Volkswirtschaften inne (Holmes et al. 2005, S.120 ff.), da Werkzeuge entscheidend die gesamte industrielle Wertschöpfungskette hinsichtlich Qualität, Zeit und Kosten beeinflussen. Trotz der Schlüsselposition steht die vormals wettbewerbbeherrschende europäische Werkzeug- und Formenbauindustrie seit geraumer Zeit aufgrund globaler Wertschöpfungsketten unter starkem Druck. Um mit Wettbewerbern aus Asien und Osteuropa konkurrieren zu können, müssen sich europäische Unternehmen mit innovativen Produkten und Dienstleistungen von der Konkurrenz abheben, eine alleinige Differenzierung über den Preis ist für Werkzeugmacher in Hochlohnländern nicht umsetzbar (Menezes 2004, S. 1-1 f.). Ausländische Konkurrenzunternehmen haben in den letzten Jahren das früher herrschende Qualitätsdefizit gegenüber europäischen Betrieben verringert. Reaktionen darauf in Europa sind unter anderem Veränderungen auf produktiver Seite, die eine zunehmende Industrialisierung mit getakteter Fertigung und definierten Schnittstellen zu Kunden umfassen (Hill et al. 2008, S. 11-14). Hierdurch soll die in den klein- und mittelständischen Unternehmen vorherrschende Unikat- oder Kleinstserienfertigung effizienter gestaltet werden. Darüber hinaus bieten neue Geschäftsmodelle die Basis, um Kunden maßgeschneiderte Lösungen anzubieten, die das physische Kernprodukt mit kundenindividuellen Dienstleistungen zu hybriden Leistungsbündeln (engl. Industrial Product Service Systems - IPS ${ }^{2}$ ) vereinen und nachhaltig den Umsatz sichern können. Derartige strategische Ansätze helfen den Werkzeugbauern, dem allgemeinen Preisverfalls entgegenzuwirken (Ittner und Wuellenweber 2004, S. 14 ff.).

Die Verbindung von innovativen Dienstleistungen und Kernprodukt zu hybriden Leistungsbündeln ist einer der meistversprechenden Wege, um den Kundennutzen zu erhöhen und Wettbewerbsvorteile zu erlangen. Allerdings bieten Werkzeugmacher bisher kaum IPS ${ }^{2}$ an, obwohl diese höhere Gewinnspannen, Wachstumschancen in gesättigten Märkten und länger anhaltende Kundenbeziehungen aufweisen - aktuell generieren Werkzeugmacher weltweit nur 15\% ihres Umsatzes mit Dienstleistungen (Schuh et al. 2009, S. 30). Darüber hinaus können $\mathrm{IPS}^{2} \mathrm{zu}$ langfristigen Partnerschaften führen, die vorteilhaft in einem sonst rein preisbasierten Wettbewerb sind (Lindahl und Ölundh 2001, S. 211-220). 
Eine weiterführende Unterstützung für IPS ${ }^{2}$ ist es, eine Schnittstelle zwischen den Geschäftsmodellen von Werkzeugbau und Kunde zu schaffen, um den Einfluss der Nutzungsphase beim Kunden auf das Werkzeug und damit dessen Einsatzverhalten erfassen zu können. Zudem muss zur Erhöhung der Produktivität und der Verfügbarkeit des Werkzeugs eine Möglichkeit zum Datenaustausch zwischen dem Kunden und dem eigenen Unternehmen geschaffen und im Geschäftsmodell berücksichtigt werden. Der Datenaustausch ist die Voraussetzung für die Umsetzung bestimmter von den Kunden nachgefragter hybrider Leistungsbündel wie die Dokumentation and Aufbewahrung der Prozessdaten. Die Gesamtheit der gewonnenen Daten erlauben eine Datenauswertung, die Rückschlüsse auf die Ausfallursachen des Werkzeugs und auf Instabilitäten des Prozesses zu schließen.

Bei der aufgezeigten Entwicklungsrichtung ist es für die Werkzeugbauunternehmen jedoch essentiell, nicht nur in die Serviceentwicklung sondern auch weiterhin in die technologische Weiterentwicklung zu investieren. Der technologische Vorsprung ist die Voraussetzung, um zusammen mit Services hybride Leistungsbündel erfolgreich am Markt zu platzieren.

\section{Literatur}

Hill A, Boos W, Ziskoven H (2008) Standardisierung im Werkzeugbau als strategischer Erfolgsfaktor. DER STAHLFORMENBAUER 25 (6): 10-16.

Holmes J, Rutherford T, Fitzgibbon S (2005) Innovation in the Automotive Tool, Die and Mould Industry. In: Wolfe D, Lucas M (Hrg.) Network Structure of an Industrial Cluster. Queens University, Toronto: 119-154.

Ittner T, Wuellenweber J (2004) Tough times for toolmakers. THE MCKINSEY QUARTERLY 2004 (2): 14-16.

Lindahl M, Ölundh G (2001) The meaning of functional sales. In: Proceedings of the 8th international seminar on life cycle engineering, Bulgaria.

Menezes J (2004) European mould making: towards a new competitive positioning. In: Proceedings of the 4th International Colloquium Tool and Die Making for the Future, Aachen.

Schuh G, Boos W, Gaus F, Schittny B (2009) Toolmaking for the future - a global study of today's situation and future trends in the tooling industry. ISBN 103 926690-19-4. 


\title{
Reducing the Complexity of Services - A new Approach of Service e-Procurement
}

\author{
Maik Herfurth' ${ }^{1}$ Peter Weiß1, Christian Rudolf2, Robert Kern ${ }^{3}$ \\ ${ }^{1}$ FZI Forschungszentrum Informatik, Karlsrube \\ 2POET AG, Karlsrube \\ ${ }^{3}$ Karlsrube Service Research Institute (KSRI)
}

The paper looks at interoperability issues in the context of e-procurement for industrial maintenance services and asset management. Today's addressed industry domain is lacking shared e-business standards which allow classification of product data, customer data, material data, etc. But still e-Solutions for product package systems are still missing attention. E-Procurement solutions like ERP solutions and catalogue data software solutions support materials and product but product service systems (PPS) are treated like exceptions and cannot be represented in a formal matter. In this paper we focus on formulization of industrial services characterized as product service systems. We focus on the specification and description of services and present our proposal for service classification of industrial maintenance services. Out of the specification and description of services we derive specific service processes and present a model process for an IT solution. We are looking at hybrid services in the field of industrial maintenance processes and develop an e-business solution with standardized processes based on service oriented architectures and a portal solution in order to exchange standardized documents and services reflecting typical service processes. We define a reference process model for the delivery of industrial service processes which can be divided into eight distinct phases describing activities from initial planning over negotiation and purchasing to service delivery, billing and payment. While typical service processes are collaborative processes and involve several actors internally at the client side and the customer side, we focus on these both parties. In our work we extend the classification model for services based on the eCl@ss classification system and develop it further to meet those challenges we discussed before. Therefore we focus on industrial maintenance services and try to include their characteristics. Industrial maintenance services are most likely hybrid services or product service systems. For the maintenance or repair of industrial objects, products like consumables, supplies or working materials are used. A strong relation between industrial services and materials is necessary to classify industrial services. We build up a relation between services and materials using an object oriented approach. In this paper we attempt to capture the variety of services by introducing a new component interactive shopping basket to the system which offers and monitors the interaction between buyer and service provider prior to the start of the service execution. The 
interactive shopping basket features the same characteristics of shopping carts used in regular online stores with an additional roundtrip component providing interactive negotiation features within the system between buyer and service provider. Within the process model indirect ${ }^{1}$ communication towards the configuration of the service to be delivered takes place controlled by the interactive shopping cart. Because of the need to specify all the details (e.g. amounts of material, delivery date or service object) to enable the service provider to estimate the costs of the desired service, an exchange of this information is necessary. According to the three roles contained in the process model, three cycles can be identified within the order process:

- Buyer-Cycle "BC" (Buyer and Interactive Shopping Cart)

- Service Provider-Cycle "SPC" (Service Provider and Interactive Shopping Cart)

- Complete cycle "CC" (Buyer and Service Provider)

The paper presents a newly approach to e-procurement for industrial maintenance services. It has been highlighted that procurement processes in industrial maintenance are complex because they require more interactions and communications between involved actors to configuration and billing of services and related technical material. Despite the fact, that IT solutions and systems for material procurement are mature and have achieved a high level of efficiency, service procurement is still characterized through inefficient and expensive transactions between customers and service providers. Most approaches and solutions on the market are often tailored to the needs of the customer view and needs. We have presented the major elements and components of a solution to improve service e-procurement through the usage of standards along the supply chain. Next steps in our research foresee to further develop the conceptualized approach through field experiments and setting up pilots to evaluate the results. In focus are services which are characterized through high standardization potential and typically ordered in context of an immediate corrective maintenance service to be rendered through a service provider. Our aim is to provide the same functionality as we find it today in material e-procurement, ordering services online through e-catalogues, fast, easy and efficient. We aim at showing that service classification standard, an agreed collaborative process and transaction standard (tailored to the needs of service procurement) will lead to significant cost savings. Cost saving can only be achieved through a joint effort of all actors, mainly service provider (supplier) and customer to exploit mutual benefit.

\footnotetext{
1 The communication is carried out asynchronously, since buyer and service provider don't talk to each other directly but alternately controlled by the interactive shopping cart.
} 


\title{
Managing Hybrid IT-Products
}

\section{Adding IT Support to the SCORE Method}

\author{
Philipp Langer', Thomas Winkler², Tilo Böhmann³, Helmut Kromar ${ }^{1}$ \\ ${ }^{1}$ Lehrstubl für Wirtschaftsinformatik, Technische Universität München \\ ${ }^{2}$ avaso GmbH, München \\ ${ }^{3} I S S$ - International Business School of Service Management Hamburg
}

While the economic impact of pure in-kind transfers tends to decrease, because of the declining differentiation possibilities, Hybrid Products (solutions) gain relevance. Hybrid Products are aligned products and services, which are being offered as integrated performance bundles (Johansson et al. 2003, S. 116-125). Therefore, their special trait is the added value of the integration of both, products and services, which helps to solve customer specific problems (Foote et al. 2001, S. 84). Consequently the value of the Hybrid Product is supposed to exceed the sum of its parts.

Hybrid Products bear an especially high relevance in the IT sector. The offering of customer specific performance bundles, consisting of hardware, software and services, is a widely used concept in this industry (Böhmann et al. 2006). With the providers facing a growing complexity in providing such Hybrid Products, customers demand an even richer product variety and individualization with reduced prices at the same time (Kratochvil und Carson 2005). A resort to overcome these contradictory demands is the concept of standardization, which is the key to realize the economy of large scale reuse (Kratochvil und Carson 2005; Miller et al. 2002; Sawhney 2006). A method of standardization known in the literature is productizing, which means that reusable components are transferred into bricks and added to the knowledge base (Sawhney 2006).

In this paper, the Score method as method to produce customer individual hybrid products is introduced first. As proof of concept, a short summary of a case study utilizing the Score method is presented. This case study is also used to motivate the need to add IT support to the method. In the main part, a data model extending the Three-Layer Architecture of Böhmann (2004) is described that is used to create an electronic Hybrid Product Catalogue, which may be used as fundamental information database for the subsequent bid process of hybrid ITproducts. 


\section{References}

Böhmann, T. (2004) Modularisierung von IT-Dienstleistungen - Eine Methode für das Service Engineering, Deutscher Universitäts-Verlag, Wiesbaden.

Böhmann, T.; Taurel, W.; Krcmar, H. (2006) Paketierung von IT-Dienstleistungen: Chancen, Erfolgsfaktoren, Umsetzungsformen. Technische Universität München, Lehrstuhl für Wirtschaftsinformatik.

Foote, N.W.; Galbraith, J.R.; Hope, Q.; Miller, D. (2001) Making solutions the answer. McKinsey Quaterly, (Nr. 3), S. 84.

Johansson, J.E.; Krishnamurthy, C.; Schlissberg, H.E. (2003) Solving the solutions problem. McKinsey Quarterly, (Nr. 3), S. 116-125.

Kratochvil, M.; Carson, C. (2005) Growing Modular: Mass Customization of Complex Products, Services And Software, Springer, Berlin.

Miller, D.; Hope, Q.; Eisenstat, R.; Foote, N.W.; Galbraith, J.R. (2002) The problem of solutions: Balancing clients and capabilities. Business Horizons, Vol. 45(Nr. 2), S. 3-12.

Sawhney, M. (2006) Going Beyond the Product: Defining, Designing and Delivering Customer Solutions. In: Going Beyond the Product: Defining, Designing and Delivering Customer Solutions. Hrsg.: Lusch, R.; Vargo, S. M.E. Sharpe, Armonk, NY 2006. 


\title{
Vergleich von Reifegradmodellen für die hybride Wertschöpfung und Entwicklungsperspektiven
}

\author{
Jörg Becker, Ralf Knackstedt, Jens Pöppelbuß \\ European Research Center for Information Systems, \\ Westfälische Wilhelms-Universität Münster
}

Die Integration von Sach- und Dienstleistungen zu hybriden Leistungsbündeln gewinnt zunehmend an Bedeutung (Rai und Sambamurthy 2006). Sowohl Konsumenten als auch industrielle Abnehmer verlangen vermehrt nach individuellen Komplettlösungen anstelle einzelner, standardisierter Sach- und/oder Dienstleistungen (Leimeister und Glauner 2008). Der Übergang von einem reinen Sachbzw. Dienstleister zu einem Anbieter hybrider Leistungsbündel bedingt einen schrittweisen Aufbau bzw. Einbeziehung zusätzlicher Kompetenzen (Müller 1998). In diesem Transformationsprozess ist es für Unternehmen unerlässlich, basierend auf einer Bestimmung ihrer Ist-Situation, Veränderungsmaßnahmen abzuleiten und zu priorisieren und deren Umsetzung einer anschließenden Fortschrittskontrolle zu unterziehen. Für diese Aufgaben auf dem Weg zum hybriden Wertschöpfer stellen Reifegradmodelle wichtige Instrumente dar.

Reifegradmodelle sind Modelle, die einen antizipierten, typischen, logischen oder erwünschten Evolutionspfad hin zu einer möglichst hohen bzw. vollkommenen Reife beschreiben (Becker et al. 2009). Wesentliche Charakteristika, die Reifegradmodelle unterscheiden, umfassen die inhaltliche Ausrichtung, die unterschiedenen Reifegradstufen, die differenzierte Beurteilung von Kompetenzobjekten anhand verschiedener Dimensionen, die adressierten Anwendungszwecke, die Art der Assessment-Unterstützung, die adressierten Nutzer und das Vorgehen bei der Modellkonstruktion (Ahlemann et al. 2005).

Anhand dieser Merkmale werden sechs für die hybride Wertschöpfung einschlägige Modelle verglichen, die im Zeitraum 1998 bis 2006 veröffentlicht wurden. Beispielsweise beschreiben Hildenbrand et al. (2006) das strategische Dienstleistungsmanagement produzierender Unternehmen anhand unterschiedlicher Grade der Dienstleistungsorientierung. Oliva und Kallenberg (2003) stellen die Transition des Unternehmensschwerpunkts von Produkten zu Dienstleistungen in einen Prozessmodell dar, der für den Produzenten neue Organisationsstrukturen und Prozesse erforderlich macht. Spath und Demuß (2006) beschreiben in ihrem Reifegradmodell für industrielle Dienstleistungen die verschiedenen Rollen, die Dienstleistungen in Kombination mit physischen Gütern des Maschinen- und Anlagenbaus einnehmen können. Müller (1998) betrachtet in seiner Arbeit vor allem die Vermarktung von industriellen Dienstleistungen und identifiziert diesbezüglich vier typische aufeinanderfolgende Kommerzialisierungssituationen. 
Der Vergleich motiviert das Aufzeigen ausgewählter Entwicklungsperspektiven, die in der weiterführenden Forschung zu Reifegradmodellen der hybriden Wertschöpfung verfolgt werden sollten. Hierzu zählen die inhaltliche Konsolidierung der Reifegrade und Dimensionen der Kompetenzobjekte, die Ergänzung der Pfadbeschreibungen, die Unterstützung des Transformationsprozesses durch Assessment-Verfahren und Konkretisierung von Gestaltungsempfehlungen mittels Verweis auf Verfahren und Instrumente und schließlich die ausführliche Dokumentation des Konstruktionsprozesses.

\section{Literatur}

Ahlemann F, Schroeder C, Teuteberg F (2005) Kompetenz- und Reifegradmodelle für das Projektmanagement: Grundlagen, Vergleich und Einsatz. ISPRIArbeitsbericht, Nr. 01/2005.

Becker J, Knackstedt R, Pöppelbuß J (2009) Entwicklung von Reifegradmodellen für das IT-Management - Vorgehensmodell und praktische Anwendung. In: Wirtschaftsinformatik, 51(3):249-260.

Hildenbrand K, Gebauer H, Fleisch E (2006) Strategische Ausrichtung des Servicegeschäfts in produzierenden Unternehmen. In: Barkawi, K; Baader, A; Montanus, S (Hrsg.): Erfolgreich mit After Sales Services - Geschäftsstrategien für Servicemanagement und Ersatzteillogistik. Berlin, Heidelberg, S. 73-94.

Leimeister JM, Glauner C (2008) Hybride Produkte - Einordung und Herausforderungen für die Wirtschaftsinformatik. In: Wirtschaftsinformatik, 50(3):248-251.

Müller R (1998) Kommerzialisierung industrieller Dienstleistungen. Rosch-Buch, Schesslitz.

Oliva R, Kallenberg R (2003) Managing the transition form products to services. In: International Journal of Service Industry Management, 14(2):160-172.

Rai A, Sambamurthy V (2006) Editorial Notes - The Growth of Interest in Service Management: Opportunities for Information Systems Scholars. . In: Information Systems Research, 17(4):327-331.

Spath D, Demuß L (2006) Entwicklung hybrider Produkte - Gestaltung materieller und immaterieller Leistungsbündel. In: Bullinger, H-J; Scheer, A-W (Hrsg.): Service Engineering - Entwicklung und Gestaltung innovativer Dienstleistungen. 2. Aufl., Berlin, Heidelberg, S. 463-502. 
Integriertes Ertrags-/Risikomanagement in automatisierten Geschäftsprozessen 



\title{
Vorwort zum Track
}

\section{Integriertes Ertrags-/Risikomanagement in automatisierten Geschäftsprozessen}

\author{
Günter Müller', Dennis Kundisch², Stefan Sackmann³, Frank Romeiket \\ ${ }^{1}$ Instituts für Informatik und Gesellschaft, Universität Freiburg \\ 2Lehrstubl für Wirtschaftsinformatik 2, \\ Information Management \& E-Finance, Universität Paderborn \\ ${ }^{3}$ Lehrstubl für Wirtschaftsinformatik,
Universität Halle-Wittenberg \\ ${ }^{4}$ RiskNET GmbH, Oberaudorf
}

Die Wettbewerbsfähigkeit und der wirtschaftliche Erfolg eines Unternehmens werden heutzutage zunehmend von der Flexibilität der Geschäftsprozesse und deren Unterstützung durch IT-Dienste bestimmt. Eine Service-Orientierung bietet Unternehmen diesbezüglich neue Optionen und erlaubt, Geschäftsprozesse weiter $\mathrm{zu}$ automatisieren und noch flexibler an den jeweiligen Kontext anzupassen. Den Chancen stehen jedoch v. a. operationelle Risiken gegenüber, die sich aus automatisierten, kontextspezifischen Anpassungen der IT-Infrastruktur, der Geschäftsprozessabläufe und der Interaktion verschiedener Akteure ergeben.

Die wirtschaftlichen Potentiale werden nur realisiert werden können, wenn auch die Möglichkeit besteht, sowohl die damit verbundenen Erträge als auch die Risiken integriert zu bewerten und zu steuern. Einen viel versprechenden Ausgangspunkt hierfür bietet die Einnahme einer geschäftsprozessorientierten Sicht, da Geschäftsprozesse für Unternehmen sowohl die Verbindung zum Umfeld, beispielsweise zu Kunden oder Lieferanten, als auch zur Applikations- und Infrastrukturebene darstellen. Im Fokus des Tracks stehen daher Konzepte, Methoden und Anwendungen, die eine Planung, Durchführung und ökonomische Bewertung von technischen und/oder organisatorischen Maßnahmen im Sinne eines integrierten Ertrags- und Risikomanagement in automatisierten Geschäftsprozessen ermöglichen.

Aus den eingereichten Beiträgen wurden fünf zur Präsentation und Publikation im Tagungsband angenommen. Vier Beiträge nehmen dabei eher eine Management-orientierte Sichtweise ein: Stefan Hörmann et al. finden in ihrem Beitrag 
"Eine quantitative Perspektive auf die Priorität von Risikofaktoren in ITProjekten" spannende Indizien, dass die Bedeutung technikbezogener Risikofaktoren in IT-Projekten bislang in der Literatur als vergleichsweise zu gering eingeschätzt wurde. André Miede et al. schlagen in ihrem Beitrag "Attacks on the Internet of Services - The Security Impact of Cross-organizational Service-based Collaboration" eine Taxonomie von betriebswirtschaftlichen Gefahren und Angriffen im Kontext von Services zur Unterstützung des IT Risikomanagements vor. Wolfgang Böhmer diskutiert im Beitrag „Managementsysteme sind Balance-Systeme Diskussion relevanter Kennzahlen eines ISMS gemäß ISO/IEC 27001:2005“ Indikatoren, welche im Rahmen eines Informationssicherheitsmanagementsystem zur Absicherung der Unternehmenswerte zum Einsatz kommen können. Stefan Kronschnabl beschäftigt sich in seinem Beitrag „Konzeption eines Modells zur Bestimmung des optimalen Investitionsbeitrags in IT-Sicherheits- bzw. Business Continuity Maßnahmen“ mit einem normativen Modell, mit dessen Hilfe optimale Investitionsbeiträge in IT-Sicherheits- bzw. Business Continuity Maßnahmen unter Einbezug von Compliance-Anforderungen bestimmt werden können. Eher der Design Science-orientierten Forschung ist der Beitrag "Integrating Compliance into Business Processes: Process Fragments as Reusable Compliance Controls" von David Schumm et al. zuzuordnen. Die Autoren entwickeln einen interessanten, auf Prozessfragmente basierenden Ansatz, mit dem Anforderungen der Compliance bei der Modellierung unter Ausnutzung der Wiederverwendbarkeit von Prozessfragmenten vorteilhaft umgesetzt werden können.

Ohne die Mitwirkung der Wirtschaftsinformatik-Community wäre die Durchführung dieses Tracks nicht möglich gewesen. Daher möchten wir uns vor allem bei den Gutachtern Bernhard Bauer (Universität Augsburg), Wolfgang Böhmer (TU Darmstadt), Roland Gabriel (Ruhr-Universität Bochum), Dogan Kesdogan (Universität Siegen), Oliver Prokein (Bâloise-Holding), Martin Reichenbach (Commerzbank AG), Jan vom Brocke (Universität Liechtenstein) und Steffen Zimmermann (Universität Innsbruck) für die fundierte und konstruktive Auseinandersetzung mit den eingereichten Beiträgen bedanken und freuen uns auf die Konferenz und spannende Diskussionen.

Im November 2009

Günter Müller, Dennis Kundisch, Stefan Sackmann, Frank Romeike 


\title{
Integrating Compliance into Business Processes
}

\section{Process Fragments as Reusable Compliance Controls}

\author{
David Schumm, Frank Leymann, Zhilei Ma, Thorsten Scheibler, Steve Strauch \\ Institute of Architecture of Application Systems, University of Stuttgart
}

Companies increasingly have to pay attention to compliance concerns addressing business processes. Flexibly reacting to changing requirements coming from laws, regulations, and internal guidelines becomes a necessary part of business process management. Compliance refers therein to the entirety of all measures that need to be taken in order to adhere to laws, regulations and guidelines within the company, subsumed as compliance sources. In order to comply, companies need to perform profound changes in their organizational structure, business processes, IT systems and applications that drive their business. Consequently, compliance also has an impact on IT systems, applications and supporting infrastructure, as they have to support the execution, monitoring and checking of compliance issues.

Yet, in the field of Business Process Management (BPM) there is currently no agreed upon solution for enabling a flexible management of compliance requirements resulting from the interpretation of various compliance sources. There is no ultimate solution that allows to integrate compliance into processes or IT systems, and which specifies, how the execution of processes can be monitored to validate a compliant execution. The integration of compliance requirements thus often results in hard-wired changes and tangled code. Our approach overcomes the drawbacks of the current solutions and aims at ensuring a faster and more consistent specification and integration of compliance controls in business processes. A compliance control in this sense is the mechanism that actually realizes a compliance requirement.

In this paper we exemplify realizing compliance requirements employing the notion of process fragments, and we show its characteristics and its practical application using a scenario common in industry. In doing so, we discuss how a fragment can be identified, which design considerations need to be taken into account, we discuss efficient storage and retrieval, and which ways of integration into business processes are feasible (cf. Figure 1). 


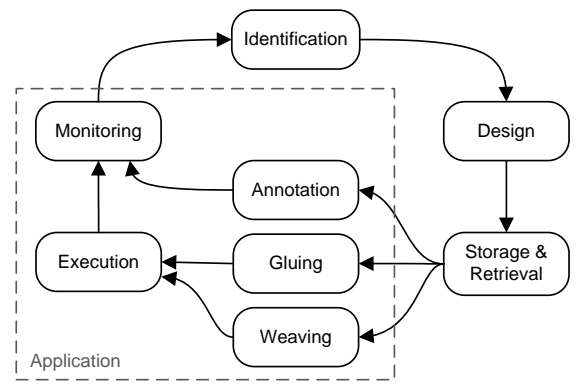

Figure 1: Compliance Fragment Life Cycle

A process fragment in our work is defined as a connected graph, however with significantly relaxed completeness and consistency criteria compared to an executable process graph. A process fragment is made up of activities, activity placeholders (so-called regions) and control edges that define control dependency among them. A process fragment may define a context (e.g., variables) and it may contain a process start or process end node, but it is not required to do so. It may contain multiple entering and leaving control edges for integration into a process or with other process fragments. A process fragment has to consist of at least one activity and there must be way to complete it to an executable process graph.

However, process fragments are not capable of realizing all compliance requirements concerning business processes. Process fragments are capable specifying or constraining control flow and data flow within a process, but they cannot ensure compliance of the applications and humans which are involved in the process. For specifying compliance controls that are not expressible as process fragments we use textual annotation, which we see as additional type of compliance controls.

Our approach has three significant contributions: Firstly, the approach is providing support for (re-)integrating compliance requirements into a process. We achieve this by presenting a way to integrate compliance requirements that are realized by process fragments. Secondly, we envision process fragments as novel language for stating constraints concerning the execution behaviour of process instances. This concept allows precisely stating compliant behaviour in terms of control flow and data flow. Thirdly, by employing transformations this constraint language can be used in monitoring in order to achieve a correlation of the actual execution and the expected, i.e. compliant execution, based on events which actually occur. The concept presented in this paper allows linkage of business processes, process fragments, textual annotations, execution information and compliance sources with each other. This linkage supports demonstrating a lawful implementation of compliance requirements in case of an audit. 


\title{
Eine quantitative Perspektive auf die Priorität von Risikofaktoren in IT-Projekten
}

\author{
Stefan Hörmann, Michael Schermann, Helmut Kromar \\ Lehrstubl für Wirtschaftsinformatik, Technische Universität München
}

Sowohl die Praxis als auch die Wissenschaft argumentiert, dass Risikomanagement essenziell für den Erfolg von IT-Projekten ist (Schmidt et al. 2001; Wallace et al. 2004). Ziel dieser Arbeit ist zur Beantwortung der Frage, welche Mechanismen der Rangfolge von Risikofaktoren in IT-Projekten zu Grunde liegen, beizutragen. Dazu analysieren wir das Risikodatenarchiv von ALPHA1, einem großen, multinationalen Softwareunternehmen und konsolidieren die einzelnen Risikoberichte in einer Risikodatenbank.

Das Risikomanagement bei ALPHA orientiert sich an einem Standardprozess, der die vier Phasen „Risikoidentifikation“, „Risikobewertung“, „Risikomaßnahmenplanung“ sowie „Risikoüberwachung“ umfasst. Die Ergebnisse des gesamten Prozesses werden in einer oder mehreren Kalkulationstabellen gespeichert, von denen wir insgesamt 1548 zur Analyse heranziehen konnten. Davon war es uns möglich, 1222 auszuwerten. Die Untersuchungsvariablen waren dabei „Auswirkung“, „Eintrittswahrscheinlichkeit“ und „Risikoexposition“ (Auswirkung x Eintrittswahrscheinlichkeit). Wir treffen die Annahme, dass sich die Bedeutung eines Risikofaktors in der Variable „Risikoexposition“ widerspiegelt.

Um einen Vergleich zu bestehenden Rangfolgen ziehen zu können, verknüpfen wir die Risikofaktoren mit den von Wallace et al. (2004) vorgeschlagenen Risikodomänen „Technisches Teilsystem“, „Soziales Teilsystem“ und „Projektmanagement". Anhand der Gegenüberstellung ist zu erkennen, dass die Risikobewertungen der Projektmanager von ALPHA erheblich von den Priorisierungen der anderen beiden Studien abweichen (vgl. Tabelle).

Bei der Begründung dieser Diskrepanz könnte zum einen die Kontrollierbarkeit der Risikofaktoren eine Rolle spielen. Risikofaktoren, die dem technischen Teilsystem zugeordnet werden können sind aus Sicht des Projektmanagers nicht kontrollierbar, da diese Risikofaktoren bereits vor Projektstart vorgegeben sind. Ebenso wird die Bewertung der Risikofaktoren auch als organisationales und politisches Instrument benutzt. Daher kann die Bewertung durch die Projektmanager als mikropolitisch beeinflusst bezeichnet werden. Mikropolitische Mechanismen führen dazu, dass die aus Unternehmensperspektive wichtigsten Risikofaktoren berichtet werden. Die beiden Dimensionen „Kontrollierbarkeit“ und „Mikropolitischer Einfluss" bieten erste Ansätze, die die zugrunde liegenden Mechanismen der

\footnotetext{
${ }^{1}$ Name geändert.
} 
Risikobewertung von Projektmanagern erklären könnten. Wir argumentieren zudem, dass die Diskrepanz auf verschiedene Perspektiven der Risikobedeutung zurückzuführen ist.

Tabelle 1: Vergleich der Rangfolgen

\begin{tabular}{|c|c|c|c|c|c|c|}
\hline Rang & ALPHA & & Schmidt et al. (2001) & & Kappelman et al. (2006) & \\
\hline 1 & $\begin{array}{l}\text { Inadequate } \\
\text { Technical } \\
\text { Infrastructure }\end{array}$ & $\mathrm{T}$ & $\begin{array}{l}\text { Lack of Effective Project } \\
\text { Management skills }\end{array}$ & $\mathrm{P}$ & $\begin{array}{l}\text { Lack of Top Management } \\
\text { Support }\end{array}$ & $\mathrm{S}$ \\
\hline 2 & $\begin{array}{l}\text { Customer } \\
\text { Expectations }\end{array}$ & $\mathrm{S}$ & $\begin{array}{l}\text { Lack of Top Management } \\
\text { commitment }\end{array}$ & $\mathrm{S}$ & $\begin{array}{l}\text { Lack of Documented } \\
\text { Requirements }\end{array}$ & $\mathrm{P}$ \\
\hline 3 & $\begin{array}{l}\text { Core Development } \\
\text { Dependencies }\end{array}$ & $\mathrm{T}$ & $\begin{array}{l}\text { Lack of Required Skills in } \\
\text { Project Personnel }\end{array}$ & $\mathrm{P}$ & Weak Project Manager & $\mathrm{P}$ \\
\hline 4 & $\begin{array}{l}\text { Complex System } \\
\text { Architecture }\end{array}$ & $\mathrm{T}$ & $\begin{array}{l}\text { Not Managing Change } \\
\text { Properly }\end{array}$ & $\mathrm{P}$ & $\begin{array}{l}\text { No Change Control Process } \\
\text { (Change Management) }\end{array}$ & $\mathrm{P}$ \\
\hline 5 & $\begin{array}{l}\text { Post Go Live } \\
\text { Approach Not } \\
\text { Defined }\end{array}$ & $\mathrm{P}$ & $\begin{array}{l}\text { No Planning or } \\
\text { Inadequate Planning }\end{array}$ & $\mathrm{P}$ & $\begin{array}{l}\text { No Stakeholder Involvement } \\
\text { and/or Participation }\end{array}$ & $S$ \\
\hline 6 & $\begin{array}{l}\text { Customer Financial } \\
\text { Obligations }\end{array}$ & $\mathrm{S}$ & $\begin{array}{l}\text { Misunderstanding the } \\
\text { Requirements }\end{array}$ & $\mathrm{P}$ & $\begin{array}{l}\text { Ineffective Schedule Planning } \\
\text { and/or Management }\end{array}$ & $\mathrm{P}$ \\
\hline 7 & $\begin{array}{l}\text { Expected } \\
\text { Performance Issues }\end{array}$ & $\mathrm{T}$ & Artificial Deadlines & $\mathrm{P}$ & $\begin{array}{l}\text { Weak Commitment of Project } \\
\text { Team }\end{array}$ & $\mathrm{P}$ \\
\hline 8 & $\begin{array}{l}\text { Customer Inability } \\
\text { to Undertake Project }\end{array}$ & $\mathrm{S}$ & $\begin{array}{l}\text { Failure to Gain User } \\
\text { Commitment }\end{array}$ & $\mathrm{S}$ & $\begin{array}{l}\text { Communication Breakdown } \\
\text { among Stakeholders }\end{array}$ & S \\
\hline 9 & $\begin{array}{l}\text { Non-T\&M Payment } \\
\text { Terms }\end{array}$ & $\mathrm{S}$ & $\begin{array}{l}\text { Lack of Frozen } \\
\text { Requirements }\end{array}$ & $\mathrm{P}$ & $\begin{array}{l}\text { Team Members Lack Requisite } \\
\text { Knowledge and/or Skills }\end{array}$ & $\mathrm{P}$ \\
\hline 10 & Functionality Gaps & $\mathrm{T}$ & $\begin{array}{l}\text { Lack of People Skills in } \\
\text { Project Leadership }\end{array}$ & $\mathrm{P}$ & $\begin{array}{l}\text { Subject Matter Experts are } \\
\text { Overscheduled }\end{array}$ & $\mathrm{P}$ \\
\hline
\end{tabular}

T: Technisches Teilsystem, S: Soziales Teilsystem, P: Projektmanagement

Insgesamt trägt unsere Forschungsarbeit zur Entwicklung des Projektrisikomanagements bei, indem ein erstes Modell vorgeschlagen wird, das die Ergebnisse bestehender Studien miteinander verbindet und neue Ansätze liefert, die Mechanismen hinter der Risikowahrnehmung von IT-Projektmanagern zu verstehen.

\section{Literatur}

Schmidt R, Lyytinen K, Keil M, Cule P (2001) Identifying Software Project Risks: An International Delphi Study. Journal of Management Information Systems, Vol. 17 (2001): 5-36.

Wallace L, Keil M, Rai A (2004) How Software Project Risk Affects Project Performance: An Investigation of the Dimensions of Risk and an Exploratory Model. Decision Sciences, Vol. 35 (2004): 289-321. 


\title{
Attacks on the Internet of Services
}

\section{The Security Impact of Cross-organizational Service-based Collaboration}

\author{
André Miede', Tobias Ackermann², Nicolas Repp ${ }^{1}$, \\ Daniel F. Abawi', Ralf Steinmetz', Peter Buxmann² \\ ${ }^{1}$ Multimedia Communications Lab (KOM), Technische Universität Darmstadt \\ ${ }^{2}$ Chair of Information Systems, Technische Universität Darmstadt \\ ${ }^{3}$ Department of Industrial Engineering and Management, \\ Hochschule für Technike und Wirtschaft des Saarlandes
}

Composite, digital business processes and the increasing application of Serviceoriented Architectures ( $S O A$ ) make it possible that parts of these business processes are outsourced to third party organizations, which can be distributed all over the world. Examples are trading processes in investment banking, where market data or credit ratings are bought from external providers, or customer creation processes where data has to be checked against external watch-lists or ratings.

The cross-organizational collaboration allows to increase quality or to perform the processes at lower costs. The integration of third party services into servicebased software systems is a value potential because it supports the services' consumers to create new functionality. The SOA paradigm and the underlying technology ease the integration and make it cheaper. (Becker et al. 2009, pp. 623-624)

The Internet of Services (IoS) is a business model which uses the Internet as a medium for the retrieval, combination, and utilization of interoperable services (Cardoso et al. 2008, pp. 15-16; Schroth 2007, pp. 635-642). Multiple providers may offer and sell their services, thereby leading to market places where consumers can find third party services. The IoS provides the base for complex business networks by supporting the composition and aggregation of existing services to valueadded services. An important aspect of these market places for crossorganizational collaboration is that they support flexible and dynamic intermediation between service providers and consumers through agreements on nonfunctional requirements like cost criteria and Quality of Service (QoS) parameters.

Security issues and attacks related to the IoS have not been researched so far. Regarding an effective risk management for the IoS, the assessment of threats and potential attacks is an important starting point for further steps such as quantifying and managing risks.

This paper focuses on attacks on cross-organizational SOAs in an Internet of Services scenario. As the current state-of-the-art regarding IoS and SOA attacks is 
focused on technological issues such as Web services (Kanneganti and Chodavarapu 2008), it is necessary to broaden the view towards more business-oriented and service-specific threats in this context. In a first step, an attack taxonomy is presented, which is able to capture the already known attacks on technological aspects, the ones on service-level presented in this paper, and also additional ones on higher levels of abstraction, e.g., on business process-level.

Building on this taxonomy, the main contribution is the discussion of attacks exploiting service-specific characteristics such as loose coupling and composability, which can so far be divided into three different classes:

- Service Selection Attacks,

- Consumer-Provider Communication Analysis, and

- Loose and Malicious Compositions.

It is shown that these attacks have a fundamental impact on the general security of cross-organizational service-based workflows as they directly target systemimminent concepts. Thus, it is mandatory to address these challenges in order to make the Internet of Services a safe and attractive "place" to do business.

\section{References}

Becker A, Buxmann P, Widjaja T (2009) Value potential and challenges of serviceoriented architectures - A user and vendor perspective. In: Proceedings of the 17th European conference on information systems (ECIS), Verona.

Cardoso J, Voigt K, Winkler M (2008) Service Engineering for the Internet of Services. In: Enterprise Information Systems 19:15-27. Lecture notes in business information processing (LNBIP). Springer, Berlin.

Kanneganti R, Chodavarapu, P (2008) SOA security. Manning, Greenwich.

Schroth C (2007) The Internet of Services: Global industrialization of information intensive services. In: Proceedings of the 2nd IEEE international conference on digital information management (ICDIM), Lyon. 


\title{
Managementsysteme sind Balance-Systeme - Diskussion relevanter Kennzahlen eines ISMS gemäß ISO/IEC 27001:2005
}

\author{
Wolfgang Böhmer \\ Fachbereich Informatik, Kryptographie und Computeralgebra, \\ TU-Darmstadt
}

Die ISO27001:2005 als Informationssicherheitsmanagementsystem (ISMS) etabliert sich zunehmend als der Sicherheitsstandard in Unternehmen. Die Grundidee des ISMS basiert auf einem Management der Informationssicherheit welches ausgerichtet ist auf dem Management der Unternehmensrisiken und einen direkten Bezug zum Firmenumsatz herstellt. Bis September 2009 wurden weltweit mehr als $5822^{1}$ zertifizierte Unternehmen registriert. Zu berücksichtigen bleibt jedoch, dass die Zertifizierung nichts über Güte und Performance eines ISMS2 aussagt. Zur kontinuierlichen Verbesserung des ISMS kann der Plan-Do-Check-Act-Zyklus (PDCA-Zyklus) und für vorbeugenden und korrigierenden Maßnahmen die Norm selbst herangezogen werden; es sind aber keine Messmethoden ${ }^{3}$ vorhanden, mit denen Aussagen über die Abschätzung der Güte eines ISMS getroffen werden können.

Zur Bewertung von Managementsystemen bzw. Prozessen stehen in der Literatur unterschiedliche Methoden zur Messung der Performance zur Verfügung.

1. Nach einer Methode, entwickelt von der Carnegie Mellon University, kann die Performance über den Reifegrad der Prozesse, mittels CMMI oder SPICE (ISO/IEC 15504) gemessen werden.

2. Eine etwas neuere Methode besteht darin den Zustandsraum der Prozesse mittels Prozess Algebra zu analysieren. Erste Überlegungen wurden von [Brandt et. al. 2008] publiziert; die Tauglichkeit dieser Methode wurde für den Business Continuity Prozess (BCP) von [Böhmer et al. 2009b] publiziert.

3. Eine weitere Methode besteht in der Abschätzung der Performance anhand von geeigneten Kennzahlen (KPI). Vorschläge zur Handhabung von Kennzahlen sind z. B. bei [Alemanni et al. 2008]. Ein Kennzahlensystem für ein ISMS ist z. B. von [Tsinas et al. 2009] entwickelt worden. Ein ähnlichen Ansatz für ein Kennzahlensystem wurde von [Böhmer, 2009b] für ein Business Continuity Managementsystem (BCMS) entworfen.

${ }^{1}$ Quellenangabe http://www.iso27001 certificates.com/ (abgerufen Sept. 2009).

${ }^{2}$ Die Norm beschreibt was zu tun ist, jedoch nicht wie etwas zu tun ist.

${ }^{3}$ Die SC27 entwickelt derzeit die ISO 27004. Diese Norm wird ein Messverfahren beinhalten. Eine Publizierung ist jedoch erst in den nächsten Jahren zu erwarten. 
In diesem Beitrag wird ein Indikatormodell vorgestellt, das die beiden Schlüsselindikatoren Effektivität und Effizienz eines ISMS ins Verhältnis setzt. Es lassen sich in einer ersten Approximation vier Zustände definieren. Bei genauerer Betrachtung verhalten sich die Schlüsselindikatoren konträr und bilden einen Zielkonflikt. Durch die Einführung einer Budget-Grenze, die z. B. von Soo Hoo empirisch ermittelt wurde, kann dieser Zielkonflikt in ein 0-1 Knapsack-Problem übertragen werden. Eine Bespielrechnung zeigt die iterative Lösung des 0-1 KnapsackProblems.

\section{Literatur}

Alemanni M., Alessia G., Tornincasa S. and Vezzetti K.: “Key Performance Indicators for PLM benefits evaluation: The Alcatel Alenia space case study," Computer in Industry, Vol. 59, No. 8, pp. 833-841, 2008.

Brandt C., Engel T., Böhmer W. und Roeltgen C.: "Diskussionsvorschlag einer Lösungsskizze zur Behandlung von operationellen IT-Sicherheitsrisken nach Basel II auf der Grundlage von Anforderungen der Credit Suisse,” in MKWI München, 2008.

Böhmer W., Brandt C. and Groote, J., F.: "Evaluation of a business continuity plan using process algebra and modal logic," in 2009 IEEE Toronto International Conference - TIC-STH 2009 - SIASP 2, pp. 147-152, Ryerson University, 245 Church Street, Toronto, Ontario, Canada, 2009.

Böhmer W.: "Survivability and business continuity management system according to BS 25999," Proceedings of the Emerging Security Information, Systems and Technologie, 2009. SECUWARE '09, pp. 142-147, June, 18-23, Athen, Greece 2009.

Tsinas L., Trösken B. and Sowa S., "KPI-Framework für Informationssicherheit," KES, SecuMedia Verlag-GmbH, 55205 Ingelheim, Vol 4. pp. 6 - 12, 2009 


\title{
Konzeption eines Modells zur Bestimmung des optimalen Investitionsbeitrags in IT-Sicherheits- bzw. IT-Notfallmaßnahmen unter Berücksichtigung Compliance-bedingter Anforderungen
}

\author{
Stefan Alexander Kronschnabl
}

\author{
ibi research an der Universität Regensburg GmbH
}

Da die IT immense Bedeutung für nahezu sämtliche Geschäftsprozesse hat, muss diese durch geeignete Sicherheits- und Notfallmaßnahmen abgesichert werden (Kronschnabl 2008, S. 3). Hierbei gilt es eine Vielzahl relevanter rechtlicher und aufsichtsrechtlicher Rahmenbedingungen zu berücksichtigen. Aufgrund eines in der Regel begrenzten Budgets sind zudem je nach Bedeutung und monetärem Wert des Geschäftsprozesses sowie erwarteten Schadenswahrscheinlichkeiten geeignete Absicherungs- und Notfallmaßnahmen zu treffen. Das ITRisikomanagement kann hierzu einen wichtigen Beitrag leisten. Es gilt sinnvolle Maßnahmen zu finden und entsprechend dem Budget zu priorisieren. Ziel ist es ein Modell zu konzipieren, welches eine aussagefähige Net Present Value (NPV) Verteilung möglicher Maßnahmen liefert, um so die angemessenste Maßnahme zu identifizieren und umzusetzen.

Zur Konzeption eines Modells zur Bestimmung des optimalen Investitionsbeitrags in IT-Sicherheits- bzw. IT-Notfallmaßnahmen wurden eine Reihe von relevanten Modellen in Wissenschaft und Praxis ausgewählt und analysiert. Exemplarisch kurz aufgeführt werden die Modelle nach Gordon/Loeb (2002), Soo Hoo (2000) Cavusoglu et al. (2004), Schechter (2004) und Sonnenreich et al. (2006). Diese Modelle weisen jedoch zwei Schwächen auf: Zum einen unterstützen sie keine mehrperiodische Betrachtung. Zum anderen stellt die Komplexität der Datenbasis, auf denen die Modelle beruhen, in der Praxis ein Problem dar.

Als Grundlage für das konzipierte Modell diente schließlich das Modell von Faisst et al. (2007) in Verbindung mit dem Verfahren von Conrad (2005). Um der Realität Rechnung zu tragen, dass eine IT-Sicherheitsmaßnahme die Opportunitätskosten sinken lässt, wurde jedoch eine erweiterte Formel erarbeitet, deren Ziel es ist, die Opportunitätskosten mit und ohne IT-Sicherheitsmaßnahmen der jeweils gleichen Periode zu vergleichen. Die Unsicherheit und ihre Auswirkung wurde mithilfe der integrierten Monte-Carlo Simulation anschaulich dargestellt. Die resultierenden Diagramme stellen dabei sowohl für IT-Risikomanager als auch für Budget-Entscheider eine gute und nachvollziehbare Entscheidungsgrundlage für Investitionen dar. Budget-Entscheider haben so die Möglichkeit bestehende Modelle, wie beispielsweise einen NPV-Ansatz, weiterhin zu verwenden, IT- 
Sicherheitsexperten sind nicht mehr gezwungen, sich im Rahmen der Abschätzung sehr unsicherer Werte, wie der Angriffshäufigkeit, auf eine einzige Zahl festzulegen.

\section{Literatur}

Cavusoglu H, Mishra B, Ragunathan S (2004) A model for evaluating IT security investments. Communications of the ACM 47 (7): 87-92.

Conrad J (2005) Analyzing the risks of information security investments with Monte-Carlo Simulations. http://infosecon.net/workshop/pdf/13.pdf. Abruf am 2009-04-15.

Faisst U, Prokein O, Wegmann N (2007) Ein Modell zur dynamischen Investitionsrechnung von IT-Sicherheitsmaßnahmen. ZfB 77 (5): 511-538.

Gordon L, Loeb M (2002) The economics of information security investment. ACM Transactions on Information and System Security 5 (4): 438-457.

Kronschnabl S (2008) IT-Security Governance. Bankinnovationen Band 23. Universitätsverlag Regensburg.

Schechter S (2004) Computer security strength \& risk - a quantitative approach. Dissertatiton Cambridge. Massachusetts.

Sonnenreich W, Albanese J, Stout B (2006) Return on security investment (ROSI) - A practical quantitative model. JRPIT 38 (1): 45-55.

Soo Hoo K (2000) How Much Is Enough? A Risk-Management Approach to Computer Security. http://iis-db.stanford.edu/pubs/11900/soohoo.pdf, 2000. Abruf am 2009-01-06. 
IT in der Energiewirtschaft 



\title{
Vorwort zur Teilkonferenz
}

\section{IT in der Energiewirtschaft}

\author{
Hans-Jürgen Appelrath ${ }^{1}$, Carsten Felden ${ }^{2}$ \\ ${ }^{1}$ OFFIS, Universität Oldenburg \\ ${ }^{2}$ Professur für ABWL, insb. für Informationsmanagment/Wirtschaftsinformatik, \\ TU Bergakademie Freiberg
}

In der Energiewirtschaft vollzieht sich ein tiefgreifender Strukturwandel. Der liberalisierte Handel mit Strom, Gas, Wärme und CO2-Emissionszertifikaten, die zunehmende Nutzung der regenerativen Energiequellen Sonne, Wind und Biomasse sowie eine weitergehende Kommunikation mit Verbrauchern und dezentralen Erzeugern führen zu grundlegend neuen Anforderungen an Produktions-, Handels- und Vertriebsprozesse in der Energiewirtschaft.

Ziel des Tracks ist die Diskussion des differenzierten IT-Einsatzes in der Energiewirtschaft, um den geschilderten Herausforderungen mit Lösungen zu begegnen. Energiewirtschaftliche sowie gesetzliche Rahmenbedingungen müssen hierbei Berücksichtigung finden. Modellierung und Simulation sind wichtige Entwurfshilfsmittel, die aber durch eine praxisnahe Validierung von prototypischen Anwendungen ergänzt werden müssen. Übergeordnetes Ziel ist es, den Wertschöpfungsbeitrag der IT in der Energiewirtschaft systematisch zu untersuchen und gezielt zu verbessern, um technisch und wirtschaftlich gangbare Wege zu einer umweltgerechten und nachhaltigen Energieversorgung aufzuzeigen. Eine derartige Betrachtung des IT-Einsatzes erfordert einen in hohem Maße multidisziplinären Ansatz mit offenen Forschungsfragen an die Wirtschaftsinformatik.

Für eine kontinuierliche Diskussion innerhalb der am Themenbereich „IT in der Energiewirtschaft“ interessierten Community wurde im Sommer 2007 der Arbeitskreis „Energieinformationssysteme“ der Gesellschaft für Informatik (GI) gegründet, siehe www.energieinformationssysteme.de.

Dieser AK ist auch verantwortlich für die Durchführung dieser Teilkonferenz auf der MKWI 2010 in Göttingen (der dritten Veranstaltung des AK nach denen auf der MKWI 2008 und WI 2009) und die Herausgabe der vorliegenden Dokumentation der angenommenen Beiträge. Unser Dank gilt den Mitgliedern des unten aufgeführten Programmkomitees und Herrn Dipl.-Wirtsch.-Inform. José Manuel González für seine ergänzende organisatorische Unterstützung. 


\section{Programmkomitee:}

Prof. Dr. Dr. h. c. H.-Jürgen Appelrath, Universität Oldenburg (Vorsitz)

Prof. Dr. Carsten Felden, TU Bergakademie Freiberg (Co-Vorsitz)

Prof. Dr.-Ing. Jan-Heinrich Florin, RWE Supply \& Trading GmbH

Prof. Dr.-Ing. Michael Kurrat, TU Braunschweig

Heiner Lake, SAS Institute GmbH, Heidelberg

Dr. Till Luhmann, BTC AG

Dr. Orestis Terzidis, SAP Research

Prof. Dr. Christian Rehtanz, Universität Dortmund

Prof. Dr. Christof Weinhardt, Universität Karlsruhe (TH)

José M. González, OFFIS Oldenburg 


\title{
Economic Robustness of Scheduling Algorithms for Distributed Storage Systems
}

\author{
Klaus-Henning Ablert, Clemens van Dinther \\ Institut für Informationswirtschaft und -management (IISM), \\ Karlsruber Institut für Technologie (KIT)
}

The article analyzed the economic robustness of scheduling algorithms for distributed storage systems (DSS) under the condition of forecast uncertainty. Nine scenarios defining different levels of forecast errors for price and load forecasts are analyzed using Monte Carlo simulations (see Figure 1). The assumed forecast error scenarios base on a literature review in this area (further details in the long version of this article). As an alternative to existing scheduling algorithms building on a linear optimization model, this article presents a heuristic scheduling algorithm, which is benchmarked against the optimal result and compared with the performance of a scheduling algorithm using a linear optimization model. The optimal result is obtained through running a linear optimization model with actual data only instead of forecast data.

\begin{tabular}{|c|c|c|c|c|c|}
\hline \multirow{3}{*}{$\begin{array}{r}\text { Load } \\
\text { Forecast } \\
\text { Error } \\
\text { [MAPE] }\end{array}$} & 22.5 & $\begin{array}{c}\text { Scenario } 7 \\
\text { P: } 5-8 \%, 0.5 \\
\text { L: } 22.5-35 \%, 0.75\end{array}$ & $\begin{array}{c}\text { Scenario } 8 \\
\text { P: } 10-16 \%, 0.5 \\
\text { L: } 22.5-35 \%, 0.75\end{array}$ & $\begin{array}{c}\text { Scenario 9 } \\
\text { P: } 15-24 \%, 0.5 \\
\text { L: } 22.5-35 \%, 0.75\end{array}$ & \multirow{2}{*}{ 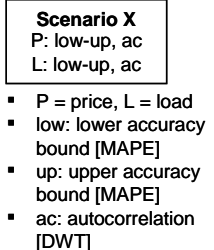 } \\
\hline & 15.0 & $\begin{array}{c}\text { Scenario } 4 \\
\text { P: } 5-8 \%, 0.5 \\
\text { L: } 15-24 \%, 0.75\end{array}$ & $\begin{array}{c}\text { Scenario } 5 \\
\text { P: } 10-16 \%, 0.5 \\
\text { L: } 15-24 \%, 0.75\end{array}$ & $\begin{array}{c}\text { Scenario } 6 \\
\text { P: } 15-24 \%, 0.5 \\
\text { L: } 15-24 \%, 0.75\end{array}$ & \\
\hline & \multirow[t]{2}{*}{7.5} & $\begin{array}{c}\text { Scenario } 1 \\
\text { P: } 5-8 \%, 0.5 \\
\text { L: } 7.5-12 \%, 0.75\end{array}$ & $\begin{array}{c}\text { Scenario } 2 \\
\text { P: } 10-16 \%, 0.5 \\
\text { L: } 7.5-12 \%, 0.75\end{array}$ & $\begin{array}{c}\text { Scenario 3 } \\
\text { P: } 15-24 \%, 0.5 \\
\text { L: } 7.5-12 \%, 0.75\end{array}$ & \\
\hline & & 5.0 & 10.0 & 15.0 & \\
\hline
\end{tabular}

Figure 1: Simulation Scenarios

Firstly, the simulations reveal that the choice of the schedule determination algorithm depends on the degree of autocorrelation of forecast errors within the available price forecast data. If the price forecast errors are autocorrelated, a linear optimization algorithm will deliver best results. Otherwise the heuristic algorithm delivers superior results (see Figure 2). 


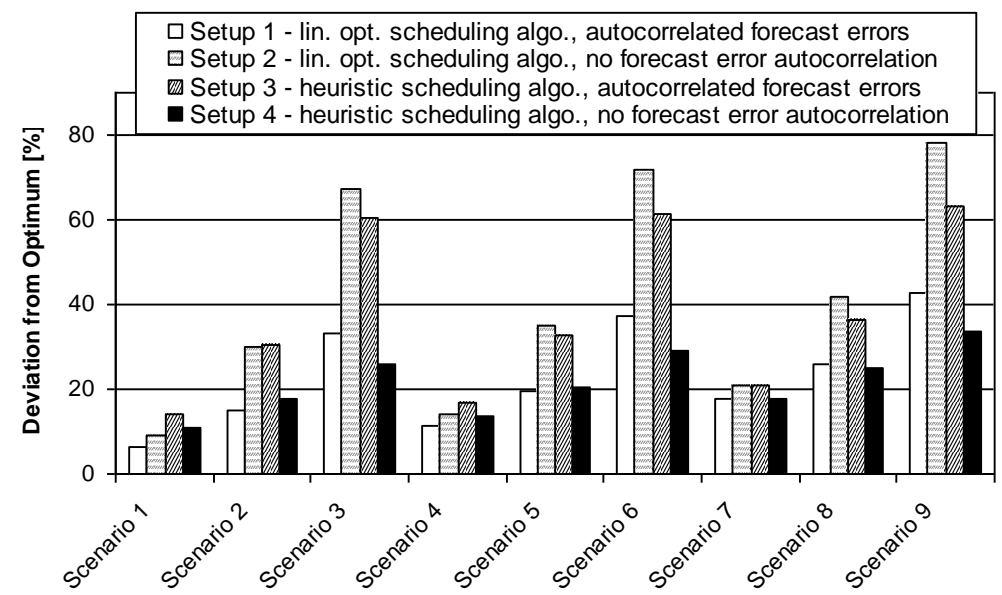

Figure 2: Results of scheduling algorithms in combination with forecast error auto correlation variations

Secondly, the results reveal that both algorithms deliver fairly robust results when varying the level of forecast accuracy. Variations of the load forecast accuracy only have a minor impact on the realized savings, whereas variations of the price forecast accuracy influence the results significantly. Still, even for very conservative scenarios assuming price forecast with a MAPE of $15 \%$ for day-ahead hourly forecasts in combination with $22.5 \%$ MAPE of load forecasts, both algorithms deviate less than $45 \%$ from the optimal result. Available forecast methods in practice achieve around 5\% (price) respectively 7.5\% (load) MAPE. Thus, the DSS scheduling algorithms show a high robustness against accuracy variations of the schedule.

Since this paper has shown the practical feasibility to economically operate DSS under forecast uncertainty, further research will have to address the question, how many DSS installations can operate profitably in a given grid. The analysis in this paper assumed that a DSS is a price taker. If a large number DSS would operate on the same or similar schedules, this would certainly have an effect on the market price in peak and off-peak hours (reduction of spreads). I.e., there must be a grid-specific limit to the number of DSS than can be run economically. 


\title{
Business-Intelligence-gestützte Vorauskalkulation in der Primärgewinnungsindustrie
}

\author{
Johannes Buder, Carsten Felden, Joel Sieber \\ Professur für ABWL, insb. für Informationsmanagement/Wirtschaftsinformatik, \\ Technische Universität Bergakademie Freiberg
}

Die europäische Braunkohleindustrie ist seit Anfang der achtziger Jahre mit rückläufigen oder stagnierenden Absatzvolumina konfrontiert. Die damit einhergehende kontinuierliche Margenerosion zwingt die Industrie zu permanenten Kostenreduzierungen. Die Braunkohlenbergbaue sind von dieser Entwicklung besonders betroffen (Konstantin 2007, S. 52). Bei stetig steigendem Wettbewerbsdruck und sinkenden Deckungsbeiträgen aus der Weiterverarbeitung ist die Tagebauplanung ein wesentlicher Wettbewerbsvorteil, um dem hohen Fixkostenblock begegnen zu können und Kostensenkungspotenziale im Gesamtprozess zu schaffen. Die Braunkohle ist zwar unabhängig von der Preisbildung am Rohstoffmarkt, da Abbau und Verstromung unmittelbar zusammenhängen, jedoch zwingen gesetzliche Normen (z. B. das EnWG), die Bevorzugung erneuerbarer Energien und der Handel mit $\mathrm{CO}_{2}$ Zertifikaten zu einer konsequenten Kostenplanung und -kontrolle. Daher rückt die Vorauskalkulation der Investitionen in den Vordergrund der Betrachtung, die neben betriebswirtschaftlichen Kennzahlen auch geologische und technische Artefakte berücksichtigt. Die Vorauskalkulation erfordert eine transparente Ermittlung der Plandaten sowie eine schnelle technische Bearbeitung. Die Verwendung impliziten Wissens erhöht zudem die Varianz der Planungsergebnisse aufgrund der Instabilität der Planungsparameter, so dass die Vergleichbarkeit der unterschiedlichen Planungsergebnisse erschwert wird.

Daher erwächst aus diesen Anforderungen bei den Braunkohlegesellschaften der Handlungsbedarf, den Planungsprozess auf Basis verlässlicher und konsistenter Daten durchzuführen. Die bisher in der Praxis übliche Verwendung von Tabellenkalkulationsprogrammen wird den genannten Anforderungen nicht gerecht, da sie in Bezug auf eine effiziente und effektive Bewertung eine Reihe von Nachteilen aufweisen. So erfolgt die Datenkonsolidierung der Quellsysteme manuell, so dass die Validität der Daten beeinträchtigt wird. Zudem werden für die Kennzahlen sowohl betriebswirtschaftliche als auch technische und geologische Daten konsolidiert. Ferner kann die Verwendung von Tabellenkalkulationsprogrammen zu einem unübersichtlichen Dateisystemansatz führen, der den Anforderungen an Validität und Konsistenz nicht entspricht. Diese Gründe dienten bereits in den 1990er Jahren als Basis der Business Intelligence (BI)-Diskussion in Unternehmen (Totok und Jaworski 1998, S. 33). Jedoch wurde bis dato in der Literatur nicht untersucht, 
ob Konzepte der BI, die in anderen Branchen in diesem Kontext diskutiert werden, in der Vorauskalkulation im Tagebau einen adäquaten Lösungsansatz bieten.

Es ist daher das Ziel des Beitrages ein multidimensionales Datenmodell zu entwerfen, welches den Vorausplanungsprozess im Tagebau effektiv und effizient unterstützt und damit einen BI gestützten Lösungsansatz zur Prozesstransparenz und Kostenreduktion begründet. BI bietet in diesem Kontext einen Mehrwert, da das bisher implizite Wissen in Form von Schätzparametern systematisch erfasst wird, um so den Anforderungen der Kostenkontrolle im Vorauskalkulationsprozess zu entsprechen. Bieten bestehende Ansätze eine ausschließlich mengenorientierte Betrachtung, wird in diesem Beitrag aufgezeigt, dass eine Verwendung multidimensionaler Datenstrukturen ein Kennzahlensystem ermöglicht, der die von Slaby und Wilke (2006) entwickelte wertgrößenorientierte Prozesssicht zulässt, um eine effektive Kostenkontrolle zu ermöglichen. Somit werden finanzmathematischen Kennzahlen im Datenmodell abzubilden.

Die Abbildung der Vorauskalkulation erfolgt in multidimensionalen Strukturen, um die unterschiedlichen Betrachtungsweisen der physisch gespeicherten Daten zu repräsentieren. Die multidimensionale Datenstruktur trennt zwischen den Dimensionstabellen, welche die beschreibenden Elemente der Faktentabellen enthält. Die Faktentabelle ist Basis der zuvor formulierten Kennzahlen zur Abbildung der Vorauskalkulation. Der gewählte Ansatz unterstützt umfangreiche Online Analytical Processing (OLAP)-Anforderungen, wie die mathematisch/statistische Berechnung ohne manuelle Programmierung als auch die Mehrbenutzerfähigkeit. Daher stellt das BI-Konzept mit seinem multidimensionalen Datenmodell in einem Data Warehouse eine Verbesserung dar, um den Prozess der Vorauskalkulation effizienter zu erfüllen und leistungsfähiger zu gestalten sowie die Nachvollziehbarkeit zu erhöhen

\section{Literatur}

Konstantin P (2007) Praxishandbuch Energiewirtschaft, Springer, Berlin.

Slaby D, Wilke FL (2006) Bergwirtschaftslehre Teil II- Wirtschaftslehre der Bergbauunternehmen und Bergbaubetriebe. Verlag der TU Bergakademie

Totok A, Jaworski R (1998) Modellierung von multidimensionalen

Datenstrukturen mit ADAPT- Ein Fallbeispiel. http://home.arcor.de/andreas. totok/pdf/Arbeitsbericht_ADAPT.pdf. 


\title{
IT-gestützte Marktforschung
}

\section{Möglichkeiten einer internationalen webbasierten Markt- beobachtung und -analyse des Marktes für energie- effizientes Bauen}

\author{
Iris Kaiser \\ Lehrstubl für Wirtschaftsinformatik II, \\ Friedrich-Alexander-Universität Erlangen-Nürnberg
}

Durch die Liberalisierung des Energiemarktes, anhaltend hohe Rohstoffpreise und wachsende politische Einflussnahme steigt die Dynamik von Marktentwicklungen im Bereich der erneuerbaren Energien sowie energieeffizienter Systeme in der Baubranche zunehmend. In Anbetracht der aktuellen Finanzkrise können sich erneuerbare Energien als Konjunkturtriebkraft beweisen. Der Ausbau von Windkraft, Biomasse, Solarenergie, Erdwärme und Wasserkraft ist außerdem eine wichtige Maßnahme zum Klimaschutz. Dies wird durch den Einsatz von neuen Materialen, Technologien und Konzepten im Rahmen des energieeffizienten Bauens unterstützt.

Aufgrund der hohen Dynamik, ist es für ein Unternehmen das erfolgreich in diesem Markt agieren will, von enormer Relevanz kontinuierlich und tagesaktuell Kenntnis von neuen Entwicklungen zu haben und dieses Wissen gezielt einzusetzen. Bisher ist dies schwer, da nur teilweise bekannt ist, welche Aspekte großen Einfluss auf die Marktentwicklungen haben und deren internationale Beobachtung manuell nahezu unmöglich ist. Wie wichtig allerdings detailliertes Wissen ist, zeigt sich insbesondere darin, dass es sich hier um einen sehr vielschichtigen Wachstumsmarkt mit unterschiedlichen Akteuren und ausgeprägten Unterschieden in Kenntnisstand sowie Motiven (Umwelt vs. Wirtschaftlichkeit) der Kunden handelt.

Gleichzeitig ist im Internet eine sehr große Menge an Daten zu diesem Markt frei zugänglich. Werden diese Daten identifiziert, bezogen und strukturiert, können sie die Basis für ein breites und tiefes Marktwissen in einem Unternehmen bilden.

Um diesen spezifischen Anforderungen erfolgreich zu begegnen, ist dabei der Einsatz von IT unumgänglich. Eine Einsatzmöglichkeit bildet die Bereitstellung eines Informationsportals mit Marktinformationen für das Management eines Unternehmens. So kann mit Hilfe der qualitativen und quantitativen Daten eine Wissensbasis über den Markt für energieeffizientes Bauen aufgebaut werden, die frühzeitig Entwicklungsprognosen und Handlungsempfehlungen bietet. Durch diese systematische Analyse des Marktes, des Wettbewerbs und der unterschiedlichen Technologien lassen sich dabei strategische Wettbewerbsvorteile erzielen. 
Die wichtigsten Einflussfaktoren auf den Markt für energieeffizientes Bauen wurden im Rahmen einer internationalen Studie erhoben. Als Ergebnis lässt sich folgendes Modell über die Relevanz des Themas „energieeffizientes Bauen“ in der Bevölkerung, aufstellen (siehe Abbildung 1).

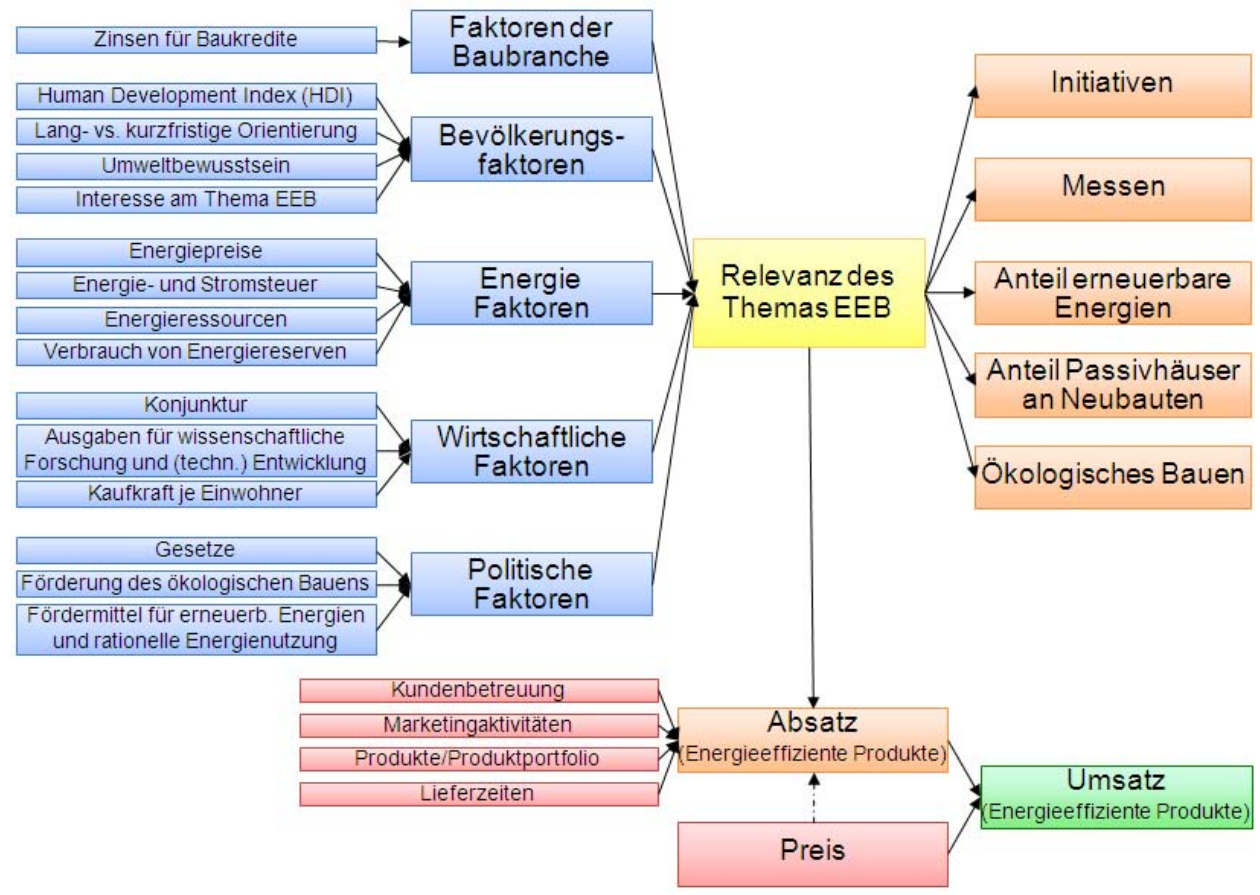

\section{Abbildung 1: Modell zur Bewertung der Marktattraktivität}

Die praktische Umsetzung des theoretischen Modells in einem Managementinformationssystem zur kontinuierlichen Beobachtung der Markt-, Wettbewerbs- und Technologieentwicklung birgt dabei verschiedene Herausforderungen bezüglich der Datenqualität und der Informationsaufbereitung. Da die Präzision und Korrektheit der Ergebnisdarstellung einen immensen Einfluss auf die Nutzerakzeptanz des Systems beim Anwender hat, muss diesen entsprechend entgegen gekommen werden. 
24. PuK-Workshop:

Planung/Scheduling und Konfigurieren/Entwerfen 



\title{
Vorwort zur Teilkonferenz
}

\section{PuK-Workshop: Planung/Scheduling und Konfigurieren/Entwerfen}

\author{
Jürgen Sauer', Stefan Edelkamp², Bernd Schattenberg 3 \\ ${ }^{1}$ Department für Informatik, Universität Oldenburg \\ ${ }^{2}$ Technologie-Zentrum Informatik, Universität Bremen \\ ${ }^{3}$ Institut für Künstliche Intelligenz, Universität Ulm
}

Der PuK-Workshop ist zum einen das jährliche Treffen der Fachgruppe Planen, Scheduling und Konfigurieren, Entwerfen im Fachbereich Künstliche Intelligenz (KI) der GI. Weit wichtiger ist aber seine Funktion als Vermittlungspunkt von unterschiedlichen Anwendungsbereichen und Lösungsansätzen aus den Bereichen Planen, Scheduling und Konfigurieren. Dabei wird meist der Schwerpunkt auf die von der KI geprägten Lösungsverfahren gelegt.

Mit der Anbindung an die MKWI bietet sich die Gelegenheit, diese Themen einem größeren Interessentenkreis zu präsentieren, da vor allem Fragen der Planung und des Scheduling von betriebswirtschaftlichen Prozessen aber auch die Konfiguration von Produkten für die Wirtschaftsinformatik besonders interessant sind.

Somit sollen beim 24. PuK Praktiker und Forscher aus den Bereichen KI und Wirtschaftsinformatik angesprochen werden, die sich mit Problemstellungen der Planung, des Scheduling und der Konfiguration sowie den dazu nötigen Systemund Lösungsansätzen beschäftigen.

Das Themenspektrum der Beiträge umfasst dementsprechend mindestens:

- Praktische Anwendungen in den Bereichen Planung, Scheduling, Konfiguration, z. B. Planung in der Supply Chain, Produktkonfiguration, Planung/Scheduling von Produktion oder Transport

- Architekturen für Planungs- und Konfigurationssysteme

- Wissensrepräsentation und Problemlösungstechniken für diese Problemstellungen, u. a. Heuristiken, Meta-Heuristiken, Verteiltes Problemlösen, Constraint basierte Techniken, Soft Computing Verfahren, reaktive Verfahren und Benutzerintegration

- Lernverfahren für Planen und Konfigurieren

Zusätzlich wird wie in früheren Workshops ein Themenschwerpunkt gesetzt, in diesem Jahr sind es Praktische Anwendungen, d. h. speziell Praktiker sind aufgefordert, die in der praktischen Nutzung befindlichen Systeme zu präsentieren, um 
zum einen den Einfluss der Forschung in der Praxis zeigen zu können und zum anderen Anregungen für Forschungsaktivitäten diskutieren zu können.

Die eingereichten und akzeptierten Papiere decken wie in der Vergangenheit auch alle Themenbereiche der Fachgruppe ab, wobei z. B. am Einsatz von Schwarmbasierten Verfahren in unterschiedlichen Anwendungsbereichen die Intention der Fachgruppe deutlich bestätigt wird. So werden Schwarmbasierte Ansätze in den Beiträgen von Edelkamp et al. (Finding the Needle in the Haystack with Swarm Search), Laroque (Parameteroptimierung von Materialflusssimulationen durch Partikelschwarmalgorithmen), Heeren (Swarm Intelligence in der dynamischen Tourenplanung) und Brodersen et al. (Optimizing the Berth Allocation Problem using a Genetic Algorithm and Particle Swarm Optimization) für unterschiedliche Planungsproblemstellungen verwendet. Bogon et al. (An Agent-based Approach for Dynamic Combination and Adaptation of Metaheuristics) stellen einen Ansatz zur einfachen Kombination von Lösungsverfahren dar, Bidot et al. (Verbal Plan Explanations for Hybrid Planning) stellen aktuelle Ergebnisse zur Erläuterung von Plänen vor, während Günther (Workforce Management an einem Praxisbeispiel aus der Produktion. Einführung einer Softwarelösung bei der Allgaier Werke $\mathrm{GmbH}$ ) den praktischen Einsatz der Personaleinsatzplanung zeigt. Schließlich wird der Konfigurationsbereich durch die Beiträge von Hotz (Construction of Configuration Models) und Mandl et al. (Personalized Configuration of Mobile Phones) abgedeckt.

Abschließend danken wir allen GutachterInnen und freuen uns auf eine interessante Konferenz.

Jürgen Sauer, Stefan Edelkamp, Bernd Schattenberg 


\title{
Construction of Configuration Models
}

\author{
Lothar Hotr. \\ HITeC e.V., Universität Hamburg
}

Knowledge-based configuration has its origin in the task of configuring physical components like drive systems (Ranze et al., 2002) or elevators (Marcus et al., 1988). For example in (Günter, 1995) configuration is defined as "the composition of technical systems from parameterisable objects to a configuration, that fulfills a certain task" or Stefik defines in (Stefik, 1995) configuration tasks as tasks that "select and arrange instance of parts from a set". The focus is set on the composition of parts to aggregates and thus, on the compositional relation has-parts.

Naturally, in all approaches descriptions of objects are composed, not the physical objects themselves. By doing so, configuration can be understood as model construction (Hotz, 2009). From the configuration point of view, model construction deals with the composition of arbitrary artifacts on the basis of a logical theory. Hereby, a strict separation of the logical theory, i.e. the knowledge base or configuration model, and the logical model, i.e. the configuration or better construction, is issued. Starting from a knowledge base a configuration system composes a construction that is consistent with the knowledge base, i.e. a logical model of the knowledge base is created.

Following this understanding of the configuration task the above mentioned has-parts relation is more a has relation, which is applied to various domains e.g. services (Tiihonen et al., 2006), where e.g. a client has a certain insurance demand, or to software (Hotz et al., 2006), where a software component has a certain feature, to scene interpretation (Hotz, 2006), where a certain scene description has observed or hypothized objects or actions. The has relation determines what part descriptions are to be integrated in a resulting construction (i.e. a system description). By taking up such a perspective sometimes considered conceptual mismatches (Tiihonen et al., 2006), which may come up, when using configuration systems in non-physical domains, are avoided.

Taking a further step, one may look at configuration models as a type of software that is constructed during a knowledge-acquisition process. Thus, the questions arise: "Can the construction of configuration models be supported by configuration tools?" or "What are the parts that are composed in such an approach?" or "How does a configuration model that enables the configuration of configuration models (i.e. a meta-configuration model) look like?".

An application of such a meta-configuration model is naturally to support the knowledge-acquisition process needed for knowledge-based configuration systems. In a first phase of a knowledge-acquisition process, the typically tacit knowledge 
about a domain is extracted by applying knowledge-elicitation methods and high interaction between a knowledge engineer and the domain expert (knowledgeelicitation phase). A model sketch is the result, which in turn is formalized during the domain-representation phase. During this phase a configuration model is created. The configuration model has to be expressed with the facilities of a configuration language. The meta-configuration model can be used to check such configuration models for being consistent with the configuration language. Thus, by using the meta-configuration model as a knowledge base of a configuration system, the domain-representation phase can be supported similarly to a configuration process.

In the full paper, we elaborate answers to the mentioned questions by first presenting a construction language, i.e. the Component Description Language CDL, which enables the description of domain objects. We than investigate in a concept for a configurator that enables the configuration of arbitrary configuration models, i.e. a meta configurator and its meta-configuration model.

\section{References}

Günter A (1995) Wissensbasiertes Konfigurieren. Infix, St. Augustin.

Hotz L, Wolter K, Krebs T, Deelstra S, Sinnema M, Nijhuis J, MacGregor. J (2006) Configuration in Industrial Product Families - The ConIPF Methodology. IOS Press, Berlin.

Hotz L (2006). Configuring from Observed Parts. In C. Sinz and A. Haag, editors, Configuration Workshop, 2006, Workshop Proceedings ECAI, Riva del Garda.

Hotz. L (2009) Frame-based Knowledge Representation for Configuration, Analysis, and Diagnoses of technical Systems (in German), volume 325 of DISKI. Infix.

Marcus S, Stout J, McDermott J. (1988) VT: An Expert Elevator Designer that uses Knowledgebased Backtracking. AI Magazine, pages 95-112.

Ranze C, Scholz T, Wagner T, Günter A, Herzog O, Hollmann O, Schlieder C, Arlt V. (2002) A Structure-based Configuration Tool: Drive Solution Designer - DSD. In Eighteenth national conference on Artificial intelligence, pages 845852, Menlo Park, CA, USA. American Association for Artificial Intelligence.

Stefik. M (1995) Introduction to Knowledge Systems. Morgan Kaufmann, San Francisco, CA.

Tiihonen J, Heiskala M, Paloheimo KS, Anderson A (2006) Configuration of Contract Based Services. In C. Sinz and A. Haag, editors, Configuration Workshop, 2006, Workshop Proceedings ECAI, Riva del Garda 


\title{
Personalized Product Configuration
}

\author{
Alexander Felfernig', Monika Mandl', Juha Tiibonen², Monika Schubert ${ }^{1}$ \\ IInstitute of Applied Informatics, \\ Graz University of Technology \\ 2Software Business and Engineering Institute, \\ Helsinki University of Technology
}

Configuration technologies are well established as a foundation of mass customization which is a production paradigm that supports the manufacturing of highlyvariant products under pricing conditions similar to mass production. A side-effect of the high diversity of products offered by a configurator is that customers (users) in many cases have the problem of not understanding the set of offered options in detail and are often overwhelmed by the complexity of those options. In order to improve the quality of configuration processes, we combine knowledge-based configuration with collaborative and content-based recommendation algorithms. In this paper we present an environment that supports personalized configuration of mobile phones and corresponding subscriptions (RecoMobile). The RecoMobile user interface provides personalized suggestions for selecting the phone and subscription features. In order to demonstrate the applicability of our approach, we conducted an empirical study to compare configurator versions with recommendation support with configurator versions without recommendation support for feature settings. The results of our evaluation showed important improvements in terms of user satisfaction, quality of the configuration process, and trust in a product decision. 



\title{
Parameteroptimierung von Materialflusssimulationen durch Partikelschwarmalgorithmen
}

\author{
Christoph Laroque', Barthold Urban', Markus Eberling² \\ ${ }^{1}$ Heinz Nixdorf Institut, \\ 2Institut für Informatik, \\ (beide) Universität Paderborn
}

Die Wirtschaftsinformatik bietet insb. im Bereich Operations Reasearch unterschiedliche Methoden, um ein weites Spektrum an Problemstellungen zu lösen. Typische Probleme sind Traveling-Salesman, Scheduling, Standortplanung, etc. Sie werden heute über Modellen abgebildet und mit Algorithmen, u.a. der Simulation und Optimierung, unter Berücksichtigung von Restriktionen auf Optimalität hin gelöst.

Eine etablierte Methode zur Planung, Absicherung und Verbesserung von Produktionsprozessen ist die Materialflusssimulation als Anwendungsgebiet der ereignisdiskreten Simulation (Law und Kelton 2000). Typische Probleme, die in dieser Domäne untersucht werden, sind Absicherungen von Produktionsprogrammen, Losgrößenplanung, Layoutplanung, Dimensionierung von Puffern, Personaleinsatzplanung, etc. Insbesondere eignet sich Simulation, wenn es schwierig, sehr kostenaufwendig oder schlicht unmöglich wäre, das Realsystem direkt zu verändern. Lange Zeitabschnitte lassen sich durch Simulation in verkürzter Zeit betrachten, erlauben Sensitivitätsanalysen des Modells und eine grafische Animation zur Absicherung von Steuerungsregeln.

Ziel der hier vorgestellten Arbeit war eine Machbarkeitsstudie für die Kombination der Simulation \& Optimierung, die unter Verwendung der Gruppe der Partikelschwarmalgorithmen (Kennedy und Eberhart 1995) möglichst schnell eine gute Modellkonfiguration eines Simulationsmodells ermöglicht. Dies ist vor allem im Hinblick auf Situationen interessant, in denen die konventionelle, mathematische Modellierung und Optimierung nicht anwendbar sind. Konkret wurde die wurde die Machbarkeitsstudie am Beispiel der Simulationssoftware d3FACT insight und der Partikelschwarmoptimierung (PSO) durchgeführt.

Es wurde ein Werkzeug implementiert in dem Simulationsmodelle eingebunden werden können, und über eine Schnittstelle zu d $\mathrm{d}^{3} \mathrm{FACT}$ insight optimiert werden können - gesteuert von der PSO. Da die Anzahl der Iterationen des Algorithmus entscheidend für den gesamten Rechenaufwand in Verbindung mit der Simulation ist, wurden einige Modifikationen vorgenommen, die sich bei Vorun- 
tersuchungen als geeignet erwiesen. Des Weiteren wurde die ursprüngliche PSO in ein 3-Phasen-Verfahren unterteilt.

Das vorgestellte, kombinierte Verfahren aus Simulation und Metaheuristik unterscheidet sich von herkömmlichen Optimierungsansätzen in dem Punkt, dass die Evaluierung der Fitness eines Partikels nicht durch eine mathematische Funktion berechnet wird, sondern durch einen Simulationslauf in $\mathrm{d}^{3} \mathrm{FACT}$ insight ermittelt werden muss. Als Kernproblematik bleibt die Tatsache bestehen, dass die Simulationsläufe die meiste Zeit und Rechenkapazität in Anspruch nehmen. Es gilt daher, deren Anzahl bestmöglich zu minimieren.

Neben der Machbarkeit war als zweiter Untersuchungsgegenstand zu bestimmen, wie sich die vorgeschlagenen Modifikationen der PSO auf Laufzeit und Lösungsqualität sowohl bei einer mathematischen Testfunktion als auch bei der Simulation durch $\mathrm{d}^{3} \mathrm{FACT}$ insight auswirken. Fokus war die Konfiguration des Algorithmus in der Form, dass eine möglichst geringe Iterationszahl für eine möglichst gute Lösung notwendig ist. Es wurde eine kurze Parameterstudie durchgeführt, um die Parameter des Algorithmus zu untersuchen und anzupassen.

Durch die implementierte Kombination aus PSO und $\mathrm{d}^{3} \mathrm{FACT}$ insight konnte erfolgreich gezeigt werden, dass sich Simulationsmodelle durch PSO so parametrieren lassen, dass eine optimale Parametrierung hinsichtlich einer Zielfunktion automatisch erreicht wird. Das Testmodell weicht in seiner Komplexität noch weit von den in der Realität zu betrachtenden Modellen ab. Mit dem geschaffenen Prototyp ist aber die prinzipielle Machbarkeit gezeigt. Es bleibt in kommenden Arbeiten zu prüfen, ob sich auch komplexere Modelle in vertretbarer Zeit parametrieren lassen.

Erste Erkenntnisse über das Verhalten und die Laufzeit der 3-Phasen-PSO wurden gesammelt und zeigen, dass dieser Entwurf weiter hinsichtlich der Laufzeit verbessert werden kann. Potenziale liegen in einer intensiveren Nutzung der Selektion, bzw. in einer besseren Anpassung der Schwarmparameter in den einzelnen Phasen. Die Qualität der Lösungen konnte bereits durch die hier beschriebenen Modifikationen signifikant verbessert werden.

\section{Literatur}

Law AM, Kelton WD (2000) Simulation Modeling and Analysis. McGraw-Hill.

Kennedy J, Eberhart RC (1995) Swarm Intelligence. MK-Publishers. 


\title{
Optimizing the Berth Allocation Problem using a Genetic Algorithm and Particle Swarm Optimization
}

\author{
Ole Björn Brodersen', Leif Meier², Matthias Schumann ${ }^{1}$ \\ ${ }^{1}$ Professur für Anwendnungssysteme und E-Business, \\ Georg-August-Universität Göttingen \\ 2 Procter \& Gamble Service GmbH, \\ Schwalbach am Taunus Frankfurt
}

Today's increasing number of container shipments requires highly efficient logistics processes at container terminals (CT). Forecasts published by the Federal Ministry of Transport, Building and Urban Affairs estimate a four times higher transshipment of standard containers in German ports in 2025 than today (Tiefensee 2007). CT therefore represent an important interface in the global transport chain and have to fulfill two man functions: The transshipment of containers from mode to mode as well as the temporary storage of containers (Saanen 2004). In order to improve CT performance, process optimization becomes more and more important, as terminal extensions and technical modifications are highly cost intensive and underlie strategic long term restrictions.

The increasing importance of the container flow especially demands for an effective berth management in CT. The Berth Allocation Problem (BAP) defines, where and when vessels will be allocated at the quay. As a result of the decomposition, this decision also has significant influence on subsequent processes and container handling nodes, such as the crane allocation and container storage locations (Meier and Schumann 2007).

This paper focuses on the BAP model described by Guan and Cheung (2004) and presents the solutions found by their proposed Composite Heuristic $(\mathrm{CH})$ applied to realistic data sets based on observations of a German port. As the goal of this paper is to improve the provided results we use two different metaheuristics for comparison purposes. As a well-known and in a variety of optimization problems applied solution method we use a Genetic Algorithm (GA). For continuous optimization problems the Particle Swarm Optimization (PSO) shows promising results. Therefore in this work we present a modification of the PSO based on the PSO proposed in (Brodersen and Schumann 2007) which is applicable to this kind of combinatorial optimization problem.

We find that the modification of the PSO provides best results compared to the $\mathrm{GA}$ and the $\mathrm{CH}$. Furthermore the $\mathrm{CH}$ seems not to be the appropriate choice for 
future investigations because Guan and Cheung propose a limitation of 15 vessels for the TS subprocedure of the $\mathrm{CH}$. This limitation will not meet future requirements especially when looking at the forecasts of (Tiefensee 2007). Not only regarding the presented results of the investigated BAP, PSO (and GA) provide better solutions but will also be applicable to even more complex problems in the future.

\section{References}

Brodersen O, Schumann M (2007) Einsatz der Particle Swarm Optimization zur Optimierung universitärer Stundenpläne. Arbeitsberichte des Instituts für Wirtschaftsinformatik, Professur für Anwendungssysteme und E-Business, Universität Göttingen 5.

Guan Y, Cheung RK (2004) The berth allocation problem: models and solution methods. OR Spectrum 1: 75-92.

Meier L, Schumann R (2007) Coordination of interdependent planning systems, a case study. Lecture Notes in Informatics, Beiträge der 37. Jahrestagung der Gesellschaft für Informatik e.V. (GI): 389-396.

Tiefensee W. (2007) Deutsche Seehäfen weiter auf Wachstumskurs Umschlagsaufkommen wird sich bis 2025 mehr als verdoppeln. Presseerklärung des Bundesministeriums für Verkehr, Bau und Stadtentwicklung (BMVBS) vom 2. Mai 2007 109/2007. 


\title{
Finding the Needle in the Haystack with Heuristically Guided Swarm Tree Search
}

\author{
Stefan Edelkamp1, Peter Kissmann², \\ Damian Sulewski1, Hartmut Messerschmidt ${ }^{1}$ \\ 1Technologie-Zentrum Informatik, Universität Bremen \\ ${ }^{2}$ Fakultät für Informatik, TU Dortmund
}

\begin{abstract}
In this paper we consider the search in large state spaces with high branching factors and an objective function to be maximized. Our method portfolio, we refer to as Heuristically Guided Swarm Tree Search, is randomized, as it consists of several Monte-Carlo runs, and guided, as it relies on fitness selection. We apply different search enhancement such as UCT, lookaheads, multiple runs, symmetry detection and parallel search to increase coverage and solution quality. Theoretically, we show that UCT, which trades exploration for exploitation, can be more successful on several runs than on only one.

We look at two case studies. For the Same Game we devise efficient node evaluation functions and tabu color lists. For Morpion Solitaire the graph to be searched is reduced to a tree. We also adapt the search to the graphics processing unit. Morpion Solitaire the results are close and in the Same Game the results are state-of-the-art.
\end{abstract}

\section{Overview}

Morpion Solitaire is a pen-and-paper longest path state space problem played on an infinite grid with some set of marked intersections taken as the initial state. In each move k-1 intersections are covered and a new one is produced by placing a (horizontal, vertical, or diagonal) line having k-1 edges. (The usual setting is a Greek cross with 36 marked intersections and $k=5$.) Edges of every two lines must not overlap. In the disjoint model two lines on the same alignment must not touch each other, in the touching model they can.

Same Game is an interactive computer puzzle played on an $\mathrm{n} \times \mathrm{m}$ grid covered with $\mathrm{n}^{*} \mathrm{~m}$ balls in $\mathrm{k}$ colors. (Usually, $\mathrm{n}=\mathrm{m}=15$ and $\mathrm{k}=5$ ). Balls can be removed, if they form a connected group of $1>1$ elements. The reward of the move is $(1-2)^{2}$. If a group of balls is removed, the ones on top of these fall down. If a column becomes empty, those to the right move to the left, so that all non-empty columns are aligned. Clearing the complete board yields a bonus reward of 1000 points. The objective is to maximize the total reward. 
Both optimization problems are known to be NP-hard. In order to find optimized solutions to these two games, we sped-up search by using what we call Heuristically Guided Swarm Tree Search integrating search heuristics, duplicate and symmetry detection, tabu and lookahead strategies as well as single root and set-based UCT (short for upper confidence bounds applied to trees).

In random depth-first trials, also known as Monte-Carlo runs, successors are drawn at random. The game is played to the end, and the achieved result is recorded. If it is better than the current high-score, both the score and the stack contents are stored for keeping track of the best solution found so far.

In uninformed swarm tree search, the probability of a successor to be selected is 1 divided by the number of successors, yielding a uniform distribution. Hence, at every sampled state, we determine the number of successors succs and a random number in $\{0, \ldots$, succs -1$\}$. Unfortunately, in state spaces with large branching factors, uniformed random runs often end up with inferior solution paths, so that improving them over time requires large amounts of time and space.

When problem-specific knowledge is encoded into the random sampling process, much better solutions can be obtained. Such knowledge can be thought of as the result of an evaluation (a.k.a. fitness or heuristic) function from states to some positive numbers. In Heuristically Guided Swarm Tree Search we, therefore, apply non-uniform sampling, which is best thought of as a fitness selection according to the relative strength of the successors' evaluation.

In both cases, we combine the computation of successors with their evaluation. In Morpion Solitaire we use a simple function that prefers dense line arrangements and touching lines. As rewards in the Same Game are immediate, we apply an evaluation function that takes into account the current and the remaining score- The algorithm to compute all successors in $\mathrm{O}(\mathrm{nm})$ has been fine-tuned by using a stack instead of a recursive implementation.

We also exploit parallel hardware as available in multi-core CPUs and graphics cards on current personal computer systems. The full paper is structured as follows. First, we introduce to the essence of guided runs and further search enhancements, including UCT. We look at different search heuristics and analyze the possible impact of having several independent runs. Next, we present a simple implementation of Parallel UCT along with some pseudo code. This parallelization is extended to work on sets, which are seeded with exploration results obtained on the GPU. Finally, we review related work, show experimental results, and conclude. 


\title{
Verbal Plan Explanations for Hybrid Planning
}

\author{
Julien Bidot', Susanne Biundo', Tobias Heinroth², \\ Wolfgang Minker², Florian Nothdurft², Bernd Schattenberg ${ }^{1}$ \\ ${ }^{1}$ Institute of Artificial Intelligence, \\ 2Institute of Information Technology, \\ (both) Ulm University
}

State-of-the-art AI planning systems are able to generate complex plans thanks to their efficient reasoning engines. In a large number of application domains, the plans are automatically executed by systems such as autonomous robots. In this context, it is not necessary to make these automated systems understand what they are actually doing during execution and why they are doing that. In other words, these systems do not need to understand the underlying semantics of the plans they execute and how these plans were generated.

However, there are a significant number of key application domains, such as disaster relief mission support or project planning, where plans are supposed to be executed by a human user who is not necessarily a planning expert, an application expert, or both. In addition, for real-world applications, the plans and the plan generation are often complex. In order to unlock a part of the application potential of the AI planning technology, it is essential that the user trusts the technology. Increasing trust in AI planning systems requires the design and implementation of user-friendly interfaces and the development of plan explanation methods that allow for taking into consideration the user's queries related to some plan components about their meaning and relevance for the plan and giving back the appropriate information that answers these queries.

The verbal communication by speech constitutes the most natural form of communication for humans. By means of natural language dialogs in this work, we focus on the explanation of plans that are generated by a refinement-based hybrid planning system called PANDA. Contrary to most approaches presented in the literature that try to provide explanations when backtracking occurs in failure situations during search, we assume in this work that the plans for which explanations are looked for are consistent. We present a domain-independent approach to enabling verbal human queries and producing verbal plan explanations.

PANDA is based on a formal framework that combines hierarchical task network planning and partial-order causal-link planning. This framework uses an ADL-like representation of states and basic actions (primitive tasks). States, preconditions, and effects of tasks are specified through formulae of a fragment of first-order logic. 
Abstract tasks can be refined by so-called decomposition methods. The methods provide task networks (partial plans) that describe how the corresponding task can be solved. Partial plans may contain abstract and primitive tasks, ordering constraints, and causal links. With that, hierarchies of tasks and associated methods can be used to encode the various ways of accomplishing an abstract task. Ordering constraints state an ordering between tasks, while causal links represent logical dependencies between tasks via preconditions and effects. PANDA can address complex planning domains because of its great flexibility and expressivity.

The understanding of individual tasks of a plan is not sufficient to grasp the whole plan, since its temporal and causal structure links the tasks. Our approach consists in providing users with semantic, context-based, and logical information about plan components. Depending on the nature of this information, we distinguish between substantial knowledge and executive knowledge.

The substantial knowledge includes semantic and context-based information that is helpful for human users who are not application experts. To enable the access to this type of information, we extend the documents describing the application domains for planning.

The generation of plan explanations for a human user who is not a planning expert requires an analysis of the plan and the set of successful planning decisions that have led to the plan during search. This logical description of the plan relies on executive knowledge. Note, that context-specific information is also used to make this logical description understandable for the user. Our formal framework for refinement-based planning allows for an explicit representation of the search space explored during the plan generation process thanks to flaws and plan modifications that explain the presence of particular components of the plan, such as tasks, causal links, and ordering constraints. In this work, we assume that a user becomes a set of tasks that are partially ordered and asks for explanations that can justify the ordering of two tasks, or the temporal position of a single task. For both types of human requests, there are the same three types of explanations that can be provided: presence of abstract tasks, unsupported preconditions, and causal threats. The three types of explanations correspond to three flaw classes, respectively. The most important plan components for the analysis are causal links and ordering constraints. The analysis required to find explanations that justify the temporal position of a single task $t e_{i}$ is the most complex, since it has to identify the direct predecessor tasks and the direct successor tasks of $t e_{i}$.

The verbal communication by speech is used for the interaction between a human user and our prototype system. A spoken language dialog system integrates a speech server and a speech interface to provide the dialogue to the user. 


\title{
Workforce Management an einem Praxisbeispiel aus der Produktion
}

\author{
Einführung einer Softwarelösung \\ bei der Allgaier Werke GmbH
}

\author{
Maik Günther
}

ATOSS Software AG, München

Trotz zunehmender Anforderungen an den Personaleinsatz und der steigenden Komplexität des Planungsprozesses werden Mitarbeiter heute zum Großteil mit Tabellenkalkulationen oder auf „Papier“ verplant (ATOSS und FH Heidelberg 2006, S. 47-49). Am Beispiel der Allgaier Werke GmbH wird die Personaleinsatzplanung mit MS EXCEL ${ }^{\text {TM }}$ veranschaulicht. In diesem Zusammenhang werden auch die Schwächen dieser Lösung aufgezeigt. Die Allgaier Werke GmbH mit etwa 1.700 Mitarbeitern entschied sich, das WFM-System der ATOSS Software AG einzuführen, um die Defizite der MS EXCEL ${ }^{\text {TM}}$-basierten Planung abzustellen.

Vor Einführung des WFM-Systems bestand für das Vorgehen bei der Einsatzplanung kein einheitlicher Prozess. Jeder Bereich hatte völlig eigenständig in individuell erstellten MS EXCEL ${ }^{\text {TM}}$-Tabellen geplant. Für eine umfassende Beurteilung von MS EXCEL ${ }^{\text {TM }}$ für die Personaleinsatzplanung sein an dieser Stelle auf den Beitrag von Günther (2009) verwiesen.

Mehr als 30 Einsatzpläne - jeder mit individuellen Abkürzungen, Farben, Zeithorizonten und Anordnungen - machten eine zentrale Ad-hoc-Übersicht sowie Auswertungen über die aktuelle Planung unmöglich. Aufgrund der fehlenden Transparenz konnten Anfragen über Auslastung, Produktivität, erwarteten Zeitsaldenverläufen und Arbeitsbelastung einzelner Mitarbeiter nicht oder nur mit erheblichem Aufwand beantwortet werden. Auch war es wegen der Intransparenz äuBerst schwierig, Mitarbeiter bei einer Überdeckung in andere Abteilungen zu verleihen oder bei einer Unterdeckung aus anderen Abteilungen zu holen. Oft wurden derartige Umbesetzungen oder Änderungen auf Zuruf geklärt und nicht im Plan nachgetragen. Die individuellen Tabellenpläne waren häufig nicht aktuell. Da diese Art der Planung keine Integration in die Zeitwirtschaft ermöglicht, gab es auch keine Online-Information über Abweichungen vom Plan. So war es täglich die Regel, dass Springer erst 30 Minuten nach Arbeitsbeginn zugeteilt werden konnten. Zudem waren in der Planung keine aktuellen und erwarteten Zeitsalden bekannt. Vermeidbare Überschreitungen der Flexikonten waren die Regel.

Vor Einführung der Personaleinsatzplanung wurden klare Ziele definiert. Man will die Transparenz im Unternehmen erhöhen und schneller auf Abweichungen 
reagieren. Maßnahmen sollen besser gesteuert und Prozesse detaillierter analysiert werden können. Zudem soll der Planungsaufwand reduziert und der Einsatz von Springern erheblich beschleunigt werden.

Das eingeführte WFM-System, die ATOSS Startup Edition (ASE), ist modular aufgebaute und vollständig in Java implementiert. Am Markt existiert eine Vielzahl an Anbietern für WFM-Lösungen, die sich in ihrer funktionalen Tiefe stark unterscheiden. Wesentliche Unterschiede zur ASE liegen bei vielen Anbietern neben funktionalen Details in der fehlenden Integration von Personaleinsatzplanung und Zeitwirtschaft, in der nicht vorhandenen Plattformunabhängigkeit sowie in nur rudimentär anpassbaren Oberflächen, Bedien- und Berechtigungskonzepten.

Da bei der Allgaier Werke GmbH bereits das Zeitwirtschaftsmodul der ASE eingesetzt wurde, entschied man sich für das ASE-Modul Personaleinsatzplanung, dass voll in die Zeitwirtschaft integriert ist. Die gesteckten Ziele wurden erreicht und der realisierte Nutzen kann wie folgt zusammen gefasst werden:

- Reduzierung des administrativen Aufwandes um rund 12 Stunden pro Woche in der Personalabteilung.

- Reduzierter Aufwand für die Teamleiter bei der Planerstellung.

- Vereinheitlichung des Planungsprozesses und der genutzten Softwarelösung.

- Deutliche Steigerung der Flexibilität in der Personaleinsatzplanung.

- Schnelle Reaktion auf Bedarfsänderungen und ungeplante Abwesenheiten.

- Prognose und Überwachung von Zeitsalden und Auslastungen.

- Transparenz über den vollen Prozess der Leistungserbringung.

\section{Literatur}

ATOSS Software AG, FH Heidelberg (Hrsg.) (2006) Standort Deutschland 2006. Zukunftssicherung durch intelligentes Personalmanagement.

Günther M (2009) Workforce Management in der Logistik. In: Pradel U-H, Süssenguth W, Piontek J, Schwolgin AF (Hrsg.) Praxishandbuch Logistik. Erfolgreiche Logistik in Industrie, Handel und Dienstleistungsunternehmen. Dt. Wirtschaftsdienst, Köln. 


\title{
Swarm Intelligence in der dynamischen Tourenplanung
}

\author{
Menno Heeren \\ Osterhörn 14, 26736 Krummbörn
}

Mit diesem Beitrag soll gezeigt werden, wie den mit der Dynamik verbundenen Herausforderungen bei der Tourenplanung mit Hilfe von Swarm Intelligence begegnet werden kann. Dazu orientieren wir uns an der Fragestellung des dynamischen Travelling Salesman Problem (DTSP), da es uns die Evaluation des Ansatzes durch zur Verfügung stehende Benchmarks ermöglicht. Die Dynamik in einer TSP-Instanz ergibt sich hierbei aus den sich ändernden Reisezeiten zwischen zwei Städten. Um nun etwaige lange Reisezeiten zu vermeiden, ist eine schnelle Reaktion auf diese Änderung von Bedeutung.

Wir schlagen einen SI-Ansatz vor, der in der Lage ist, diese Dynamik zu handhaben. Dabei wird die Möglichkeit zur positiven Vorhersage von Wegen als Teil einer guten Lösung genutzt, um SI-Systeme in die Lage zu versetzen, schnell alternative Wege zu erkennen. So werden in Abhängigkeit des Ereignisses und der mit ihm verbundenen Stärke der Änderung SI-basierende Entscheidungsprozesse in der Weise modifiziert, dass eine positive Vorhersage von Wegen, welche als Teil einer guten Lösung wahrscheinlich sind, möglich wird.

Dieser SI-Ansatz wird mit drei anderen Ansätzen in einem experimentellen Test für das dynamische TSP verglichen. 



\title{
An Agent-based Approach for Dynamic Combination and Adaptation of Metaheuristics
}

\author{
Tjorben Bogon, Andreas D. Lattner, Yann Lorion, Ingo J. Timm \\ Professur für Wirtschaftsinformatik und Simulation, \\ Goethe Universität Frankfurt am Main
}

Many organizations have to face optimization problems in their day-to-day business. Such problems usually have some optimization functions like minimizing the make span in job-shop scheduling or minimizing the overall sum of all routes in Vehicle Routing Problems (Lenstra and Kan 2006). These problems are known to be NP-complete and thus, in the general case, it is not tractable to perform an uninformed search in the solution space to find the optimal solution due to complexity.

As a trade-off between computational time and solution quality heuristic approaches have been introduced. These approaches do not exhaustively search the solution space but try to find a good solution by applying some domain specific heuristics or metaheuristics. Most of these metaheuristics start with a fixed set of parameters which does not change during runtime. Different metaheuristics are more suitable for different types of problems than others because of their character. Genetic Algorithms (GA), for example, are a fast optimization technique (Koza 1995). Depending on the binary construction of the different solutions and the easy way to mutate a solution, the genetic algorithm creates, in comparison to other metaheuristics, a new solution with a fast computation of the next generation. The disadvantage of this technique is that the best solution is found not as often as with other approaches (Koza 1995). The metaheuristic Particle Swarm Optimization (PSO) has been introduced by (Eberhart and Kennedy 1995). It also performs a randomized parallel search in the solution space. In comparison to PSO the computation of the next generation's individuals is faster in genetic algorithms but PSO focuses earlier to a specific minimum. Depending on the problem (i.e., the fitness landscape), different metaheuristics or varying parameters lead to better or worse efficiency in the optimization process. In standard metaheuristics no dynamic changes (i.e., no adaptation) in parameter settings during the optimization process are intended. If an optimization starts, it will run until the termination criterion is reached. Finding the right termination criterion is another task when using metaheuristics. If the optimization terminates too early, a good solution may not be found. If the computation runs too long, computation time is wasted without relevant increase in the solution quality. We want to face these problems of missing dynamics during the optimization process and of finding adequate parameter sets for a combination of advantages of metaheuristics in this paper. 
In this work we present a new approach based on (Lorion et al. 2008) for analysing and using metaheuristics in a more efficient and easier way. The extension to new metaheuristics is simple through the few interfaces that have to be implemented. We also give an example how new metaheuristics can be integrated, enhanced and controlled during runtime. The underlying agent structure guarantees flexibility and autonomous computation of metaheuristics in a wide field of technology. In order to demonstrate the usability of the framework and the possibilities using the framework for research we adapt the Particle Swarm Optimization and test how a dynamic switch of the parameters influences the optimization process. We are at the beginning of the development of this framework and some features depend on our own systems in the current status. The agents communicates with an own language and have no official agent communication language. We avoid to use an agent framework like $J A D E$ in the first step because we want to build an easy agent structure which can be adapted and reused easily on all systems. The first experiments with this framework have been done and demonstrated the flexibility as well as the possibility to change parameters dynamically in a metaheuristic.

\section{References}

Eberhart, R., and Kennedy, J. (1995). "A New Optimizer using Part Swarm Theory." Proceedings of the SixthInternational Symposium on Micro Maschine and Human Science, 39-43.

Lenstra, J. K., and Kan, A. H. G. R. (2006). "Complexity of vehicle routing and scheduling problems." Networks, 11, 221 - 227.

Lorion, Y., Bogon, T., Timm, I. J., and Drobnik, O. "An Agent Based Parallel Particle Swarm Optimization - APPSO." Presented at Swarm Intelligence Symposium SIS 2009, Nashville, TN.

Koza, J. R. (1995). "Survey of genetic algorithms and genetic programming"WESCON/'95. Conference record. 'Microelectronics Communications Technology Producing Quality Products Mobile and Portable Power Emerging Technologies. City: Dept. of Comput. Sci., Stanford Univ., CA: San Francisco. 
RFID, NFC, Ad Hoc Netze und Web 2.0:

Logistik, mobile Informationsverarbeitung und neue Wertschöpfungsnetzwerke 



\title{
Vorwort zur Teilkonferenz
}

\section{RFID, NFC, Ad Hoc Netze und Web 2.0: Logistik, mobile Informationsverarbeitung und neue Wertschöpfungsnetzwerke}

\author{
Michael H. Breitner, Günter Woblers \\ Institut für Wirtschaftsinformatik, \\ Leibniz Universität Hannover
}

RFID, NFC und AHN ermöglichen heute revolutionäre mobile Prozesse und vielfältige Anwendungen. Wir lesen wiederholt, dass die Standardisierung vollzogen ist und die meisten Pilotprojekte erfolgreich verlaufen sind.

Die Theorie könnte etwa so lauten: Klassische, weitverbreitete Anwendungen sind Waren-/Personenerkennung und Waren-/Personenverfolgung. U. a. werden zukünftig Gesundheitskarten, Personalausweise und Pässe teils mit Transpondern versehen sein. Zusammen mit dem Einsatz von Wikis, Microblogging und Blogs in Unternehmen erweitern sich die Möglichkeiten der Informationssammlung und Informationsverarbeitung erheblich. In der Warenlogistik können Waren und deren Teilprodukte in ihrem Lebenszyklus verfolgt (Lesen eines Transponders) und zusätzlich anfallende Informationen immer wieder hinzugefügt werden (Schreiben eines Transponders). Schreiben und Lesen sind oft automatisch, berührungslos und unbemerkt möglich: Die verwendete Hard- und Software wird dabei schnell billiger, u. a. durch Masseneinsatz und Standardisierung, so dass sich immer mehr Prozesse wirtschaftlich durch RFID, NFC und AHN unterstützen lassen. Insbesondere die Zusammenarbeit in dynamischen, anreizbasierten Wertschöpfungsnetzen kann hiervon profitieren, da die flexible unternehmensübergreifende Prozessintegration noch große Effizienzsteigerungspotentiale birgt. So können z. B. logistische Prozesse in den Organisationen entlang einer Lieferkette automatisiert, umfassend überwacht sowie Entscheidungen besser unterstützt werden als bisher.

Mit anderen Worten: alles scheint möglich und RFID \& Co. sind kaum noch aufzuhalten. Der sehr gute Beitrag von Ralf God und Hartmut Hintze von der Technischen Universität Hamburg-Harburg „Drahtlose Kommunikation in der Flugzeugkabine für effiziente Arbeitsabläufe und Passagierdientstleistungen“ illustriert stellvertretend für alle die große Herausforderung, dass ohne die Zusammenarbeit mehrerer Interessengruppen entlang der Wertschöpfungskette keine effizienten Gesamtlösungen möglich sind. Die weiteren guten Beiträge z.B. von Christian Scharfe und Co-Autoren (Einsatzmöglichkeiten der RFID-Technologie im Techni- 
schen Außendienst - Eine Analyse am Beispiel der IBM Deutschland Gmbh) und von Tobias Rhensius und Günther Schuh (Prozessorientierte RFIDEinsatzplanung) von der RWTH Aachen verdeutlichen, dass unabhängig vom Einsatzfeld die Neugestaltung der Geschäftsprozesse das Schlüsselelement für eine erfolgreiche Innovation ist.

Werfen wir allerdings einen genaueren Blick auf die Praxis, dann wandelt sich das Bild erheblich: Die E-Gesundheitskarte sollte 2006 eingeführt sein, als neuester Termin für eine flächendeckende bundesweite Einführung wird Ende 2010 (Bitkom) genannt! Im Handelsblatt vom 27.11.2009 lautet eine Schlagzeile: „RFIDAnwender ziehen selten an einem Strang - Handelsketten wollen ihre Kosten durch den Einsatz von RFID-Chips senken. Doch weil nur wenige Konsumgüterhersteller ihre Produkte mit den Chips ausstatten, müssen die automatischen Lieferketten oft gestoppt werden. Die Technologie verkommt zur Insellösung." Das ist mehr als vorsichtige Kritik und beschreibt einen fatalen Zustand im Handel. Die Metro wird nicht müde, die Vorteile herauszustellen. Allerdings werden gerade in der Konsumgüterindustrie die Möglichkeiten, die RFID heute bietet, nicht genutzt. Der Hemmschuh für eine umfassende Marktdurchdringung der Technologie ist schlicht die mangelnde Zusammenarbeit zwischen den Unternehmen.

Die RFID \& Co.-Technologie bleibt damit bis auf Weiteres ein wichtiges Forschungsfeld, das sich in immer neuen Projekten beweisen kann und muss. Die Forschung hat damit eine Rolle übernommen, die ihr nicht selbstverständlich zukommt. Sie muss durch weitere umfassende Innovationen einen Weg bereiten, an dessen Ende die schlichte Kraft der Überzeugung von den Unternehmen nicht mehr ignoriert werden kann.

Track Chairs und Programmkomitee:

Prof. Dr. Michael H. Breitner, Universität Hannover (Koordinator)

Prof. Dr. Elgar Fleisch, Universität St. Gallen

Prof. Oliver Günther, Ph.D., HU Berlin

Dr. Paul Hofmann, SAP Labs, Palo Alto

Dr. Werner Knop, deister electronic GmbH, Barsinghausen

Prof. Dr. Franz Lehner, Universität Passau

Prof. Dr. Günter Müller, Universität Freiburg

Dr. Volker Schäffer, Seeburger AG, Bretten

Dr. Jens Strüker, Universität Freiburg

Dr. Orestis Terzidis, SAP Research Center, Karlsruhe

Dr. Frederic Thiesse, Universität St. Gallen

Dr. Günter Wohlers, nisss GmbH Hannover und München

Dr. Holger Ziekow, HU Berlin 


\title{
Drahtlose Kommunikation in der Flugzeugkabine für effiziente Arbeitsabläufe und Passagierdienstleistungen
}

\author{
Ralf God', Hartmut Hintre? ${ }^{2}$ \\ ${ }^{1}$ Institut für Flugzeng-Kabinensysteme, \\ Technische Universität Hamburg \\ ${ }^{2}$ RFID Laboratory, Hamburger Logistik. Institut GmbH
}

\section{Einleitung und Problemstellung}

Der Gebrauch und die kontinuierliche Weiterentwicklung von Informations- und Kommunikationstechnik stellen einen entscheidenden Punkt für die Zukunft unserer Informationsgesellschaft dar. Themen wie Mobilität, nahtlose Konnektivität, Funknetzwerke und der Gebrauch von tragbaren elektronischen Geräten spielen in zunehmendem Maße in der Kabine von Verkehrsflugzeugen eine wichtige Rolle.

Wenn man eine Anforderungsanalyse für den Einsatz drahtloser Kommunikationsarchitektur in der Flugzeugkabine durchführt, so kann man diese als geschlossenes System mit den drei Interessengruppen Flugzeughersteller, Fluggesellschaft und Passagier betrachten. Dabei stellt man fest, dass sich Ausgangslage und Anforderungen dieser drei Gruppen hinsichtlich des Einsatzes von Funktechnologie im Flugzeug voneinander unterscheiden:

Der Flugzenghersteller muss die kundenspezifische Kabineninstallation und deren Rekonfigurationsmöglichkeit im Betrieb vereinfachen. Prozesse zum Unterhalt und zur Wartung der Kabine müssen effizient gestaltet werden. Eine Steigerung der Netzwerkleistung in der Kabine soll bei gleichzeitiger Einsparung von Kabelgewicht erreicht werden. Eine besondere Herausforderung stellt dabei die gefordert hohe Netzwerk- und Informationssicherheit dar.

Die Fluggesellschaft muss die Arbeitsabläufe und logistischen Prozesse in der Kabine verbessern. Durch erweiterte und automatisierte Kommunikations- und Informationsprozesse soll die Arbeitsbelastung der Kabinenbesatzung begrenzt und mögliche Fehlerquellen sollen reduziert werden. Weiterhin ist es für die Fluggesellschaft extrem wichtig, die Service-Interaktion mit dem einzelnen Passagier während der Flugreise und das Kundenbeziehungsmanagement kontinuierlich zu verbessern. Ziel ist es dabei, die Kundenbindung nachhaltig zu stärken und weiteren Umsatz durch zusätzlich erbrachte Dienstleistungen zu generieren.

Der Passagier möchte an Bord Kommunikations-, Informations- und Unterhaltungsdienstleistungen zu angemessenen Kosten nutzen. Dabei erwartet er für seine 
tragbaren elektronischen Geräte (PEDs, Portable Electronic Devices) ein ähnlich leistungsfähiges Angebot wie er dieses vom Flughafen her kennt, und welches sich in der Kabine nahtlos fortsetzt.

\section{Smart Cards, RFID und NFC in der Flugzeugkabine}

In der Flugzeugkabine betrachten wir kontaktlose Schnittstellen auf Basis von Smart Cards, RFID und NFC als wichtige und nützliche Technologieplattform zur Verbesserung von Prozessen, an denen Gegenstände und Personen beteiligt sind. Die einzelnen Elemente dieser Technik sind am Boden erprobt und die erforderlichen Standards sind weltweit verbreitet. Für die Zulassung im Flugzeug existieren bereits erste Empfehlungen seitens der Behörden. Weiterhin beinhaltet die Technologie gut beherrschte Sicherheitsfunktionalitäten, welche kontinuierlich weiterentwickelt werden. Die Integration dieser kontaktlosen Kommunikationsschnittstellen in die Flugzeugkabine würde eine nahtlos anschlussfähige Infrastruktur für am Boden genutzte tragbare elektronische Geräte, Smart Cards und RFID-Transponder bieten können.

Eine Herausforderung dabei ist, dass diese Infrastruktur als Plattformlösung verstanden werden muss, deren gesamte Wertschöpfung sich auf die drei Interessengruppen Flugzeughersteller, Fluggesellschaft und Passagier verteilt. In künftigen Arbeiten soll daher der übergeordnete Nutzen dieser Gesamtlösung in der Flugzeugkabine weiter herausgearbeitet und bewertet werden. Parallel dazu sollen für die Kabine Wege zur Entwicklung und zum Test betrachtet werden. Die Einführung und Zertifizierung einer solchen Lösung würden den letzten Schritt vor der Kommerzialisierung einer solchen Technologieplattform im Verkehrsflugzeug darstellen. 


\title{
Datengeneratoren für die Unterstützung von Design-Science-Projekten im Umfeld RFID- basierter Analyseanwendungen
}

\author{
Xuanpu Sun, Henning Baars \\ Lehrstubl für ABWL und Wirtschaftsinformatik I, \\ Universität Stuttgart
}

Seit einigen Jahren wird verstärkt der Einsatz von Radio Frequency Identification (RFID) im Kontext der Logistik diskutiert (Curtin et al. 2007, S. 87-110). Ein wachsender Forschungszweig beschäftigt sich mit dem Design und der Evaluation innovativer Logistik-Anwendungssysteme, die auf den Möglichkeiten von RFID zur Erhöhung von Datenqualität und Datenumfang aufsetzen (Baars et al., 2009). Hierbei ist insbesondere die Evaluation entsprechender Lösungsentwürfe eine Herausforderung. Dies gilt vor allem für Anwendungen, die Datenhaltungssysteme mit historisierten und angereicherten RFID-Daten vorsehen, um damit Freiheitsgrade bei der Datennutzung zu schaffen (Baars und Sun 2009; Chawathe et al. 2004, S. 1189-1195).

In diesem Beitrag wird vorgeschlagen, zur Unterstützung entsprechender Design-Science-Projekte einen Generator zu entwickeln, der es erlaubt, Datenpools für die Visualisierung und Exploration unterschiedlichster RFID-Analyseszenarios zu erstellen. Die flexible Erzeugung konsistenter, nachvollziehbarer und überzeugender Demonstrationsdaten ist jedoch mit einer sehr hohen Komplexität verbunden. Der im Kontext eines mehrjährigen Projektes konzipierte und prototypisch realisierte Datengenerator zeigt Problemklassen und Lösungsansätze auf.

Das Anforderungsgerüst an den Generator wurde über Analyseszenarien definiert, die auf Basis der Literatur sowie einer Serie von Fallstudien abgeleitet wurden. Der Grundansatz der Lösung besteht dabei in der Manipulation von Kennzahlenwerten in einem relational-multidimensionalen Analyse-Datenmodell, wobei die Einhaltung der sachlogischen Konsistenz durch eine separate Komponente („Hauptprozess“) realisiert wird. Grundsätzlich können in die dabei erzeugten Daten Ergebnisse zu drei Analyseszenario-Typen eingearbeitet werden (Szenarios, die sich aus einer spezifischen Konstellation von Parametern ergeben; Szenarios, die von der Konfiguration des Logistiksystems abhängen; Szenarios, die aus Inputmengen resultieren). Hierbei werden drei Arten von Kennzahlen berücksichtigt (additive wie „Dauer“, nicht-additive wie „Minimale Temperatur" und alphanumerische wie „Status“). Zur Erhöhung der Flexibilität wurde ein komponentenorientiertes Design gewählt. Die Umsetzbarkeit der Szenarios wurde u.a. anhand eines Textilbeispiels erprobt, bei dem Ausschüsse und Streckenalternativen analysiert werden können. 
Der Datengenerator bzw. der damit erzeugte Datenpool im Kontext eines übergeordneten Design-Science-Projektes wird zur Evaluation einer entwickelten Analyselösung eingesetzt.

Weiterer Untersuchungsbedarf besteht bezüglich der Frage, ob der Datengenerator auch in einem Praxisumfeld als Werkzeug für die Informationsbedarfsanalyse sowie gegebenenfalls auch in anderen Einsatzdomänen (Lehre, Weiterbildung, Vertrieb) genutzt werden kann. Darüber hinaus wird hier angeregt, der Idee eines gezielten Einsatzes von Datengeneratoren im Kontext von Design-ScienceVorhaben stärkere Aufmerksamkeit zu widmen.

\section{Literatur}

Baars H, Gille D, Strüker J (2009) Evaluation of RFID applications for logistics: a framework for identifying, forecasting and assessing benefits. European Journal of Information Systems (EJIS) 18 (6). In Druck.

Baars H, Sun X (2009) Multidimensional Analysis of RFID Data in Logistics. In: Proceedings of the 42. Hawaii International Conference on System Sciences (HICCS-42), Hawaii.

Chawathe SS, Krishnamurthy V, Ramachandran S, Sarma S (2004) Managing RFID Data. In: Proceedings of the 30th VLDB Conference, Toronto, Canada.

Curtin J, Kauffman RJ, Riggins FJ (2007) Making the 'MOST' out of RFID technology: a research agenda for the study of the adoption, usage and impact of RFID. Information Technology and Management. 8(2):87-110. 


\title{
Einsatzmöglichkeiten der RFID-Technologie im Technischen Außendienst
}

\section{Eine Analyse am Beispiel der IBM Deutschland GmbH}

\author{
Christian Scharfe', Daniel Fischer², Bernd Markscheffel2, \\ Dirk Stelzer², Ramón Somoza ${ }^{1}$ \\ ${ }^{1}$ IBM Deutschland CSS GmbH, Erfurt \\ Institut für Wirtschaftsinformatik, \\ Fachgebiet Informations- und Wissensmanagement, \\ Technische Universität Ilmenau
}

Die Anwendung der RFID-Technologie wird bisher überwiegend aus Sicht des Supply-Chain-Managements diskutiert (BSI 2004, S. 84-89). Einsatzmöglichkeiten von RFID-Systemen im Technischen Außendienst wurden bisher nur in wenigen Veröffentlichungen erörtert (z. B. Hanhart et al. 2005a, S. 62-73). Hierbei beschränken sich die Autoren auf die Skizzierung von Chancen und Risiken des RFID-Einsatzes. Aussagen zur Wirtschaftlichkeit fehlen mit Ausnahme der Publikation von Lampe et al. (2005, S. 53-74) zum Werkzeugmanagement in der Flugzeugwartung. Gerade im Technischen Außendienst werden aber große Potenziale vermutet, mit Hilfe der RFID-Technologie die Geschäftsprozesse zu beschleunigen und effizienter zu gestalten.

In diesem Beitrag werden Einsatzmöglichkeiten der RFID-Technologie im Technischen Außendienst der IBM Deutschland GmbH vorgestellt und deren Wirtschaftlichkeit bewertet. Dazu führten die Prozessverantwortlichen in Zusammenarbeit mit dem Fachgebiet Informations- und Wissensmanagement der TU Ilmenau ein Projekt durch. Das Projekt wurde in die Phasen Prozessanalyse, Prozessverbesserung und Prozessbewertung gegliedert, deren Ergebnisse und die daraus resultierenden Schlussfolgerungen werden ausführlich im Beitrag diskutiert.

Im Rahmen der Prozessanalyse wird der Ist-Prozess des Technischen Außendienstes der IT-Service Delivery für Hardware-Produkte der IBM Deutschland $\mathrm{GmbH}$ beschrieben. Anschließend werden Schwachstellen des Ist-Prozesses erörtert, deren Ursachen primär in den manuellen Eingaben sowie im Fehlen weiterführender Informationen, wie z. B. der Wartungshistorie, liegen.

Zur Beseitigung der ermittelten Schwachstellen werden Einsatzmöglichkeiten der RFID-Technologie diskutiert, die vor allem auf einer automatischen Erfassung von Zeiten und Ersatzteilnummern, der Identifikation der zu reparierenden Komponenten sowie der Automatisierung des Reportings beruhen. Ausgehend davon wird der durch RFID-Einsatz veränderte Soll-Prozess dargestellt. 
Die Grundlage für die Bewertung der Einsatzmöglichkeiten der RFIDTechnologie bildete eine Kosten-Nutzen-Analyse, wobei nicht monetär bestimmbare Nutzenvorteile unberücksichtigt bleiben. Durch den Einsatz der RFIDTechnologie kann die Bearbeitungszeit um fast 20\% gesenkt werden. Bei einer durchschnittlichen Anzahl von 180.000 Service-Requests im Jahr ergibt sich somit ein Einsparpotenzial von ca. 4,8 Mio € pro Jahr. Dem stehen Infrastruktur- und Integrationskosten in Höhe von ca. 7,5 Mio $€$ als einmalige Kosten sowie Hardware- und Wartungskosten von ca. 2,5 Mio € als jährliche Kosten gegenüber. Unter Nutzung der Kapitalwertmethode (Kruschwitz 2008, S. 43 ff.) ergibt sich bei einem kalkulatorischen Zinssatz von $10 \%$ und unter Vernachlässigung der Abschreibungen eine Amortisation des RFID-Einsatzes im 4. Jahr.

Die Ausführungen zeigen, welche Einsatzmöglichkeiten es für die RFIDTechnologie im Technischen Außendienst der IBM Deutschland GmbH gibt und welche Einsparungen erreichbar sind. Zur Überprüfung des ermittelten Nutzens bzw. der Kosten wird in einem nächsten Schritt die prototypische Umsetzung eines Piloten angestrebt. Dieser Pilot wird wichtige Aufschlüsse über die Nutzbarkeit der RFID-Technologie im Technischen Außendienst liefern und - positive Ergebnisse vorausgesetzt - die Basis für eine Detailplanung darstellen.

\section{Literatur}

BSI (2004) Risiken und Chancen des Einsatzes von RFID-Systemen. http://www.bsi.bund.de/fachthem/rfid/RIKCHA.pdf. Abruf am 2007-01-30.

Finkenzeller K (2006) RFID-Handbuch. Hanser, München.

Hanhart D, Jinschek R, Kipper U, Legner C, Österle H (2005a) Mobile und Ubiquitous Computing in der Instandhaltung - Bewertung der Anwendungsszenarien bei der Fraport AG. HMD 244:62-73.

Kruschwitz L (2008) Investitionsrechnung. Oldenbourg, München.

Lampe M, Strassner M, Fleisch E (2005) RFID in Movable Asset Management. In: Roussos G (Hrsg.): Ubiquitous and Pervasive Commerce - New Frontiers for Electronic Business, Berlin. 


\title{
Prozessorientierte RFID-Einsatzplanung
}

\section{Entwurf eines Beschreibungsmodells für RFID- Anwendungen}

\author{
Günther Schub, Tobias Rhensius \\ Forschungsinstitut für Rationalisierung (FIR) e. V., \\ RWTH Aachen
}

Neben anderen Technologien wie NFC, AHN, WLAN, GSM bzw. UMTS oder GPS gilt RFID als Basistechnologie auf dem Weg zum Internet der Dinge. Durch die Ausstattung von Objekten mit elektronischen, sensorischen und aktorischen Komponenten werden diese in die Lage versetzt, innerhalb vordefinierter Grenzen autonom Entscheidungen zu treffen und Aktivitäten auszulösen.

Praktiker wie Wissenschaftler bescheinigen RFID ein enormes betriebswirtschaftliches Potenzial. Die große Zahl an RFID-Komponenten, Speziallösungen und Produkten ergeben sich diverse Kombinations- und Einsatzmöglichkeiten, die - verstärkt durch die hohe Innovationsgeschwindigkeit auf Anbieterseite - für Anwender nicht transparent und selbst für Fachleute kaum zu überblicken sind. Fehlendes Know-how in Bezug auf die Technologie aber auch fehlende Referenzlösungen und die nur unzureichend methodisch abgesicherte systematische Einsatzplanung für RFID-Anwendungen stehen einer weiteren Verbreitung im Weg.

Zielsetzung des Beitrags ist der Entwurf eines Beschreibungsmodells für RFID-Anwendungen, das es Anwendern ermöglicht, ausgehend von den Anforderungen aus den eigenen Prozessen systematisch Einsatzmöglichkeiten von RFID zu identifizieren und die Umsetzbarkeit einer technischen Lösung zu evaluieren.

Aufbauend auf der Analyse technischer Klassifikationen für RFID-Systeme und anwendungsorientierte Systematisierungen werden RFID-Anwendungen definiert als RFID-System (bestehend aus mindestens einem Transponder, einem Schreib-Lesegerät und der zugehörigen Software) zur Unterstützung eines betrieblichen Prozesses.

Anschließend wird zunächst ein Partialmodell für den Prozess, der mit RFID unterstützt werden soll, entwickelt. Tabelle 1 visualisiert das entwickelte Teilmodell mit Hilfe eines morphologischen Kastens. Die exemplarisch dargestellte Merkmalskombination ist charakteristisch für RFID-Systeme zur Unterstützung des Behältermanagements oder auch für Anwendungen in der Produktionsautomatisierung, in denen Ladungsträger mit RFID-Transpondern ausgestattet sind. 
Tabelle 1: Teilmodell RFID-Prozess

\begin{tabular}{|c|c|c|c|c|c|}
\hline Merkmal & \multicolumn{5}{|c|}{ Ausprägungen } \\
\hline Hauptfunktion & Lokalisierung & Übe & wachung & $\begin{array}{r}\text { Automati } \\
\text { Transakt }\end{array}$ & $\begin{array}{l}\text { Objektinfor- } \\
\text { mation }\end{array}$ \\
\hline Art der Objekte & Mobile Objel & & Immobil & le Objekte & Personen \\
\hline Integrationsreichweite & \multicolumn{3}{|c|}{ Lokal } & \multicolumn{2}{|c|}{ Kollaborativ } \\
\hline Kreislauf & \multicolumn{3}{|c|}{ Geschlossen } & \multicolumn{2}{|c|}{ Offen } \\
\hline Automatisierungsgrad & \multicolumn{3}{|c|}{ Vollautomatisch } & \multicolumn{2}{|c|}{ Teilautomatisch } \\
\hline Anbringung Transponder & \multicolumn{3}{|c|}{ Fest } & \multicolumn{2}{|c|}{ Abnehmbar } \\
\hline Prozesscharakteristik & \multicolumn{3}{|c|}{ Strukturiert/ Geführt } & \multicolumn{2}{|c|}{ Unstrukturiert/ Ungeführt } \\
\hline
\end{tabular}

Analog dazu wird ein Modell zur Beschreibung der eingesetzten Technik aus Perspektive der Anwender entwickelt. Das zweite Partialmodell abstrahiert dementsprechend von konkreten technischen Ausprägungen (z. B. Frequenz) und nutzt die resultierenden Leistungseigenschaften. Tabelle 2 stellt exemplarisch eine charakteristische Merkmalskombination für RFID-Systeme auf Basis der beschriebenen Merkmale und Ausprägungen dar. Dieses typische Profil kommt beispielsweise in einfachen Automatisierungsanwendungen zum Einsatz, wobei diese Kombination sowohl auf Low-frequency (LF) als auch mit High-frequency (HF) Komponenten realisierbar ist. Die zentrale Datenhaltung (Data-on-Network) und die einzelne Erfassung der Objekte pro Lesevorgang ermöglichen zudem den Einsatz (preiswerter) Read-only oder WORM Transponder, die nicht pulkfähig sind.

Tabelle 2: Teilmodell RFID- Technik

\begin{tabular}{|c|c|c|c|}
\hline Merkmal & \multicolumn{3}{|c|}{ Ausprägungen } \\
\hline Reichweite & $0-15 \mathrm{~cm}$ & $15-100 \mathrm{~cm}$ & $>1 \mathrm{~m}$ \\
\hline Datenhaltung & \multicolumn{2}{|c|}{ Data-on-Network } & Data-on-Tag \\
\hline Objekte pro Lesevorgang & \multicolumn{2}{|c|}{ Einzeln } & Pulklesungen \\
\hline Zusatzfunktionen & Keine & Sensorik & Kryptographie \\
\hline
\end{tabular}

Die Anwendbarkeit der Modelle wird anschließend mit Hilfe einer Fallstudie zum RFID-basierten Bettenmanagement überprüft. Die entwickelten Modelle leisten damit einen Beitrag zur Systematisierung des Untersuchungsbereichs. Aufbauend auf den Beschreibungsmodellen kann dann durch die Ableitung typischer Merkmalskombinationen eine Gestaltungshilfe für die Planung von RFID-Anwendungen geleistet werden. 


\title{
Modellierung eines Anwendungssystems zur Behälterlokalisation und Behälterreservierung auf Basis des Architekturstils REST
}

\author{
Thomas Barton, Harriet Bach \\ Fachbereich Informatik, Fachhochschule Worms
}

Der Einsatz von RFID-Technologien im Behältermanagement reduziert die Kosten und erlaubt eine Lokalisation von Behältern in Echtzeit (Fleisch, Christ und Dierkes 2005; Franke und Dangelmaier 2006; Golabeck 2007; Günther, Kletti und Kubach 2008; Strüker, Gille und Faupel 2008; Thoroe, Melski und Schumann 2009). Die Behälterlokalisation und -reservierung von beliebigen Positionen aus ist somit eine interessante Anwendung für ein mobiles Informationssystem und besitzt zwei Anwendungsfälle: „Informationen zu Behälter abfragen“ und „Behälter reservieren“. Werden die Standards des Web auf die Entwicklung eines solchen Informationssystems übertragen, bietet sich die Verwendung des Architekturstils REST (Representational State Transfer) an (Fielding 2000; Liu und Deters 2008; Tilkov 2009). REST bietet u.a. die Verwendung einer gemeinsamen Schnittstelle, eine einfache Wiederverwendbarkeit und eine gute Skalierbarkeit (Fielding und Taylor 2002; Pautasso, Zimmermann und Leymann 2008). Bei der Planung des Anwendungssystems sind die Identifizierung von Ressourcen und ihre Modellierung wesentliche Tätigkeiten und werden in diesem Beitrag beschrieben.

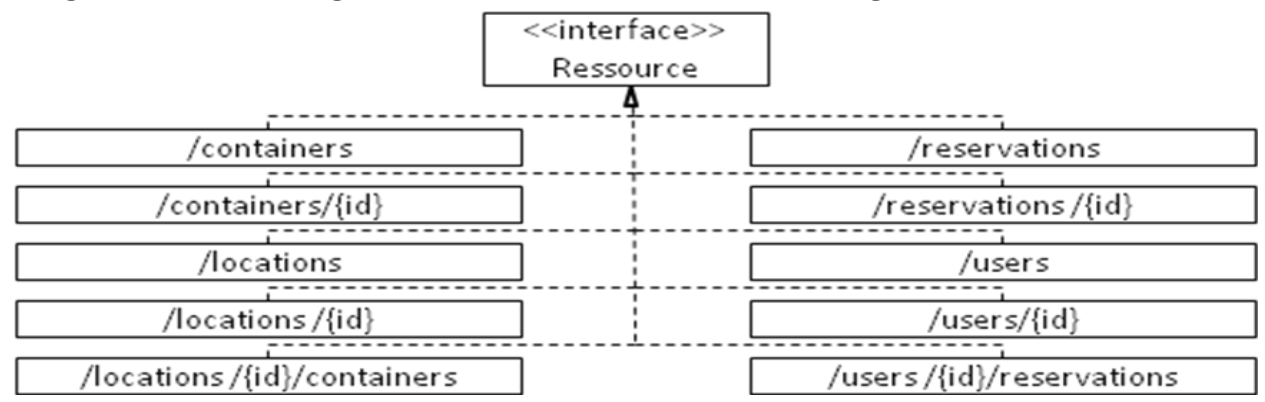

\section{Abbildung 1: Ressourcenmodell}

Zur Entwicklung des Anwendungssystems werden i.w. folgende Ressourcen benötigt: Die Behälter („,containers“) werden als eine Ressource angesehen. Die Stationen im Produktionsprozess und die Läger in der Instandhaltung werden als Standorte („locations“") modelliert. Zur Benutzerverwaltung, welche bei der Behälterre- 
servierung verwendet wird, wird eine Ressource Benutzer („users“) benötigt. Die Reservierungen stellen eine Ressource „reservations“ dar.

\section{Literatur}

Fielding RT (2000) Architectural styles and the design of network-based software architectures. Ph.D. thesis. University of California. Irvine.

Fielding RT, Taylor RN (2002) Principled Design of the Modern Web Architecture. ACM Transactions on Internet Technology, 2:2: 115-150.

Fleisch E, Christ O, Dierkes M (2005) Die betriebswirtschaftliche Vision des Internets der Dinge. In: Fleisch E, Mattern F (Hrsg.) Das Internet der Dinge Ubiquitous Computing und RFID in der Praxis: Visionen, Technologien, Anwendungen, Handlungsanleitungen. Springer.

Franke W, Dangelmaier W (2006) (Hrsg.) RFID - Leitfaden für die Logistik. Gabler.

Golabeck K (2007) RFID im Behältermanagement. In: Koschke R, Herzog O, Rödiger KH, Ronthaler M. INFORMATIK 2007 - Informatik trifft Logistik (Band 1). volume P-109 of Lecture Notes in Informatics. 87-90. Gesellschaft für Informatik e.V..

Günther O, Kletti W, Kubach U (2008) RFID in Manufacturing. Springer.

Liu D, Deters R (2008) Management of service-oriented systems. Service Oriented Computing and Applications. 2:51-64.

Pautasso C, Zimmermann O, Leymann F (2008) RESTful Web Services vs. "Big” Web Services: Making the Right Architectural Decision. In: Proceedings of the 17th International World Wide Web Conference. Beijing. China. 805-814.

Strüker J, Gille D, Faupel T (2008) RFID Report 2008 - Optimierung von Geschäftsprozessen in Deutschland. IIG-Telematik. Albert-LudwigsUniversität Freiburg. VDI nachrichten. Düsseldorf.

Tilkov S (2009) REST und HTTP - Einsatz der Architektur des Web für Integrationsszenarien. dpunkt. Heidelberg.

Thoroe L, Melski A, Schumann M (2009) The impact of RFID on management of returnable containers. Electron Markets. 19:115-124. 


\section{Autorenverzeichnis}

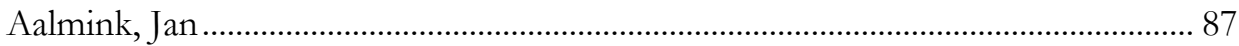

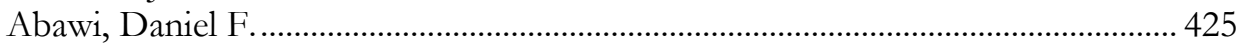

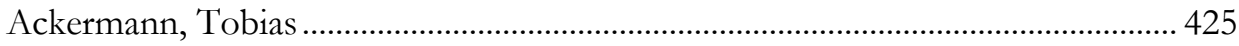

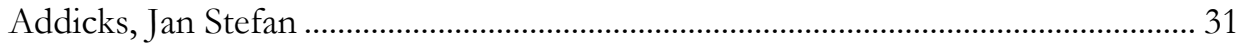

Ahlemann, Frederik ……………………………………………………….... 15

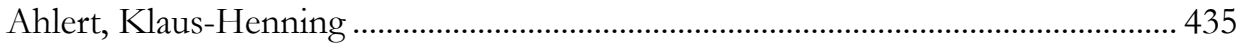

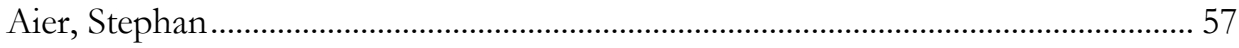

Allgaier, Matthias …………………………………………………………..... 303

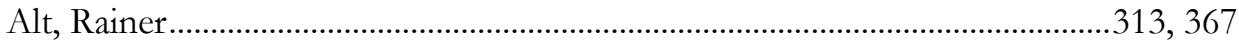

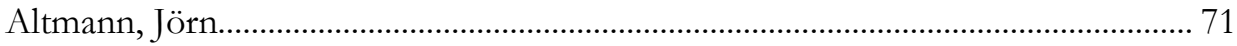

Amberg, Michael ……………………………………………......37, 41, 111, 237

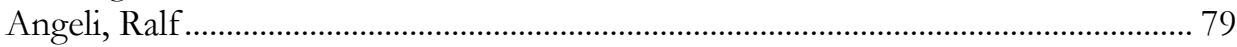

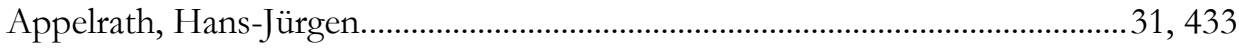

Baars, Henning...……………………………………………………...215, 469

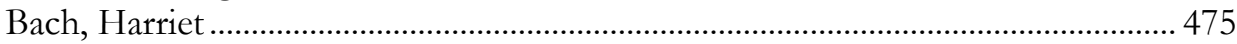

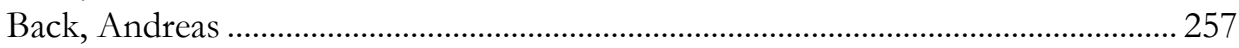

Baecker, Oliver ………………………………………………………...183, 373

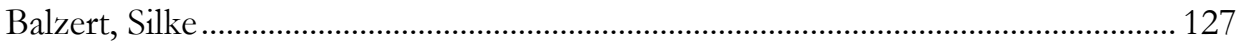

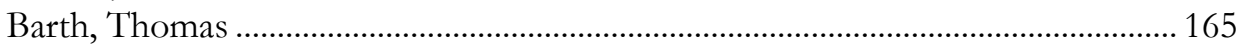

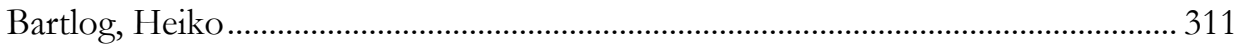

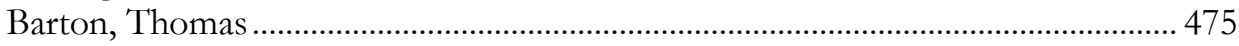

Bartsch, Stefan ........................................................................................... 53, 143

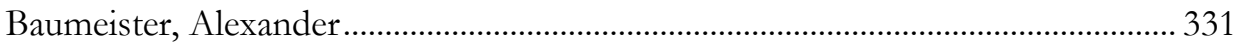

Baumöl, Ulrike ………………………………………………………………. 45

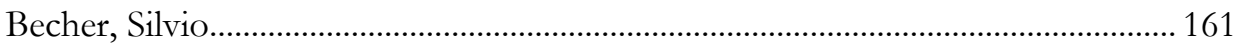

Becker, Jörg …………………………………….......17, 55, 263, 291, 407, 415

Belkius, Bartosch …………………………………………………………..... 111

Benlian, Alexander ………………………………….................................. 99, 101

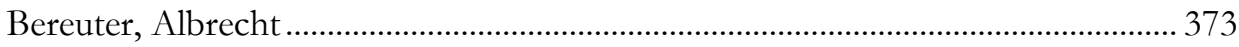

Berkovich, Marina ………………………………………………….... 107, 401, 405

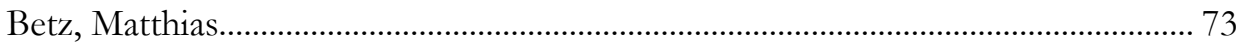

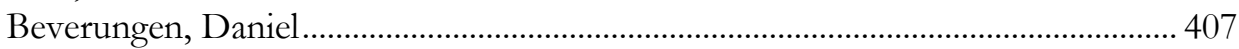

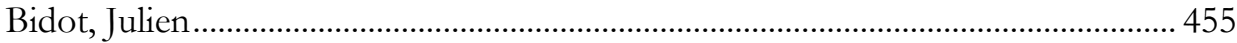

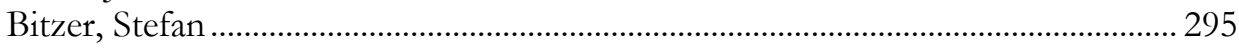

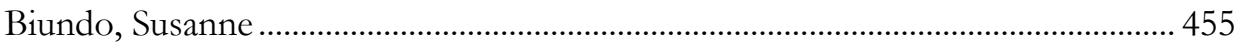

Blattmann, Olivier …………………………………………………………. 247

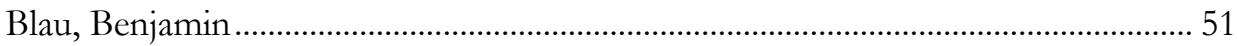

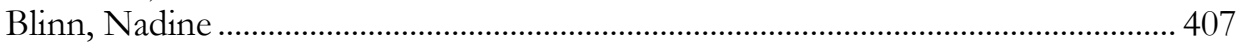

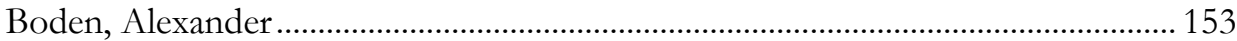




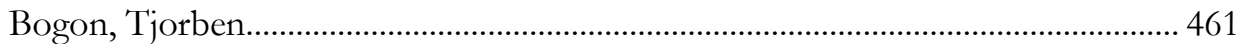

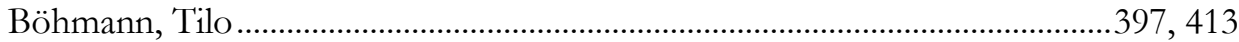

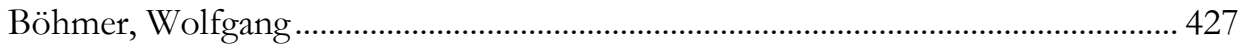

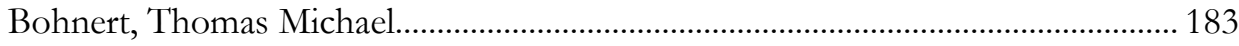

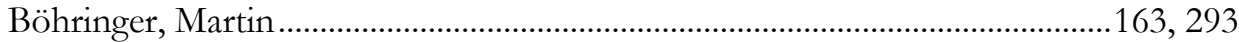

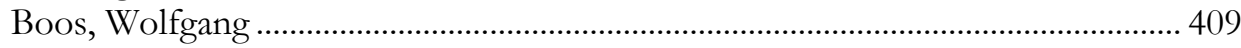

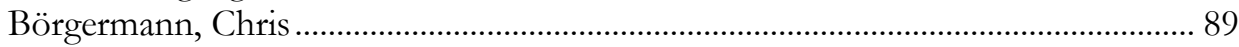

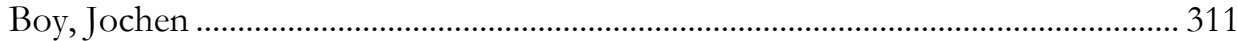

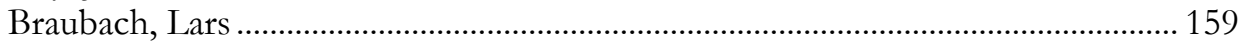

Breitner, Michael H.........................................................117, 125, 249, 281, 456

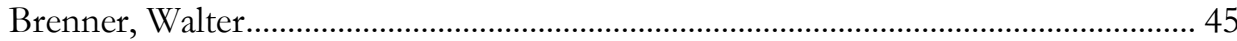

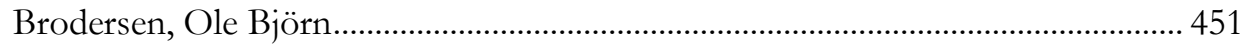

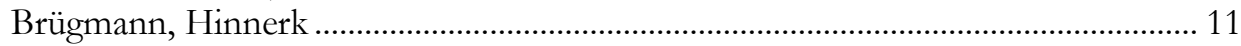

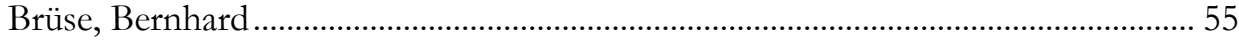

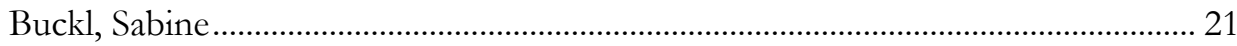

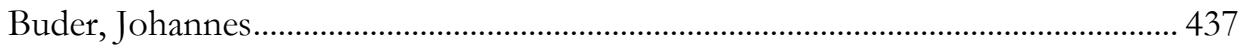

Bukvova, Helena...............................................................................283, 287

Bullinger, Angelika C............................................................................151, 245

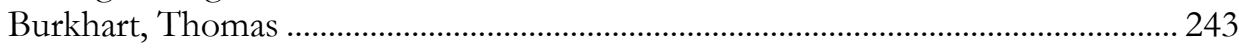

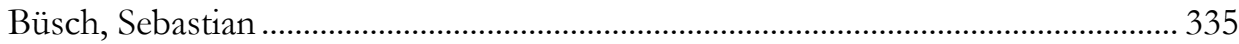

Buxmann, Peter .............................................................................. 95, 105, 425

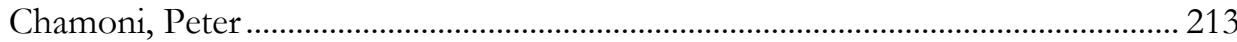

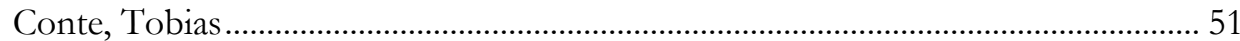

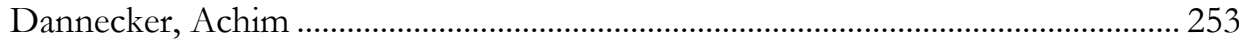

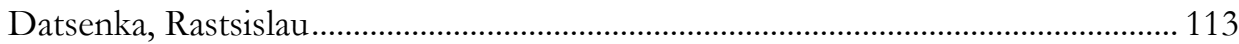

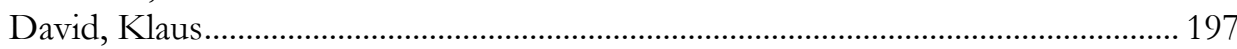

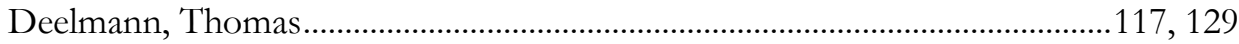

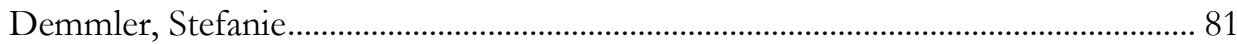

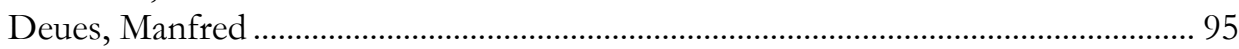

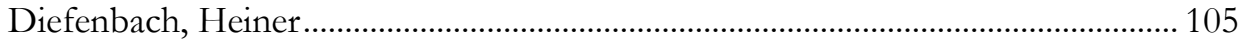

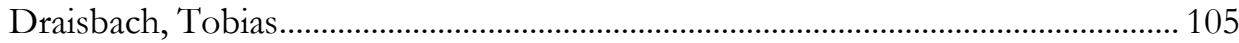

Draxler, Sebastian...................................................................................139, 153

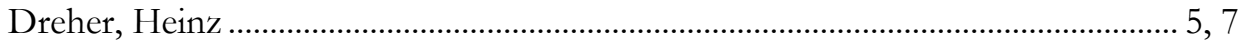

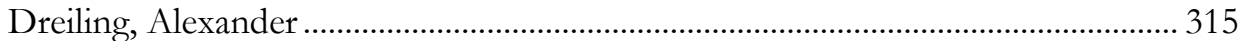

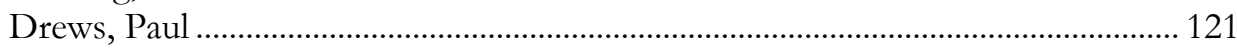

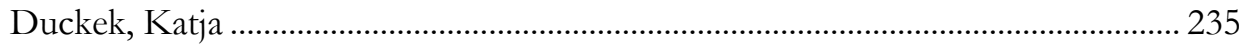

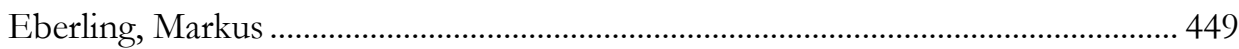

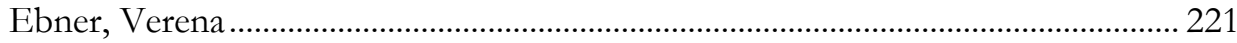

Edelkamp, Stefan................................................................................443, 453

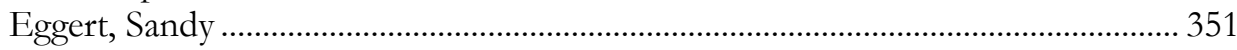




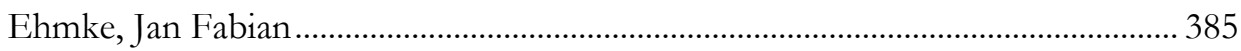

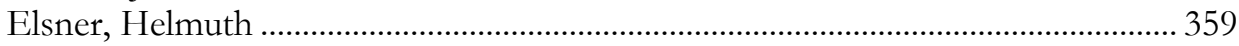

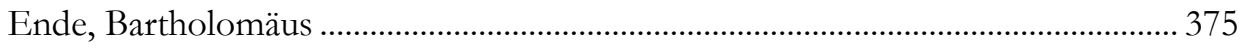

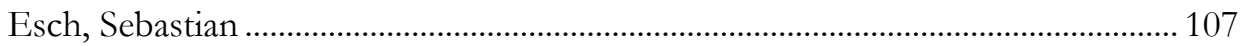

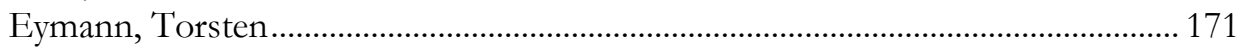

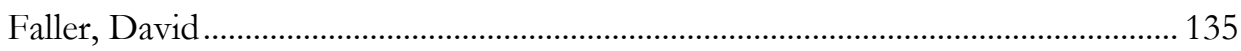

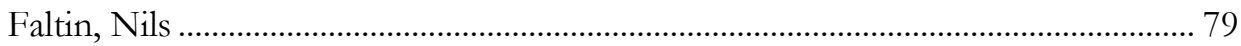

Felden, Carsten ……………………………………………………….....433, 437

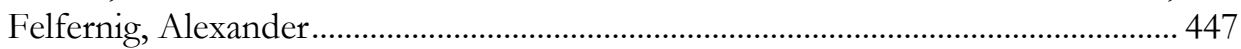

Fellmann, Michael ………………………………………………………....403, 407

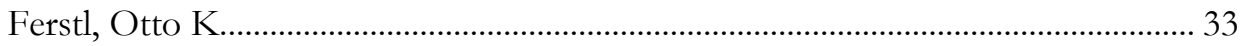

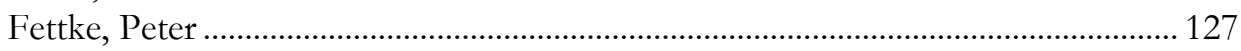

Fink, Corinna ........................................................................................................ 91

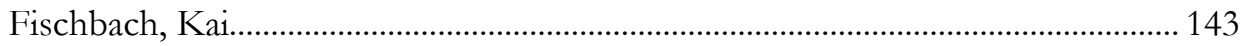

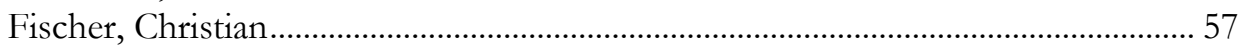

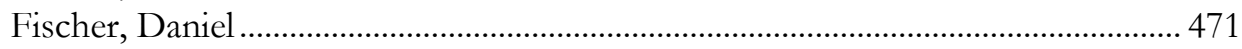

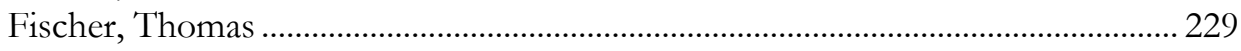

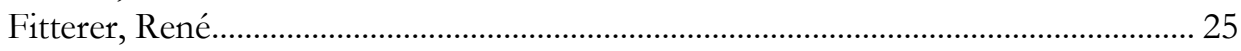

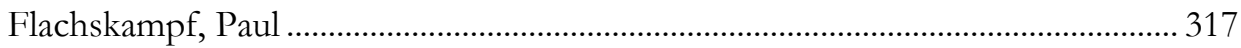

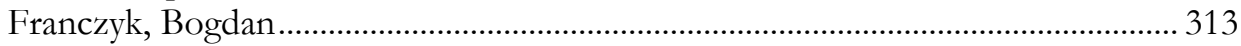

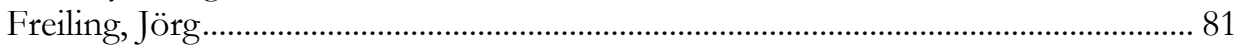

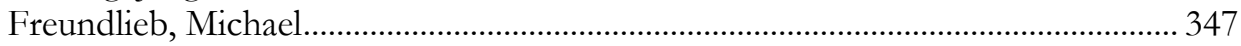

Frick, Norbert ………………………………………………………....309, 323

Fricke, Gerald.......................................................................................................... 349

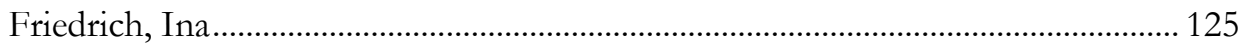

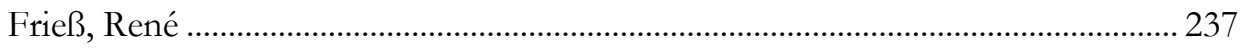

Funk, Burkhard....................................................................................201, 205

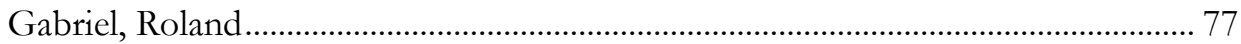

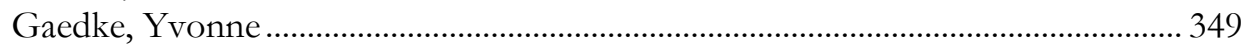

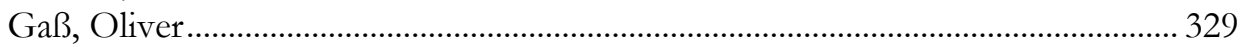

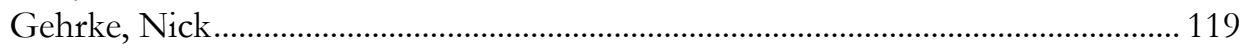

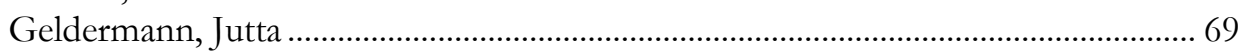

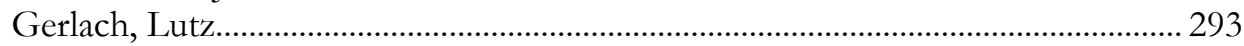

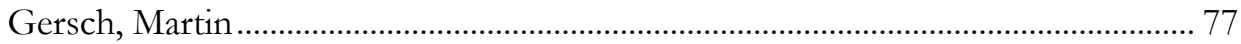

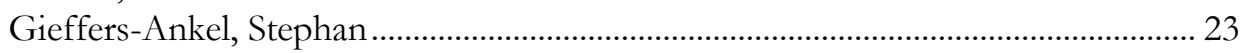

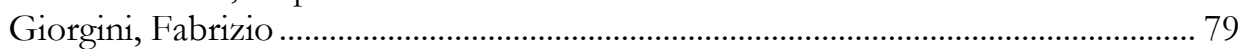

Gluchowski, Peter ………………………………………………....163, 213, 217

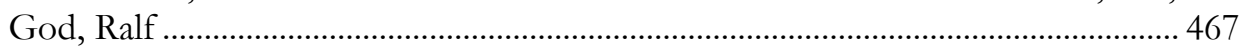

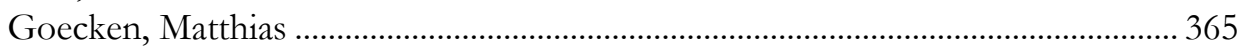

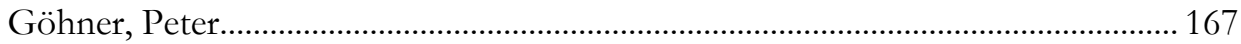

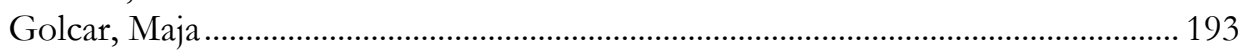


Gómez, Jorge Marx....................................................................195, 201, 209, 343

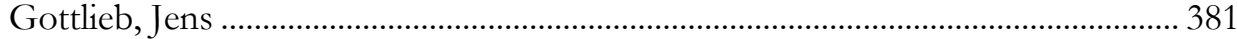

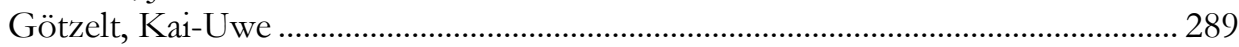

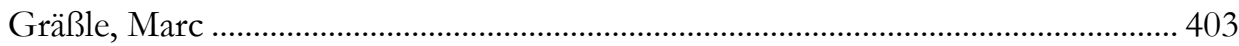

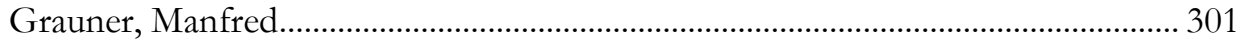

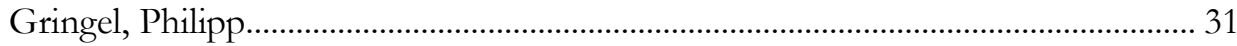

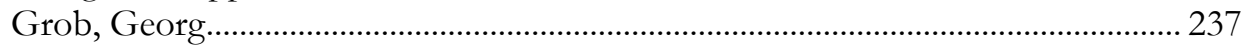

Gronau, Norbert ……………………………………………….... 95, 273, 321

Grüter, Marcel...…………………………………………………………….... 247

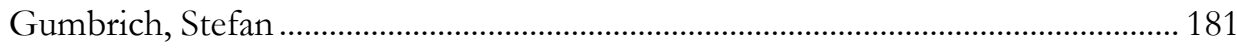

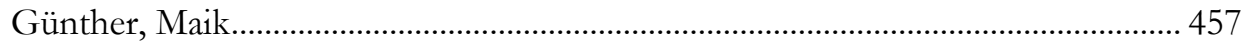

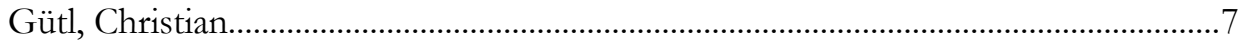

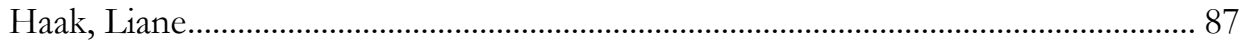

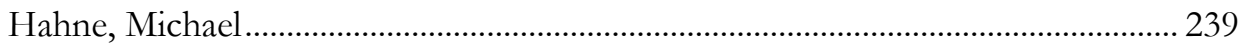

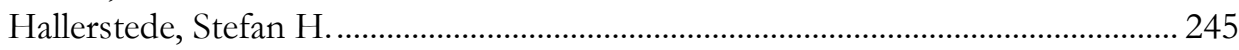

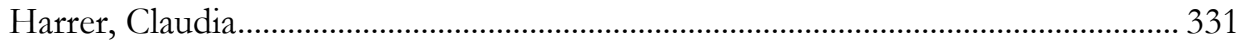

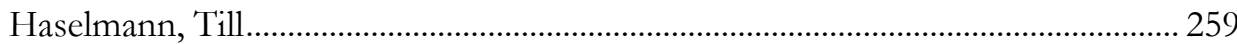

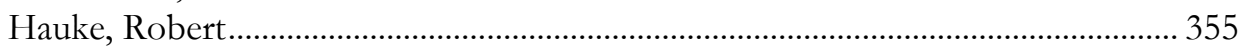

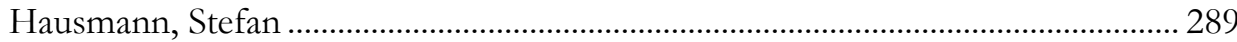

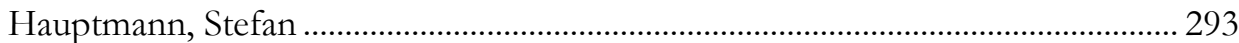

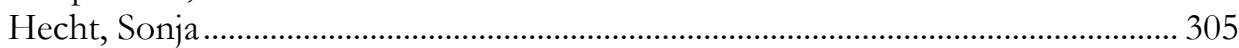

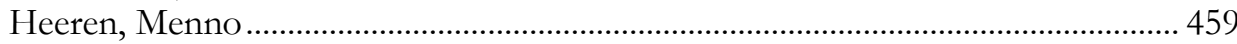

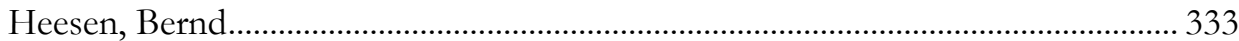

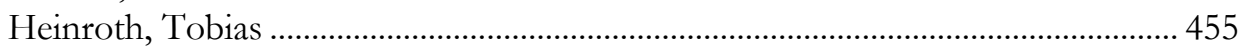

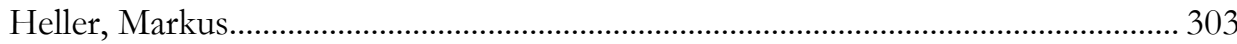

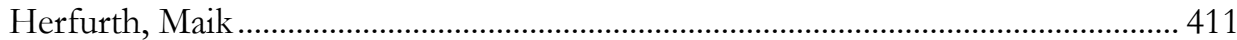

Herzwurm, Georg ……………………………………………....... 95, 109, 327

Hess, Thomas ………………………………………………….... 95, 99, 101

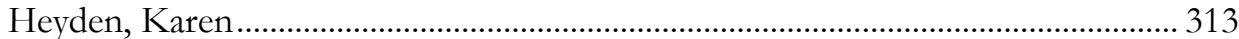

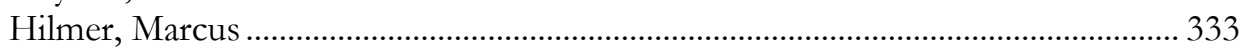

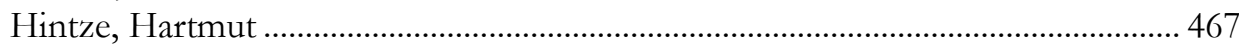

Hoffmann, Holger...........................................................................................185, 193

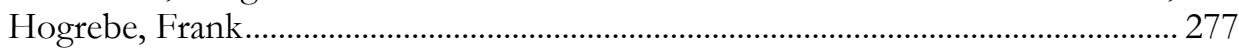

Hörmann, Stefan ........................................................................................................ 423

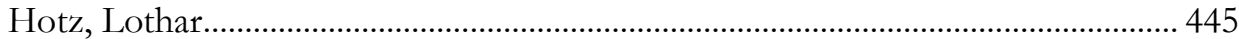

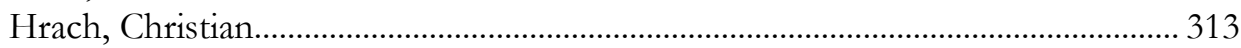

Huemer, Christian …………………………………………………………. 235

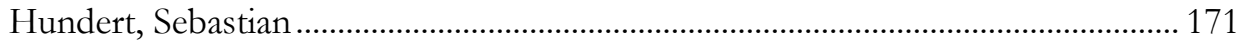

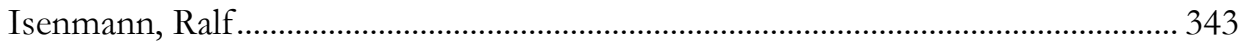

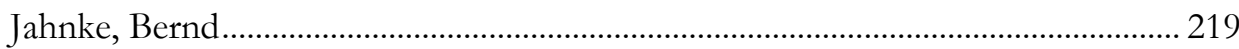




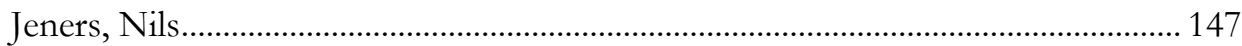

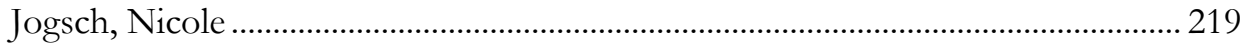

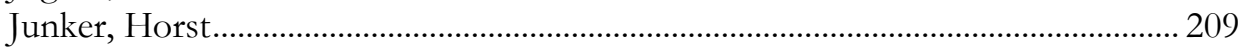

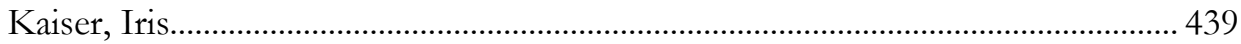

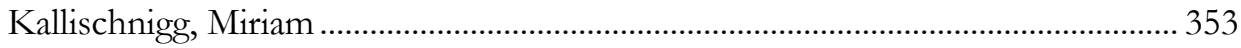

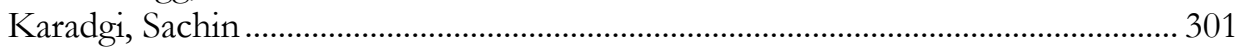

Kemper, Hans-Georg .............................................................................213, 215

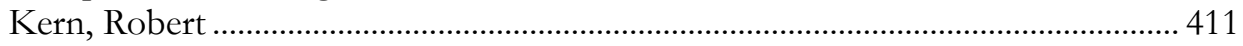

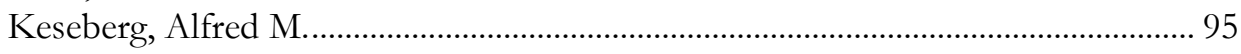

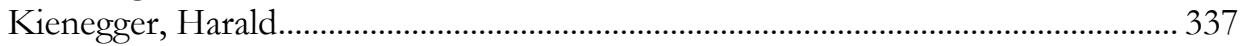

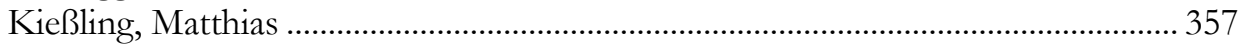

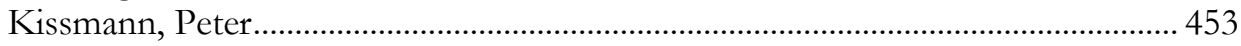

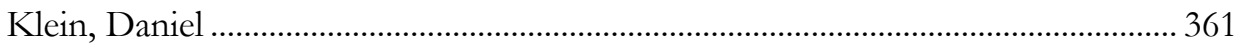

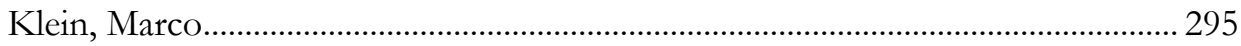

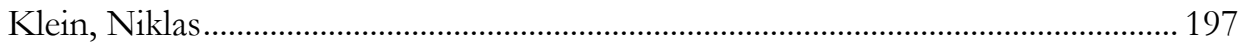

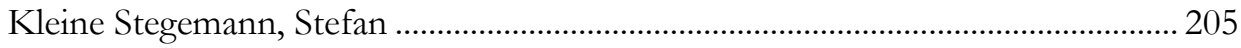

Knackstedt, Ralf ………................................................................. 291, 407, 415

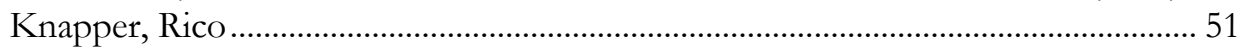

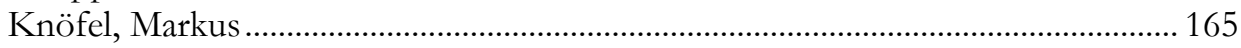

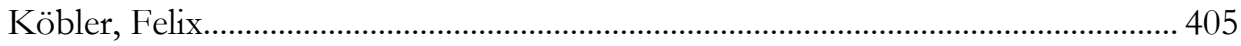

Koch, Michael.........................................................................................135, 141

Kolbe, Lutz M.......................................................................61, 67, 69, 357

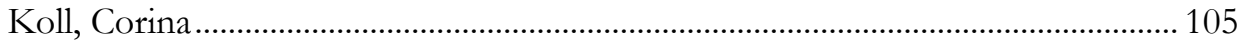

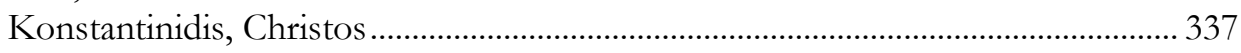

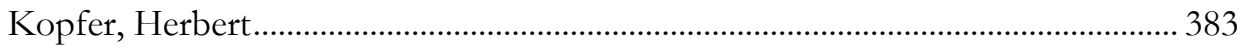

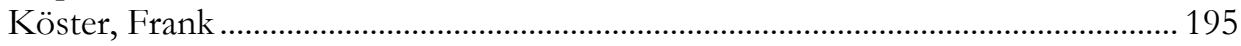

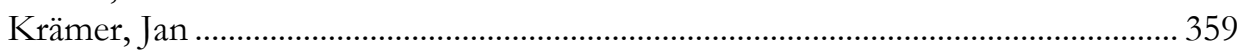

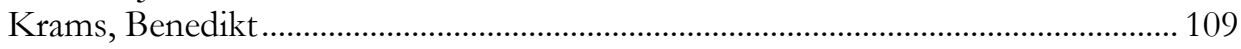

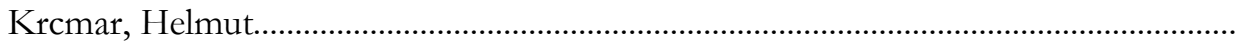

49,107 177, 179, 185, 189, 193, 263, 305, 321, 337, 401, 405, 413, 423

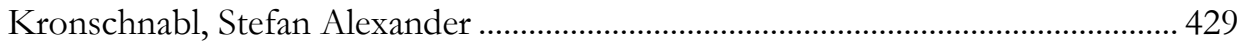

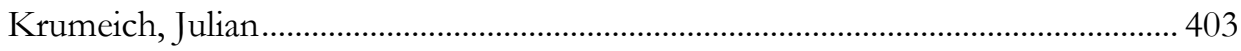

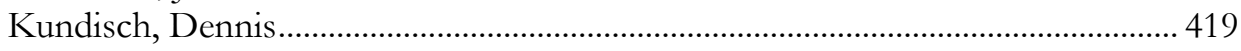

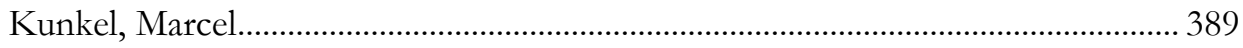

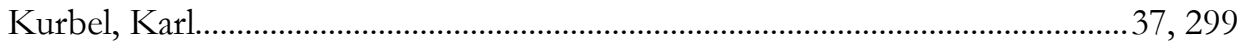

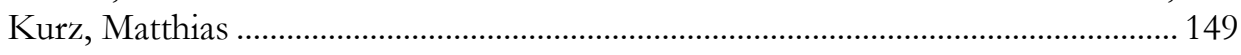

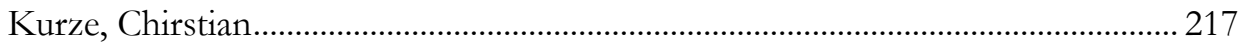

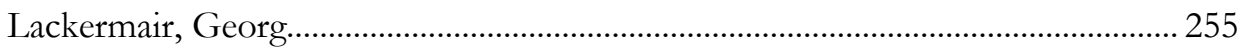

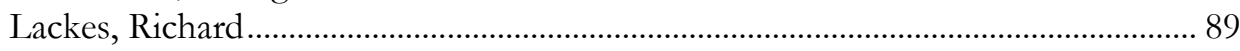

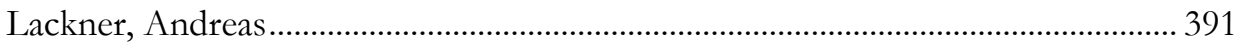

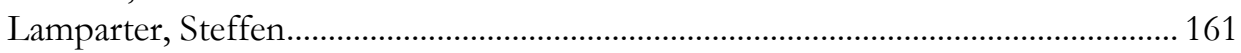




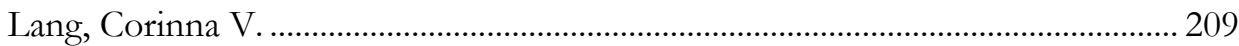

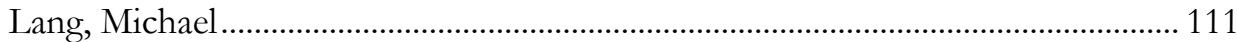

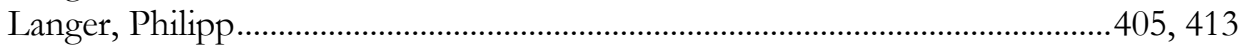

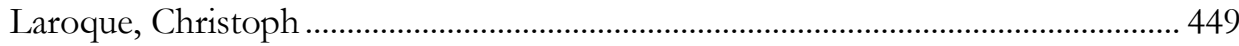

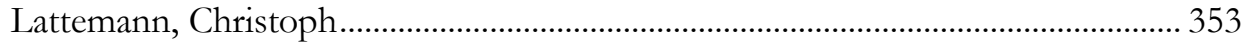

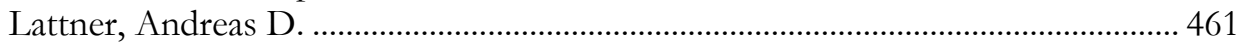

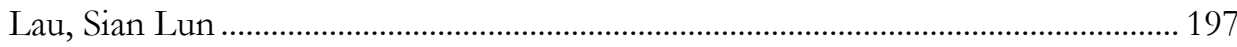

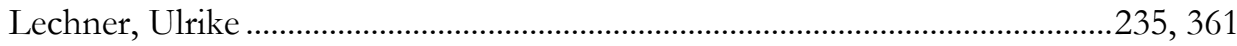

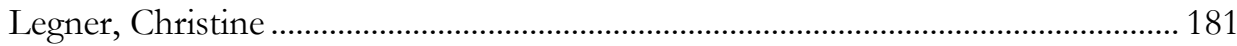

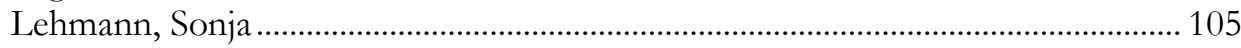

Lehr, Christian ...................................................................................... 83, 287

Leimeister, Jan Marco ……….......................... 83, 107, 177, 179, 185, 397, 401, 405

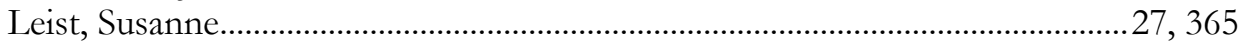

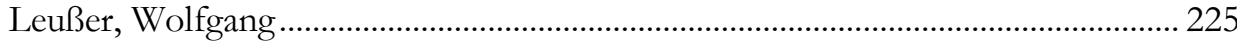

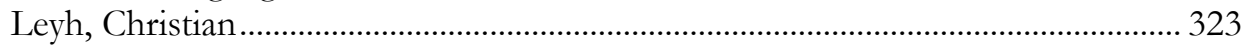

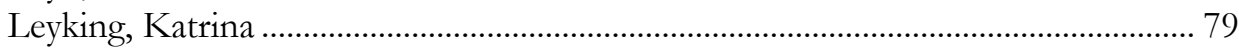

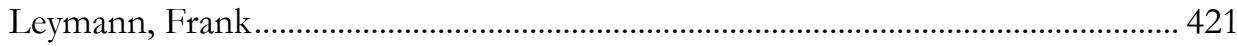

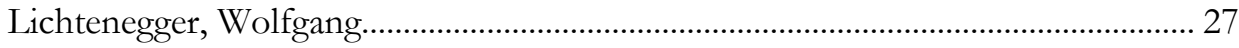

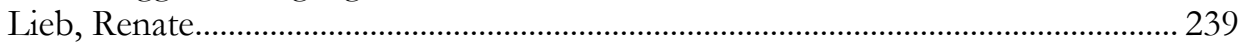

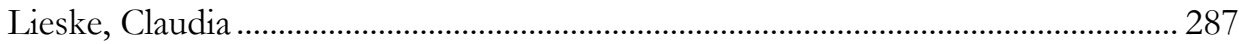

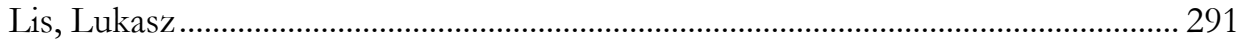

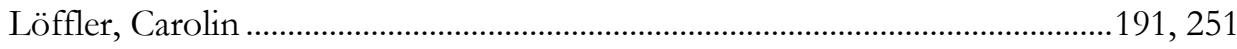

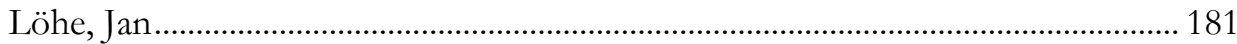

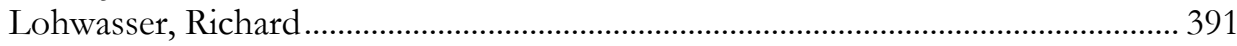

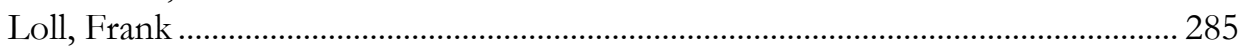

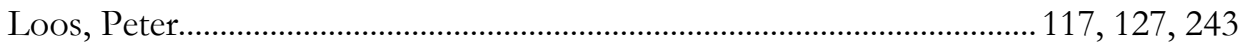

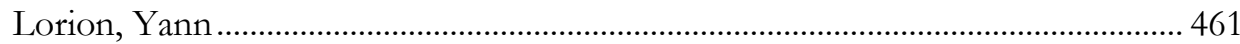

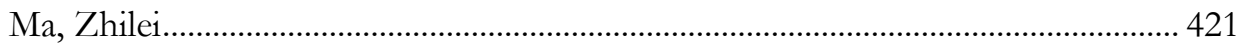

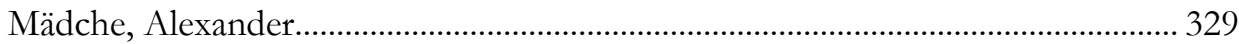

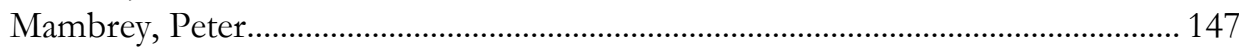

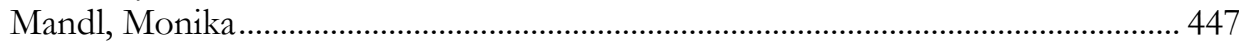

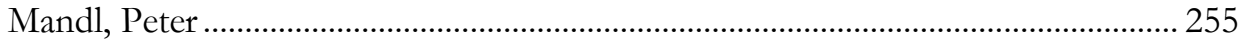

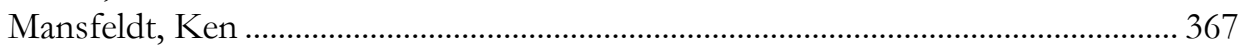

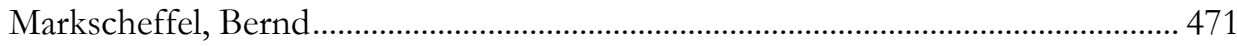

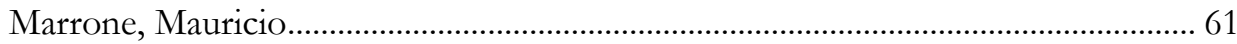

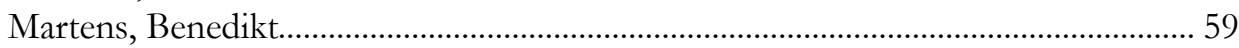

Mattfeld, Dirk Christian ............................................................... 381, 385, 393

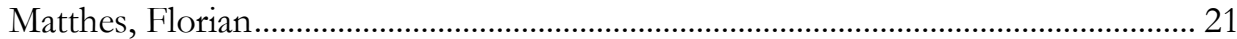

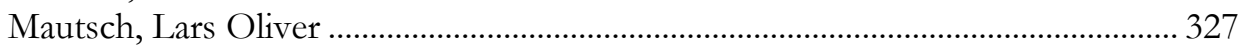

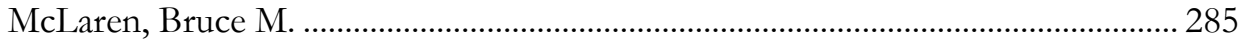

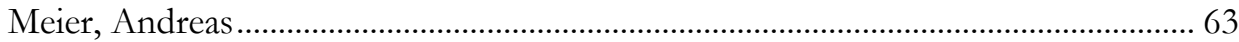

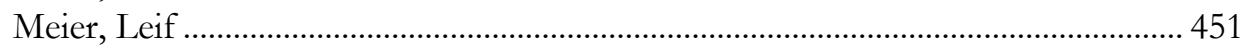




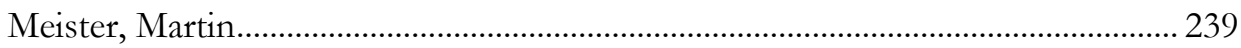

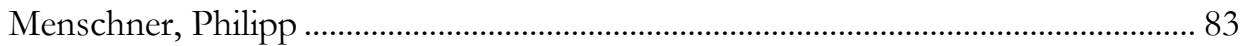

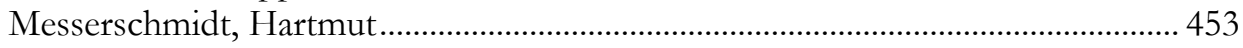

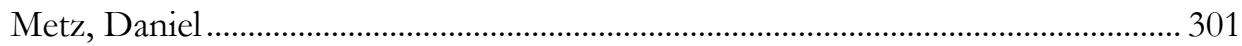

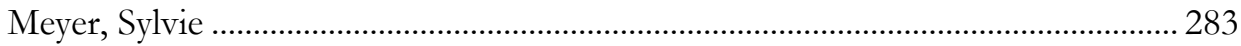

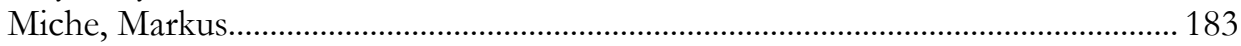

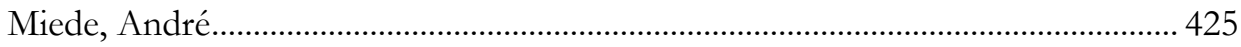

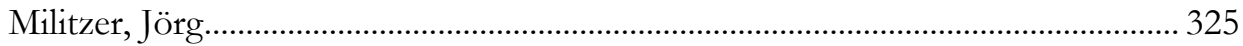

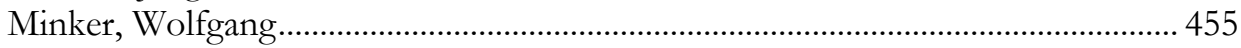

Mochol, Malgorzata ………………………………………………………………..... 81

Möslein, Kathrin M...................................................................................245, 399

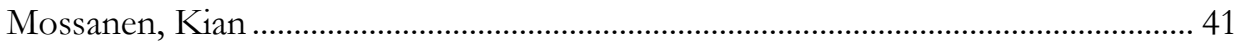

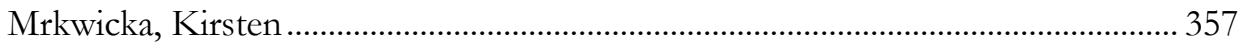

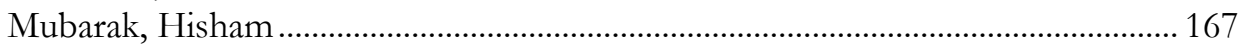

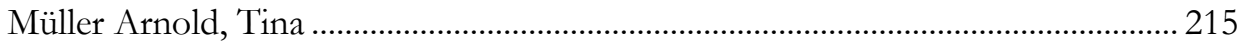

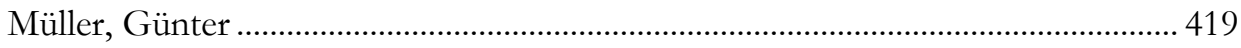

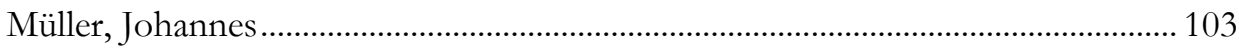

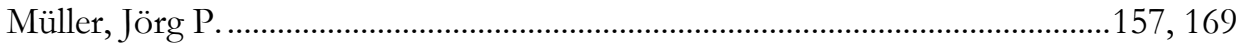

Müller, Jürgen...................................................................................................... 391

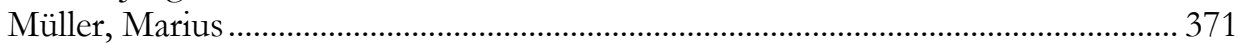

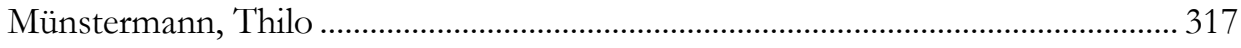

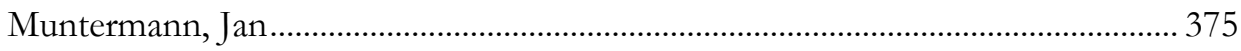

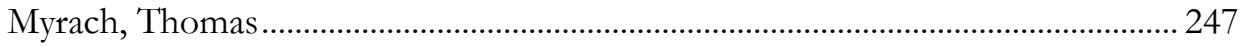

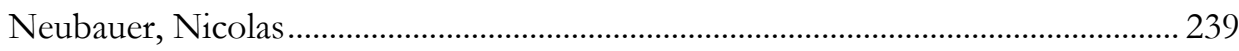

Neyer, Anne-Katrin …………………………………………………......245, 399

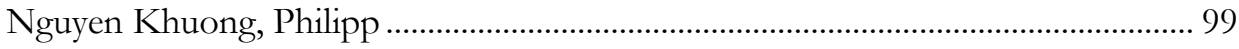

Nicolescu, Valentin ………………………………………………………………. 179

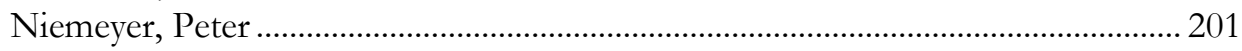

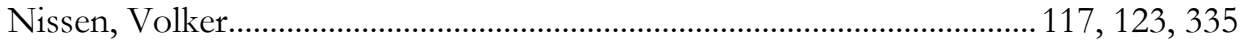

Nothdurft, Florian ...................................................................................................... 455

Nowakowski, Philipp ……………………………………………………….....

Nüttgens, Markus …………………………………………………………277, 407

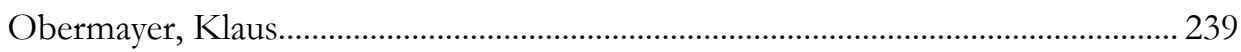

Ochab, Bartek …………………………………………………………………. 239

Opuszko, Marek ……………………………………….......................................... 145

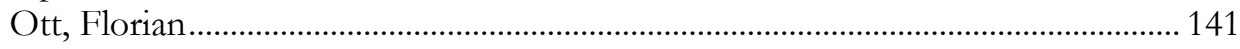

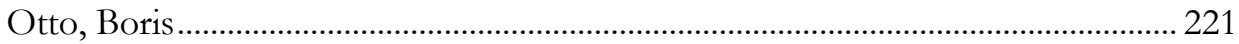

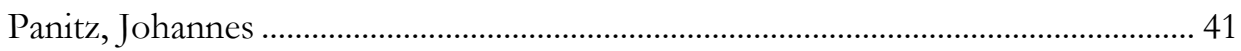

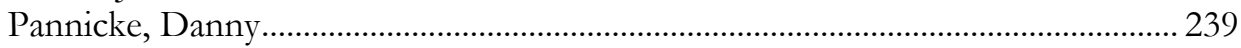

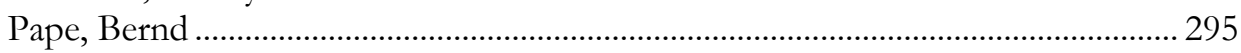




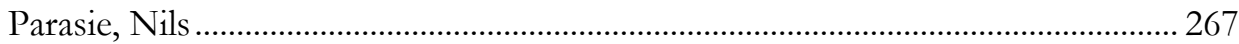

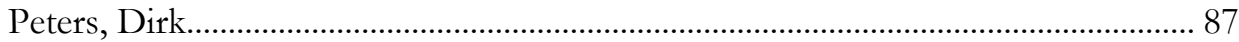

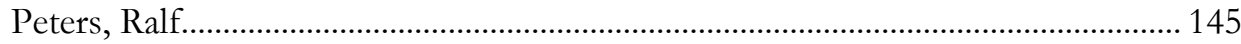

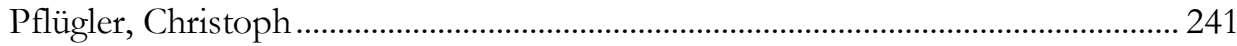

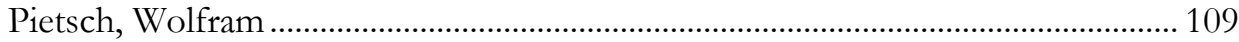

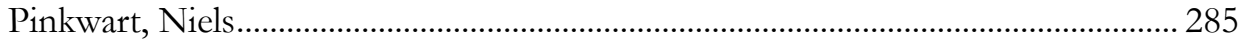

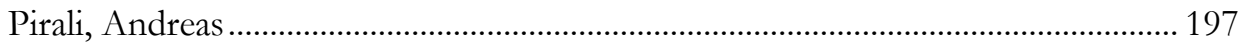

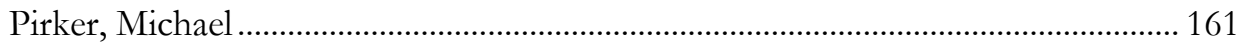

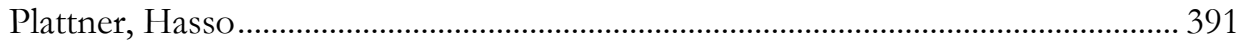

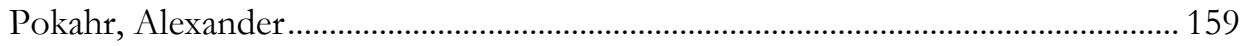

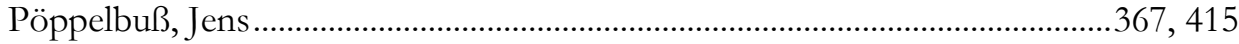

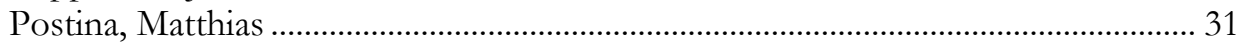

Pühler, Maximilian ..................................................................................189, 193

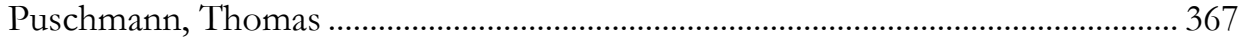

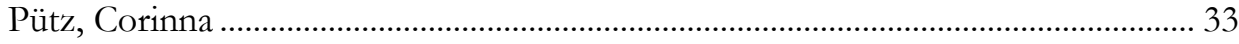

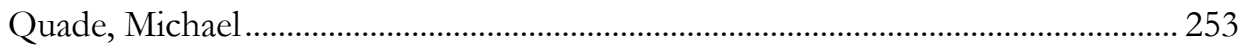

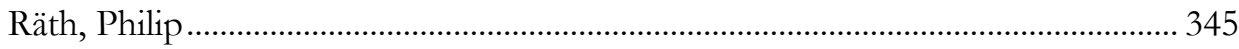

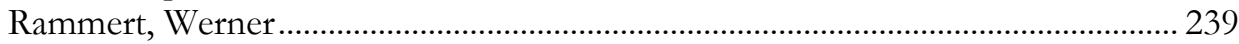

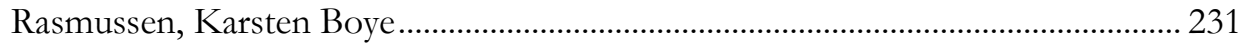

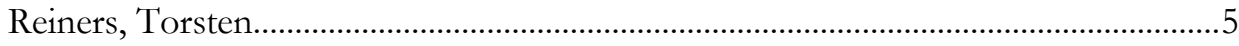

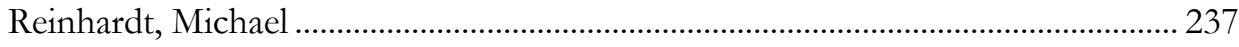

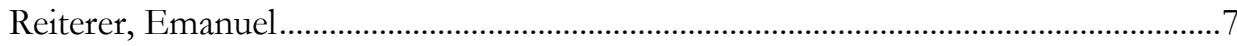

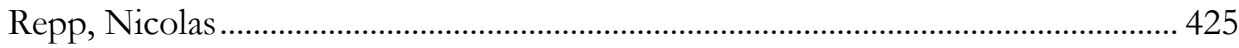

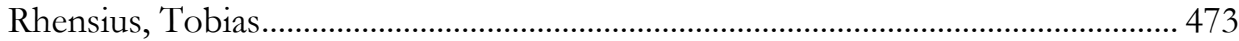

Richter, Alexander.................................................................................141, 151

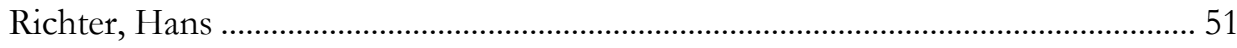

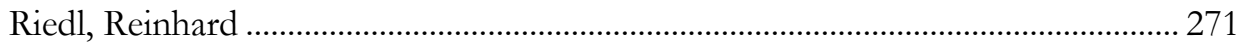

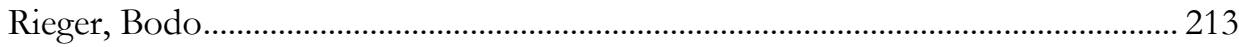

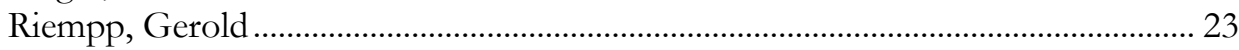

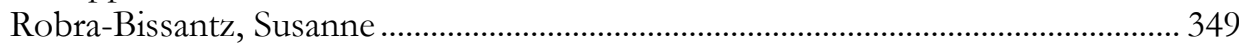

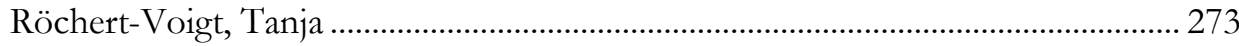

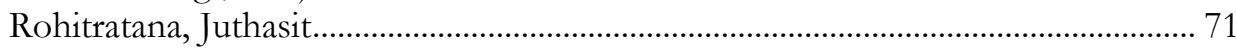

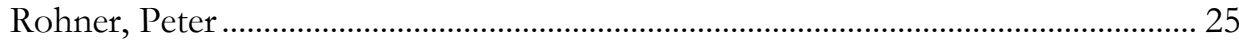

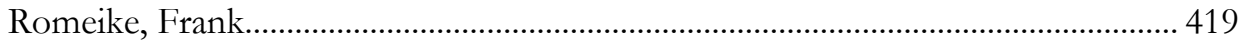

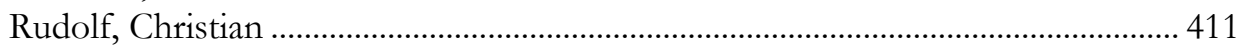

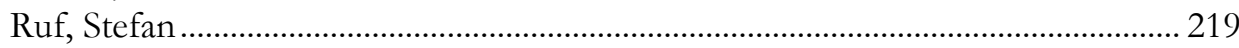

Ruhland, Johannes ......................................................................................145, 229

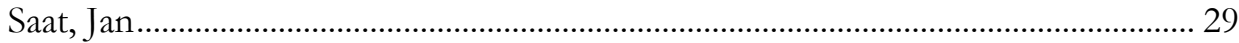

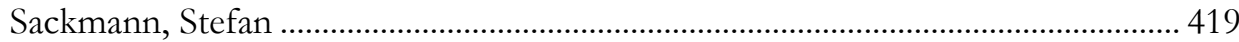

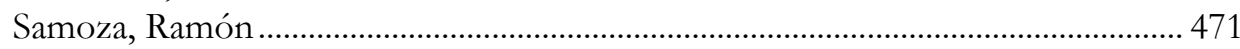




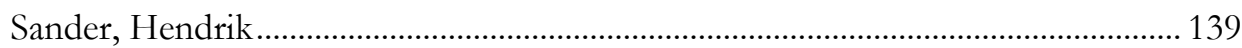

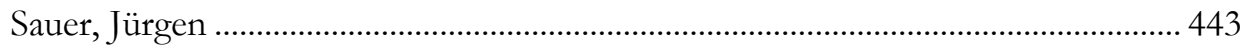

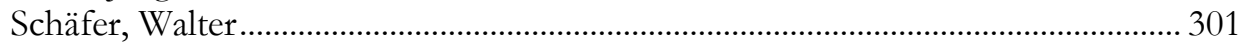

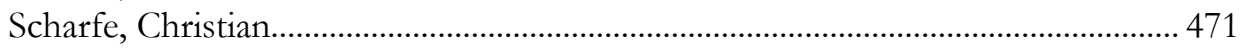

Schattenberg, Bernd .....................................................................................443, 455

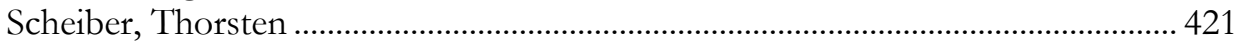

Schenk, Birgit .................................................................................................137, 269

Schermann, Michael ...........................................................................189, 193, 423

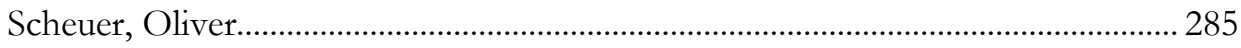

Schiefer, Josef........................................................................................................ 227

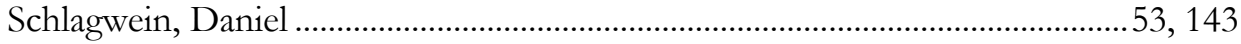

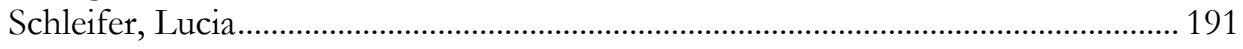

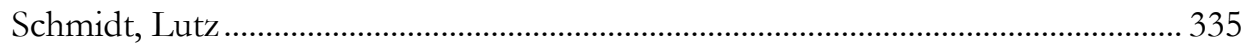

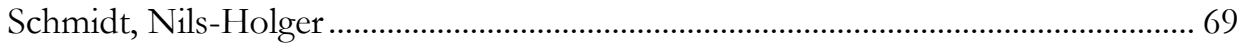

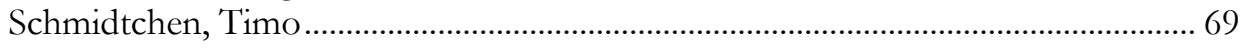

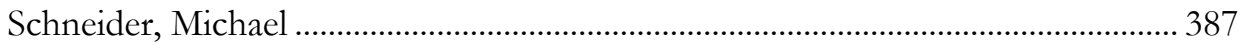

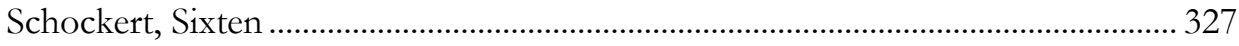

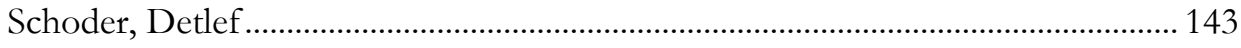

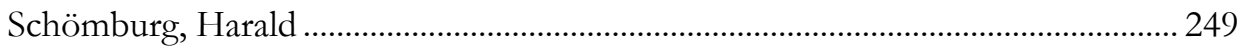

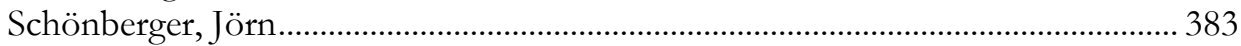

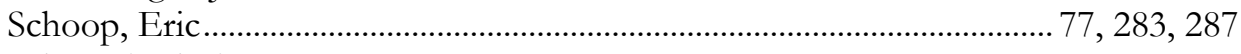

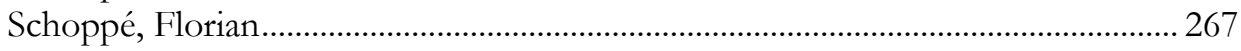

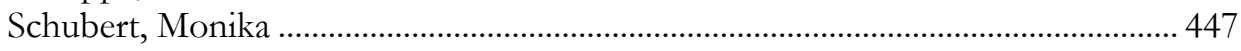

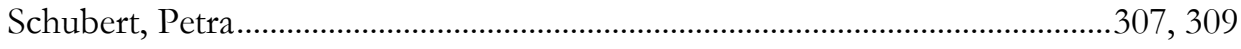

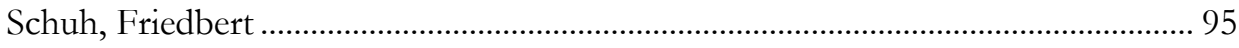

Schuh, Günther.................................................................................................409, 473

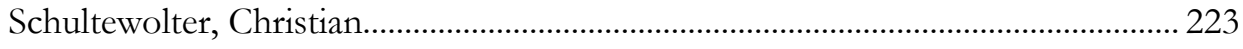

Schumacher, Peter.......................................................................................................... 37

Schumann, Matthias......................................................................................225, 451

Schumm, David ............................................................................................... 421

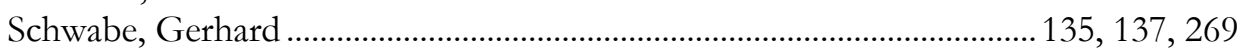

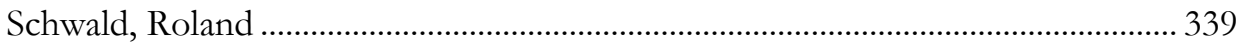

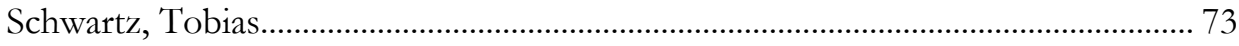

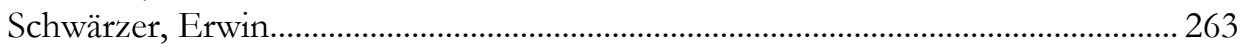

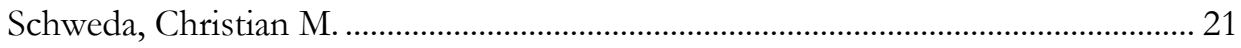

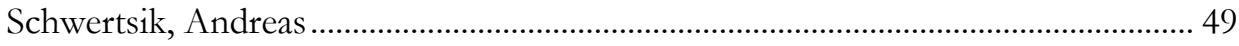

Schwind, Michael...…………………………………………………….......387, 389

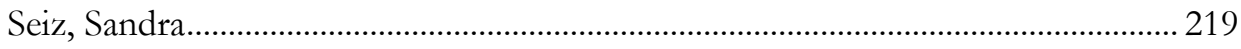

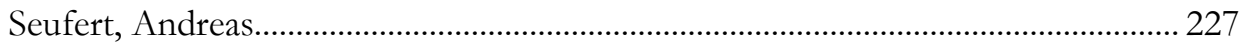

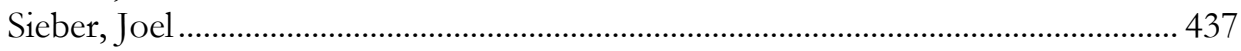

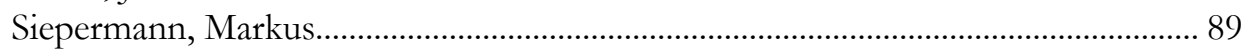

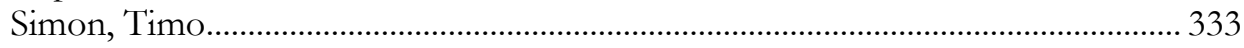

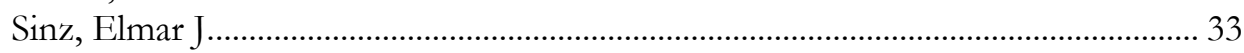




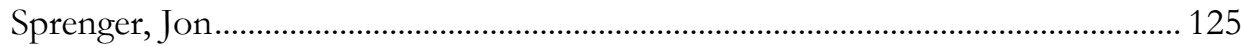

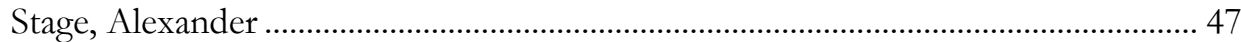

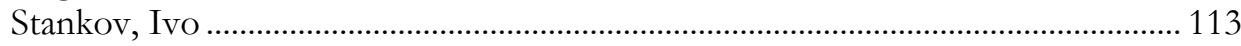

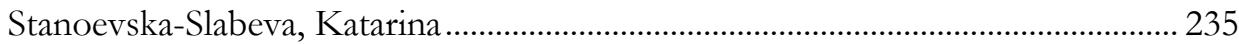

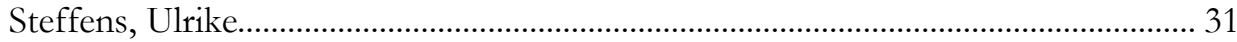

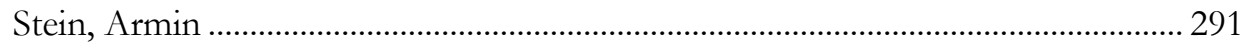

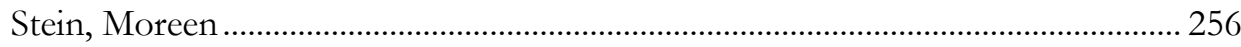

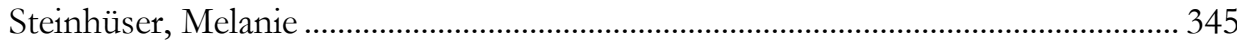

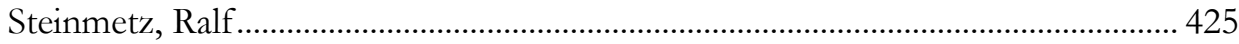

Stelzer, Dirk ........................................................................................... 19, 471

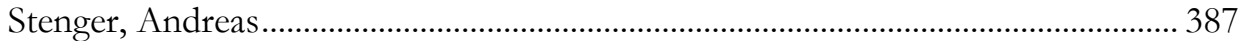

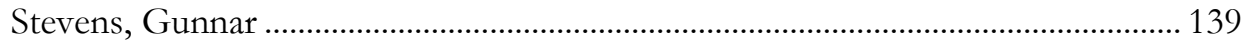

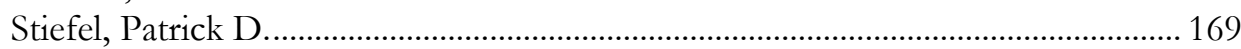

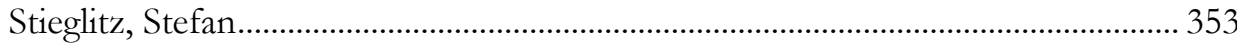

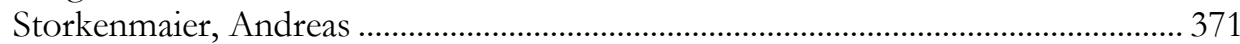

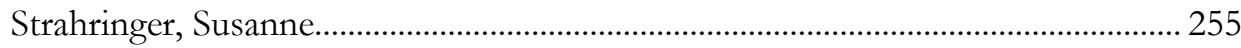

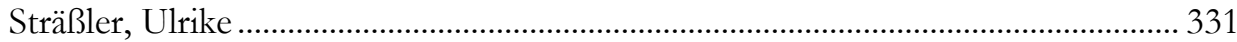

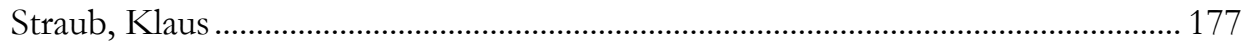

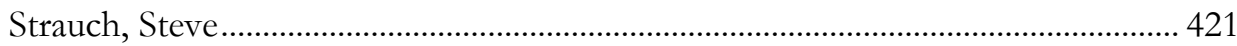

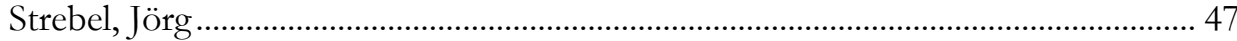

Stuckenschmidt, Heiner ...........................................................................................

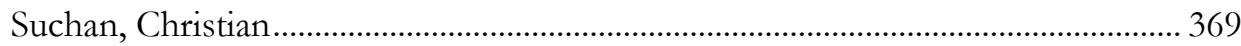

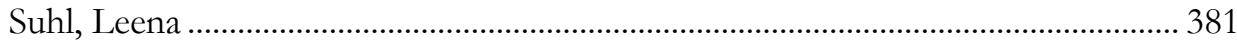

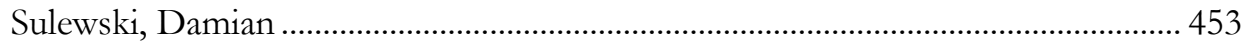

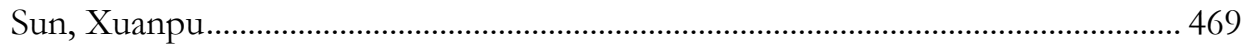

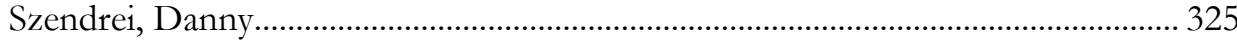

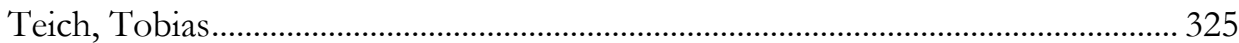

Teuteberg, Frank ............................................................... 59, 201, 203, 207, 347

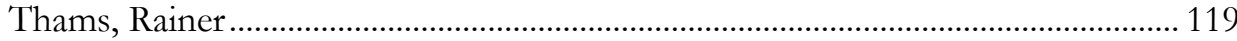

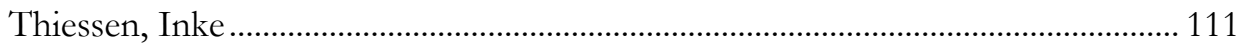

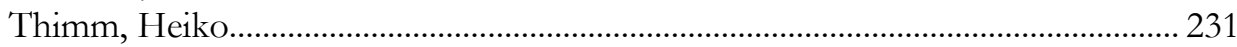

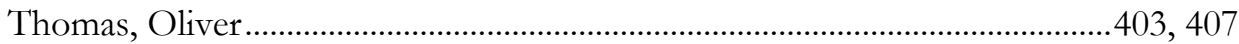

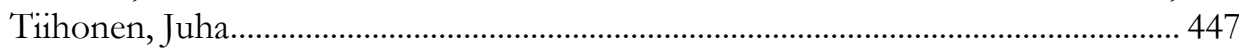

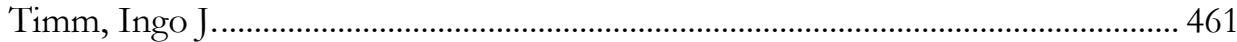

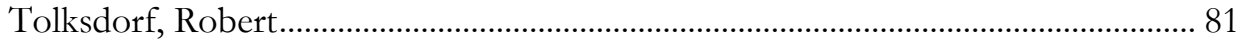

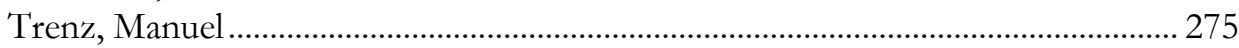

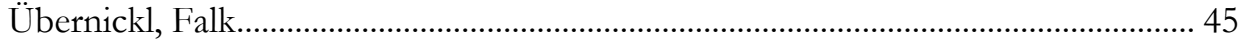

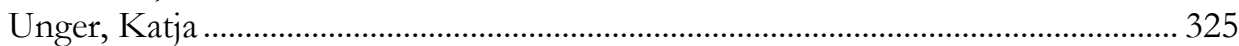

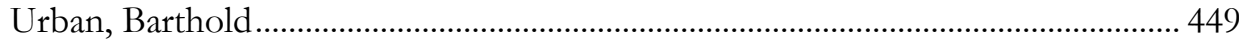

Van Dinther, Clemens .................................................................................................. 435 


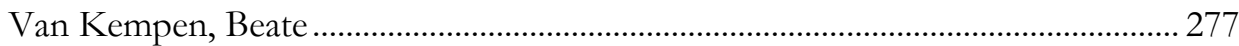

Veit, Daniel..............................................................................................267, 275

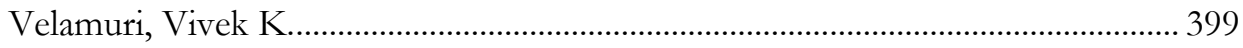

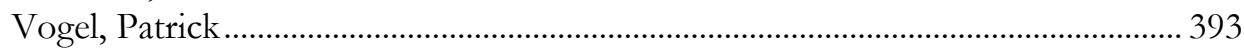

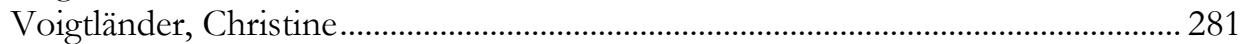

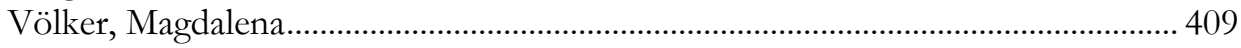

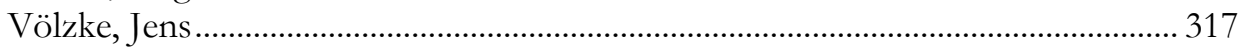

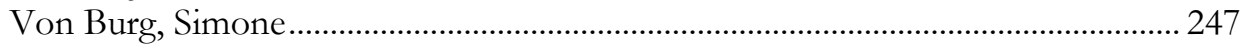

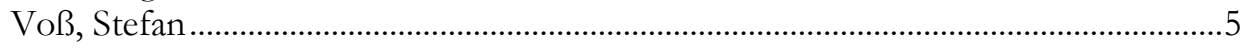

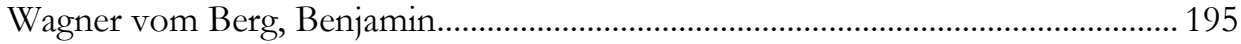

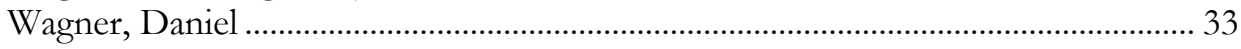

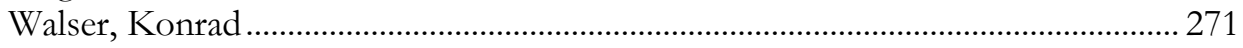

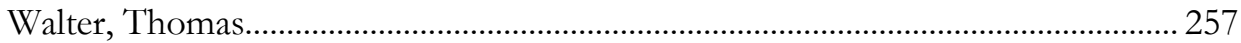

Weber, Peter................................................................................................... 77,287

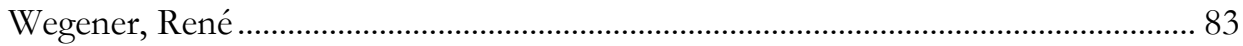

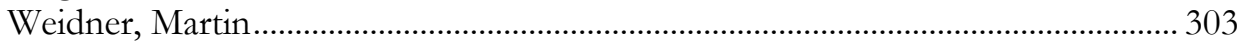

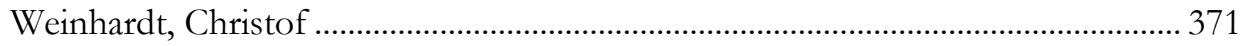

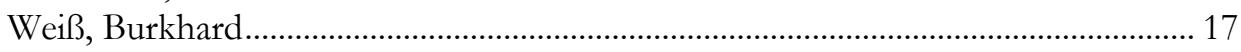

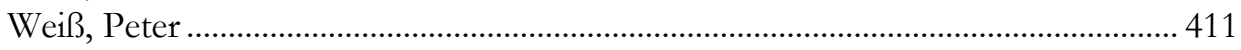

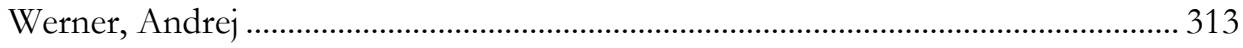

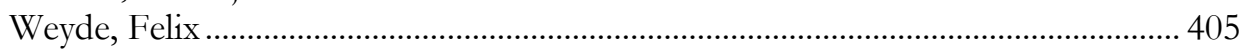

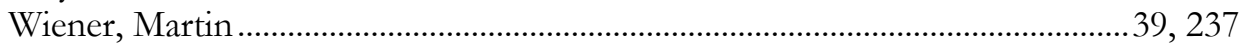

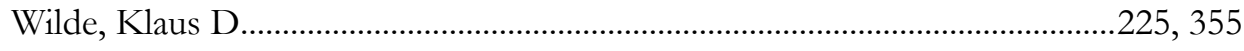

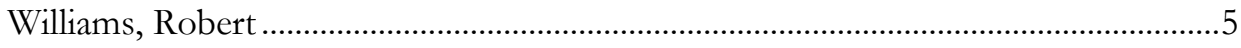

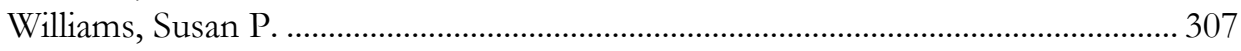

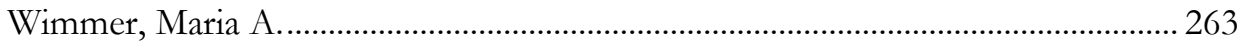

Winkelmann, Axel ............................................................................ 17, 259, 323

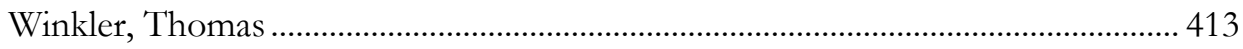

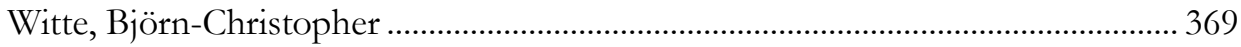

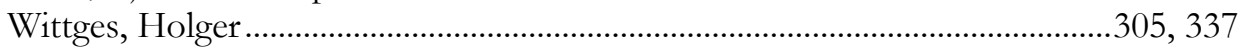

Wittstruck, David ...............................................................................203, 207

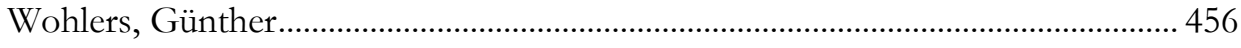

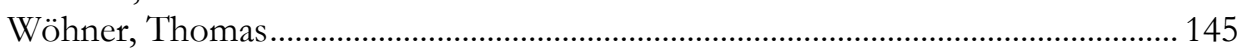

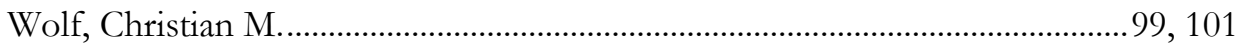

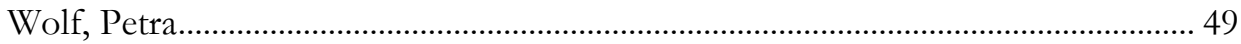

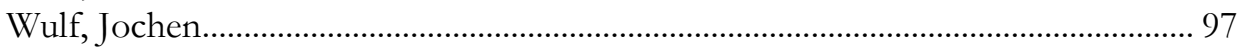

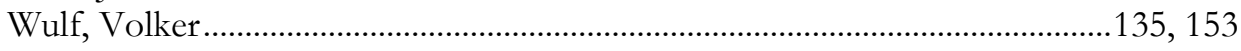

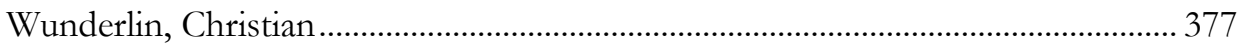

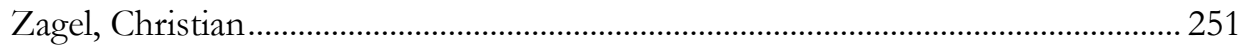

Zarnekow, Rüdiger....................................................................... 67, 97, 239

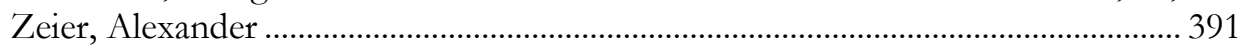


Zellner, Gregor ............................................................................................................. 365

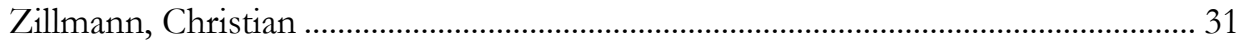

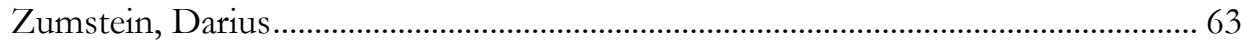


$\mathrm{M}$ oderne Informations- und Kommunikationssysteme leisten einen wesentlichen Beitrag zum Erfolg vieler Unternehmen in einer immer stärker vernetzten Wirtschaft. Darüber hinaus besitzen sie auch bei der Vernetzung von Betrieben mit öffentlichen Verwaltungen sowie effizienten Verwaltungsprozessen große Bedeutung und sind zunehmend auch im privaten Bereich relevant. Mit der alle zwei Jahre stattfindenden Multikonferenz Wirtschaftsinformatik gibt es im regelmäßigen Rhythmus einen Treffpunkt, bei dem die neuesten wissenschaftlichen Entwicklungen der Wirtschaftsinformatik im breiten Rahmen präsentiert werden und auch ein Austausch mit der Praxis erfolgt. Die Tagung im Jahr 2010 spannt einen Bogen von aktuellen Herausforderungen des Informationsmanagements über die Weiterentwicklung ausgewählter Techniken und Methoden der Wirtschaftsinformatik zu dem breiten Feld der Anwendungen. Ziel der Veranstaltung ist es, mit Vorträgen und Diskussionen sowohl innerhalb dieser Themenfelder als auch themenübergreifend den Austausch der wissenschaftlichen Fachgruppen zu fördern und die Diskussion mit der Praxis anzuregen.

\section{Sponsoren}
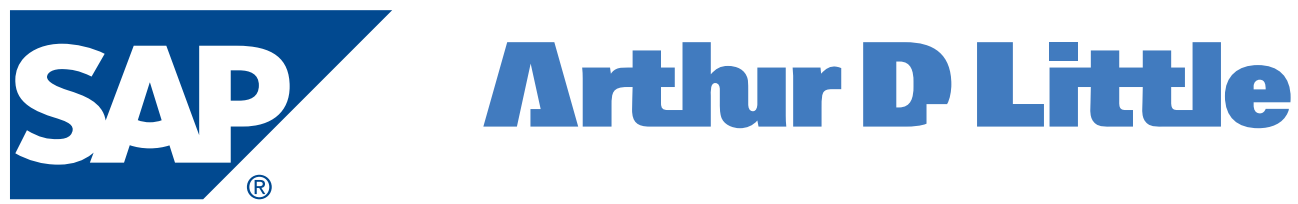

Deutsche Bank

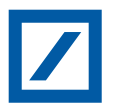

\section{PRICENATERHOUSECOOPERS T: \\ SENACOR}

\section{Medienpartner}

\section{WIRTSCHAFTS INFORMATIK}

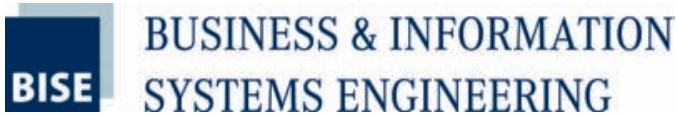

Wirtschaftsinformatik \& Management

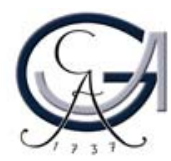

GEORG-AUGUST-UNIVERSITÄT GÖTTINGEN 
$\mathrm{M}$ oderne Informations- und Kommunikationssysteme leisten einen wesentlichen Beitrag zum Erfolg vieler Unternehmen in einer immer stärker vernetzten Wirtschaft. Darüber hinaus besitzen sie auch bei der Vernetzung von Betrieben mit öffentlichen Verwaltungen sowie effizienten Verwaltungsprozessen große Bedeutung und sind zunehmend auch im privaten Bereich relevant. Mit der alle zwei Jahre stattfindenden Multikonferenz Wirtschaftsinformatik gibt es im regelmäßigen Rhythmus einen Treffpunkt, bei dem die neuesten wissenschaftlichen Entwicklungen der Wirtschaftsinformatik im breiten Rahmen präsentiert werden und auch ein Austausch mit der Praxis erfolgt. Die Tagung im Jahr 2010 spannt einen Bogen von aktuellen Herausforderungen des Informationsmanagements über die Weiterentwicklung ausgewählter Techniken und Methoden der Wirtschaftsinformatik zu dem breiten Feld der Anwendungen. Ziel der Veranstaltung ist es, mit Vorträgen und Diskussionen sowohl innerhalb dieser Themenfelder als auch themenübergreifend den Austausch der wissenschaftlichen Fachgruppen zu fördern und die Diskussion mit der Praxis anzuregen.

\section{Sponsoren}
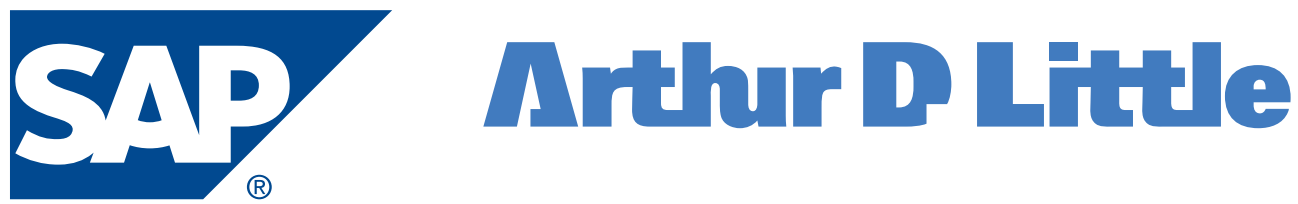

Deutsche Bank

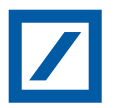

\section{PRICENATERHOUSECOOPERS T: \\ SENACOR}

\section{Medienpartner}

\section{WIRTSCHAFTS INFORMATIK}

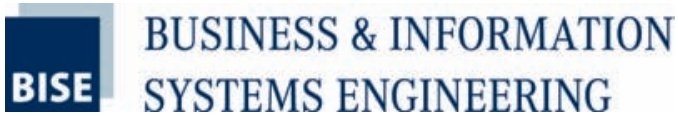

Wirtschaftsinformatik \& Management

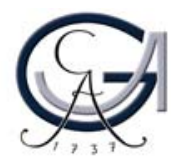

GEORG-AUGUST-UNIVERSITÄT GÖTTINGEN 